\title{
Samarium doped alkaline earth halides as red-emitting scintillators and phosphors
}

by

Laura Catherine Dixie

A thesis

submitted to the Victoria University of Wellington

in fulfilment of the requirements for a Doctorate of Philosophy in Physics

Victoria University of Wellington

2013 



\section{Abstract}

This thesis is concerned with the manufacture, spectroscopic characterisation, and radiation detection performance of three rare earth doped alkaline earth halides; these were designed for scintillation or phosphor detection of $x$-rays and $\gamma$-rays. The materials are transparent polycrystals of lanthanum or praseodymium stabilised cubic barium chloride $\left((\mathrm{La}, \mathrm{Pr})_{0.125} \mathrm{Ba}_{0.875} \mathrm{Cl}_{2.125}\right), \mathrm{BaCl}_{2}-\mathrm{SrCl}_{2}$ solid solutions, or single crystals of $\mathrm{CaF}_{2}$. The primary dopant investigated was $\mathrm{Sm}^{2+}$ since this has a red emission in all the materials which is well matched to the spectral sensitivity of silicon photodiodes. The cubic structure of the polycrystalline materials is essential for optical transparency, and so the structural stability of the materials has been investigated using $x$ ray diffraction and thermal analysis. For $\mathrm{CaF}_{2}$ large single crystals were unintentionally produced without following the usual BridgmanStockbarger or Czochralski methods. All of the materials showed predominantly $\mathrm{Sm}^{2+}$ ions, and only in $\mathrm{CaF}_{2}$ could evidence of $\mathrm{Sm}^{3+}$ ions also be seen.

The spectroscopy of the $4 \mathrm{f}^{5} 5 \mathrm{~d}^{1} \rightarrow 4 \mathrm{f}^{6}$ red emission, including lifetimes, and absorption of $\mathrm{Sm}^{2+}$ ions in all these materials is reported; a strong thermal cross over to $4 \mathrm{f}^{6} \rightarrow 4 \mathrm{f}^{6}$ emission is observed and successfully modelled. A time correlated single photon counted system has been built to measure the scintillation decay time of these materials. The system yields decay times in excellent agreement with the literature values. The performance of the materials as scintillators is limited to varying degrees by the formation of colour centres which slow the electron-hole recombination process after $x$-irradiation. $\mathrm{Ba}_{0.3} \mathrm{Sr}_{0.7} \mathrm{Cl}_{2}: \mathrm{Sm}$ was found to be a bright and fast $\mathrm{x}$-ray phosphor. The integrated intensity (per x-ray half thickness of material) of the radioluminescence is $\sim 30 \%$ that of the commercial material, the scintillation lifetime is $\sim 30 \mu$ s (c.f. milliseconds for $\mathrm{Gd}_{2} \mathrm{O}_{2} \mathrm{~S}: \mathrm{Tb}^{3+}$ ) and the imaging resolution is $6 \mathrm{LP} / \mathrm{mm}$ (c.f. 4.2 LP/mm for $\mathrm{Gd}_{2} \mathrm{O}_{2} \mathrm{~S}: \mathrm{Tb}^{3+}$ ). $\mathrm{CaF}_{2}: \mathrm{Sm}^{2+}$ was shown to be a red-emitting scintillator with a decay time of $\leq 1 \mu$ s and a light output of 15,000 photons $/ \mathrm{MeV}$ when cooled by dry ice. The $\mathrm{x}$-ray imaging resolution was high at $8.5 \mathrm{LP} / \mathrm{mm}$. Several of the materials have been tested for performance as neutron detecting phosphors by adding neutron capture elements such as gadolinium or lithium, the strongest emission observed was $6 \%$ the integrated intensity of the standard material ${ }^{6} \mathrm{LiI}\left(\mathrm{Eu}^{2+}\right)$. 



\section{Acknowledgements}

I would like to take the opportunity to thank everyone who made the completion of this thesis possible. First and foremost I am grateful to my primary supervisor A/Prof. Andy Edgar for not only his support, guidance, enthusiasm for this research but also for many coffees, BBQs and the odd kayaking trip. I also would like to thank my co-supervisor Dr. Murray Bartle for his advice, broad knowledge and willingness to help. I would like to thank Dr. Nicola Winch who has provided friendship, entertainment and made this time highly enjoyable. I would like to thank the mechanical workshop for creating various pieces of equipment (and repairs) along with afternoon tea. I also would like to thank Chris Varoy who provided valuable instruction in the laboratory.

I would also like to thank and acknowledge A/Prof. Mike Reid (University of Canterbury) who performed crystal field calculations described in this work and Dr. John Campbell (University of Canterbury) who kindly lent his collection of samarium doped alkaline earth fluoride crystals for preliminary investigations.

I would also like to acknowledge the funding I received from the Ministry of Science and Innovation (now Ministry of Business, Innovation and Employment) in the form of a PhD stipend. I am grateful for a travel grant from the Faculty of Science (VUW). I would also like to acknowledge the Australian Institute of Nuclear Science and Engineering for facilitating and funding a trip to the ANSTO nuclear reactor.

Finally, I would like to thank my family and friends, especially Manu, who have provided support and encouragement. 



\section{Contents}

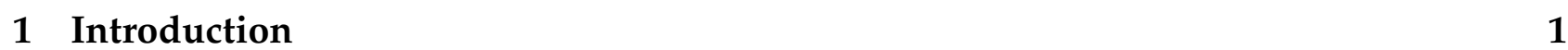

1.1 Scintillators and phosphors for $x$-ray and $\gamma$-ray detection . . . . . . . . . . 1

1.2 Preview of this thesis . . . . . . . . . . . . . . . . . 4

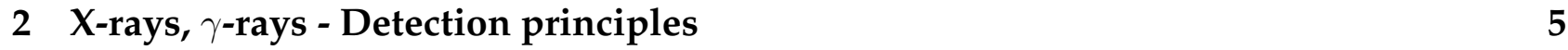

2.1 Origins and discovery $\ldots \ldots \ldots \ldots \ldots \ldots$

2.2 Applications $\ldots \ldots \ldots \ldots \ldots \ldots \ldots \ldots$

2.3 Methods of detection $\ldots \ldots \ldots \ldots \ldots$

2.3 .1 Ionisation chamber detectors $\ldots \ldots \ldots \ldots$. . . . . . . . 10

2.3 .2 Semiconductor detectors . . . . . . . . . . . . . . . . . . . . 11

2.3 .3 Scintillators . . . . . . . . . . . . . . . . . . . . . 12

2.3 .4 Comparison of $x$-ray and $\gamma$-ray detectors $\ldots \ldots \ldots$. . . . . . . . 13

2.4 Photodetectors . . . . . . . . . . . . . . . . . . . . . . . 16

2.4 .1 Photomultiplier tubes . . . . . . . . . . . . . . 17

2.4 .2 Silicon based detectors . . . . . . . . . . . . . . . . 18

2.4 .3 Comparison of photodetectors . . . . . . . . . . . . . . 19

3 Scintillators \& phosphors - Principles \& performance 21

3.1 Introduction . . . . . . . . . . . . . . . . . . 21

3.2 Scintillation mechanism . . . . . . . . . . . . . . . . . 22

3.2.1 Absorption of $x$-rays or $\gamma$-rays . . . . . . . . . . . . 23

3.2 .2 Thermalisation . . . . . . . . . . . . . . . . 25

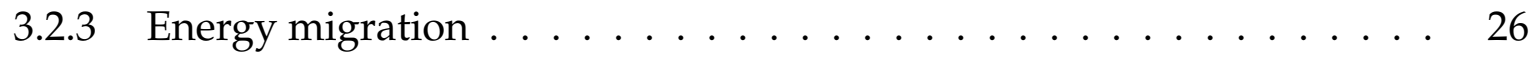

3.2 .4 Luminescence . . . . . . . . . . . . . . . . . . . 27 
3.3 Performance measures $\ldots \ldots \ldots \ldots \ldots \ldots \ldots \ldots \ldots$

$3.3 .1 \quad$ Efficiency and light yield $\ldots \ldots \ldots \ldots \ldots \ldots$

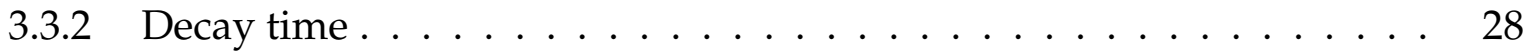

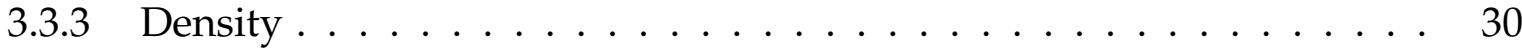

3.3 .4 Emission wavelength $\ldots \ldots \ldots \ldots \ldots$

$3.3 .5 \quad$ Mechanical and chemical properties $\ldots \ldots \ldots \ldots \ldots$

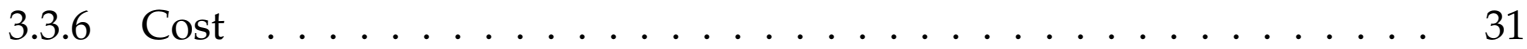

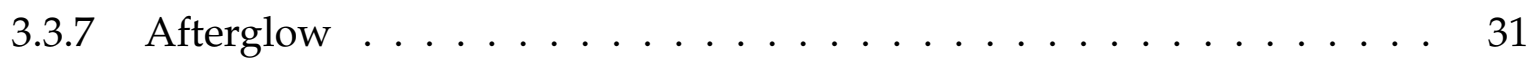

3.3 .8 Temperature stability $\ldots \ldots \ldots \ldots \ldots \ldots \ldots$

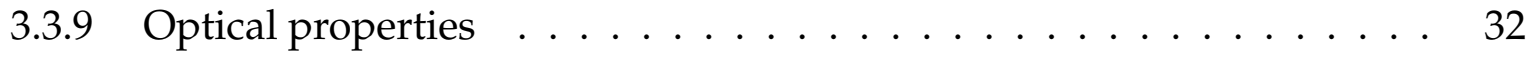

3.3 .10 Radiation hardness . . . . . . . . . . . . . . . . . . . . . 32

3.3 .11 Proportionality of response $\ldots \ldots \ldots \ldots$. . . . . . . . . . 32

3.4 Commercial Scintillators $\ldots \ldots \ldots \ldots$. . . . . . . . . . . . . . . . . . 32

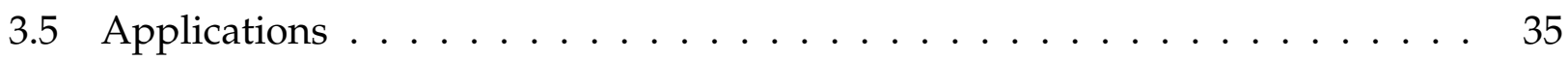

4 Scintillators and Phosphors: Spectroscopy and materials science 37

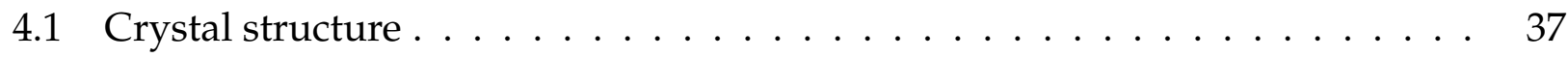

$4.1 .1 \quad$ X-ray diffraction $\ldots \ldots \ldots \ldots \ldots \ldots \ldots \ldots \ldots$

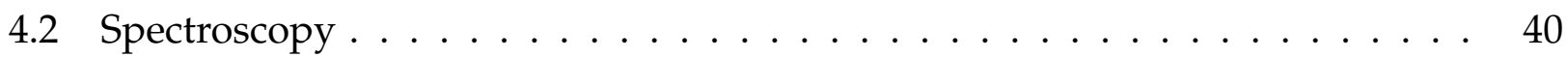

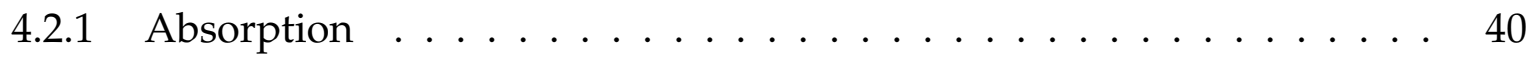

4.2 .2 Fluorescence. . . . . . . . . . . . . . . . . . . . . 42

4.3 Spectroscopy of rare earth ions in crystals $\ldots \ldots \ldots \ldots \ldots$. . . . . . 44

4.3 .1 Free ions . . . . . . . . . . . . . . . . . . . . 44

$4.3 .2 \quad$ Crystal field splitting $\ldots \ldots \ldots \ldots \ldots \ldots \ldots$

4.3 .3 Selection rules . . . . . . . . . . . . . . . . . . . . . 47

$4.3 .4 \quad$ Electron-phonon interaction $\ldots \ldots \ldots \ldots \ldots \ldots$

4.4 Characteristics of some rare earth ions $\ldots \ldots \ldots \ldots \ldots$

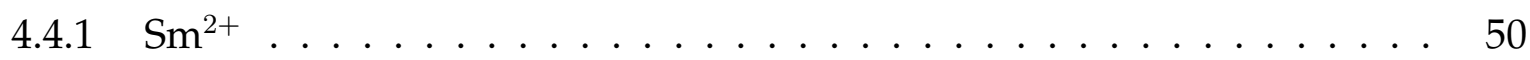

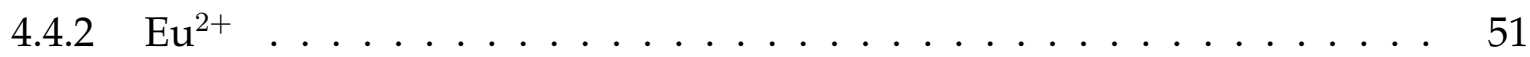

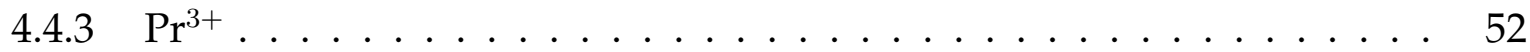

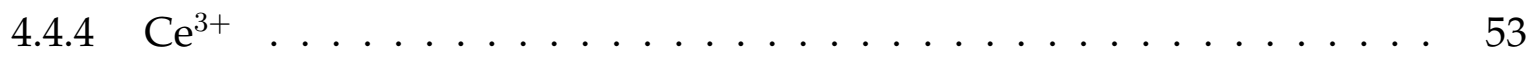


4.5 Thermally stimulated luminescence $\ldots \ldots \ldots$. . . . . . . . . . . 53

4.6 Electron spin resonance $\ldots \ldots \ldots \ldots \ldots$

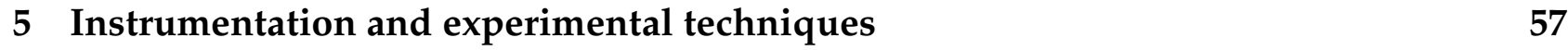

5.1 Sample preparation . . . . . . . . . . . . . . . . 57

5.2 X-ray diffraction . . . . . . . . . . . . . . . . . . . 59

5.3 Thermal analysis $\ldots \ldots \ldots \ldots \ldots \ldots \ldots$

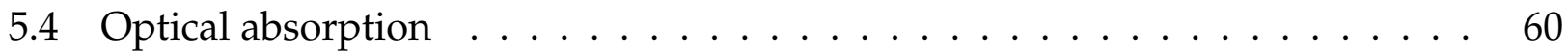

5.5 Photoluminescence $\ldots \ldots \ldots \ldots$. . . . . . . . . . . . . . 61

5.6 Photoluminescent lifetimes . . . . . . . . . . . . . . . . . . 61

$5.7 \quad$ Variable temperature measurements $\ldots \ldots \ldots$. . . . . . . . . . . 63

5.8 Thermally stimulated luminescence $\ldots \ldots \ldots$. . . . . . . . . . . . . 64

5.9 Electron spin resonance $\ldots \ldots \ldots \ldots$. . . . . . . . . . . . . . 64

5.10 Radioluminescence . . . . . . . . . . . . . . . . . . . . 65

5.11 X-ray imaging $\ldots \ldots \ldots \ldots 6 \ldots \ldots \ldots \ldots \ldots \ldots \ldots \ldots$

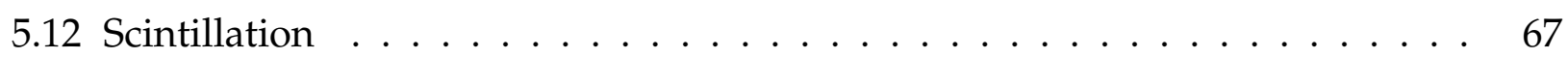

5.13 Photomultiplier tube spectral response correction $\ldots \ldots$. . . . . . . . 67

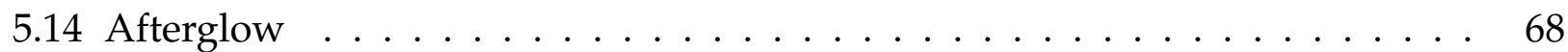

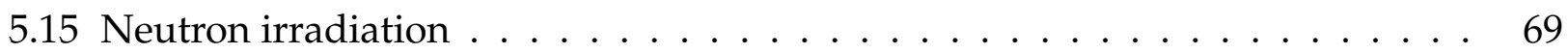

\begin{tabular}{|lll|}
\hline 6 & Time correlated single photon counting for scintillation lifetimes & 71
\end{tabular}

6.1 Introduction . . . . . . . . . . . . . . . . . 71

6.2 The advantages of time correlated single photon counting scintillation timing 74

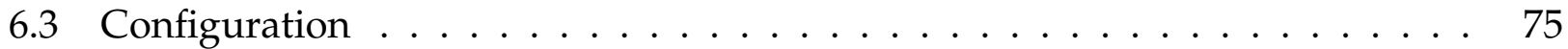

6.3 .1 Optics and hardware . . . . . . . . . . . . . . 75

6.3 .2 Photomultipliers and constant fraction discriminators . . . . . . . . 78

6.3 .3 Count rate . . . . . . . . . . . . . . . . . . . . 81

6.3 .4 Impulse response $\ldots \ldots \ldots \ldots$. . . . . . . . . . . . 82

6.3 .5 Calibration . . . . . . . . . . . . . . . . . 84

6.3 .6 Dynamic range $\ldots \ldots \ldots \ldots \ldots \ldots$

6.4 Alternative measurement technique $\ldots \ldots \ldots$. . . . . . . . . 86 
6.4 .1 The effect of signal averaging . . . . . . . . . . . . . 86

6.5 Comparison with a fast oscilloscope $\ldots \ldots \ldots$. . . . . . . . . . 88

$6.5 .1 \quad \mathrm{NaI}(\mathrm{Tl})$ scintillator $\ldots \ldots \ldots \ldots \ldots$. . . . . . . . . . . 88

6.5 .2 NE102 Scintillator . . . . . . . . . . . . . . . . 89

6.5 .3 Developmental scintillator $\ldots \ldots \ldots \ldots$. . . . . . . . . . 91

6.6 Possible improvements . . . . . . . . . . . . . . . . . . . . . . 92

$7 \quad$ Rare-earth $\left(\mathrm{La}^{3+}, \mathrm{Pr}^{3+}\right)$ stabilised cubic barium chloride 95

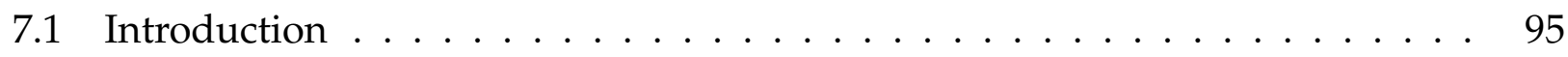

7.2 Lanthanum stabilised cubic barium chloride . . . . . . . . . . . . . . 96

$7.2 .1 \quad$ Physical properties $\ldots \ldots \ldots \ldots \ldots$. . . . . . . . . . . 96

7.2 .2 Optical spectroscopy $\ldots \ldots \ldots \ldots$. . . . . . . . . . . . . 99

7.2.3 Simulations of optical spectroscopy $\ldots \ldots \ldots$. . . . . . . . 111

$7.2 .4 \quad$ Discussion of optical spectroscopy $\ldots \ldots \ldots$. . . . . . . . . . . 119

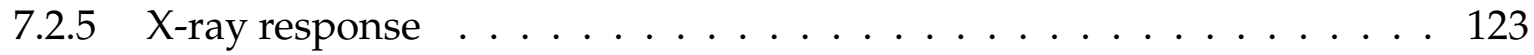

7.2 .6 Discussion of $x$-ray response $\ldots \ldots \ldots \ldots$. . . . . . . . . 128

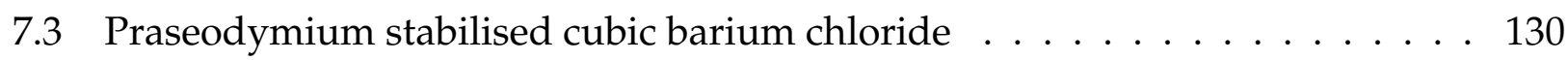

7.3 .1 Physical properties $\ldots \ldots \ldots \ldots \ldots$. . . . . . . . . . . 130

7.3 .2 Photoluminescence . . . . . . . . . . . . . . . . . . . . . . . 132

$7.3 .3 \quad$ X-ray response $\ldots \ldots \ldots \ldots \ldots \ldots \ldots$

7.4 Summary . . . . . . . . . . . . . . . . . . . . 136

8 Rare earth doped barium chloride - strontium chloride solid solutions $\quad 139$

$8.1 \quad$ Introduction $\ldots \ldots \ldots \ldots \ldots \ldots \ldots \ldots$

$8.2 \mathrm{BaCl}_{2}-\mathrm{SrCl}_{2}$ solid solutions . . . . . . . . . . . . . . . . 140

8.3 Samarium doped $\mathrm{BaCl}_{2}-\mathrm{SrCl}_{2}$ solid solutions . . . . . . . . . . . . . . 143

$8.3 .1 \quad$ Optical spectroscopy . . . . . . . . . . . . . . . . . . . 143

8.3 .2 X-ray response $\ldots \ldots \ldots \ldots \ldots \ldots \ldots \ldots$

8.4 Europium doped $\mathrm{BaCl}_{2}-\mathrm{SrCl}_{2}$ solid solutions $\ldots \ldots \ldots$. . . . . . . . 156

$8.4 .1 \quad$ Physical Properties . . . . . . . . . . . . . . . . . 158

8.4 .2 Photoluminescence . . . . . . . . . . . . . . . . . 158 
$8.4 .3 \quad$ X-ray response $\ldots \ldots \ldots \ldots \ldots \ldots \ldots \ldots \ldots$

8.5 Discussion of $x$-ray response $\ldots \ldots \ldots \ldots \ldots$

8.6 Summary . . . . . . . . . . . . . . . . . . . . . . . . 167

8.7 Phoswich application $\ldots \ldots \ldots \ldots$. . . . . . . . . . . . . 168

8.7 .1 Introduction . . . . . . . . . . . . . . . . . . . . . . 168

8.7.2 Energy discrimination with $\mathrm{Ba}_{0.3} \mathrm{Sr}_{0.7} \mathrm{Cl}_{2}: \mathrm{RE}\left(\mathrm{RE}=\mathrm{Sm}^{2+}\right.$ or $\left.\mathrm{Eu}^{2+}\right) \ldots 170$

$\begin{array}{lll}9 & \text { Samarium doped calcium fluoride } & 175\end{array}$

9.1 Introduction . . . . . . . . . . . . . . . . . . . . . . . . . . . 175

9.2 Physical properties . . . . . . . . . . . . . . . . . . . . . . . . . . . 179

9.3 Optical spectroscopy $\ldots \ldots \ldots \ldots$

9.3 .1 Absorption . . . . . . . . . . . . . . . . . . 183

9.3 .2 Photoluminescence excitation and emission spectra . . . . . . . . . 183

9.3 .3 Photoluminescent lifetimes . . . . . . . . . . . . . . . . . . 186

9.3 .4 Discussion of optical spectroscopy $\ldots \ldots \ldots \ldots \ldots \ldots$

9.4 X-ray response . . . . . . . . . . . . . . . . . . . . . . . . . . . . . . . . 192

9.4 .1 Radioluminescence . . . . . . . . . . . . . . . . . . . . . . . . 192

9.4 .2 Scintillation . . . . . . . . . . . . . . . . . . . 192

$9.5 \quad$ X-ray imaging . . . . . . . . . . . . . . . . . . . . . . . . 195

9.6 Summary $\ldots \ldots \ldots \ldots \ldots \ldots$

\begin{tabular}{ll}
\hline 10 Conclusions and outlook & 197
\end{tabular}

10.1 Conclusions . . . . . . . . . . . . . . . . . . . . . . . . . . . . 197

10.2 Outlook . . . . . . . . . . . . . . . . . . . . . . . . . 199

\begin{tabular}{lr}
\hline Appendices & 217
\end{tabular}

\begin{tabular}{|rr}
\hline A Neutron sensitivity & 219
\end{tabular}

A.1 Neutron scintillators . . . . . . . . . . . . . . . . . . . . . . . . . . 219

A.2 Neutron sensitised lanthanum stabilised cubic barium chloride . . . . . . . 221

A.2.1 Structure . . . . . . . . . . . . . . . . . . . . . . 221

A.2.2 X-ray response . . . . . . . . . . . . . . . . 222 
A.2.3 Neutron response . . . . . . . . . . . . . . . . . . . . . 222

A.3 Neutron sensitised $\mathrm{Ba}_{0.3} \mathrm{Sr}_{0.7} \mathrm{Cl}_{2}: \mathrm{RE}(\mathrm{RE}=\mathrm{Sm}$, Eu or Ce $\ldots \ldots \ldots \ldots \ldots \ldots$

A.3.1 Structure . . . . . . . . . . . . . . . . . . . . . 224

A.3.2 X-ray sensitivity . . . . . . . . . . . . . . 225

A.3.3 Neutron sensitivity . . . . . . . . . . . . . . . 226

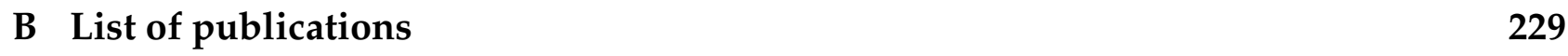




\section{List of Figures}

1.1 Typical spectral sensitivity versus wavelength for a photomulitplier and a silicon avalanche photodiode $\ldots \ldots \ldots \ldots$

2.1 Schematic depiction of $\gamma$-ray production and $x$-ray production . . . . . . . . 6

2.2 Representative $x$-ray spectrum and possible inner electron transitions . . . . 7

2.3 Comparison of mass attenuation coefficients for lead, water, cortical bone and adipose tissue ........................ 8

2.4 Depiction of a basic ionisation chamber detector and plot of gas detector current output against anode voltage showing different operational regimes . 10

2.5 Depiction of a typical germanium semiconductor detector $\ldots$. . . . . . . . 12

2.6 Depiction of a typical scintillator detector $\ldots \ldots \ldots$. . . . . . . . . . . 13

2.7 Diagram of a photomultiplier tube . . . . . . . . . . . . . . 17

2.8 Structure of a silicon avalanche photodiode $\ldots \ldots \ldots$. . . . . . . . . 18

2.9 Typical spectral sensitivity versus wavelength for a photomultiplier tube and

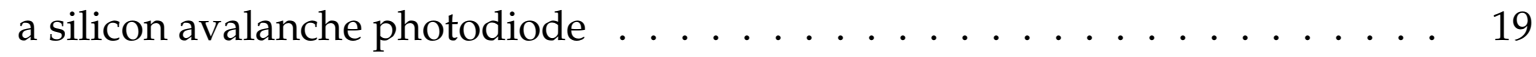

3.1 Diagram of scintillation mechanism for a rare earth doped material . . . . . 23

$3.2 \quad$ Overall $\gamma$-ray absorption and contribution from Rayleigh scattering, photoelectric effect, Compton scattering and pair production for iron . . . . . . . . . . . 24

3.3 Selected crystal defects depicted in $\mathrm{NaCl} \ldots \ldots$. . . . . . . . . . . 26

$3.4 \quad$ Energy level diagram of an ideal blue emitting scintillator and an ideal red emitting scintillator $\ldots \ldots \ldots \ldots \ldots \ldots$

4.1 Cubic fluorite structure $\ldots \ldots \ldots \ldots$. . . . . . . . . . . . . . . 38

4.2 Simulated XRD patterns for $\mathrm{CaF}_{2}$ and cubic $\mathrm{BaCl}_{2} \ldots \ldots \ldots$ 
4.3 Schematic plot of absorption for two level system . . . . . . . . . . . . 41

4.4 Simple depiction of photoluminescence process $\ldots \ldots \ldots$. . . . . . . . 42

4.5 Simple example of cross relaxation . . . . . . . . . . . . . . . . . . 43

4.6 Depiction of energy levels of $4 \mathrm{f}^{6}$ configuration $\ldots \ldots \ldots$

4.7 Dieke diagram $\ldots \ldots \ldots \ldots \ldots$. . . . . . . . . . . . . . . . . . . . . . . .

4.8 Depiction of the effect of Stokes shift $\ldots \ldots \ldots \ldots$

4.9 Schematic energy level diagrams for $\mathrm{Sm}^{2+}, \mathrm{Eu}^{2+}, \mathrm{Pr}^{3+}$ and $\mathrm{Ce}^{3+}$ ions . . . . 52

4.10 Splitting of ground state in a magnetic field and recorded ESR signal . . . . . 56

5.1 Schematic of radio frequency induction furnace configuration $\ldots . . . .58$

5.2 Schematic diagram of the spectrophotometer . . . . . . . . . . . . . 60

5.3 Schematic diagram of the Fluorolog used $\ldots \ldots$. . . . . . . . . . . . 62

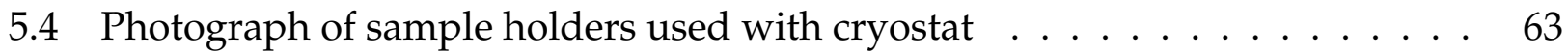

5.5 Schematic diagram of the electron spin resonance (ESR) spectrometer used. . 65

5.6 Schematics of the $\mathrm{x}$-ray imaging configurations used $\ldots . . \ldots 6$

5.7 Quantum efficiency of the photomultipliers used . . . . . . . . . . . 68

5.8 Photograph of the neutron beam configuration . . . . . . . . . . . . . 69

6.1 Real scintillation event as recorded on an oscilloscope . . . . . . . . . . 72

6.2 Depiction of the idea behind time correlated single photon counting . . . . 73

6.3 Depiction of the difference between constant level and constant fraction trig-

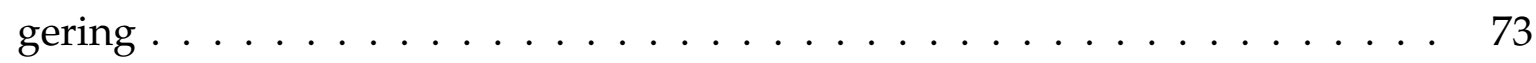

6.4 Block diagram of the TCSPC configuration $\ldots \ldots \ldots$

6.5 Photograph of the configuration used in our system $\ldots \ldots$. . . . . . . 76

6.6 Diagrams of optical arrangement options $\ldots \ldots$. . . . . . . . . . . 77

6.7 Photographs of photomultiplier appendages for reducing light intensity . . . 78

6.8 Signal and dark count frequencies as a function of voltage for the stop photo-

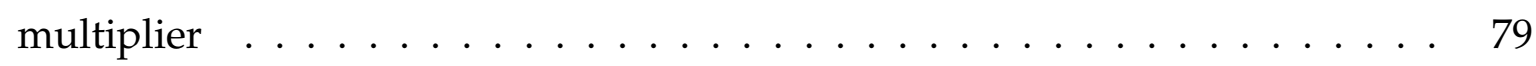

6.9 The signal and dark count frequencies as a function of voltage and discriminator level for the start photomultiplier $\ldots \ldots \ldots \ldots$. . . . . . . . 80

6.10 Depiction of the pulse rate considerations $\ldots \ldots \ldots$. . . . . . . . 81

6.11 Instrumental response function $\ldots \ldots \ldots$. . . . . . . . . . 82 
6.12 Background spectrum $\ldots \ldots \ldots \ldots$

6.13 Calibration of time to pulse height converter $\ldots \ldots \ldots$. . . . . . . 85

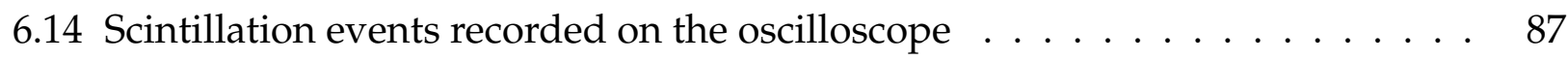

6.15 Photograph of $\mathrm{NaI}(\mathrm{Tl})$ single crystal scintillator $\ldots \ldots$. . . . . . . . . . 88

6.16 Decay profiles for a commercial $\mathrm{NaI}(\mathrm{Tl})$ recorded using an oscilloscope and the time correlated single photon counting system . . . . . . . . . . . . . 89

6.17 Photograph of plastic NE102 scintillator $\ldots \ldots \ldots$. . . . . . . . . . . 90

6.18 Decay profiles for NE102 plastic scintillator recorded using an oscilloscope and the time correlated single photon counting system . . . . . . . . . . 9 91

6.19 Longer timescale NE102 scintillation profile showing evidence of after-pulsing 92

6.20 Decay profiles for $\mathrm{Ba}_{0.3} \mathrm{Sr}_{0.7} \mathrm{Cl}_{2}: \mathrm{Eu}^{2+}$ recorded using an oscilloscope and the time correlated single photon counting system . . . . . . . . . . . . 93

$7.1 \quad$ Powder x-ray diffraction patterns for $\mathrm{Ba}_{1-x} \mathrm{La}_{x} \mathrm{Cl}_{2+x} \ldots \ldots$. . . . . . . . 97

7.2 Powder x-ray diffraction patterns for $\mathrm{Ba}_{0.875} \mathrm{La}_{0.125} \mathrm{Cl}_{2.125}: \mathrm{Sm}$ and lattice parameter variation with samarium concentration $\ldots \ldots \ldots$. . . . . . . . . . . 98

7.3 Photograph of a $\mathrm{Ba}_{0.875} \mathrm{La}_{0.125} \mathrm{Cl}_{2.125}: \mathrm{Sm}(0.02 \%)$ sample $\ldots \ldots$. . . . . . . 98

7.4 Absorption spectra at 12 and $300 \mathrm{~K}$ for and schematic energy level diagram showing the relevant transitions $\ldots \ldots \ldots \ldots$. . . . . . . . . 100

7.5 Photographs of a $\mathrm{Ba}_{0.875} \mathrm{La}_{0.125} \mathrm{Cl}_{2.125}: \mathrm{Sm}(0.02 \%)$ sample showing colour change with temperature . . . . . . . . . . . . . . . . . 101

7.6 Photograph of a sample showing strong red photoluminescence . . . . . . . . 101

7.7 Excitation and emission spectra for LSCBC:Sm (0.01\%) at $300 \mathrm{~K}$ and $12 \mathrm{~K}$. . 102

7.8 Diagram of the radiative transitions observed from the photoluminescence spectra at low temperatures and high temperatures . . . . . . . . . . . . 104

7.9 Photoluminescence spectra for LSCBC:Sm (0.01\%) at $12 \mathrm{~K}, 170 \mathrm{~K}$ and $290 \mathrm{~K}$. 105

7.10 Anti-stokes vibronic sideband of the ${ }^{5} \mathrm{D}_{0} \rightarrow{ }^{7} \mathrm{~F}_{0}$ transition shown between $30 \mathrm{~K}$

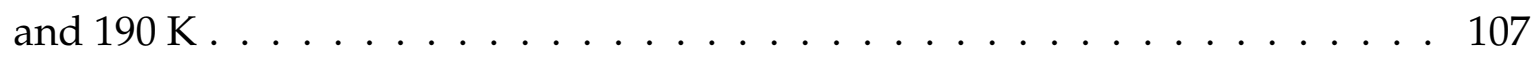

7.11 Integrated light output from the $4 \mathrm{f}^{5} \mathrm{~d}^{1}$ configuration, the ${ }^{5} \mathrm{D}_{1}$ state, the ${ }^{5} \mathrm{D}_{0}$ state in LSCBC:Sm ${ }^{2+}$ as a function of temperature $\ldots . . . . . . .108$ 
7.12 Diagram of energy levels and transitions relevant to the recorded photoluminescent lifetimes in LSCBC:Sm${ }^{2+}$. . . . . . . . . . . . . . . . . . . . . . . 109

7.13 Photoluminescent decays of the $4 \mathrm{f}^{6}\left({ }^{5} \mathrm{D}_{0} \rightarrow{ }^{7} \mathrm{~F}_{1}\right)$ and $4 \mathrm{f}^{5} 5 \mathrm{~d}^{1} \rightarrow 4 \mathrm{f}^{6}$ transitions

for LSCBC:Sm (0.01\%) at various temperatures between $12 \mathrm{~K}$ and $350 \mathrm{~K}$. . . 110

7.14 Fitted lifetimes of $4 \mathrm{f}^{6}\left({ }^{5} \mathrm{D}_{0} \rightarrow{ }^{7} \mathrm{~F}_{1}\right)$ and $4 \mathrm{f}^{5} 5 \mathrm{~d}^{1} \rightarrow 4 \mathrm{f}^{6}$ transitions as a function of

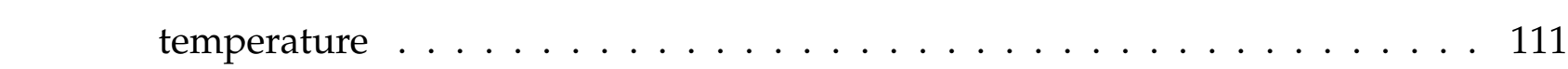

7.15 Simulated and experimental absorption spectra for LSCBC:Sm ${ }^{2+} \ldots 112$

7.16 High energy cut-off of the $4 \mathrm{f}^{5} 5 \mathrm{~d}^{1}$ emission band variation with temperature . 114

7.17 Diagram of the five lowest excited states and the transitions occurring between

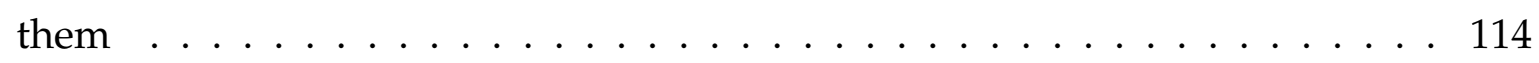

7.18 Summary of energy levels and transition parameters used for photoluminescence simulation . . . . . . . . . . . . . . . . . . . 118

7.19 Comparison of simulated and experimental photoluminescent emission intensities and lifetimes as a function of temperature . . . . . . . . . . . . . 120

7.20 Schematic diagram of the energy levels of the $\mathrm{Sm}^{2+}$ ion in LSCBC and the radia-

\begin{tabular}{|c|}
\hline tive and non-radiative transitions occurring between them as the temperature \\
\hline
\end{tabular}

is increased $\ldots \ldots \ldots \ldots \ldots \ldots$. . . . . . . . . . . . . . . . . . . . . . . .

7.21 Radioluminesence of LSCBC and LSCBC:Sm . . . . . . . . . . . . . 124

7.22 Scintillation events for undoped LSCBC $\ldots \ldots$. . . . . . . . . . . . . . 125

7.23 Powder x-ray diffraction patterns for $\mathrm{La}_{0.6125} \mathrm{M}_{0.6125} \mathrm{Ba}_{0.875} \mathrm{Cl}_{2}: \mathrm{Sm},(\mathrm{M}=\mathrm{Li}, \mathrm{K}$,

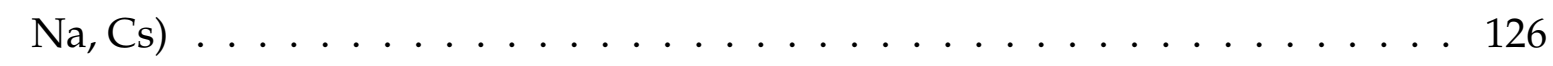

7.24 LSCBC:Sm and undoped LSCBC afterglow profiles . . . . . . . . . . . . 127

7.25 Thermally stimulated luminescence glow curves for LSCBC and LSCBC:Sm . 128

7.26 Spectra of the emission from undoped and samarium doped LSCBC at the peak of the thermally stimulated luminescence glow curves . . . . . . . . . 129

7.27 Depiction of possible colour centres LSCBC lattice . . . . . . . . . . . . 130

7.28 Powder x-ray diffraction patterns for $\mathrm{Ba}_{0.875} \mathrm{La}_{0.125-z} \operatorname{Pr}_{z} \mathrm{Cl}_{2.25}$ for $z=0.125,0.01$

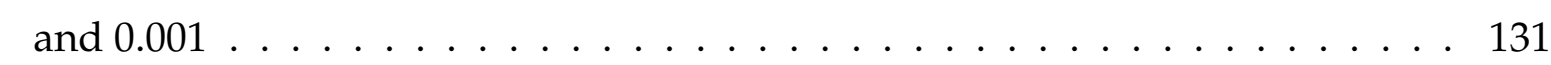

7.29 Photograph of a sample of LSCBC with $0.1 \% \mathrm{Pr}^{3+}$ and $12.4 \% \mathrm{La}^{3+}$. . . . . . 132 
7.31 Energy levels and transitions observed for $\mathrm{Pr}^{3+}$.

7.32 Photoluminescent excitation and emission spectra at $12 \mathrm{~K}$ and $300 \mathrm{~K}$ for a 0.1 $\% \operatorname{Pr}^{3+}$ sample.

7.33 Photoluminescent lifetimes of the ${ }^{3} \mathrm{H}_{4}{ }^{3} \mathrm{~F}_{4}$ and ${ }^{3} \mathrm{H}_{6}$ excited states of the $\mathrm{Pr}^{3+}$ ions in LSCBC .

7.34 Radioluminescence of $\operatorname{Pr}^{3+}$ doped LSCBC for various $\operatorname{Pr}^{3+}$ concentrations . . 135

7.35 Afterglow profiles of LSCBC:Pr ${ }^{3+} \ldots \ldots \ldots \ldots$. . . . . . . . . . 136

8.1 Heating and cooling heat flow curves for $\left(\mathrm{x} \cdot \mathrm{BaCl}_{2}\right)-\left((1-\mathrm{x}) \cdot \mathrm{SrCl}_{2}\right)$ mixtures . . . 141

8.2 Powder x-ray diffraction patterns for cubic $\mathrm{BaCl}_{2}, \mathrm{Ba}_{0.3} \mathrm{Sr}_{0.7} \mathrm{Cl}_{2.0}$ and cubic $\mathrm{SrCl}_{2} 142$

8.3 Photographs of $\mathrm{Ba}_{0.3} \mathrm{Sr}_{0.7} \mathrm{Cl}_{2}$ additionally doped with samarium or europium 142

8.4 X-ray half-thickness for $\mathrm{Ba}_{0.3} \mathrm{Sr}_{0.7} \mathrm{Cl}_{2}, \mathrm{Gd}_{2} \mathrm{O}_{3} \mathrm{~S}: \mathrm{Tb}^{3+}$ and $\mathrm{SrCl}_{2} \ldots \ldots$. . . . . . 143

8.5 Absorption spectra $\mathrm{Ba}_{0.3} \mathrm{Sr}_{0.7} \mathrm{Cl}_{2}: \mathrm{Sm}$ at $12 \mathrm{~K}$ and $300 \mathrm{~K} \ldots \ldots$. . . . . . . . . . 144

8.6 Schematic diagram of the excited state energy levels of $\mathrm{Sm}^{2+}$ with the absorption transitions indicated $\ldots \ldots \ldots$. . . . . . . . . . . . . . . . . 144

8.7 Photoluminescence excitation and emission spectra of $\mathrm{Ba}_{0.3} \mathrm{Sr}_{0.7} \mathrm{Cl}_{2}: \mathrm{Sm}^{2+}$ at $298 \mathrm{~K}$ and at $12 \mathrm{~K} \ldots \ldots \ldots \ldots \ldots \ldots$

8.8 An expanded view of the emission spectrum at $12 \mathrm{~K}$ alongside a diagram of the transitions observed $\ldots \ldots \ldots \ldots$. . . . . . . . . . . . 147

8.9 Integrated intensity of $4 \mathrm{f}^{5} 5 \mathrm{~d}^{1}, 4 \mathrm{f}^{6}\left({ }^{5} \mathrm{D}_{0}\right)$ and total photoluminescence emission as a function of temperature $\ldots \ldots \ldots \ldots$. . . . . . . . . . 147

8.10 Decay profiles of the ${ }^{5} \mathrm{D}_{0}$ emission at $12 \mathrm{~K}, 50 \mathrm{~K}$ and $100 \mathrm{~K} \ldots 148$

8.11 Decay profiles of the $4 \mathrm{f}^{5} 5 \mathrm{~d}^{1}$ emission at $200 \mathrm{~K}, 300 \mathrm{~K}$ and $375 \mathrm{~K}$. . . . . . . . . 148

8.12 Summary of lifetime variation with temperature for both the $4 f^{5} 5 d^{1}$ and ${ }^{5} D_{0}$ emissions . . . . . . . . . . . . . . . . . . . . . . . . . 149

8.13 Shift in absorption spectra from LSCBC:Sm ${ }^{2+}$ to $\mathrm{Ba}_{0.3} \mathrm{Sr}_{0.7} \mathrm{Cl}_{2}: \mathrm{Sm}^{2+} \ldots 150$

8.14 Summary of the energy levels and lifetimes used the photoluminescence simulation of $\mathrm{Ba}_{0.3} \mathrm{Sr}_{0.7} \mathrm{Cl}_{2}: \mathrm{Sm}^{2+} \ldots \ldots \ldots \ldots \ldots \ldots \ldots$

8.15 Simulated integrated intensity of emission and photoluminescent lifetimes alongside experimental data . . . . . . . . . . . . . . . . 152

8.16 Radioluminescence of $\mathrm{Ba}_{0.3} \mathrm{Sr}_{0.7} \mathrm{Cl}_{2}: \mathrm{Sm}^{2+} \ldots \ldots \ldots \ldots$. . . . . . . . 153 
8.17 Scintillation events of undoped and samarium doped $\mathrm{Ba}_{0.3} \mathrm{Sr}_{0.7} \mathrm{Cl}_{2} \ldots \ldots$

8.18 Pulse height analysis spectra for $\mathrm{Ba}_{0.3} \mathrm{Sr}_{0.7} \mathrm{Cl}_{2}$ and $\mathrm{Ba}_{0.3} \mathrm{Sr}_{0.7} \mathrm{Cl}_{2}: \mathrm{Sm}^{2+}$. . . . . 155

8.19 Thermally stimulated luminescence glow curves for undoped $\mathrm{Ba}_{0.3} \mathrm{Sr}_{0.7} \mathrm{Cl}_{2}$ and

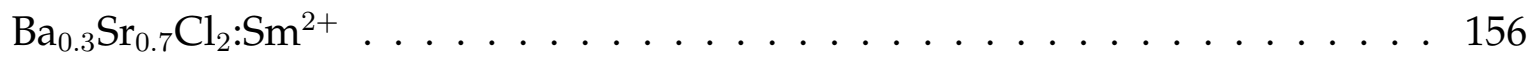

8.20 Emission spectra at the peak temperature of the glow curves for $\mathrm{Ba}_{0.3} \mathrm{Sr}_{0.7} \mathrm{Cl}_{2}$ and $\mathrm{Ba}_{0.3} \mathrm{Sr}_{0.7} \mathrm{Cl}_{2}: \mathrm{Sm}^{2+} \ldots \ldots \ldots \ldots \ldots \ldots \ldots \ldots$

8.21 X-ray images taken with $\mathrm{Ba}_{0.3} \mathrm{Sr}_{0.7} \mathrm{Cl}_{2}: \mathrm{Sm} \ldots \ldots \ldots \ldots$. . . . . . . 157

8.22 Photoluminescence excitation and emission spectra and emission lifetime of $\mathrm{Ba}_{0.3} \mathrm{Sr}_{0.7} \mathrm{Cl}_{2}: \mathrm{Eu} \ldots \ldots \ldots \ldots \ldots \ldots \ldots \ldots \ldots$

8.23 Radioluminescence for $\mathrm{Ba}_{0.3} \mathrm{Sr}_{0.7} \mathrm{Cl}_{2}: \mathrm{Eu}^{2+}$. . . . . . . . . . . . . . . . 159

8.24 Scintillation decay profile and pulse height analysis for $\mathrm{Ba}_{0.3} \mathrm{Sr}_{0.7} \mathrm{Cl}_{2}: \mathrm{Eu}^{2+} \quad$. 160

8.25 Thermally stimulated luminescence glow curves and peak temperature emission spectrum for $\mathrm{Ba}_{0.3} \mathrm{Sr}_{0.7} \mathrm{Cl}_{2}: \mathrm{Eu}^{2+}$

8.26 Depiction of possible $\mathrm{V}_{k}$-F-centre recombination mechanism in $\mathrm{Ba}_{0.3} \mathrm{Sr}_{0.7} \mathrm{Cl}_{2}$. 162

8.27 Depiction of possible TSL mechanism in $\mathrm{Ba}_{0.3} \mathrm{Sr}_{0.7} \mathrm{Cl}_{2} \ldots \ldots \ldots$

8.28 Depiction of possible TSL mechanism in $\mathrm{Ba}_{0.3} \mathrm{Sr}_{0.7} \mathrm{Cl}_{2} \ldots \ldots 6$

8.29 Original phoswich concept for directional sensing . . . . . . . . . . . . . 168

8.30 Commercial example of image subtraction $\ldots$. . . . . . . . . . . . 170

$8.31 \mathrm{Ba}_{0.3} \mathrm{Sr}_{0.7} \mathrm{Cl}_{2}$ phoswich energy discrimination $\ldots \ldots \ldots \ldots$. . . . . . 171

$8.32 \mathrm{Ba}_{0.3} \mathrm{Sr}_{0.7} \mathrm{Cl}_{2}$ phoswich set-up $\ldots \ldots \ldots \ldots \ldots \ldots \ldots$

8.33 Low and high energy images of 8 pin integrated circuit chip . . . . . . . . . 173

9.1 The lifetime of the dominant emission from $\mathrm{Sm}^{2+}$ ions in various alkaline earth halide lattices as a function of temperature $\ldots \ldots \ldots$. . . . . . . 176

9.2 Depiction of the energy levels for $\mathrm{Sm}^{2+}$ in a cubic crystal site . . . . . . . . . . 177

9.3 Photograph of $\mathrm{CaF}_{2}: \mathrm{Sm}$ samples $\ldots \ldots \ldots$. . . . . . . . . . . . 179

9.4 X-ray diffraction patterns for $\mathrm{CaF}_{2} \ldots \ldots \ldots \ldots \ldots$

9.5 X-ray half thickness of $\mathrm{CaF}_{2}$ and $\mathrm{NaI}(\mathrm{Tl}) \ldots \ldots \ldots \ldots$

9.6 Photographs of $\mathrm{CaF}_{2}$ doped with samarium and europium . . . . . . . . 181

9.7 Electron spin resonance spectra of $\mathrm{CaF}_{2}: \mathrm{Eu}^{2+} \ldots \ldots \ldots$. . . . . . . . . 182

9.8 Electron spin resonance spectra of $\mathrm{Ba}_{0.7} \mathrm{Sr}_{0.3} \mathrm{Cl}_{2}: \mathrm{Eu}^{2+} \ldots \ldots \ldots \ldots$ 
9.9 Absorption spectrum of $\mathrm{CaF}_{2}: \mathrm{Sm}$ at room temperature $\ldots \ldots \ldots$. . . . . 184

9.10 Photoluminescence excitation and emission spectra of $\mathrm{CaF}_{2}: \mathrm{Sm}$ and energy level diagram . . . . . . . . . . . . . . . . . . . . . 184

9.11 Temperature dependence of photoluminescence emission spectra of $\mathrm{CaF}_{2}: \mathrm{Sm} \quad 185$

9.12 Photoluminescence emission of $\mathrm{CaF}_{2}: \mathrm{Sm}^{3+}$ showing different sites for the $\mathrm{Sm}^{3+}$

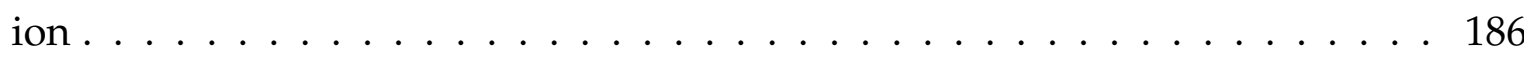

9.13 Photoluminescent lifetimes of $\mathrm{CaF}_{2}: \mathrm{Sm}$ at $12 \mathrm{~K}$ and $300 \mathrm{~K} \ldots$. . . . . . . . 187

9.14 Photoluminescent lifetimes of $\mathrm{CaF}_{2}: \mathrm{Sm}$ for various samarium concentrations 187

9.15 Photoluminescence intensity and lifetime as a function of temperature for

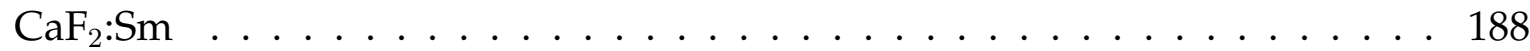

9.16 Electron spin resonance spectra for and $\mathrm{F}$ centre and that recorded for $\mathrm{CaF}_{2}: \mathrm{Sm}$ and $\mathrm{CaF}_{2}: \mathrm{Eu} \ldots \ldots \ldots \ldots \ldots \ldots$. . . . . . . . . . . . . . . . . . . . . . . . .

9.17 Radioluminescence of $\mathrm{CaF}_{2}: \mathrm{Sm} \ldots \ldots \ldots$. . . . . . . . . . . 193

9.18 Scintillation decays for $\mathrm{CaF}_{2}: \mathrm{Sm} \ldots \ldots \ldots$. . . . . . . . . . . . 193

9.19 Pulse height analysis spectra of $\mathrm{CaF}_{2}: \mathrm{Sm}$ compared to NaI(Tl) $\ldots . \ldots$ 9.20 X-ray and optical images . . . . . . . . . . . . . . . . . . 195

9.21 X-ray and image of standard lead grid . . . . . . . . . . . . . . 196

A.1 Neutron capture cross sections for thermal neutrons plotted against atomic number. . . . . . . . . . . . . . . . . . . . 220

A.2 Powder XRD patterns of LSCBC neutron sensitised samples . . . . . . . . . . 222

A.3 Radioluminescence of neutron sensitised samples . . . . . . . . . . . . . . 223

A.4 Example of the response to the neutron beam and the fitting of an afterglow profile . . . . . . . . . . . . . . . . . . . 224

A.5 X-ray diffraction patterns of various $\mathrm{Ba}_{0.3} \mathrm{Sr}_{0.7} \mathrm{Cl}_{2}: \mathrm{RE}$ neutron sensitised samples.225 A.6 Radioluminescence spectra of various neutron sensitised samples. . . . . . . . 226

A.7 Example of response to neutron beam of a $\mathrm{Sm}^{2+}$ and $\mathrm{Gd}^{3+}$ co-doped sample of

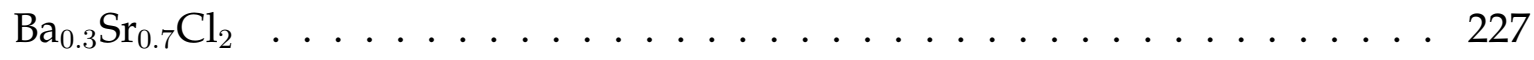





\section{List of Tables}

2.1 Comparison of some performance characteristics for $\gamma$-ray detectors . . . . 15

$3.1 \quad$ Selected properties of commonly used scintillators . . . . . . . . . . . . . . 34

4.1 Summary of selection rules for rare earth ion transitions . . . . . . . . . . . . . 48

$5.1 \quad$ Selected properties of the XP2020Q and R5929 photomultipliers used . . . . . 67

6.1 Double Gaussian instrumental response fit parameters . . . . . . . . . . . . 83

6.2 Calibration factors for use with time to pulse-height converter and MCA . . . 84

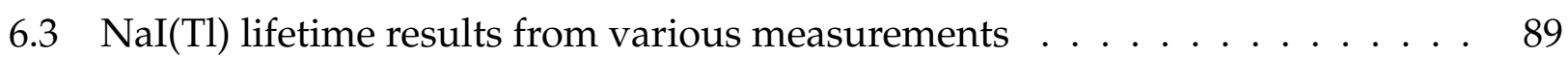

6.4 NE102 lifetime results from various measurements . . . . . . . . . . . . . . . 90

7.1 Selection rules applied to intra $4 \mathrm{f}^{6}$ configuration transitions for a cubic site of

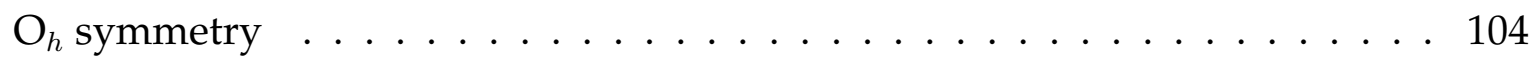

7.2 Integrated intensity of radioluminescence emission of LSCBC:Sm relative to that from $\mathrm{Gd}_{2} \mathrm{O}_{3} \mathrm{~S}: \mathrm{Tb}^{3+} \ldots \ldots \ldots \ldots$. . . . . . . . . . . . . . 124

7.3 Afterglow of undoped and samarium doped LSCBC . . . . . . . . . . . . . 126

7.4 Parameters of fits to glow curves for undoped and samarium doped LSCBC . 128

7.5 Afterglow of LSCBC:Pr${ }^{3+} \ldots \ldots \ldots \ldots \ldots \ldots$

8.1 Fitted Kitis parameters to glow curves for $\mathrm{Ba}_{0.3} \mathrm{Sr}_{0.7} \mathrm{Cl}_{2}$ and $\mathrm{Ba}_{0.3} \mathrm{Sr}_{0.7} \mathrm{Cl}_{2}: \mathrm{Sm}^{2+} 155$

8.2 Fitted Kitis parameters to glow curves for $\mathrm{Ba}_{0.3} \mathrm{Sr}_{0.7} \mathrm{Cl}_{2}, \mathrm{Ba}_{0.3} \mathrm{Sr}_{0.7} \mathrm{Cl}_{2}: \mathrm{Sm}^{2+}$ and

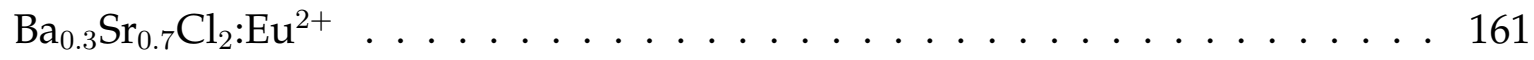

9.1 Parameters used for easyspin electron spin resonance simulation . . . . . . . 182

9.2 The crystal radii of $\mathrm{Ba}^{2+}, \mathrm{Sr}^{2+}, \mathrm{Ca}^{2+}, \mathrm{Sm}^{2+}, \mathrm{Sm}^{3+}, \mathrm{Eu}^{2+}$ and $\mathrm{Eu}^{3+} \ldots \ldots 189$ 
9.3 Parameters of double exponential decays fitted to scintillation profiles _ . . . 193

A.1 Summary of structure and neutron sensitivity for LSCBC samples investigated 223

A.2 Summary of structure and neutron sensitivity for $\mathrm{Ba}_{0.3} \mathrm{Sr}_{0.7} \mathrm{Cl}_{2}$ samples investi-

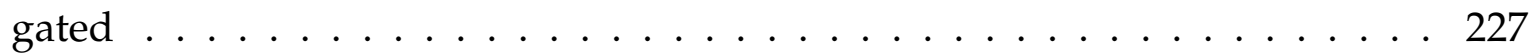




\section{Chapter 1}

\section{Introduction}

\subsection{Scintillators and phosphors for $x$-ray and $\gamma$-ray detection}

Scintillators and x-ray phosphors are materials of high atomic number which convert high energy photons ( $\gamma$-rays and x-rays) into tens of thousands of photons of lower energy, generally in the visible range. The word scintillation comes from Latin where 'scintilla' means a spark. If the light emission happens on a fast time scale $(\lesssim 1 \mu \mathrm{s})$ the material is called a scintillator, if the timescale is longer the material is called a phosphor [1].

Historically the importance of scintillators and phosphors was first appreciated by Wilhelm Röntgen in 1895. He discovered that a hitherto unknown radiation, which we now know as $\mathrm{x}$-ray radiation, passed through a human hand. The difference in absorption of the $\mathrm{x}$ rays between bone and tissue revealed the internal bone structure, and an image could be formed on photographic film. However the film was not very sensitive to the x-rays making the imaging process highly inefficient and requiring long irradiation times. The need for a converter material, a phosphor, which could efficiently absorb an x-ray and convert the energy to many more visible photons for subsequent recording on a visible-light sensitive photographic film was immediately seen by Röntgen, and he initiated the search for efficient scintillators/x-ray phosphors. Within a year of his discovery, calcium tungstate had been discovered to be suitable for this purpose and subsequently it was in use.

Since then many scintillators and phosphors have been discovered, and have found appli- 
cations in medical diagnostics, industrial inspections, security and in scientific research [2]. Scintillators are the primary radiation detectors used in medical diagnostics and scientific research, whilst phosphors are used in radiography, security and industrial component inspection [3,4]. There are varying requirements for different applications; the choice of scintillator is based on the overall performance envelope and usually requires a compromise among the many characteristic properties to best fit a given application [5].

Scintillators/phosphors can be crystalline solids, amorphous solids, liquids or gases and may be organic or inorganic. The most commonly used scintillators for $\gamma$-ray and x-ray detection are inorganic solid compounds. Most are comprised of a halide, oxide or sulphide crystal with an additional luminescent dopant, the "activator". The absorption of $\gamma$-rays/x-rays is determined by the properties of the crystal. The stopping power of the crystal for the high energy photons is proportional to $\rho Z_{\text {eff }}^{4}$ (where $\rho$ is the density and $Z_{\text {eff }}$ is the effective atomic number) [6], and thus compounds of high density and high effective atomic number are used. The transfer of energy from the lattice to the activator is determined by both the lattice and luminescent dopant and is difficult to predict. The character of the final luminescence (wavelength, decay time, etc.) depends on the particular activator. The decay time of the luminescence, an important scintillator characteristic, depends on the probability of the final radiative transition. Therefore, dopants which show transitions that are allowed by Laporte's rule (i.e. the final and initial states are of different parity) are commonly used. The inorganic luminescent dopants which show such transitions are mainly those which form cations with open outer shells. In recent years there has been a focus on utilising the allowed $4 \mathrm{f}^{n-1} 5 \mathrm{~d}^{1}$ $\rightarrow 4 \mathrm{f}^{n}$ transitions of rare earth ions because decay times are expected to be in the $10-100$ ns range [7]. In particular $\mathrm{Ce}^{3+}$ has attracted a lot of attention, and lifetimes down to a few nanoseconds have been found [1].

Scintillators/phosphors are always coupled to a photodetector to convert the visible light produced into an electrical signal. Historically photomultiplier tubes (PMTs) have been used and work well; however more recently there has been significant interest in the use of silicon based photodiode photodetectors. Benefits of the silicon based photodetectors include reduction of cost, size and ease of use. Silicon photodiodes are at least a factor of $10-50$ times cheaper than PMTs, and this can be a significant factor in large installations such as 


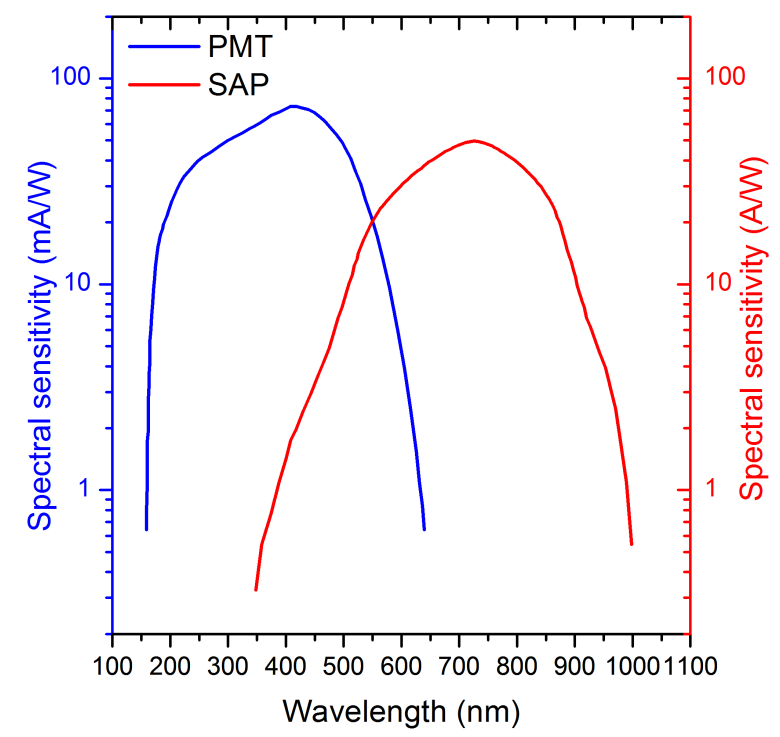

Figure 1.1: Typical spectral sensitivity versus wavelength for the commonly used XP2020Q PMT [9] and a silicon avalanche photodiode (S2383) [10].

positron emission tomography (PET) scanners which can have hundreds of photodetectors. Also silicon is insensitive to magnetic fields, making combined magnetic resonance imaging (MRI)/PET scans feasible. This would be a safer and more efficient technique than the currently used PET/CT as no x-ray dose would be received and the scans could be done simultaneously [8]. Silicon photodiodes are not only cheaper than PMTs per photodetector but also do not require an expensive high voltage power supply [8].

While silicon photodiodes offer significant benefits, the two types of photodetectors are not readily interchangeable electrically or optically, as they have very different quantum efficiency curves. When coupling to a PMT the ideal emission wavelength is typically in the blue around $400 \mathrm{~nm}$, whereas when using a photodiode-based detector the ideal emission is above 600 $\mathrm{nm}$ in the red region [11, 12]. Figure 1.1] shows wavelength responses typical of the two types of photodetectors. The PMT sensitivity peaks at $400 \mathrm{~nm}$ while the photodiode sensitivity peaks at $725 \mathrm{~nm}$. As PMTs have historically been used, scintillator research and development has primarily been focused on blue emission [13]. There is a lack of available red emitting scintillators and phosphors that can be used with silicon photodiodes [11, 14, 15]. This has been recognised in the literature, for example by Schotanus et al. (1992) "Unfortunately very few scintillation crystals are known with a significantly large light output in the wavelength region above $500 \mathrm{~nm}$ for effective readout with photodiodes" [14]. In 1998 Moses et al. attempted to address this, "We are extending this search to cover longer emission wavelengths, since 
applications utilising silicon photodiodes as optical detectors would benefit from scintillators that emit in the 550 to $1050 \mathrm{~nm}$ wavelength region" [11]. Still in 2002, Rodnyi et al. noted there was still a need of red emitting materials, "Commonly used silicon photodiodes exhibit an efficient sensitivity in the range from 500 to $1000 \mathrm{~nm}$, this generates a need for long wavelength scintillators" [15]. While there have been some developments of red-emitting scintillators such as that of $\mathrm{Lu}_{2} \mathrm{~S}_{3}: \mathrm{Ce}^{3+}$ by Huber et al [16] this remains a current issue, as noted by Yanagida in 2012 [6]. A longer wavelength will also reduce losses in fibre so is desirable for applications requiring the use of fibre optics [11]. Thus the motivation for this thesis is to explore materials which may fulfil the requirements for red or near infra red scintillators and phosphors.

\subsection{Preview of this thesis}

This thesis describes work on the development of red-emitting scintillators and phosphors, and is organised as follows. Chapter 2 presents some background to the detection of $\gamma$-rays and x-rays. Chapter 3 reviews the scintillation process and the performance and requirements of scintillators and phosphors in various applications. Chapter 4 discusses some of the spectroscopy and materials science involved in the development and characterisation of scintillators. Chapter 5 describes the experimental techniques and equipment used. Chapter 6 details the building of a time correlated single photon counting system and the performance of the system. Chapters 7 - 9 relate the investigation into the scintillation properties of various rare earth doped materials. In Chapter 7 the results for lanthanum stabilized cubic $\mathrm{BaCl}_{2}: \mathrm{Sm}^{2+}$ are presented. In Chapter 8 the results for $\mathrm{Ba}_{0.3} \mathrm{Sr}_{0.7} \mathrm{Cl}_{2}: \mathrm{RE}^{2+}(\mathrm{RE}=\mathrm{Sm}$ or $\mathrm{Eu}$ ) are presented. In Chapter 9 the scintillation properties of $\mathrm{CaF}_{2}: \mathrm{Sm}^{2+}$ are reported. Finally in Chapter 10 the results are summarised and an outlook for future work in field is provided. 


\section{Chapter 2}

\section{X-rays, $\gamma$-rays - Detection principles}

\subsection{Origins and discovery}

There are various types of ionizing radiation whose detection is of interest: $\alpha$ particles, $\beta$ particles, $\gamma$ rays, $x$-rays and neutrons. Radiation is detected by its interaction with matter and these different radiations all interact differently with matter. $\alpha$ and $\beta$ particles (helium nuclei and electrons or positrons respectively) are charged particles and interact with matter primarily through the coulomb interaction. This directly causes excitation or ionization of atoms, hence these are called directly ionizing forms of radiation. $\gamma$-rays, $x$-rays and neutrons are called indirectly ionizing radiation as the photons or particles have no charge, and so interact with matter indirectly via electromagnetic or nuclear interactions. This thesis focuses on $\gamma$-ray and x-ray detection.

$\gamma$-rays and $x$-rays form part of the continuous spectrum of electromagnetic radiation. Generally x-rays are of lower energies $(124 \mathrm{eV}-124 \mathrm{keV}$, or wavelengths of $\sim 10 \mathrm{~nm}-100 \mathrm{pm}$ ) compared to $\gamma$-rays ( $\gtrsim 124 \mathrm{keV}$ or $\lesssim 10 \mathrm{pm}$ ). However, the nominal distinction between the two arises from the origin of the radiation. $\gamma$-rays generally originate inside the nucleus while x-rays generally originate from the strongly bound electron shells. A $\gamma$-ray is a result of a nuclear reaction such as $\alpha$ decay, $\beta$ decay, fission and fusion which all leave the nucleus in an excited state; a $\gamma$-ray is then emitted as the nucleus decays to a lower energy state. This is schematically depicted in figure 2.1(a), $\gamma$-rays emitted from nuclear decays have fixed 


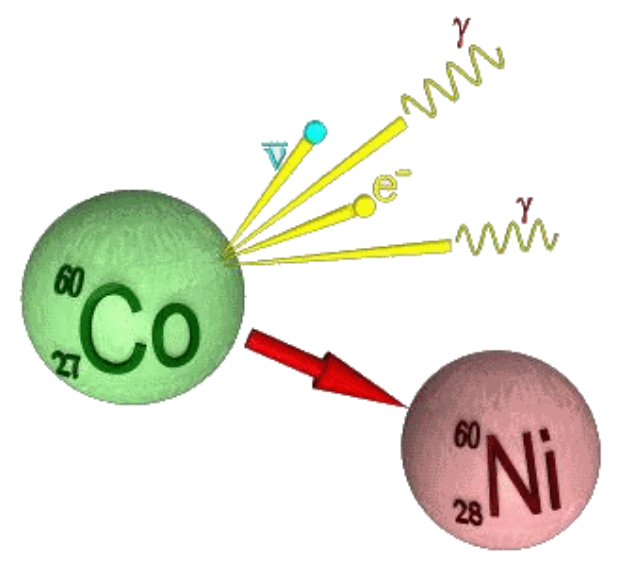

(a)

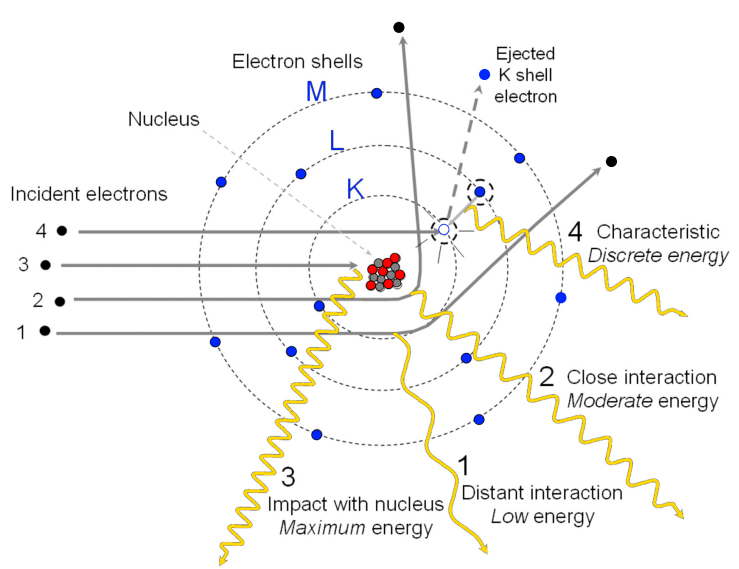

(b)

Figure 2.1: Schematic depiction of (a) $\gamma$-ray production [17] and (b) x-ray production [18].

energies characteristic of the decay.

The production of $x$-rays is depicted in figure 2.1(b). The paths of incident electrons 1 to 3 illustrate that when a fast moving electron comes close to a positive nucleus it experiences a strong coulombic force and is decelerated and deflected from its path. The attractive force to the nucleus is much greater than the repulsion from the electron cloud due to the density of charges in the nucleus. As for any charged particle undergoing acceleration the electron will lose energy by radiating one or more photons. When the deceleration is large, such as when a high speed electron impinges on a solid material, the radiation will have a continuous distribution of energy centered on energies corresponding to wavelengths of $\lambda \sim 1 \AA$. These very high energy photons are called x-rays and the process by which they are produced is the "bremsstrahlung" or "braking" process. Alternatively the electron may interact with an electron in an inner shell (such as incident electron 4 in figure 2.1(b)), ejecting this electron from the shell, in which case an electron from a higher energy shell will drop down to fill the hole emitting a characteristic x-ray in the process.

Figure 2.2(a) shows a representative x-ray spectrum produced for incident electrons on copper. The maximum energy for x-rays occurs in the above process when an electron gives up all its kinetic energy as radiation on its first collision. This gives a well defined maximum on the x-ray spectra labelled $E_{\max }$, and shown with the vertical dotted line in figure 2.2(a). Normally the electrons undergo more than one collision and give off multiple photons of various energies as well as losing some energy to the lattice as heat, and this leads to a smooth 


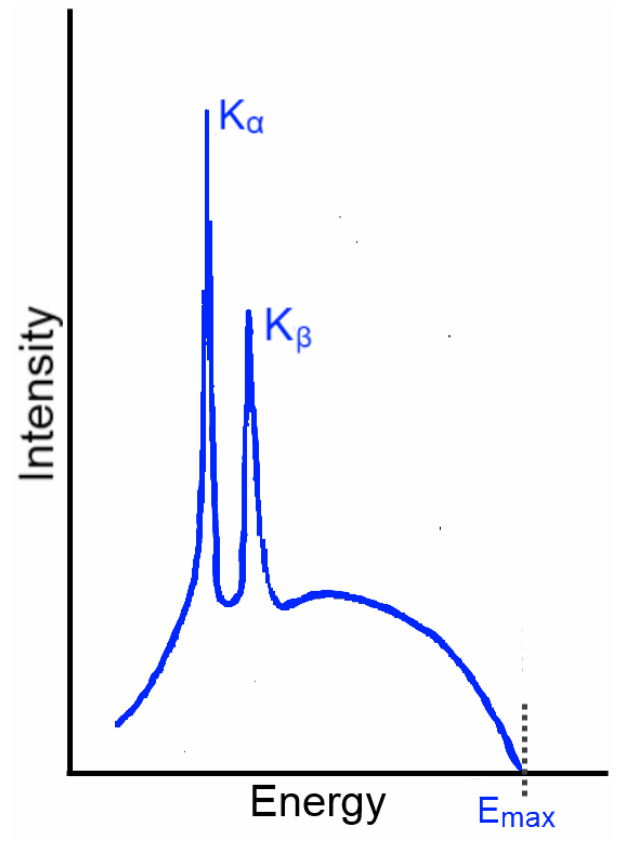

(a)

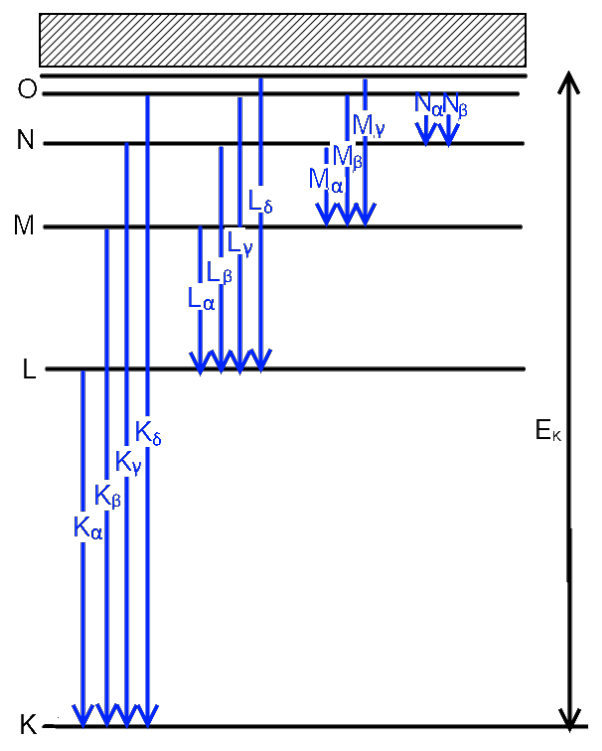

(b)

Figure 2.2: (a) A representative x-ray spectrum [4]. (b) The possible inner electron transitions as labelled [4].

distribution of $x$-ray energies up to the maximum. The sharp lines in figure 2.2(a) occur when a high energy electron interacts with an inner shell electron transferring a large amount of energy, sufficient to remove an inner shell electron, for example from the K-shell. This leaves a vacant hole in the K-shell which can then be filled by any electron in a higher shell dropping down and emitting a photon of appropriate energy. The possibilities for this transition are shown in figure 2.2(b). The L shell electrons have the highest probability of dropping down followed by those in the M shell. This leads to the sharp lines corresponding to the energy difference between the $\mathrm{K}$ shell and the $\mathrm{L}$ or $\mathrm{M}$ shell observable in figure $2.2(\mathrm{a})\left(\mathrm{K}_{\alpha}\right.$ and $\mathrm{K}_{\beta}$ respectively) [4].

\subsection{Applications}

The detection of different types of radiation is an essential part of a wide range of applications of radiation sciences. Most people are familiar with the use of x-rays in radiography and mammograms to image the human body; these are just two of a very broad range of applications. In radiation oncology, x-rays or $\gamma$-rays are used to kill malignant cells as part of cancer treatment. Fluoroscopy uses x-rays to obtain real-time moving images of the internal 


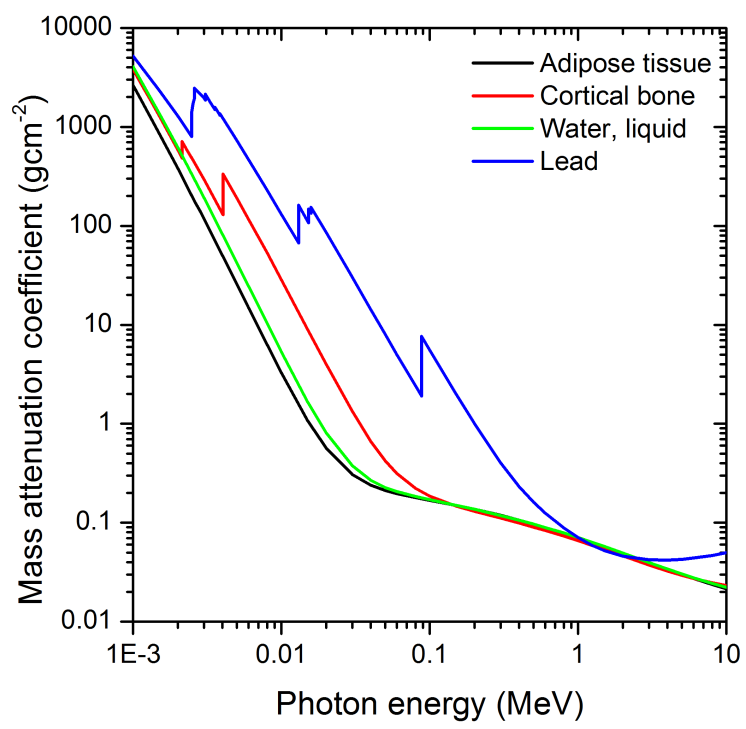

Figure 2.3: Mass attenuation coefficients for adipose tissue (black), cortical bone (red), lead (blue), water (green) and lead (red) [19]. The sharp discontinuities in the lead and bone curves are due to absorption edges.

structures of a patient. In x-ray astronomy the x-rays emitted from astronomical objects are detected, providing information on the gaseous reactions occurring. In positron emission tomography (PET), the $\gamma$-rays emitted due to positron-annihilation reactions are detected to provide three dimensional imagery of internal processes in the human body. In a research context the energy of $\gamma$-rays emitted in high energy particle collisions provides information on the energies of the particles involved and the interactions that have occurred. X-ray diffraction provides structural analysis of chemical compounds. X-ray fluorescence is used for analysis of chemical elements by observing the $\mathrm{x}$-ray emissions which are characteristic to elements. It is also important to be able to detect $\gamma$-radiation for security reasons; $\gamma$-rays are a common by-product of nuclear reactions and the detection of a high flux of $\gamma$-rays can indicate the presence of harmful radioactive materials. These dangerous materials can also potentially be identified based on the energy spectrum of the $\gamma$-rays emitted. Furthermore, $\gamma$-rays and x-rays themselves are harmful to humans so it is important to be able to detect them and measure the dose rate. All these various applications require the detection of $\mathrm{x}$-rays or $\gamma$-rays and offer significant advantages compared to any other method, or are the only feasible technique. For example, consider a x-ray image of a fractured arm; there is no other technique which can tell if it is fractured or not which is as fast and inexpensive and is painless. 
While there are a wide array of applications for x-rays and $\gamma$-rays, the most common is non-invasive or non-destructive imaging of internal structures. $\gamma$-ray and $x$-ray beams are attenuated as they pass through a material with the attenuation depending on the thickness of the material, the atomic number of the material and the $x$-ray/ $\gamma$-ray energy. This is described by the mass attenuation coefficient $(\mu)$, a measure of the attenuation of a $\gamma$-ray or $x$-ray beam of a given energy per unit thickness of a given material.

The mass attenuation coefficient is unique to each element; Figure 2.3 shows the mass attenuation coefficient for lead, water, cortical bone and tissue - representative materials which commonly need to be distinguished in $x$-ray $/ \gamma$-ray imaging. As the atomic mass and/or thickness increases the absorption increases. After passing through an object the spatial variation of the x-ray beam intensity in the plane normal to the beam thus provides a projected image of the internal structure. This is the principle behind x-ray imaging.

\subsection{Methods of detection}

While $\mathrm{x}$-rays and $\gamma$ rays have different origins they are both electromagnetic radiation, and are identical for detection purposes. Hence we use the term $\gamma$-rays here in a broad sense to encompass $x$-rays. $\gamma$-rays are detected by observing the effect they have on matter. The electromagnetic nature of $\gamma$-rays means they interact strongly with the charged electrons in the atoms of all matter, and this is how they are detected. The key process by which a $\gamma$-ray interacts with matter is ionization, where it gives up part or all of its energy to an electron. This highly energetic electron collides with other atoms and liberates many more electrons, leaving behind positive ions. The liberated charge is collected to register the presence of the $\gamma$-ray and measure its energy. The final result can be an electrical pulse with a voltage proportional to the energy deposited in the detecting medium for "direct" detectors or, as for scintillators ("indirect" detectors), a light pulse which results when the electrons and holes recombine [20]. There are three main techniques of detecting $\gamma$-rays: ionisation chamber detection, semi-conductor detection and scintillation detection; these are described briefly in what follows. 


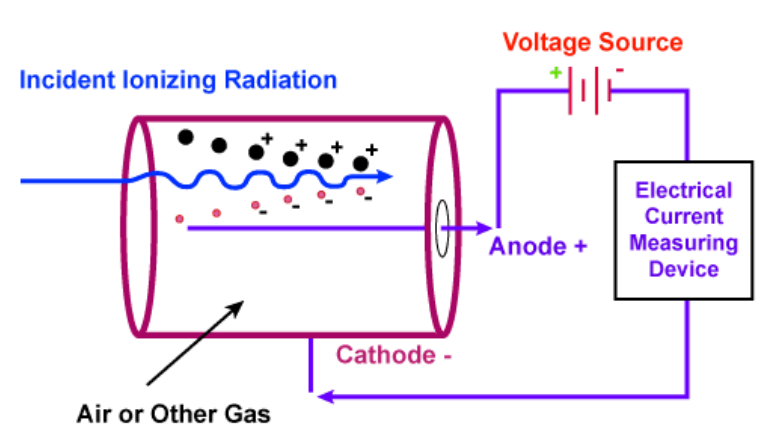

(a)

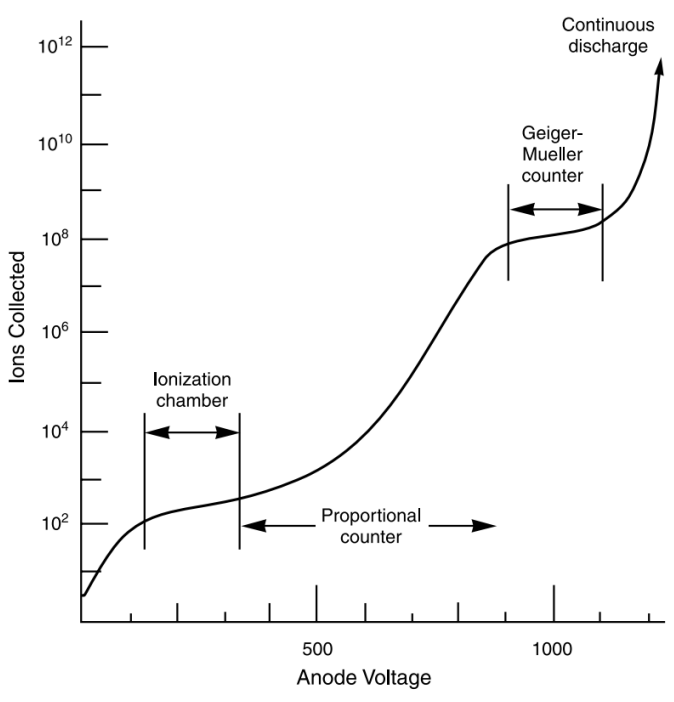

(b)

Figure 2.4: (a) Depiction of a basic ionisation chamber detector [21]. (b) Plot of ionisation chamber detector current output against anode voltage showing different operational regimes [22].

\subsubsection{Ionisation chamber detectors}

Ionisation chamber detectors are the simplest detectors and basically consist of a metal chamber filled with gas and containing a positively biased anode wire in the centre. This is depicted in figure 2.4(a), A $\gamma$-ray which interacts with the gas will produce free electrons and ions via ionization. The electrons are attracted to the anode and produce an electrical pulse. For most applications a noble gas, such as argon, is used in a sealed chamber at pressure (8 - $10 \mathrm{~atm})$ [20]. The pressure increases the chance of ionization and hence sensitivity. The performance characteristics depend critically on the voltage applied as shown in figure 2.4(b). At low anode voltages some electrons may recombine with gas ions before reaching the anode and subsequently not all $\gamma$-rays are detected. However as the voltage is increased a level is reached such that nearly all electrons are collected, and in this region the detector is known as an ionisation chamber [20,23]. Operated in this regime, the detectors are used for dosimetry. As the voltage is increased the electrons have a higher acceleration towards the anode and may cause further ionisations in an "avalanche" effect depending on how energetic they are. The resulting anode pulse amplitude depends approximately linearly on the energy of the $\gamma$-ray and this region is therefore known as the proportional counter region [20, 23], and may be used for low resolution spectroscopy. At even higher voltages the space charge produced 
by residual ions inhibits further ionisation, and so the charge deposited saturates. This region is known as the Geiger-Mueller counter region, where the electron multiplication is large, such that no further amplification is required for pulse counting [23]. The charge collected is independent of the initial energy and provides no information on the $\gamma$-ray energy. On further increase of the voltage, continuous discharge and breakdown occurs [22].

\subsubsection{Semiconductor detectors}

A semiconductor (or solid state) detector is made of a semiconductor doped to have a hole rich region $(\mathrm{P})$, an intrinsic region $(\mathrm{I})$ and an electron rich region $(\mathrm{N})$ in a sandwich structure, this is known as the PIN diode structure and is depicted in figure 2.5. The I region is the $\gamma$-ray sensitive region. Incident $\gamma$-rays ionise atoms and the resulting electrons and holes are pulled to their respective electrodes by the large applied electric field. The consequent charge pulse can then be integrated by a pre-amplifier and converted to a voltage pulse with an amplitude proportional to the $\gamma$-ray energy [23].

The most popular early designs used lithium-drifted germanium $(\mathrm{Ge}(\mathrm{Li}))$ as the detection medium. The lithium served to inhibit trapping of charge at impurity sites in the crystal lattice during the charge collection process as this lowers the efficiency. In recent years, manufacturers have produced hyperpure germanium (HPGe) crystals, essentially eliminating the need for the lithium doping and simplifying the operation of the detector [20,24]. Another popular solid-state detector material for photon spectroscopy is lithium drifted silicon ( $\mathrm{Si}(\mathrm{Li})$ ) [20]. The lower atomic number of silicon compared to germanium reduces the efficiency, but this type of detector has been widely used in the measurement of x-ray spectra in the 1 to 50 $\mathrm{keV}$ energy range and finds some application in x-ray fluorescence measurements.

Both silicon and germanium detectors have to be cooled in order to suppress the thermal excitation of electrons across the energy gap of $\sim 0.7-1 \mathrm{eV}$. In an attempt to avoid this operational constraint the possibility of room-temperature semiconductor materials such as $\mathrm{CdTe}, \mathrm{HgI}_{2}$, and $\mathrm{GaAs}$ has been extensively researched [24]. Their higher average atomic numbers than silicon or germanium provide greater photoelectric efficiency per unit volume of material and they do not require cumbersome cooling. However, these detector materials have limited 


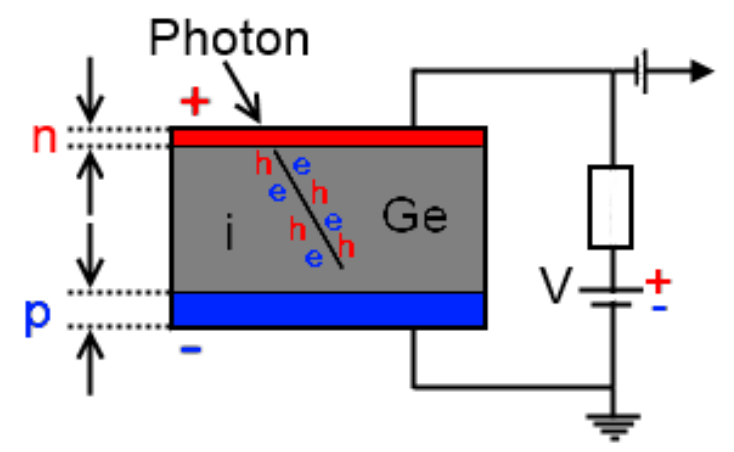

Figure 2.5: Depiction of a typical germanium semiconductor set-up [25].

applications largely because it has not been possible to produce crystals sufficiently large for the detection efficiencies needed [20].

Amorphous selenium is another semiconductor detector which requires no cooling. It is used for mammography but the low atomic mass offers poor attenuation of $\gamma$-rays so cannot be used for applications with higher energies. Because the selenium is used in the amorphous form large plates can be made by evaporation. This is in contrast to other semi-conductor materials which cannot be made into large area detectors easily [26].

\subsubsection{Scintillators}

A scintillator offers indirect measurement of the radiation as opposed to the two direct methods discussed prior, that is to say the output when $\gamma$-rays or x-rays are detected is a light pulse which must undergo further conversion to an electrical pulse by a photodetector. The sensitive volume of a scintillation detector is the entire scintillator volume viewed by a photodetector which responds to the $\gamma$-ray-induced light emissions. When $\gamma$-rays penetrate a scintillator material they generate a large number of electrons and holes, corresponding to ionisations in a gas, but in the solid the 'ionisation' is in the form of an excess of carriers (electrons, holes) in the conduction and valence bands. Electrons and holes subsequently recombine with the emission of one or more photons. In a pure inorganic scintillator crystal, the recombination with the emission of a photon is generally an inefficient process. Furthermore, the emitted photons are usually too high in energy to lie in the range of wavelengths to which photodetectors are sensitive. For this reason small amounts of luminescent impurities are added to most scintillators to enhance the emission of visible photons. Crystal 


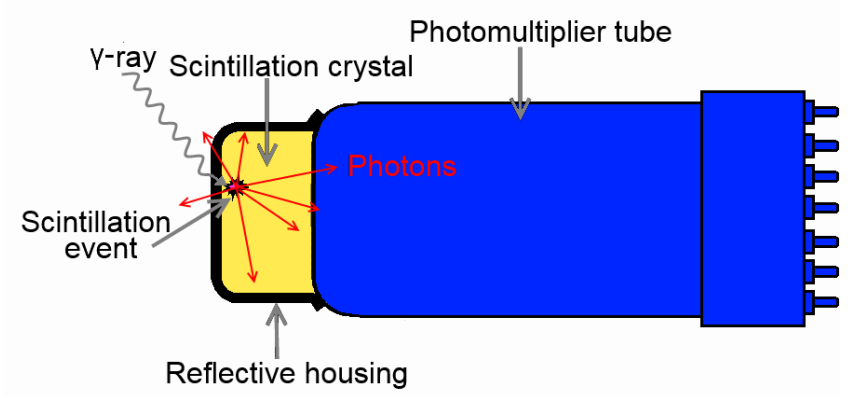

Figure 2.6: Depiction of a typical scintillator detector, in this case the photodetector is a photomultiplier tube.

de-excitations channelled through these impurities give rise to luminescence that can activate the photodetector. One important consequence of luminescence through impurities is that the bulk scintillator crystal must be transparent to the scintillation light. Figure 2.6 shows the typical arrangement of components in a scintillation detector. In this case a photomultiplier tube (PMT) is used as the photodetector.

A common scintillator encountered in $\gamma$-ray measurements is thallium-doped sodium iodide $\mathrm{NaI}(\mathrm{Tl})$. The scintillation light is emitted isotropically; so the scintillator is typically surrounded with reflective material (such as powdered $\mathrm{MgO}$ ) to minimize the loss of light. The scintillator is optically coupled by direct contact to the photodetector which provides amplification and conversion to an electrical pulse. The magnitude of the pulse is proportional to the initial amount of energy deposited by the $\gamma$-ray provided there are no light losses from the scintillator. However the deposited energy shows variation even for a monoenergetic $\gamma$-ray source; this is due to statistical fluctuations associated with how the $\gamma$-ray transfers its energy to the material [20]. Some other common inorganic scintillation materials are caesium iodide (CsI), zinc sulphide (ZnS), lithium iodide (LiI) and more recently bismuth germanate $\left(\mathrm{Bi}_{4} \mathrm{Ge}_{3} \mathrm{O}_{12}\right)$ and $\mathrm{LaBr}_{3}: \mathrm{Ce}^{3+}[22,24,27]$.

\subsubsection{Comparison of $x$-ray and $\gamma$-ray detectors}

There are three main specifications on which the performance of a $\gamma$-ray detector is judged. The first being the absolute efficiency; the number of photons emitted by the scintillator per $\gamma$-ray emitted by the source. The second is the energy resolution; the variation in the pulse 
size for a mono-energetic $\gamma$-ray source. The third is the response time which limits the count rate achievable. Some typical performance characteristics are summarised in table 2.1.

The absolute efficiency of ionisation chamber detectors is much lower than that of semiconductors and scintillators. This is because the low atomic numbers of the gases used mean there is a low probability that a $\gamma$-ray will interact with the gas. Silicon, germanium and selenium based detectors also suffer from low absorption coefficients - particularly at high energies. However, a larger issue for the semi-conductor detectors is insufficient electron-hole collection. To maximise the collection of electrons and holes and increase their drift velocity both silicon and germanium detectors require relatively high voltages $(\sim \mathrm{kVs})$. This results in an additional noise in the current response and leads to problems of surface conductance and voltage breakdown. Scintillators have the highest absolute efficiency owing to their high density and atomic number.

Semiconductor detectors achieve higher energy resolution than scintillators or ionisation chamber detectors. This is because the energy required to create an e-h pair in a semiconductor is small (less than $\sim 3 \mathrm{eV})$, compared to a scintillator $(\sim 10 \mathrm{eV})$ or a gas detector $(\sim 30 \mathrm{eV})$. This means statistical fluctuations in energy deposited in the detector have much less effect as there are more charge carriers $[22-24,32]$. Optical absorption of emitted photons before they escape the crystal also lowers the energy resolution of scintillators. While scintillator detectors will never achieve the energy resolution of germanium detectors the recently discovered $\mathrm{LaBr}_{3}: \mathrm{Ce}^{3+}$ offers much higher resolution than previously achieved with inorganic scintillators [27].

Scintillators offer superior timing responses than semiconductors and ionisation chambers. Even operating at high voltages, silicon and germanium detectors have response times of over $100 \mathrm{~ns}$. This is much slower than a fast scintillator such as $\mathrm{LaBr}_{3}: \mathrm{Ce}^{3+}$ which has a decay time of $\sim 30 \mathrm{~ns}$. Also, for semiconductors, the rise time of the output pulse depends on the position at which the electron-hole pairs were created. This complicates the measurement for timing applications as one cannot use a leading edge trigger or even a standard constant fraction discriminator trigger. Ionisation chamber detectors operating in the proportional or Geiger-Mueller mode are only suitable for low count rate applications as there is a long dead-time required for the space charge to dissipate. A fast response is critical for timing 


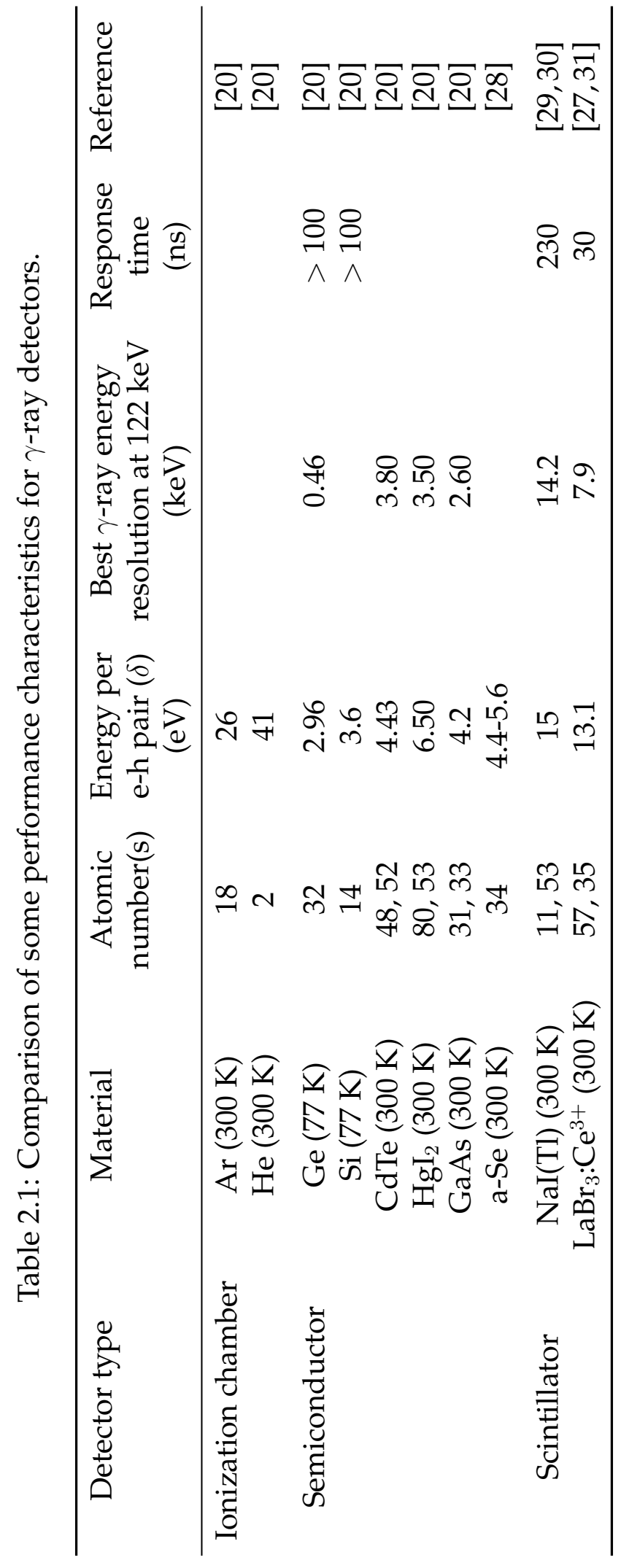


applications such as PET and those requiring photon counting. Scintillators are almost exclusively used for these purposes [20,23,24,32,33].

Both ionisation chambers and scintillators can be made into very large area detectors. In particular, a scintillator can be powdered and bound in a resin to make a large sheet. This cannot be done for a semiconductor; semiconductors are very size constrained by the size of available ingots. Silicon and germanium detectors also require cumbersome cooling to liquid nitrogen temperatures. Semiconductors which are not limited to low temperature operation show inferior resolution to germanium and have not yet been made into sufficiently large crystals for widespread use.

While ionisation chamber detectors are very simple to use and inexpensive, their applications are basically limited to dosimetry. Geiger-Mueller counters are used for personal dosimetry [34]. Very large ionisation chambers, operated in the proportional counter region, are used in hospitals for absolute dosimetry measurements. High resolution $\gamma$-ray spectroscopy is generally performed with semiconductors, particularly germanium, as these offer the highest energy resolution. However, as discussed in section 2.2 these are a very small part of the varied applications. Almost all other applications utilise phosphors or scintillators [20, 23, 24, 32]. While amorphous selenium is now widely used for mammography, generally all other radiography is performed by $\mathrm{x}$-ray phosphors coupled with a complementary metaloxide-semiconductor (CMOS) or charge-coupled device (CCD). Applications such as PET, calorimetry and others utilise scintillators because the light output, timing, large detector size and operating conditions make scintillators the best choice.

\subsection{Photodetectors}

As mentioned above, a scintillator provides an indirect detection of the $\gamma$-ray as it must be coupled with a photodetector. Photomultiplier tubes (PMTs) or silicon avalanche photodiodes (SAPs) are used almost exclusively to detect the emission from a scintillator under $\gamma$-ray irradiation; the advantages of each are discussed in what follows. 


\subsubsection{Photomultiplier tubes}

PMTs are very sensitive light detectors that are useful in low intensity applications such as fluorescence spectroscopy [35]. As shown in figure 2.7] a PMT consists of an evacuated glass tube. On the surface of one end is a photocathode coating of a low work function material such as Sb-Cs or Sb-K-Cs. At the other end of the tube is the anode, and between the two is a set of $\sim 8-16$ dynodes. The cathode is generally held at a high negative voltage (-1000 to $-2000 \mathrm{~V})$; a resistive voltage divider then distributes the voltage to the dynodes, and leaves the anode at $0 \mathrm{~V}$. The divider design influences the frequency response and can be adjusted to suit the application. When photons of an appropriate wavelength are incident on the photocathode an electron may be ejected via the photoelectric effect. This electron is accelerated down the tube by the electric field towards the first dynode, which is usually $100 \mathrm{~V}$ positive with respect to the cathode. When this electron strikes the surface it produces several secondary electrons which are then accelerated to the next dynode (held at a higher voltage). This continues in an avalanche effect down the PMT until finally approximately $10^{6}$ electrons per incident photon have been produced and these hit the anode creating a large charge pulse.

The low work function of the cathode means it is also possible to get thermal (i.e. non-optical) emission of electrons at room temperature, leading to undesirable noise (dark noise) in measurements, so the PMT may need to be cooled in high sensitivity experiments. Noise also arises due to the discrete nature of electric current; this is called shot noise. The gain of a PMT is given by $\delta^{n}$ where $n$ is the number of dynodes (typically 8-16) and $\delta$ is the secondary

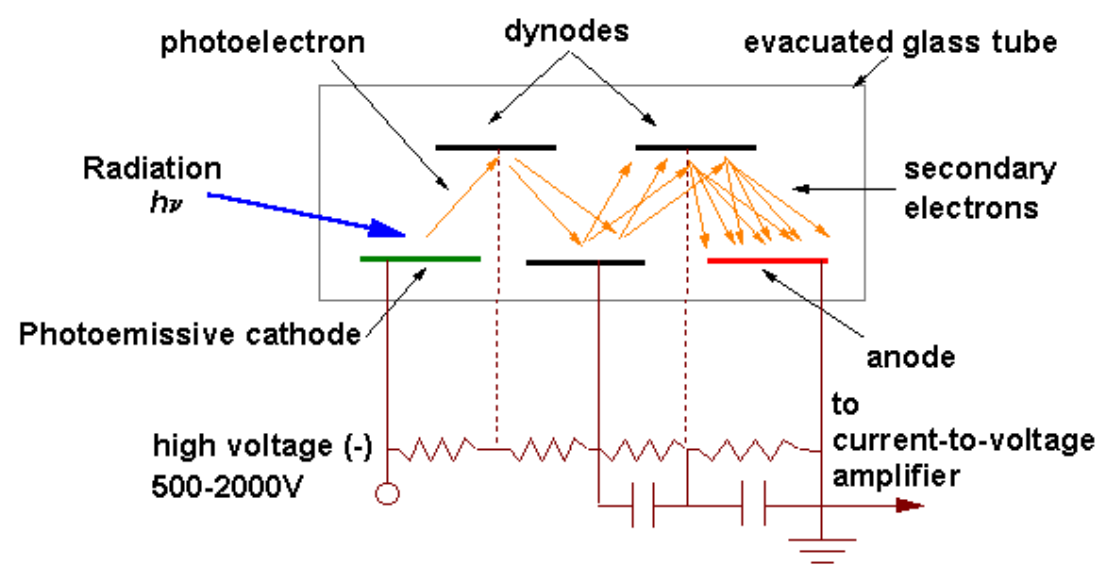

Figure 2.7: A diagram of a photomultiplier tube [35]. 
emission coefficient for each dynode (typically 2-3) [36]. The spectral sensitivity (S) represents the photocurrent from the photocathode per watt of incident power; this depends on the wavelength of the light $(\lambda)$ and is given by

$$
S=\mu \lambda \frac{e}{h c}
$$

Where $h$ is the Planck constant, $c$ is the speed of light in a vacuum, $e$ is the elementary charge and $\mu$ is the quantum efficiency of the PMT. The quantum efficiency is given by ratio of the numbers of photo-ejected electrons to the incident photons. The peak quantum efficiency for a PMT generally between 400 and $450 \mathrm{~nm}$ and is $\sim 0.30$.

\subsubsection{Silicon based detectors}

Silicon based photodiode detectors can be used directly for radiation detection in the form of PIN diodes as discussed above, but, they can also be used for detecting light from a scintillator when fabricated as simple PN diodes or avalanche photodiodes. Figure 2.8 shows a schematic of a silicon avalanche photodiode detector (SAP). A silicon photodiode is essentially a PN junction consisting of a positively doped $(\mathrm{P})$ region and a negatively doped $(\mathrm{N})$ region with a depletion region between. Incident light excites electrons into the conduction band leaving holes in the valence band. The electrons and holes drift to the cathode/anode respectively and if a circuit is connected current will flow that is proportional to the light intensity. While these

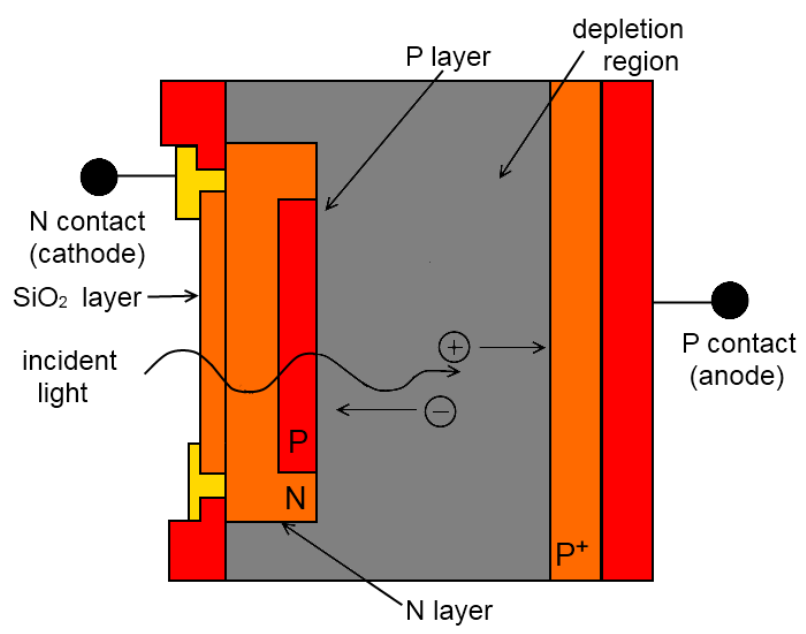

Figure 2.8: Structure of a silicon avalanche photodiode [37]. 


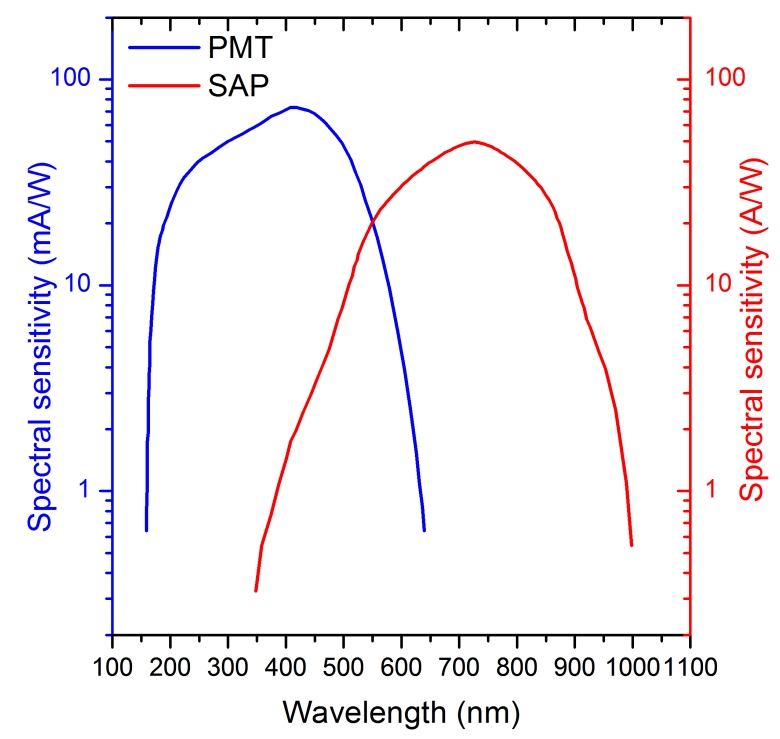

Figure 2.9: Typical spectral sensitivity versus wavelength for the commonly used XP2020Q PMT [9] and a silicon avalanche photodiode (S2383) [10]. This figure is repeated from figure 1.1

detectors are useful, the lack of internal gain hinders their performance. SAPs are specialised silicon photodiodes designed to operate in reverse mode and offer internal gain. A large reverse voltage is applied to the junction and creates a strong electric field. Electron-hole pairs that experience this field gain enough energy to create further electron-hole pairs (impact ionisation). In this multiplicative manner the SAP achieves internal gain. Unfortunately all silicon based detectors suffer from substantial inherent thermal noise. This is because the small band gap of silicon allows for thermal excitation of electrons, and this is registered as dark current.

\subsubsection{Comparison of photodetectors}

SAPs offer higher spectral sensitivities than PMTs and over a wider range of wavelengths as can be seen in figure 2.9 , but the sensitivity is entirely located in the red/near infra-red region on the spectrum.

SAPs are rugged, compact and insensitive to shock and vibration whereas PMTs are much larger, fragile and contain a vacuum which may degrade over time. SAPs offer a higher dynamic range (the intensity range over which the response is linear) of $>10^{6}$ as compared to PMTs $\left(\sim 10^{4}\right)$ which are limited by electron build up at the anode [38. SAPs do not require as high voltage for operation as PMTs. SAPs are particularly of interest for medical systems 
based on magnetic resonance imaging (MRI) as they are insensitive to magnetic fields which deflect the electrons in PMTs. SAPs recover instantly from high light exposure compared to PMTs which require a significant recovery time or may be damaged permanently.

PMTs do however offer far superior internal gain $\left(\sim 10^{6}\right)$ than SAPs $(\sim 350)$. This means PMTs are much more appropriate for single photon counting applications than SAPs. The dark current in SAPs and the lack of gain prevents SAPs performing well in single counting. For most other applications spectral sensitivity is more important than gain in determining overall performance and the SAP is superior [38-40]. 


\section{Chapter 3}

\section{Scintillators \& phosphors - Principles \& performance}

\subsection{Introduction}

In this chapter we discuss some attributes of the current inorganic commercial scintillators, their applications and limitations. We discuss the scintillation mechanism, with a focus on rare earth activation. There are many different scintillation mechanisms with many complexities, this is a large area of current research, so the following merely provides a general overview.

The development of photomultiplier tubes around 1934 stimulated research into scintillators. Since then there has been a constant market for scintillators and consistent research into materials which may show improvements in one of the many defining characteristics. High energy physics research is a large driver of developments. Extensive work on production processes of Bismuth Germanate (BGO) was carried out to allow it to be used in the Large Electron Positron Collider (LEP) where a high density material with a lifetime shorter than $1 \mu \mathrm{s}$ was required. During the period 1990 to 2000, much research went into scintillators to be used for the Large Hadron Collider (LHC). These scintillators needed to be of very high density and have fast lifetimes but the light output was not critical, and this program lead to the discovery of $\mathrm{PbWO}_{4}$. Eighty thousand $\mathrm{PbWO}_{4}$ crystals are in use in the Compact Muon Solenoid and 
another 18,000 in ALICE (both part of the LHC). Today with scintillators in demand for many applications, new scintillator materials are constantly being investigated.

As mentioned earlier x-ray phosphors are scintillators for which the luminescence has a microsecond or longer time-scale decay. These are used in applications where the signal is time integrated such as computed tomography (CT), scanners rather than pulse counting applications. Security scanning systems at airports and ports worldwide use $\mathrm{CdWO}_{4}$ coupled with silicon photodiodes. CT scanners generally make use of $\mathrm{Gd}_{2} \mathrm{O}_{2} \mathrm{~S}$ : $\left(\mathrm{Ce}^{3+}\right.$ or $\left.\mathrm{Pr}^{3+}\right)$ coupled with silicon photodiodes [6]. X-ray phosphors are the basis of most scientific x-ray imaging systems [36].

\subsection{Scintillation mechanism}

In very general terms a scintillator or phosphor can be thought of a wavelength shifter, absorbing incident $\mathrm{x}$-rays or $\gamma$-rays and re-emitting the energy as UV or visible range photons. The process is complex and can be divided into four stages as depicted in figure 3.1] [41]. In the first stage a scintillator or phosphor absorbs an x-ray or $\gamma$-ray photon which, through the photoelectric effect or Compton scattering, creates primary electrons and holes. In the second stage these relax to produce numerous secondary electrons, holes, photons, plasmons and other electronic excitations. These secondary electrons and holes then de-energize through electron-phonon interaction to give electron-hole pairs with near band gap energy. The third stage involves the transport of the electron-hole pairs through the material to a luminescent centre (trap) and the excitation of the luminescent centre. The final stage concerns the resulting luminescence [13]. These four stages are described in more detail in the following subsections. Materials which have a high light output and short lifetime under photoexcitation may have a very low light output and/or long lifetime under x-ray or $\gamma$-ray excitation. This is due to the energy losses and delays in the energy migration processes which are absent in photoluminescence where the luminescent centre is directly and intentionally excited. 


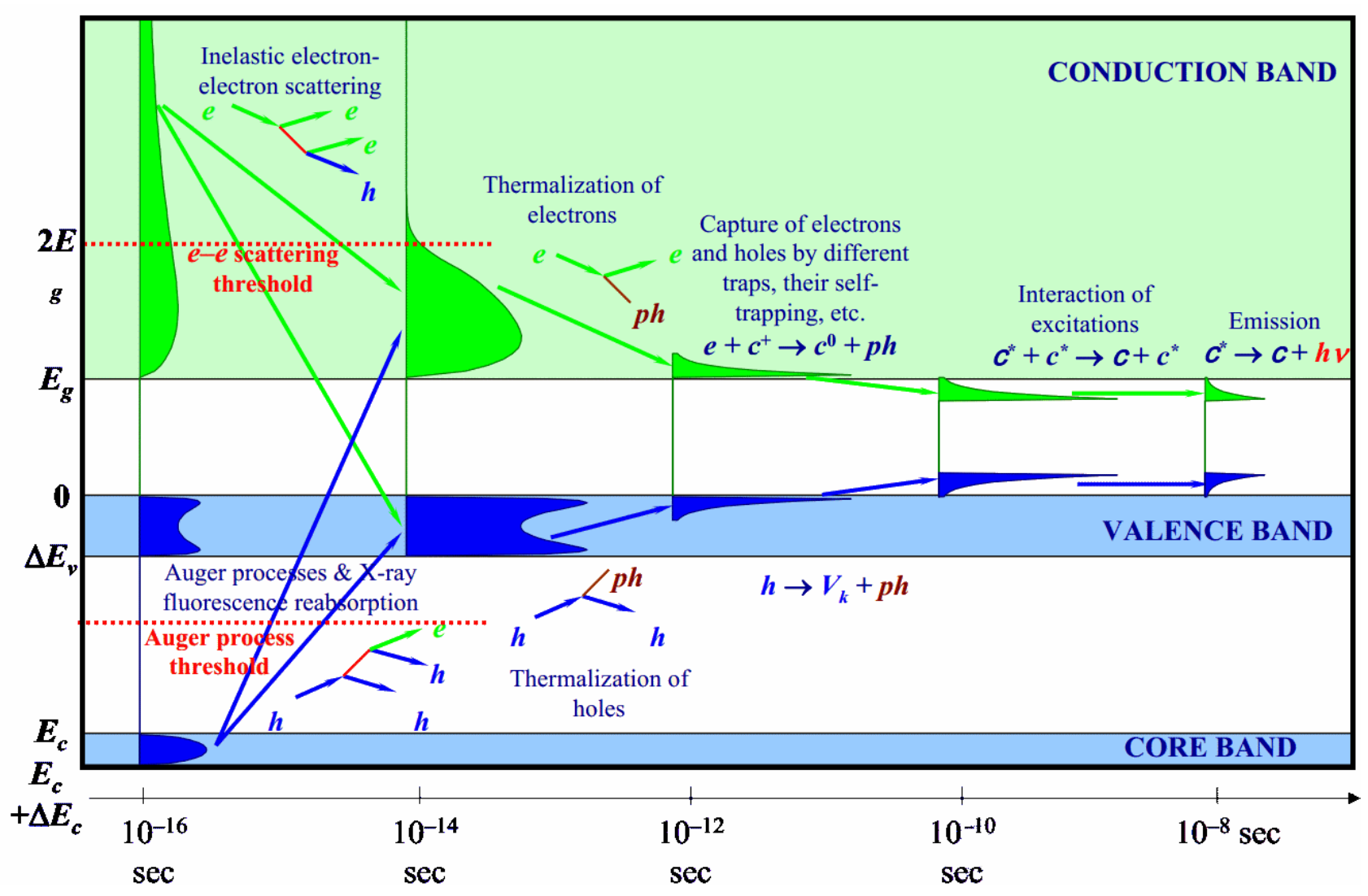

Figure 3.1: Diagram of scintillation mechanism for a rare earth doped material [41].

\subsubsection{Absorption of $\mathbf{x}$-rays or $\gamma$-rays}

As discussed in chapter 2 the distinction between x-rays and $\gamma$-rays is not of particular importance for detection purposes. We therefore again use the term $\gamma$-rays in a broad sense encompassing $\mathrm{x}$-rays. As high energy photons, $\gamma$-rays interact with matter as for any electromagnetic radiation, a quantum of energy may be completely or partially absorbed, or a scattering event may occur. If a monochromatic $\gamma$-ray beam of intensity $\mathrm{I}_{0}$ is incident on a material of thickness $x$, the beam intensity will be reduced to $I$ following the Lambert-Beer law

$$
I=I_{0} \exp (-\mu x)
$$

where $\mu$ is the linear absorption coefficient of the material. $\mu$ is dependent on the atomic species and the density $(\rho)$ of the material, and also varies with $\gamma$-ray energy. Shown in figure 3.2 is the contribution to overall $\gamma$-ray absorption of the Rayleigh scattering, the photoelectric effect, Compton scattering and pair production for iron. Within the range of keVs - MeVs the dominant process is the the photoelectric effect, and above $\sim 1000 \mathrm{keV}$ Compton scattering 


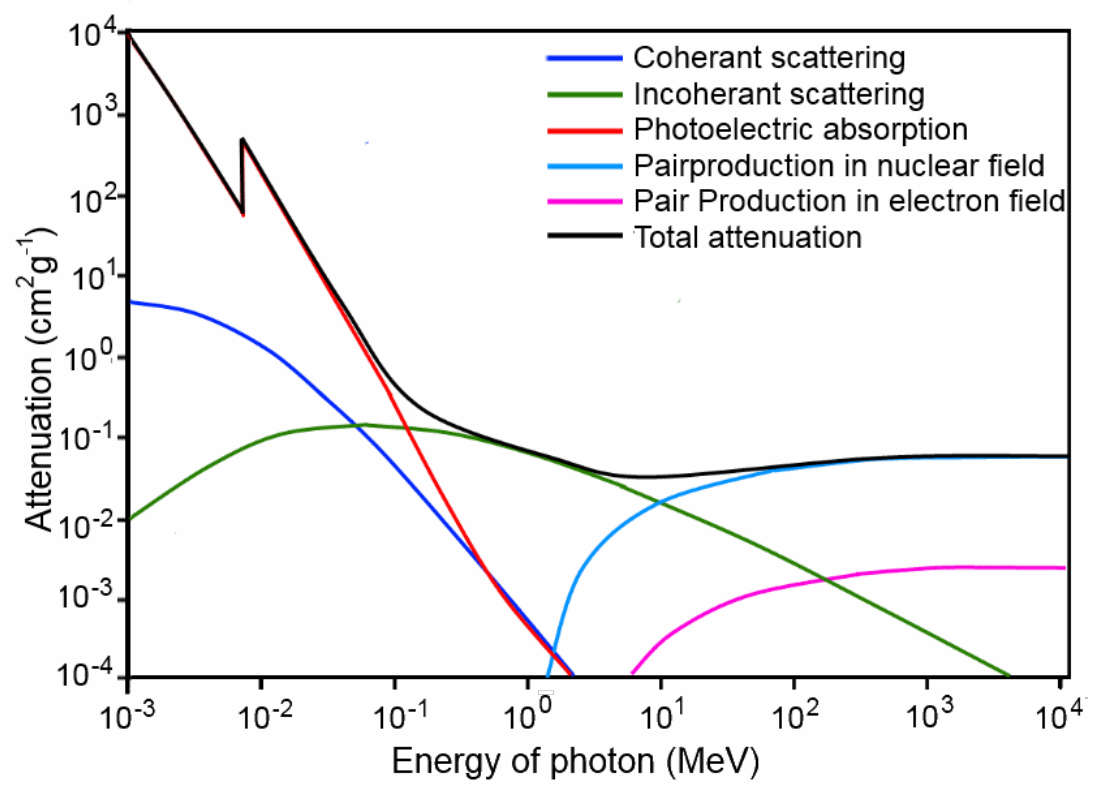

Figure 3.2: Overall $\gamma$-ray absorption and contribution from Rayleigh scattering, photoelectric effect, Compton scattering and pair production for iron [42].

dominates. Pair production may occur if the $\gamma$-ray energy is over $1.02 \mathrm{MeV}\left(2 \mathrm{~m}_{e}\right)$, but in practice requires much higher $\gamma$-ray energies.

\subsubsection{The photoelectric effect and Compton scattering}

The photoelectric effect occurs when a photon transfers all of its energy to a bound electron providing it with sufficient ionization energy to escape, and any excess energy appears as kinetic energy.

Compton scattering is the near elastic scattering of $\gamma$-rays by electrons. The photon scatters off the electron and in doing so transfers sufficient energy for the electron to be ionized, the $\gamma$-ray then continues on a deflected path with less energy. By treating the photon and electron collision as elastic, the conservation of energy and momentum give the Compton effect equation

$$
\Delta \lambda=\frac{h}{m_{e} c}(1-\cos (\theta))
$$

Where $\Delta \lambda$ is the difference in wavelength between the incident and scattered photons, $h$ is the Planck constant, $m_{e}$ is the electron rest mass, $c$ is the speed of light and $\theta$ is the angular difference in the paths of the incident and scattered photons. This equation gives the increase in wavelength (decrease in energy) of the scattered photon compared to the incident photon. 
By setting the angle to $180^{\circ}$ the wavelength change is maximised giving the "Compton edge" in $\gamma$-ray spectroscopy.

Absorption of $\gamma$-rays is most efficient for materials of high atomic number, and the absorption coefficient increases as the $\gamma$-ray energy decreases (provided the energy is sufficient to excite a K shell electron, below which there is a discontinuity). The cross section for the photoelectric effect $\left(\kappa_{P E}\right)$ per atom of atomic number $Z$, for a photon of energy $E(\mathrm{eV})$ is approximately [43]

$$
\kappa_{P E} \sim \frac{Z^{n}}{E^{3.5}}
$$

with $\mathrm{n}$ varying from 4 to 5 .

For Compton scattering the cross section $\left(\kappa_{C}\right)$ per atom is approximately [43]

$$
\kappa_{C} \sim \frac{Z}{E^{0.5}}
$$

Regardless of which interaction occurs the incident $\gamma$ ray ionizes an inner shell electron from the atom creating a highly energetic electron in the conduction band and hole in a core band in less than a picosecond. These are the 'primary' or 'hot' electrons and holes. The core hole rapidly thermalises to the valence band through an Auger process mechanism.

\subsubsection{Thermalisation}

At this stage the high mobility high energy electron and lower mobility hole now move through the crystal lattice colliding with more atoms and ionizing more electrons (outer shell electrons) to the conduction band, with corresponding holes in the valance band. This avalanche continues creating more and more 'secondary' electron hole pairs of much lower energy until they have insufficient energy to ionize any further electrons. The average energy required to ionize an atom is empirically 2 - 3 times the band gap of the material [12, 41]. This all happens very quickly, over $\sim 1$ picosecond [41]. 


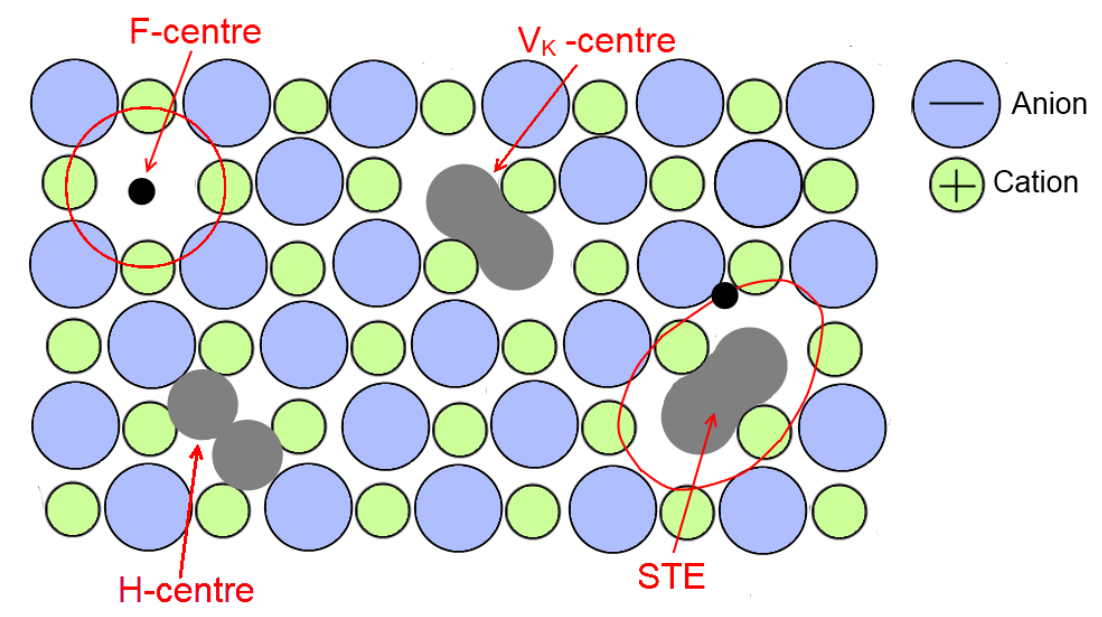

Figure 3.3: Selected crystal defects depicted in $\mathrm{NaCl}$ [12].

\subsubsection{Energy migration}

The energy migration stage which covers the transfer of energy from a multitude of electron and hole pairs in the conduction band and valence band respectively to excited luminescent centres is the most variable and difficult to predict, yet critical to the performance of the scintillator [41]. Ideally during this stage luminescent centres become excited due to impact excitation by hot electrons, sequential electron-hole capture (or sequential holeelectron capture). However before this can happen the electrons and holes may be become trapped.

There are many different types of trapping which may occur, for example by defects and impurities in the lattice, self-trapping by the crystal lattice, and the formation of impuritybound excitons [44]. Some trapping centres are depicted in figure 3.3 for a simple cubic lattice. An anion vacancy might bind an electron, this is an "F-centre". An "H-centre" is formed when a lattice distortion due to a hole binds a second anion at a single anion site forming a $\mathrm{X}_{2}^{-}$pseudomolecule, this has an overall neutral charge. Alternatively if the lattice distortion binds a hole to an anion which subsequently bonds to neighbouring anion this also forms a $\mathrm{X}_{2}^{-}$pseudomolecule but with a overall positive charge, this is a $\mathrm{V}_{K}$ centre. A different type of defect are excitons, spatially correlated electron-hole pairs. If an electron becomes localized on a $\mathrm{V}_{K}$ centre this is a self-trapped exciton [12,41,43]. All these traps have various energy levels and different stabilities. Eventually the trap may decay, either to release an electron (or hole) which may then excite the luminescent centre, or the trap may decay non-radiatively or 
radiatively via electron-hole recombination. The time scale of this stage varies greatly from picoseconds to milliseconds.

\subsubsection{Luminescence}

The final stage in the scintillation process is the radiation emission by excited luminescent centre. This is the same process as for photoluminescence which will be discussed in the next chapter.

\subsection{Performance measures}

Scintillators are characterised by many properties including; efficiency, decay time, afterglow, temperature stability, transparency, radiation hardness, density, emission wavelength, mechanical properties, chemical stability, cost, homogeneity, and linearity of response [13].

\subsubsection{Efficiency and light yield}

An efficient phosphor/scintillator will give a high light yield for a given incident x-ray flux, and this is important for spatial resolution, signal to noise ratio [5] and reduced exposure to harmful radiation. The light yield of a phosphor is characterised by the number of visible photons emitted per $\mathrm{MeV}$ of radiation incident on the material and depends on the absorption coefficient for x-rays of the material, the efficiency of exciton creation and capture at luminescent centres, the band gap and the intrinsic emission quantum efficiency of the luminescent centres [13]. A smaller band-gap increases the light yield [43, 45]. However, there is a trade-off because the band gap must not overlap with the luminescent centre's excited states as this can quench the luminescence. Dorenbos has estimated that for $\mathrm{Ce}^{3+}$ emission the band gap can be reduced to $2.5 \mathrm{eV}$ giving a $600 \mathrm{~nm}$ emission with maximum of 150,000 photons / MeV [43].

Currently theoretical light yield predictions far exceed experimental results [46]. The light 

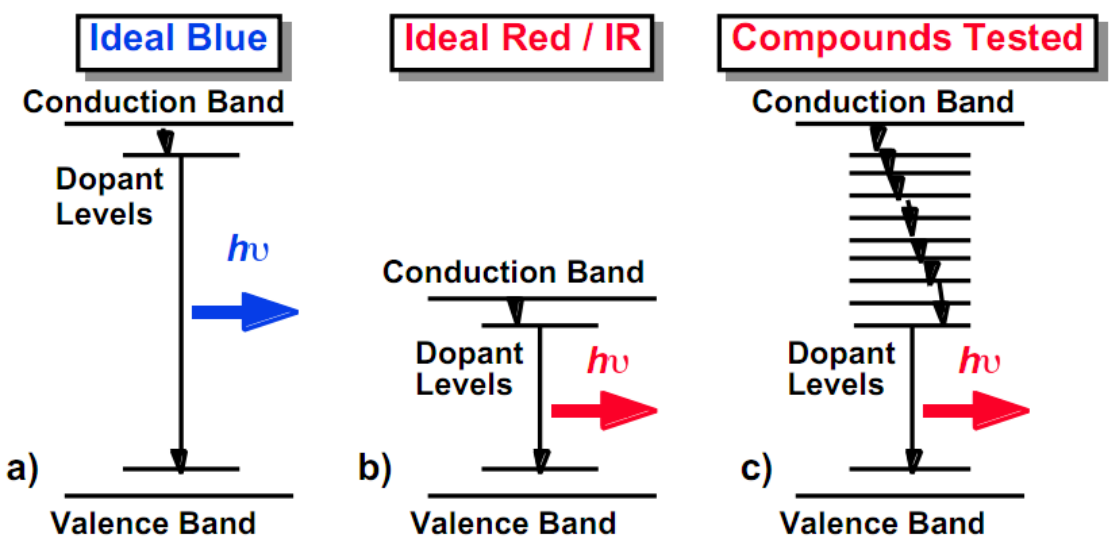

Figure 3.4: Energy level diagram of (a) an ideal blue emitting scintillator, (b) an ideal red emitting scintillator, and (c) the compounds tested by Moses et al. [11].

yield of a scintillator is estimated as [43]

$$
Y=10^{6} \frac{S Q}{\beta E_{g}}
$$

where $\beta E_{g}$ is the energy need to create one electron hole pair, $S$ is the quantum efficiency of the transport to the luminescent centre and $Q$ is the quantum efficiency of the luminescent centre. The usual channel of excitation for rare earth ions is a charge transfer process with a sequential capture of charge carriers. For scintillators with high light yield, the capture efficiency must be high.

In search of red-emitting scintillators in 1998 Moses et al. [11] tested over 1,100 compounds for scintillation in the $600 \mathrm{~nm}-900 \mathrm{~nm}$ region. Notable results included $\mathrm{Eu}^{3+}$ and $\mathrm{Sm}^{3+}$ doped $\mathrm{LuPO}_{4}, \mathrm{ScPO}_{4}$, and $\mathrm{YPO}_{4}$ showing intensities comparable to commercial phosphors but unfortunately all had decay times over $100 \mu$ s. Figure 3.4 shows the ideal set of energy levels for a red scintillator proposed by Moses et al. to give a much higher efficiency than the compounds tested.

\subsubsection{Decay time}

The decay time is another important property for a phosphor/scintillator and is generally the parameter used to distinguish between a phosphor and a scintillator. Phosphors have a long decay time and as such are used in applications involving integration under constant 
irradiation [1]. Scintillators have a significantly faster decay time $(<1 \mu \mathrm{s})$ and are used in applications involving single pulses, for example pulse counting and (anti-)coincidence measurements. A fast decay time gives a scintillator a high count rate capability and enables it to be used for coincidence counting [5], and is also important for image quality and real time imaging [3]. The decay time of a scintillator basically depends on two processes. First, the speed of the transfer of free electrons and holes from the original ionisation to the luminescence center, and secondly, the lifetime of the emitting state of the luminescent centre [47]. As for photoexcitation, the lifetime of the luminescent centre generally shows an exponential decay after excitation ceases

$$
I(t)=I_{0} \exp (-t / \tau)
$$

the time constant of this decay is known as the primary decay time $(\tau)$ and is intrinsic to the emitting site. It is the inverse of the probability of the corresponding radiative transition per unit time from the luminescent state [3] and is given by

$$
\Gamma=\frac{1}{\tau} \propto \frac{n}{\lambda_{e m}^{3}}\left(\frac{n^{2}+2}{3}\right) \sum_{f}|<f| \mu|i>|^{2}
$$

where $n$ is the refractive index of the crystal and $\lambda_{e m}$ the emission wavelength and $f$ and $i$ denote the final state and initial state respectively. The matrix element connecting both states via the dipole operator $\mu$ is only appreciable when the two states are of different parity. This is the case for transitions between $4 \mathrm{f}^{n-1} 5 \mathrm{~d}^{1}$ and $4 \mathrm{f}^{n}$ configurations as in $\mathrm{Ce}^{3+}, \mathrm{Pr}^{3+}, \mathrm{Nd}^{3+}$, $\mathrm{Sm}^{2+}$ and $\mathrm{Eu}^{2+}$ and also for transitions between $6 \mathrm{~s}^{2}$ and $6 \mathrm{~s}^{1} 6 \mathrm{p}^{1}$ configurations as in $\mathrm{Tl}^{+}, \mathrm{Pb}^{2+}$, and $\mathrm{Bi}^{3+}$ [13]. Equation 3.7 shows the decay time of an allowed transition is much faster than that for a forbidden transition due to their higher transition probabilities $\left(\left.\sum_{f}|<f| \mu|i\rangle\right|^{2}\right)$, and UV transitions tend to have faster decay times than their longer wavelength counterparts due to the $\lambda^{-3}$ factor. However the decay time under x-ray or $\gamma$-ray excitation is generally longer than under UV excitation due to limitations in the the energy transfer rate from the electron-hole pairs formed under irradiation, as opposed to direct excitation of a luminescent centre. Currently the fastest known scintillator is undoped $\mathrm{BaF}_{2}$, which has a $0.8 \mathrm{~ns}$ decay component, although it should be noted that this is the fast component of a two component 
decay with the other being $630 \mathrm{~ns}$. Undoped $\mathrm{BaF}_{2}$ is a very special scintillator in that the emission is due to an interband transition between the valence band and a hole in the nearby core band. $\mathrm{NaI}(\mathrm{Tl})$ is a well known commercial scintillators with a primary decay time of 230 ns [30].

\subsubsection{Density}

Materials with a density $(\rho)$ that is high and with large atomic numbers $(Z)$ are desirable. A high density material means the scintillator can be compact. Materials with large atomic numbers have higher $\gamma$-ray stopping power and absorb more of the incident energy, which is important for detection efficiency [5]. As mentioned above the the attenuation of a $\gamma$-ray beam depends on the energy but is is approximately proportional to $\rho \mathrm{Z}^{n}(n=4$ to 5 ) for the photoelectric effect, or $\rho \mathrm{Z}$ for Compton scattering. These $\gamma$-rays which pass through the scintillator result in reduced efficiency, in addition these $\gamma$-rays can interact with the detector and add to the noise in the system [3].

\subsubsection{Emission wavelength}

The emission of the scintillator should match the peak sensitivity of the photodetector. Standard photomultiplier tubes have maximum quantum efficiency between 280 and $500 \mathrm{~nm}$. In contrast the quantum efficiency of silicon diodes photodetectors peaks between 500 and $900 \mathrm{~nm}[13]$. This was discussed in section 2.4.

\subsubsection{Mechanical and chemical properties}

In some industrial applications scintillators may be in a severe environment and require shock resistance and mechanical hardness [5, 13]. It is also desirable that the materials be chemically stable in atmospheric conditions. Many materials are hygroscopic and hence require protection. The importance of this depends strongly on the application; for example $\mathrm{NaI}(\mathrm{Tl})$ is very commonly used despite its hygroscopic nature meaning it requires encapsulation to protect it from the environment [5]. 


\subsubsection{Cost}

Low cost materials are desirable, especially for applications requiring mass production. The availability and cost of raw materials, production method and any extra requirements such as hermetic sealing of hygroscopic materials all contribute to the overall cost [13]. The environmental energy cost is also relevant. Materials requiring high formation temperatures and energy intensive processes are less desirable.

\subsubsection{Afterglow}

Afterglow is delayed luminescence which occurs due to belated thermal release of electrons and holes trapped by lattice defects. Afterglow is highly temperature dependent as the traps cannot be released without sufficient thermal energy. In photon counting applications a long afterglow reduces the signal to noise ratio and increases the dead time of the detector. Long afterglow is also a significant hindrance to any real time imaging as it leads to "ghosting" [3]. Afterglow is generally reported as the fraction of luminescence still occurring a certain time after the material receives a specified dose of $\mathrm{x}$-irradiation. For example the light output of $\mathrm{NaI}(\mathrm{Tl})$ drops to $1 \%, 0.6 \%$ and $0.2 \%$ when measured 10, 20 and $50 \mathrm{~ms}$ respectively after a short $\gamma$-ray pulse.

\subsubsection{Temperature stability}

Scintillators are generally used at room temperature. However the operation temperature will fluctuate as other nearby electronic components warm and the ambient temperature varies. If the pulse amplitude is important (eg. in $\gamma$-ray spectroscopy) then the scintillator must operate with a constant quantum efficiency over the range of expected temperatures. The photoluminescent efficiency tends to decrease as temperature increases due to the thermal Boltzmann population of excited states increasing the probability of non-radiative transitions which may quench the radiative transitions [13]. The temperature coefficient for performance specification is particularly important for oil-well logging applications where the temperature may rise to well over $100{ }^{\circ} \mathrm{C}$. 


\subsubsection{Optical properties}

The transparency, transmission and refractive index are often considered the most important properties for a scintillator [3, 13]. These properties are important as the visible scintillation photons need to be efficiently transported to the photodectector. Any scattering will lengthen their path and increase the chance of absorption [3, 13, 44]. It is desirable to match the refractive index of the scintillator and the detector to prevent reflections at the interface. For both silicon photodetectors and photomultiplier tube windows this is around $n=1.5$ [13] [5]. Refractive index matched optical grease is frequently used to provide higher optical coupling between the scintillator and photodetector.

\subsubsection{Radiation hardness}

A scintillator interacts with ionising radiation in order to function, but this radiation has the potential to permanently change the scintillation efficiency which is undesirable. Studies have shown that the most important effect of radiation damage is to reduce the optical transmission [13].

\subsubsection{Proportionality of response}

For high energy resolution it is necessary to have a linear response to the incident $\gamma$-ray energy over as wide a range as possible. This means the scintillator can discriminate between different energy $\gamma$-rays [5,13]. In recent years there has been a lot of research into the non-linearity of many scintillators, which appears difficult to predict [48, 49].

\subsection{Commercial Scintillators}

The main scintillation properties of some commercially used scintillators are tabulated in table 3.1 (which has been partially reproduced from reference [12]). Also included are those which have reached the highest standards in a particular measure, for example $\mathrm{BaF}_{2}$ for its 
very short lifetime. The lower section of the table includes some red-emitting scintillators which are currently available.

Some of the scintillators in table 3.1 (e.g. $\mathrm{NaI}, \mathrm{LaCl}_{3}$, etc.) require doping with an activator substance in order to obtain optimum scintillation properties. $\mathrm{NaI}(\mathrm{Tl})$ has been used extensively in SPECT and to a lesser extent in PET, although it is both hygroscopic and fragile. However the scintillator most commonly used in PET scanners using block-detectors is $\mathrm{Bi}_{3} \mathrm{Ge}_{4} \mathrm{O}_{12}$, which has a high effective atomic number [52]. $\mathrm{Bi}_{3} \mathrm{Ge}_{4} \mathrm{O}_{12}$ is not hygroscopic and does not have long-lived secondary scintillation components [41]. $\mathrm{SrI}_{2}: \mathrm{Eu}^{2+}$ utilises the strongly allowed $\mathrm{Eu}^{2+}$ luminescence resulting in one of the highest known light outputs, but suffers from a long decay intrinsic to $\mathrm{Eu}^{2+}$ luminescence and the extreme hygroscopy of $\mathrm{SrI}_{2}$. $\mathrm{BaF}_{2}$ exhibits core valence luminescence and is one of the fastest known scintillators but also has a much longer component along with the 0.8 ns decay component [43].

The scintillator options for use with silicon photodiodes include certain plastic scintillators, but due to low density and low effective atomic mass these show poor $\gamma$-ray absorbance and degrade under $\gamma$-irradiation. $\operatorname{CsI}(\mathrm{Tl})$ is the most commonly used material for silicon photodiodes; it has a high light output but a relatively slow lifetime of $980 \mathrm{~ns}$ and the emission is green $(540 \mathrm{~nm})$ [45, 53]. $\mathrm{Gd}_{2} \mathrm{O}_{2} \mathrm{~S}:\left(\mathrm{Ce}^{3+}\right.$ or $\left.\mathrm{Pr}^{3+}\right)$ is also widely used; this offers a high density $\left(7.54 \mathrm{gcm}^{-3}\right)$ and faster lifetime than $\mathrm{CsI}(\mathrm{Tl})(\sim 300 \mathrm{~ns})$ but the emission is only at $510 \mathrm{~nm}$. $\mathrm{Lu}_{2} \mathrm{~S}_{3}: \mathrm{Ce}^{3+}$ has a faster $32 \mathrm{~ns}$ decay but suffers from a lower light output $(28,000$ photons $/ \mathrm{MeV})$, uses rare and expensive, radioactive constituent chemicals, and has a high refractive index which causes light losses at the scintillator-detector interface [16]. Cerium doped YAG $\left(\mathrm{Y}_{3} \mathrm{Al}_{5} \mathrm{O}_{12}\right)$ is another red emitting scintillator with a fast component (100 ns) to its luminescent decay but it also has a second slow component and an even lower light output (16,700 photons/MeV) [54]. ZnSe(Te) has a very high light out-put of 55,000 photons $/ \mathrm{MeV}$ but the decay is too long at $50 \mu \mathrm{s}$ for some phosphor applications and all scintillation applications [14]. 


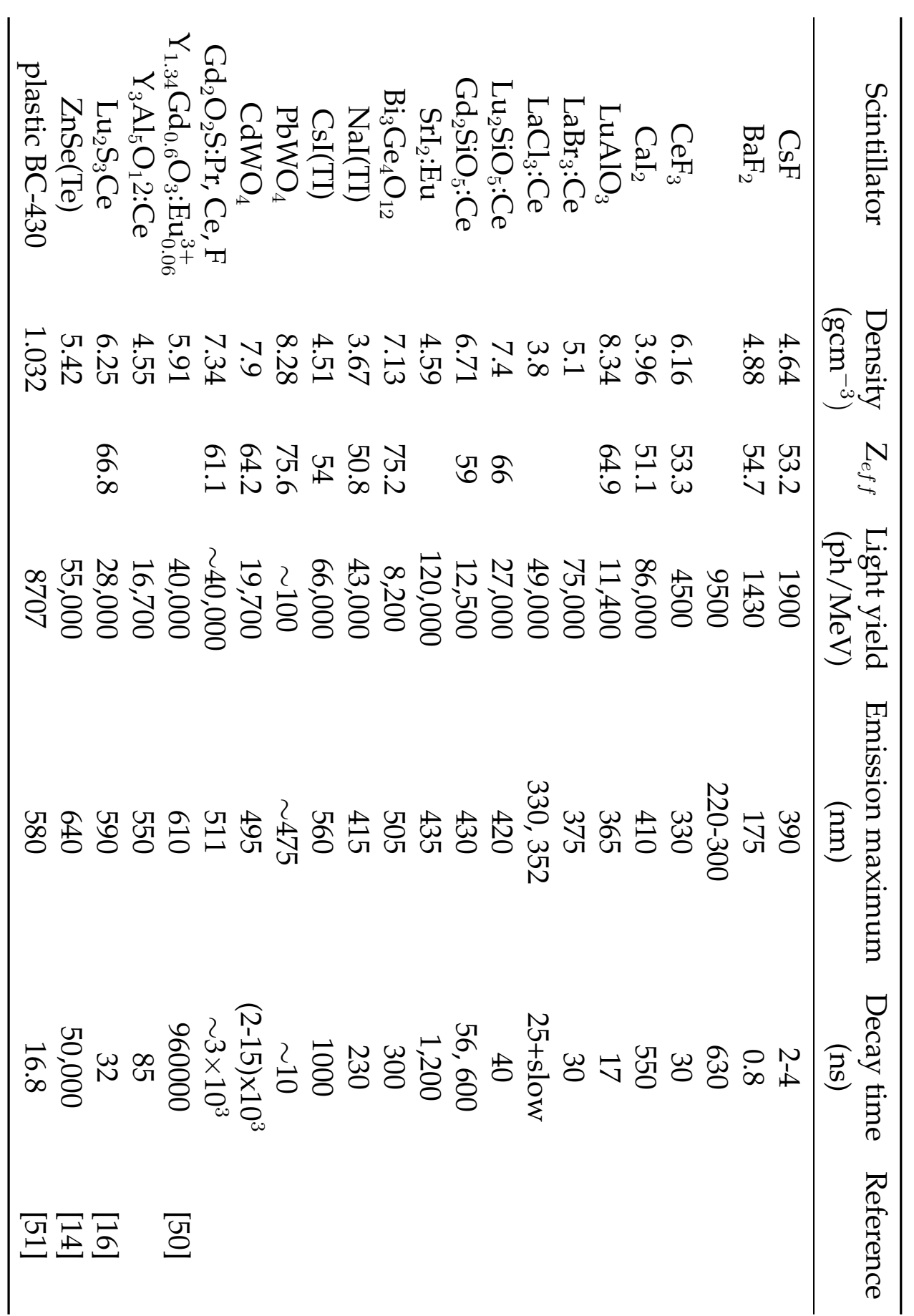




\subsection{Applications}

As discussed in chapter 2, scintillators and phosphors are used in many different applications. They are the primary radiation detectors used in medical diagnostics, radiography and industrial component inspection [3,4] but recently the largest market for scintillators is actually high energy physics calorimetry [55]. However, this is likely to be a one-off market event with the completion of the CERN large hadron accelerator.

In security, baggage scanners at airports use phosphors coupled to silicon photodetectors to image the contents of bags as they pass through the system. For this application it is important that afterglow is minimised to prevent ghosting or 'smeared' images which occur when the previous image has not fully decayed before the next appears [46]. For this reason $\mathrm{Gd}_{2} \mathrm{O}_{2} \mathrm{~S}: \mathrm{Pr}^{3+}$ or $\mathrm{CdWO}_{4}$ is generally used as it has low afterglow. Checking structural properties such as concrete foundations and aeroplane parts without damaging their integrity is another important application. In this application the attenuation of $\gamma$-rays through the object is measured highlighting any defects. In high energy physics research scintillators are used for calorimetry (determining the energy of photons), the collision of proton beams produces high particles and protons which need to be detected. In this case it is critical that the light output is proportional to the incident photon energy, that the scintillator has a high stopping power and is very radiation hard [12]. In order to evaluate bore holes, in oil exploration scintillators are used to detect the gamma ray emission intensity hence rock type, this helps indicate the likelihood of oil. For this application the materials and detectors must be very rugged and able to withstand high temperatures.

Medical applications such as positron emission tomography (PET) scans, computed tomography $(\mathrm{CT})$ scans and the newer single positron emission computed tomography (SPECT) scans all require the use of a scintillator/phosphor coupled with a detector. In a PET scan a patient ingests a biologically active molecule (fluorodeoxyglucose) with a radioactive tracer $\left({ }^{18} \mathrm{~F}\right)$ incorporated. The ${ }^{18} \mathrm{~F}$ decays inside the body giving out a positron which quickly meets an electron; the two annihilate and produce two identical gamma emissions (511 keV) in opposite directions. These are coincidence-detected on opposing sides of a ring of detectors. The concentrations of ${ }^{18} \mathrm{~F}$ imaged indicate tissue metabolic activity and the possibility of 
cancer metastasis. In this application the timing is critical as the time of flight difference indicates where the decay occurred and hence where the ${ }^{18} \mathrm{~F}$ has accumulated; thus this application requires a scintillator with a fast decay [41,46], the scintillator must also have a high detection efficiency for $511 \mathrm{keV}$ gamma rays and a high scintillation light yield. Most PET scanners use $\mathrm{BGO}\left(\mathrm{Bi}_{4} \mathrm{G}_{3} \mathrm{O}_{12}\right)$ which has a light output of 8,200 photons $/ \mathrm{MeV}$ and a 300 ns decay time [46]. Single-photon emission computed tomography (SPECT) is similar to a PET scan but the radioactive tracer emits a single gamma ray which is detected. Generally ${ }^{99} \mathrm{Tc}$ is used which has a longer half-life than that of ${ }^{18} \mathrm{~F}$ (6 hours compared to $\sim 2$ hours). This technique is much cheaper but less accurate than PET [46].

This differs from the requirements for a CT scan; in CT scans the patient is x-irradiated from one side and the resulting attenuated beam detected on the other side; using scanning a $3 \mathrm{~d}$ image is built up. For this technique the decay time is not as important as the luminescence is generally integrated to form an image, however the afterglow (prolonged luminescence after irradiation) must be minimised to prevent artefacts from previous images being included in the next one. This is a fast and accurate technique but exposes patients to a high $\gamma$-ray dose [46]. A single CT scan can give a patient a x-ray dose five times that of the average annual dose [56]. The dose the patient receives would be reduced with increased scintillator sensitivity. CT scanners generally use single crystals of $\mathrm{CaWO}_{4}$ as these show minimal afterglow of $0.005 \% 3$ milliseconds after irradiation [46,57]. 


\section{Chapter 4}

\section{Scintillators and Phosphors: Spectroscopy}

\section{and materials science}

In this chapter we discuss the background to the materials and techniques discussed in later chapters.

\subsection{Crystal structure}

The crystal structure of a solid describes the unique arrangement of the atoms or molecules which exhibits long-range order and symmetry. The unit cell is the smallest arrangement of atoms which can be translated in space to form the periodic bulk structure [58]. All crystal structures fall into one of seven crystal lattice classes according to their symmetry. The most symmetric hence simple system is the cubic system, this exhibits four threefold rotational axes oriented at $109.5^{\circ}$ (the tetrahedral angle) with respect to each other. There are many different cubic crystal structures; figure 4.1 shows the cubic structure of $\mathrm{CaF}_{2}$ of particular interest in this work. While the fluorite crystal structure derives its name from being the structure calcium fluoride naturally forms in, many other compounds of the form $\mathrm{AX}_{2}$ also form in the same structure. The lattice can be visualised as a simple cubic structure of anions with every second cube filled by a cation at the body centre position.

Most commercial scintillators are single crystals. This is because while these are difficult 


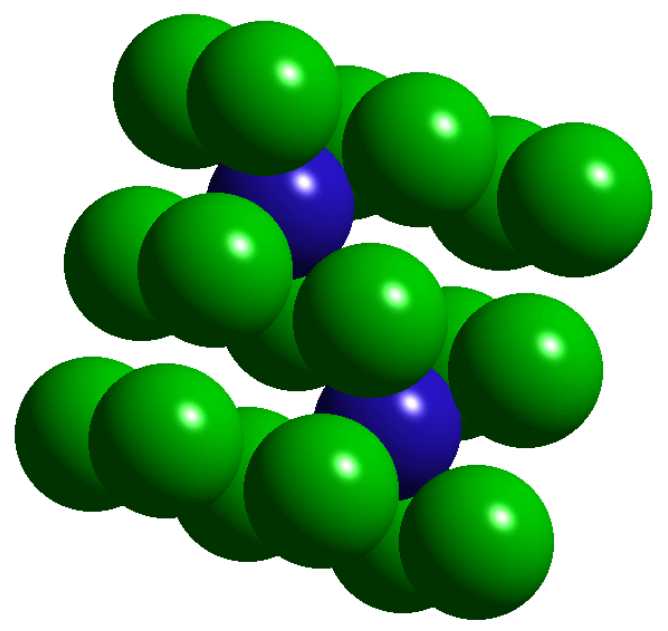

Figure 4.1: Cubic fluorite structure, a simple cubic lattice of anions (green) with half the cubes filled with a cation (blue).

and expensive to grow they are highly transparent; an important property for a scintillator. However, in this work we study primarily polycrystalline ceramics as these are much easier, faster, and cheaper to produce. These will only be transparent if the crystal structure is cubic. Cubic structures are unique in that the refractive index is isotropic; in other structures the refractive index for propagation varies along different axes. In a polycrystalline materials the grains are all randomly aligned, and so for non-cubic structures, scattering occurs at the grain boundaries where there is a refractive index mismatch. For cubic materials there is no mismatch so transparent polycrystalline materials can be made. Ceramic polycrystalline materials can be made by melting or sintering constituent chemicals, whereas single crystals are slowly grown at closely controlled temperatures at typical rates of tens - hundreds of $\mu \mathrm{m}$ per hour. The first polycrystalline ceramic scintillator to be developed was $(\mathrm{Y}, \mathrm{Gd})_{2} \mathrm{O}_{3}: \mathrm{Eu}^{3+}$, a cubic material specifically made for high performance medical detectors and commercially introduced in General Electrics premium x-ray computed tomography scanner in 1988. While this has been successfully used, the long luminescent lifetime of $\mathrm{Eu}^{3+}$ is a limitation for fast acquisition [3,59].

\subsubsection{X-ray diffraction}

The standard way to determine the crystal structure of a material is to use $x$-ray diffraction (XRD). In XRD a source of monochromatic plane $x$-ray waves irradiates the crystal, and each 


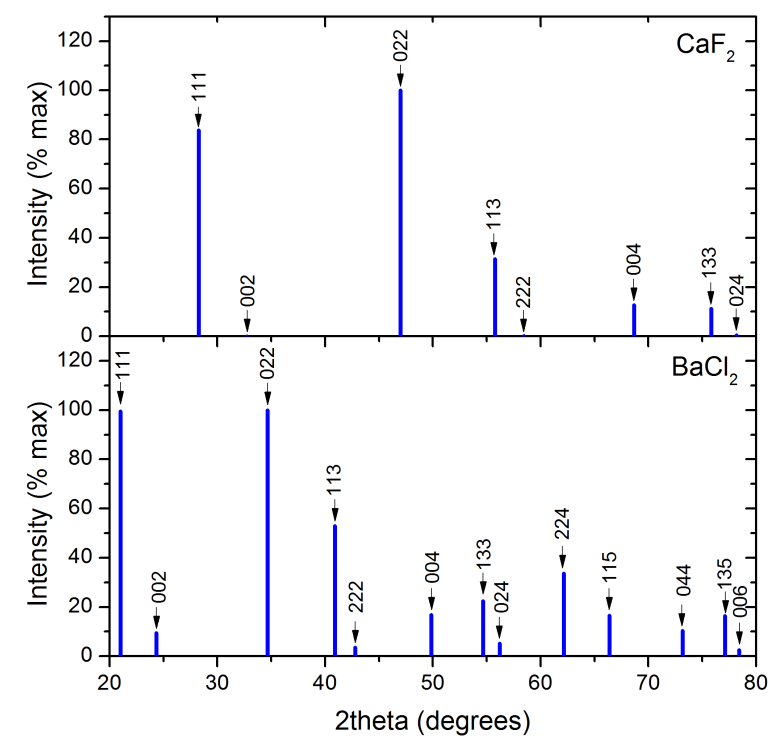

Figure 4.2: Simulated XRD patterns for $\mathrm{CaF}_{2}$ (top) and cubic $\mathrm{BaCl}_{2}$ (bottom); both materials share the same fluorite structure.

atom acts as a centre of scattering. A detector records the intensity of the diffracted beam as a function of angle. The angle of incidence and detection $(\theta)$ are kept equal and varied, typically from $10^{\circ}$ to $20^{\circ}$. The scattering from planes of atoms (of spacing $d$ ) interfere to give a high intensity beam when Bragg's law

$$
\lambda=2 d \sin (\theta)
$$

is satisfied, where $\lambda$ is the $x$-ray wavelength. Every compound has a unique diffraction pattern, and the peaks correspond to reflections off certain planes in the crystal. These planes are labelled by Miller indices; Miller indices are the reciprocals of the fractional intercepts which the plane makes with crystallographic axes [60].

Shown in figure 4.2 are the simulated XRD patterns for $\mathrm{CaF}_{2}$ (top) and cubic $\mathrm{BaCl}_{2}$ (bottom) both of which share the same fluorite structure. Despite having the same structure the patterns look quite different at first glance. The same Miller indices lines are observed, but at different positions. This is due to the difference in the lattice parameter ( $d$ in equation 4.1 ). $\mathrm{CaF}_{2}$ has a lattice parameter of $5.46 \AA$ while that of $\mathrm{BaCl}_{2}$ is much larger at at $7.34 \AA$ [61] so from equation 4.1 the lines spacing is reduced for $\mathrm{BaCl}_{2}$.

Only lines corresponding to certain planes are observed, for example there is no line labelled $<100>$ but there is a $<100>$ plane in the material. The lines observed are determined by the 
structure factor $(S(h k l))$

$$
S(h k l)=\sum_{j} f_{j} \exp \left(-i 2 \pi\left(x_{j} h+y_{j} k+z_{j} l\right)\right)
$$

where the sum is over all the atoms $(j)$ in the unit cell, $(h, k, l)$ are the Miller indices of the plane under consideration and $f_{j}$ is the atomic scattering factor of the atom $j$. The structure factor thus consists of a geometric factor $\left(\exp \left(-i 2 \pi\left(x_{j} h+y_{j} k+z j_{l}\right)\right)\right)$ and a atomic scattering factor. The atomic scattering factor reflects the ability of a atom to scatter the incident x-rays, and so is larger for elements of higher atomic mass. The geometric factor represents whether constructive or destructive interference will occur [60]. In very generalised terms the XRD patterns of $\mathrm{CaF}_{2}$ and $\mathrm{BaCl}_{2}$ reflect a strong contribution from the heavier cations, with a weaker contribution from the lighter anions.

In a cubic crystal the lattice constant a is related to the Miller indices $(h, k, l)$ and the interplanar distance $d$ by the relation [60]

$$
d=\frac{a}{\sqrt{h^{2}+k^{2}+l^{2}}}
$$

This can then be combined with Bragg's law (equation 4.1) to give

$$
\frac{\lambda}{2 a}=\frac{\sin \theta}{\sqrt{h^{2}+k^{2}+l^{2}}}
$$

Thus a plot of $\sin (\theta)$ against $\sqrt{h^{2}+k^{2}+l^{2}}$ gives a linear relationship from which the lattice parameter $(a)$ can be found provided the x-ray wavelength is known. Such plots have been used here to determine the cubic lattice parameter.

\subsection{Spectroscopy}

\subsubsection{Absorption}

Absorption spectra are useful in determining the energy level scheme of a material and finding at which wavelengths it is desirable to pump in order to get luminescence [62]. When 


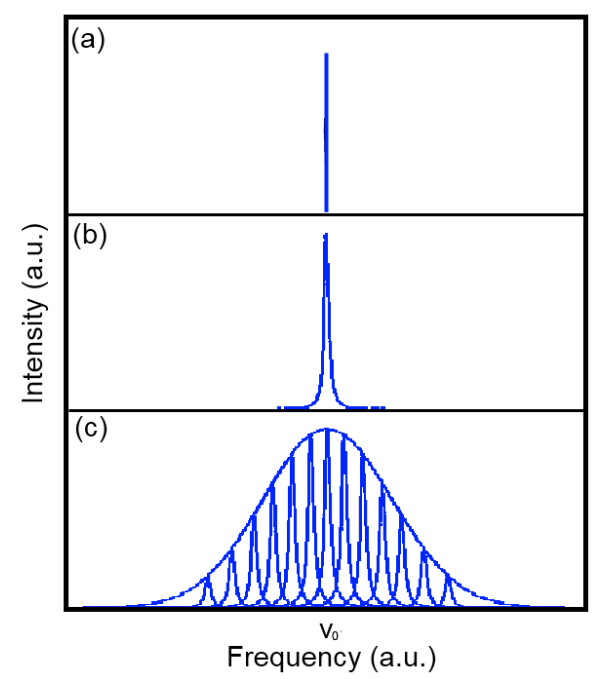

Figure 4.3: Schematic plot of absorption for two level system: (a) shows the expectation with no broadening, (b) the natural broadening and (c) the result of natural and inhomogeneous broadening [36].

light of intensity $I_{0}$ is incident on a material of thickness $x$ the transmitted intensity is given by the Lambert-Beer law

$$
I=I_{0} \exp (-\alpha x)
$$

When scattering can be considered negligible $\alpha$ is the linear absorption coefficient of the material. In a simple two level system with population densities of $\mathrm{N}$ in the ground state and $\mathrm{N}^{\prime}$ in the excited state the expression for $\alpha$ can be written

$$
\alpha=\sigma(\nu)\left(N-N^{\prime}\right)
$$

furthermore if $N^{\prime}$ is far less than $N$ this can be approximated as

$$
\alpha=\sigma(\nu) N
$$

in which $\sigma$ is the transition cross section. The transition cross section parametrises the absorption of incoming light of frequency $\nu$, and is proportional to the square of the matrix element $\left|<\phi_{e}\right| \mathcal{H}\left|\phi_{g}>\right|$, in which $\phi_{g}$ and $\phi_{e}$ are eigenfunctions corresponding to the ground and excited states respectively and $\mathcal{H}$ is the interaction Hamiltonian between the incoming light and system [63].

Figure 4.3 (a) shows the expected absorption frequency for such a two level system where $\nu_{0}$ is 


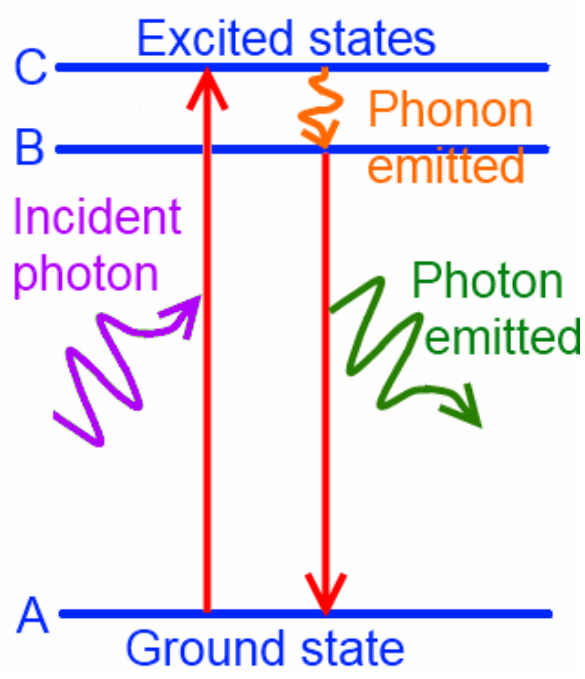

Figure 4.4: Simple depiction of photoluminescence process.

the energy difference between the levels divided by Plancks constant. However an absorption spectrum is not simply a delta function, but a Gaussian curve as shown in figure 4.3 (c). The line broadening has two main causes; the broadening shown in figure 4.3 (b) is called natural broadening and arises from Heisenberg's uncertainty principle. The larger effect is that of inhomogeneous broadening which is due to the various absorbing centres having slightly different resonant frequencies due to small distortions in the crystal environment that are always present. The measured absorption, as seen in figure 4.3 (c) is actually the convolution of peaks from all the different centres weighted by concentration [36]. Most systems comprise of multiple levels which leads to multiple absorption peaks of varying intensity depending on the transition probabilities.

\subsubsection{Fluorescence}

Studying photoluminescence spectra provides insight into the electronic structure of a material. In the current context, photoluminescence spectroscopy helps in the understanding of the last stage in a scintillation process where the residue of the ionisation energy is emitted as light. Photoluminescence refers to the absorption of a photon by a luminescent centre and subsequent re-emission of a lower energy photon from the resulting excited state. This is depicted in figure 4.4. The absorption part of the process is referred to as excitation and the return to the ground state as emission. This is a much simplified depiction, and in reality 


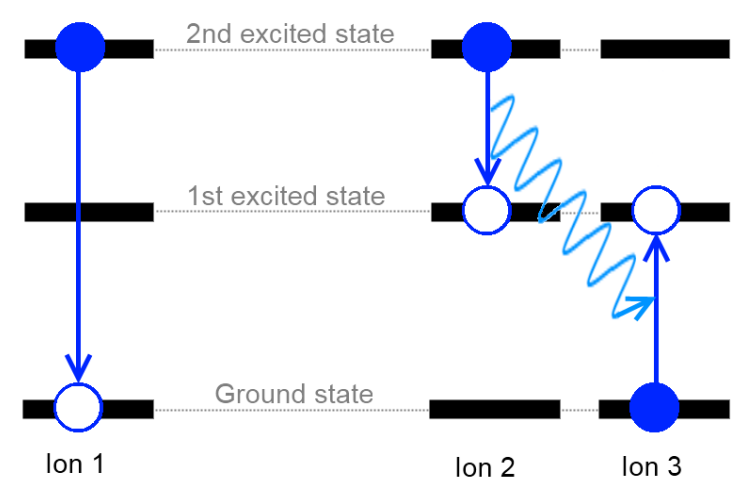

Figure 4.5: Simple example of cross relaxation for a three level luminescent centre [64].

there are generally a multitude of excited states and non-radiative relaxation processes occur alongside and in competition with the radiative transitions.

\subsubsection{Concentration quenching}

Increasing the concentration of the optically active ion increases the intensity of luminescence at first in proportion to the concentration, however, this is only true up to a critical concentration. Above this critical concentration the luminescence yield decreases, essentially due to very efficient energy transfer between luminescent centres ("cross-relaxation"). There are two mechanisms by which this may occur. The first occurs when the concentration is high enough that the distance between the luminescent centres is small enough for energy transfer between the centres to be more probable than emission. In this case the energy migrates from centre to centre through the material until it eventually reaches a defect or impurity in the crystal (quenching trap) and is lost by infra-red emission or multi-phonon relaxation [36].

The other mechanism occurs by resonant energy transfer between two identical adjacent centres [36], and is illustrated in figure 4.5 for a 3 level system. For the isolated centre (ion 1), emission from the second excited state dominates, but when two ions are close (ions 2 and 3) some energy can be transferred from the ion in the higher state (ion 2) to that in the ground state (ion 3) leaving both ions in the first excited state. Emission then occurs from this lower state and the higher level emission has been quenched.

Concentration quenching can also be recognised by a decrease in the lifetime of the lumi- 
nescence. This is because quenching involves energy transfer processes which happen on a much faster time-scale than luminescence, so there is a significantly decreased probability of slower luminescence. (There is also potential that a very high concentration could lead to clusters of luminescent centres with different energy levels and decay times compared to isolated centres [36]).

\subsection{Spectroscopy of rare earth ions in crystals}

The lanthanides, also known as the rare earths, are the 14 elements after lanthanum with atomic numbers from 57 to 70 . The ground state electron configurations of the neutral atoms are $[X e] 4 f^{n} 5 d^{m} 6 s^{2}$ with $n$ varying from 1 for cerium to 13 for ytterbium as the $4 f$ electron shell is progressively filled ( $\mathrm{m}=0,1$ to allow for the extra stability of a half filled electron shell). The common trivalent state is formed by the ionisation of the $5 \mathrm{~d}^{1} 6 \mathrm{~s}^{2}$ electrons or one 4f electron and the $5 d^{1} 6 s^{1}$ electrons leaving [Xe] $4 f^{n}$. Lanthanide ions are used as luminescent centres in a wide variety of materials such as glasses, polymers and crystals. While they are most commonly found in the trivalent oxidation state some rare earth ions also occur in the divalent state, most notably $\mathrm{Eu}^{2+}, \mathrm{Sm}^{2+}$ and $\mathrm{Yb}^{2+}$.

\subsubsection{Free ions}

There are many excited states within the $4 \mathrm{f}^{n}$ configuration over a range of $\sim 40,000 \mathrm{~cm}^{-1}$. The next highest configurations are the $4 \mathrm{f}^{n-1} 5 \mathrm{~d}^{1}, 4 \mathrm{f}^{n-1} 6 \mathrm{~s}^{1}$ and $4 \mathrm{f}^{n-1} 6 \mathrm{p}^{1}$; for trivalent ions these are normally well above the excited $4 \mathrm{f}^{n}$ energy levels [63]. Within a configuration the splitting of energy levels of the free ions arises predominantly from the coulomb interaction and spin orbit coupling, these effects are depicted in figure 4.6 for the $4 \mathrm{f}^{6}\left(\mathrm{Sm}^{2+}, \mathrm{Eu}^{3+}\right)$ configuration along with the effect of a crystal field. The coulomb interaction is the largest effect; it splits each configuration into levels specified by $L$ (the orbital angular momentum quantum number) and $S$ (the total spin quantum number). Spin-orbit coupling of the individual orbital angular momenta $l_{i}$ and $s_{i}$ between electrons is then considered, and can be expressed in terms of the total angular spin momenta $(\underline{L}$ or $\underline{S})$. Mostly the energy separation of the different $L S$ terms 


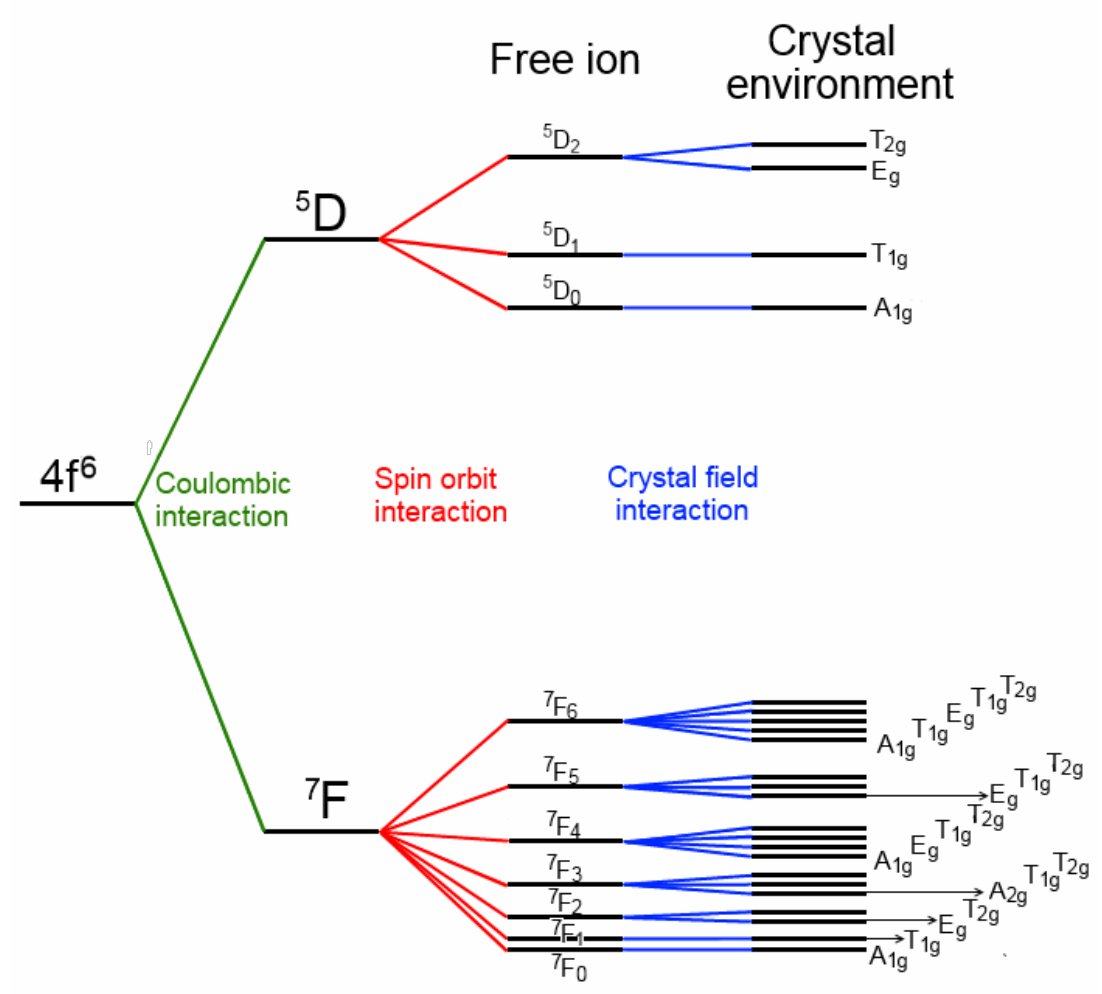

Figure 4.6: Depiction of energy levels of $4 \mathrm{f}^{6}$ configuration for a cubic crystal field as split by the coulombic interaction, spin orbit interaction and crystal field interaction. The free ion states are labelled as ${ }^{2 S+1} L_{J}$.

is much larger than the spin orbit coupling energy so there is little mixing of the $L S$ terms, and if this is neglected, as in the Russell-Saunders approximation, the degeneracy of the free ion term is lifted and the term split into multiplets labelled by the total angular momentum (J) quantum number $J$.

$$
\underline{J}=\underline{L}+\underline{S}
$$

where $J=L+S, L+(S-1), . ., L-S$. This partially lifts the degeneracy of the $L S$ term. The resulting $(2 J+1)$ degeneracy for each multiplet labelled by ${ }^{2 S+1} L_{J}$ is the starting point of most theoretical considerations of the rare earth ions in solids [63].

\subsubsection{Crystal field splitting}

As depicted in figure 4.6 when the free ion is located in a crystal the degeneracy of the energy levels is further lifted due to the influence of surrounding ions and the electric field they produce. Figure 4.7 shows energy levels of the $4 \mathrm{f}^{n}$ configuration for all the trivalent rare earth ions in $\mathrm{LaCl}_{3}$. The crystal field effect is seen in the thickness of the lines representing 


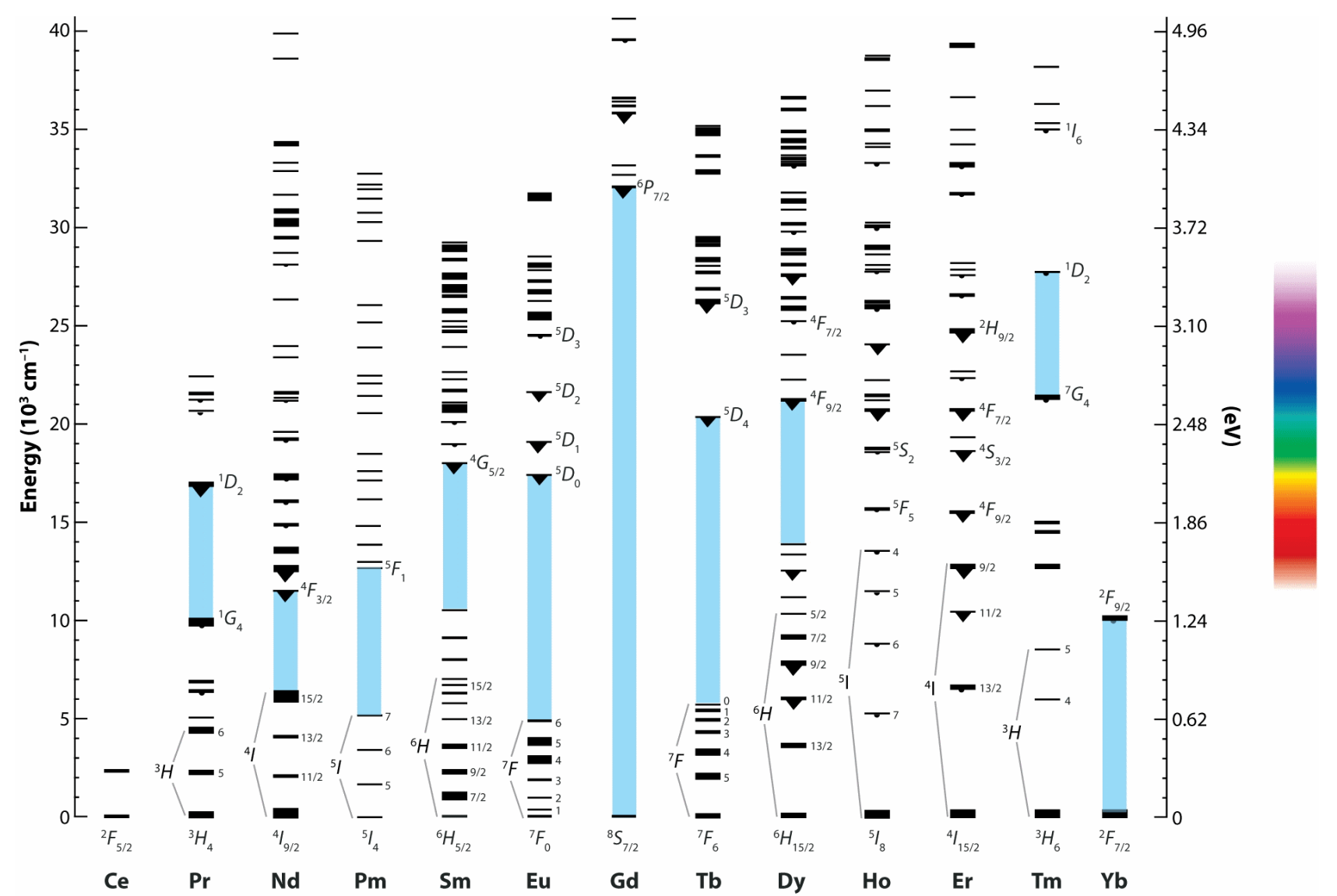

Figure 4.7: The Dieke diagram shows the energy levels of all trivalent rare earth ions in $\mathrm{LaCl}_{3}$ [65].

the energy levels while coulomb interactions and spin-orbit coupling cause the splittings. The effect of the crystal field is much weaker than that of spin-orbit coupling for the $4 \mathrm{f}^{n}$ configurations because of the shielding from the crystal field afforded by the $5 s^{2}$ and $5 p^{6}$ outer shells. For rare earth ions in crystals the crystal field effect is normally weak, meaning the free ion energy levels are only slightly perturbed, so the free ion wavefunctions can be used as basis functions to which crystal field Hamiltonian $\left(H_{C F}\right)$ can be applied as a perturbation over the ${ }^{2 S+1} L_{J}$ states [36]. The crystal field splitting depends on the symmetry of the site, for example figure 4.6 shows the $4 \mathrm{f}^{6}$ configuration splitting pattern for a cubic site of $\mathrm{O}_{h}$ symmetry. The ${ }^{7} \mathrm{~F}_{1}$ level is a degenerate singlet, but if the symmetry is reduced, for example by a interstitial ion, to $\mathrm{C}_{4 v}$ then the degenerate ${ }^{7} \mathrm{~F}_{1}$ level is split into two levels $\left(\mathrm{A}_{2}\right.$ and E).

For divalent ions there is an added complexity. The $4 \mathrm{f}^{n-1} 5 \mathrm{~d}^{1}$ configuration energies are much lower in energy than those the trivalent ions; they are closer to and sometimes overlap the $4 \mathrm{f}{ }^{n}$ energy levels. These outer orbitals have a much larger spatial extent and so interact 
strongly with the lattice. Hence they shift dramatically with variations in the crystal lattice unlike the $4 \mathrm{f}^{n}$ energy levels. For example the $4 \mathrm{f}^{6} 5 \mathrm{~d}^{1} \rightarrow 4 \mathrm{f}^{7}$ transition of $\mathrm{Eu}^{2+}$ varies from red in $\mathrm{Ca}_{2} \mathrm{SiS}_{4}: \mathrm{Eu}^{2+}[66]$ to blue in $\mathrm{CaF}_{2}: \mathrm{Eu}^{2+}$ [67].

\subsubsection{Selection rules}

The $4 \mathrm{f}^{n}$ states of lanthanide ions all have the same parity, this is means electric dipole transitions between them are forbidden by Laporte's rule (the parity must change). Therefore, only magnetic dipole transitions are expected to occur. However when the rare earth ion is at a site that lacks inversion symmetry there can be an admixture of the $4 \mathrm{f}^{n-1} 5 \mathrm{~d}^{1}$ states of opposite parity into mainly the excited $4 \mathrm{f}^{n}$ states, this allows for "forced" electric dipole transitions [63]. The electric dipole process is of orders of magnitude larger compared to the magnetic dipole process, and hence even a small amount of this mixing can result in the emission spectrum being dominated by forced electric dipole transitions [63, 68]. Transitions between the $4 \mathrm{f}^{n-1} 5 \mathrm{~d}^{1}$ excited configuration and the $4 \mathrm{f}^{n}$ ground state do show a change in parity and as such are electric dipole allowed and very strong.

Group theory is used in spectroscopy in order to interpret spectra with few or no calculations. By considering the symmetry properties of the active centre, which are shared by the corresponding Hamiltonian, it is possible to determine the number of and degeneracy of energy levels, predict how these energy levels will split under a reduction in symmetry and establish selection rules for optical transitions between these levels [36]. The probability of a radiative transition between two states $\left(\phi_{i}\right.$ and $\left.\phi_{f}\right)$ is proportional to $<\phi_{f}|\mu| \phi_{i}>\left.\right|^{2}$ where $\mu$ may be the electric dipole operator $\left(\mu=\sum_{n} e r_{n}\right)$ or the magnetic dipole operator $\left(\underline{\mu}=\sum_{n} \frac{e}{2 m}\left(\underline{l_{n}}+2 \underline{s_{n}}\right)\right)$. This probability then indicates the intensity of the relevant transition. The operator $\mu$ transforms as an irreducible representation $\left(\Gamma_{\mu}\right)$ of the symmetry group. The generalised Wigner-Eckart theorem then establishes the selection rule: The matrix element $\left|<\phi_{f}\right| \mu\left|\phi_{i}>\right|^{2}$ is zero unless the direct product between the irreducible representation $\Gamma_{i}$ and $\Gamma_{\mu}$ contains the representation $\Gamma_{f}$, that is unless

$$
\Gamma_{i} \times \Gamma_{\mu} \subset \Gamma_{f}
$$


Table 4.1: Summary of selection rules for rare earth ion transitions [63, 68].

\begin{tabular}{ccccc}
\hline Process & $\mathrm{S}$ & $\mathrm{L}$ & $\mathrm{J}$ & Parity \\
\hline Electric Dipole & $\Delta \mathrm{S}=0$ & $\Delta \mathrm{L}=0, \pm 1$ & $\Delta \mathrm{J}=0, \pm 1$ (but not J=J'=0) & opposite \\
Magnetic Dipole & $\Delta \mathrm{S}=0$ & $\Delta \mathrm{L}=0$ & $\Delta \mathrm{J}=0, \pm 1$ & same \\
\hline
\end{tabular}

[36] where $\phi_{f}$ and $\phi_{i}$ transform as $\Gamma_{f}$ and $\Gamma_{i}$ respectively. The direct product between two irreducible representations is a representation itself which is usually reducible. The product representation will have a character given by

$$
\chi^{\text {product }}(R)=\chi^{1}(R) \times \chi^{\mu}(R)
$$

To determine if the transition is allowed the direct product is calculated, the result reduced and examined for $\Gamma_{2}$. Multiplication tables are available in the literature for the direct products between irreducible representations of the point symmetry groups [36].

The selection rules for the different processes which come from Laporte's rule and the WignerEckart theorem are summarised in table 4.1 where $S$ is the spin quantum number, $L$ the azimuthal quantum number and $J$ the total angular momentum quantum number.

\subsubsection{Electron-phonon interaction}

The emission observed from rare earth ions can be easily classified into two categories; sharp line emission which arises from $4 \mathrm{f}^{n} \rightarrow 4 \mathrm{f}^{n}$ transitions and broad band emission which arises from $4 \mathrm{f}^{n-1} 5 \mathrm{~d}^{1} \rightarrow 4 \mathrm{f}^{n}$ transitions.

\subsubsection{Sharp line emission}

Because of the shielding afforded to the $4 \mathrm{f}^{n}$ electrons by the $5 \mathrm{~s}^{2}$ and $5 \mathrm{p}^{6}$ orbitals from the crystal field, electron-phonon coupling is weak for the $4 \mathrm{f}^{n}$ states and as such these are characterised by sharp line emission spectra. Because these are weak magnetic dipole transitions they have long lifetimes ( $\sim \mathrm{ms})$. However, when the site lacks inversion symmetry, a mixing of opposite parity $5 \mathrm{~d}$ states into the $4 \mathrm{f}^{n}$ states occurs. The amount of admixture is small and hence does not affect the energy levels significantly but the transitions become 
more allowed and the lifetime decreases $(\sim 10-100 \mu \mathrm{s})$ [63, 68]. Sharp line emission generally dominates the spectra for the trivalent ions from $\mathrm{Pr}^{3+}$ to $\mathrm{Yb}^{3+}$.

\subsubsection{Broadband emission}

For some rare earth ions, the $4 \mathrm{f}^{n-1} 5 \mathrm{~d}^{1}$ configuration state are relatively low lying and overlap the excited states of the $4 \mathrm{f}^{n}$ configuration (eg. $\mathrm{Ce}^{3+}, \mathrm{Pr}^{3+}, \mathrm{Sm}^{2+}, \mathrm{Eu}^{2+}$ ). This can lead to intense broad band emission and absorption between the ground state of the $4 \mathrm{f}^{n}$ configuration and the $4 \mathrm{f}^{n-1} 5 \mathrm{~d}^{1}$ states. These $4 \mathrm{f}^{n-1} 5 \mathrm{~d}^{1} \rightarrow 4 \mathrm{f}^{n}$ transitions are also commonly seen in the trivalent ion $\mathrm{Ce}^{3+}$ and less frequently for $\mathrm{Pr}^{3+}$ and $\mathrm{Nd}^{3+}$ ions. Because the parity changes these transitions are strong and have much faster lifetimes $(\sim 10-1000 \mathrm{~ns})$. The $5 \mathrm{~d}^{1}$ level is an outer orbital which couples strongly to the crystal field and so these transitions have large crystal field splittings and broad phonon sidebands (which can dominate the zero-phonon line) [63,68]. This type of emission is almost always seen for $\mathrm{Ce}^{3+}$ and $\mathrm{Eu}^{2+}$ ions. Others such as $\mathrm{Sm}^{2+}$ or $\mathrm{Pr}^{3+}$ ions may show broadband emission, sharp line emission or a combination depending on the crystalline environment.

\subsubsection{Stokes shift}

While excitation and emission are in a sense inverse processes there is generally a shift in frequency between the two called the Stokes' shift. Figure 4.8 shows a system which is excited from the ground state at the equilibrium position $Q_{0}$. Once in the excited state it quickly relaxes non-radiatively down to the excited equilibrium position $Q_{0}^{\prime}$. From here fluorescence is observed as the state returns to a vibronic state of the ground state at $Q_{0}^{\prime}$ before quickly relaxing non-radiatively back to $Q_{0}$. As shown in figure 4.8 (b) this causes the emitted energy to be less than the absorbed energy. An anti-Stokes' shift is also possible where the emitted frequency is greater that the absorbed frequency. This is also depicted in figure 4.8 if one reverses the directions of the arrows. A system may be in a vibronic state of the ground state at $Q_{0}^{\prime}$ due to thermal energy when absorption of a photon occurs where again thermal energy can allow the occupation of a vibronic state at $Q_{0}$. Radiative emission can then occur leaving the system at the equilibrium point of the ground state. The system is cooled in this 


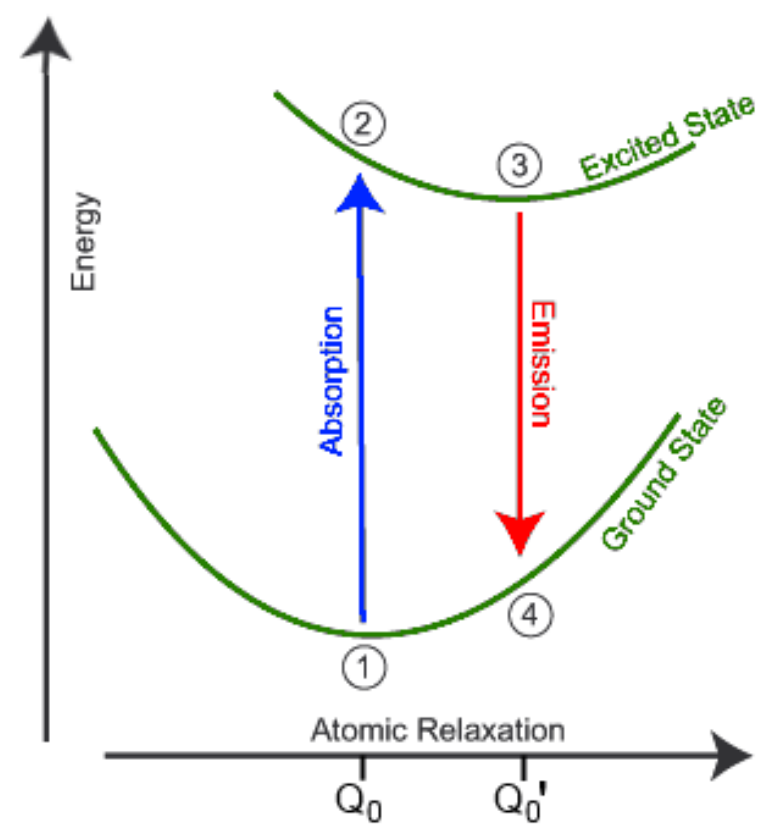

Figure 4.8: A photon is absorbed exciting the higher energy state, this relaxes to the lowest excited state before emitting a lower energy photon to return to the lower state [69].

process and the emitted light is of a higher frequency than that absorbed. It is desirable for scintillators to have a large Stokes shift to reduce the possibility of an emitted photon being reabsorbed, as this lowers efficiency and increases luminescence time scales.

\subsection{Characteristics of some rare earth ions}

The following sections discuss the general spectroscopy of the particular rare earth ion studied in this work.

\subsection{1 $\mathrm{Sm}^{2+}$}

Samarium is most commonly found in the trivalent state, but can also be stable as a divalent ion. Both $\mathrm{Sm}^{3+}$ and $\mathrm{Sm}^{2+}$ can be used as luminescent centres for x-ray phosphor applications. In addition to these scintillation or x-ray phosphor applications, samarium doped materials are of interest in a range of other applications. A schematic energy level diagram for the $\mathrm{Sm}^{2+}$ ion is shown in figure 4.9(a). Inhomogeneous broadening of the sharp singlet $\rightarrow$ singlet, ${ }^{5} \mathrm{D}_{0} \rightarrow{ }^{7} \mathrm{~F}_{0}$ transition and the ease with which $\mathrm{Sm}^{2+}$ can be photo-ionised means that the samarium-doped material has been widely used in studies of persistent hole-burning [70] 
in which case $\mathrm{Sm}^{2+}$ ions are ionised semi-permanently to $\mathrm{Sm}^{3+}$. The reverse process can be achieved with $\mathrm{x}$-irradiation, that is $\mathrm{Sm}^{3+}$ can be reduced to $\mathrm{Sm}^{2+}$, and this process has been proposed for applications in photoluminescence-detected x-ray storage phosphors [71] and in micro-beam radiation therapy dosimetry [72].

While $\mathrm{Sm}^{3+}$ always exhibits magnetic dipole allowed $4 \mathrm{f}^{6} \rightarrow 4 \mathrm{f}^{6}$ transitions which show little variation between host lattices, the emission from $\mathrm{Sm}^{2+}$ varies dramatically between host lattices. This is because the lowest energy levels of the $4 \mathrm{f}^{5} 5 \mathrm{~d}^{1}$ configuration are strongly perturbed by the crystal field and thus may overlap some, many, or none of the $4 \mathrm{f}^{6}$ excited state energy levels [63,68]. This leads to variation in the emission from comprising only $4 \mathrm{f}^{6}$ $\rightarrow 4 \mathrm{f}^{6}$ lines to much stronger, electric dipole allowed, broad $4 \mathrm{f}^{5} 5 \mathrm{~d}^{1} \rightarrow 4 \mathrm{f}^{6}$ transitions in the red region depending on the crystal. This emission can potentially be used for a scintillator coupled to a silicon photodetector.

Interest in the optical properties of $\mathrm{Sm}^{2+}$ dates back to 1960 when the development of masers created interest in divalent rare earth ions in alkaline earth lattices. Accordingly in 1963 Axe et al [73] undertook to derive the selection rules of $4 \mathrm{f}^{6} \rightarrow 4 \mathrm{f}^{6}$ fluorescence for such crystals. They applied this theory to $\mathrm{SrF}_{2}: \mathrm{Sm}^{2+}$ and $\mathrm{SrCl}_{2}: \mathrm{Sm}^{2+} . \mathrm{SrF}_{2}: \mathrm{Sm}^{2+}$ showed no luminescence at room temperature, however $\mathrm{SrCl}_{2}: \mathrm{Sm}^{2+}$ showed a broad emission around $700 \mathrm{~nm}$ at room temperature. This broad band was ascribed to $4 \mathrm{f}^{5} 5 \mathrm{~d}^{1}$ fluorescence and decreased in intensity as the temperature was reduced, disappearing entirely by $4.2 \mathrm{~K}$. At lower temperatures emission from the ${ }^{5} \mathrm{D}_{0}$ level of the $4 \mathrm{f}^{6}$ configuration was observed.

\subsection{2 $\mathrm{Eu}^{2+}$}

$\mathrm{Eu}^{2+}$ luminescence has been extensively studied in many compounds. $\mathrm{Eu}^{2+}$ is isoelectronic with $\mathrm{Gd}^{3+}$ so as can be seen in figure 4.7 there are very few $4 \mathrm{f}^{7} \rightarrow 4 \mathrm{f}^{7}$ transitions, and as such these are very rarely seen. Figure $4.9(\mathrm{~b})$ shows the typical $\mathrm{Eu}^{2+}$ ion energy level structure. The $\mathrm{Eu}^{2+}$ ion generally exhibits broad and strong $4 \mathrm{f}^{6} 5 \mathrm{~d}^{1} \rightarrow 4 \mathrm{f}^{7}$ transitions around $420 \mathrm{~nm}$ with a lifetime ranging from 500 - 1000 ns [74]. Very high quantum efficiencies have been observed for chloride and $\mathrm{CaF}_{2}$ crystals. Because it exhibits such strong luminescence, $\mathrm{Eu}^{2+}$ is used as the luminescent centre in many scintillators, light outputs of 120,000 photons/MeV have 


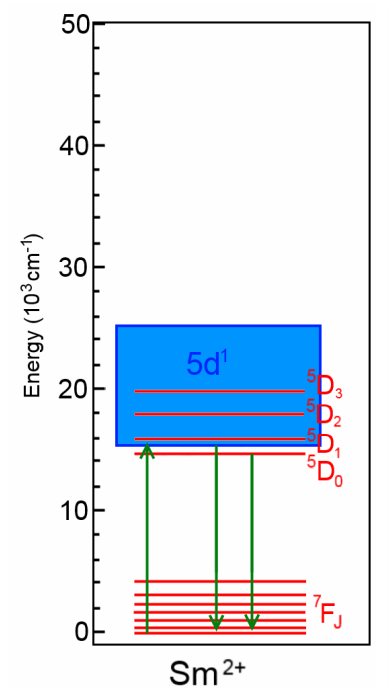

(a)

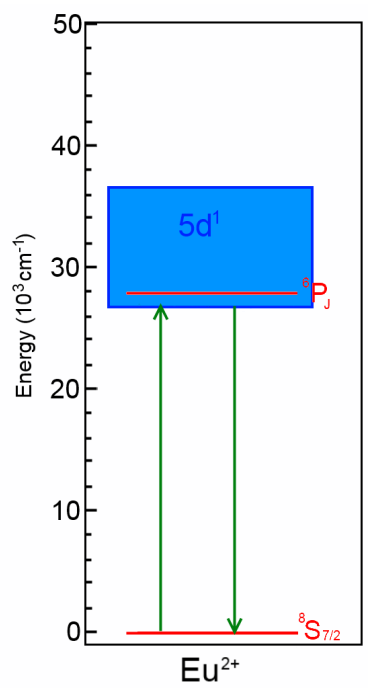

(b)

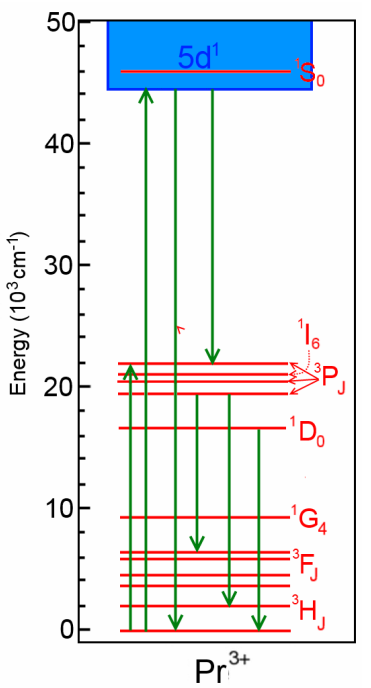

(c)

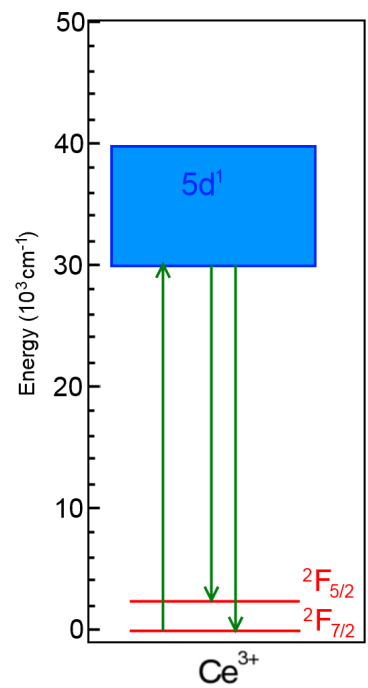

(d)

Figure 4.9: Schematic energy level diagrams for (a) $\mathrm{Sm}^{2+}$ ions, (b) $\mathrm{Eu}^{2+}$ ions (adapted from reference [78]), (c) $\mathrm{Pr}^{3+}$ ions (adapted from reference [79]) and (d) $\mathrm{Ce}^{3+}$ ions (adapted from reference $[\overline{80}])$. The red lines indicate levels of the $4 \mathrm{f}^{n}$ configuration and the blue boxes the excited $4 \mathrm{f}^{n-1} 5 \mathrm{~d}^{1}$ excited configuration energy levels. The green arrows indicate absorption and emission transitions commonly observed.

been reported for $\mathrm{SrI}_{2}: \mathrm{Eu}^{2+}[75]$ making it one of the brightest scintillators. Unfortunately the lifetime of $1200 \mathrm{~ns}$ and extreme hygroscopy limits the applications.

\subsection{3 $\operatorname{Pr}^{3+}$}

$\operatorname{Pr}^{3+}$ has two electrons in the $4 \mathrm{f}$ shell, and again the emission depends strongly on the host lattice. The energy level structure is shown in figure 4.9(c). Emission is most commonly seen from the $4 \mathrm{f}^{2}\left({ }^{3} \mathrm{P}_{0}\right)$ state; this can be green as in $\mathrm{Gd}_{2} \mathrm{O}_{3}: \mathrm{Pr}^{3+}\left({ }^{3} \mathrm{P}_{0} \rightarrow{ }^{3} \mathrm{H}_{4}\right)$, or red $\left({ }^{3} \mathrm{P}_{0} \rightarrow\right.$ ${ }^{3} \mathrm{H}_{6},{ }^{3} \mathrm{~F}_{2}$ ) as in $\mathrm{LiYF}_{4}: \operatorname{Pr}$ [68]. Being a lighter rare earth, the $4 \mathrm{f}$ orbitals are more spatially diffuse allowing more mixing of opposite parity states for sites lacking inversion symmetry, and this leads to a relatively fast emission from the ${ }^{3} P_{0}$ state (tens of $\mu s$ ) [68]. Emission can also be observed from the ${ }^{1} D_{2}$ state, and this is generally in the red or near infra-red region. $\mathrm{Pr}^{3+}$ is also known to show very fast luminescence from $4 \mathrm{f}^{1} 5 \mathrm{~d}^{1} \rightarrow 4 \mathrm{f}^{2}$ transitions in the UV region [76]. This occurs when the crystal field is strong enough to bring the lowest $4 \mathrm{f}^{1} 5 \mathrm{~d}^{1}$ level into the vicinity of the ${ }^{1} S_{0}$ level. If the lowest $4 \mathrm{f}^{1} 5 \mathrm{~d}^{1}$ level is even below the ${ }^{1} \mathrm{~S}_{0}$ level quantum cutting may be observed in which one excitation photon in results in the emission of two photons of lower energy [77]. 


\subsection{4 $\mathrm{Ce}^{3+}$}

$\mathrm{Ce}^{3+}$ has just one electron in the $4 \mathrm{f}$ shell. The spin-orbit interaction splits this into a ${ }^{2} \mathrm{~F}_{5 / 2}$ ground state with a ${ }^{2} \mathrm{~F}_{7 / 2}$ state about $2000 \mathrm{~cm}^{-1}$ higher. As seen in figure 4.7 there are no higher energy $4 \mathrm{f}$ states. The next lowest level belongs to the $5 \mathrm{~d}^{1}$ orbital and is around 30000 $\mathrm{cm}^{-1}$ above the ground state; this is depicted in figure $4.9(\mathrm{~d})$. The $5 \mathrm{~d}^{1} \rightarrow 4 \mathrm{f}^{1}$ transitions are parity allowed leading to two strong broad band emissions $\left(5 \mathrm{~d}^{1} \rightarrow{ }^{2} \mathrm{~F}_{5 / 2}\right.$ and $\left.5 \mathrm{~d}^{1} \rightarrow{ }^{2} \mathrm{~F}_{7 / 2}\right)[68]$. Because the $5 \mathrm{~d}$ orbital is strongly influenced by the host lattice the wavelength of these transitions vary dramatically between materials from $\mathrm{LaBr}_{3}: \mathrm{Ce}^{3+}[27]$ at $375 \mathrm{~nm}$ to $590 \mathrm{~nm}$ in $\mathrm{Lu}_{2} \mathrm{~S}_{3}: \mathrm{Ce}^{3+}$ [16]. $\mathrm{Ce}^{3+}$ is one of the most studied rare earth ions particularly for scintillation because of the fast lifetime ( $\sim 10 \mathrm{~ns})$, and also because of the simplicity of the energy levels. Dorenbos [81-85] has compiled data on the energy levels of $\mathrm{Ce}^{3+}$ in a wide variety of host lattices. From this he formed empirical rules to predict the $4 \mathrm{f}^{5} 5 \mathrm{~d}^{1} \rightarrow 4 \mathrm{f}^{n}$ emission wavelength of any trivalent rare earth ion in a crystal given the emission wavelength of another trivalent ion in the same lattice.

\subsection{Thermally stimulated luminescence}

Thermally stimulated luminescence (TSL) is a popular technique for determining the electron trapping characteristics of insulators and semiconductors. The luminescence is monitored as the previously irradiated sample is heated at a linear rate. The plot of recombination luminescence intensity vs. temperature is called the glow curve. The number of peaks indicates the number of different trapping centres and the position and shape indicate the trap depth [86]. The traps determine the time scale of the energy migration phase of the scintillation process. Shallow traps also play a role in the afterglow process. The mean time an electron will spend in a trap $(\tau)$ depends on the trap depth $(E$, which is the energy difference between the trap level and the conduction band), the temperature $(T)$ and the 'attempt frequency' $(s)$ according to

$$
\tau=s^{-1} \exp \frac{E}{k T}
$$


If $n$ is the number of trapped electrons, the rate of release of electrons is

$$
\frac{\mathrm{d} n}{\mathrm{~d} t}=\frac{-n}{\tau}=\frac{-n}{s^{-1} \exp \left(\frac{E}{k T}\right)}=-n s \times \exp \left(\frac{-E}{k T}\right)
$$

At temperatures such that the thermal energy is much less than the trap depth the lifetime of the trap is long and no luminescence is seen. As the temperature and thermal energy are increased the lifetime becomes shorter, and luminescence is observed as the trap population is depleted. Eventually as the thermal energy continues to increase the luminescence decreases as the trap population tends to zero, an so a peak is observed in the emission as the temperature is increased. Assuming the number of electrons released is proportional to the luminescence intensity (i.e. assuming electrons are not re-trapped, referred to as "first order kinetics") allows the intensity of the thermoluminescence at temperature $T$ to be written as [87]

$$
I(T)=-\frac{\mathrm{d} n}{\mathrm{~d} t}=s n \times \exp \left(\frac{-E}{k T}\right)
$$

which can be used to derive the intensity as a function of time as

$$
I(t)=\frac{\mathrm{d} n}{\mathrm{~d} t}=n_{0} s \times \exp \left(-\frac{E}{k T(t)}\right) \exp \left(-s \int_{0}^{t} \exp \left(-\frac{E}{k T\left(t^{\prime}\right)}\right) \mathrm{d} t^{\prime}\right)
$$

where $n_{0}$ is the number of trapped electrons at $t=0$. For a linear heating rate this can be expressed as

$$
I(T)=\frac{1}{\beta} \frac{\mathrm{d} n}{\mathrm{~d} t}=n_{0} \frac{s}{\beta} \exp \left(-\frac{E}{k T}\right) \exp \left(\frac{-s}{\beta} \int_{T_{0}}^{T} \exp \left(-\frac{E}{k T^{\prime}}\right) \mathrm{d} T^{\prime}\right)
$$

This is the Randall-Wilkins first order expression of a single glow peak [88, 89]. The above is difficult to evaluate or relate to measurement. Kitis et al [90] have showed that equation 4.15 can be well approximated by

$$
I(T)=I_{m} \exp \left[1+\frac{E}{k T} \frac{T-T_{m}}{T_{m}}-\frac{T^{2}}{T_{m}^{2}} \exp \left(\frac{E}{k T} T-T_{m} T_{m}\right)(1-\Delta)-\Delta_{m}\right]
$$

with

$$
\Delta=\frac{2 k T}{E}
$$




$$
\Delta_{m}=\frac{2 k T_{m}}{E}
$$

with $\mathrm{T}_{m}$ the temperature at the maximum of the glow curve and $\mathrm{I}_{m}$ the intensity at the maximum. Least squares fitting to equation 4.16 has been used here to determine the trap depths.

\subsection{Electron spin resonance}

Electron spin resonance (ESR) (also known as electron paramagnetic resonance) is a spectroscopic technique for studying materials with unpaired electrons. ESR is useful for providing information regarding chemical identification, crystal sites, crystal defects and crystallinity. This information may not be available from optical spectroscopy. The basic concept of ESR is analogous to nuclear magnetic resonance (NMR); however, in ESR it the electron spins which are excited and not the nuclear spins.

An ESR spectrum records the absorption of microwaves by a sample in a magnetic field. A free electron allows a simple description of the concepts as described here. In a magnetic field (of strength $\left.B_{0}\right)$ the electron's magnetic moment will aligns either parallel $\left(m_{s}=-\frac{1}{2}\right)$ or antiparallel $\left(m_{s}=\frac{1}{2}\right)$ to the field splitting a degenerate energy level into two as depicted in figure $4.10(\mathrm{a})$, The energy levels are

$$
E_{s}=m_{s} g \mu_{B} B_{0}
$$

where $g_{e}$ is the the electron's "g-factor" and $\mu_{B}$ is the Bohr magneton. In ESR the microwaves of a fixed frequency are shone on a sample as the magnetic field is changed. When the incident microwave energy $(h \nu)$ is equal to the energy difference between the two levels $\left(g \mu_{B} B_{0}\right)$ a transition is induced, and the microwaves are strongly absorbed. An absorption peak is seen at the magnetic field value which satisfies

$$
h \nu=m_{s} g \mu_{B} B_{0}
$$

where $\mathrm{h}$ is Planck's constant and $\nu$ is the microwave frequency. Equation 4.20 can be solved 


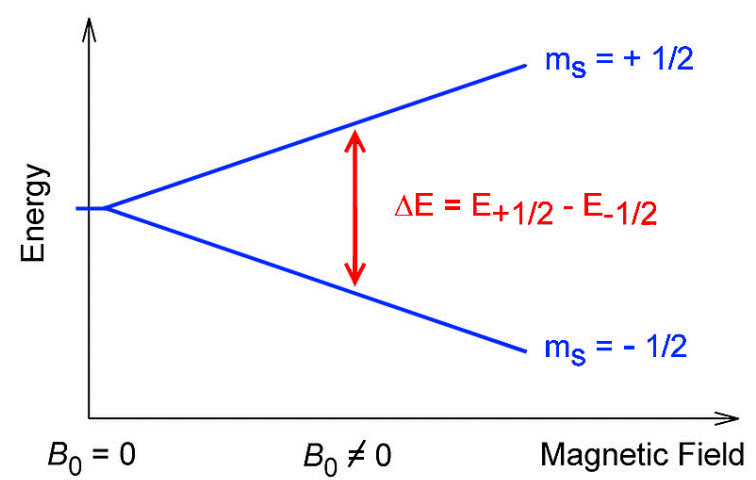

(a)

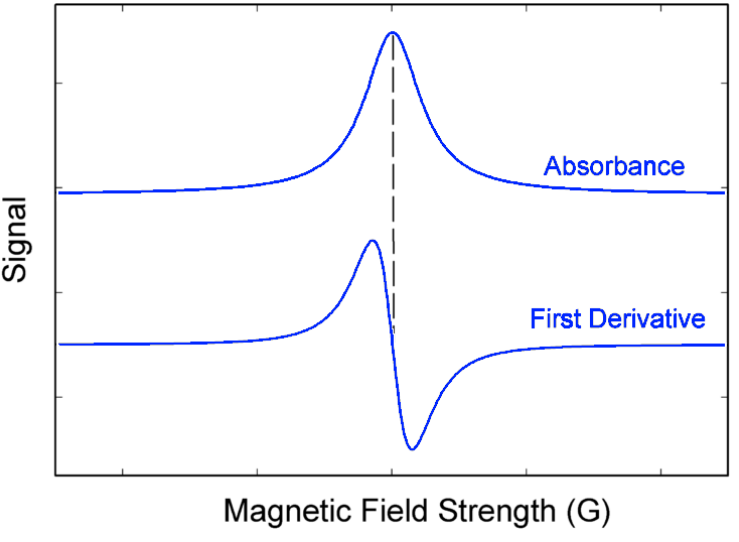

(b)

Figure 4.10: (a) Splitting of ground state in a magnetic field [91]. (b) Absorbance and first derivative spectrum [91].

for a wide range of frequency and magnetic field values, however ESR measurements are generally made with microwaves in the $X$ band $(9-10 \mathrm{GHz})$ region, with fields corresponding to about $\sim 350 \mathrm{mT}$. As the microwave detectors have high noise levels at low frequency, the magnetic field is usually modulated at $10-100 \mathrm{kHz}$ and the change in absorption is detected using a lock-in amplifier. This means signals appear as first derivatives. The two different forms of the measurement are shown in figure $4.10(\mathrm{~b})$.

For a more complex system, higher spin values $\left(S \geq \frac{1}{2}\right)$ and the magnetic interaction between the nuclei and the unpaired electrons (the hyperfine interaction and super hyperfine interaction) splits the ground state (and excited states) into many more states, between which transitions can be observed. This leads to a complex ESR spectrum with many lines. 


\section{Chapter 5}

\section{Instrumentation and experimental}

\section{techniques}

\subsection{Sample preparation}

As the chemicals used were hygroscopic, they were stored in a nitrogen or argon atmosphere glove box with low water vapour and oxygen content (at most 5 parts per million of each vapour). To prepare samples, the required powders were weighed into either a platinumrhodium alloy or glassy carbon crucible in the glove box; total sample weights were between 2 and $15 \mathrm{~g}$. The crucible and contents were then transferred to another nitrogen atmosphere glove box with a vertical radio frequency (RF) induction furnace attached (a schematic of the furnace configuration is shown in figure 5.1). The susceptor coils are powered by an Ameritherm $3 \mathrm{~kW}$ power supply operating at $\sim 485 \mathrm{kHz}$. The crucible was put into a graphite susceptor and lowered into the silica furnace tube which was then sealed off from the glove box and put under argon gas flow. A defined heating pattern was applied using a computerised temperature controller. The heating pattern used depended on the properties of the material being prepared. In general the powders were heated above the highest constituent chemical's melting point and held in the molten phase for 15 - 30 minutes to ensure the materials were fully melted and mixed. The liquid would then be cooled at $\sim 100^{\circ} \mathrm{C} /$ hour to room temperature. Through the expected solid to liquid phase transition 


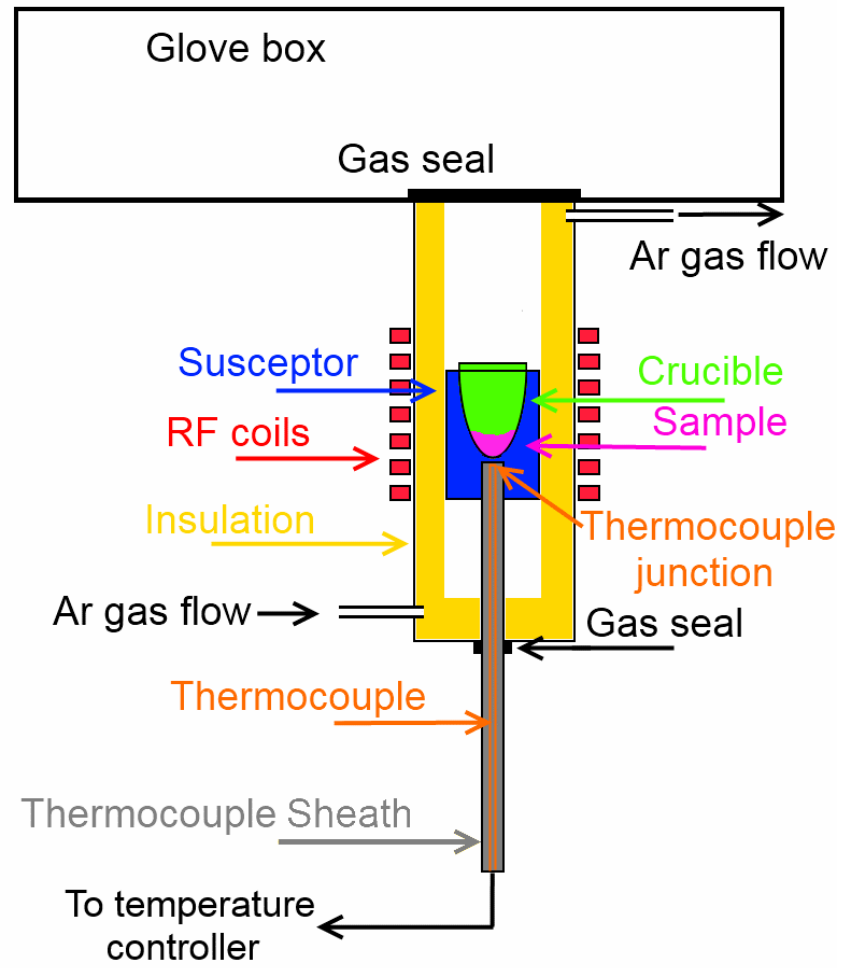

Figure 5.1: Schematic of radio frequency induction furnace configuration.

temperature the cooling rate was slowed to $\sim 10{ }^{\circ} \mathrm{C} /$ hour. If there were multiple phases possible, the cooling rate was increased to $\sim 1800{ }^{\circ} \mathrm{C} /$ hour through the range of stable temperatures of the undesired phase in order to supress it.

For most sample preparation a type K control thermocouple was used. The thermocouple is as close to the crucible as possible to ensure the temperature of the crucible is reliably measured. The furnace was calibrated for each type of crucible and thermocouple by filling the crucible with $\mathrm{Al}_{2} \mathrm{O}_{3}$ powder (which has a high thermal conductivity) and putting a second calibration thermocouple inside the crucible. An attempt to replicate the lid on the crucible was made using graphite with a hole for the second (top) thermocouple in it. The calibrations were closely linear. The gas flow was generally argon ( $<5$ ppm water, $<5$ ppm oxygen) which went through oxygen and water traps to remove any residual water or oxygen before reaching the furnace.

Almost all chemicals were sourced from Sigma Aldrich and had a specified purity of $\geq$ 99.999\% $\left(\mathrm{BaCl}_{2}, \mathrm{SrCl}_{2}\right), \geq 99.995\left(\mathrm{SrCl}_{2} \cdot 6 \mathrm{H}_{2} \mathrm{O}\right), \geq 99.99 \%\left(\mathrm{CeCl}_{3}, \mathrm{EuCl}_{2}, \mathrm{LaCl}_{3}, \mathrm{SmCl}_{3}, \mathrm{TbCl}_{3}\right.$, $\left.\mathrm{PrCl}_{3}, \mathrm{GdCl}_{3}, \mathrm{Eu}, \mathrm{Sm}\right), \geq 99.9 \%\left(\mathrm{CaF}_{2}, \mathrm{SmF}_{3}\right)$ or $\geq 99.0 \%\left(\mathrm{LiCl}, \mathrm{PbF}_{2}\right)$. In order to dehydrate $\mathrm{SrCl}_{2} \cdot 6 \mathrm{H}_{2} \mathrm{O}$ to $\mathrm{SrCl}_{2}$, the $\mathrm{SrCl}_{2} \cdot 6 \mathrm{H}_{2} \mathrm{O}$ was heated to $400{ }^{\circ} \mathrm{C}$ over 4 hours and then held at 
$400{ }^{\circ} \mathrm{C}$ under argon gas flow for a further 3 hours (full dehydration occurs by $325^{\circ} \mathrm{C}$ [92]). Dehydration was confirmed by noting that the mass change corresponded to the loss of 6 molecules of water per mole $\mathrm{SrCl}_{2} \cdot 6 \mathrm{H}_{2} \mathrm{O}$, and no additional mass loss was seen on further heating.

\subsection{X-ray diffraction}

X-ray diffraction (XRD) patterns are unique to the crystals present and as such can be used for structural identification. Powder x-ray diffraction was performed using two different X-ray diffractometers; either a Philips PW1730 diffractometer or a PANalytical X'pert PRO MPD diffractometer. Both diffractometers utilise copper tubes with both $\mathrm{K}_{\alpha}(1.54056 \AA)$ and $\mathrm{K}_{\beta}(1.3926 \AA)$ lines present. As the samples were hygroscopic, sealed sample holders which were loaded in the inert glove box atmosphere were used for both diffractometers. Patterns obtained were compared to those of known compounds from the International Centre of Diffraction Data database.

\subsection{Thermal analysis}

Differential Scanning Calorimetry (DSC) and Thermal Gravimetric Analysis (TGA) are both techniques which allows one to identify phase transition temperatures of a material. In this work, DSC was used to observe phase transition temperatures and monitor the suppression of phase transitions. TGA was also used for these purposes, as well as for monitoring the hydration and dehydration of materials as a function of time and temperature. Simultaneous DSC and TGA was carried out on a TA Q600 thermogravimetric calorimeter under nitrogen gas flow. This is capable of reaching temperatures of $1500^{\circ} \mathrm{C}$ and has a calorimetric accuracy of $\pm 2 \%$ Care was taken to minimise the time of exposure to air when loading the sample into the calorimeter and the furnace was purged for $\sim 10$ mins with inert gas before the sample was loaded. 


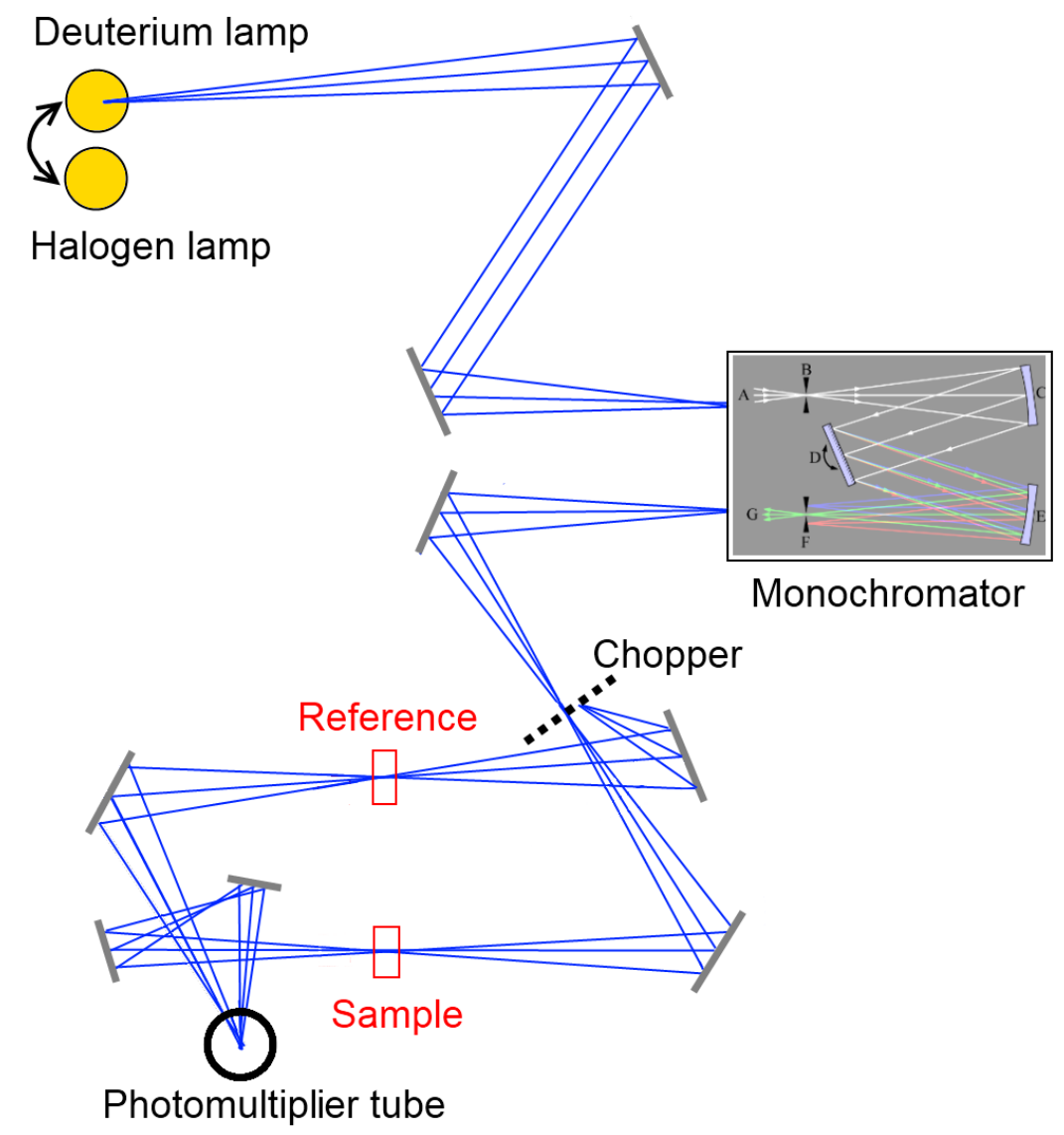

Figure 5.2: A schematic diagram of the Shimadzu UV-Vis 2100 spectrophotometer.

\subsection{Optical absorption}

Spectrophotometers are used to measure the optical absorption of a material as a function of wavelength, providing provide further information on the electronic structure and the optical properties of a material. A Shimadzu UV-Vis 2100 Spectrophotometer was used to record the absorbance of samples from $200-800 \mathrm{~nm}$. This is a dual beam system with a double monochromator of the Czerny-Turner type; a schematic is shown in figure 5.2. Either a halogen lamp or a deuterium lamp are used as the light source, the system automatically switches between the two at $\sim 370 \mathrm{~nm}$. The resolution of the system can be adjusted from 0.1 - $5 \mathrm{~nm}$ by adjusting the entrance and emission slits in tandem. The measurable absorbance range is 0 to 5 . The absorbance measured is

$$
A=-\log _{10} \frac{I}{I_{0}}
$$


where $I$ is the transmitted light intensity and $I_{0}$ the incident light intensity; this measurement depends on the thickness of the material. Data was therefore transformed into the thicknessindependent extinction coefficient $\left(\alpha_{e x}\right)$ values using the measured thickness, as this can be directly related to physical theories. The extinction coefficient is a measure of both absorption and scattering in the material per unit length and is defined as

$$
I=I_{0} e^{-z \alpha_{e x}}
$$

with $I$ and $I_{0}$ as before and $z$ is the thickness of material the beam passes through. Then from equations 5.1 and 5.2

$$
\alpha_{e x}=\frac{A}{z} \ln (10)
$$

\subsection{Photoluminescence}

The photoluminescence spectra of the samples were obtained using a Horiba Jobin Yvon Fluorolog 3 spectrometer; a schematic of this system is shown in figure 5.3 . The light source is a $450 \mathrm{~W}$ xenon arc lamp operated in continuous wave mode; this provides a broadband source from 240 - $900 \mathrm{~nm}$. Both the excitation and emission monochromators are double monochromators of the Czerny-Turner type. The excitation and emission slits are adjustable to achieve a bandpass of $0.3-14 \mathrm{~nm}$ for excitation and emission separately. Both excitation and emission monochromators have an uncertainty of $0.5 \mathrm{~nm}$ in the wavelength. Photon counting detection is performed by a R928P photomultiplier which is sensitive from 185 - 900 $\mathrm{nm}$.

\subsection{Photoluminescent lifetimes}

For a simple system the time dependence of the photoluminescence intensity can be described as an exponential decay of the form

$$
I(t)=I_{0} \exp \left(\frac{-t}{\tau}\right)
$$




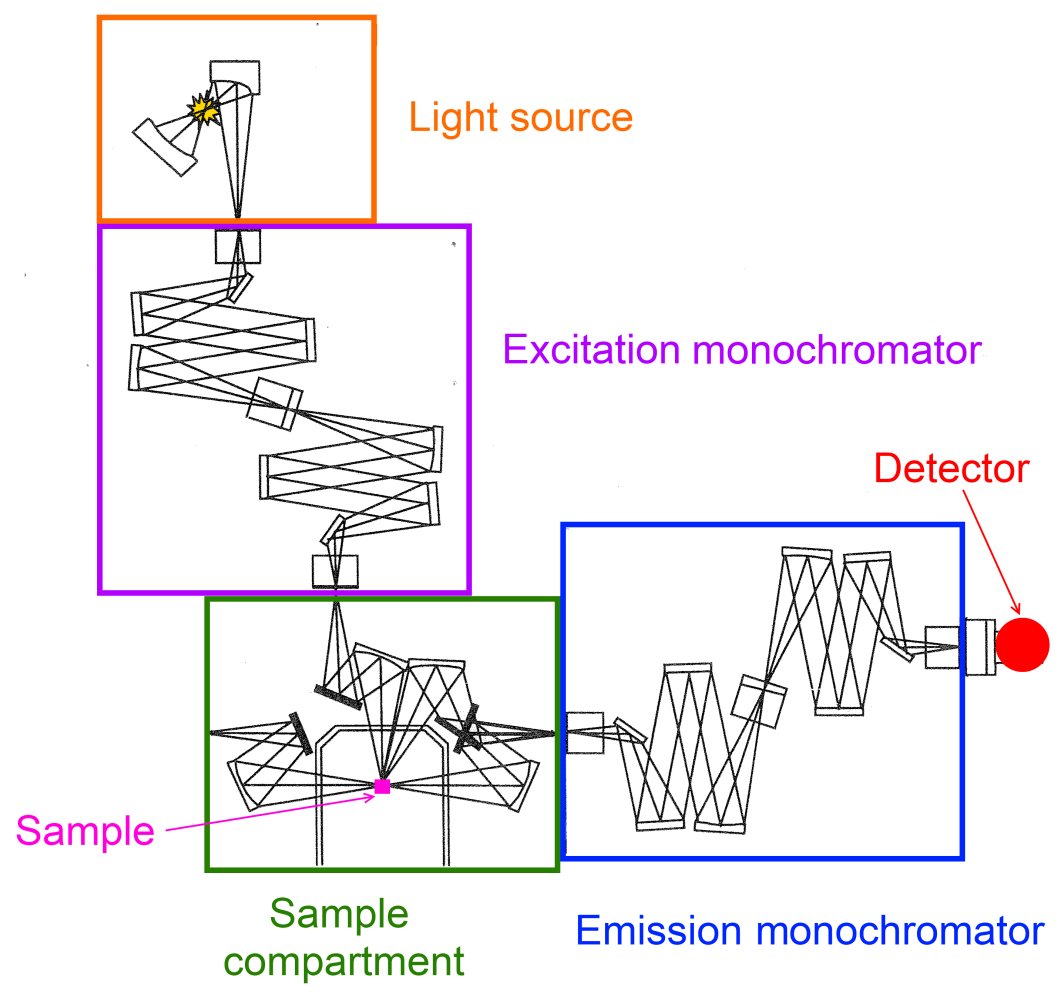

Figure 5.3: Schematic diagram of the Fluorolog used [93].

where $I_{0}$ is the initial photoluminescence intensity when excitation ceases and $\tau$, the decay constant, is the photoluminescent lifetime. The photoluminescent lifetime is given by the inverse of the probability of the transition. Two systems were used to measure photoluminescent lifetimes; both utilise the Horiba Jobin Yvon Fluorolog 3 spectrometer but with variable external pulsed excitation sources instead of the xenon lamp. For short lifetimes $(\lesssim 1 \mu \mathrm{s})$ a time correlated single photon counting (TCSPC) setup was used. NanoLEDs were used as the excitation source as these have a narrow pulse width $(\sim 1 \mathrm{~ns})$. Various wavelengths were used: $265 \mathrm{~nm}(<1 \mathrm{~ns}), 295 \mathrm{~nm}(<1 \mathrm{~ns}), 341 \mathrm{~nm}(<1 \mathrm{~ns}), 463 \mathrm{~nm}(1.3 \mathrm{~ns})$, $592 \mathrm{~nm}(1.4 \mathrm{~ns}), 632 \mathrm{~nm}(1.3 \mathrm{~ns}), 670 \mathrm{~nm}(\leq 200 \mathrm{ps})$ and $742 \mathrm{~nm}(1.5 \mathrm{~ns})$. These illuminate the sample causing photoluminescence and the emitted photons then pass through the double monochromator to the detector of the Fluorolog 3. Deconvolution with the nanoLED pulse profile can be performed allowing resolution of lifetimes down to 100 ps. For longer lifetimes ( $\gtrsim 1 \mu \mathrm{s})$ a Multichannel Scaling single-photon-counting spectroscopy (MCS) system was used. SpectraLEDs were used as the excitation source; the pulse duration from these is adjustable from $100 \mathrm{~ns}$ to milliseconds. The spectraLEDs used had peak wavelengths of $370 \mathrm{~nm}, 465 \mathrm{~nm}$ and $498 \mathrm{~nm}$. The fluorolog emission monochromator and detector were again utilised. 


\subsection{Variable temperature measurements}

A RMC 22 closed cycle cryostat was used to obtain temperatures from $12 \mathrm{~K}$ to $380 \mathrm{~K}$. This uses a 4 stage Gifford-McMahon refrigeration cycle with helium gas as the refrigerant. Objects may be cooled by attaching them to a metallic cold plate inside a vacuum chamber which is in thermal contact with the helium vapour chamber. The cryostat was used for photoluminescence and photoluminescent lifetime measurements in the fluorolog and absorption measurements in the spectrophotometer. Figure 5.4 shows the sample holders which were attached to the cold plate for use in the fluorolog and spectrophotometer. Both are made of oxygen free high conductivity (OFHC) copper and are thermally connected to the cold finger with an indium gasket. The temperature of the cold finger was monitored by a Lakeshore DT-400 PN diode connected to a Lakeshore 321 temperature controller, this controller operates a heater in the cryostat to allow stable temperature measurements and heating. We also monitored the temperature much closer to the sample with a second Lakeshore DT-400 PN diode mounted on the sample holder as seen in figure 5.4 , the temperature from this diode was read with the aid of a constant current source set to $10 \mu \mathrm{A}$ and a digital voltmeter. Reported temperatures are those observed from the DT-400 PN diode close to the sample. However, in general there was less than $2{ }^{\circ} \mathrm{C}$ difference in the temperatures. This indicates good thermal contact and conductivity of the sample holders.

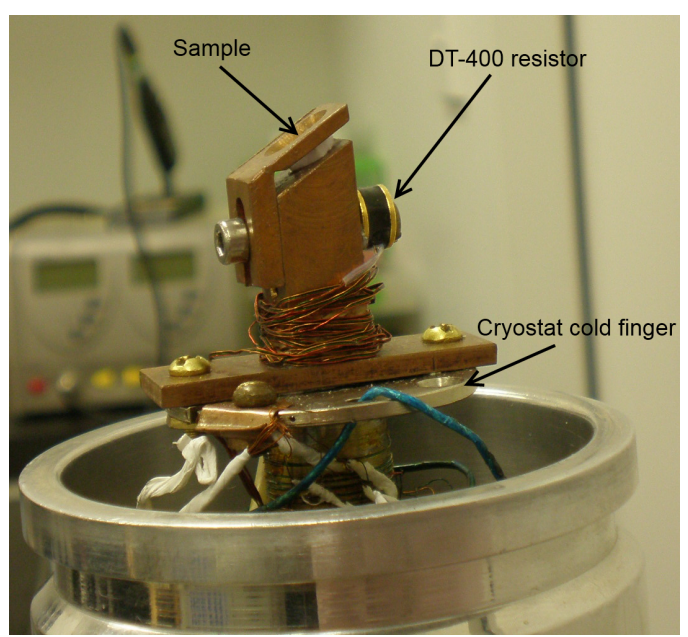

(a)

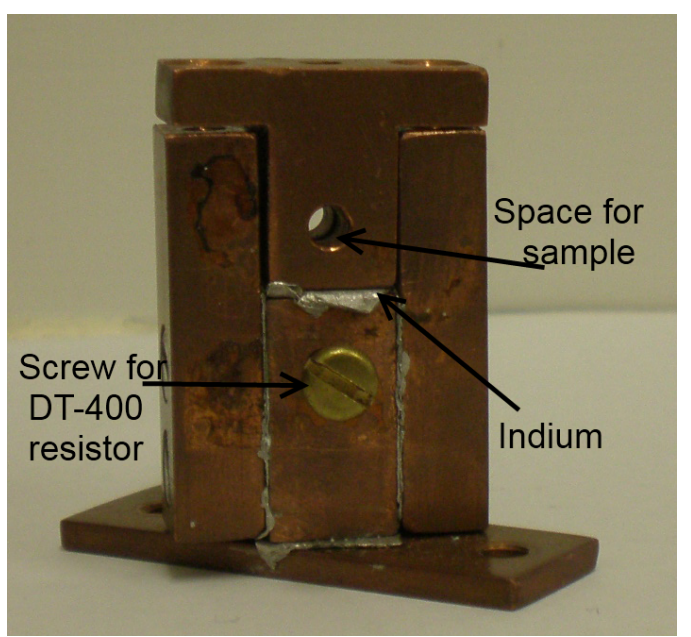

(b)

Figure 5.4: Cryostat sample holders; (a) used in the fluorolog for fluorescent measurements, and (b) used in the spectrophotometer for absorption measurements (b). 


\subsection{Thermally stimulated luminescence}

Thermally stimulated luminescence (TSL) refers to the phenomenon of energy being stored in 'traps' in a material then released as luminescence as the material is heated. TSL measurements were carried out on the set-up described by Quilty et al. [94]. An emulsion is created of powdered samples and silicone oil, this is spread onto the combined sample holder, heating element and sensor which consists of two back-to-back PT100 precision resistors. These have dimensions of approximately $2 \times 5 \mathrm{~mm}^{2}$ and temperatures from $30-450{ }^{\circ} \mathrm{C}$ could be reliably obtained. The samples were irradiated by a Philips PW 1720 x-ray generator, generally operated with a tungsten tube and no filtering. As the sample was heated post-irradiation, the emitted light was guided to the detector via a $600 \mu \mathrm{m}$ fibre optic cable. The detector used was either a photomultiplier (PMT), or a CCD spectrometer; the latter offered spectral resolution but at a cost of a decrease in the signal to noise ratio. The PMT was a peltier-cooled Electron Tubes Limited 9558QB PMT which was connected to a Stanford SR445A preamplifier (with a gain of 125) and thence to a Stanford SR400 two-channel gated photon counter. The spectrometer used was an Ocean Optics USB2000 CCD spectrometer, with a blaze angle of $500 \mathrm{~nm}$, a range of $200-850 \mathrm{~nm}$, a slit width $60 \mu \mathrm{m}$ and a resolution of $1.5 \mathrm{~nm}$. All reported results have been corrected for the spectral response of the detector. The correction was achieved by recording the emission of a calibrated tungsten lamp. The correction ends at 300 $\mathrm{nm}$ as the lamp used is uncalibrated below $300 \mathrm{~nm}$.

\subsection{Electron spin resonance}

Figure 5.5 shows a diagram of the custom build electron spin resonance (ESR) spectrometer used. A Gunn diode generates microwaves of frequencies $9-9.5 \mathrm{GHz}$ (X-band). The microwave frequency is monitored by a frequency counter. The microwave's path is split by a "magic $\mathrm{T}^{\prime}$; half the intensity is directed though a wave guide to the cavity and the other half is directed to an impedance load. The impedance load is adjusted so that no power is reflected from it. The coupling in the cavity is also adjusted so that in the absence of ESR, power is also not reflected from the cavity. When ESR absorption occurs the cavity is mis-matched 


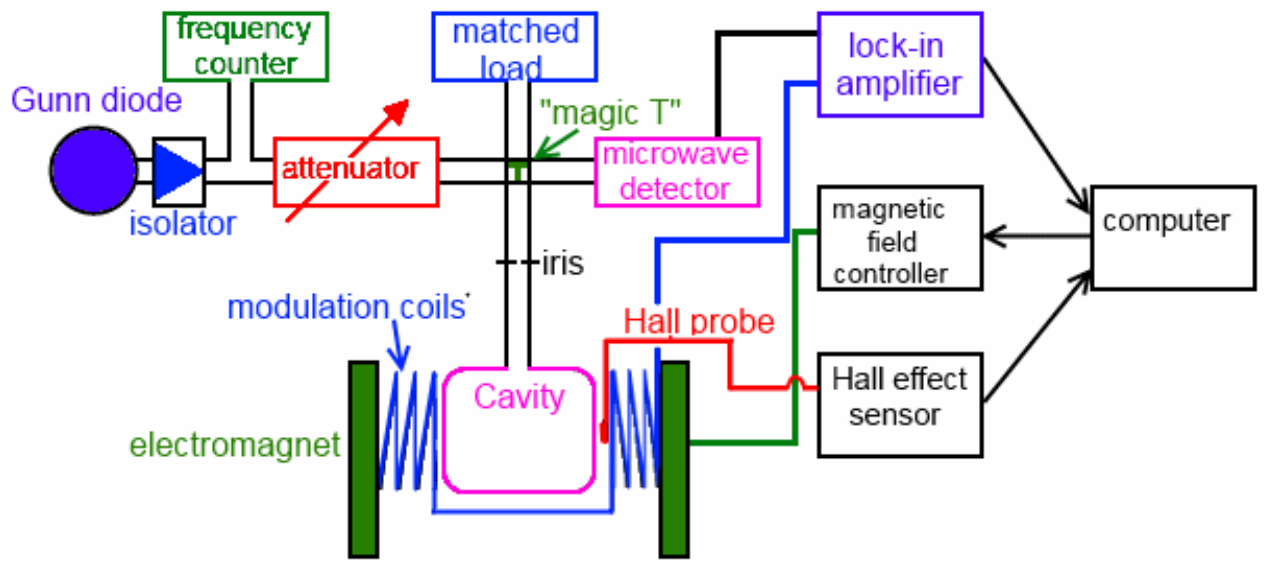

Figure 5.5: Schematic diagram of the electron spin resonance (ESR) spectrometer used [95].

and some power is reflected. At the "magic T" this signal is divided in two; half the intensity is directed to the microwave detector and the other half to a isolator which protects the Gunn diode. The signal is modulated, generally at $51 \mathrm{kHz}$, and detected by a lock in amplifier before being recorded by computer software. The cavity is in a large electromagnet capable for fields between 0 and $600 \mathrm{mT}$. The field strength is monitored by a Hall probe attached to the electromagnet.

\subsection{Radioluminescence}

Radioluminescence is the radiative de-excitation of luminescent centres that occurs when $\mathrm{x}$-rays are absorbed by the material and this energy is transferred to luminescent centres. To record radioluminescence, both Philips PW1700 and PW1730 X-ray generator sets were used as the x-ray source; most measurements were carried out using a tungsten tube. A $0.7 \mathrm{~mm}$ aluminium filter was used in the beam to eliminate the soft (low energy) x-ray intensity. A 600 $\mu \mathrm{m}$ fibre optic cable was used to guide the emitted light to the detector. An Ocean Optics USB 4000 spectrometer was used as the detector. This has a range of $200-1100 \mathrm{~nm}$ and is blazed at $300 \mathrm{~nm}$. The entrance slit width is $50 \mu \mathrm{m}$ and the resolution $2 \mathrm{~nm}$. All reported results have been corrected for the spectral response of the detector; the correction was performed as above for the USB2000 spectrometer. 


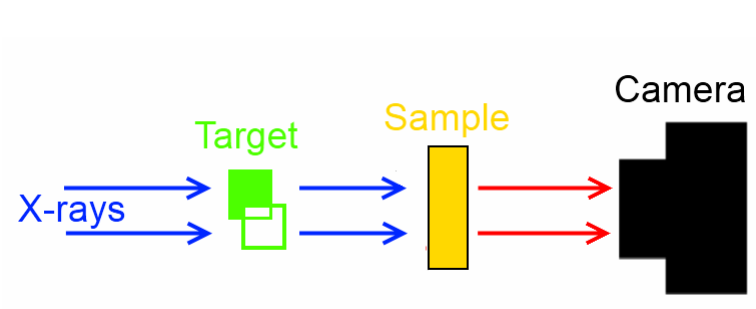

(a)

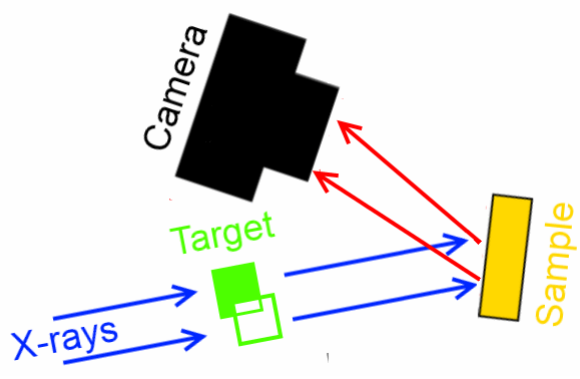

(b)

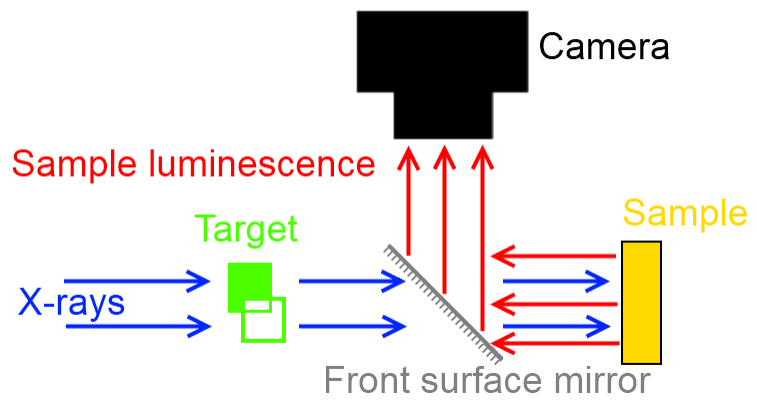

(c)

Figure 5.6: Schematics of the x-ray imaging configurations used; (a) straight through irradiation and detection (b) front surface irradiation and (c) front surface irradiation with a mirror.

\subsection{X-ray imaging}

Recently Winch and Edgar [96] introduced a new read-out technique for storage phosphors using a digital camera rather than the traditional raster-scanned laser technique. With slight modification this set-up was adapted for x-ray imaging based on the prompt phosphors reported in this thesis. Figure 5.6 shows diagrams of the imaging configurations used.

As the x-ray beam passes through the imaging target, the beam is attenuated according to the composition of the target. The spatial variation of intensity of this beam causes a luminescent image to be formed on the sample. This image is then captured with a digital camera. The exposure time used on the camera can be varied to suit the intensity of the luminescence. The cameras used were a Canon EOS 5D Mark II digital SLR camera or a SBIG ST-2000XM CCD camera. The front surface mirror is made of mica which has a very low cross section for x-ray absorbance so that the x-ray beam passes through unaffected to the phosphor. The phosphor then forms an image of the target in visible light which reflected by the mirror into the camera. The x-ray source used was a de Goetzen Xgenus dental x-ray set with a tungsten tube operated at $70 \mathrm{kV}, 8 \mathrm{~mA}$, and with a $2 \mathrm{~mm}$ aluminium filter. 
Table 5.1: Selected properties of the XP2020Q [9] and R5929 photomultipliers used.

\begin{tabular}{ccc}
\hline Property & XP2020Q & R5929 \\
\hline Anode pulse rise time (ns) & 1.6 & 15 \\
Transit time (ns) & 28 & 60 \\
Cathode & bi-alkali & multi-alkali \\
Spectral range (nm) & $150-650$ & $300-900$ \\
\hline
\end{tabular}

\subsection{Scintillation}

To observe scintillation, ${ }^{137} \mathrm{C}$ s sources were mainly used as the gamma ray source, these emit $0.66 \mathrm{MeV}$ gamma rays and have a strength of $\sim 10 \mu \mathrm{Ci}$. Photomultipliers (PMTs) were used to detect the light emitted under gamma irradiation. Two PMTs were used, a Photonis XP2020Q and a Hamamatsu R5929; these two PMTs have different properties some of which are tabulated in table 5.1. The fast response time of the XP2020Q meant it was the PMT of choice for timing and high frequency pulse counting applications. However when working with red emissions the XP2020Q lacks sensitivity, and so we used the R5929. A Tektronix $1 \mathrm{GHz} 7104$ fast oscilloscope recorded the signals from the PMTs with or without signal averaging of scintillation events. A time correlated single photon counting (TCSPC) system was built and used for scintillation lifetime measurements; this is described in more detail in chapter 6 .

Pulse height analysis spectra were recorded using the R5929 PMT. Samples were optically coupled to the PMT with optical grease and covered in multiple layers of white teflon tape to concentrate scintillation light on the photocathode. The PMT signal was amplified with a fast pre-amplifier (Ortec 9305), then further amplified and integrated with a linear amplifier (CI1410) before being recorded on a pulse height analyser (Fastcom MCA3).

\subsection{Photomultiplier tube spectral response correction}

To compare scintillators which which emit in different regions of the spectrum it is necessary to account for the different quantum efficiencies of the photodetector at the different emission wavelengths. The spectral response of both a Hamamatsu R5929 PMT and a Photonis 


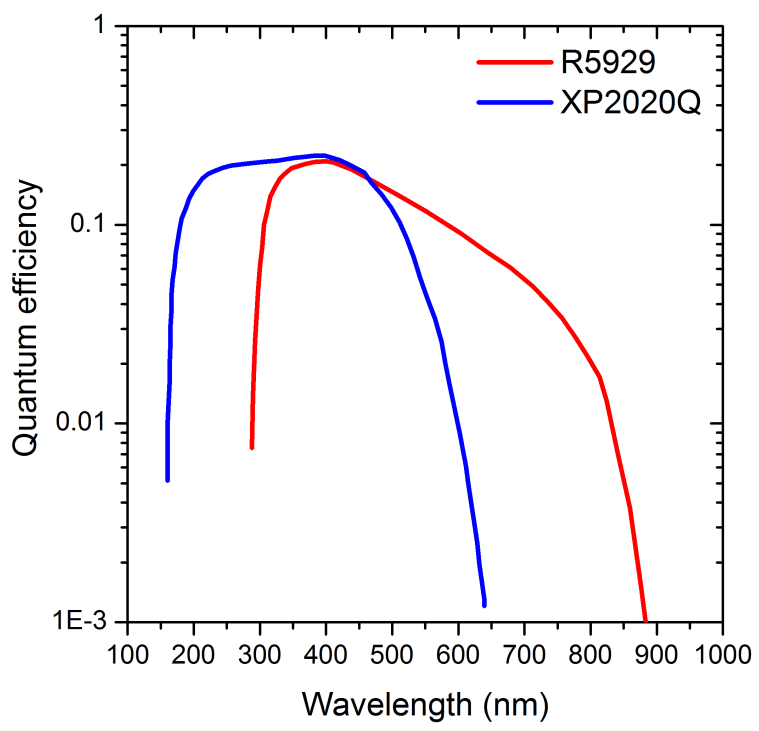

Figure 5.7: Quantum efficiency curves of Hamamatsu R5929 [97] and Photonis XP2020Q [9] photomultiplier tubes.

XP2020Q PMT are shown in figure 5.7 there is a clear variation with wavelength for each PMT. For narrow line emissions, corrections for the PMT spectral sensitivity are straight forward. However, for the measurements of broadband emissions, a more complex process is required. Corrected spectra for the emissions to be compared were first recorded on the fluorolog spectrometer. These were fitted with single Gaussians and the integrated area normalised. Each Gaussian was then multiplied by the spectral response of the PMT and the result was integrated. The resulting integrals give the relative expected intensities for the different emissions detected by the PMT. The ratio of these integrals was taken as the correction factor.

\subsection{Afterglow}

Afterglow was measured on the novel set up described by Bartle et al. [98]. In this set-up the sample to be measured is optically coupled to a photomultiplier tube (PMT), this whole arrangement is passed through a Smiths-Detection DEXA (dual energy X-ray absorption) scanner via a conveyor belt system. An object which passes through the scanner experiences a brief intense pulse of x-rays. For our measurements, the conveyor belt speed gave a pulse that was $12 \mathrm{~ms}$ long. By recording the signal from the PMT and deconvolving this with the Gaussian x-ray pulse shape one can measure the afterglow of the material. Two PMTs 


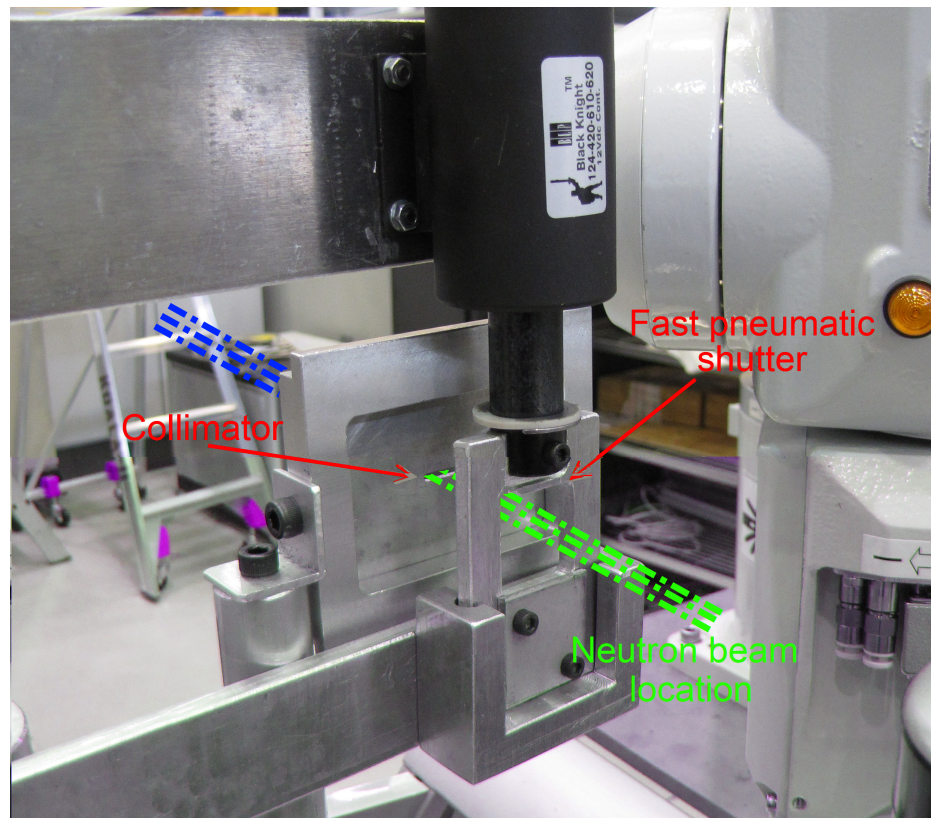

Figure 5.8: A photograph of the collimator and pneumatic shutter of the Koala beam. The estimated neutron beam position has been drawn for clarity.

were used in this set-up; an ADIT B38B01S for blue emitting materials and a Hamamatsu R5929 for red emissions. The PMT signals were recorded on a Tektronix TDS 2022 digital oscilloscope.

\subsection{Neutron irradiation}

Neutron irradiation was performed at the Australian Nuclear Science and Technology Organization's (ANSTO) OPAL reactor located at Lucas Heights, Sydney. The Koala line was used; this is normally used for Laue diffraction but allowed us access to a beam of thermal neutrons of intensity $\sim 2.5 \times 10^{6}$ neutrons $\mathrm{cm}^{-2} \mathrm{~s}^{-1}$. The experimental set-up is very similar to that described by Bartle et al. [98]. The beam passed through a collimator $(10 \mathrm{~mm} \times 2 \mathrm{~mm})$ before reaching a sample. A fast pneumatic shutter made of ${ }^{10} \mathrm{~B}$ impregnated $\mathrm{Al}$ was used to shut the beam off from the sample in about $1 \mathrm{~ms} .{ }^{10} \mathrm{~B}$ has a very high cross section for neutron capture, so that once the shutter is in place effectively no neutrons will reach the sample. The collimator, shutter and beam path can be seen in figure 5.8. The samples were optically coupled with optical grease to a perspex light guide $100 \mathrm{~mm}$ long which was wrapped in Al foil then black tape. The other end of the light guide was coupled to a Hamatsu R5929 photomultiplier tube (PMT). The sample and PMT all sat on a stage which could be adjusted 
until the sample position was such as to have the beam entirely located on the sample. The difference in signal from the PMT with the shutter open and then closed provided a measure of the emission intensity; this was compared to that for a commercial neutron scintillator. The afterglow profile was also recorded by monitoring the signal as the shutter was closed. To make the recordings a digital Tektronix TDS 2022 oscilloscope was used. 


\section{Chapter 6}

\section{Time correlated single photon counting for scintillation lifetimes}

\subsection{Introduction}

Time correlated single photon counting (TCSPC) is a technique for determining the time dependence of the light intensity of pulsed luminescence, and was originally introduced for scintillation applications by Bollinger and Thomas in 1961 [99]. TCSPC is a broadly applicable technique used in a wide array of applications such as ultra-fast recording of optical waveforms (e.g. fluorescence lifetime measurements), detection and identification of single molecules, fluorescence correlation spectroscopy, DNA sequencing, optical tomography, photon correlation experiments, fluorescence lifetime imaging, and fluorescence resonance energy transfer [100]. In the following discussion the focus will be on the measurement of the decay time of scintillation luminescence, where the scintillation is gamma-ray induced and the detectors used are photomultiplier tubes (PMTs).

A single scintillation event consists of a multitude of photons. The basic definition of a scintillation decay time $(\tau)$ is that the probability that a photon is emitted at time $t$ after the excitation is given by

$$
P(t)=P_{0} \exp \left(\frac{-t}{\tau}\right)
$$

Where $\tau$ is mean time for mean lifetime of the excited state. Figure 6.1 shows a typical 


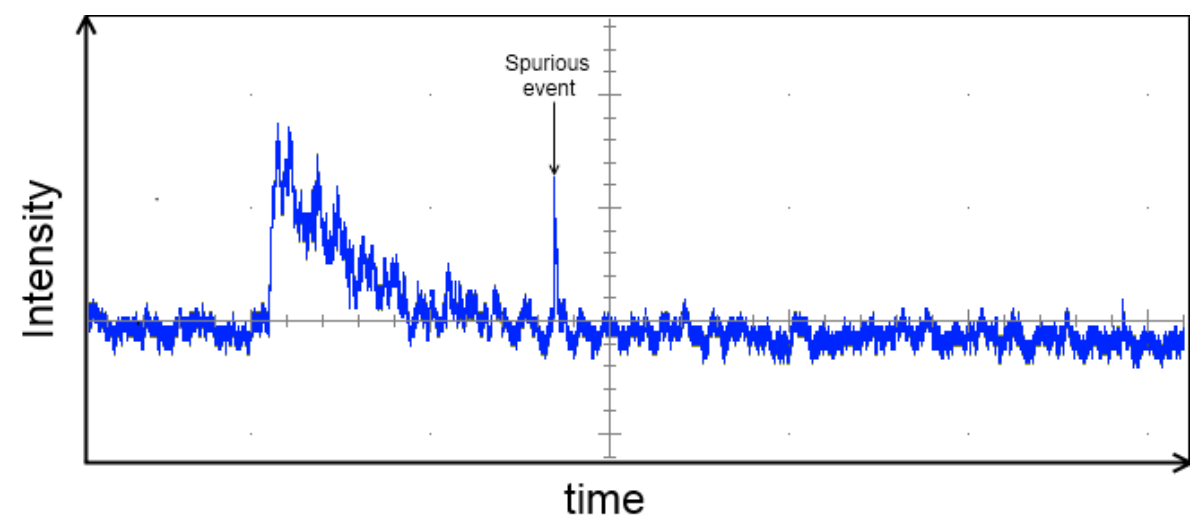

Figure 6.1: Real scintillation event as recorded on an oscilloscope.

scintillation decay as recorded on an oscilloscope. Each photon detected causes a sharp response 'spike', these add up to form the overall decay shape. As can be seen in figure 6.1 the result is far from the ideal smooth decay one might imagine.

To measure the lifetime of the luminescence requires the recording of the arrival times of these photons. The principles of TCSPC lifetime measurements are illustrated in figure 6.2 In TCPSC two detectors are used, the 'start' detector and the 'stop' detector. The optics are arranged so that the majority of photons from each scintillation event reach the start detector but on average less than one reaches the stop detector. When the start detector detects the leading edge of the large start pulse, a timing unit is triggered. This timing unit is then stopped when the stop detector detects a single photon; the time interval between the start and stop signals is recorded and sent to a time-to-amplitude converter (TAC). By doing this for many excitations, a histogram of the time difference between the start and stop signals can be built which reproduces the scintillation decay shape. The technique relies on the fact that for the highly attenuated stop beam, the intensity is so low that the chance of more than one photon being detected is negligible [99], but in consequence, the majority of start signals have no stop signal. This means the acquisition times for such a measurement are long as the information from a large proportion of the scintillation events is discarded.

It is important to note the detector output signals do not directly trigger the timing unit; instead they are used to trigger constant fraction discriminators (CFD)s, the outputs of which in turn start and stop the timing on the TAC. A CFD is used as this generates a very narrow output pulse only when the input pulse reaches a specified fraction of its maximum amplitude as depicted in figure 6.3 (b). This means the timing of the output with respect 


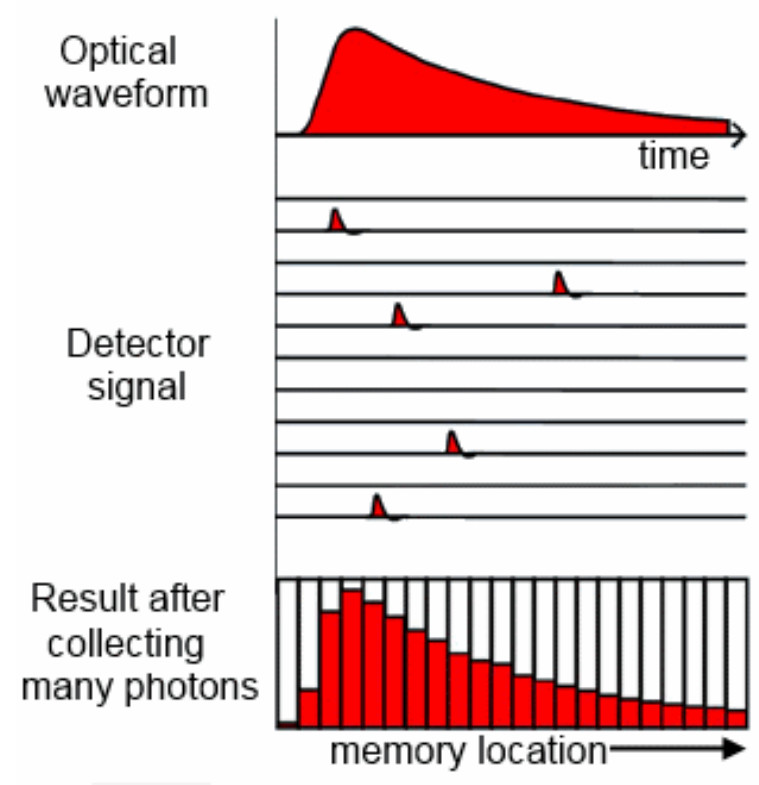

Figure 6.2: The scintillation pulse consisting of many photons (top) is used to start the timing unit and an attenuated single photon signal from the same pulse is used to stop the timing unit. This time difference is recorded for many events (middle) to build up a histogram representing the original waveform (bottom) [100].

to the pulse arrival does not change with pulse amplitude ("timewalk") as it would for a constant level trigger as shown in 6.3 (a). The TAC generates a pulse which has an amplitude that is proportional to the time difference between the start and stop signals. This pulse is then recorded in a channel ("bin"), with a channel number corresponding to the size of the pulse by the multichannel analyser (MCA). The bin number is linearly related to the pulse height.

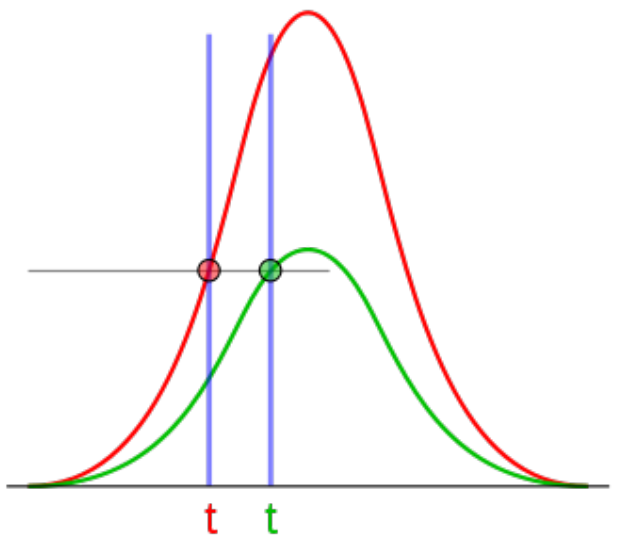

(a)

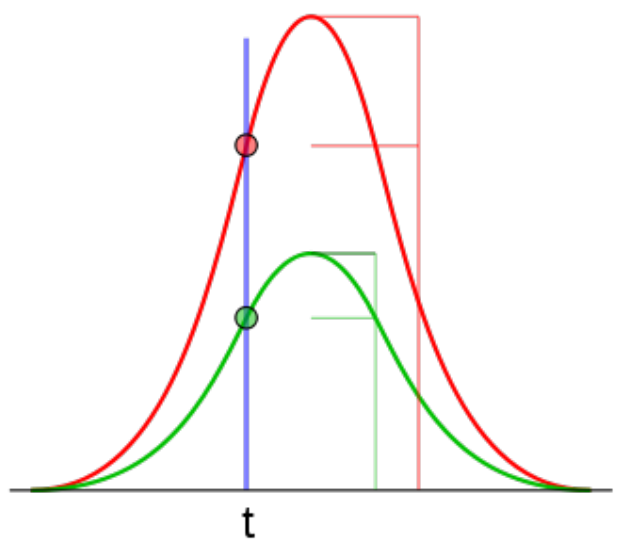

(b)

Figure 6.3: (a) Constant level triggering and (b) constant fraction triggering. 


\subsection{The advantages of time correlated single photon count- ing scintillation timing}

There are a wide variety of techniques available for time resolved detection of optical signals. The lifetime of a light pulse can be recorded using either time-domain techniques or frequency domain techniques. In time-domain techniques the signal intensity as a function of time is recorded, whereas with a frequency domain technique the phase and amplitude of the signal are measured as a function of excitation frequency. The two are related by a Fourier transform and so provide equivalent information. However, in practice time-domain techniques generally prove easier for lifetime measurements, and are in fact the only option for processes with random start times such as scintillation. Further categorising of the measurement technique is based on photon counting vs. analogue techniques. With photon counting techniques, the signal comprises a distribution of pulses from which those from photons can be distinguished by pulse height spectra, by using a level discriminator. "Dark current" noise, such as that from thermally ejected cathode electron, is thus discriminated against, which is not possible with analogue techniques.

Using a photon counting technique is beneficial as it is the frequency of the pulses which gives the intensity as opposed to the amplitude of the pulses. This means the result is unaffected by noise, amplitude jitter and long term stability issues which are detrimental to analog systems. Photon counting is also beneficial when wait times between signals are greater than the pulse width detected, as is generally the case in gamma-ray induced scintillation. In this situation an analogue system accumulates a considerable noise signal, and is subject to background drift which leaves photon counting systems unaffected.

TCSPC is a time-domain photon counting technique. Two general techniques for photon counting in the time domain are Multichannel Scaling (MCS) or TCSPC. MCS systems advance through many channels (time intervals) at a high speed and count the number of detected pulses during each time interval into the corresponding memory channels. This technique is limited by the speed of the memory of the MCS. TCSPC has no such limitation; the time difference between the start and stop signals is recorded as a count in the channel 
corresponding to that time interval. The channel width can be as little as picoseconds, offering very high timing resolution. In fact TCSPC is able to offer higher $(\sim \times 10)$ time resolution than any analogue system because the resolution is not limited by the single electron response (SER) time of system, which is around 5 ns in most PMTs [101]. Instead in TCSPC because the detector outputs trigger CFDs, which have very fast rise times, the accuracy is limited by the jitter in the leading edge of the photomultiplier output pulse. This jitter is caused by differences in the time between absorption of a photon at the photocathode and the corresponding electron pulse reaching the anode and is around 0.5 ns for most PMTs [101].

An area in which analogue techniques are potentially superior is in light intensity and acquisition time. The maximum light intensity for an analogue system is virtually unlimited when the PMT pulses are integrated to give a steady DC current, at least until the anode current becomes comparable with the dynode resistor current. With photon counting the maximum is $10-100 \mathrm{MHz}$ [100]; this is limited by the SER. The average time interval between photon pulses must be larger than the SER time in order for the single photons to be distinguished. The count rates in TCSPC systems are further limited by the large amount of dead time required to process each photon. However, modern systems still achieve rates of several million photons per second. Corresponding to the higher count rates achievable, analogue techniques offer shorter acquisition times. Despite this one drawback, TCSPC is the most accurate and most commonly used technique to measure scintillation decay times.

\subsection{Configuration}

\subsubsection{Optics and hardware}

TCSPC systems with high timing resolution can be set up from standard Nuclear Instrumentation Modules (NIMs). Shown in figure 6.4 is a block diagram of the configuration and shown in figure 6.5 is a photograph of the actual arrangement which was built and tested. Fourteen ${ }^{137} \mathrm{C}$ s sources are used as the excitation source; these emit gamma rays of $0.66 \mathrm{MeV}$. The detectors and operators are well shielded by thick $(5 \mathrm{~cm})$ blocks of lead. The start and 


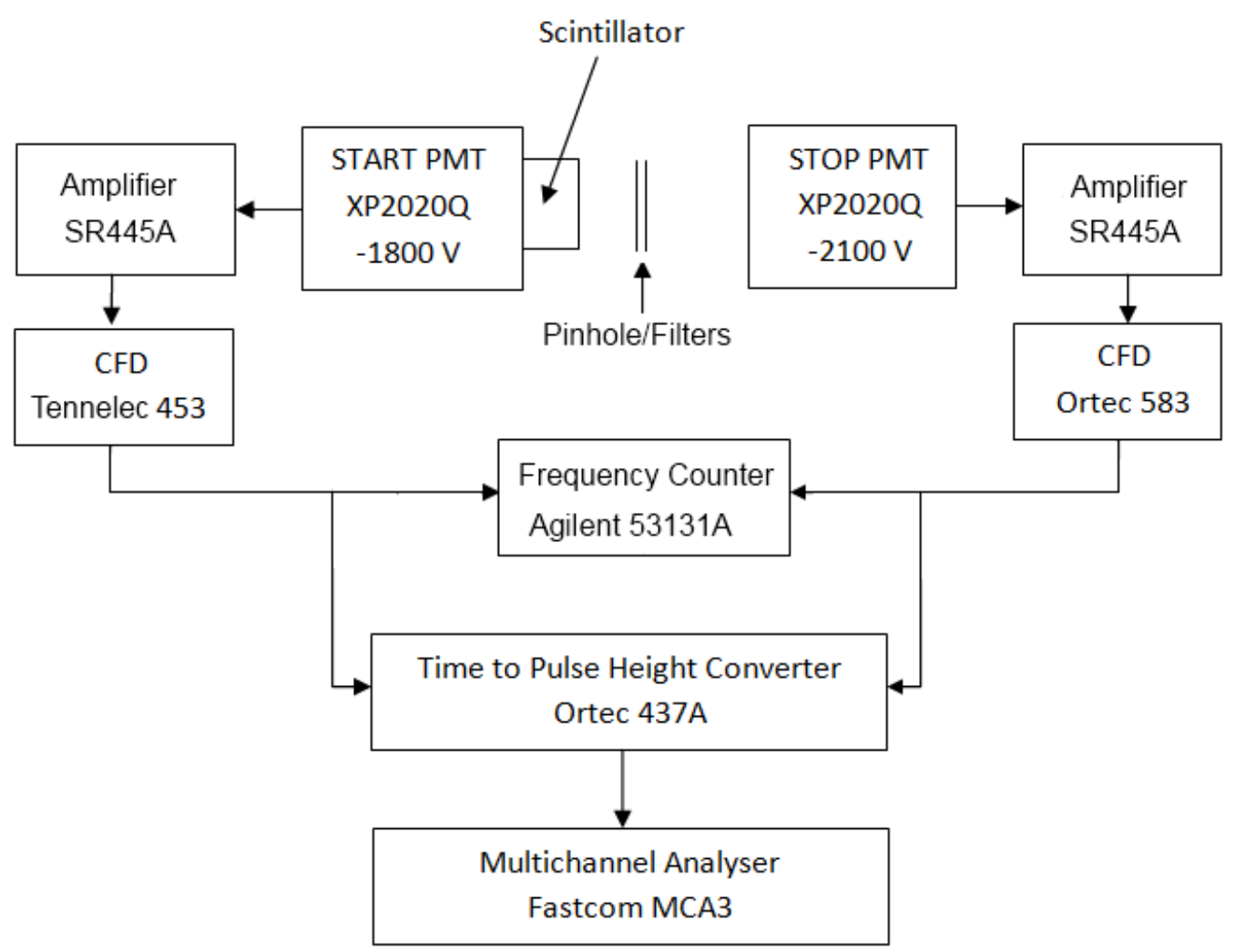

Figure 6.4: Block diagram of the configuration used in our system.

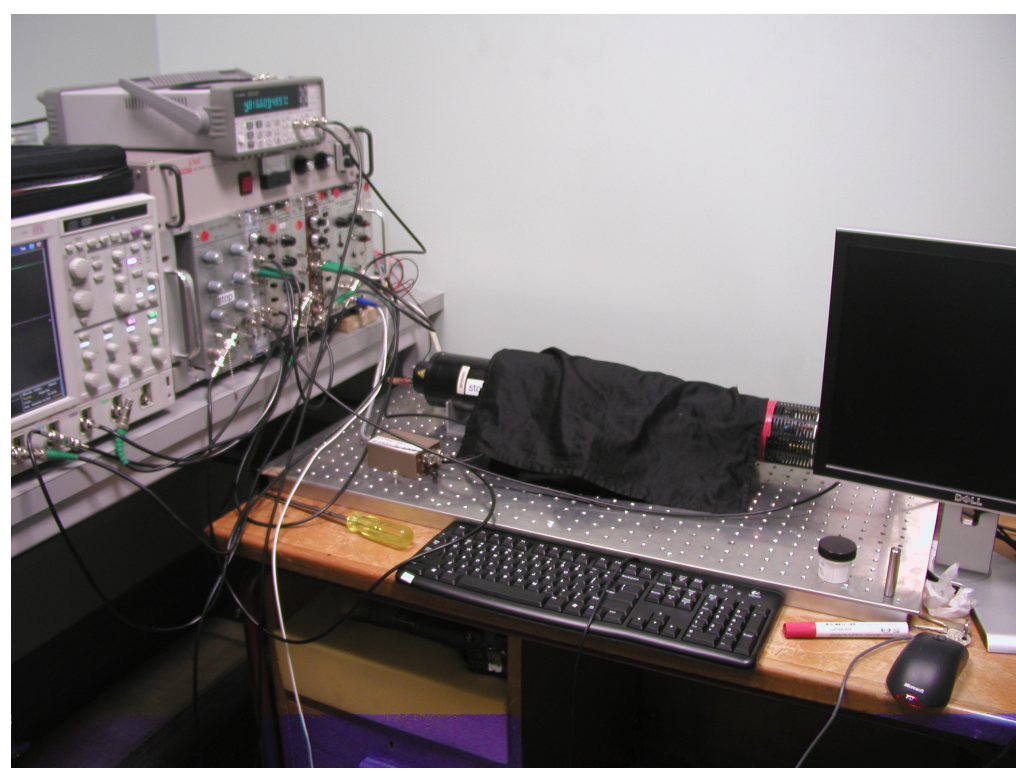

Figure 6.5: Photograph of the configuration used in our system, the oscilloscope and NIM bin can be seen on the left and the PMTs in the centre under the black cloth. 


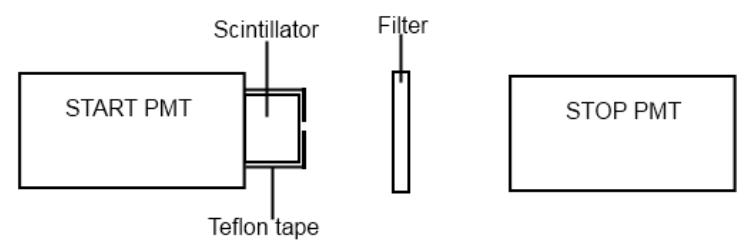

(a)

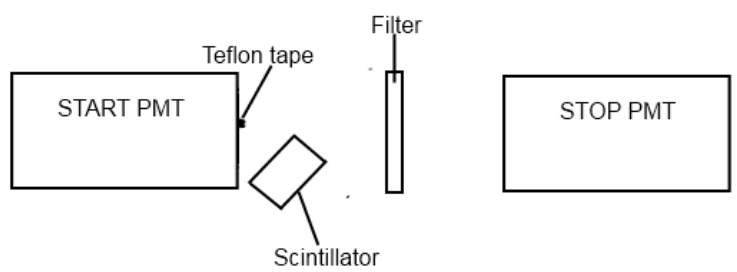

(b)

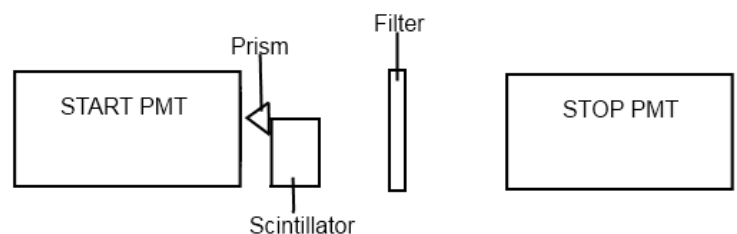

(c)

Figure 6.6: The arrangement of the scintillator to be measured depends on the optical and physical properties of the material, suggestions are shown in (a) for a transparent scintillator, in (b) for a non-transparent scintillator and (c) a non-transparent scintillator which has a uniform composition.

stop PMTs are aligned concentrically but anti-parallel. If the scintillator to be measured is transparent this can be optically coupled to the start PMT with optical grease; the other side can then be partially covered in reflective Teflon tape with a hole to allow some photons to be transmitted in the opposite direction to the stop PMT; this is depicted in figure 6.6(a). If the scintillator is not transparent it may be placed in the PMT tube holder on an angle towards the start PMT and a small white reflector placed on the PMT to reflect some photons to the stop PMT as depicted in figure 6.6(b). Alternatively a prism may be used to reflect some light at $180^{\circ}$ as depicted in figure 6.6(c), this arrangement can only be used for homogeneous scintillators as the recorded stop photons will have originated in only a small portion of the scintillator.

To further reduce the stop signal to around 1 photon per 100 scintillation events, neutral density filters, pinholes and/or interference filters (for wavelength selectability) can be placed in front of the stop detector. Figure 6.7 shows a neutral density filter and a pinhole aperture mounted in one inch square holders; these can be slotted into the attachment to the PMT 


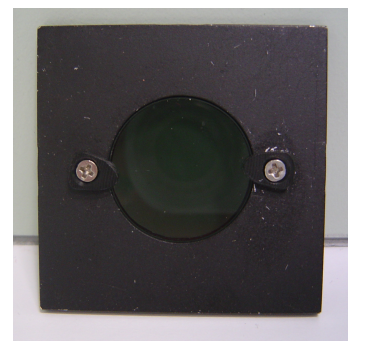

(a)

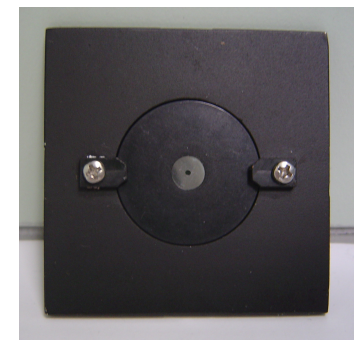

(b)

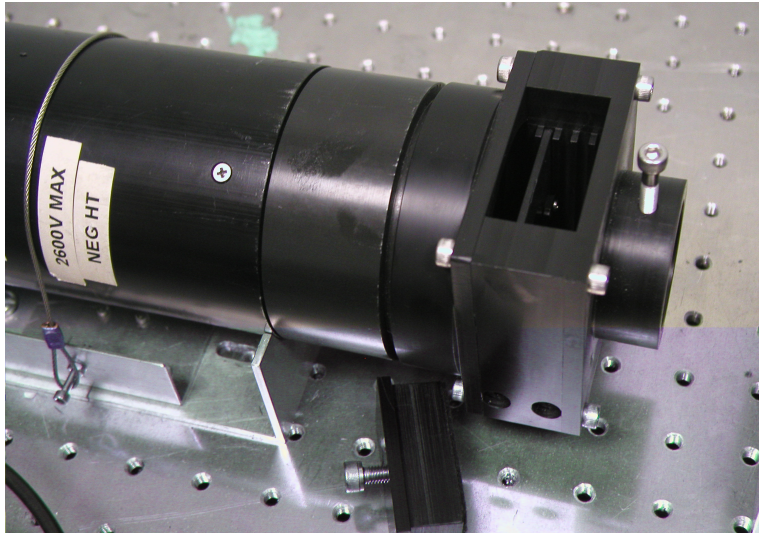

(c)

Figure 6.7: (a) Neutral density filter in holder , (b) Pinhole aperture in holder, both to be used to cut down the light intensity to a single photon event level for the stop detector and (c) the attachment to the PMT these fit into.

shown in figure 6.7(c) in the light path from the scintillator to the stop PMT.

The large scintillation pulses from the start PMT (XP2020Q) are fed to one unit of a fast quad pre-amplifier (Stanford Research $445,350 \mathrm{MHz}$ ) with a $\times 5$ gain to make them large enough to trigger the CFD (Tennelec 453). The single photon pulses from the stop PMT (XP2020Q) are fed to a different channel of the same fast preamplifier, cascaded with a second unit (total gain $\times 25$ ) before the CFD (Ortec 483). Fast pre-amplifiers are needed to make the signal detectable but not change the timing characteristics. The CFD outputs are used to trigger a time to pulse-height converter (Ortec 437A). This outputs a pulse which has an amplitude which is proportional to the time difference between the start and stop signals. The TAC output then drives a multichannel analyser (Fastcom MCA3) which measures the input voltage pulse amplitudes and sorts them into a histogram of number of events versus pulse-height. The time range is set on the time to pulse height converter and spans the range from $200 \mathrm{~ns}$ to 80 $\mu \mathrm{s}$.

\subsubsection{Photomultipliers and constant fraction discriminators}

For single photon counting the voltage on the PMT or the CFD setting must be optimised. The signal increases with PMT voltage but so does the dark count at a different rate. For the CFD setting a lower setting increases both the signal and dark count at different rates. To find the optimal operating settings one can either vary the CFD setting for a fixed PMT voltage or 


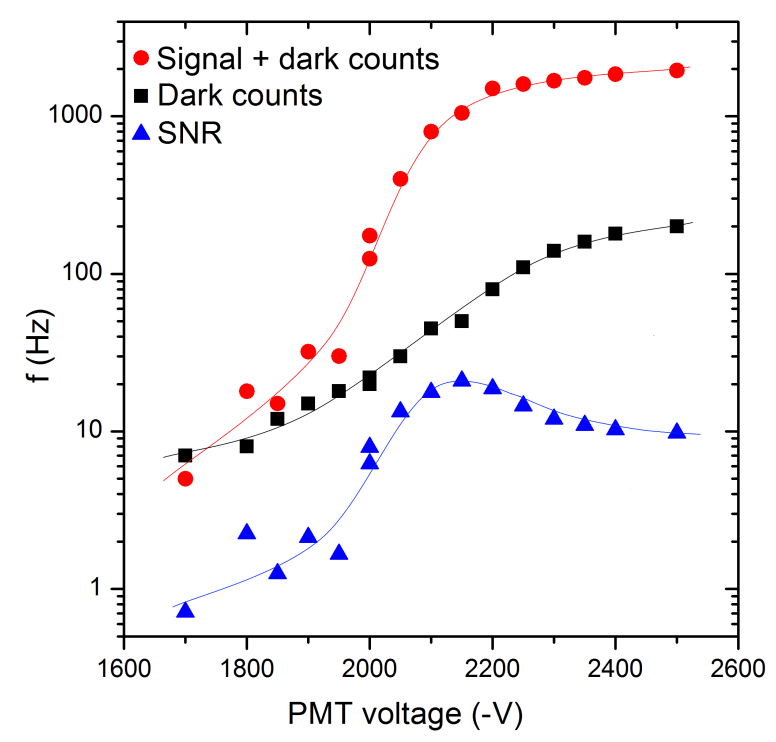

Figure 6.8: The change in frequency of counts on stop PMT with the LED on (red circles), the LED off (black squares) and the signal to noise ratio (SNR) (blue triangles) as a function of PMT voltage. The lines are provided as a guides to the eye.

vary the PMT voltage for a fixed CFD setting and observe the SNR variation; we chose the latter.

A low forwards current was applied to a LED to form a weak light source, and neutral density filters were then used to further reduce the intensity of the light until single photons became distinguishable. The frequency of detected pulses from the CFD (with the CFD setting fixed at $100 \mathrm{mV}$ ) was recorded as a function of PMT voltage with the LED on (signal + dark counts) and off (dark counts) as shown in figure 6.8. The 'knee' of the graph corresponding to optimal SNR occurs around $-2100 \mathrm{~V}$. This is the voltage setting used for all experiments. The sensitivity of the system is limited by dark counts on the stop PMT. For an XP2020Q operated at -2000 V Photonis quote 900 counts /s [102], but in TCSPC this is reduced by using the CFD. We measure a background rate of 30 counts/s with the ${ }^{137} \mathrm{Cs}$ sources present but no scintillator. These counts are due to scintillation in the glass envelope as well as cosmic rays.

The amplitude of the start pulse will vary for each scintillator measured and will depend on the light output and decay time of the scintillator. Because of this, optimisation of the PMT and discrimination threshold should be specific to each particular scintillator, but in practice, as the start PMT works from much larger scintillation pulses as opposed to single photons, optimisation of the settings is not so critical. We have optimised the PMT voltage as 


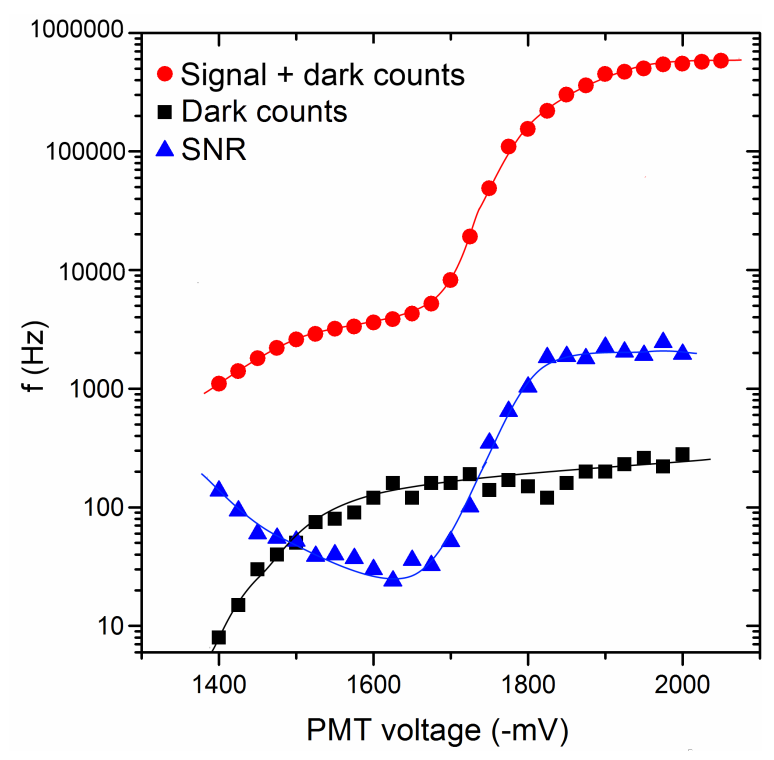

(a)

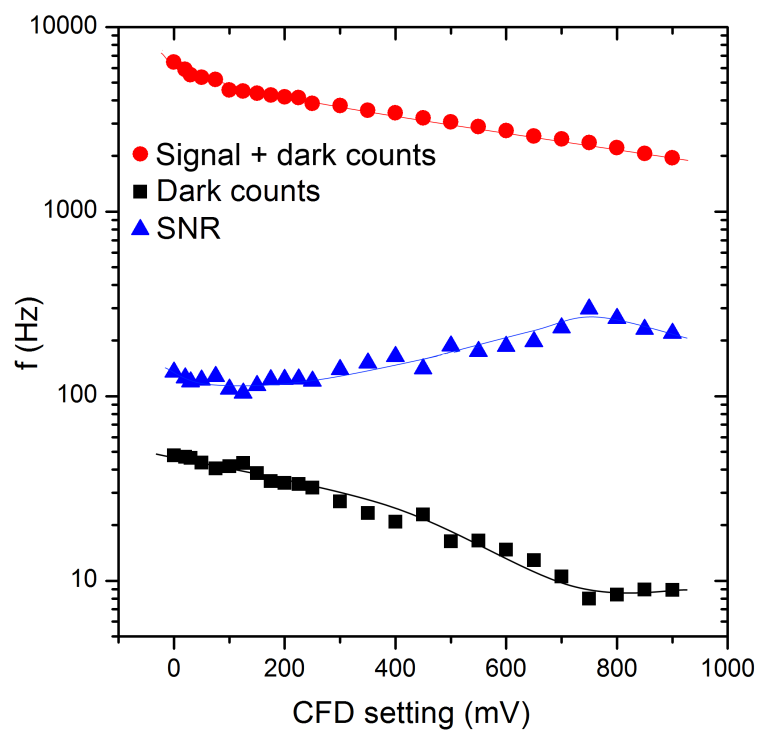

(b)

Figure 6.9: (a) The change in frequency of counts on start PMT with LED on (red circles), the LED off (black squares) and signal to noise ratio (SNR) (blue triangles) as a function of PMT voltage (b) and as a function of CFD setting. The lines are provided as guides to the eye.

for the stop PMT above, but without a CFD or neutral density filters. Again the frequency of detected pulses was recorded with the LED on and off and the SNR calculated as the PMT voltage was varied this is shown in figure 6.9(a). From this the optimal voltage (the knee) was observed to be $-1800 \mathrm{~V}$. This voltage was used in all further work. While the start and stop PMTs are the same models we are operating them in quite different regimes; one is detecting single photons while the other is detecting a larger pulse resulting from multiple single photon events. Furthermore the output of the stop PMT is being discriminated and that of the start PMT is not and hence, the difference in optimal voltage.

With the start PMT set at $-1800 \mathrm{~V}$ and a CFD added before the frequency counter we recorded the variation in the SNR as the discriminator setting was varied; this is shown in figure 6.9(b). There is very little variation visible in this SNR curve. This means that the time consuming process of optimising the settings for each trial scintillator can be avoided. Instead one can adjust the CFD setting, while monitoring the PMT output and CFD output on an oscilloscope, until pulses corresponding to scintillation events exclusively trigger the CFD. This is far more practical and as can be seen from figure 6.9(b) this will have little effect on the SNR achieved. 


\subsubsection{Count rate}

When the system is in operation a frequency counter (Agilent 53131A $225 \mathrm{MHz}$ Universal Frequency Counter/Timer) is used to ensure the counts rates are appreciable and to avoid "pile up". Pile up is the name given to the situation where the light attenuation in the stop beam is inadequate and more than one stop photon is detected from one start pulse. The TAC converts on the first pulse detected, thus leading to a shortening of the recorded lifetime. Significant pile up begins to occur if over $5 \%$ of start pulses result in a stop signal [100]. The "Hit rate" $(H)$ is the fraction of start pulses resulting in a stop, signal corrected for dark current pulses, and is given by

$$
H=\frac{f_{\text {stop }}(S)-f_{\text {stop }}(N S)}{f_{\text {start }}(S)-f_{\text {start }}(N S)}
$$

Where $f_{\text {stop(start) }}$ refers to the frequency of the stop(start) rates and S(NS) refers to '(no) scintillator in place'. For our configuration this is

$$
H=\frac{f_{\text {stop }}(S)-30}{f_{\text {start }}(S)}
$$

The frequency counter is also used to ensure the time between start signals is on average longer than the time range of the measurement. Figure 6.10 depicts the situation when the time between start signals (shown at top) is shorter than the measurement time range; a single photon is detected (shown at bottom) but could be from either start pulse. This means for an $80 \mu$ s range (the longest available) the start signal rate can be no greater than $12500 \mathrm{~s}^{-1}$ which is greater than the rate measured for $\mathrm{NaI}(\mathrm{Tl})$.

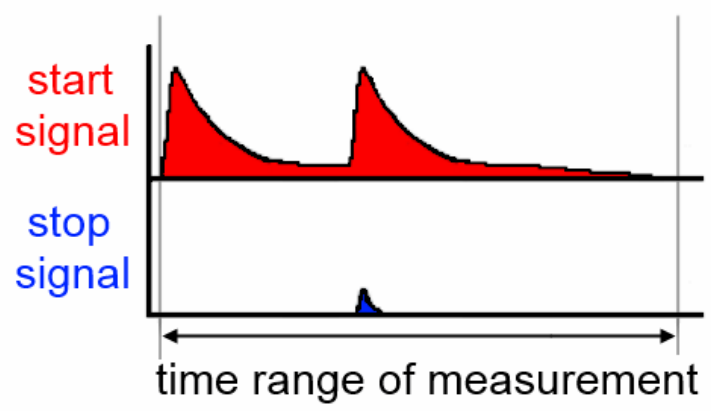

Figure 6.10: Depiction of why if the pulse rate is higher than the measurement time range a single photon cannot be accurately associated with the first pulse. 


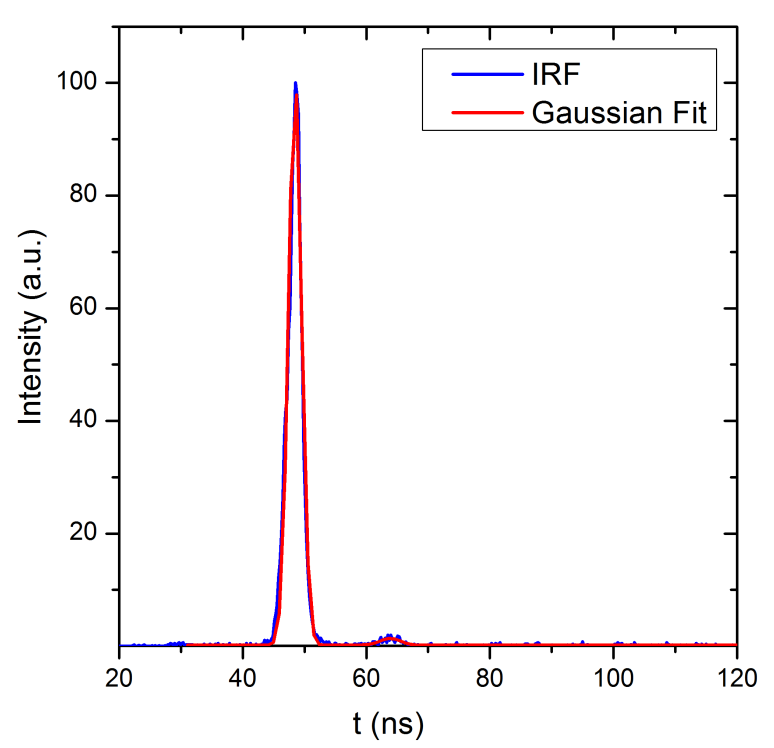

(a)

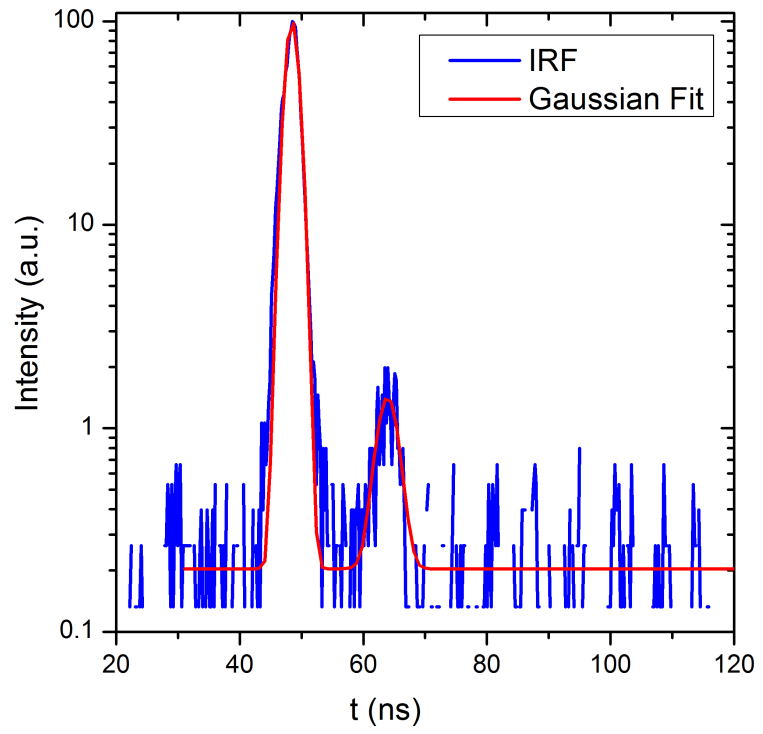

(b)

Figure 6.11: The instrument response of the system fitted with two Gaussians (red line) shown on (a) a linear scale and (b) a log scale.

\subsubsection{Impulse response}

The instrumental response is shown in figure 6.11 on both linear and log scales. This was recorded by replacing the scintillator with a light guide (Perspex). Perspex does not scintillate, but under $\gamma$-irradiation Cherenkov radiation is produced which has a lifetime of a few picoseconds, and so it can be regarded as a delta function, and used to determine the instrument response [103]. The effective time resolution of the system is characterised by the instrument response function (IRF) which is shown in figure 6.11. The IRF is determined by temporal dispersion in the optical configuration, transit time spread in the PMT and timing jitter in the recording electronics. The decays recorded with the system will be a convolution of the scintillation decay and the IRF. The main Gaussian of the IRF has a full width half maximum (FWHM) value of $2.5 \mathrm{~ns}$. For fast scintillation decays ( $\tau<100 \mathrm{~ns}$ ) deconvolution will be required to extract the true decay. There is a second peak in the IRF which occurs 15 ns after the main peak. This may be an after-pulse; these are spurious pulses that appear in the wake of true pulses at defined intervals. There are two main causes (a) ionization of residual gases and (b) luminous reactions. After-pulses due to the ionisation of residual gases occur hundreds to thousands of ns after the original pulse [102]. After-pulses due to luminous reactions occur within 15-100 ns of the main pulse. These are due to the PMT dynodes emitting photons when bombarded with electrons, in some PMTs these photons can 


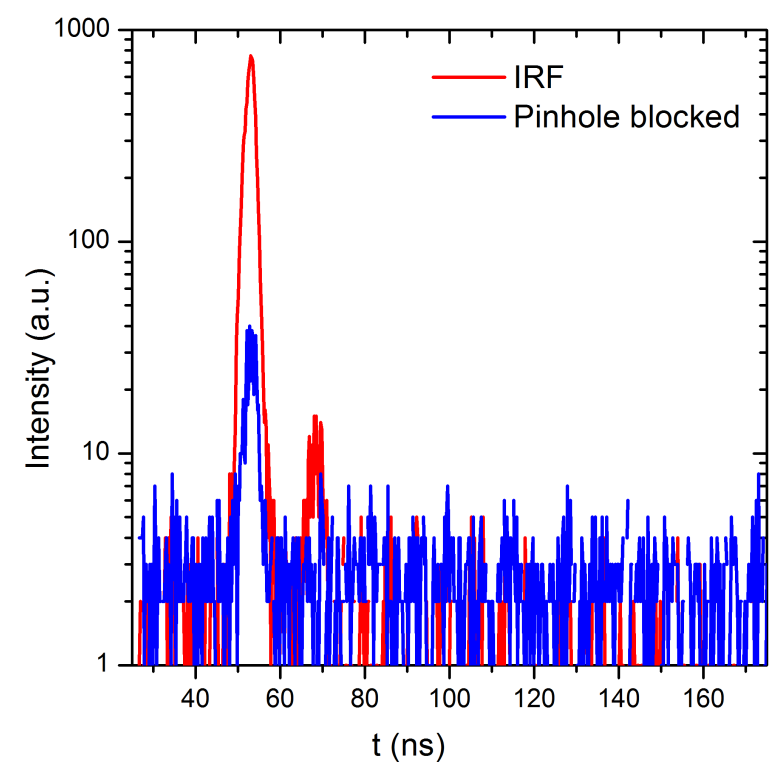

Figure 6.12: Background spectrum (blue) shown over the IRF (red) so as to show the contribution to the IRF due to the background.

Table 6.1: Double Gaussian instrumental response fit parameters.

\begin{tabular}{cc}
\hline Parameter & Value \\
\hline$y_{0}$ & 0.20 \\
$A_{1}$ & 100 \\
$t_{1}(\mathrm{~ns})$ & 48 \\
$w_{1}(\mathrm{~ns})$ & 1.1 \\
$A_{2}$ & 1.2 \\
$t_{2}(\mathrm{~ns})$ & 63 \\
$w_{1}(\mathrm{~ns})$ & 1.7 \\
\hline
\end{tabular}

strike the photocathode causing a delayed pulse (after pulse). Luminous after pulses are the most likely cause of the second peak in the IRF. From figure 6.11 the integrated area under this peak is less than 1 percent that of the main response and as such should not significantly affect any results, in addition both peaks can be allowed for in deconvolution processes.

The IRF has been fitted with two Gaussians, which are time shifted with respect to each other, to approximate the response for deconvolution purposes. The fitted curve is

$$
I=y_{0}+A_{1} \exp \left(\frac{-1}{2}\left(\left(t-t_{1}\right) / w_{1}\right)^{2}\right)+A_{2} \exp \left(\frac{-1}{2}\left(\left(t-t_{2}\right) / w_{2}\right)^{2}\right)
$$

and the fitted parameters are given in table 6.1 .

As well as the IRF we record a spectrum with the same settings as used for the IRF but with 
Table 6.2: Calibration factors for use with time to pulse-height converter and MCA.

\begin{tabular}{cccc}
\hline Multiplier & Calibrated channels per ns & $\begin{array}{c}\text { Nominal range } \\
\text { (ns) }\end{array}$ & $\begin{array}{c}\text { Actual range } \\
(\mathrm{ns})\end{array}$ \\
\hline $\mathrm{x} 1$ & 19.1 & 200 & 214 \\
& 9.77 & 400 & 419 \\
$\mathrm{x} 10$ & 4.41 & 800 & 929 \\
& 7.94 & 500 & 516 \\
& 3.84 & 1000 & 1070 \\
& 1.87 & 2000 & 2190 \\
$\mathrm{x} 100$ & 0.958 & 4000 & 4280 \\
& 0.471 & 8000 & 8700 \\
& 0.735 & 5000 & 5570 \\
& 0.369 & 10000 & 11100 \\
& 0.181 & 20000 & 22600 \\
& 0.0872 & 40000 & 47000 \\
& 0.0380 & 80000 & 94000 \\
\hline
\end{tabular}

the optical path from the perspex to the stop PMT blocked. This allows the measurement of the spurious signal due to gamma rays interacting with PMT or electronic crosstalk. This is the background spectrum and is shown in figure 6.12 .

\subsubsection{Calibration}

The time to pulse height converter font panel specifies nominal settings for the time interval which generates a $10 \mathrm{~V}$ pulse. These settings apeared to be significantly in error, and so a calibration was carried out. A function generator was used to pulse two light emitting diodes (LEDs) at a known time difference. The time difference between these pulses after passing through the pre-amplifiers and CFDs was observed on a fast $(1 \mathrm{GHz})$ oscilloscope (Tektronix 7104). The time difference recorded on the oscilloscope was compared to the peak channel on the multichannel analyzer (MCA) spectrum. This was done for all time range settings on the time to pulse-height converter. The calibration plots are shown in figure 6.13, and the correction factors obtained are tabulated in table 6.2. The linear trend lines are all good fits and the calibration error varies between 7 and $15 \%$ to that expected from the nominal settings. 


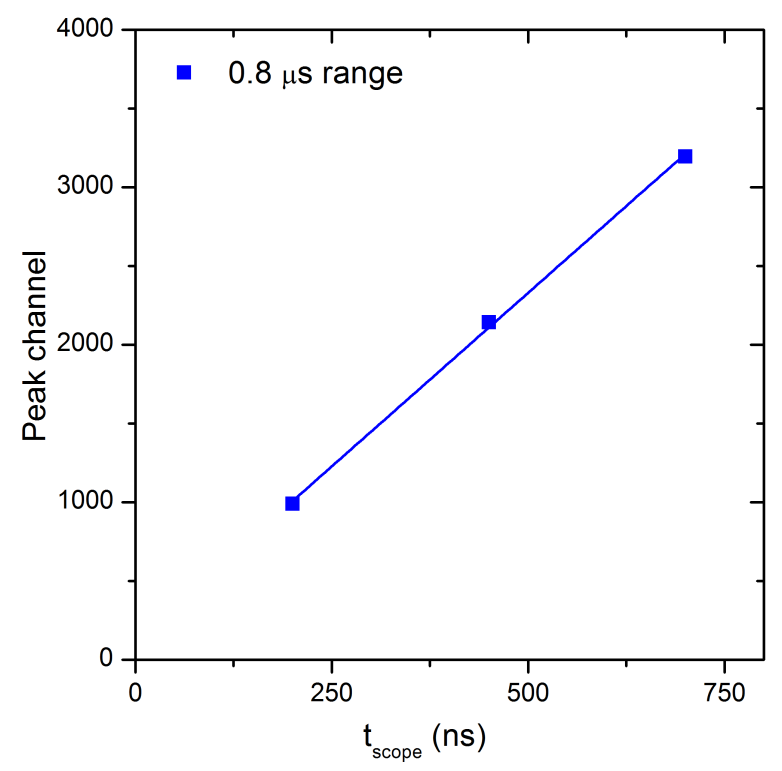

(a)

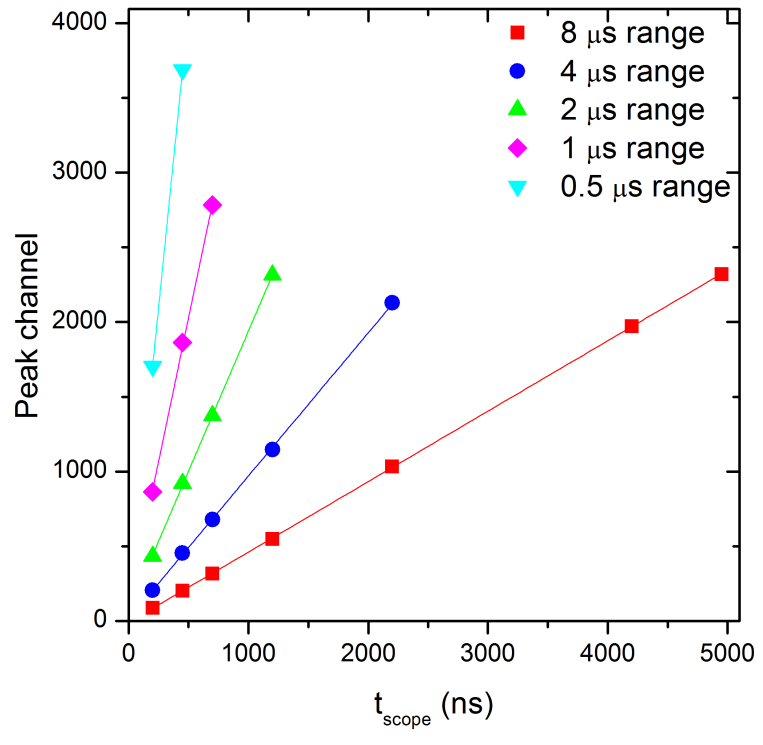

(b)

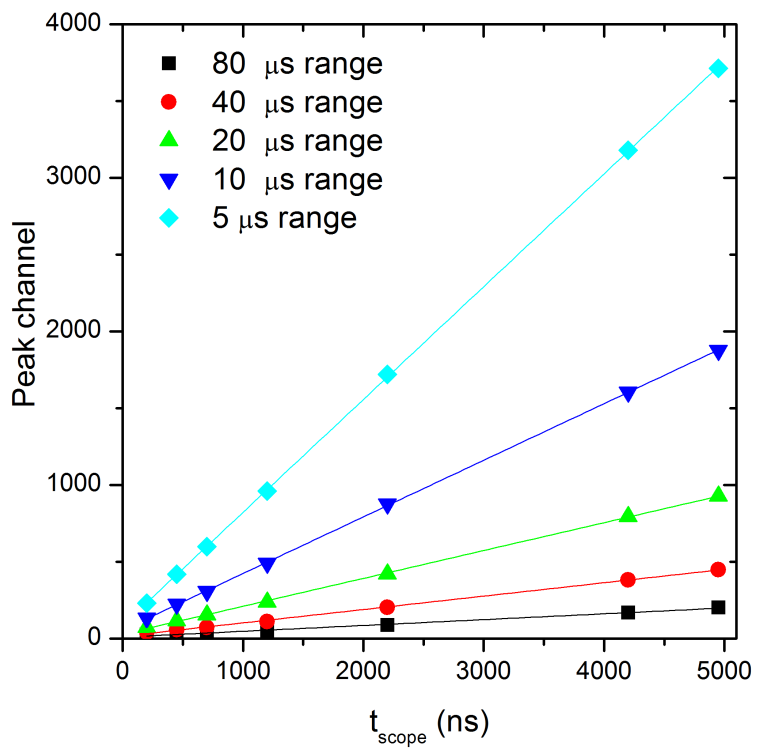

(c)

Figure 6.13: Calibration of the time to pulse height converter on the $(a) \times 1$ multiplier $(b) \times$ 10 multiplier and $(c) \times 100$ multiplier time settings. 


\subsubsection{Dynamic range}

The dynamic range is the time range the analogue to digital converter (ADC) operates over. The ADC used in the setup described here is a Fastcom MCA3, which has 4096 bins. To measure a decay component of $\tau$ accurately one needs the bin width to be less than or equal to $\frac{\tau}{10}$; however the dynamic range needs to be 3 times the longest decay component [101]. The shortest time range usable on the time to pulse height converter is 200 ns, so over 4096 bins this gives the fastest recordable decay time of $0.5 \mathrm{~ns}$. While the system can also record decays out to $27 \mu$ s the two components cannot be recorded in the same spectra, however one has the option of recording two spectra and simultaneously fitting both data sets.

\subsection{Alternative measurement technique}

The main alternative to using a TCSPC set-up for measuring scintillation lifetimes is to use a fast oscilloscope. However even fast oscilloscopes (such as the Tektronix 7104) have digitisation problems, since only a discrete number of levels are available to represent the continuous signal. Most oscilloscopes are based on either a 8-bit or a 16-bit analog-to-digital converter (ADC); fast (GHz) oscilloscopes generally only have 8-bit converters. An 8-bit converter provides only 256 levels [104]. This means the recorded decays have high levels of 'quantisation noise'.

\subsubsection{The effect of signal averaging}

For scintillation events it is desirable to record the decay over 3 orders of magnitude (or 1 in 1000). This is difficult on a fast oscilloscope as, with only 8 bits, one can achieve a signal that is at best defined to 1 part in 256. Signal averaging is normally used to improve "noisy" signals; signal averaging takes the mean of the digitised values. Figure 6.14 shows a single scintillation event and an average of 2000 such events. The single decay shows marked fluctuations due to the statistical nature of photon emission. In figure 6.14(a) the quantisation of the data is particularly evident for signals between $1 \mathrm{mV}$ and $10 \mathrm{mV}$; there are only three 


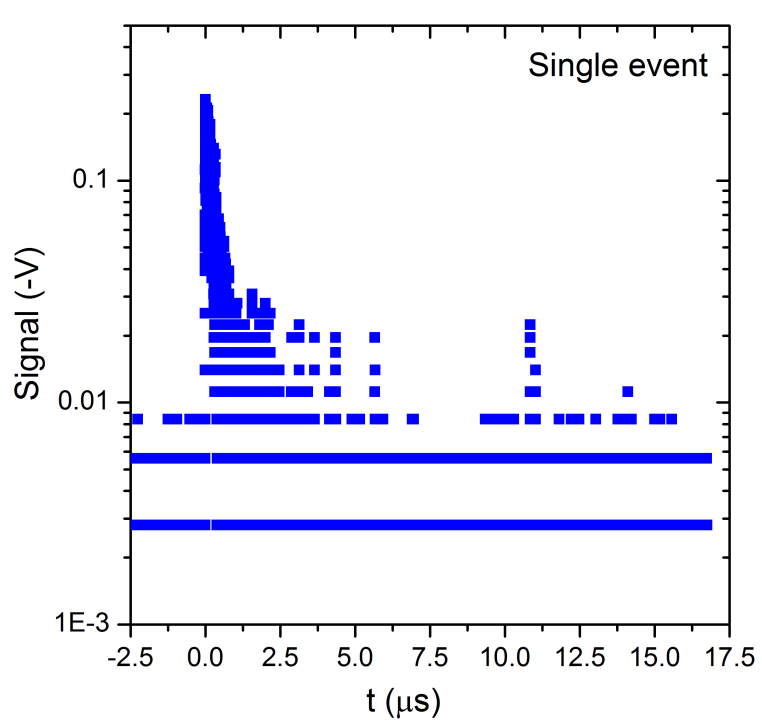

(a)

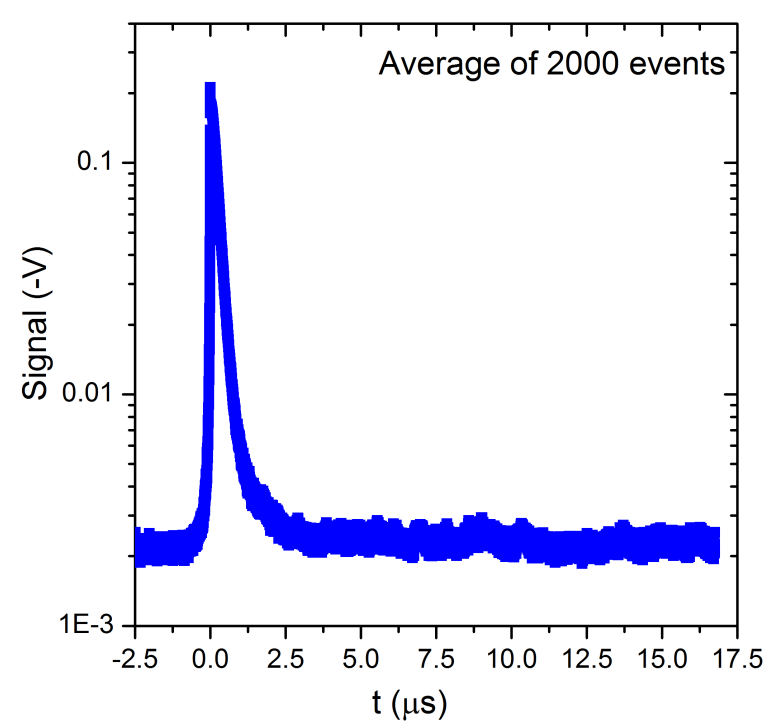

(b)

Figure 6.14: (a) Typical single scintillation event and (b) the signal average of 2000 such events.

values over this order of magnitude change in signal. As seen in figure 6.14 signal averaging removes the quantised look of the data, but whether the decay is more accurately represented is not clear.

In both figure 6.14(a) and figure 6.14(b) the signal is essentially the DC background from $t=7.0 \mathrm{~ms}$ to $t=17 \mathrm{~ms}$. The DC background of the single event signal is $-2.3 \mathrm{mV}$ and the standard deviation around this value is 0.00158 . For the averaged event the DC background signal is $-2.1 \mathrm{mV}$ and the standard deviation of the the signal around this value is 0.000134 . The averaged signal is comprised of the mean value from of 2000 events, and so for a non quantised signal the SNR should be $\sim \sqrt{2000}$ times better than for a single event. However the standard deviation is only $\sim \sqrt{140}$ times better. Signal averaging is far less effective on a quantised signal. Therefore, while signal averaging does improve the quantised look of the data and does improve accuracy this is not at the expected rate due to quantisation. This makes it difficult to achieve high accuracy over the whole decay with an oscilloscope, and this particularly limits measurements of longer lifetimes. 


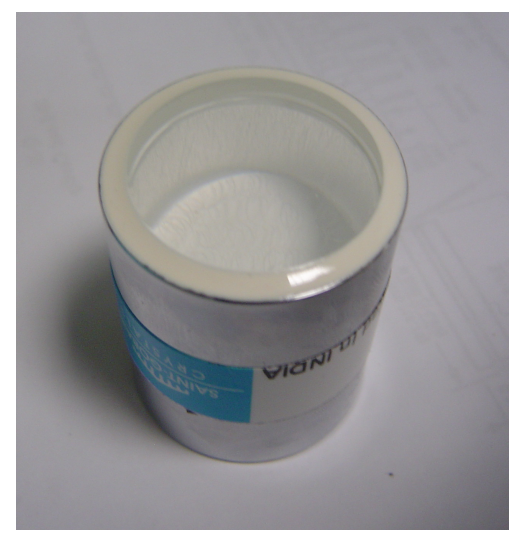

Figure 6.15: Photograph of $\mathrm{NaI}(\mathrm{Tl})$ single crystal scintillator.

\subsection{Comparison with a fast oscilloscope}

It is of interest to compare our TCSPC results with those from a fast oscilloscope to confirm firstly that we record similar decay times with the two systems and furthermore to compare the performance of the two systems. We want to record decays over three orders of magnitude in order to resolve long lifetime components.

\subsubsection{NaI(Tl) scintillator}

$\mathrm{NaI}(\mathrm{Tl})$ is a commercially used scintillator and at $20^{\circ} \mathrm{C}$ has a reported dominant lifetime component of $230 \mathrm{~ns}$ with a slower $1100 \mathrm{~ns}$ component in a 4:1 intensity ratio at room temperature [30]. The decay times are temperature dependent. Figure 6.15] shows a single crystal of $\mathrm{NaI}(\mathrm{Tl})$ from St Gobain and figure 6.16(a) shows the scintillation profile recorded on a $1 \mathrm{GHz}$ fast oscilloscope (Tektronix 7104). The signal recorded is an average of 2052 events, triggered from a CFD. This has been fitted with a double exponential decay of the form

$$
Y=Y_{0}+A_{1} \exp \left(\frac{-t}{\tau_{1}}\right)+A_{2} \exp \left(\frac{-t}{\tau_{2}}\right)
$$

with lifetime components of $\tau_{1}=236 \mathrm{~ns}$ and $\tau_{2}=936 \mathrm{~ns}$ in an integrated intensity ratio of 10:1. At $23{ }^{\circ} \mathrm{C}$ (the temperature of our measurements) the principal decay time specified by the St Gobain is $237 \mathrm{~ns}$ [105].

Shown in figure 6.16(b) is the decay of the same $\mathrm{NaI}(\mathrm{Tl})$ crystal recorded on the TCSPC system, this has been fitted with the same from of double exponential decay with lifetime 


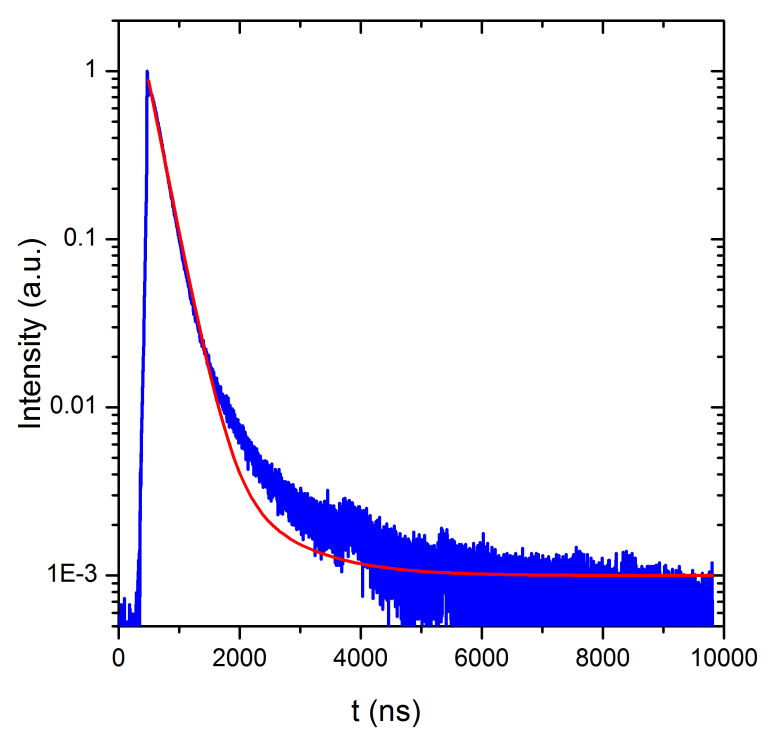

(a)

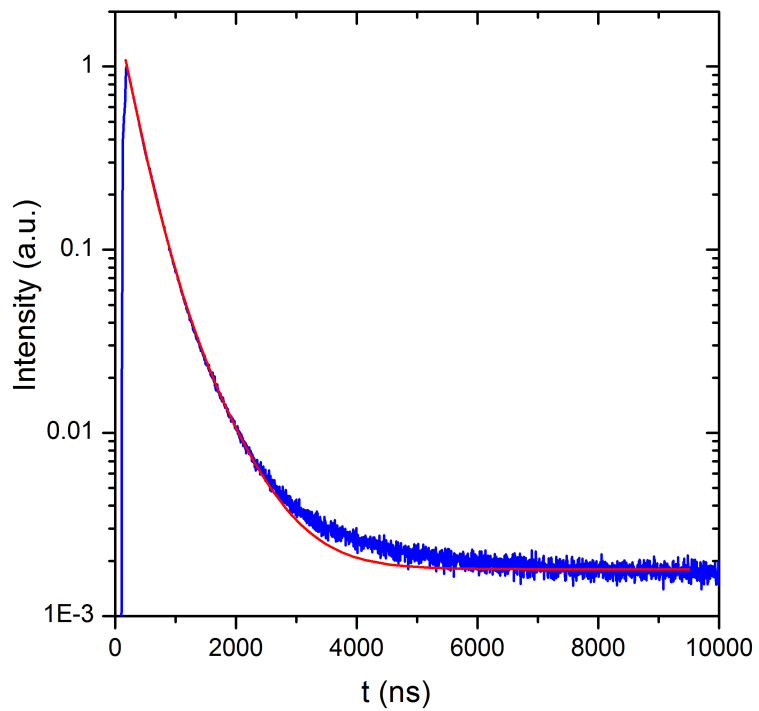

(b)

Figure 6.16: The decay profile for a commercial $\mathrm{NaI}(\mathrm{Tl})$ single crystal recorded using (a) the fast oscilloscope and (b) the TCSPC system, also shown in red are the double exponential fits.

Table 6.3: $\mathrm{NaI}(\mathrm{Tl})$ lifetime results from various measurements.

\begin{tabular}{ccccc}
\hline & Literature value [30] & St Gobain [105] & TCSPC & Oscilloscope \\
\hline fast component (ns) & 230 & 237 & 236 & 236 \\
longer component (ns) & 1100 & & 900 & 936 \\
integrated intensity ratio & $4: 1$ & & $3: 1$ & $10: 1$ \\
\hline
\end{tabular}

components of $\tau=236$ and $900 \mathrm{~ns}$ in an integrated intensity ratio of 3:1. The decay was recorded over two days using the TAC set to $5.5 \mathrm{~ns} /$ channel. Table 6.3 summarises the results found from literature, TCSPC and oscilloscope measurements. The two methods give the same decay time and agree with the literature, which instills faith in the TCSPC system.

\subsubsection{NE102 Scintillator}

The plastic scintillation, NE102, shown in figure 6.17, is a very fast plastic scintillator which has a stated lifetime of just $2.4 \mathrm{~ns}[51,106]$. The lifetime of a commercial NE102 (BC400 equivalent) was measured on both a fast oscilloscope and with the TCSPC sytem. The averaged pulse profile recorded on the fast oscilloscope is shown in figure 6.18(a) for 2052 events. The decay recorded on the TCSPC configuration is shown in figure 6.18(b). That 


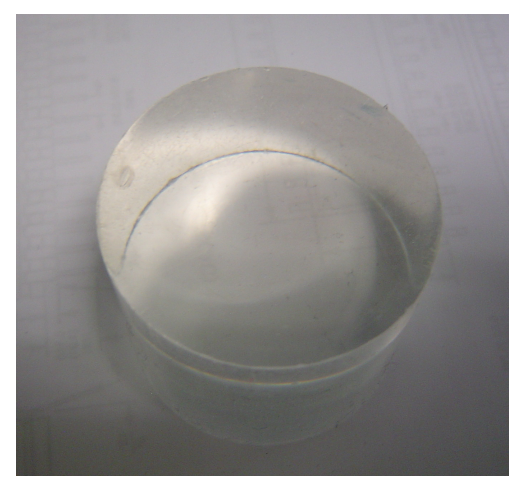

Figure 6.17: Photograph of plastic NE102 scintillator.

Table 6.4: NE102 lifetime results from various measurements.

\begin{tabular}{cccc}
\hline & St Gobain [51] & TCSPC & Oscilloscope \\
\hline fast component $(\mathrm{ns})$ & 2.4 & 2.5 & 6.7 \\
longer component (ns) & & 20 & \\
integrated intensity ratio & & $12: 1$ & \\
\hline
\end{tabular}

recorded on the scope has been fitted with a single exponential of the form

$$
Y=Y_{0}+A \exp \left(\frac{-t}{\tau}\right)
$$

with $\tau=6.7 \mathrm{~ns}$. That of the TCSPC has been fitted prior to deconvolution with a single exponential of the same form with $\tau=6 \mathrm{~ns}$. The anaylsis process can be improved by deconvolution with the IRF; this was performed using deconvolution software (DAS6) software, which performs a convolution of a test decay with the IRF and compares the result to the recorded decay in an iterative process which converges on the best fit. A double exponential decay of the form

$$
Y=Y_{0}+A_{1} \exp \left(\frac{-t}{\tau_{1}}\right)+A_{2} \exp \left(\frac{-t}{\tau_{2}}\right)
$$

was fitted for the actual decay; with $\tau_{1}=2.5 \mathrm{~ns}$ and $\tau_{2}=20 \mathrm{~ns}$ in an approximate 12:1 integrated intensity ratio. The decay curve and fitted curve can be seen along with the instrumental response in figure $6.18(\mathrm{~b})$.

The results obtained for the NE102 scintillator are summarised in table 6.4 which shows that the decay time recorded with the TCSPC system is in excellent agreement with the manufacturer's data.

When looking at the plastic scintillation profile recorded using the TCSPC system on a longer 


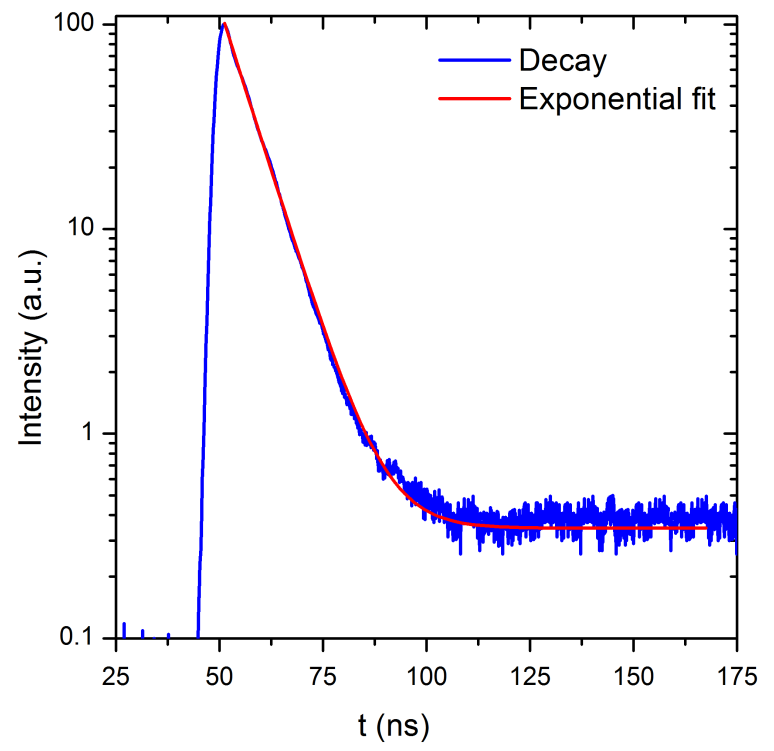

(a)

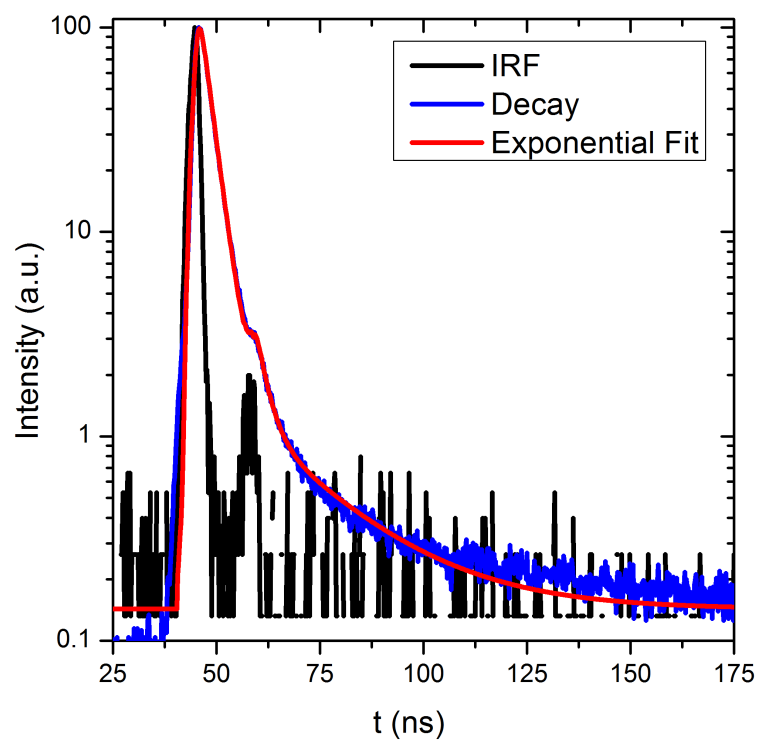

(b)

Figure 6.18: The decay profile for NE102 plastic scintillator recorded using (a) the fast oscilloscope and (b) the TCSPC system, also shown in red are the single exponential fits.

timescale as in figure 6.19 there is clear evidence of after-pulses. The after-pulse peaks visible in figure 6.19 occur $265,370,510$ and 734 ns after the main pulse with those at 265 ns and 734 ns being the dominant after-pulse peaks. These time-scales are appropriate for after-pulses caused by ionization of residual gases in the PMT. Some commonly observed ions are $\mathrm{H}^{+}$, $\mathrm{H}_{2}^{+}, \mathrm{He}^{2+}, \mathrm{He}^{+}, \mathrm{N}^{+}, \mathrm{O}^{+}$and $\mathrm{CH}_{4}^{+}$which typically occur $\sim 250,300,300,400,850,850$ and $1000 \mathrm{~ns}$ after the main pulse respectively (although this does vary with the particular PMT design) [102, 107]. Thus the main after-pulses can likely be assigned to $\mathrm{H}^{+}$, and $\mathrm{N}^{+}$and/or $\mathrm{O}^{+}$. Residual gases may left in the PMT after evacuation, desorbed by the structural materials or may be $\mathrm{He}$ or $\mathrm{H}_{2}$ that has migrated through the glass envelope, which may be ionized by electrons. The hydrogen gas may have permeated the glass envelope of the PMT whereas any larger molecules such as nitrogen or oxygen must be from the original manufacturing process. Residual gases are found in most PMT's which have been in use for some time.

\subsubsection{Developmental scintillator}

$\mathrm{Ba}_{0.3} \mathrm{Sr}_{0.7} \mathrm{Cl}_{2}$ doped with $\mathrm{Eu}^{2+}$ is a developmental x-ray phosphor discussed in this thesis. The lifetime of $\mathrm{Eu}^{2+}$ luminescence is in general much longer than the decays discussed above at $\sim$ $1 \mu \mathrm{s}$ in most compounds. Thus $\mathrm{Ba}_{0.3} \mathrm{Sr}_{0.7} \mathrm{Cl}_{2}: \mathrm{Eu}^{2+}$ offers a longer timescale over which to test 


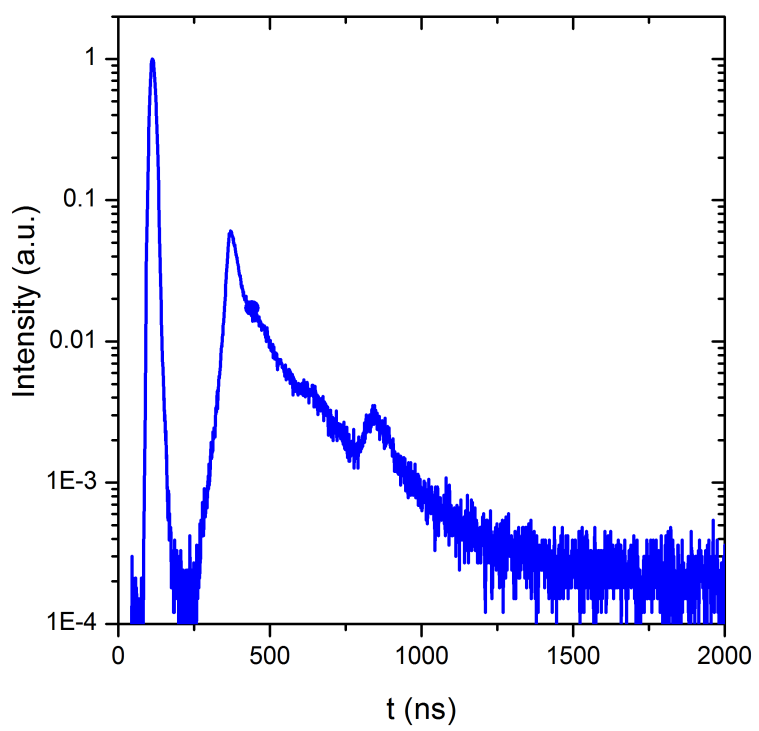

Figure 6.19: Longer timescale NE102 scintillation profile showing evidence of after-pulsing.

the system. Shown in figure 6.20(a) is the decay recorded on the fast oscilloscope (a) (average of 2052 events). The lifetime recorded on the TCSPC system is also shown in figure 6.20(b) Both scintillation profiles have been fitted with single exponential decays of the form

$$
Y=Y_{0}+A \exp \left(\frac{-t}{\tau}\right)
$$

and the fitted lifetimes are both $1.6 \mu$ s showing good agreement. The photoluminescent lifetime recorded under UV excitation is $1.0 \mu$ s (see chapter 8) and one expects a longer decay time for scintillation due to additional energy transfer processes, so these are reasonable results. Figure 6.20(b) clearly shows the significantly improved dynamic range and S/N ratio achieved with the TCSPC system compared to the oscilloscope (figure 6.20(a),

\subsection{Possible improvements}

Cooling the PMT is a well known and commonly used technique to reduce dark counts. This would help to lower the background and improve the SNR of the recorded decay curves enabling much longer decay times to be resolved accurately. While the temperature effect on each PMT is different (even those of the same model), the dark counts for a bi-alkali cathode roughly double with a 5 degree increase in temperature [108]. A decrease in temperature is easily achievable with thermoelectric cooling or alternatively with liquid nitrogen, dry ice or 


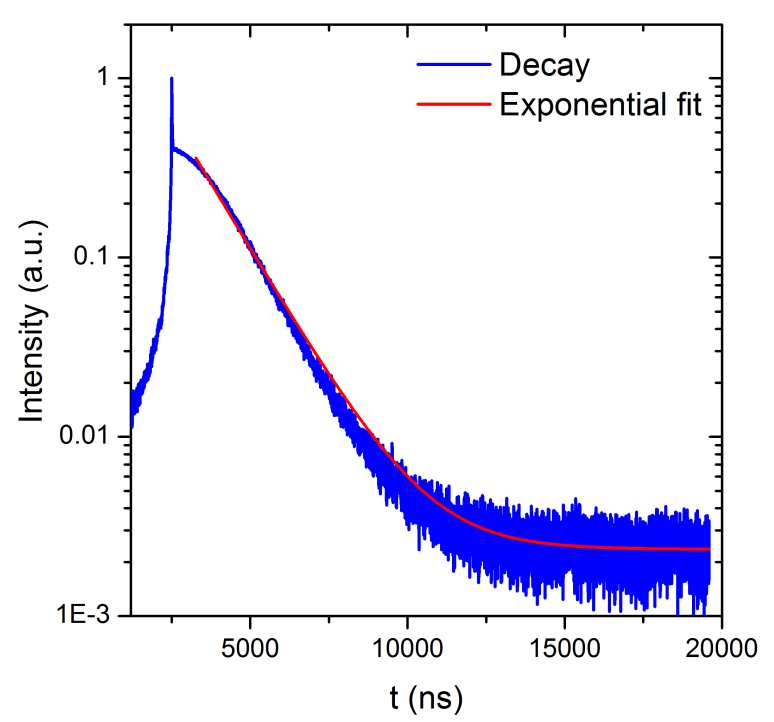

(a)

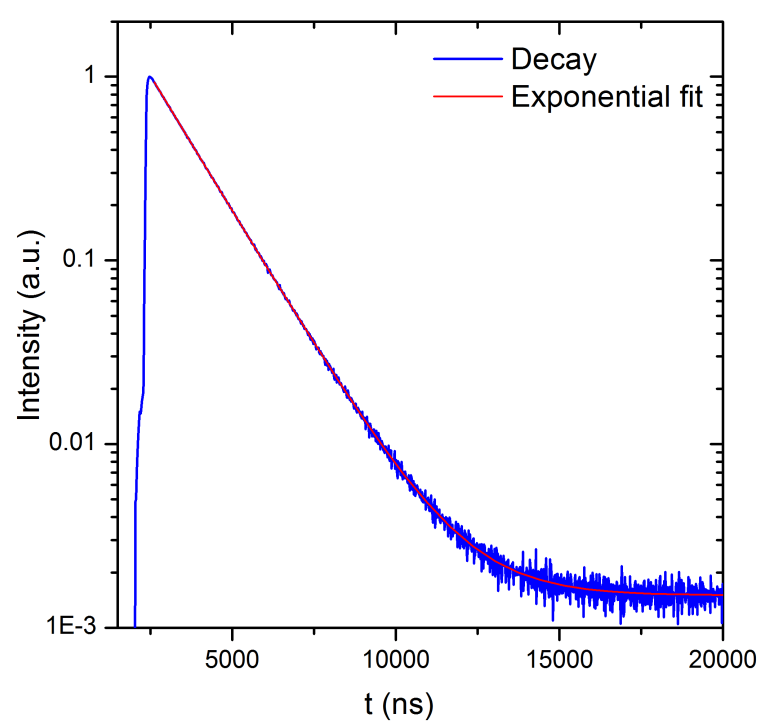

(b)

Figure 6.20: The decay profile for $\mathrm{Ba}_{0.3} \mathrm{Sr}_{0.7} \mathrm{Cl}_{2}: \mathrm{Eu}^{2+}$ recorded using (a) the fast oscilloscope and (b) the TCSPC system, also shown in red are the single exponential fits.

water cooling.

Using a different radioactive source may yield faster acquisition times and higher SNR. ${ }^{137} \mathrm{Cs}$ decays via the emission of a $0.66 \mathrm{MeV}$ gamma ray; this is a relatively high energy $\gamma$-ray. In general the higher the energy the more likely the $\gamma$-ray is to pass through the scintillator without being absorbed. Using a lower energy $\gamma$-ray source would likely increase the $\gamma$-ray absorption rate which would correspond to an increase in counting rate. This would also decrease the number of $\gamma$-rays which may interact directly with the PMT.

In 1979 a modified TCPSC technique [103] was introduced in which a separate fast scintillator is coupled to the start detector, and the excitation source is ${ }^{22} \mathrm{Na}$ which produces two positron-annihilation gamma rays per event that travel in opposite directions. Thus the start timing pulse is the same for all scintillators measured. This also allows for intensity comparisons. This technique has become quite common for fast scintillation lifetime measurements. However, the requirement that both the fast 'start' scintillator and the one to be measured absorb gamma rays from the same event leads to an approximately five fold SNR deterioration [103]. Hallam [103] notes that for intense scintillators, the signal-to-background ratio is limited by the strength of the radioactive source, whereas, for weak scintillators the signal-to-background ratio is dominated by photomultiplier noise. Thus in order to retain maximum SNR, so as to cover the full range of potential scintillators we have opted to use 
the original technique with the start and stop signals coming from the same scintillator. Using multiple ${ }^{137} \mathrm{C}$ s sources which are relatively weak $(\sim 10 \mu \mathrm{Ci}$ each $)$ as our excitation source allows us to change the strength of the source, depending on the scintillator to be measured.

The modified technique of Hallam [103] does offer the advantage of allowing for intensity comparisons. It would be very easy to adapt our system for this, using the NE102 scintillator as the start pulse and sandwiching a ${ }^{22} \mathrm{Na}$ source between the NE102 and the scintillator to be measured. 


\section{Chapter 7}

\section{Rare-earth $\left(\mathrm{La}^{3+}, \mathrm{Pr}^{3+}\right)$ stabilised cubic barium chloride}

\subsection{Introduction}

Alkali earth halides have shown to be promising scintillator and phosphor host lattices showing very high light yields when doped with a rare earth luminescent ion [109-111]. $\mathrm{BaCl}_{2}$ is particularly interesting as it has a high average atomic number and can form in three different crystal structures, all of which have been suggested as x-ray detection or imaging materials. At room temperature $\mathrm{BaCl}_{2}$ normally forms in the orthorhombic phase which in undoped form has been found to be a fast scintillator [112-114]. It exhibits a two component (1.5 and $85 \mathrm{~ns}$ ) decay in a 34:66 integrated intensity ratio but has a relatively low light output, at $2 \%$ that of $\mathrm{NaI}(\mathrm{Tl})$ [113]. Small europium doped $\mathrm{BaCl}_{2}$ nano-crystals of hexagonal structure can be formed in glass ceramics, and this forms the basis for a class of transparent high-resolution storage phosphors [115]. The third phase is the high temperature cubic fluorite phase which is only stable for pure $\mathrm{BaCl}_{2}$ between $925^{\circ} \mathrm{C}$ and the melting point at $962{ }^{\circ} \mathrm{C}$.

Previous work by Edgar et al. [116-118] demonstrated that the cubic (fluorite) phase of $\mathrm{BaCl}_{2}$ can be stabilised at room temperature by doping with $12.5 \%$ (by mole fraction) $\mathrm{LaCl}_{3}$ or $\mathrm{CeCl}_{3}$. The fluorite structure can be visualised as a simple cubic lattice of anions with every 
second interstitial space filled by a cation. It was argued that in the lanthanum/cerium stabilised cubic material, the added $\mathrm{LaCl}_{3} / \mathrm{CeCl}_{3}$ leads to $\mathrm{La}^{3+} / \mathrm{Ce}^{3+}$ ions substituted for $\mathrm{Ba}^{2+}$ ions at cation sites and additional $\mathrm{Cl}^{-}$ions occupying the interstitial vacancies inherent in the fluorite lattice.

The cerium stabilised cubic barium chloride (CSCBC) showed a good light yield of 7200 photons/MeV and a fast decay of 50 ns with the emission comprising two peaks at 348 and $373 \mathrm{~nm}$ in the UV part of the spectral range [116]. When additionally doped with $\mathrm{Eu}^{2+}$ ions, the lanthanum stabilised cubic barium chloride (LSCBC) was shown to be a highly efficient $x$-ray phosphor. The radioluminescence output was comparable to a commercial x-ray phosphor $\left(\mathrm{Gd}_{2} \mathrm{O}_{2} \mathrm{~S}: \mathrm{Tb}^{3+}\right)$. As typical for the $\mathrm{Eu}^{2+}$ ion, this emission was in the $\mathrm{UV} /$ blue region of the spectral range $(\sim 400 \mathrm{~nm})[118]$.

The stabilised cubic phase of $\mathrm{BaCl}_{2}$ provides a moderate density $\left(3.917 \mathrm{gcm}^{-3}\right.$ [119]) material from which transparent ceramics can be made. The previous work [116-118] showed that additional doping with rare earth ions results in luminescent centres and this combination forms the basis for a good scintillator. We therefore investigate samarium doped LSCBC in the hope of observing fast efficient scintillation based on luminescence from the $4 f^{5} 5 d^{1}$ configuration to the $4 \mathrm{f}^{6}$ ground state of the $\mathrm{Sm}^{2+}$ ion. This emission will likely be in the red spectral region, ideal for detection by a silicon photodetector.

\subsection{Lanthanum stabilised cubic barium chloride}

\subsubsection{Physical properties}

Polycrystalline samples of $\mathrm{Ba}_{1-x-y} \mathrm{La}_{x} \mathrm{Sm}_{y} \mathrm{Cl}_{2+x+y}(5 \leq \mathrm{x} \leq 0.125,0 \leq \mathrm{y} \leq 0.1)$ were formed by melting anhydrous $\mathrm{BaCl}_{2}, \mathrm{LaCl}_{3}$ and $\mathrm{SmCl}_{3}$ powders in a platinum crucible. The melt was slow cooled at $5^{\circ} \mathrm{C} / \mathrm{hr}$ through the melting point of pure $\mathrm{BaCl}_{2}\left(962^{\circ} \mathrm{C}\right)$ then quickly cooled at $30{ }^{\circ} \mathrm{C} / \mathrm{min}$ through the cubic to orthorhombic phase transition $\left(925^{\circ} \mathrm{C}\right)$ to optimise retention of the cubic phase, then slow cooled below $850^{\circ} \mathrm{C}$ to minimise cracking. Powder $\mathrm{x}-$ ray diffraction (XRD) patterns are shown in figure 7.1 for $0.1 \%$ samarium doping and various lanthanum doping values, along with the expected patterns for orthorhombic $\mathrm{BaCl}_{2}$ and 


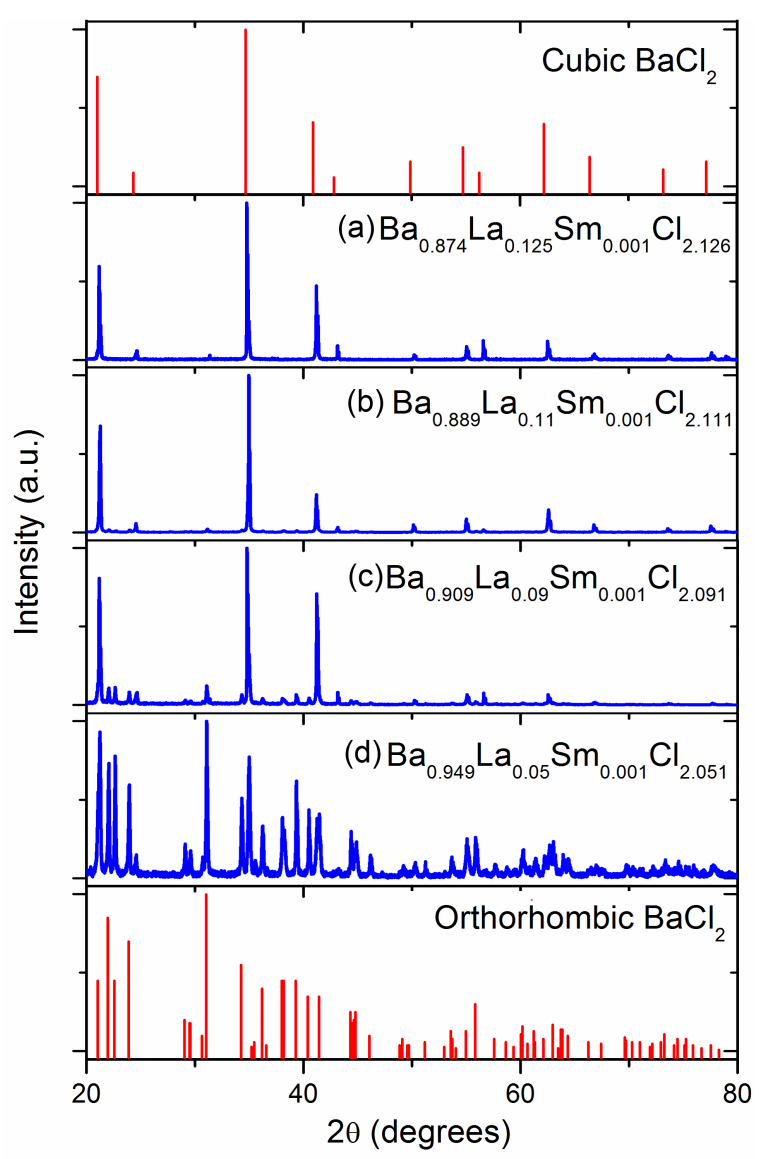

Figure 7.1: Powder x-ray diffraction patterns: powder diffraction file pattern for cubic $\mathrm{BaCl}_{2}$, experimental patterns for (a) $12.5 \% \mathrm{La}^{3+}$ doped $\mathrm{BaCl}_{2}$, (b) $11 \% \mathrm{La}^{3+}$ doped $\mathrm{BaCl}_{2}$, (c) $9 \%$ $\mathrm{La}^{3+}$ doped $\mathrm{BaCl}_{2}$, (d) $5 \% \mathrm{La}^{3+}$ doped $\mathrm{BaCl}_{2}$ and (e) the powder diffraction file pattern for orthorhombic $\mathrm{BaCl}_{2}$.

cubic $\mathrm{BaCl}_{2}$. For $11 \%$ and $12.5 \%$ lanthanum (figure 7.1(c) and (d)) the material has retained the high temperature cubic phase with minimal impurity phases. When the lanthanum content was reduced to $9 \%$ (c), the XRD pattern showed weak orthorhombic lines, indicating a composite of cubic and orthorhombic phases; the intensity of the orthorhombic phase lines increases in the $5 \%$ pattern (a). Thus all further work was carried out on samples with one in eight $(12.5 \%)$ of the cations being $\mathrm{La}^{3+}$. These results are in keeping with previous studies of LSCBC: $\left(\mathrm{Eu}^{2+}, \mathrm{Ce}^{3+}\right)$ and CSCBC which all showed optimal transparency for $\sim 12.5 \%$ stabiliser ion concentrations [116-118].

With additional $\mathrm{SmCl}_{3}$ doping the material still retains the cubic phase for samarium concentrations of up to $10 \%$. Figure 7.2(a) shows the powder XRD patterns for LSCBC:Sm for samarium concentrations of $0 \%, 1 \%, 2 \%$ and $10 \%$; the patterns are all cubic and show no evidence of the orthorhombic phase or any other crystal structure. The lattice parameter for the LSCBC:Sm samples was calculated from the XRD patterns and is shown in figure 


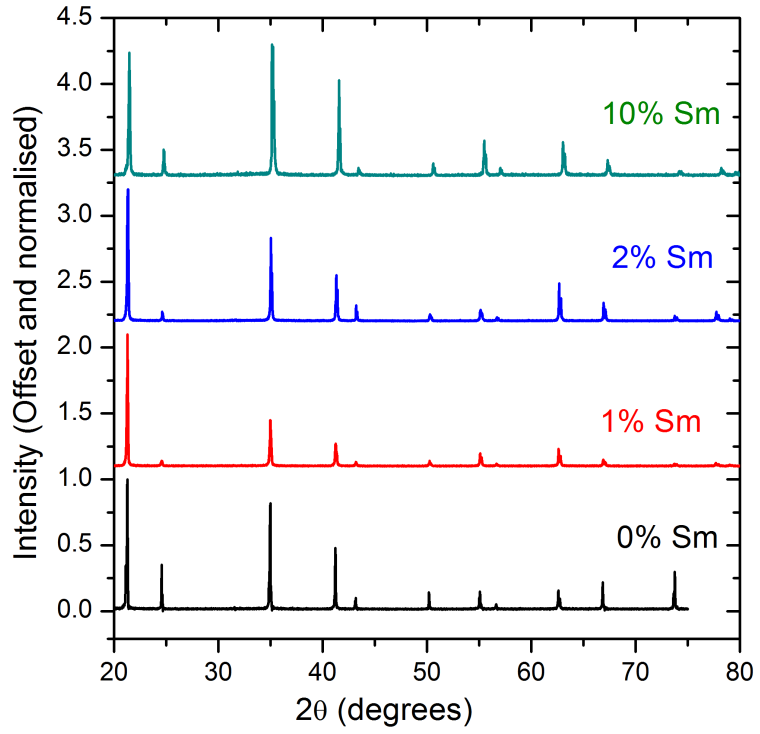

(a)

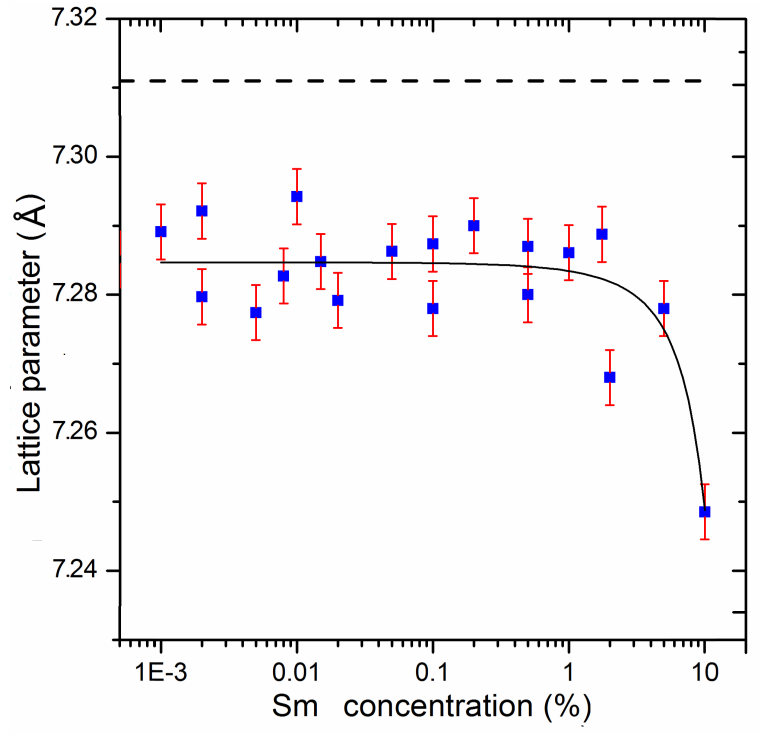

(b)

Figure 7.2: (a) Powder x-ray diffraction patterns for LSCBC:Sm for samarium concentrations of $10 \%, 2 \%, 1 \%$ and $0 \%$. (b) Lattice parameter variation with samarium concentration for LSCBC:Sm, the dashed line represents the lattice parameter for pure cubic $\mathrm{BaCl}_{2}$.

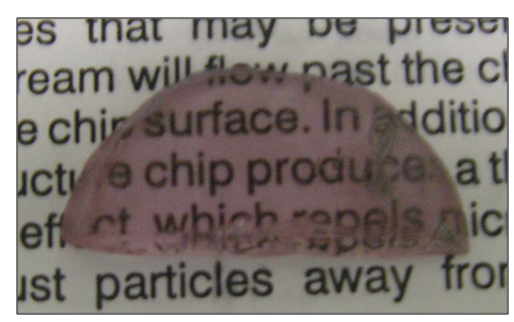

Figure 7.3: Photograph of a LSCBC:Sm (0.02\%) sample showing the high transparency of the polycrystalline material obtained due to the cubic structure.

7.2(b) along with the lattice parameter for pure cubic $\mathrm{BaCl}_{2}$. The $\mathrm{La}^{3+}$ ion (ionic radius 1.032 $\AA[120,121])$ is smaller than the $\mathrm{Ba}^{2+}$ ion $(1.35 \AA$ ) it substitutes for, so the lattice parameter is reduced to $7.285 \AA$ for $\mathrm{LSCBC}$, cf. $7.3110 \AA$ for pure $\mathrm{BaCl}_{2}$. Since $\mathrm{Sm}^{2+}$ is also a smaller ion (1.27 $\AA$ ) than the $\mathrm{Ba}^{2+}$ ion $(1.35 \AA)$, additional samarium doping further reduces the lattice parameter as can be seen in figure 7.2(b). There is an excess of $\mathrm{Cl}^{-}$ions in the material arising mostly from the $\mathrm{LaCl}_{3}$ doping but also the $\mathrm{SmCl}_{3}$ doping (generally at lower concentrations). It would seem likely these would sit at interstitial sites as found in LSCBC:Eu ${ }^{2+}[118]$ and would likely be spatially associated with the $\mathrm{La}^{3+}$ ions to provide charge compensation.

The resulting materials are transparent and range from colourless $(\sim 0.001 \% \mathrm{Sm})$ to a light purple colour $(\sim 0.1 \% \mathrm{Sm})$ to black $(\sim 10 \% \mathrm{Sm})$ for a few millimetres thickness. An example can be seen in figure 7.3 which shows a LSCBC:Sm (0.02\%) sample. The optical transparency is excellent. 


\subsubsection{Optical spectroscopy}

\subsubsection{Absorption}

The absorption spectrum of LSCBC:Sm is shown in figure 7.4(a) at $12 \mathrm{~K}$ and $300 \mathrm{~K}$. The absorption spectra comprise strong characteristic $\mathrm{Sm}^{2+} 4 \mathrm{f}^{6} \rightarrow 4 \mathrm{f}^{5} 5 \mathrm{~d}^{1}$ absorption bands superposed on a rising background. The background is not evident in the excitation spectra and is not correlated with samarium concentration, so is unlikely to be associated with the samarium. The absorption bands show a close resemblance to those of cubic $\mathrm{SrCl}_{2}: \mathrm{Sm}^{2+}$ [122] which suggests that in LSCBC the predominant site is also of cubic or near cubic symmetry. The only band which is not common is the broad band at $\sim 41,000 \mathrm{~cm}^{-1}$ which must arise from a different electronic centre. Following the scheme given by Karbowiak et al. [122] for $\mathrm{SrCl}_{2}: \mathrm{Sm}^{2+}$ the absorption bands have been labelled A-F in figure 7.4(a) and the corresponding transitions are depicted in figure $7.4(\mathrm{c})$. The description of the $4 \mathrm{f}^{5} 5 \mathrm{~d}^{1}$ configuration levels is based on the superposition of the lowest $4 \mathrm{f}^{5}$ configuration energy levels with the $5 \mathrm{~d}^{1}$ configuration levels. For a cubic site, the $5 \mathrm{~d}^{1}$ configuration is split by the crystal field into a upper $T_{2 g}$ and a lower $E_{g}$ state. The $\mathrm{T}_{2 g}$ is split by spin orbit interaction into a $\Gamma_{7 g}$ doublet and $\Gamma_{8 g}$ quartet; the $\mathrm{E}_{g}$ state is not split and is of $\Gamma_{8 g}$ symmetry. This provides three energy levels for the electron in the $5 \mathrm{~d}^{1}$ orbital. The other five electrons can then be found in any state of the $4 \mathrm{f}^{5}$ configuration which is primarily split by the coulombic interaction, however, within the UV-Visible energy range only the lowest two states are relevant (the ${ }^{6} \mathrm{H}_{J}$ and ${ }^{6} \mathrm{~F}_{J}$ ). The ${ }^{6} \mathrm{H}_{J}$ and ${ }^{6} \mathrm{~F}_{J}$ state are further split by spin orbit and crystal field interactions, which contribute to the width of the absorption bands [122].

As the temperature is reduced from $300 \mathrm{~K}$ to $12 \mathrm{~K}$ the sample changes colour from purple to green as shown in figure 7.5. The apparent colour change can be explained by figure 7.4(b) which shows in an expanded view the absorption spectrum in the visible light range. At room temperature the transmission in the visible range is primarily in the red and to a lesser extent in the blue and blue-green region, giving the purple colouration, while at low temperatures the narrowing of the absorption lines means that a transmission window in the green part of the spectrum opens up, and so green dominates the perceived transmission and scattering spectrum. 


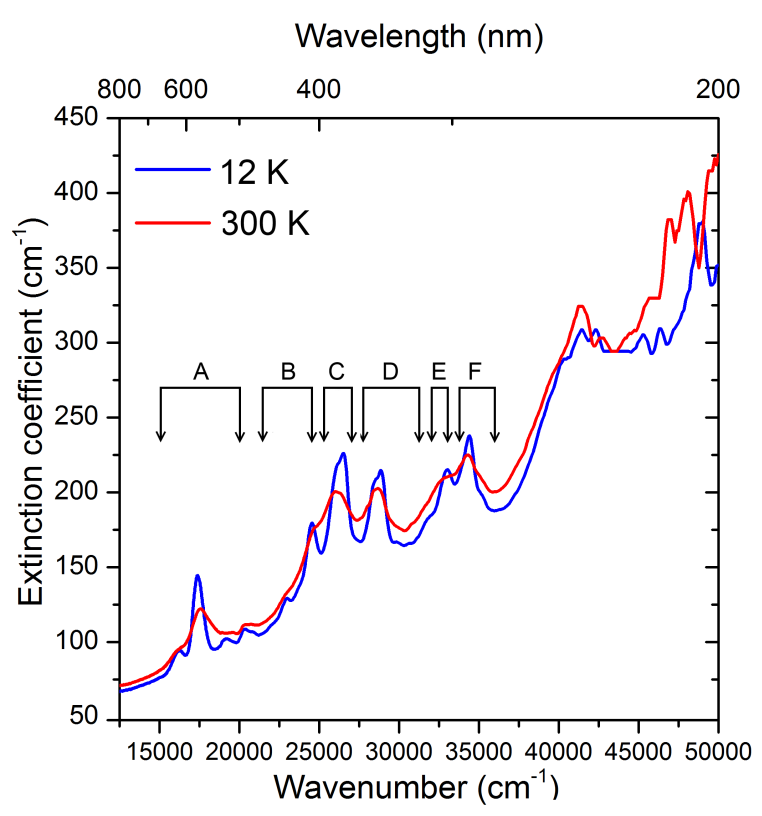

(a)

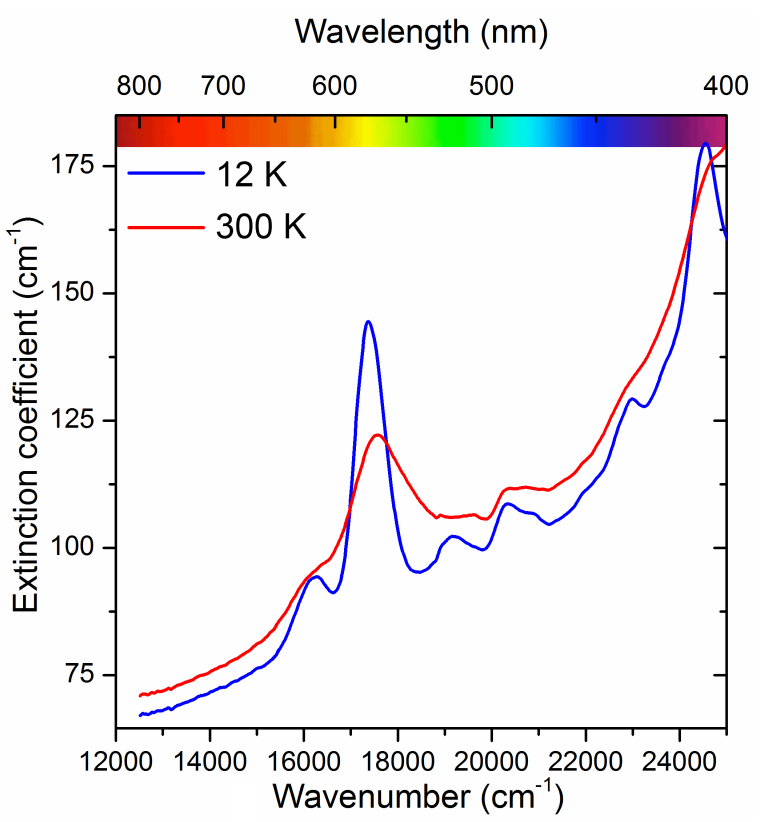

(b)

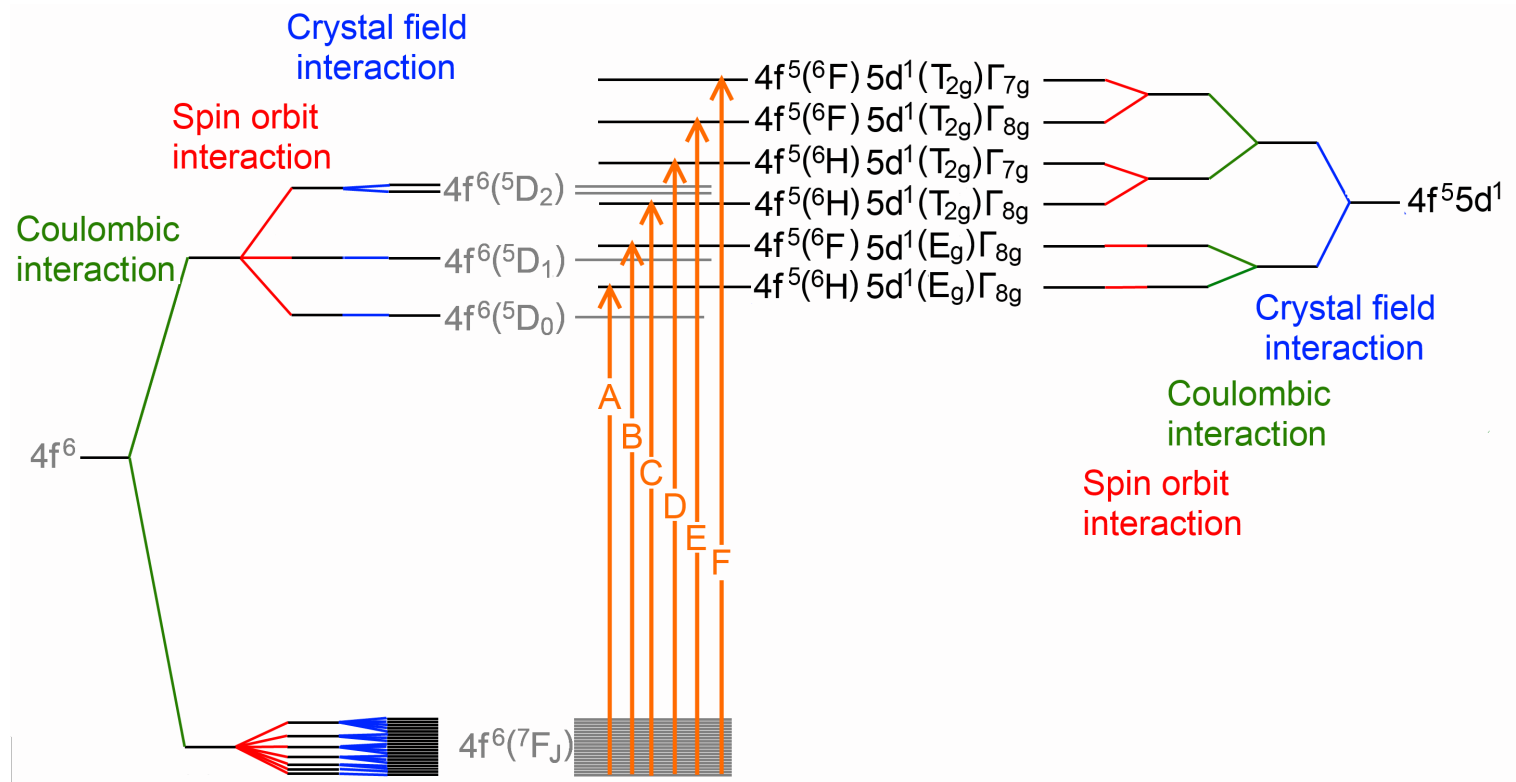

(c)

Figure 7.4: (a) Absorption spectra at 12 and $300 \mathrm{~K}$ for LSCBC:Sm (0.5 \%). (b) An expanded view of the visible region of the absorption spectrum. The resolution of both spectra is $2 \mathrm{~nm}$. (c) A schematic diagram of the excited state energy levels of $\mathrm{Sm}^{2+}$, the transitions corresponding to the absorption spectrum are indicated with arrows. 

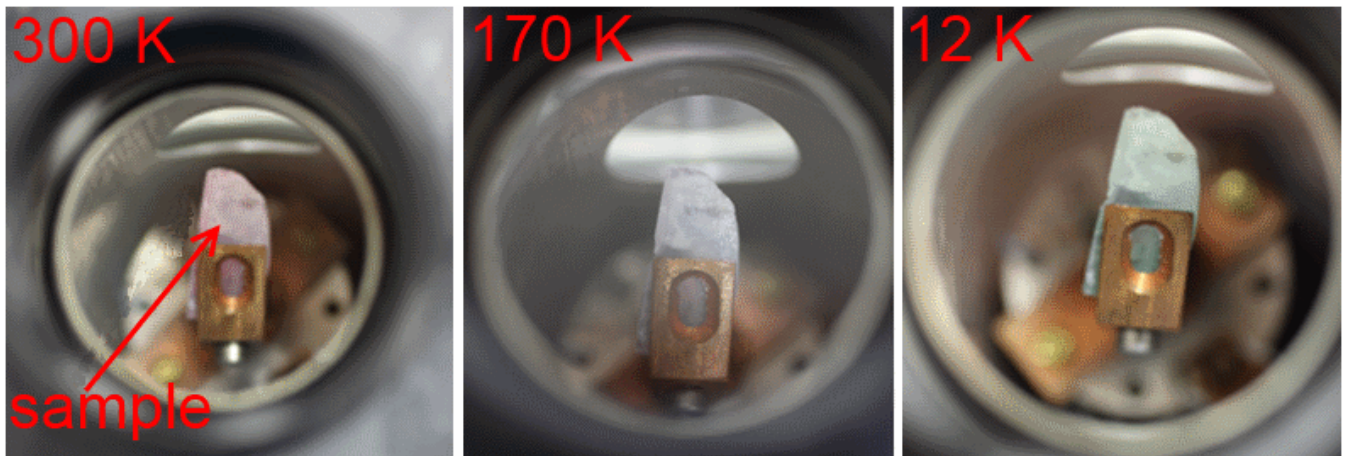

Figure 7.5: Photographs of a $0.02 \%$ samarium sample. A change in colour from red (left, 300 $\mathrm{K})$ to green (right, $12 \mathrm{~K}$ ) is evident.

\subsubsection{Photoluminescence and excitation spectra}

All samples showed red photoluminescence visible under a UV mineral lamp as shown in figure 7.6. This was especially intense for samarium concentrations in the range of $0.002 \%$ to $0.1 \%$. Photoluminescence spectra are shown in figure 7.7 at $300 \mathrm{~K}$ and $12 \mathrm{~K}$. The spectra are typical of divalent samarium despite the dopant material being $\mathrm{SmCl}_{3}$. This means there is some auto-reduction of the $\mathrm{Sm}^{3+}$ ions occurring the melt. This is often observed in alkaline earth halides and halo-fluoride lattices where $\mathrm{Sm}^{2+}$ appears in measurable concentrations even under conditions where no deliberate reducing agent was added to the system [123]. While there is no indication of $\mathrm{Sm}^{3+}$ ions in either the absorption or photoluminescence spectra, the $\mathrm{Sm}^{3+}$ ion $4 \mathrm{f}^{5} \leftrightarrow 4 \mathrm{f}^{5}$ transitions would likely be swamped by the much stronger $4 \mathrm{f}^{6} \leftrightarrow 4 \mathrm{f}^{5} 5 \mathrm{~d}^{1}$ transitions any $\mathrm{Sm}^{2+}$ ions present and the reduction may be not be complete.

At least some $\mathrm{Sm}^{3+}$ ions must be gaining an electron from some other species in the melt in

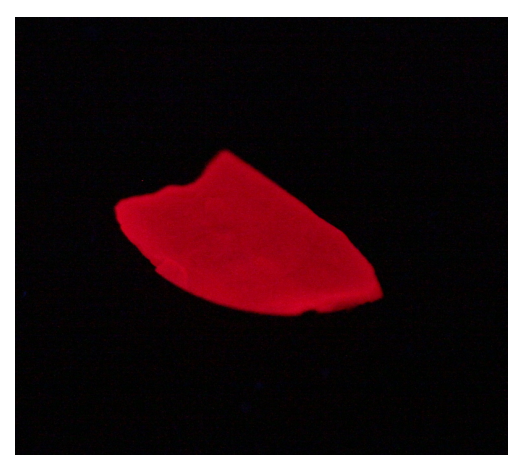

Figure 7.6: A sample showing strong red photoluminescence under $365 \mathrm{~nm}$ excitation from a UV mineral lamp. 


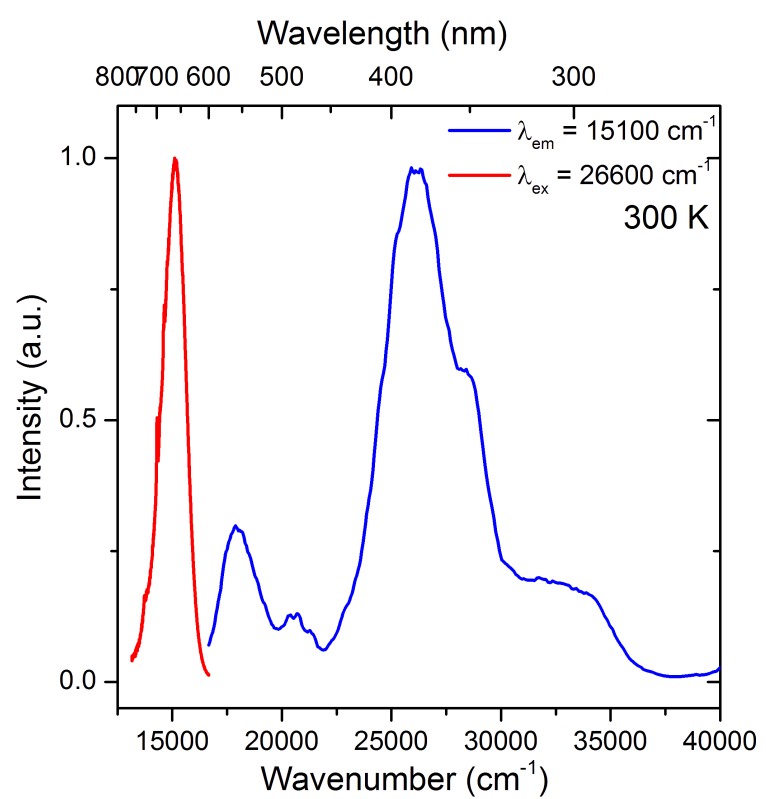

(a)

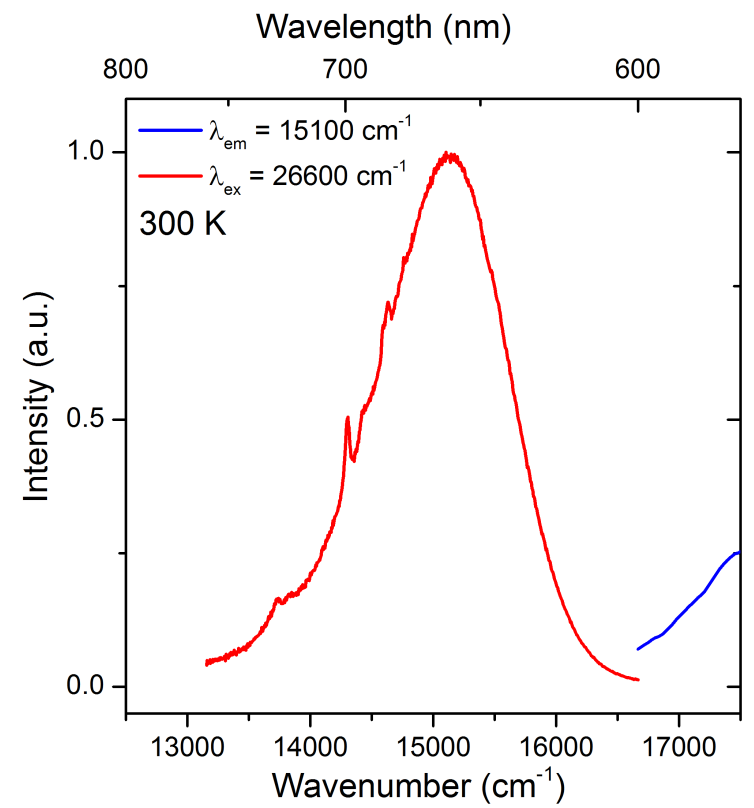

(c)

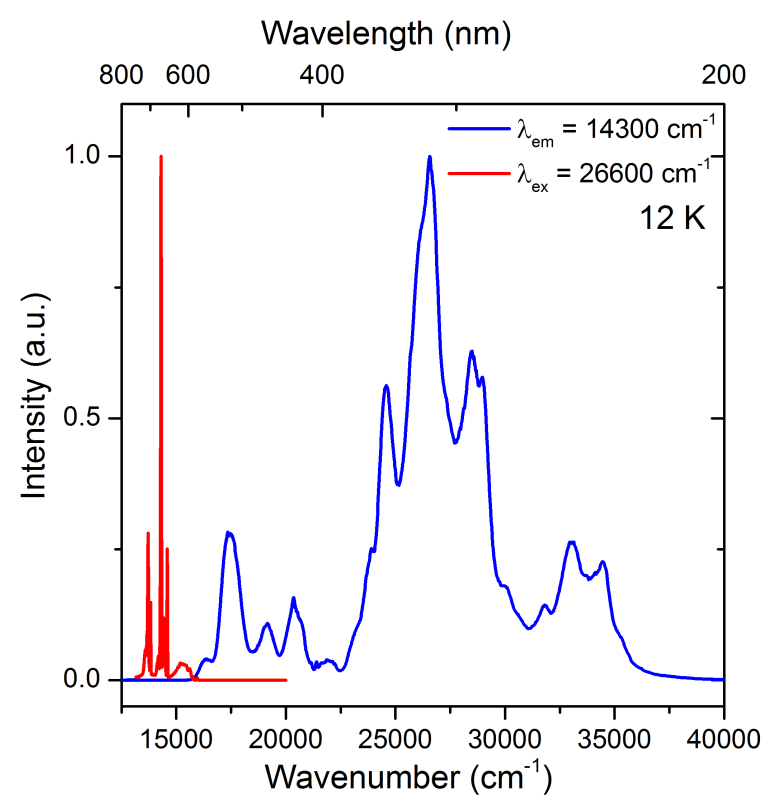

(b)

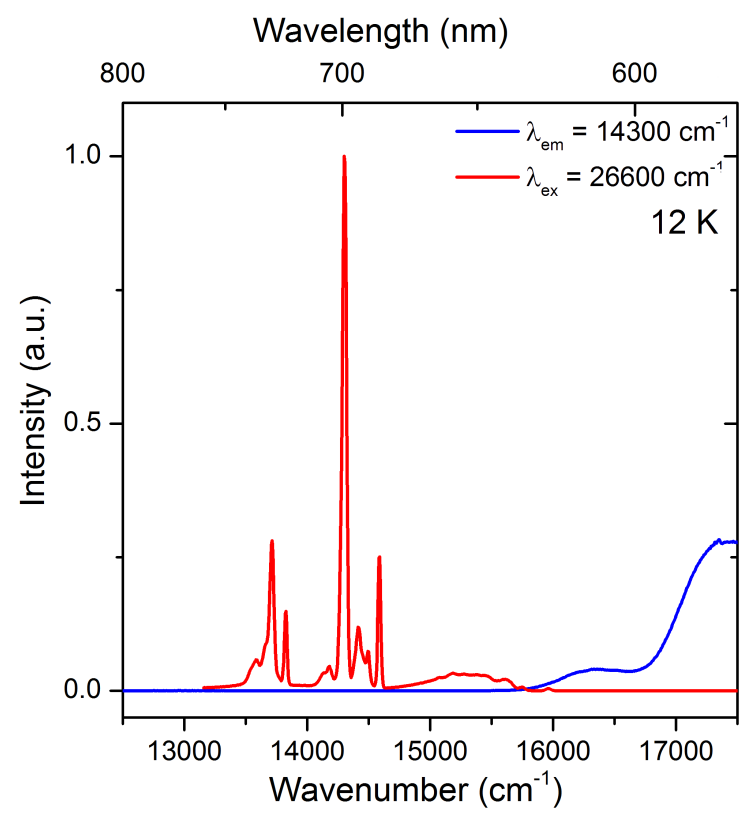

(d)

Figure 7.7: Excitation (blue) and emission (red) spectra for LSCBC:Sm (0.01 \%) at (a) 300 $\mathrm{K}$ and (b) $12 \mathrm{~K}$. An expanded view of the emission spectra at (c) $300 \mathrm{~K}$ and (d) $12 \mathrm{~K}$. The resolution is $1 \mathrm{~nm}$ for all spectra. 
order to be reduced

$$
S m^{3+}+e^{-} \rightarrow S m^{2+}
$$

The melt must have an excess of $\mathrm{Cl}^{-}$ions (one for each $\mathrm{La}^{3+}$ or $\mathrm{Sm}^{3+}$ ion), these could potentially donate an electron to the $\mathrm{Sm}^{3+}$ then boil off as molecular chlorine $\left(\mathrm{Cl}_{2}\right)$ :

$$
2 \mathrm{Cl}^{-} \rightarrow \mathrm{Cl}_{2}+2 e^{-}
$$

To examine this possibility we checked for the loss of chlorine using a mass spectrometer as a sample was heated to $1000{ }^{\circ} \mathrm{C}$ and seen none. Therefore the reduction must occur via some other mechanism. The flow through gas system in the furnace is likely subject to small air leaks in the multiplicity of joints. Therefore, in the furnace the crucible containing the materials sits in a carbon susceptor, the atmosphere is predominantly argon with some leak induced nitrogen and oxygen. Above $\sim 400{ }^{\circ} \mathrm{C}$ the leak induced oxygen will react with the hot carbon of the susceptor to give carbon dioxide.

$$
\mathrm{C}+\mathrm{O}_{2} \rightarrow \mathrm{CO}_{2}
$$

On further heating to above $800^{\circ} \mathrm{C}$ the carbon dioxide further reacts with the carbon susceptor to give carbon monoxide [124]

$$
\mathrm{C}+\mathrm{CO}_{2} \rightarrow 2 \mathrm{CO}
$$

Carbon monoxide is a strong reducing agent, and so now at least some of the $\mathrm{Sm}^{3+}$ ions are reduced. Unlike the $\mathrm{Sm}^{3+}$ ions, we do not expect the $\mathrm{La}^{3+}$ ions to undergo any reduction because the third ionization energy of lanthanum is low (19.2 eV compared to $23.4 \mathrm{eV}$ for samarium [119]), and so $\mathrm{La}^{2+}$ ions are rarely seen and are generally unstable [125, 126].

The photoluminescent emission of LSCBC:Sm${ }^{2+}$ shows very strong temperature dependence as evident in figure 7.7. Marked temperature dependence is also seen for the similar materials $\mathrm{BaCl}_{2}$ [127, 128], $\mathrm{BaClF}: \mathrm{Sm}^{2+}$ [129] (both orthorhombic), $\mathrm{SrF}_{2}: \mathrm{Sm}^{2+}$ [130] and $\mathrm{SrCl}_{2}: \mathrm{Sm}^{2+}$ [73] (both cubic). The thermal dependence of the luminescence arises due to the proximity of the excited $4 \mathrm{f}^{6}$ states and lowest levels of the $4 \mathrm{f}^{5} 5 \mathrm{~d}^{1}$ configuration as depicted schematically in figure 7.8. At room temperature a broad band emission corresponding to the electric 


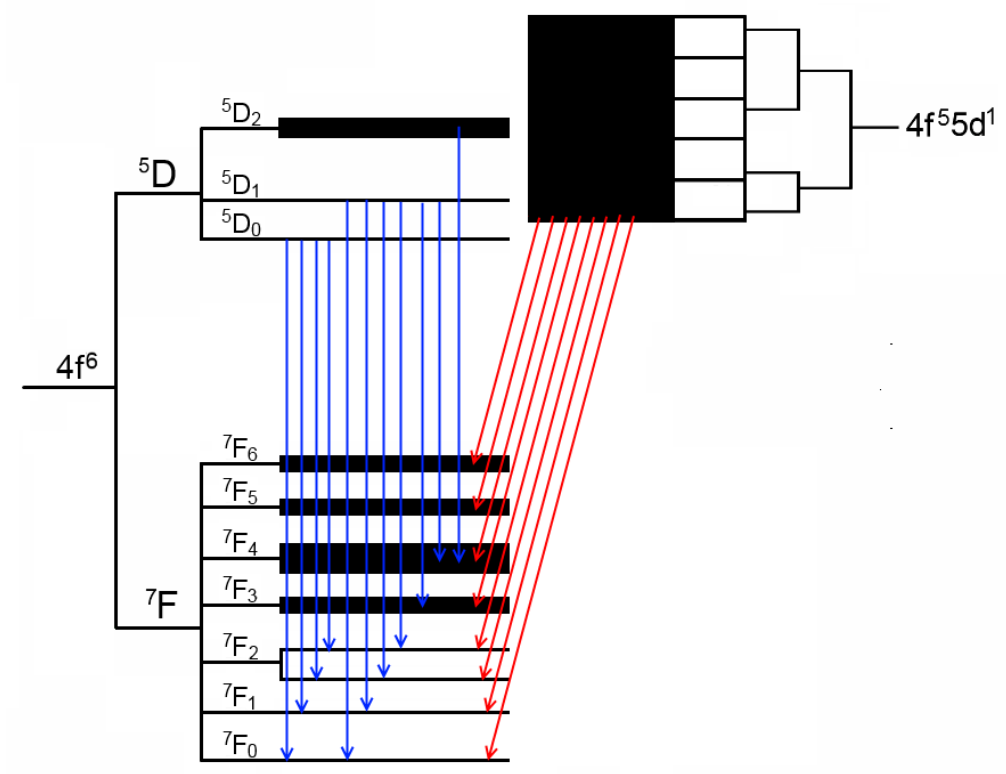

Figure 7.8: Diagram of the radiative transitions observed from the photoluminescence spectra at low temperatures (blue) and high temperatures (red).

Table 7.1: Selection rules applied to intra $4 \mathrm{f}^{6}$ configuration transitions for a cubic site of $\mathrm{O}_{h}$ symmetry, the magnetic dipole allowed transitions are indicated with a Y.

Final state

\begin{tabular}{|c|c|c|c|c|c|c|c|c|c|c|c|}
\hline \multirow[b]{2}{*}{ Initial State } & \multirow{2}{*}{$\frac{{ }^{7} \mathrm{~F}_{0}}{\left(\mathrm{~A}_{1 g}\right)}$} & \multirow{2}{*}{$\frac{{ }^{7} \mathrm{~F}_{1}}{\left(\mathrm{~T}_{1 g}\right)}$} & \multicolumn{2}{|c|}{${ }^{7} \mathrm{~F}_{2}$} & \multicolumn{3}{|c|}{${ }^{7} \mathrm{~F}_{3}$} & \multicolumn{4}{|c|}{${ }^{7} \mathrm{~F}_{4}$} \\
\hline & & & $\left(\mathrm{E}_{g}\right)$ & $\left(\mathrm{T}_{2 g}\right)$ & $\left(\mathrm{A}_{2 g}\right)$ & $\left(\mathrm{T}_{1 g}\right)$ & $\left(\mathrm{T}_{2 g}\right)$ & $\left(\mathrm{A}_{1 g}\right)$ & $\left(\mathrm{E}_{g}\right)$ & $\left(\mathrm{T}_{1 g}\right)$ & $\left(\mathrm{T}_{2 g}\right)$ \\
\hline${ }^{5} \mathrm{D}_{0} \quad\left(\mathrm{~A}_{1 g}\right.$ & & $\mathrm{Y}$ & & & & $\mathrm{Y}$ & & & & $\mathrm{Y}$ & \\
\hline${ }^{5} \mathrm{D}_{1} \quad\left(\mathrm{~T}_{1 g}\right)$ & Y & Y & Y & Y & & Y & Y & Y & Y & Y & Y \\
\hline
\end{tabular}

dipole allowed $4 \mathrm{f}^{5} 5 \mathrm{~d}^{1} \rightarrow 4 \mathrm{f}^{6}$ transitions, as shown with red arrows in figure 7.8 , are dominant. As the material is cooled to $12 \mathrm{~K}$ the sharp line transitions $\left(4 \mathrm{f}^{6} \rightarrow 4 \mathrm{f}^{6}\right)$, as shown with blue arrows in figure 7.8, become the dominant feature. This dramatic change is not observed in the excitation or absorption spectra; these are dominated by $4 f^{6} \rightarrow 4 f^{5} 5 d^{1}$ transitions at all temperatures.

Due to shielding from the crystal field by the outer 5 s and $5 p$ orbitals, the $4 \mathrm{f}^{6}$ levels of $\mathrm{Sm}^{2+}$ do not show dramatic shifts between host lattices and as such the sharp line emissions observed can be assigned to transitions according to the literature [73, 127, 131], and this is shown above the spectra in figure 7.9 which shows the emission temperature dependence in more detail.

Table 7.1 indicates the intra $4 \mathrm{f}^{6}$ configuration transitions which are magnetically dipole allowed (all intra $4 \mathrm{f}^{6}$ configuration transitions are electric dipole forbidden as the parity 


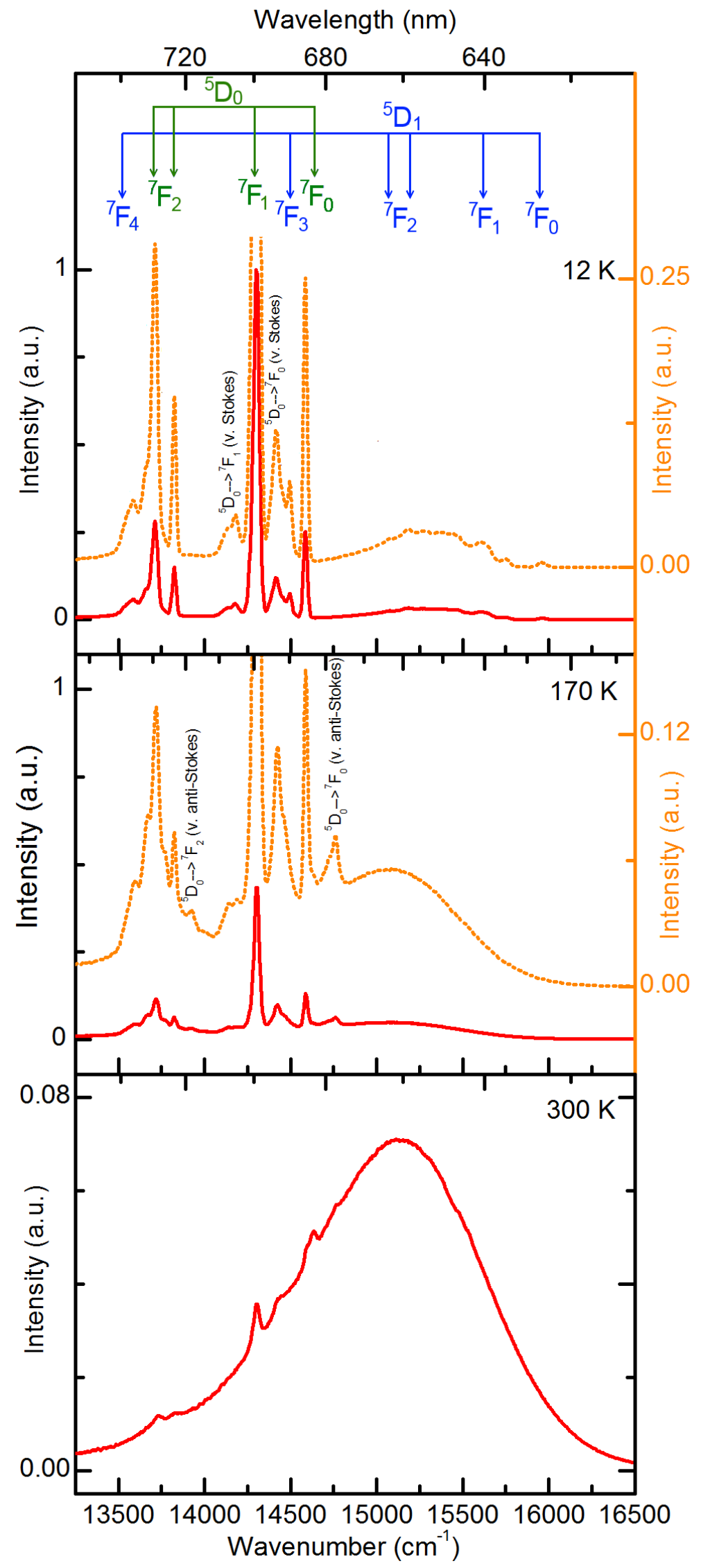

Figure 7.9: Photoluminescence for LSCBC:Sm (0.01\%) at $12 \mathrm{~K}$ (top), $170 \mathrm{~K}$ (middle) and $290 \mathrm{~K}$ (bottom) all excited at $385 \mathrm{~nm}$ and normalised to the $14300 \mathrm{~cm}^{-1}$ peak value at $12 \mathrm{~K}$. The low temperature spectra expanded to the right axis scale are also shown (dotted lines). The resolution is $1 \mathrm{~nm}$. Shown above the spectra is a bar pattern indicating the transitions observed from the ${ }^{5} \mathrm{D}_{J}$ states. The label v. (anti-)Stokes refers to lines identified as vibronics. 
does not change) for a cubic site of $\mathrm{O}_{h}$ symmetry. From table 7.1, the only magnetic dipole allowed transition from the ${ }^{5} \mathrm{D}_{0}$ state within the range of our spectra is the ${ }^{5} \mathrm{D}_{0} \rightarrow{ }^{7} \mathrm{~F}_{1}$. At $12 \mathrm{~K}$ the strongest emission corresponds to this ${ }^{5} \mathrm{D}_{0} \rightarrow{ }^{7} \mathrm{~F}_{1}$ transition, which occurs at 14300 $\mathrm{cm}^{-1}(699 \mathrm{~nm})$. However, weaker lines originating from transitions from the ${ }^{5} \mathrm{D}_{0}$ to various ${ }^{7} \mathrm{~F}_{J}$ states can also be observed and identified. This is not unusual for an impure material; even very slight perturbations in the crystal field relax the transition rules markedly. In particular, the ${ }^{5} \mathrm{D}_{0} \rightarrow{ }^{7} \mathrm{~F}_{0}$ transition is observed as a singlet at $14585 \mathrm{~cm}^{-1}(686 \mathrm{~nm})$ and has a full width half maximum (FWHM) line width of $30 \mathrm{~cm}^{-1}$ at $12 \mathrm{~K}$. The observation of a single line corresponding to this transition indicates there is likely only one site for the $\mathrm{Sm}^{2+}$ ion; a mixture of sites would lead to more than one line. The splitting between similar sites for the ${ }^{5} \mathrm{D}_{0} \rightarrow{ }^{7} \mathrm{~F}_{0}$ can be down to a few $\mathrm{cm}^{-1}$ making it possible two emissions are contained within the one line. We take further evidence of a single site from the two doublets which arise from ${ }^{5} \mathrm{D}_{0,1} \rightarrow{ }^{7} \mathrm{~F}_{2}$ transitions; the ${ }^{7} \mathrm{~F}_{2}$ state is split by the crystal field so any change in site and symmetry is exaggerated for these levels, the fact that we see only doublets for these lines suggests a single site occupied by the $\mathrm{Sm}^{2+}$ ions [132].

Multiple lines originating from transitions from the ${ }^{5} \mathrm{D}_{1}$ to various ${ }^{7} \mathrm{~F}_{J}$ states can also be observed and identified, indicating there is a significant population in the ${ }^{5} \mathrm{D}_{1}$ state at $12 \mathrm{~K}$. Shown at the top of figure 7.9 is a bar pattern indicating the transitions observed; this shows the same splitting for the ${ }^{7} \mathrm{~F}_{J}$ states determined by the transitions from the ${ }^{5} \mathrm{D}_{0}$ and ${ }^{5} \mathrm{D}_{1}$ states, indicating they have been correctly identified. At $15750 \mathrm{~cm}^{-1}(635 \mathrm{~nm})$ a weak single line can be seen at low temperatures. This emission is of too higher energy to originate from the ${ }^{5} \mathrm{D}_{0}$ state. It also cannot originate from ${ }^{5} \mathrm{D}_{1}$ state as we have accounted for all the expected emissions from this state, and the assigned transitions are consistent with literature. We attribute this to a $4 \mathrm{f}^{6}\left({ }^{5} \mathrm{D}_{2} \rightarrow{ }^{7} \mathrm{~F}_{4}\right.$ transition) based on the expected energy of this transition, however no other lines from this level could be detected. Those lying above about 16,000 $\mathrm{cm}^{-1}$ would be masked by the strong $4 \mathrm{f}^{6} \rightarrow 4 \mathrm{f}^{5} \mathrm{~d}^{1}$ absorption which occurs above this energy as seen in the absorption spectra and excitation spectra shown in figures 7.4 and $7.7(\mathrm{~b})$, In summary, all of the observed sharp line emission can be attributed to ${ }^{5} \mathrm{D}_{0,1,2} \rightarrow{ }^{7} \mathrm{~F}_{0,1,2,3,4}$ transitions of a single (cubic) site.

A very weak broad band emission band can also be observed at $12 \mathrm{~K}$ centred at $15280 \mathrm{~cm}^{-1}$ 


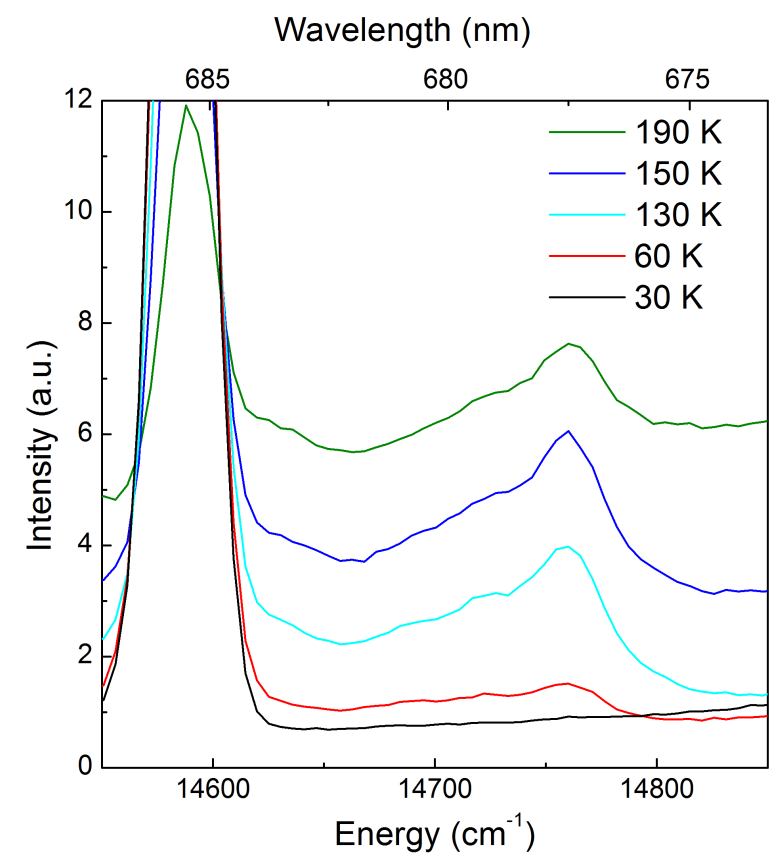

Figure 7.10: Anti-stokes vibronic sideband of the ${ }^{5} \mathrm{D}_{0} \rightarrow{ }^{7} \mathrm{~F}_{0}$ transition shown between $30 \mathrm{~K}$ and $190 \mathrm{~K}$.

$(655 \mathrm{~nm})$, which we associate with emission from the lowest $4 \mathrm{f}^{5} 5 \mathrm{~d}^{1}$ levels. With increasing temperature, the intensity of this band first increases slightly then falls to a minimum at $\sim 90 \mathrm{~K}$ before rising again. Above $\sim 90 \mathrm{~K}$, the sharp line spectra decrease markedly in intensity, so that at room temperature the broad band emission totally dominates the overall spectrum with the maximum at $15130 \mathrm{~cm}^{-1}(660 \mathrm{~nm})$, as seen in figure 7.9 (lower). The lines marked "v. anti-Stokes" in figure 7.9 (centre, $170 \mathrm{~K}$ ) are assigned to one-phonon antiStokes hot sidebands of the $4 \mathrm{f}^{6} \rightarrow 4 \mathrm{f}^{6}$ zero-phonon transitions by virtue of their temperature dependence, an example of which is shown in figure 7.10 . They are not present in the $12 \mathrm{~K}$ spectra, but increase in intensity markedly above $50 \mathrm{~K}$. Above $\sim 200 \mathrm{~K}$ broadening becomes too great to follow their behaviour. Figure 7.10 shows the ${ }^{5} \mathrm{D}_{0} \rightarrow{ }^{7} \mathrm{~F}_{0}$ anti-Stokes vibronic band; there is a corresponding Stokes vibronic band labelled "v. Stokes" in figure 7.9 (upper) for the ${ }^{5} \mathrm{D}_{0} \rightarrow{ }^{7} \mathrm{~F}_{0}$ transition, and we associate a weak band on the low energy side of the ${ }^{5} \mathrm{D}_{0}$ $\rightarrow{ }^{7} \mathrm{~F}_{1}$ with a Stokes vibronic for that line. The anti-Stokes equivalent cannot be distinguished from stronger bands in the expected position. The distribution of intensity within the two vibronic bands (for the ${ }^{5} \mathrm{D}_{0} \rightarrow{ }^{7} \mathrm{~F}_{1}$ and ${ }^{5} \mathrm{D}_{0} \rightarrow{ }^{7} \mathrm{~F}_{0}$ transitions) differs, but this is not unexpected given that the latter transition is forbidden and so different coupling strengths to the different phonons is possible. Individual component phonon frequencies are not resolved, but the range of frequencies is $\sim 100-180 \mathrm{~cm}^{-1}$. 


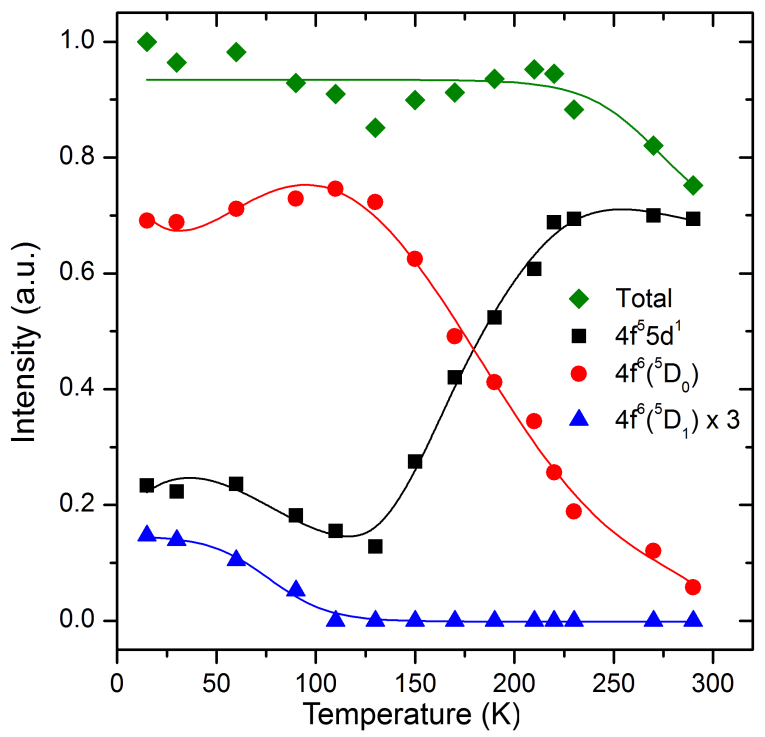

Figure 7.11: Integrated light output from the $4 \mathrm{f}^{5} \mathrm{~d}^{1}$ configuration, the ${ }^{5} \mathrm{D}_{1}$ state, the ${ }^{5} \mathrm{D}_{0}$ state in LSCBC: $\mathrm{Sm}^{2+}$ as a function of temperature. The total light output is also shown, all values have been normalised to the total at $12 \mathrm{~K}$. The solid lines are provided as a guide to the eye.

Figure 7.11 shows the integrated intensity of the luminescence from the ${ }^{5} \mathrm{D}_{1},{ }^{5} \mathrm{D}_{0}$ states and $4 \mathrm{f}^{5} 5 \mathrm{~d}^{1}$ configuration as a function of temperature. This was calculated by performing a least squares fitting of Gaussian curves to all the observed bands, followed by an integration over all the components associated with a particular emitting level to the various sub-levels of the ${ }^{7} \mathrm{~F}_{J}$ ground multiplet. We also show the sum of all the intensities. Below $200 \mathrm{~K}$ this is approximately constant but above $200 \mathrm{~K}$ there is a gradual decline in total intensity with increasing temperature. The constant intensity (below $\sim 200 \mathrm{~K}$ ) indicates that the non-radiative de-excitation processes from the excited states (the $4 \mathrm{f}^{6}\left({ }^{5} \mathrm{D}_{J}\right)$ levels and all the radiating $4 \mathrm{f}^{5} 5 \mathrm{~d}^{1}$ excited configuration levels) to the ground ${ }^{7} \mathrm{~F}_{J}$ multiplet are insignificant in that temperature range. The origins of the temperature dependences are discussed in more detail in later sections.

\subsubsection{Photoluminescent lifetimes}

The lifetimes of the $4 \mathrm{f}^{5} 5 \mathrm{~d}^{1}$ states show complex behaviour with different relaxation rates ranging over several orders of magnitude. The temperature dependence of the $\mathrm{Sm}^{2+}$ ion luminescence is much more complicated than for other rare earth ions due to the interaction between the $4 \mathrm{f}^{6}$ excited states and the close lying lowest $4 \mathrm{f}^{5} 5 \mathrm{~d}^{1}$ levels; this also affects the lifetime of the states. While radiative transition probabilities are temperature independent, the 


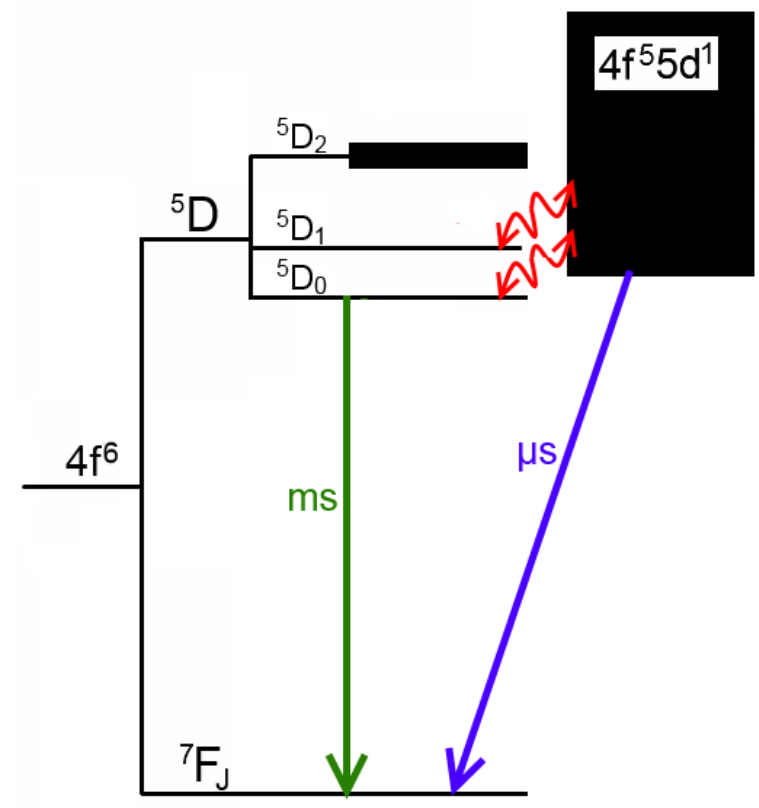

Figure 7.12: Diagram of energy levels and transitions relevant to the recorded photoluminescent lifetimes in LSCBC: $\mathrm{Sm}^{2+}$.

probabilities of non-radiative transitions involving phonon emission are strongly temperature dependant. The transitions relevant to the main emissions are shown in figure 7.12. As indicated, the ${ }^{5} \mathrm{D}_{0}$ state has a radiative lifetime of the order of milliseconds, and the $4 \mathrm{f}^{5} 5 \mathrm{~d}^{1}$ configuration has a lifetime of the order of microseconds. If no non-radiative transitions occur the decays would be recorded as such, however, when non-radiative transitions occur the recorded lifetimes change. For example, the effect of the non-radiative transition $4 f^{6}\left({ }^{5} D_{0}\right)$ $\rightarrow 4 \mathrm{f}^{5} 5 \mathrm{~d}^{1}$ (shown as the lower red arrow in figure 7.12$)$, at moderate temperatures $(\sim 100 \mathrm{~K}$ $250 \mathrm{~K})$ will be to decrease the lifetime of the ${ }^{5} \mathrm{D}_{0}$ state by providing a faster decay route, but increase the lifetime of the $4 f^{5} 5 d^{1}$ level as it may be extended by the link to the $4 f^{6}\left({ }^{5} D_{0}\right)$ and $4 \mathrm{f}^{6}\left({ }^{5} \mathrm{D}_{1}\right)$ states.

Figure 7.13(a) shows the decay of the $4 \mathrm{f}^{6}\left({ }^{5} \mathrm{D}_{0} \rightarrow{ }^{7} \mathrm{~F}_{1}\right)$ transition intensity for a $0.01 \%$ Sm sample at various temperatures from $12 \mathrm{~K}$ to $230 \mathrm{~K}$. The decay can be seen to change dramatically above $110 \mathrm{~K}$. The decays have been fitted with single exponential (with a baseline) fits, but while these are a good representation of the decay at low temperatures, as the temperature increases and non-radiative processes become significant the decay deviates from a single exponential. However the single exponential still provides a reasonable representation of the data and is a good fit over at least one order of magnitude. The broad band emission decay is shown in figure $7.13(\mathrm{~b})$ at $250 \mathrm{~K}, 300 \mathrm{~K}$, and $350 \mathrm{~K}$. Here the data is clearly not well fitted by a 


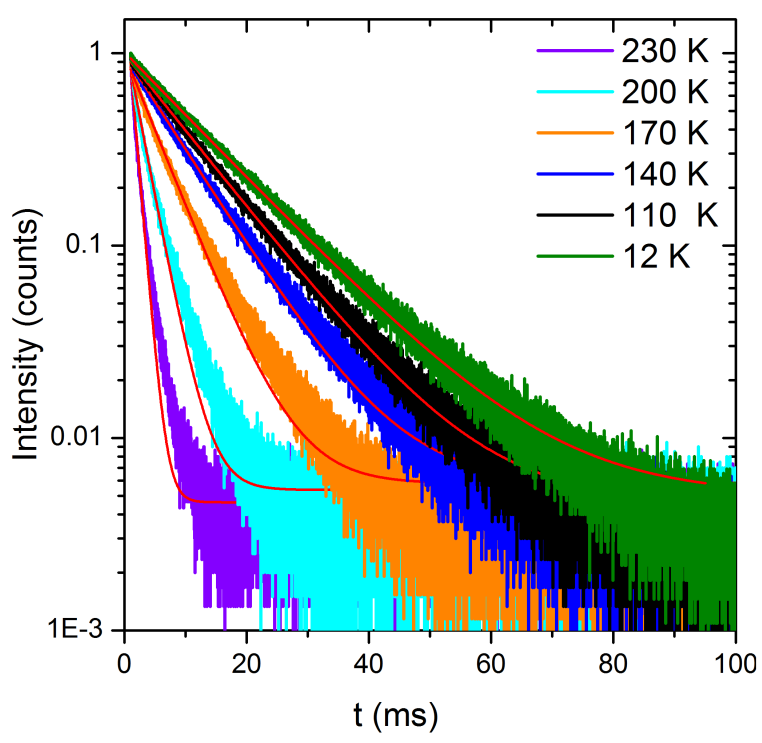

(a)

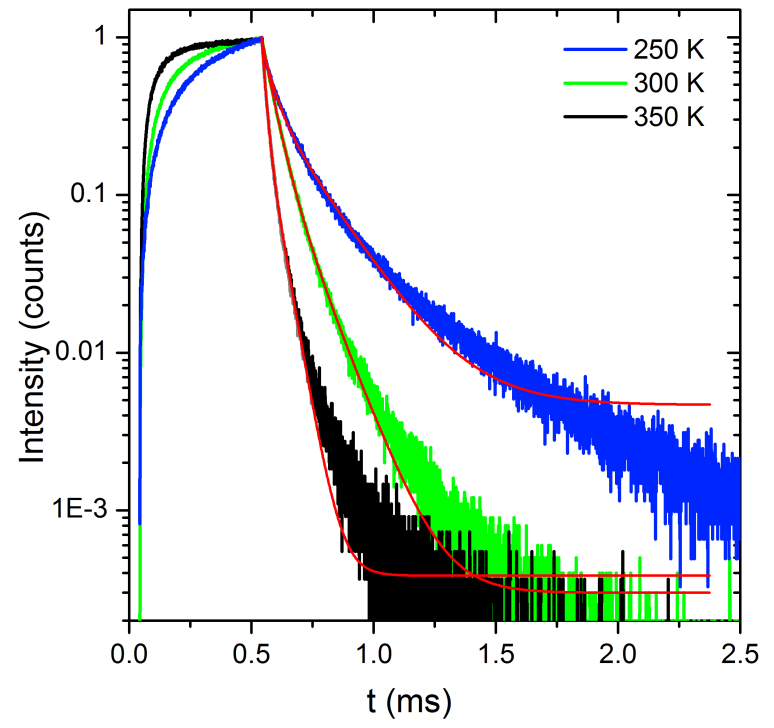

(b)

Figure 7.13: Photoluminescent decays of LSCBC:Sm (0.01\%) for; (a) the $4 \mathrm{f}^{6}\left({ }^{5} \mathrm{D}_{0} \rightarrow{ }^{7} \mathrm{~F}_{1}\right)$ transition between 12 and $230 \mathrm{~K}$, and (b) the $4 \mathrm{f}^{5} 5 \mathrm{~d}^{1} \rightarrow 4 \mathrm{f}^{6}$ transition between 250 and $350 \mathrm{~K}$. All decays were recorded at $14300 \mathrm{~cm}^{-1}$ and excited with a pulsed $375 \mathrm{~nm}$ spectra LED. In (a) the bandpass was $1 \mathrm{~nm}$ and in (b) the bandpass was $2 \mathrm{~nm}$. The first section in (b) shows the response as the LED is turned on for $0.5 \mathrm{~ms}$.

single exponential, instead double exponentials (with a baseline) have been fitted; however this still does not completely describe the decay indicating the complex processes underlying the temperature dependence.

Figure 7.14 shows the temperature dependence of the principal lifetime for the two predominant emissions, the $4 \mathrm{f}^{5} 5 \mathrm{~d}^{1} \rightarrow{ }^{7} \mathrm{~F}_{J}$ at higher temperatures and the ${ }^{5} \mathrm{D}_{0} \rightarrow{ }^{7} \mathrm{~F}_{1}$ at lower temperatures. These correspond to the dominant component (by integrated intensity) of the decay profile. The ${ }^{5} \mathrm{D}_{0}$ emission lifetime reduces from $12 \mathrm{~ms}$ at $12 \mathrm{~K}$ to around $2 \mathrm{~ms}$ at $150 \mathrm{~K}$ where the emission becomes too weak to measure separately from the broad band. This is because the $4 \mathrm{f}^{5} 5 \mathrm{~d}^{1}$ states have become thermally accessible and the corresponding electric dipole decay route is much more probable. The $4 \mathrm{f}^{5} 5 \mathrm{~d}^{1}$ emission lifetime is around $100 \mu \mathrm{s}$ at $370 \mathrm{~K}$, on cooling this increases up to about $2 \mathrm{~ms}$, this is far longer than one would expect for a parity allowed $5 \mathrm{~d}^{1} \rightarrow 4 \mathrm{f}^{6}$ transition, and is likely indicative of an excitation route through the $4 f^{6}\left({ }^{5} D_{1}\right)$ and $4 f^{6}\left({ }^{5} D_{0}\right)$ excited states. The intensity of the emission from the $4 f^{6}\left({ }^{5} D_{1}\right)$ was insufficient to obtain a decay profile. Lauer and Fong [128] report a lifetime $2.15 \mathrm{~ms}$ and 1.45 ms for this state in orthorhombic $\mathrm{BaCl}_{2}$ and $\mathrm{BaBr}_{2}$ respectively. A literature value could not be found for a fluorite lattice; this emission is not commonly observed. No concentration 


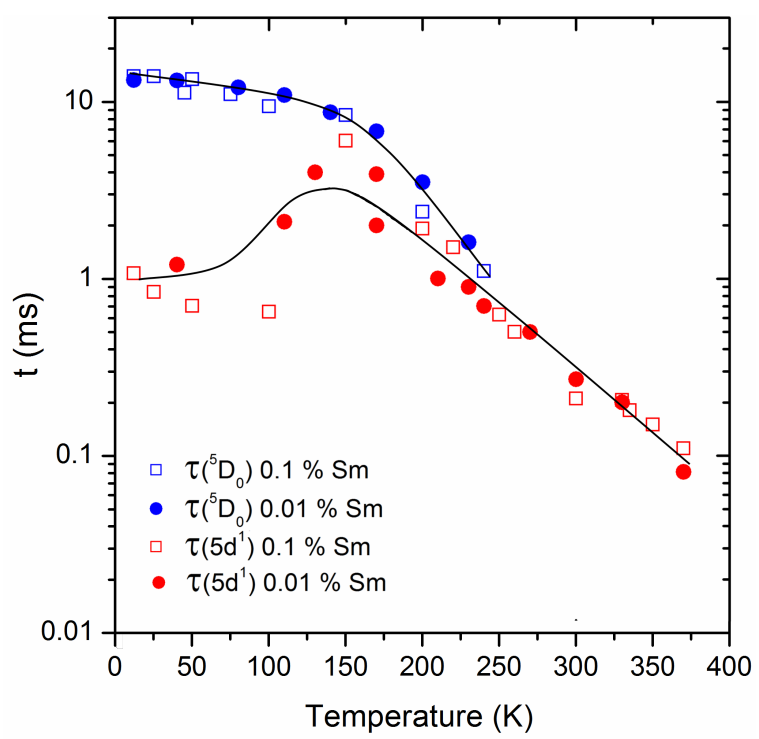

Figure 7.14: Lifetime of ${ }^{5} \mathrm{D}_{0} \rightarrow{ }^{7} \mathrm{~F}_{1}$ transition and $4 \mathrm{f}^{5} 5 \mathrm{~d}^{1} \rightarrow{ }^{7} \mathrm{~F}_{J}$ transitions for LSCBC:Sm ${ }^{2+}$ recorded at 699 and $660 \mathrm{~nm}$ respectively as a function of temperature. All emissions were excited by a $375 \mathrm{~nm}$ pulsed LED. The solid black lines are provided as a guide to the eye.

dependence for the photoluminescent lifetimes of LSCBC:Sm samples with up to $1 \%$ Sm was observed.

\subsubsection{Simulations of optical spectroscopy}

The optical absorption spectra (figure 7.4(a) are very similar to those of cubic $\mathrm{SrCl}_{2}: \mathrm{Sm}^{2+}$ with the exception of the marked vibronic structure reported for that case but missing from the transitions in LSCBC:Sm ${ }^{2+}$ [122]. We expect broader line widths for the $4 \mathrm{f}^{5} 5 \mathrm{~d}^{1} \rightarrow 4 \mathrm{f}^{6}$ transitions in LSCBC:Sm${ }^{2+}$ than $\mathrm{SrCl}_{2}: \mathrm{Sm}^{2+}$ because of the $\mathrm{La}^{3+}$ ion and $\mathrm{Cl}^{-}$ion defects, and so it is not surprising that we see no vibronic structure. We do observe vibronics in the sharp-line $4 \mathrm{f}^{6} \rightarrow 4 \mathrm{f}^{6}$ spectra because the $4 \mathrm{f}^{6}$ energy levels are far less susceptible to crystal field perturbations than the $4 \mathrm{f}^{5} 5 \mathrm{~d}^{1}$ energy levels and so vibronics close to the parent zero phonon line may be resolved.

In the case of $\mathrm{SrCl}_{2}$ Karbowiak et al [122] used a semi-empirical Hamiltonian to simulate the spectra with regard to line intensities and energies, and adjusting line widths to match the observed spectra. A collaborator for this work and co-author of the Karbowiak paper, Dr. Mike Reid, has used the same process and basic energy parameters for the $4 \mathrm{f}^{5} 5 \mathrm{~d}^{1}$ configuration taken from table II of that reference to fit the LSCBC:Sm ${ }^{2+}$ spectra, but with 


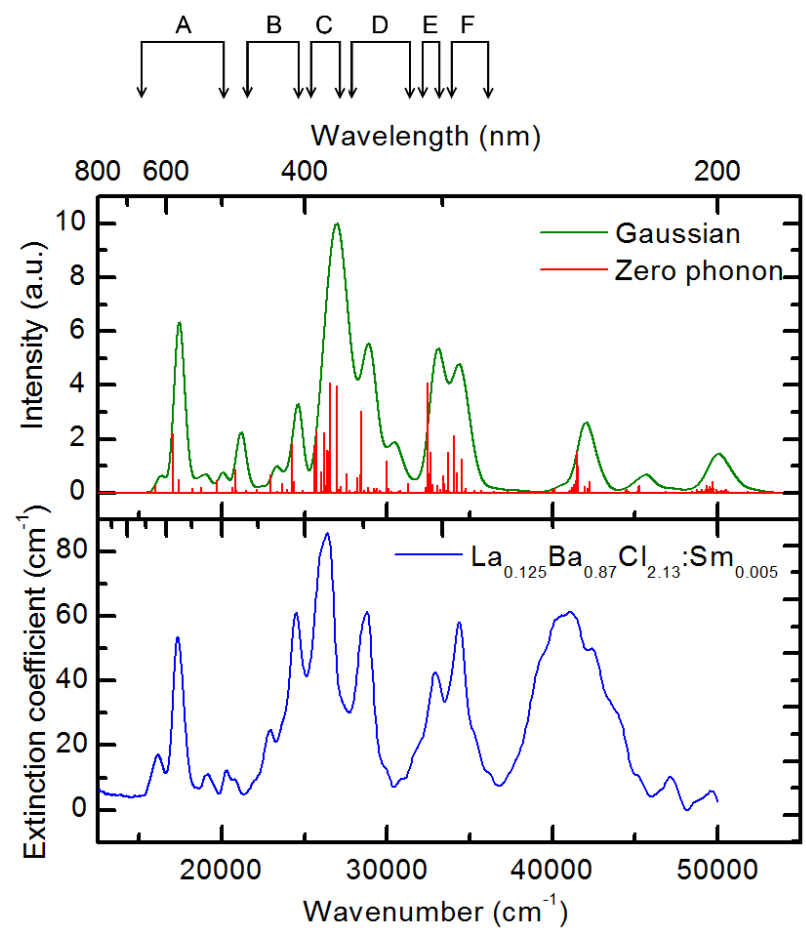

(a)

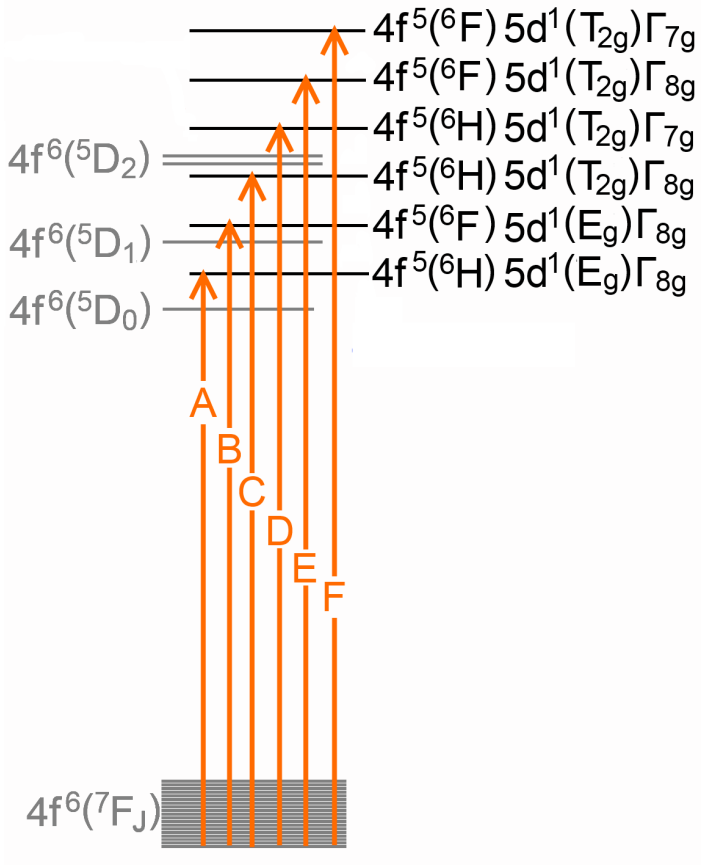

(b)

Figure 7.15: (a) The simulated (top) and experimental (bottom) absorption spectra for LSCBC:Sm ${ }^{2+}$ showing the high correlation. The experimental spectrum has had a rising background subtracted. (b) Schematic of the energy levels between which the absorption transitions occur.

adjusted values for the spin orbit $\zeta(\mathrm{dd})\left(=1075 \mathrm{~cm}^{-1}\right)$ and crystal field parameter $\mathrm{B}_{4}(=-16400$ $\mathrm{cm}^{-1}$ ). These values are 1.25 and 0.8 of the values used for $\mathrm{SrCl}_{2}$ respectively. The parameter $\delta \mathrm{E}(\mathrm{fd})$ was also adjusted, this determines the overall energy displacement between the $4 \mathrm{f}^{6}$ and $4 \mathrm{f}^{5} 5 \mathrm{~d}^{1}$ configurations, and was changed from $9506 \mathrm{~cm}^{-1}$ in $\mathrm{SrCl}_{2}$ to $11706 \mathrm{~cm}^{-1}$ so as to achieve a good match to the observed spectrum.

The simulated spectrum is in good agreement with that observed, as shown in figure 7.15(a), the absorption bands have again been labelled $\mathrm{A}$ to $\mathrm{F}$ and the corresponding transitions are shown in figure $7.15(\mathrm{~b})$. The only substantive discrepancy is the broad band centred at around $41,000 \mathrm{~cm}^{-1}$ in the experimental spectrum which is not predicted by the simulation, not present in the photoluminescent excitation spectra and is not observed in $\mathrm{SrCl}_{2}: \mathrm{Sm}^{2+}$, and which we therefore attribute to some other electronic centre present in LSCBC. The energy of the lowest $4 \mathrm{f}^{5} 5 \mathrm{~d}^{1}$ level is predicted to be $\sim 15700^{-1}$ from this calculation. We note that a more complete description of the $4 \mathrm{f}^{5} 5 \mathrm{~d}^{1}$ states would permit different bond lengths for the $4 \mathrm{f}^{5} 5 \mathrm{~d}^{1}(\mathrm{E})$ and $4 \mathrm{f}^{5} 5 \mathrm{~d}^{1}\left(\mathrm{~T}_{2}\right)$ states [133], but this has not been necessary here. 
Previous work on $\mathrm{Sm}^{2+}$ luminescence in alkali earth halides [127-129] has explained the strong temperature dependences of the photoluminescence intensities and lifetimes on the basis of thermally induced crossovers between the two configurations $\left(4 \mathrm{f}^{6}\right.$ and $\left.4 \mathrm{f}^{5} 5 \mathrm{~d}^{1}\right)$. The $4 \mathrm{f}^{5} 5 \mathrm{~d}^{1}$ configuration has usually been represented as a single level, assumed to be the lowest, but with LSCBC:Sm we find this does not adequately describe the population dynamics observed through the temperature dependence of the photoluminescence spectra and photoluminescent lifetimes. Instead we must include several levels of the $4 \mathrm{f}^{5} 5 \mathrm{~d}^{1}$ configuration to achieve satisfactory results. This has been done previously for the $\mathrm{Eu}^{2+}$ ion (e.g. see Duan et al. [134]) but not the $\mathrm{Sm}^{2+}$ ion, probably because of the complexity and the lack of knowledge of the $4 \mathrm{f}^{5} 5 \mathrm{~d}^{1}$ crystal field energy-level structure. For LSCBC:Sm the level structure has been calculated by Dr. Reid and basic levels are shown schematically in figure 7.17 .

The positions of the ${ }^{5} \mathrm{D}_{0,1}$ energy-levels can be found from the low temperature photoluminescent spectra. However the broad band emission observed contains contributions from many close lying $4 \mathrm{f}^{5} 5 \mathrm{~d}^{1}$ states. The emission extends over $>2000 \mathrm{~cm}^{-1}$ but the energy levels are separated by as little as $130 \mathrm{~cm}^{-1}$ and therefore we cannot measure the $4 \mathrm{f}^{5} 5 \mathrm{~d}^{1}$ level energies directly. Our crystal field calculation described above gives an estimate for the splittings between the lowest $4 f^{5} 5 d^{1}$ levels. The precise value of relative shift between the $4 f^{6}$ and $4 f^{5} 5 d^{1}$ configurations is critical for thermal crossover calculations but as $4 \mathrm{f}^{5} 5 \mathrm{~d}^{1}$ zero phonon lines are not observed, this shift cannot be accurately determined from optical absorption spectra. We have obtained an estimate for the lowest $4 \mathrm{f}^{5} 5 \mathrm{~d}^{1}$ level by monitoring the high energy cut-off of the $4 \mathrm{f}^{5} 5 \mathrm{~d}^{1}$ emission band as a function of temperature, and extrapolating this to $\mathrm{T}=0$ so that contributions to the absorption edge from thermally excited states in the ${ }^{7} \mathrm{~F}_{J}$ ground multiplet are eliminated; this provides an estimate of the zero-phonon line position. Shown in figure 7.16 is the plot of cut-off wavenumber vs. temperature, the linear fit indicates a zero-phonon position of $15500 \mathrm{~cm}^{-1}$. This estimate is consistent with the estimate of $\sim 15,700$ $\mathrm{cm}^{-1}$ used for the calculation by Dr. Reid. The estimates are similar and both result in a picture with several $4 f^{5} 5 d^{1}$ levels sandwiched between the ${ }^{5} \mathrm{D}_{0}$ and ${ }^{5} \mathrm{D}_{1}$ levels. Here we have used the estimate of $15,500 \mathrm{~cm}^{-1}$ as a trial value in modelling the thermal behaviour of the intensities, and then refined it to obtain an improved match at $15520 \mathrm{~cm}^{-1}$.

To describe the dynamics we assume that the populations of the relevant levels $\left(\mathrm{n}_{\mathrm{i}}, \mathrm{i}=\right.$ 


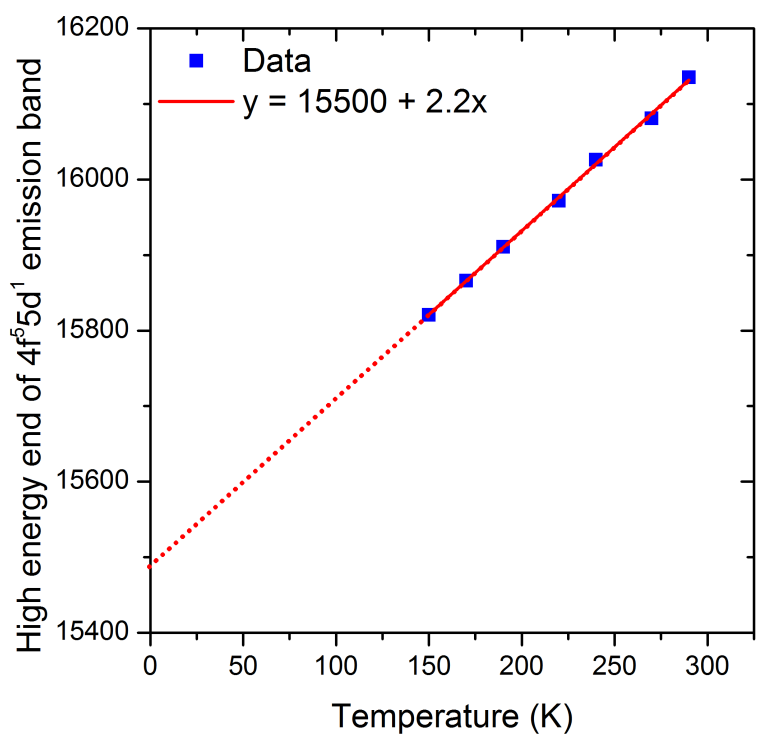

Figure 7.16: The high energy cut-off of the $4 \mathrm{f}^{5} 5 \mathrm{~d}^{1}$ emission band variation with temperature, this should give the lowest $4 \mathrm{f}^{5} 5 \mathrm{~d}^{1}$ configuration energy level at $\mathrm{T}=0 \mathrm{~K}$. The data has been fitted with a linear trend-line and extrapolated to $\mathrm{T}=0 \mathrm{~K}$.

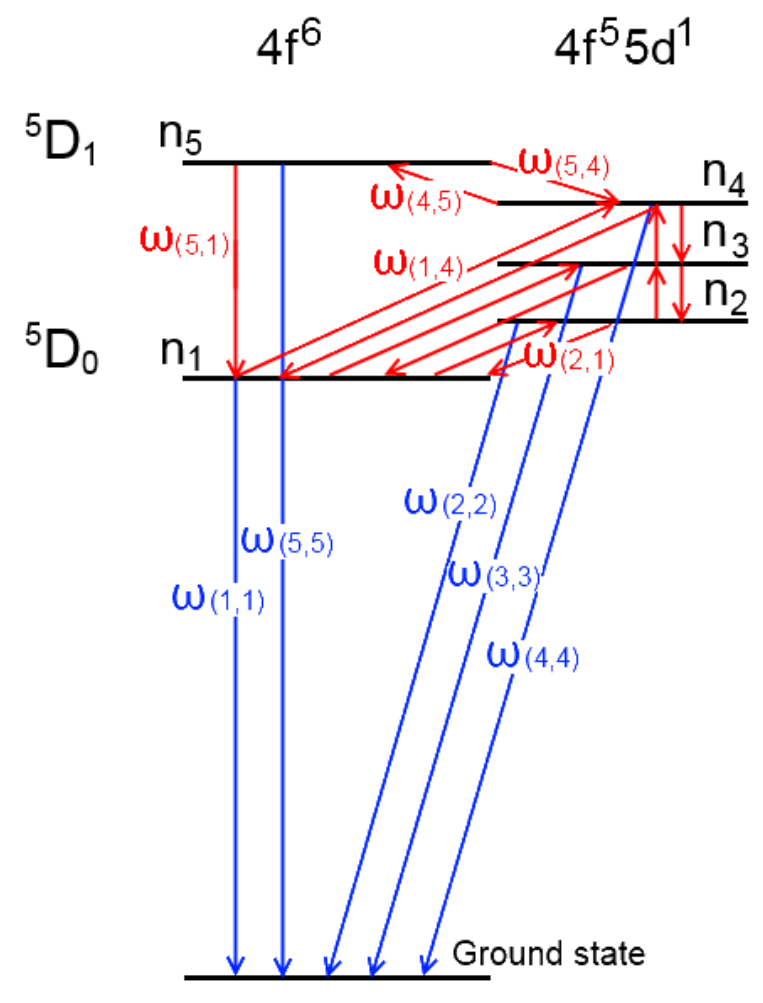

Figure 7.17: Diagram of the five lowest excited states labelled with their populations $\left(\mathrm{n}_{\mathrm{i}}\right)$. Radiation transitions observed are indicated with blue arrows. Non-radiative transitions expected to occur are indicated with red arrow. The transitions rates between levels $\mathrm{i}$ and $\mathrm{j}$ are represented by $\omega_{i, j}$ as indicated on some arrows. 
$1,2,3,4,5)$, which are schematically depicted in figure 7.17 , can be described by a set of rate equations as follows,

$$
\frac{d \mathrm{n}_{\mathrm{i}}}{d t}=-\mathrm{n}_{\mathrm{i}} \sum_{\mathrm{j}=1}^{5} \omega_{\mathrm{ij}}+\sum_{\mathrm{j}=1, \mathrm{j} \neq \mathrm{i}}^{5} \mathrm{n}_{\mathrm{j}} \omega_{\mathrm{ji}}+\mathrm{p}_{\mathrm{i}}
$$

where $\omega_{\mathrm{ij}}$ are the transition rates between levels $\mathrm{i}$ and $\mathrm{j}$ and $\mathrm{p}_{\mathrm{i}}$ is the excitation rate into level $\mathrm{i}$. The first term describes the decay routes from level i to other levels $(j)$, the second term the transitions into level i from other levels $(\mathrm{j})$, and the third the excitation into level i via other processes. In matrix form

$$
\frac{d \mathbf{N}}{d t}=\mathbf{W N}+\mathbf{P}
$$

where

$$
\mathrm{w}_{\mathrm{n}, \mathrm{l}}= \begin{cases}\omega_{\mathrm{l}, \mathrm{n}}, & \text { if } \mathrm{n} \neq \mathrm{l} \\ -\sum_{\mathrm{k}} \omega_{\mathrm{n}, \mathrm{k}}, & \text { if } \mathrm{n}=1\end{cases}
$$

where $\omega_{n, n}$ is the radiative transition probability for level $n$. Then if $\mathbf{W}$ is diagonalizable there is a transformation $\mathbf{A}$ which diagonalises $\mathbf{W}$ such that $\mathbf{W}=\mathbf{A}^{-\mathbf{1}} \mathbf{D A}$ (or $\mathbf{D}=\mathbf{A W A} \mathbf{A}^{-\mathbf{1}}$ ) with $\mathrm{D}$ being diagonal. Therefore we can write equation 7.6 as

$$
\begin{gathered}
\frac{d \mathbf{N}}{d t}=\mathbf{A}^{-1} \mathbf{D A N}+\mathbf{P} \\
\mathbf{A} \frac{d \mathbf{N}}{d t}=\mathbf{A} \mathbf{A}^{-1} \mathbf{D A N}+\mathbf{A P} \\
\mathbf{A} \frac{d \mathbf{N}}{d t}=\mathbf{D A N}+\mathbf{A P}
\end{gathered}
$$

and as $\mathbf{W}$ is time independent so is $\mathbf{A}$, therefore we have

$$
\frac{d \mathbf{A} \mathbf{N}}{d t}=\mathbf{D A N}+\mathbf{A P}
$$

or by letting $\mathbf{A N}=\mathbf{M}$ and $\mathbf{A P}=\mathbf{U}$

$$
\frac{d \mathbf{M}}{d t}=\mathbf{D M}+\mathbf{U}
$$

Here we have rewritten the populations in their 'normal modes' $\left(\mathrm{m}_{\mathrm{i}} \mathrm{s}\right)$ which represent linear combinations of populations $\left(\mathrm{N}_{\mathrm{i}} \mathrm{s}\right)$ such that the decay of the $\mathrm{M}_{\mathrm{i}}$ is a single exponential. 
Under continuous wave excitation (such as in the fluorolog) the system is in equilibrium therefore

$$
\frac{d \mathbf{N}}{d t}=0
$$

which means

$$
\mathbf{A} \frac{d \mathbf{N}}{d t}=\frac{d \mathbf{A N}}{d t}=\frac{d \mathbf{M}}{d t}=\mathbf{D M}+\mathbf{U}=0
$$

So under continuous wave excitation the populations of the normal modes are the solutions of

$$
\mathrm{DM}=-\mathrm{U}
$$

or

$$
\mathbf{M}=-\mathbf{D}^{-1} \mathbf{U}
$$

which as $\mathbf{D}$ is diagonal has the solutions

$$
\mathrm{m}_{\mathrm{l}}=\frac{\mathrm{u}_{\mathrm{l}}}{\mathrm{d}_{\mathrm{l}, \mathrm{l}}}
$$

Furthermore $\mathbf{A N}=\mathbf{M}$ or $\mathbf{N}=\mathbf{A}^{-1} \mathbf{M}$ therefore

$$
\mathbf{N}=\mathbf{A}^{-1} \mathbf{M}=-\mathbf{A}^{-1} \mathbf{D}^{-1} \mathbf{U}
$$

which has the solutions

$$
\mathrm{n}_{\mathrm{l}}=\sum_{\mathrm{n}} \mathrm{a}_{\mathrm{l}, \mathrm{n}}^{-1} \frac{\mathrm{u}_{\mathrm{n}}}{\mathrm{d}_{\mathrm{n}, \mathrm{n}}}
$$

Therefore we can solve for the populations of each level as a function of temperature. By multiplying the population of level $\mathrm{i}$ by the radiative transition rate of level $\mathrm{i}$ we find the relative integrated intensity of emission from that level.

In order to find the lifetimes of the radiative levels we consider the decay of population after an excitation pulse. Returning to equation 7.12, this time the system is not in equilibrium, and so $\frac{d \mathbf{M}}{d t} \neq 0$, however, we consider the kinetics after an excitation pulse so that $\mathbf{U}=0$. In this situation the solutions to equation 7.12 becomes

$$
\frac{d \mathbf{M}}{d t}=\mathbf{D M}
$$


which has the solutions

$$
\mathrm{m}_{\mathrm{l}}=\mathrm{m}_{1,0} \exp \left(\mathrm{d}_{1,1} t\right)
$$

Where $\mathrm{m}_{\mathrm{n}, 0}$ represents initial population of mode $\mathrm{n}$ at $t=0$. Therefore the population of level $l$ as a function of time is given by

$$
\mathrm{n}_{\mathrm{l}}=\sum_{\mathrm{n}} \mathrm{a}_{1, \mathrm{n}}^{-1} \mathrm{~m}_{\mathrm{n}, 0} \exp \left(\mathrm{d}_{\mathrm{n}, \mathrm{n}} t\right)
$$

By fitting the simulated population as a function of time with an exponential decay we obtain the dominant decay component of the levels as a function of temperature.

We use energy level values based from the calculated electric-dipole oscillator strengths for the $4 \mathrm{f}^{5} 5 \mathrm{~d}^{1}$ levels. For the non-radiative rates, we use the analytic expression first developed by Rebane [135], and discussed by Henderson and Imbusch [63] and Struck and Fonger [136]. In this treatment, the non radiative rate $\mathrm{w}_{\mathrm{i}, \mathrm{j}}$ between levels $\mathrm{i}$ and $\mathrm{j}$ is given by

$$
\mathrm{w}_{\mathrm{NR}(\mathrm{i}, \mathrm{j})}=R^{2} \mathrm{e}^{(-S(2 m+1))}\left(\frac{1+m^{\frac{p}{2}}}{m}\right) I_{p}(2 S \sqrt{m(m+1)})
$$

where $R$ is an electronic matrix element, $S$ is the Huang-Rhys factor (a dimensionless constant which characterizes the difference in electron-lattice coupling between two states), $p$ is the number of phonons of frequency $\omega$ required to bridge the gap between levels $i$ and $j, m$ is the mean thermal occupancy [63]

$$
m=\exp \left(\frac{-\hbar \omega}{k T}\right)
$$

in the phonon mode, and $I_{p}$ is the modified Bessel function of the second kind. A series expansion of the Bessel function leads to the useful form [63],

$$
W n r_{(h, f)}=R^{2} \mathrm{e}^{(-S(2 m+1))} \frac{S^{p}}{p !}(1+m)^{p}\left(1+\frac{S^{2} m(m+1)}{p+1}\right)
$$

We make some assumptions to simplify the model 


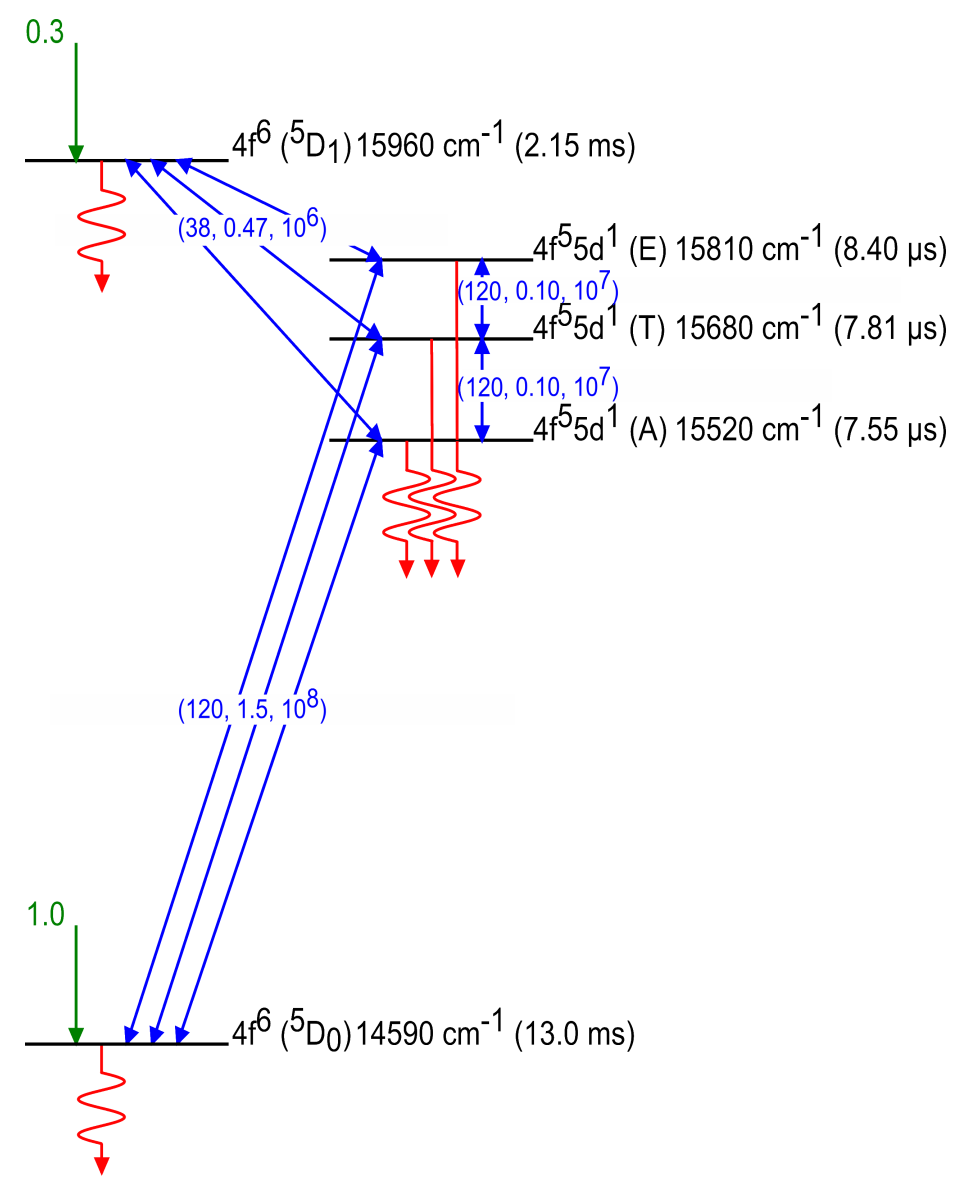

Figure 7.18: The calculated energy levels of LSCBC:Sm, described as orbital (configuration) wavenumber (radiative lifetime). The expected non-radiative transitions are shown with blue arrows and labelled as (phonon frequency $(\omega)$, Huang-Rhys factor (S), electronic matrix element $(R)$ ).

1. Only the ${ }^{5} \mathrm{D}_{0,1}$ levels of the $4 \mathrm{f}^{6}$ configuration and the three lowest levels of the $4 \mathrm{f}^{5} 5 \mathrm{~d}^{1}$ configuration are included in the numerical calculation as this seems to be the minimal set required to explain our results.

2. The direct non-radiative transition ${ }^{5} \mathrm{D}_{1} \rightarrow{ }^{5} \mathrm{D}_{0}$ is neglected, this is reasonable as the energy gap $\left(1370 \mathrm{~cm}^{-1}\right)$ requires a large number of phonons $(p)$, and this is reflected in equation 7.25 by the $p$ ! term in the denominator.

3. We use just two phonon frequencies: $120 \mathrm{~cm}^{-1}$ for transitions within the $4 \mathrm{f}^{5} 5 \mathrm{~d}^{1}$ configuration and $4 \mathrm{f}^{5} 5 \mathrm{~d}^{1} \leftrightarrow 4 \mathrm{f}^{6}\left({ }^{5} \mathrm{D}_{0}\right)$ transitions. This is a single-frequency estimate consistent with the values reported for $\mathrm{SrCl}_{2}: \mathrm{Sm}^{2+}$ by Karbowiak et al. [122] of 81, 116, $213 \mathrm{~cm}^{-1}$ for $4 \mathrm{f}^{5} 5 \mathrm{~d}^{1} \leftrightarrow 4 \mathrm{f}^{6}\left({ }^{5} \mathrm{D}_{0}\right)$ transitions and the observation of vibronics in the $100-180 \mathrm{~cm}^{-1}$ range on the $4 \mathrm{f}^{6} \leftrightarrow 4 \mathrm{f}^{6}$ photoluminescent spectra. For $4 \mathrm{f}^{5} 5 \mathrm{~d}^{1} \leftrightarrow 4 \mathrm{f}^{6}\left({ }^{5} \mathrm{D}_{1}\right)$ we use $40 \mathrm{~cm}^{-1}$ based on measurements on orthorhombic $\mathrm{BaCl}_{2}: \mathrm{Sm}$; Lauer and Fong [128] report a 
vibronic sideband of the ${ }^{5} \mathrm{D}_{1} \rightarrow{ }^{7} \mathrm{~F}_{0}$ transition starting at $38 \mathrm{~cm}^{-1}$.

4. We take radiative lifetimes for the ${ }^{5} \mathrm{D}_{0}$ and ${ }^{5} \mathrm{D}_{1}$ states of $13 \mathrm{~ms}$ and $2.15 \mathrm{~ms}$ respectively. These are based on our measurements of the ${ }^{5} \mathrm{D}_{0}$ emission at low temperature and on the reported rate for the $4 \mathrm{f}^{6}\left({ }^{5} \mathrm{D}_{1}\right)$ level of orthorhombic $\mathrm{BaCl}_{2}$ [128].

5. We assume that the ${ }^{5} \mathrm{D}_{0}$ and the ${ }^{5} \mathrm{D}_{1}$ state respectively are populated by non-radiative decay from higher states in the proportion 1:1 respectively, chosen by trial and error to best reproduce the observed intensity ratios at the lowest temperature of measurement.

6. We take calculated radiative lifetimes for the $4 \mathrm{f}^{5} 5 \mathrm{~d}^{1}$ states from the crystal field calculation.

Figure 7.18 summarises the parameters and energy-level positions used in the numerical calculation and figure 7.19(a) shows the predicted temperature dependence of the integrated intensity of emission from each level based on those parameters. A comparison between the simulated and experimental intensities, which are repeated in figure 7.19(c), shows excellent agreement between the trends observed and calculated numerically. The experimental observation of a reduced total intensity at temperatures close to $300 \mathrm{~K}$ is not reproduced in the numerical calculation since non-radiative decay from any of the excited states to the ground states is not included in the model, but would only require a minor amendment (e.g. see Duan et al [134]).

We then extend our simulation to include the lifetime of each state by fitting the simulated population as a function of time with an exponential decay. The results of this are shown figure $7.19(\mathrm{~b})$. Comparing these results to the observed lifetimes shown again in figure $7.19(\mathrm{~d})$ shows an excellent match to the general trend above $\sim 100 \mathrm{~K}$. The decrease in lifetime at the lowest temperature for the lowest $5 \mathrm{~d}^{1}$ level is reproduced but with less satisfactory agreement. Below $\sim 20 \mathrm{~K}$ the simulation appears to suffer from numerical inaccuracies.

\subsubsection{Discussion of optical spectroscopy}

Previous work by Edgar et al. [118] has shown (on the basis of density measurements) that the most likely way in which $\mathrm{LaCl}_{3}$ is incorporated into the cubic $\mathrm{BaCl}_{2}$ lattice is with the $\mathrm{La}^{3+}$ 

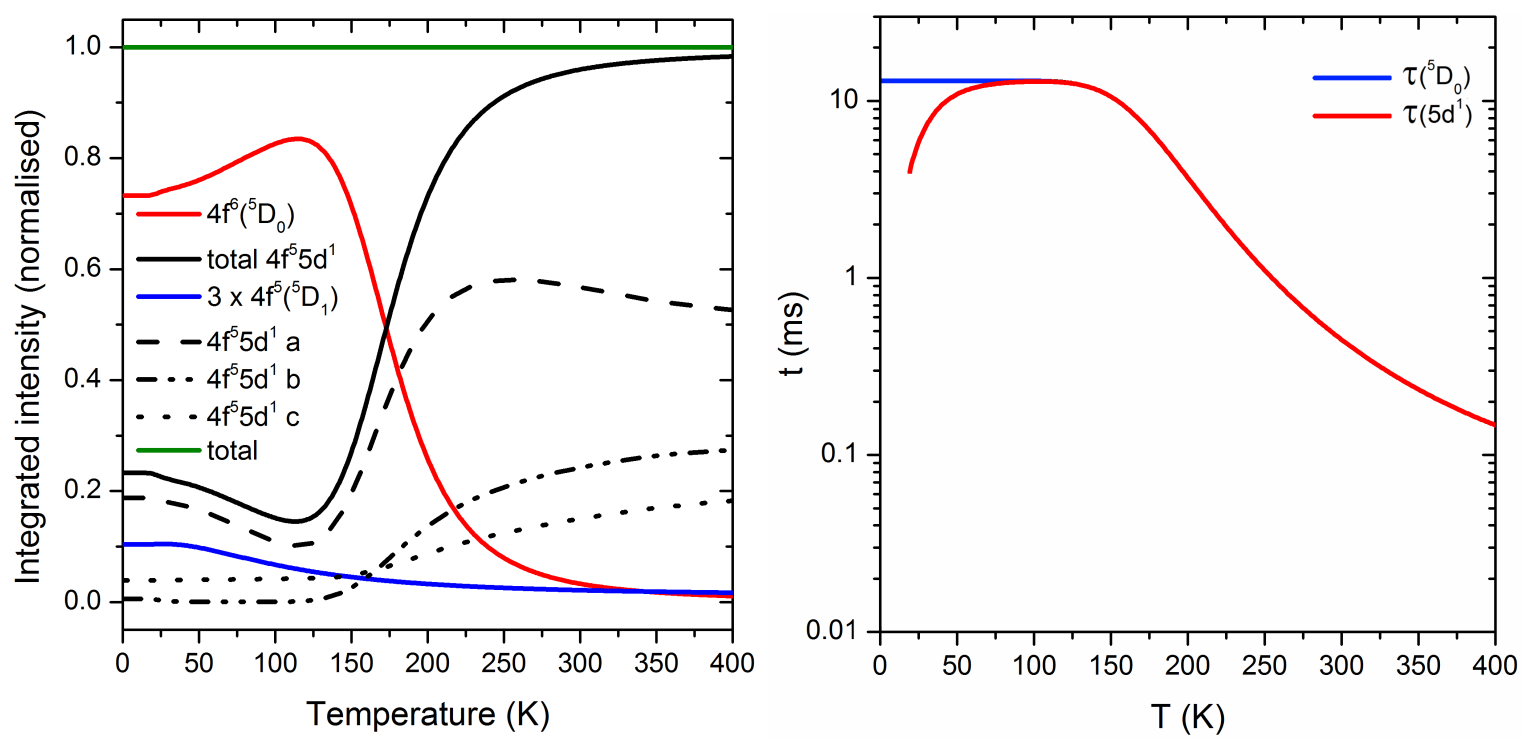

(a)

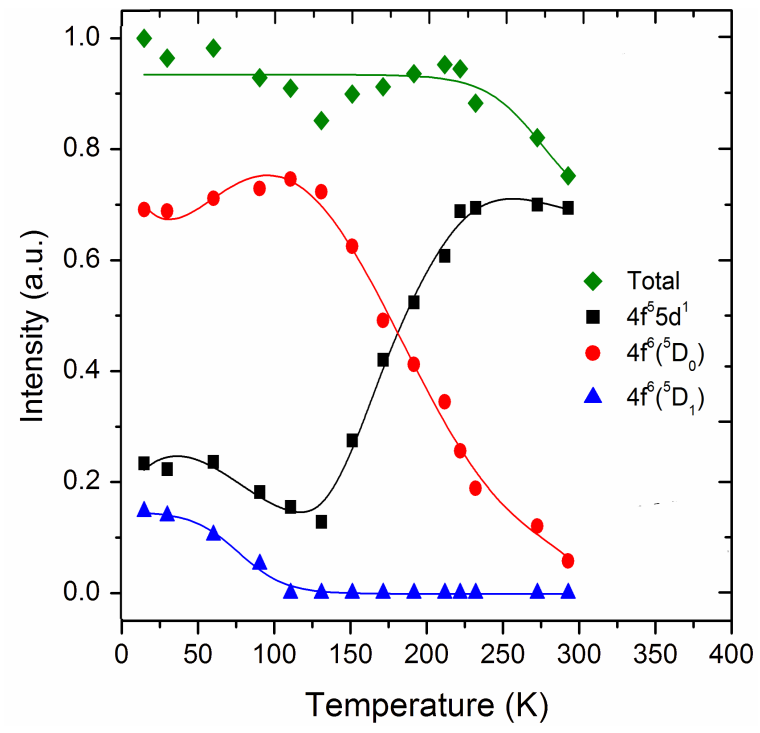

(c) (b)

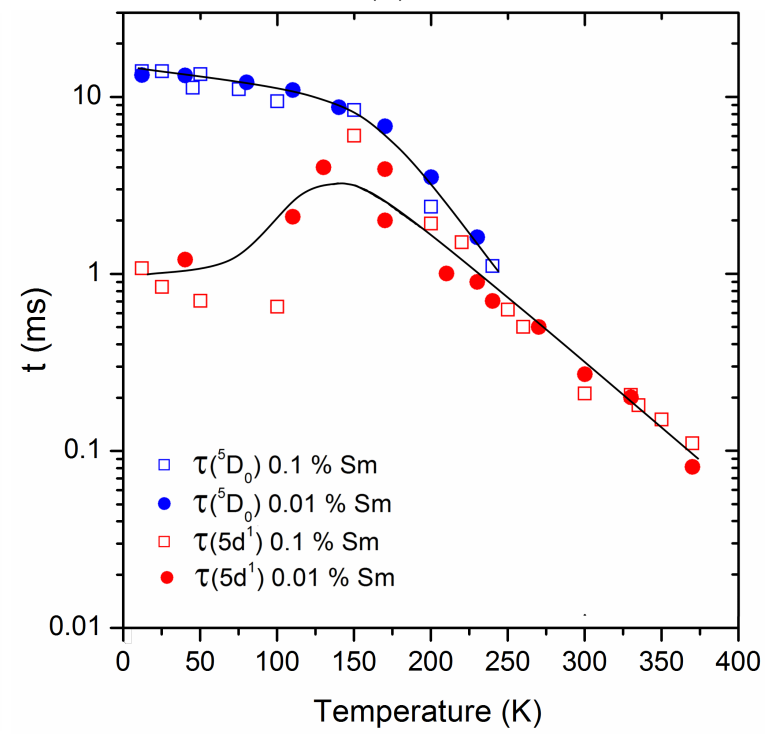

(d)

Figure 7.19: (a) Simulated emission intensity from each state as a function of temperature and (b) simulated lifetime of the ${ }^{5} \mathrm{D}_{0}$ and lowest $5 \mathrm{~d}^{1}$ levels as a function of temperature for LSCBC:Sm ${ }^{2+}$. (c) Experimental integrated intensity of emission from ${ }^{5} \mathrm{D}_{0},{ }^{5} \mathrm{D}_{1}$ and $4 \mathrm{f}^{5} 5 \mathrm{~d}^{1}$ configuration as a function of temperature (repeated from figure 7.11). (d) Lifetime of ${ }^{5} \mathrm{D}_{0} \rightarrow$ ${ }^{7} \mathrm{~F}_{1}$ transition and $4 \mathrm{f}^{5} 5 \mathrm{~d}^{1} \rightarrow{ }^{7} \mathrm{~F}_{J}$ transitions for LSCBC:Sm${ }^{2+}$ (repeated from figure 7.14). 
ions substituting for $\mathrm{Ba}^{2+}$ ions at cation sites, and with an extra $\mathrm{Cl}^{-}$ion per $\mathrm{La}^{3+}$ ion providing charge compensation and located at an interstitial vacancy [117]. Recall that in the fluorite lattice, cations occupy half the anion cubes and half are empty. Therefore, $\left(\frac{1}{8}\right)$ th of the empty interstitial sites are occupied with a $\mathrm{Cl}^{-}$ion for $12.5 \% \mathrm{La}^{3+}$ ion doping levels. The $\mathrm{Sm}^{2+}$ ions substitute for $\mathrm{Ba}^{2+}$ ions at cation sites due to the charge and radius match. Each $\mathrm{Sm}^{2+}$ ion occupying a $\mathrm{Ba}^{2+}$ ion site is surrounded by 6 empty interstitial sites in a perfect lattice. The probability of none of these containing a $\mathrm{Cl}^{-}$ion is $\left(\frac{7}{8}\right)^{6}$ or $\sim 45 \%$. Therefore we expect more than half the $\mathrm{Sm}^{2+}$ ions to have non-cubic symmetry considering the nearest interstitial shell. Further, each $\mathrm{Ba}^{2+}$ ion site in the fluorite lattice has 12 nearest neighbour cation sites, with $12.5 \%$ of the cations being $\mathrm{La}^{3+}$ ions there is there is only a $\left(\frac{7}{8}\right)^{12}$ or $\sim 20 \%$ chance of a cubic environment as far as the first nearest neighbour cation shell is concerned. Despite this lack of probability of a cubic site we observe absorption spectra which are well-described by a single site of cubic symmetry, and the photoluminescence showed only one $4 \mathrm{f}^{6}\left({ }^{5} \mathrm{D}_{0} \rightarrow{ }^{7} \mathrm{~F}_{0}\right)$ singlet photoluminescence line, indicative of a cubic site. Thus we are led to deduce that either the perturbation of the cubic crystal field by nearby $\mathrm{Cl}^{-}$interstitial ions and $\mathrm{La}^{3+}$ ions is so weak that it leads only to broadening and not splitting of the PL spectra, or that the defect ions are not randomly distributed.

A possible mechanism for the latter is clustering of $\mathrm{La}^{3+}-\mathrm{Cl}^{-}$pairs. Such pairs are commonly found in trivalent rare-earth doped alkaline earth halides and indeed as larger clusters [137]. There is no evidence for large scale phase modifications such as superlattice lines in the XRD pattern. These would appear as a second pattern at small angles due to the large superlattice constant. As an alternative to block formation, interstitial $\mathrm{Cl}^{-}$ions may be repelled from the $\mathrm{Sm}^{2+}$ ions as a result of the ionic radius mismatch. While only a singlet $4 \mathrm{f}^{6}\left({ }^{5} \mathrm{D}_{0} \rightarrow{ }^{7} \mathrm{~F}_{0}\right)$ transition was observed this was stronger relative to the $4 \mathrm{f}^{6}\left({ }^{5} \mathrm{D}_{0} \rightarrow{ }^{7} \mathrm{~F}_{1}\right)$ transition than one would expect for a cubic site lacking inversion symmetry (for a simple site of $\mathrm{O}_{h}$ symmetry the $4 \mathrm{f}^{6}\left({ }^{5} \mathrm{D}_{0} \rightarrow{ }^{7} \mathrm{~F}_{0}\right)$ transition is electric-dipole and magnetic dipole forbidden). Thus we favour the interpretation in which the perturbation on the cubic crystal field provided by nearby lattice defects is small. There is some evidence for this as part of a general pattern in the alkaline earth halides as both the ionic radius of the cation and anion increase from the $\mathrm{Ca}^{2+}$ ion to the $\mathrm{Ba}^{2+}$ ion and from the $\mathrm{F}^{-}$ion to the $\mathrm{I}^{-}$ion [138]. Also, the reduction process 


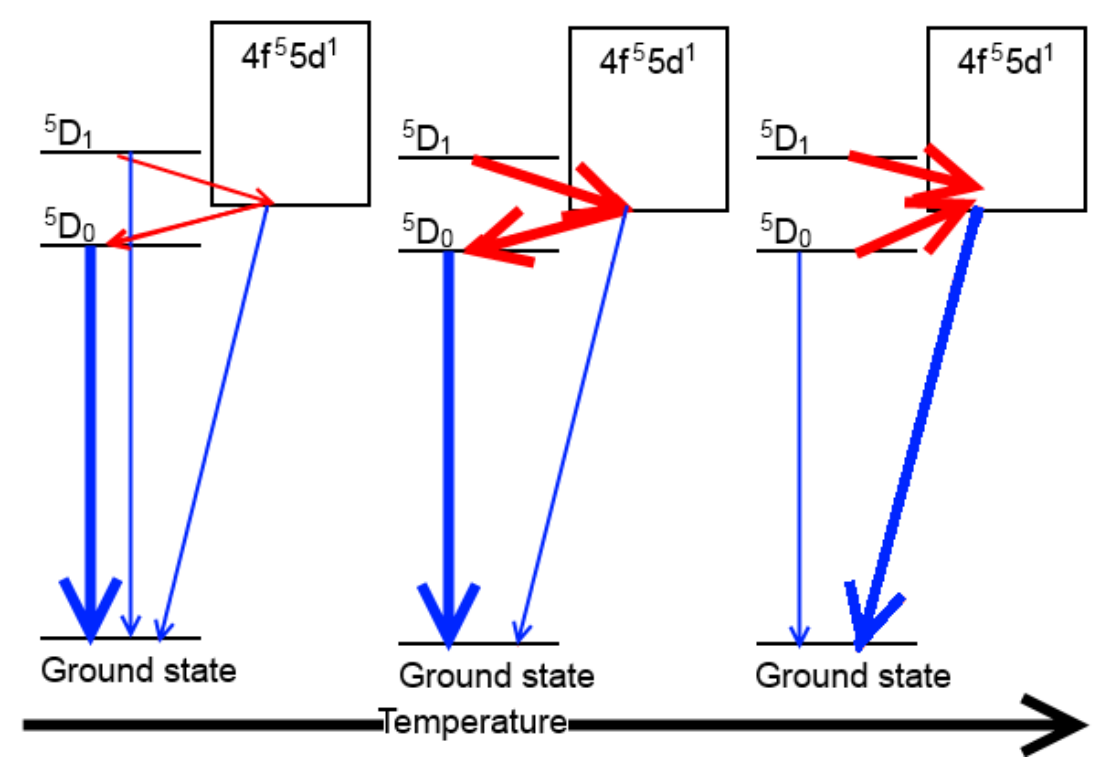

Figure 7.20: A schematic diagram of the energy levels of the $\mathrm{Sm}^{2+}$ ion in LSCBC and the radiative and non-radiative transitions occurring between them as the temperature is increased. The dominant processes are indicated with the larger arrows. Radiative transitions shown in blue and non-radiative transitions are shown in red.

may have simply reduced the number of $\mathrm{Cl}^{-}$ions.

The numerically calculated intensities and lifetimes (figure 7.19) help explain the processes which occur as the sample temperature is raised. The dominant processes are depicted schematically in figure 7.20 in the three main temperature regimes. At the lowest temperatures $(\sim 12-50 \mathrm{~K})$, the $4 \mathrm{f}^{6}\left({ }^{5} \mathrm{D}_{1}\right)$ level shows radiative emission, but this rapidly decreases with temperature as it is depopulated by non-radiative transitions to the $4 \mathrm{f}^{5} 5 \mathrm{~d}^{1}(\mathrm{~A}),(\mathrm{T})$, and (E) levels. They in turn are subject to internal relaxation and to decay to the $4 \mathrm{f}^{6}\left({ }^{5} \mathrm{D}_{0}\right)$ level. The ${ }^{5} \mathrm{D}_{0}$ level emission is largely temperature independent in this regime as thermal excitation to the $4 \mathrm{f}^{5} 5 \mathrm{~d}^{1}$ levels is improbable, which is shown schematically on the left of figure 7.20 . The $4 \mathrm{f}^{5} 5 \mathrm{~d}^{1}$ emission first shows a small rise with increasing temperature to $80 \mathrm{~K}$ as these levels are populated from the $4 \mathrm{f}^{6}\left({ }^{5} \mathrm{D}_{1}\right)$ level. The intensity then decreases as the temperature is raised to $\sim 140 \mathrm{~K}$ as this population is lost to the $4 \mathrm{f}^{6}\left({ }^{5} \mathrm{D}_{0}\right)$ level. The $4 \mathrm{f}^{6}\left({ }^{5} \mathrm{D}_{0}\right)$ emission shows a corresponding increase up to $140 \mathrm{~K}$, which is shown schematically in the centre of figure 7.20 . However, at around $140 \mathrm{~K}$, the $4 \mathrm{f}^{5} 5 \mathrm{~d}^{1}$ levels start to become populated by thermal excitation from the $4 \mathrm{f}^{6}\left({ }^{5} \mathrm{D}_{0}\right)$ level, leading to a dramatic decline in the emission from the $4 f^{6}\left({ }^{5} D_{0}\right)$ level and a corresponding increase in emission from the $4 f^{5} 5 d^{1}$ levels. The deexcitation route through the $4 \mathrm{f}^{5} 5 \mathrm{~d}^{1}$ levels with their short radiative lifetime provides a faster decay route for the $4 \mathrm{f}^{6}\left({ }^{5} \mathrm{D}_{1}\right)$ population than through the slow direct radiative decay, which 
is shown schematically on the right of figure 7.20. This is the underlying reason behind the dominance of the broad band emission at high temperature and that of narrow bands at low temperatures. The crossover between the two regimes at around $175 \mathrm{~K}$ is nicely reproduced in the numerical calculations, and is sensitive to the energy difference between the electronic levels. The good agreement achieved for the crossover encourages confidence in the process used to estimate the energy origin of the broad $4 \mathrm{f}^{5} 5 \mathrm{~d}^{1}$ band. The measured and numerically simulated lifetimes also provide evidence of these phenomena. The $4 \mathrm{f}^{5} 5 \mathrm{~d}^{1}$ levels lifetimes are limited by the feeding route. At the lowest temperatures this is from the $4 \mathrm{f}^{6}\left({ }^{5} \mathrm{D}_{1}\right)$ level (which has a shorter lifetime than that of the $4 \mathrm{f}^{6}\left({ }^{5} \mathrm{D}_{0}\right)$ level), then as the temperature increases, more population comes from the $4 \mathrm{f}^{6}\left({ }^{5} \mathrm{D}_{0}\right)$ level and so the lifetime increases. As thermal population of the $4 \mathrm{f}^{5} 5 \mathrm{~d}^{1}$ levels becomes more probable due to temperature increase, the lifetime abruptly decreases, and we expect the decrease would continue with temperature increase until the calculated intrinsic radiative rate of the $4 \mathrm{f}^{5} 5 \mathrm{~d}^{1}$ levels is reached $(\sim 8$ $\mu \mathrm{s})$.

\subsubsection{X-ray response}

\subsubsection{Radioluminescence}

The radioluminescence generally follows the photoluminescence for the higher $\mathrm{Sm}^{2+}$ concentrations, but for concentrations below $\sim 0.05 \%$ a new and strong emission band appears in the blue region of the spectrum, as shown in figure 7.21 . This new band is not observed in photoluminescence and is present in samples with no samarium doping. This emission overlaps with the $\mathrm{Sm}^{2+}$ ion absorption bands seen in figure 7.4 and is likely to be absorbed by the $\mathrm{Sm}^{2+}$ ions before it can exit the lattice, this is likely why the emission is not observed in higher concentration samples. A further effect evident in figure 7.21 is the shift in the peak of the broad band $\mathrm{Sm}^{2+}$ ion fluorescence to lower energies in the more concentrated samples and a decrease in intensity, which is indicative of re-absorption and concentration quenching.

The relative $\mathrm{x}$-ray phosphor efficiency has been determined by comparing the spectra of LSCBC:Sm ${ }^{2+}$ with that of a standard $\mathrm{Gd}_{2} \mathrm{O}_{2} \mathrm{~S}: \mathrm{Tb}^{3+}$ phosphor from the Kansei company. The 
Table 7.2: Integrated intensity of radioluminescence emission relative to that from $\mathrm{Gd}_{2} \mathrm{O}_{3} \mathrm{~S}: \mathrm{Tb}^{3+}$.

\begin{tabular}{cccc}
\hline Sm concentration (\%) & Blue band (\%) & Red band (\%) & Total (\%) \\
\hline 0 & 8.5 & - & 8.5 \\
0.002 & 6.5 & 4.4 & 11 \\
0.01 & 3.5 & 5.5 & 9.0 \\
0.1 & 0.1 & 1.3 & 1.4 \\
1 & 0.1 & 0.5 & 0.6 \\
\hline
\end{tabular}

relative efficiencies for the broad red band and broad blue band are tabulated in table 7.2 as percentages of that of $\mathrm{Gd}_{2} \mathrm{O}_{2} \mathrm{~S}: \mathrm{Tb}^{3+}$. The spectral sensitivity of the spectrometer has been corrected for in these estimates. It should be noted that the $\mathrm{Gd}_{2} \mathrm{O}_{2} \mathrm{~S}: \mathrm{Tb}^{3+}$ is a $140 \mu \mathrm{m}$ layer of power in an organic binder resin while our samples are $1 \mathrm{~mm}$ thick polycrystals; the $\mathrm{x}$-ray half thicknesses at the peak of $x$-ray spectrum are $0.48 \mathrm{~mm}$ and $0.18 \mathrm{~mm}$ respectively so both materials are 1 - 2 half thicknesses.

\subsubsection{Scintillation}

Since the material is a relatively good x-ray phosphor, it is also of interest to investigate the scintillation performance. However, although scintillation events can be observed these are followed by a very long phosphorescent persistence, as shown in figure 7.22 which shows a

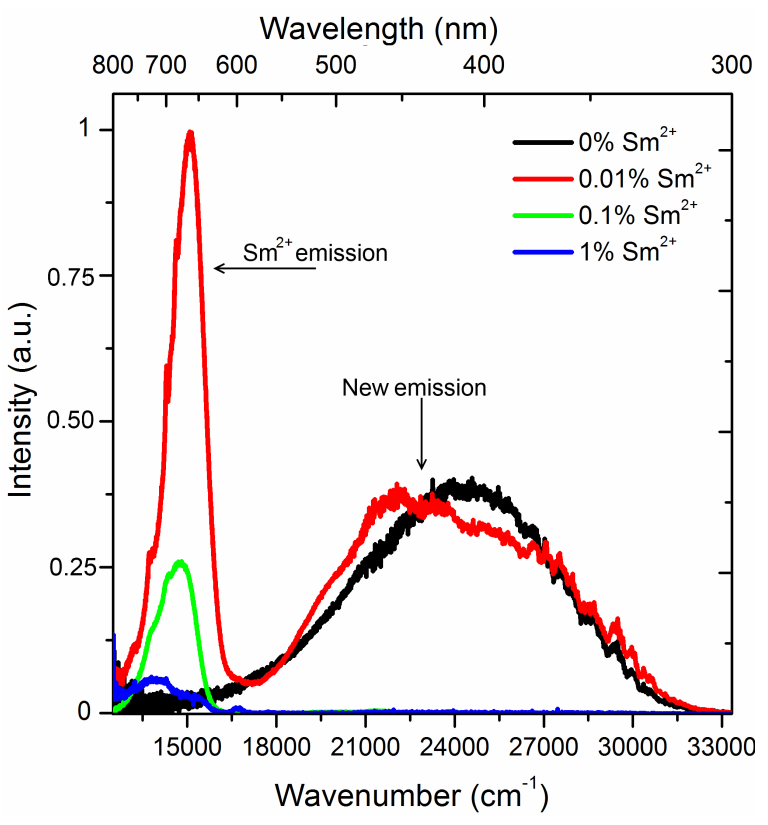

Figure 7.21: Radioluminesence of LSCBC and LSCBC:Sm for $0.01 \%, 0.1 \%$ and $1 \%$ samarium concentrations, recorded under $x$-irradiation of $35 \mathrm{kV}$ from a copper tube with a $2 \mathrm{~mm}$ aluminium filter. 


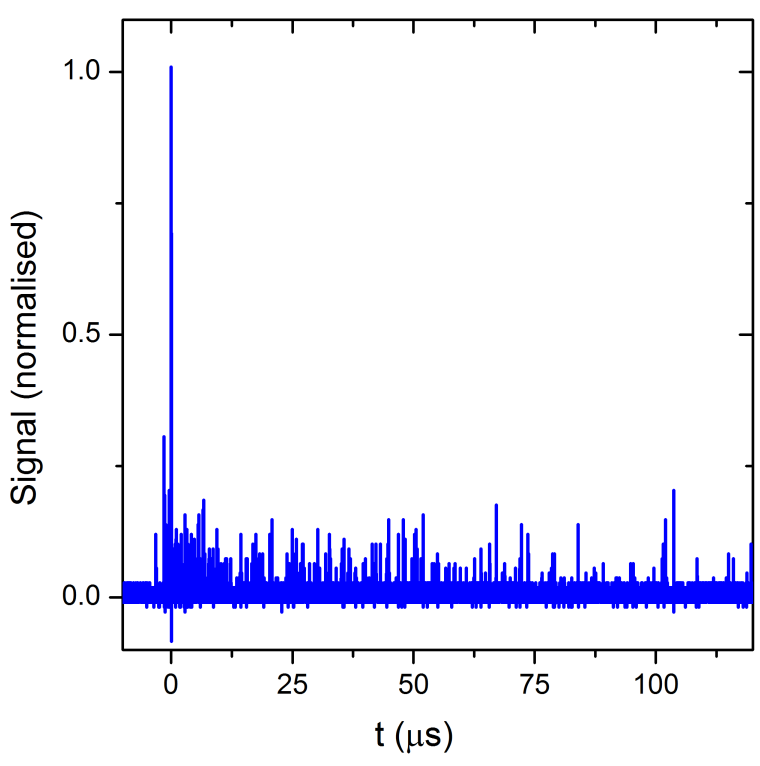

(a)

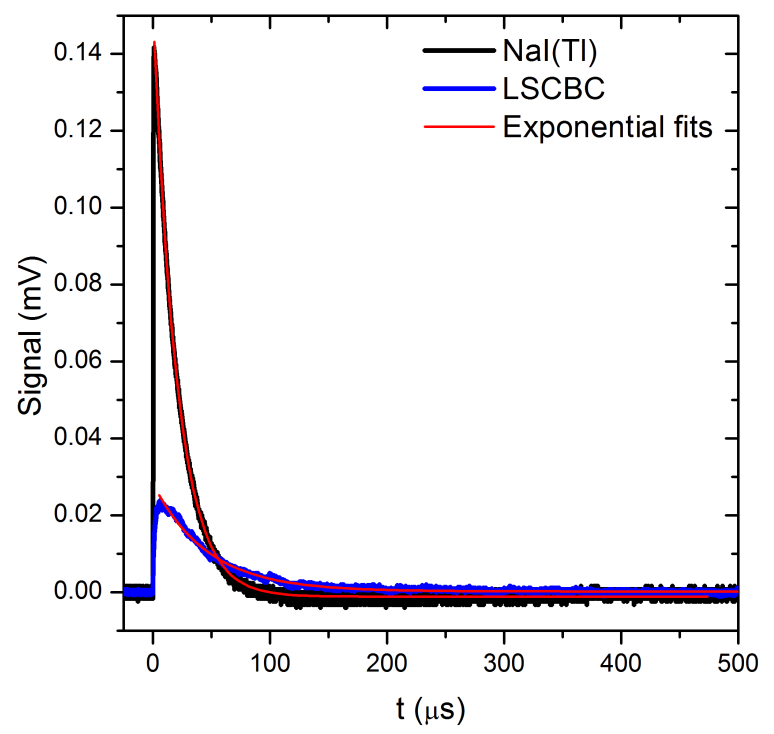

(b)

Figure 7.22: Single scintillation event of undoped LSCBC recorded direct from photomulitplier tube (a). Average of 1000 scintillation events of both undoped LSCBC (blue) and NaI(Tl) (black), these have been integrated by a spectroscopy amplifier (CI 1410) with a time constant of $7 \mu$ s.

single scintillation event of the undoped material extending well over $100 \mu$ s. Figure $7.22(\mathrm{~b})$ compares the average of 1000 scintillation events with that of $\mathrm{NaI}(\mathrm{Tl})$; the integrated area of the LSCBC curve is about half that of the $\mathrm{NaI}(\mathrm{Tl})$, indicating that the light output is relatively high but is extended over a long decay time. The samarium doped samples showed similar pulses although no scintillation was observed when a long pass filter was used to filter out the blue emission.

We attempted doping with other alkali halides with monovalent cations to provide charge compensation for the $\mathrm{La}^{3+}$ ions in an attempt to remove the $\mathrm{Cl}^{-}$ion interstitials as these may slow the scintillation process significantly through colour centre generation. For example, $\mathrm{H}$ centres comprising a $\mathrm{Cl}^{-}$ion interstitial may combine with a lattice $\mathrm{Cl}^{-}$ion to form a $\mathrm{Cl}_{2}^{-}$ molecular ion representing a trapped charge. The release of this trapped hole and subsequent recombination emission via a $\mathrm{Sm}^{2+}$ ion would involve a delay depending on the trap energies. Figure 7.23 shows the powder XRD patterns formed from samples which had $6.25 \% \mathrm{La}^{3+}$ and $6.25 \% \mathrm{MCl}(\mathrm{M}=\mathrm{Li}, \mathrm{K}, \mathrm{Na}, \mathrm{Cs})$. None of these materials showed a strong presence of the cubic phase (shown at bottom of figure 7.23), and most peaks could be attributed to the orthorhombic phase (shown at top of figure 7.23). Since these materials are not transparent, they have not been investigated further. 


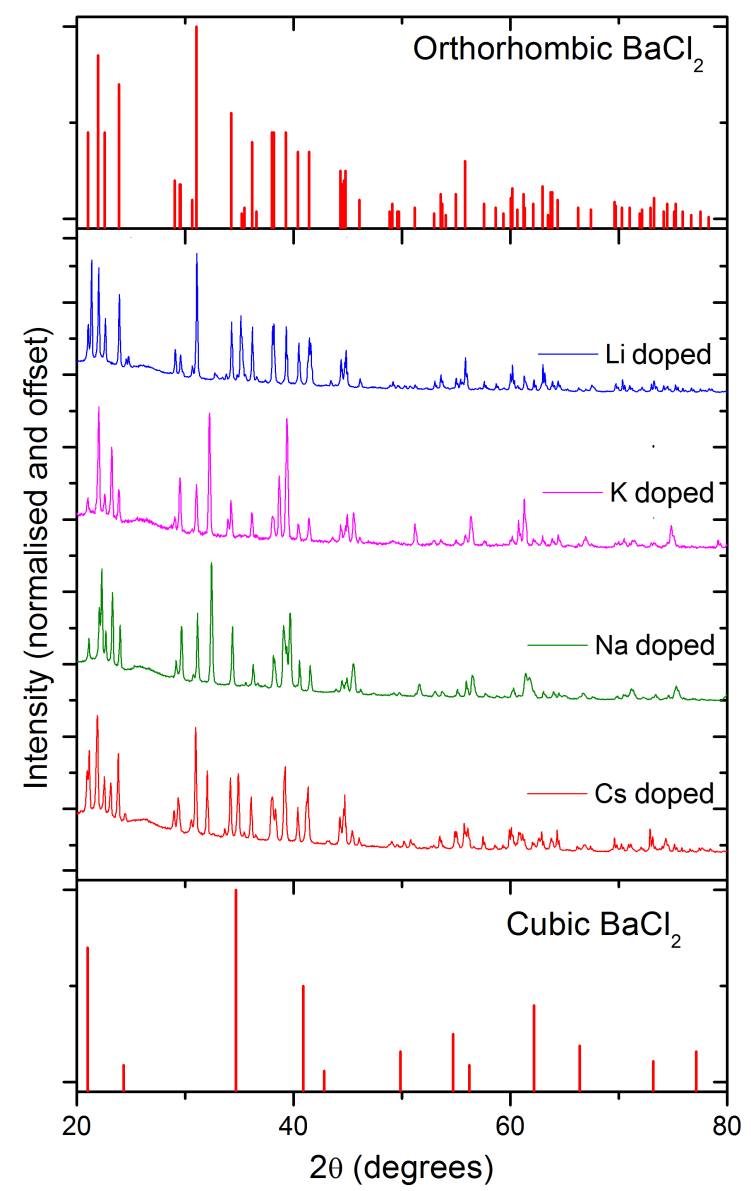

Figure 7.23: Powder x-ray diffraction patterns for $\mathrm{La}_{0.6125} \mathrm{M}_{0.6125} \mathrm{Ba}_{0.875} \mathrm{Cl}_{2}$ :Sm, $(\mathrm{M}=\mathrm{Li}, \mathrm{K}$, $\mathrm{Na}, \mathrm{Cs})$. The resulting patterns indicate non-cubic materials. The powder pattern file for orthorhombic $\mathrm{BaCl}_{2}$ is shown at the top and for cubic $\mathrm{BaCl}_{2}$ at the bottom.

Table 7.3: Afterglow of undoped and samarium doped LSCBC.

\begin{tabular}{cccc}
\hline Sample & $10 \mathrm{~ms}$ & $20 \mathrm{~ms}$ & $50 \mathrm{~ms}$ \\
\hline LSCBC:Sm $(0 \%)$ & 12.3 & 6.10 & 2.30 \\
LSCBC:Sm $(0.01 \%)$ & 9.8 & 4.3 & 1.4 \\
LSCBC:Sm $(0.5 \%)$ & 1.3 & 0.5 & 0.4 \\
NaI $(\mathrm{Tl})$ & 1.00 & 0.60 & 0.20 \\
\hline
\end{tabular}

\subsubsection{Afterglow}

The afterglow was recorded for the undoped and samarium doped material as shown in figure 7.24. Samarium doping significantly reduces the afterglow. Inspection of figure 7.24 indicates there are two processes which are reducing the afterglow of LSCBC with samarium doping, first small amounts of samarium reduce the number of trapped electrons but not the rate of release of traps. This is indicated by the approximately common gradients but lower starting point of the $0.01 \%$ Sm sample afterglow curve compared to that of the 


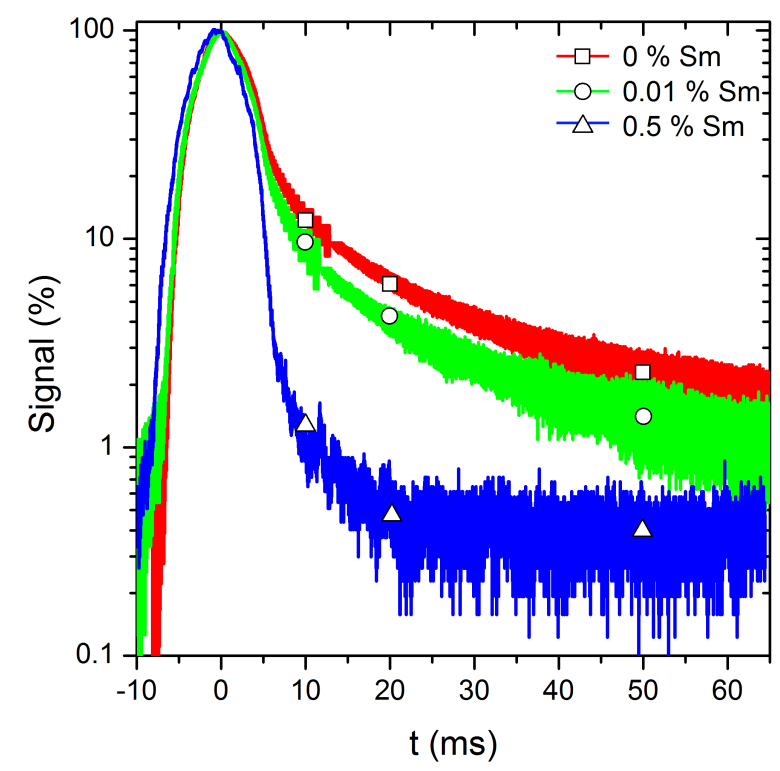

Figure 7.24: LSCBC:Sm and undoped LSCBC afterglow profiles. The profiles were each recorded on two channels on the oscilloscope with different gains to allow a higher signal to noise ratio as the intensity dropped below $10 \%$.

undoped material. Second, the rate of trap-related recombination appears to increase with samarium concentration. The $0.5 \%$ curve appears to not only start at a lower point but also to decay faster, that is there are fewer traps and they have a shorter lifetime. Fewer traps are formed because there is a finite probability the free charge carriers may interact directly with a samarium ion luminescent centre before being trapped. The traps decay faster as the concentration of samarium ions increases because the likelihood of a nearby samarium ion to which the electron may 'hop' to is significantly increased.

\subsubsection{Thermally stimulated luminescence}

Thermally stimulated luminescence (TSL) is a popular technique for determining the electron trapping characteristics of insulators and semiconductors. In this case we are investigating the traps which are above room temperature; these are generally the cause of afterglow in materials. Figure 7.25 shows the glow curves recorded for LSCBC and LSCBC:Sm. The glow curves indicate up to three traps are present in the both materials above $330 \mathrm{~K}$. These have been fitted with first order kinetic equations to extract the peak temperature and activation energy according to the method of Kitis [90]. The fitted parameters for LSCBC and LSCBC:Sm are shown in table 7.4. Undoped LSCBC has peaks at $370 \mathrm{~K}$ and $440 \mathrm{~K}$ with corresponding activation energies of 0.48 and $0.62 \mathrm{eV}$. LSCBC:Sm has peaks at $385 \mathrm{~K}, 410 \mathrm{~K}$ and $470 \mathrm{~K}$ 


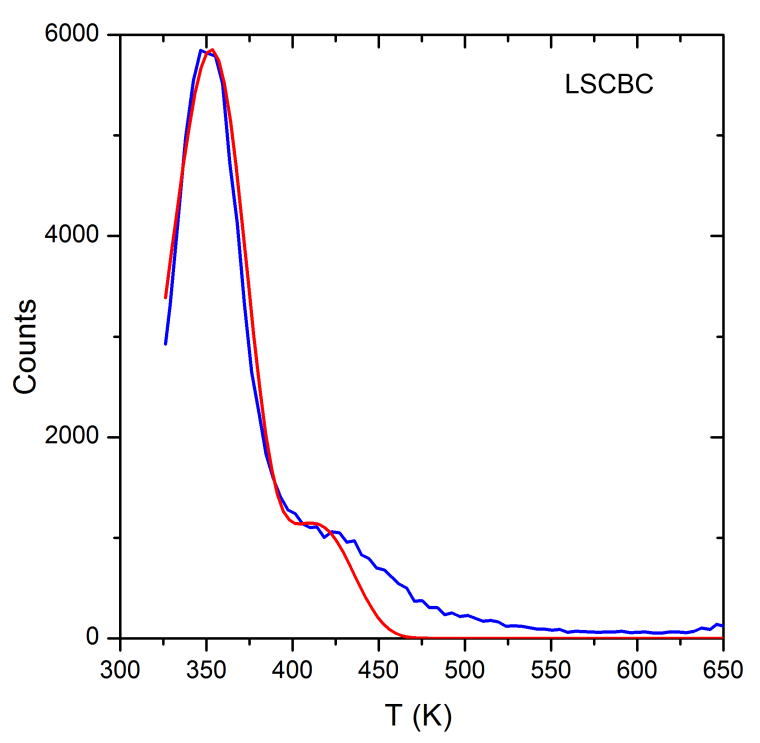

(a)

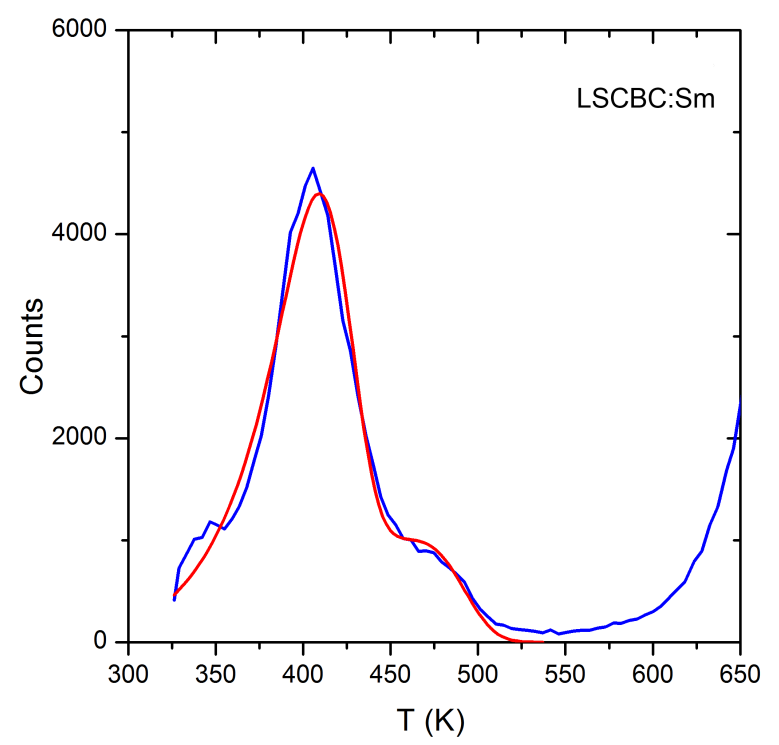

(b)

Figure 7.25: Thermally stimulated luminescence glow curves for (a) LSCBC and (b) LSCBC:Sm $(0.2 \%)$. Both were irradiated using a copper tube for 15 minutes at $30 \mathrm{kV} 25 \mathrm{~mA}$. The red lines are the fitted curves, parameters of which are given in table 7.4 .

Table 7.4: Kitis parameter fits to glow curves for undoped LSCBC and LSCBC:Sm. E refers to the trap depth and $\mathrm{T}$ the peak temperature of the glow curve corresponding to that trap.

\begin{tabular}{cccccc}
\hline Peak number & \multicolumn{2}{c}{ Undoped } & & \multicolumn{2}{c}{ Sm doped } \\
\cline { 2 - 3 } \cline { 5 - 6 } & $\mathrm{E}(\mathrm{eV})$ & $\mathrm{T}_{m}(\mathrm{~K})$ & $\mathrm{E}(\mathrm{eV})$ & $\mathrm{T}_{m}(\mathrm{~K})$ \\
\hline 1 & $0.5 \pm 0.2$ & $370 \pm 15$ & & $0.35 \pm 0.15$ & $380 \pm 20$ \\
2 & $0.6 \pm 0.2$ & $440 \pm 15$ & & $0.75 \pm 0.15$ & $410 \pm 20$ \\
3 & - & - & & $0.8 \pm 15$ & $470 \pm 20$ \\
\hline
\end{tabular}

with corresponding activation energies of $0.34 \mathrm{eV}, 0.75 \mathrm{eV}$ and $0.82 \mathrm{eV}$. To confirm that the thermally stimulated luminescence emission matched the radioluminescence we monitored the emission with a CCD detector as the temperature was raised. Both emissions matched the radioluminescence at all temperatures. Figure 7.26 shows the emission at the peak of the glow curve for both undoped and samarium doped LSCBC.

\subsubsection{Discussion of $x$-ray response}

From the photoluminescence excitation spectra and absorption spectra we see strong absorption of $\mathrm{Sm}^{2+}$ in the blue region. This overlaps with the intrinsic emission of LSCBC. Thus, it is likely that the blue emission probably indirectly excites the $\mathrm{Sm}^{2+}$ emission during $\mathrm{x}$-irradiation. The blue emission only appears for $\mathrm{x}$-irradiation or VUV excitation. It therefore 


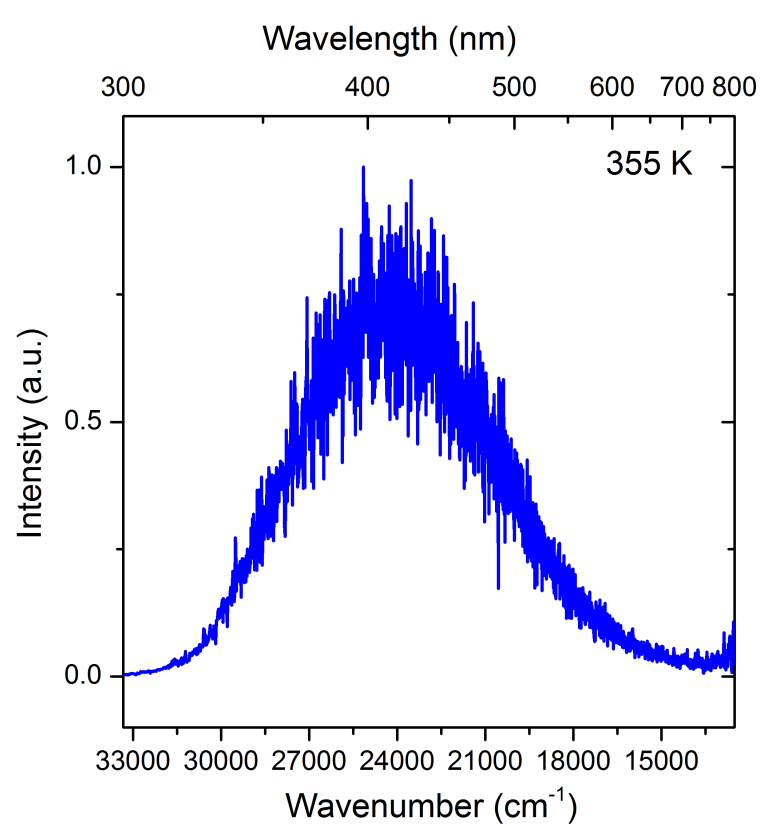

(a)

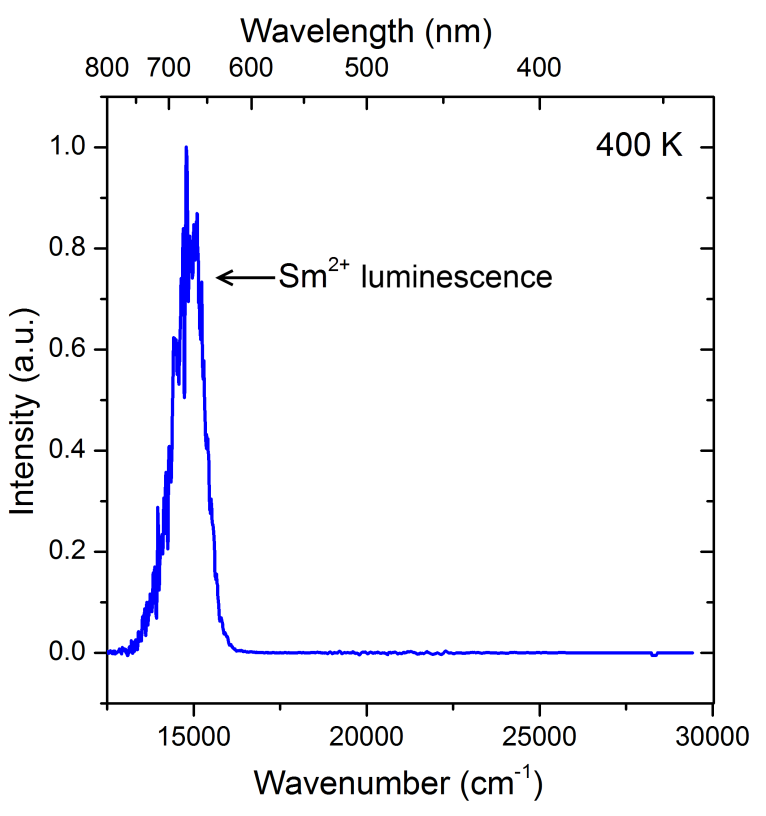

(b)

Figure 7.26: The emission at the peak of glow curves for (a) undoped LSCBC at $355 \mathrm{~K}$ and (b) LSCBC:Sm at $400 \mathrm{~K}(\mathrm{~b})$.

likely corresponds to an ionization process. It is well known that in alkali and alkaline earth halides, $\mathrm{x}$-irradiation can produce centres such as $\mathrm{F}, \mathrm{V}_{k}$ and $\mathrm{H}$ centres. A similar emission is seen for $\mathrm{SrCl}_{2}$ [76]. In LSCBC there is an excess of $\mathrm{Cl}^{-}$interstitials so $\mathrm{F}$ centres (requiring an anion vacancy) would seem improbable. However, it is very plausible that $\mathrm{H}$ centres $\left(\mathrm{Cl}_{2}^{-}\right.$ molecular ions formed from an interstitial and a regular lattice chlorine ion) will be generated from ionisation of the interstitial $\mathrm{Cl}^{-}$ion. The detached electron could subsequently be bound to the $\mathrm{H}$ centre, constituting a self trapped exciton as found in the alkaline earth fluorides [138], or may be trapped more remotely. The possible traps are depicted schematically in figure 7.27 . Here we identify the blue emission band with the recombination radiation arising from the bound excitonic state, comprising an electron bound to an $\mathrm{H}$ centre.

The recombination process can be either prompt, or delayed, since the detached electrons can be trapped elsewhere in the crystal (for example by a impurity rare earth ion), with subsequent STE recombination following thermal or optical activation to free it from the trap. This would correspond to the phosphorescence which we observe by eye after x-irradiation, and is evident on a longer time scale in the scintillation (figure 7.22(b)). Depending on the proximity of the $\mathrm{H}$ centre and the trapped electron, this may be viewed as a range of excitations ranging from excitonic for an isolated $\mathrm{H}$ centre where an x-ray generated electron 


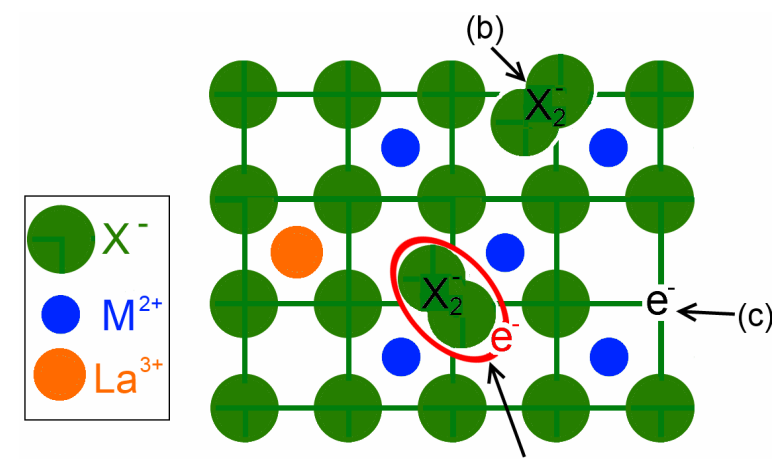

(a)

Figure 7.27: Depiction of (a) a possible self trapped exciton, (b) and (c) spatially separated $\mathrm{V}_{K}$ and $\mathrm{F}$ centres respectively. The $\mathrm{Cl}^{-}$anions are shown in green, the $\mathrm{Ba}^{2+}$ cations in blue, and the $\mathrm{La}^{3+}$ in orange.

promptly recombines, to slow recombination for a donor-acceptor pair. This provides the mechanism for the blue luminescence.

This emission may then exit the material to be detected, or it may excite the $\mathrm{Sm}^{2+}$ ions, and so the blue luminescence intensity decreases with samarium concentration. The above discussion is consistent with the TSL and afterglow observations. Several traps are observed, the shallowest of which is likely responsible for the room temperature phosphorescence.

\subsection{Praseodymium stabilised cubic barium chloride}

The next smallest lanthanide ion after $\mathrm{La}^{3+}$ and $\mathrm{Ce}^{3+}$ is $\operatorname{Pr}^{3+}$ with 2 electrons in the $4 \mathrm{f}$ shell. Following this series along the lanthanide series we investigate if the same structural stabilisation effect as seen with CSCBC and LSCBC can be achieved with $\mathrm{Pr}^{3+} . \mathrm{Pr}^{3+}$ usually shows sharp line $4 \mathrm{f}^{2} \rightarrow 4 \mathrm{f}^{2}$ line luminescence from both the ${ }^{1} \mathrm{~S}_{0}$ level and the ${ }^{3} \mathrm{P}_{0}$ levels. But in some host lattices it can instead (or as well) show very fast $4 \mathrm{f}^{1} 5 \mathrm{~d}^{1} \rightarrow 4 \mathrm{f}^{2}$ luminescence. Thus not only is $\operatorname{Pr}^{3+}$ of interest as a stabiliser of the cubic phase but also as a luminescent centre.

\subsubsection{Physical properties}

As for LSCBC:Sm anhydrous materials $\left(\mathrm{BaCl}_{2}, \mathrm{LaCl}_{3}\right.$ and $\left.\mathrm{PrCl}_{3}\right)$ were heated in an argon atmosphere with the same heating profile used for LSCBC:Sm. The compositions investigated 


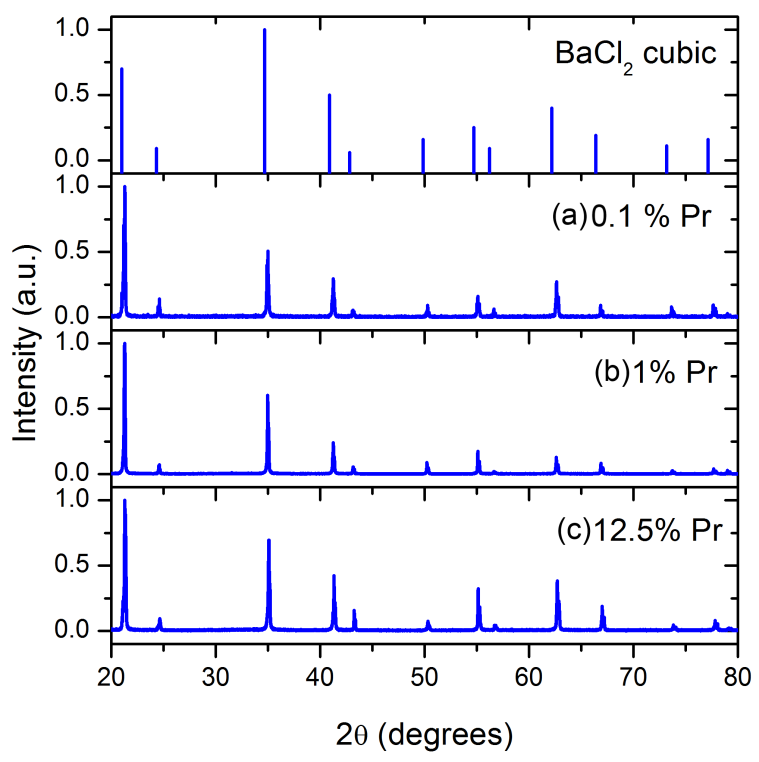

Figure 7.28: The powder XRD pattern for cubic $\mathrm{BaCl}_{2}$ (top) and experimental powder XRD patterns of praseodymium stabilised cubic $\mathrm{BaCl}_{2}$ for (a) $\mathrm{Ba}_{0.875} \mathrm{La}_{0.124} \mathrm{Pr}_{0.001} \mathrm{Cl}_{2.25}$, (b) $\mathrm{Ba}_{0.875} \mathrm{La}_{0.115} \operatorname{Pr}_{0.01} \mathrm{Cl}_{2.25}$ and (c) $\mathrm{Ba}_{0.875} \operatorname{Pr}_{0.125} \mathrm{Cl}_{2.25}$.

were $\mathrm{Ba}_{1-x-y} \mathrm{La}_{x} \operatorname{Pr}_{y} \mathrm{Cl}_{2+x+y}$ (where $\mathrm{x}+\mathrm{y}=0.125$ and $0 \leq \mathrm{x} \leq 0.1249,0 \leq \mathrm{y} \leq 0.125$ ). Powder x-ray diffraction patterns confirm the crystals have adopted the cubic phase for all $\operatorname{Pr}^{3+}$ concentrations. In the case of $12.5 \% \mathrm{Pr}^{3+}$, as there are no $\mathrm{La}^{3+}$ ions the $\mathrm{Pr}^{3+}$ ions must be acting as stabilisers of the cubic phase, we call this praseodymium stabilised cubic barium chloride (PSCBC). Figure 7.28 shows some representative XRD patterns obtained along with the expected pattern for cubic $\mathrm{BaCl}_{2}$ (top). No evidence of any non-cubic phase is evident in any of the patterns. The variation in the lattice parameter is very small as the praseodymium and lanthanum concentrations are adjusted, from $0.7285 \AA$ for LSCBC to $0.7283 \AA$ for PSCBC, (while keeping the total dopant concentration level at $12.5 \%$ ) this is to be expected as the crystal ionic radii of the $\operatorname{Pr}^{3+}$ ion $(0.99 \AA)$ is similar to that of the $\mathrm{La}^{3+}$ ion $(1.032 \AA)$ with both being much smaller than the $\mathrm{Ba}^{2+}$ ion $(1.35 \AA)$; hence the lattice contraction from the $7.3110 \AA$ of pure cubic $\mathrm{BaCl}_{2}$ [120,121]. The samples are transparent and show a slight green colouring dependent on concentration; this is due to a combination of the absorption of most blue light into the excited ${ }^{3} \mathrm{P}_{J}$ states and the human eye's strong response to green. Figure 7.29 shows an example of a colourless sample. 


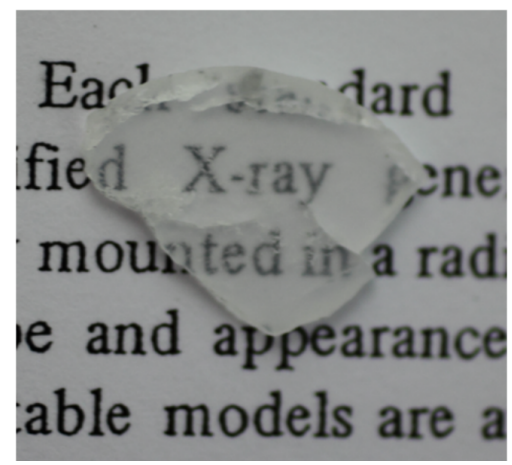

Figure 7.29: Photograph of a sample of LSCBC with $0.1 \% \mathrm{Pr}^{3+}$ and $12.4 \% \mathrm{La}^{3+}$.

\subsubsection{Photoluminescence}

Photoluminescence can be observed for all samples but concentration quenching sets in at relatively low praseodymium concentrations $\left(\gtrsim 0.2 \% \mathrm{Pr}^{3+}\right)$.

The photoluminescent spectra for a sample with $0.1 \% \operatorname{Pr}^{3+}$ is shown in figure 7.30. These spectra show the typical $4 \mathrm{f}^{2} \rightarrow 4 \mathrm{f}^{2} \operatorname{Pr}^{3+}$ transitions commonly observed, and have been labelled in figure 7.30 according to literature [77, 139, 140]. Figure 7.31 shows an every level diagram for the $\operatorname{Pr}^{3+}$ ion with the transitions observed indicated. The splittings of the lines are expected for $\operatorname{Pr}^{3+}$ as the ground state is not a singlet, and multiple sites are expected as the trivalent $\operatorname{Pr}^{3+}$ ions require charge compensation. The variation with temperature is merely an increase in amplitude and a sharpening of lines as the temperature is reduced from $300 \mathrm{~K}$ to $12 \mathrm{~K}$; this can be seen in figure 7.32 which shows the change in both the excitation and emission spectra on cooling from $300 \mathrm{~K}$ to $12 \mathrm{~K}$. We looked for $4 \mathrm{f}^{1} 5 \mathrm{~d}^{1} \rightarrow 4 \mathrm{f}^{2}$ emission as reported for the similar host lattices $\mathrm{SrCl}_{2}, \mathrm{BaCl}_{2}$ (orthorhombic) and $\mathrm{CaF}_{2}$ [76, 77] but could not find any evidence of such an emission. Such emission tends to require direct $4 \mathrm{f}^{1} 5 \mathrm{~d}^{1}$ band excitation around $230 \mathrm{~nm}\left(223 \mathrm{~nm}\right.$ for $\mathrm{CaF}_{2}, 230 \mathrm{~nm}$ for orthorhombic $\mathrm{BaCl}_{2}$ and $236 \mathrm{~nm}$ for $\mathrm{SrCl}_{2}$ [76]) and the lack of observation of a corresponding emission may be due to a lack of intensity from the xenon lamp used for excitation in the fluorolog at this wavelength.

The photo-excited lifetimes of the various emissions have been investigated and show little temperature variations and are similar to values reported in the literature for $\mathrm{CdF}_{2}$ [141]. Figure 7.33 shows the lifetime data recorded for various samples. While the temperature dependence is minimal, in contrast to LSCBC:Sm a strong concentration dependence can be observed, the lifetime of LSCBC:Pr $(0.1 \%)$ is approximately three times longer than that of 


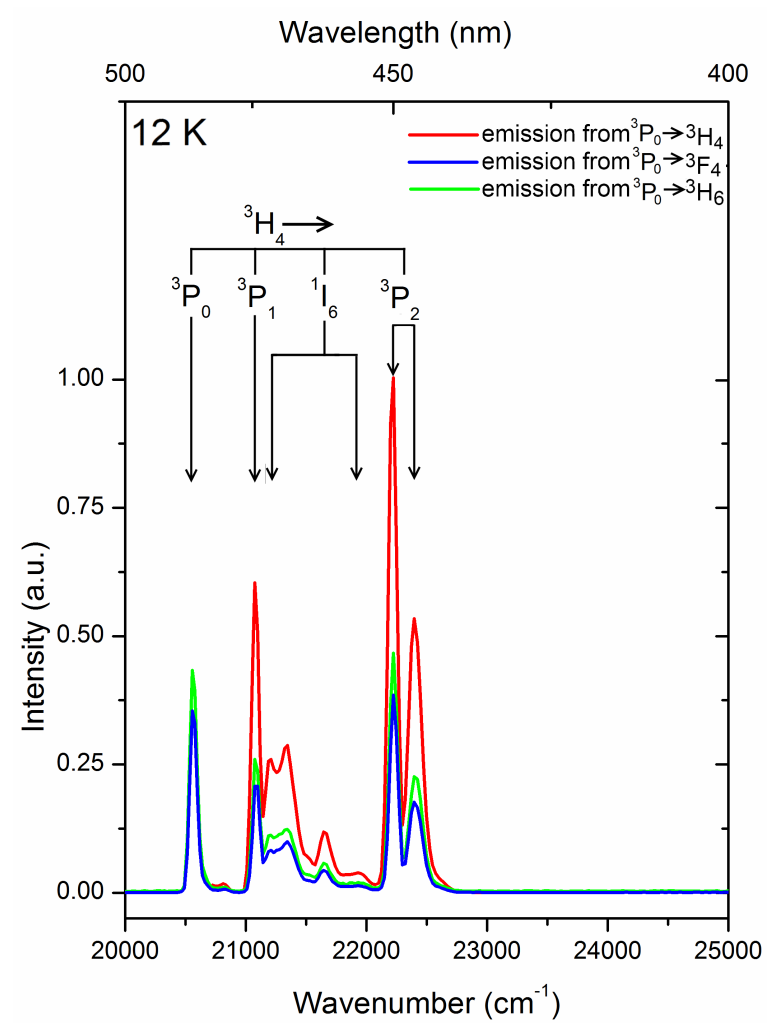

(a)

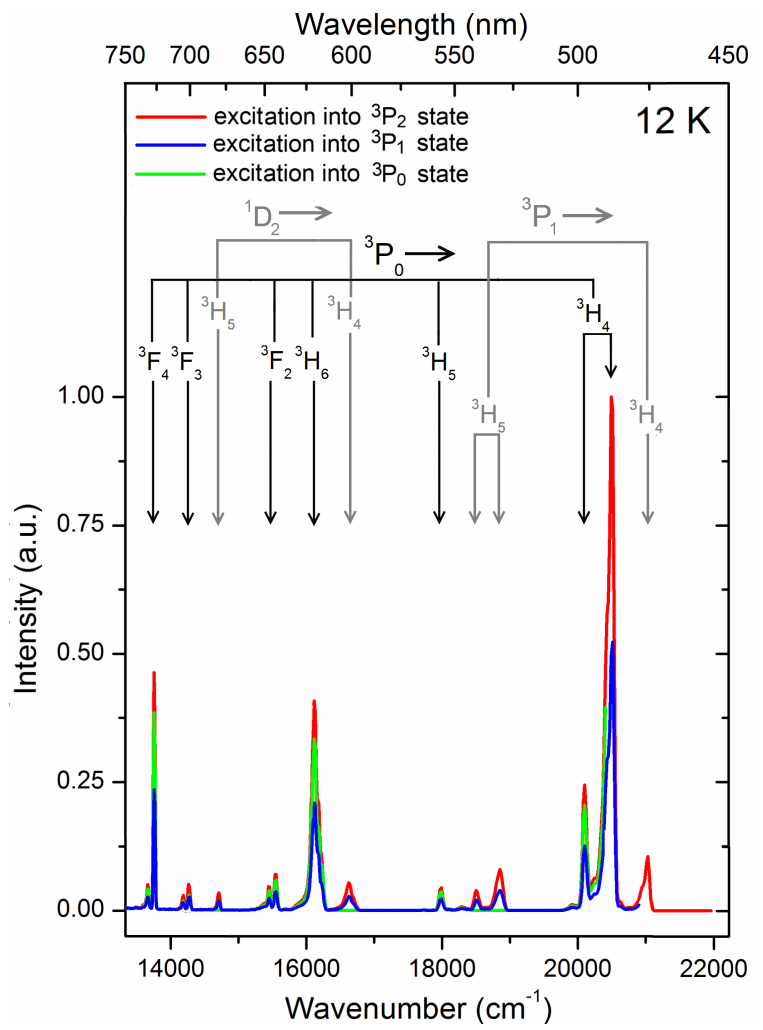

(b)

Figure 7.30: The excitation (a) and emission (b) photoluminescence spectra at $12 \mathrm{~K}$ for a sample with $0.1 \% \operatorname{Pr}^{3+}$. The $4 \mathrm{f}^{2} \rightarrow 4 \mathrm{f}^{2}$ transitions have been labelled. Both spectra have resolution of $1 \mathrm{~nm}$. The transitions corresponding to the emissions are labelled in the bar diagram above.

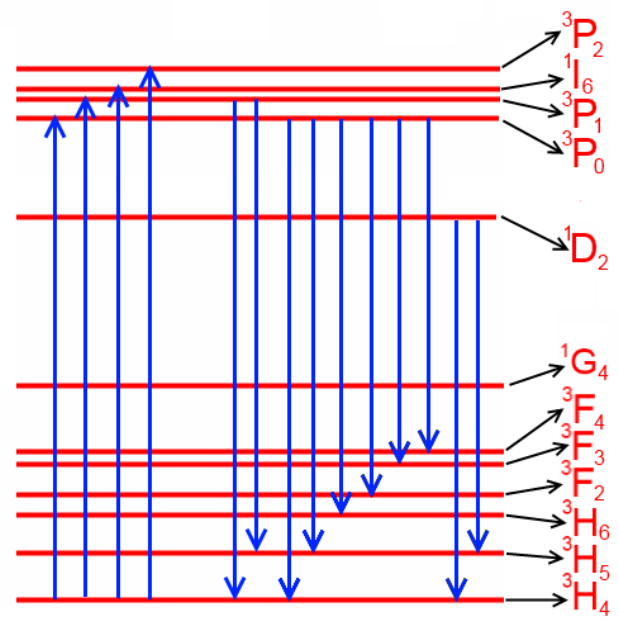

Figure 7.31: Energy levels and transitions observed for $\operatorname{Pr}^{3+}$ in $\mathrm{Ba}_{0.875} \mathrm{La}_{0.124} \mathrm{Pr}_{0.001} \mathrm{Cl}_{2.25}$. The crystal field splittings of the energy levels are not shown; these depend on $\operatorname{Pr}^{3+}$ site. 


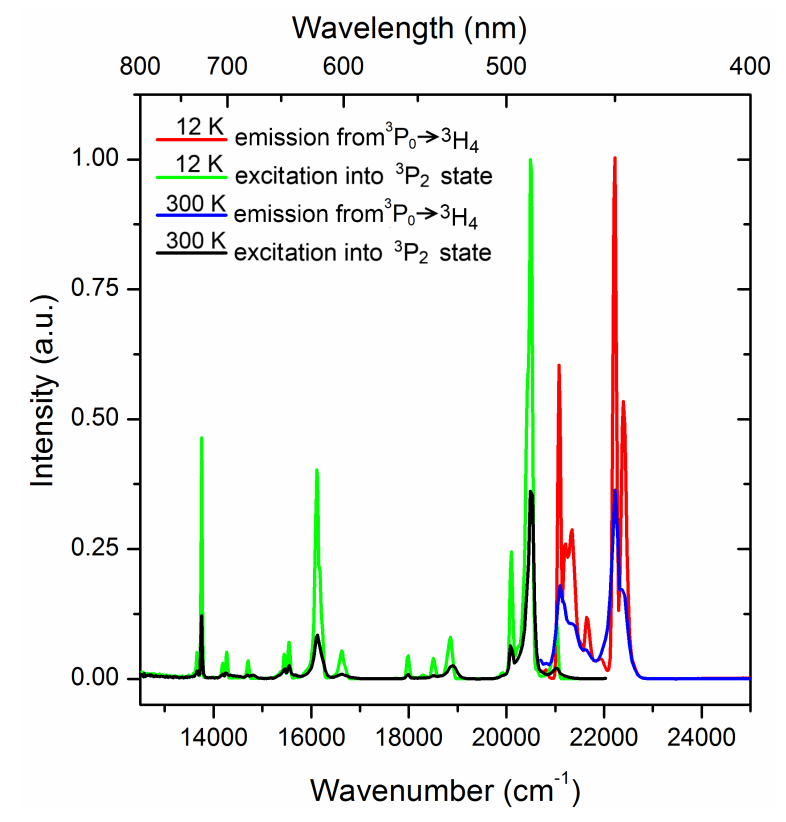

Figure 7.32: Emission and excitation spectra at $12 \mathrm{~K}$ and $300 \mathrm{~K}$ for a $\mathrm{Ba}_{0.875} \mathrm{La}_{0.124} \mathrm{Pr}_{0.001} \mathrm{Cl}_{2.25}$ sample.

the LSCBC:Pr (5\%). For the more concentrated samples the lifetime decreases further, this is likely due to clustering of the $\operatorname{Pr}^{3+}$ ions providing non-radiative energy loss routes, and this also accounts for a decrease in emission intensity with concentration.

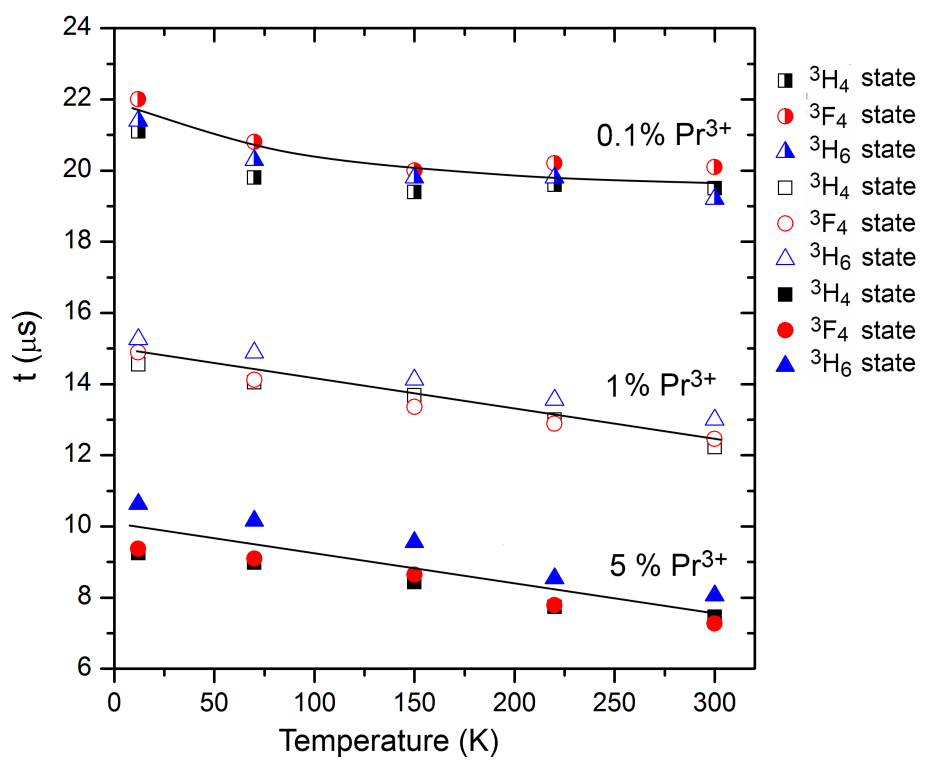

Figure 7.33: Photoluminescent lifetimes of the ${ }^{3} \mathrm{H}_{4},{ }^{3} \mathrm{~F}_{4}$ and ${ }^{3} \mathrm{H}_{6}$ excited states of the $\operatorname{Pr}^{3+}$ ions in LSCBC. The lines are guide to the eye 


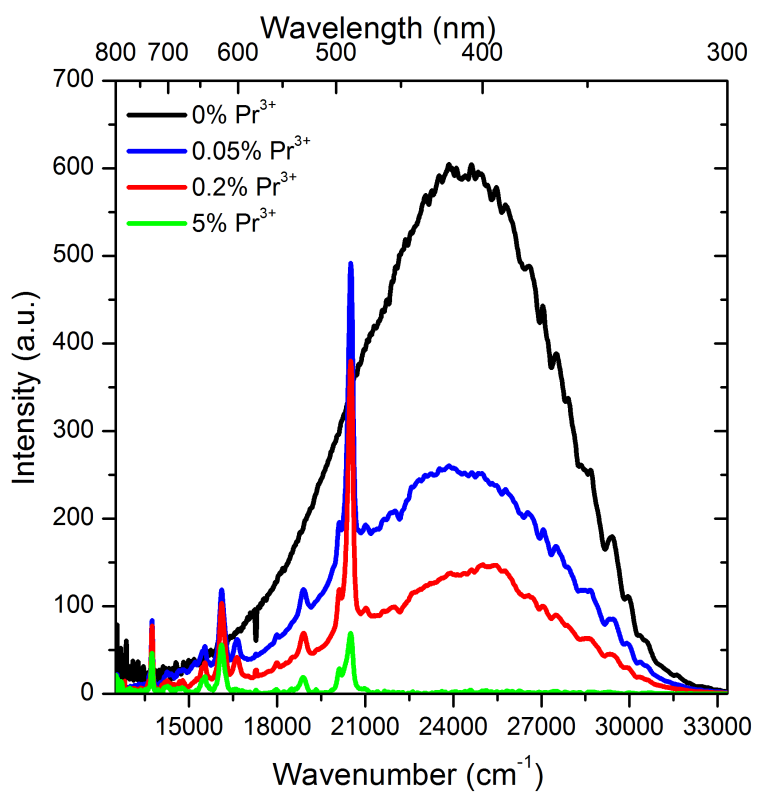

Figure 7.34: Radioluminescence of LSCBC:Pr for various $\operatorname{Pr}^{3+}$ concentrations.

\subsubsection{X-ray response}

The radioluminescence follows the photoluminescence with the addition of a strong broad band host luminescence as shown in figure 7.34, this host emission is very similar to that seen for LSCBC:Sm ${ }^{2+}$. The dip in intensity at $448 \mathrm{~nm}$ is due to absorption energy transfer to the ${ }^{3} \mathrm{P}_{2}$ level of the $\operatorname{Pr}^{3+}$ ion. Radioluminescence was too weak to be observed for samples with $>5 \% \operatorname{Pr}^{3+}$. The host emission decreases with increasing $\operatorname{Pr}^{3+}$ concentration as seen in LSCBC:Sm ${ }^{2+}$. As for UV excitation, no $4 \mathrm{f}^{1} 5 \mathrm{~d}^{1} \rightarrow 4 \mathrm{f}^{2}$ emission is observed, this is in line with previous studies [76] that indicate that the $4 \mathrm{f}^{1} 5 \mathrm{~d}^{1}$ excitation bands are too high to overlap with the excitonic emission and so energy is transferred preferentially to the $4 \mathrm{f}^{2}$ excited states and the only rare earth emissions observed are the sharp line $4 \mathrm{f}^{2} \rightarrow 4 \mathrm{f}^{2}$ transitions.

Afterglow measurements show the $\mathrm{Pr}^{3+}$ doped samples have far less afterglow than the undoped material. $\mathrm{Pr}^{3+}$ can be used to reduce afterglow and speed up processes as it provides non radiative decay routes [142]. This effect is clearly seen when comparing the percentage light output remaining 10, 20 and $50 \mathrm{~ms}$ after a x-ray pulse for various samples: as shown in figure 7.35 and tabulated in table 7.5

The photoluminescent lifetimes of the material are too long for the material to act as a scintillator. As an x-ray phosphor the brightness was measured by comparing the integrated intensity of the radioluminescence relative to a piece of the commercial material $\mathrm{Gd}_{2} \mathrm{O}_{2} \mathrm{~S}: \mathrm{Tb}^{3+}$. 
Table 7.5: Afterglow of LSCBC: $\mathrm{Pr}^{3+}$. The values given are the relative intensity (\%) of the maximum intensity of the signal remaining 10, 20 and 50 milliseconds after the excitation ceases.

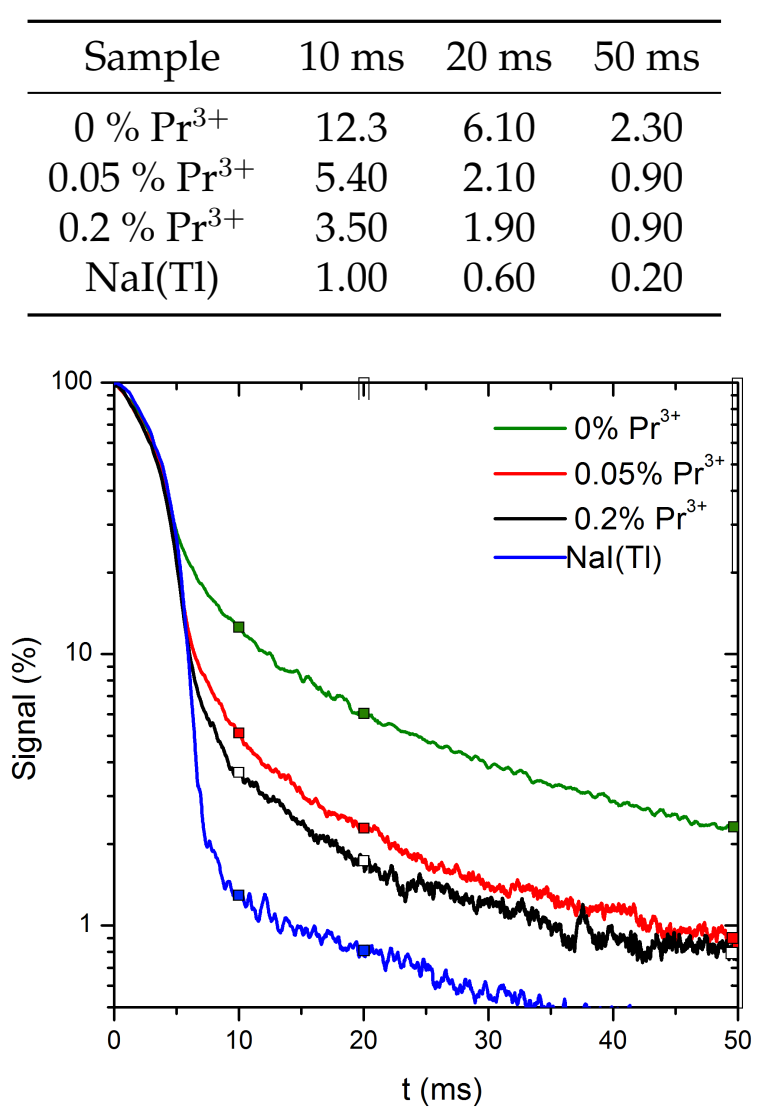

Figure 7.35: Afterglow profiles of LSCBC:Pr ${ }^{3+}$ for $0 \%, 0.05 \%$ and $2 \%$ praseodymium shown with that of $\mathrm{NaI}(\mathrm{Tl})$.

The highest relative output obtained was $2 \%$ for a $0.05 \% \operatorname{Pr}^{3+}$ sample.

PSCBC:Sm${ }^{2+}(0.1 \%)$ showed no luminescence from either the $\mathrm{Sm}^{2+}$ ions or $\mathrm{Pr}^{3+}$ ions under UV or x-ray excitation.

\subsection{Summary}

Polycrystalline samples of barium chloride containing $12.5 \%$ by cation fraction of $\mathrm{LaCl}_{3}$ adopt the simple cubic fluorite structure with no evidence for other crystalline phases. LSCBC doped with $\mathrm{SmCl}_{3}$ shows optical absorption and luminescence spectra characteristic of $\mathrm{Sm}^{2+}$ ions only. The samarium ions occupy sites of predominantly cubic symmetry with a small non-cubic perturbation generated by $\mathrm{Cl}^{-}$ion interstitials and nearby $\mathrm{La}^{3+}$ ions. The optical absorption spectra arise from transitions from the ground state of the $4 \mathrm{f}^{6}$ configuration to 
the multiple levels of crystal field split $4 \mathrm{f}^{5} 5 \mathrm{~d}^{1}$ excited configuration; the observed spectra are in good agreement with a calculation based on crystal field theory. The photoluminescence spectra arise from both sharp line transitions within the $4 \mathrm{f}^{6}$ configuration, and a broad band transition from the lowest state of the excited $4 \mathrm{f}^{5} 5 \mathrm{~d}^{1}$ configuration to the sub-states of the ground ${ }^{7} \mathrm{~F}_{J}$ term of the $4 \mathrm{f}^{6}$ configuration. The strong temperature dependence of the lines and band system can be accounted for by a model based on thermally activated crossovers between the excited states of the $4 \mathrm{f}^{6}$ and $4 \mathrm{f}^{5} 5 \mathrm{~d}^{1}$ configurations.

Under x-irradiation, luminescence corresponding to both the $\mathrm{Sm}^{2+}$ ions and the host lattice is seen. The host lattice emission is attributed to self trapped exciton emission. The $\mathrm{Sm}^{2+}$ ion emission observed under $\mathrm{x}$-irradiation is well matched to the spectral sensitivity of silicon photodiodes. The material is a bright $\mathrm{x}$-ray phosphor (up to $11 \%$ the integrated intensity of $\mathrm{Gd}_{2} \mathrm{O}_{2} \mathrm{~S}: \mathrm{Tb}^{3+}$ ) but shows poor scintillation characteristics. The scintillation observed has a very long lifetime making the material unsuitable as a scintillator for fast timing applications. However, while the lifetime is too long for scintillation, it it significantly faster than the millisecond time-scales used by commercial phosphor materials such as $\mathrm{GdO}_{2} \mathrm{~S}_{3}: \mathrm{Tb}^{3+}$. The scintillation lifetime is much longer than the UV excited lifetime as the $\mathrm{Sm}^{2+}$ ions are likely excited by the thermal release of shallow trapped electron-hole pairs. The afterglow of the samarium doped materials is very low which is important for integrated imaging systems such as CT scanners. The materials shows a response to neutron irradiation, but the light output is $\leq 1 \%$ that of the commercial material $\mathrm{LiI}(\mathrm{Eu})$; however the afterglow is low, and again this is an important property for integrated imaging systems.

Polycrystalline samples of barium chloride containing $12.5 \%$ by cation fraction of $\mathrm{PrCl}_{3}$ adopt the simple cubic fluorite structure with no evidence for other crystalline phases. Polycrystalline samples of barium chloride containing $12.5 \%$ by cation fraction of a combination of $\mathrm{LaCl}_{3}$ and $\mathrm{PrCl}_{3}$ show luminescence characteristic of $\mathrm{Pr}^{3+}$ ions. The material acts as an x-ray phosphor but does not show scintillation due to the long lifetimes of the $4 \mathrm{f}^{2}$ excited states of the $\operatorname{Pr}^{3+}$ ion from which the luminescence is observed. No luminescence is observed from the $4 \mathrm{f}^{1} 5 \mathrm{~d}^{1}$ excited states. The neutron sensitivity of these materials was also investigated, and this reported in appendix A. 



\section{Chapter 8}

\section{Rare earth doped barium chloride - strontium chloride solid solutions}

\subsection{Introduction}

In chapter 7 it was found that LSCBC:Sm${ }^{2+}$ is a bright $\mathrm{x}$-ray phosphor, but, the material is a poor scintillator. This was attributed to the effects of having a large number of $\mathrm{Cl}^{-}$interstitial ions present providing traps. The traps slow the scintillation process and hence decrease the peak light output and spread the scintillation event over a longer time-scale. Another option for a heavy ion cubic halide lattice but without the $\mathrm{Cl}^{-}$interstitial ions introduced by the $\mathrm{LnCl}_{3}\left(\mathrm{Ln}=\mathrm{La}, \mathrm{Ce}\right.$ or Pr) doping is to form a $\mathrm{BaCl}_{2}-\mathrm{SrCl}_{2}$ solid solution. One would expect similar photoluminescent and radioluminescence properties for $\mathrm{BaCl}_{2}-\mathrm{SrCl}_{2}: \mathrm{Sm}^{2+}$ as for LSCBC:Sm ${ }^{2+}$, but, potentially fewer trapping mechanisms, and so $\mathrm{BaCl}_{2}-\mathrm{SrCl}_{2}: \mathrm{Sm}^{2+}$ may show observable scintillation.

$\mathrm{SrCl}_{2}$ forms in the cubic fluorite phase structure at all temperatures but does not have a high stopping power for x-rays; however, previous studies [143] have shown the $\mathrm{SrCl}_{2}$ fluorite lattice can accommodate up to $32 \%$ cation substitution of the $\mathrm{Ba}^{2+}$ ion for the $\mathrm{Sr}^{2+}$ ion while retaining the cubic structure. More recently Gahane et al. [144] investigated the effect of substituting up to $22 \%$ of $\mathrm{Ba}^{2+}$ ions for $\mathrm{Sr}^{2+}$ ions in $\mathrm{SrCl}_{2}$ and found the emission intensity of $\mathrm{Eu}^{2+}$ ions to be significantly increased from that of $\mathrm{Eu}^{2+}$ ions in pure $\mathrm{BaCl}_{2}$ or $\mathrm{SrCl}_{2}$. As the 
$\mathrm{Ba}^{2+}$ ion has a larger atomic number $\left(\mathrm{Z}_{\mathrm{Ba}^{2+}}=56\right)$ than the $\mathrm{Sr}^{2+}$ ion $\left(\mathrm{Z}_{\mathrm{Sr}^{2+}}=38\right)$, substituting $\mathrm{Ba}^{2+}$ ions for $\mathrm{Sr}^{2+}$ ions in the $\mathrm{SrCl}_{2}$ lattice increases the stopping power for $\mathrm{x}$-rays. The higher the fraction of $\mathrm{Ba}^{2+}$ ions the greater the stopping power. Thus it is of interest to investigate the luminescence of rare earth doped $\mathrm{Ba}_{x} \mathrm{Sr}_{1-x} \mathrm{Cl}_{2}$ solid solutions which have the maximal $\mathrm{Ba}^{2+}$ ion substitution consistent with the cubic phase and hence transparency. Samarium doping is of particular interest as we expect similar emission properties to LSCBC:Sm ${ }^{2+}$, where the emission wavelength is well matched to the response of silicon photodiodes, but potentially $\mathrm{Ba}_{x} \mathrm{Sr}_{1-x} \mathrm{Cl}_{2}: \mathrm{Sm}^{2+}$ has faster scintillation decay time. Due to the similarities in host lattice, and therefore crystal fields, it will also be interesting to compare the luminescent properties of samarium in the two materials and see what effect the presence and absence of interstitial $\mathrm{Cl}^{-}$ions have.

\section{2 $\mathrm{BaCl}_{2}-\mathrm{SrCl}_{2}$ solid solutions}

$\mathrm{Ba}_{x} \mathrm{Sr}_{1-x} \mathrm{Cl}_{2}$ samples with values of $x$ between 0 and 1.0 were investigated by thermal calorimetric analysis. The heating/cooling curves for various compositions are shown in figure 8.1 . The curves shown are second iterations after the constituent compounds have previously been melted and formed in a solid solution, and so correspond to the melting patterns of the solid solution as opposed to the $\mathrm{SrCl}_{2}$ and $\mathrm{BaCl}_{2}$ powders. For $x \leq 0.30$ the heating/cooling curves show a profile similar to what one would expect for pure $\mathrm{SrCl}_{2}$. The heat flow is approximately constant as the temperature is raised until $\sim 850{ }^{\circ} \mathrm{C}$, where an abrupt transition occurs. This indicates the solid to liquid phase transition. Pure $\mathrm{SrCl}_{2}$ melts at $873{ }^{\circ} \mathrm{C}[119]$ and melting point depression is expected for an impure material. On cooling the corresponding liquid to solid transition can be seen around $850{ }^{\circ} \mathrm{C}$. The instrumental hysteresis is typical for differential scanning calorimetry (DSC) instruments and depends on the heating and cooling rates. For larger values of $x(>0.35)$ a phase transition on cooling can be observed between $650{ }^{\circ} \mathrm{C}$ and $800{ }^{\circ} \mathrm{C}$, and with larger fractions of $\mathrm{BaCl}_{2}$ this moves to higher temperatures. This represents either the separation of $\mathrm{BaCl}_{2}$ and $\mathrm{SrCl}_{2}$ crystals into a mixed phase solid of orthorhombic $\mathrm{BaCl}_{2}$ and cubic $\mathrm{SrCl}_{2}$, or alternatively, a phase transition from a cubic solid solution to an orthorhombic solid solution. The temperature of the cubic to 


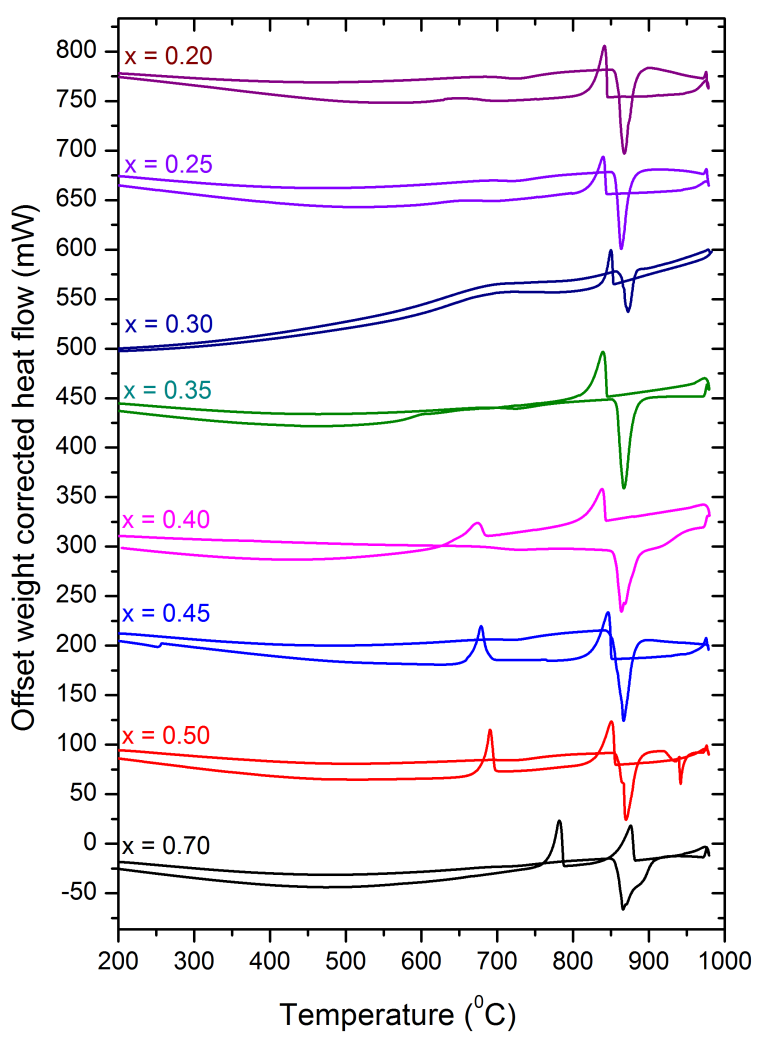

Figure 8.1: Heating and cooling heat flow curves for $\left(\mathrm{x} \cdot \mathrm{BaCl}_{2}\right)-\left((1-\mathrm{x}) \cdot \mathrm{SrCl}_{2}\right)$ mixtures ranging from $x=0.20$ to $x=0.70$. The heating and cooling rates were both $30{ }^{\circ} \mathrm{C} / \mathrm{min}$.

orthorhombic phase transition of pure $\mathrm{BaCl}_{2}$ is $925^{\circ} \mathrm{C}$ [119]. For $0.30<x<0.35$ this phase transition could be suppressed with a fast cooling rate $(30 \% \mathrm{~min})$ as is shown in figure 8.1 . This is consistent with previous studies which found the eutectic point to be at $852{ }^{\circ} \mathrm{C}$ for a $68: 32 \mathrm{Sr}^{2+}: \mathrm{Ba}^{2+}$ ratio [143]. All further work was carried out on samples prepared with a $70: 30 \mathrm{Sr}^{2+}: \mathrm{Ba}^{2+}$ ratio so as to have maximum $\mathrm{x}$-ray attenuation while retaining the cubic phase.

The samples were prepared from anhydrous $\mathrm{BaCl}_{2}$ and dehydrated $\mathrm{SrCl}_{2} \cdot 6 \mathrm{H}_{2} \mathrm{O}$ in the appropriate weight ratio. The dehydration of $\mathrm{SrCl}_{2} \cdot 6 \mathrm{H}_{2} \mathrm{O}$ was as described in chapter 5. The materials were heated to $1000{ }^{\circ} \mathrm{C}$, and the temperature was then held at $1000{ }^{\circ} \mathrm{C}$ for 15 minutes to ensure the materials fully melted. The melt was cooled to $880{ }^{\circ} \mathrm{C}$ at $\sim 4{ }^{\circ} \mathrm{C} / \mathrm{min}$ then slowly cooled from $880{ }^{\circ} \mathrm{C}$ to $820^{\circ} \mathrm{C}$ at $\sim 0.2{ }^{\circ} \mathrm{C} / \mathrm{min}$ then cooled to room temperature at $\sim 3$ ${ }^{\circ} \mathrm{C} / \mathrm{min}$. An especially slow cooling rate was used between $880{ }^{\circ} \mathrm{C}$ and $820^{\circ} \mathrm{C}$ as the solid to liquid phase transition was observed over this range in figure 8.1. A slow cooling rate below the liquid to solid phase transition relieves strain and helps prevent cracking. This cooling profile resulted in transparent materials with little or no cracking. The powder x-ray 


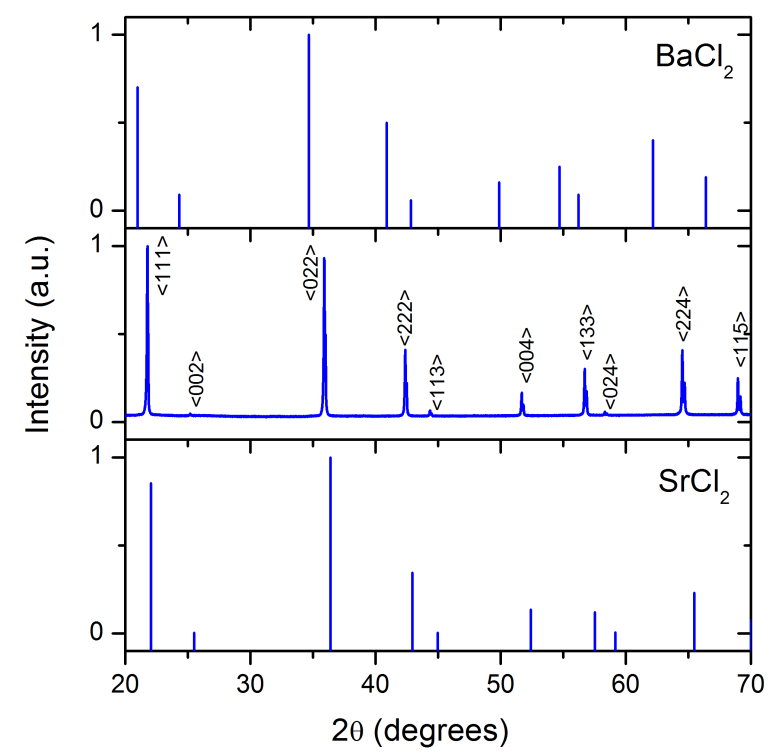

Figure 8.2: Powder x-ray diffraction file pattern (PDF) for cubic $\mathrm{BaCl}_{2}$ (top), experimental pattern for $\mathrm{Ba}_{0.3} \mathrm{Sr}_{0.7} \mathrm{Cl}_{2.0}$ (middle), and PDF pattern for cubic $\mathrm{SrCl}_{2}$ (bottom).

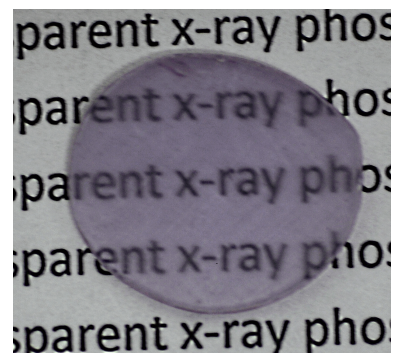

(a)

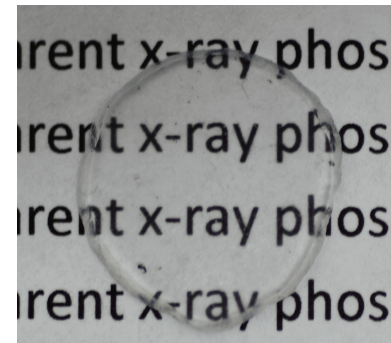

(b)

Figure 8.3: Photographs of $\mathrm{Ba}_{0.3} \mathrm{Sr}_{0.7} \mathrm{Cl}_{2}$ doped with (a) $0.1 \%$ samarium and (b) $0.5 \%$ europium showing excellent optical properties.

diffraction (XRD) pattern for a sample of $\mathrm{Ba}_{0.3} \mathrm{Sr}_{0.7} \mathrm{Cl}_{2.0}$ is shown in figure 8.2 along with the file patterns of cubic $\mathrm{BaCl}_{2}$ and $\mathrm{SrCl}_{2}$.

As can be seen in figure 8.2 the $\mathrm{XRD}$ pattern for $\mathrm{Ba}_{0.3} \mathrm{Sr}_{0.7} \mathrm{Cl}_{2.0}$ is very similar to the cubic patterns of $\mathrm{BaCl}_{2}$ and $\mathrm{SrCl}_{2}$; it represents a simple fluorite structure with the peaks found at angles between those of the pure materials. No evidence of any other phase was observed. The lattice parameter for the undoped material was found to be $7.080 \AA$ from the spacing of the XRD pattern lines as described in chapter 4 ; this falls between the values of $7.063 \AA$ and 7.311 $\AA$ for pure $\mathrm{SrCl}_{2}$ and cubic $\mathrm{BaCl}_{2}$ respectively as expected for a mixed crystal material. Figure 8.3 shows the physical appearance of two samples doped with either samarium or europium; the transparency can be seen to be excellent. The substitution of $30 \%$ of the $\mathrm{Sr}^{2+}$ ions with heavier $\mathrm{Ba}^{2+}$ ions offers a substantial increase in the effective atomic number and hence x-ray attenuation coefficient; this can be seen in figure 8.4 which shows the half- 


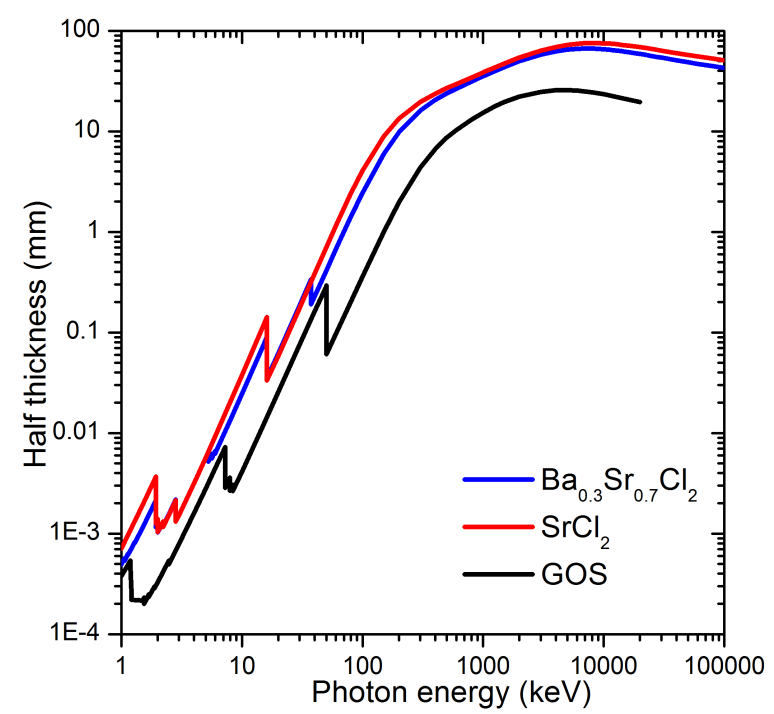

Figure 8.4: Calculated x-ray half-thickness for $\mathrm{Ba}_{0.3} \mathrm{Sr}_{0.7} \mathrm{Cl}_{2}$ (blue), $\mathrm{Gd}_{2} \mathrm{O}_{3} \mathrm{~S}$ : $\mathrm{Tb}^{3+}$ (black) and $\mathrm{SrCl}_{2}$ (red) [145].

thicknesses of $\mathrm{Ba}_{0.3} \mathrm{Sr}_{0.7} \mathrm{Cl}_{2}, \mathrm{SrCl}_{2}$ and the commercial x-ray phosphor $\mathrm{Gd}_{2} \mathrm{O}_{3} \mathrm{~S}: \mathrm{Tb}^{3+}$. The half-thicknesses were calculated from the NIST database of x-ray attenuation coefficients [145]. The half thickness of $\mathrm{Ba}_{0.3} \mathrm{Sr}_{0.7} \mathrm{Cl}_{2}$ is about $40 \%$ less than that of $\mathrm{SrCl}_{2}$ for $x$-rays in the $40-300$ keV range.

\subsection{Samarium doped $\mathrm{BaCl}_{2}-\mathrm{SrCl}_{2}$ solid solutions}

Additional doping with samarium was achieved by adding the appropriate weight of anhydrous $\mathrm{SmCl}_{3}$ to the $\mathrm{BaCl}_{2}$ and $\mathrm{SrCl}_{2}$ powders before melting. The resulting materials formed in the cubic phase with excellent optical qualities as can be seen in figure 8.3 .

\subsubsection{Optical spectroscopy}

\subsubsection{Absorption}

Figure 8.5 shows the absorption spectrum of a $0.1 \%$ samarium sample at $12 \mathrm{~K}$ and $300 \mathrm{~K}$. The spectra are very similar to those seen for LSCBC:Sm in the previous chapter and show the strong $4 \mathrm{f}^{6} \rightarrow 4 \mathrm{f}^{5} 5 \mathrm{~d}^{1}$ transitions characteristic of the $\mathrm{Sm}^{2+}$ ion. As for LSCBC:Sm the absorption bands have been labelled A-F in accordance with the scheme of Karbowiak et al [122], and the transitions corresponding to these labels are shown in figure 8.6 . 


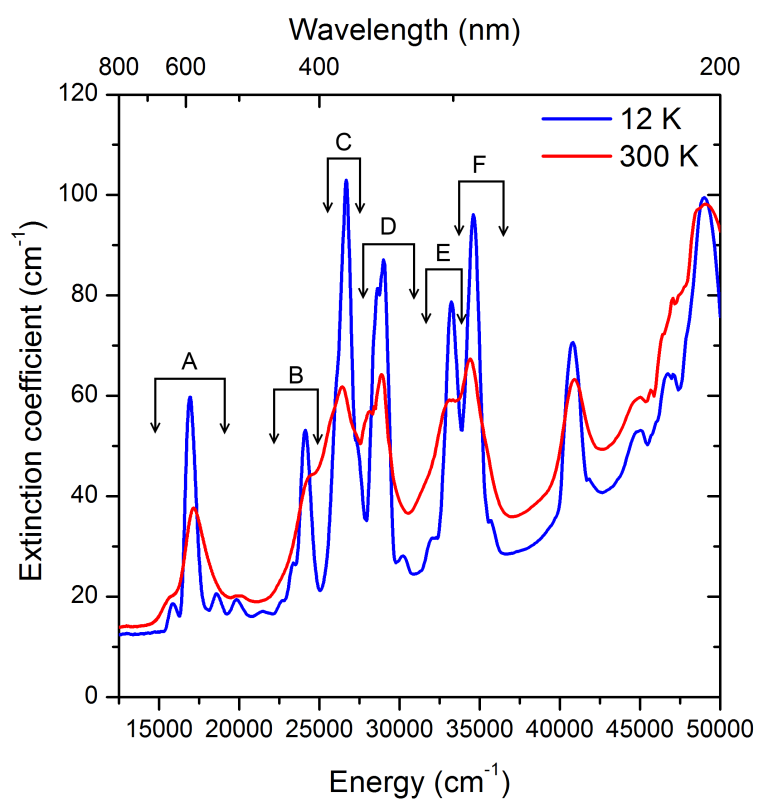

Figure 8.5: Absorption spectra of $\mathrm{Ba}_{0.3} \mathrm{Sr}_{0.7} \mathrm{Cl}_{2}: \mathrm{Sm}(0.5 \%)$ at $12 \mathrm{~K}$ and $300 \mathrm{~K}$. The resolution is $1 \mathrm{~nm}$ for both spectra. The absorption bands have been labelled $\mathrm{A}-\mathrm{F}$ in accordance with the scheme of Karbowiak et al. [122].

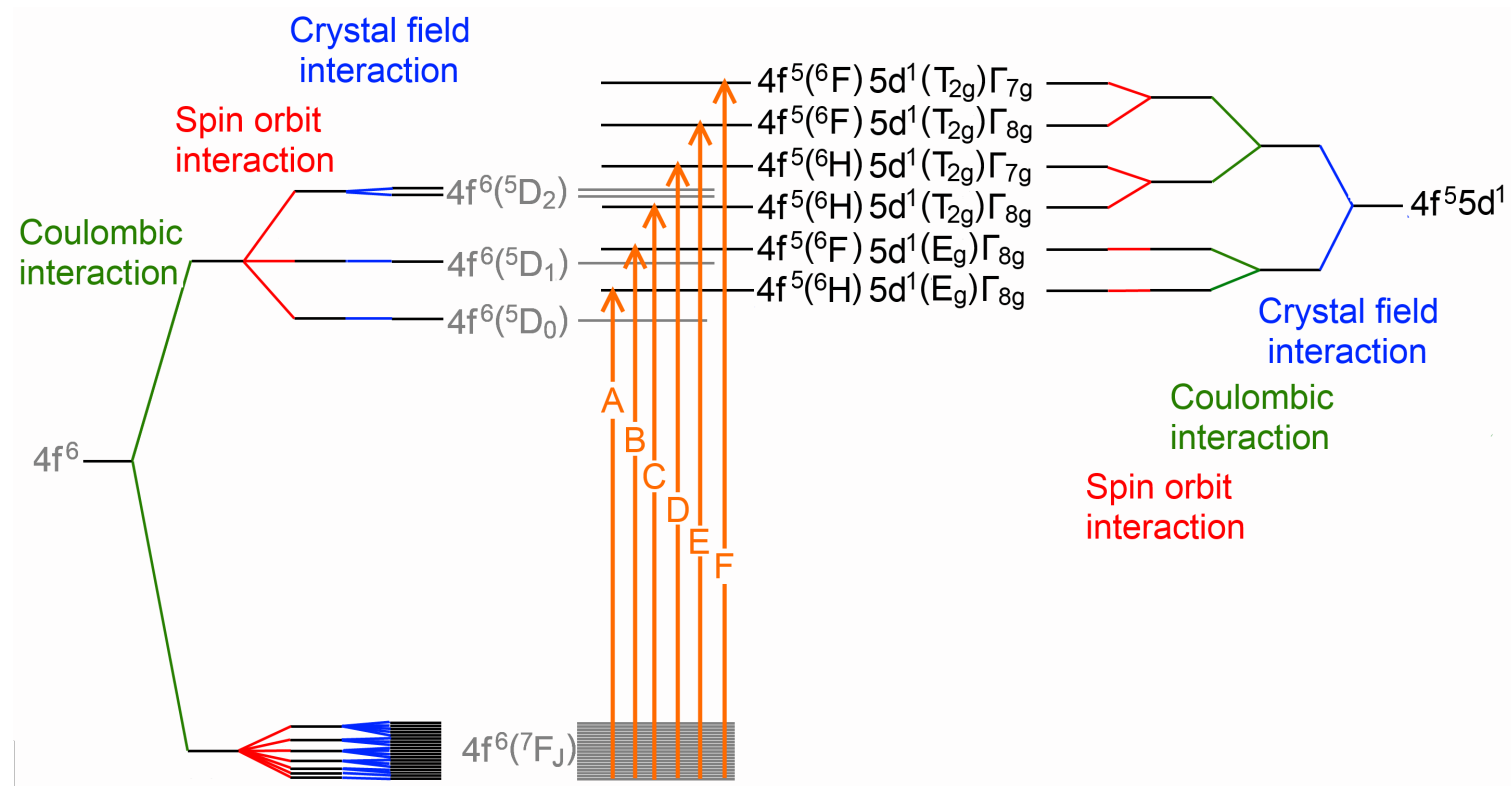

Figure 8.6: A schematic diagram of the excited state energy levels of $\mathrm{Sm}^{2+}$, the transitions corresponding to the absorption spectrum are indicated with arrows. This figure is repeated from figure $7.4(\mathrm{c})$. The ordering and labelling of the $4 \mathrm{f}^{5} 5 \mathrm{~d}^{1}$ states follows the scheme of Karbowiak et al. for $\mathrm{SrCl}_{2}: \mathrm{Sm}^{2+}$ [122]. 


\subsubsection{Photoluminescence and excitation spectra}

The samarium doped materials all show red photoluminescence for samarium concentrations from $0.01 \%$ to $1 \%$. The room temperature photoluminescence is shown in figure 8.7(a) and consists of a broad emission band corresponding to $4 \mathrm{f}^{5} 5 \mathrm{~d}^{1} \rightarrow 4 \mathrm{f}^{6}$ transitions of Sm ${ }^{2+}$ ions peaked at $14580 \mathrm{~cm}^{-1}(680 \mathrm{~nm})$. The broad band emission is similar to that seen for LSCBC:Sm ${ }^{2+}$. As for LSCBC:Sm we see absorption and photoluminescence corresponding to $\mathrm{Sm}^{2+}$ ions and not $\mathrm{Sm}^{3+}$ ions. The reduction process which results in $\mathrm{Sm}^{3+} \rightarrow \mathrm{Sm}^{2+}$ conversion occurring for $\mathrm{Ba}_{0.3} \mathrm{Sr}_{0.7} \mathrm{Cl}_{2}: \mathrm{Sm}^{2+}$ is likely the same as for LSCBC:Sm${ }^{2+}$, that is the formation of carbon monoxide reducing the trivalent samarium ions.

On cooling a dramatic change in emission to purely $4 \mathrm{f}^{6} \rightarrow 4 \mathrm{f}^{6}$ transitions is observed as seen in figure $8.7(\mathrm{~b})$ which shows the photoexcited luminescence spectra at $12 \mathrm{~K}$. This is as was observed for the similar material LSCBC:Sm${ }^{2+}$ in chapter 7. Both spectra have been normalised. Figure 8.8(a) shows the emission spectrum at $12 \mathrm{~K}$ in more detail, labelled with the intra $4 \mathrm{f}^{6}$ configuration transitions observed. Figure 8.8(b) depicts the energy level structure and the transitions observed. At $12 \mathrm{~K}$ the ${ }^{5} \mathrm{D}_{0} \rightarrow{ }^{7} \mathrm{~F}_{1}$ transition is dominant at $14295 \mathrm{~cm}^{-1}(700 \mathrm{~nm})$ and a much weaker ${ }^{5} \mathrm{D}_{0} \rightarrow{ }^{7} \mathrm{~F}_{0}$ transition can be seen at $14580 \mathrm{~cm}^{-1}$ (686 nm). Unlike in LSCBC:Sm ${ }^{2+}$, no evidence of the ${ }^{5} \mathrm{D}_{1}$ emission can be seen. Further, at room temperature the emission spectrum is entirely broad band emission and the emission band peak is at a lower energy than in LSCBC:Sm ${ }^{2+}$. These effects are all indications that the crystal field is stronger in $\mathrm{Ba}_{0.3} \mathrm{Sr}_{0.7} \mathrm{Cl}_{2}$ than in LSCBC and so the lowest $4 \mathrm{f}^{5} 5 \mathrm{~d}^{1}$ level is closer to the ${ }^{5} \mathrm{D}_{0}$ state. The relative intensity of the ${ }^{5} \mathrm{D}_{0} \rightarrow{ }^{7} \mathrm{~F}_{0}$ transition compared to the ${ }^{5} \mathrm{D}_{0} \rightarrow{ }^{7} \mathrm{~F}_{1}$ transition is much weaker than in LSCBC:Sm${ }^{2+}$, which indicates that perturbations on the cubic symmetry are of a smaller scale. In the perfect $\mathrm{O}_{h}$ symmetry case, this transition has zero probability.

The room temperature excitation spectrum (also shown in figure 8.7(a) shows broad band excitations into the $4 \mathrm{f}^{5} 5 \mathrm{~d}^{1}$ orbital excited states. On cooling to $12 \mathrm{~K}$ (figure $8.7(\mathrm{~b})$ ) the excitation bands narrow as the excited ground states become thermally unreachable.

The intensity of the emission from the two emitted levels can be plotted as a function of temperature to investigate the population dynamics occurring, and this has been done in 


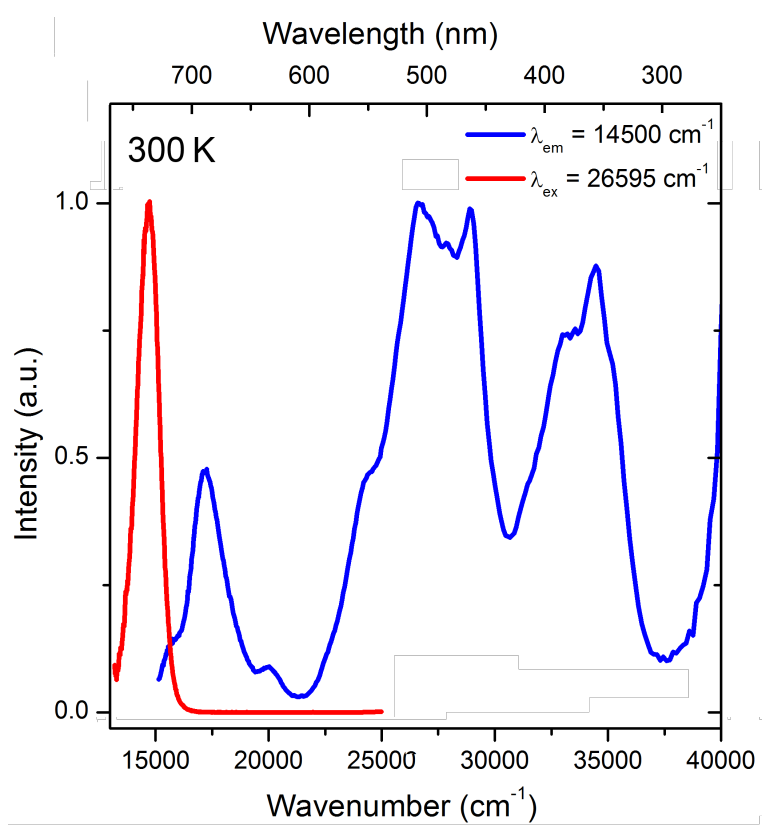

(a)

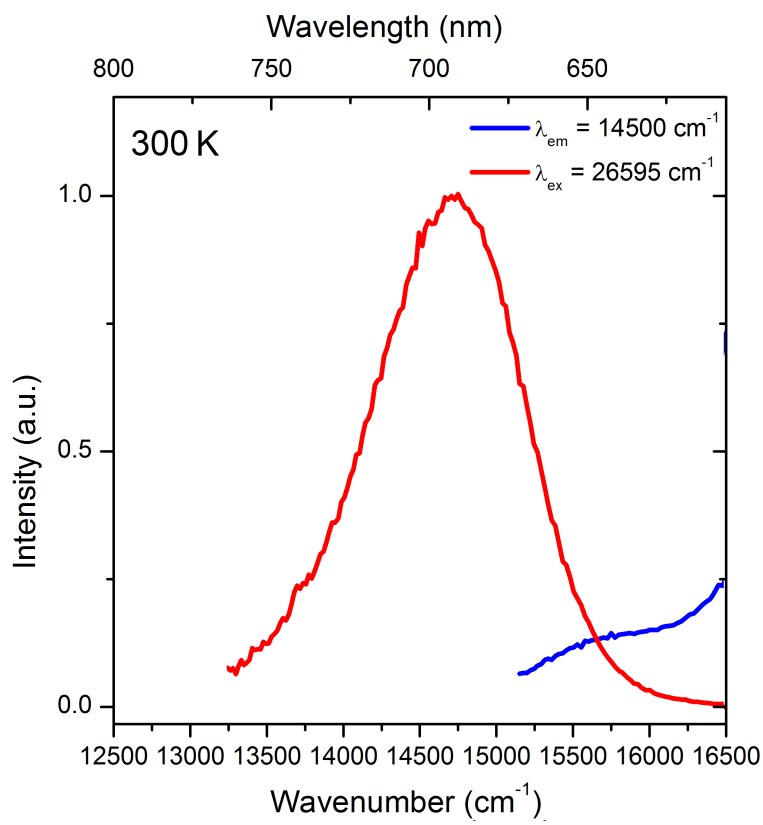

(c)

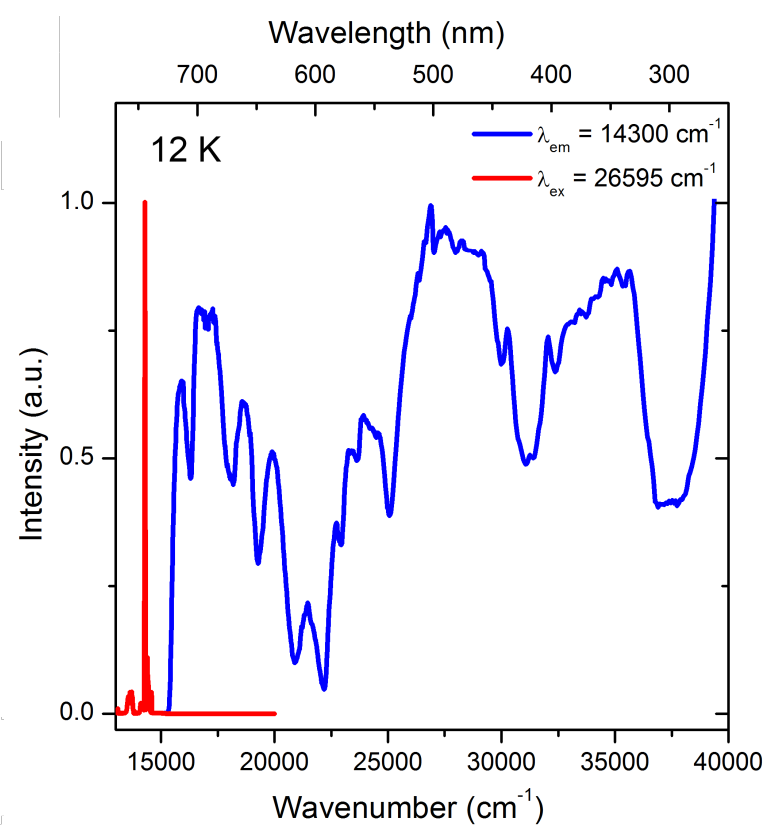

(b)

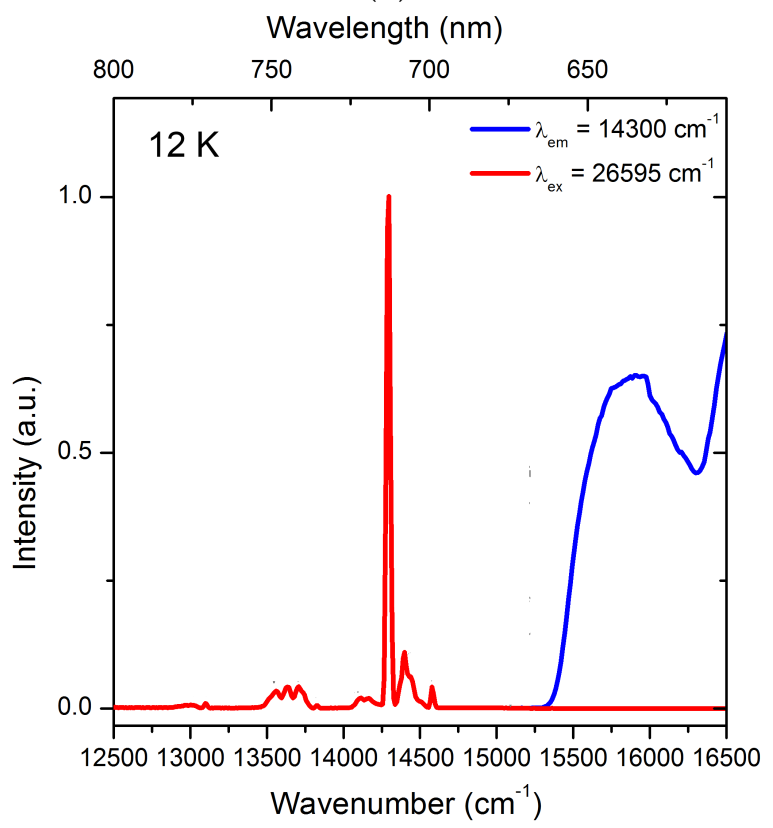

(d)

Figure 8.7: Photoluminescence excitation and emission spectra of $\mathrm{Ba}_{0.3} \mathrm{Sr}_{0.7} \mathrm{Cl}_{2}: \mathrm{Sm}^{2+}(0.1 \%)$ at (a) $298 \mathrm{~K}$ and at (b) $12 \mathrm{~K}$. Expanded views of the emission spectra at (c) $298 \mathrm{~K}$ and (d) $12 \mathrm{~K}$. The resolution of all spectra is $1 \mathrm{~nm}$. 

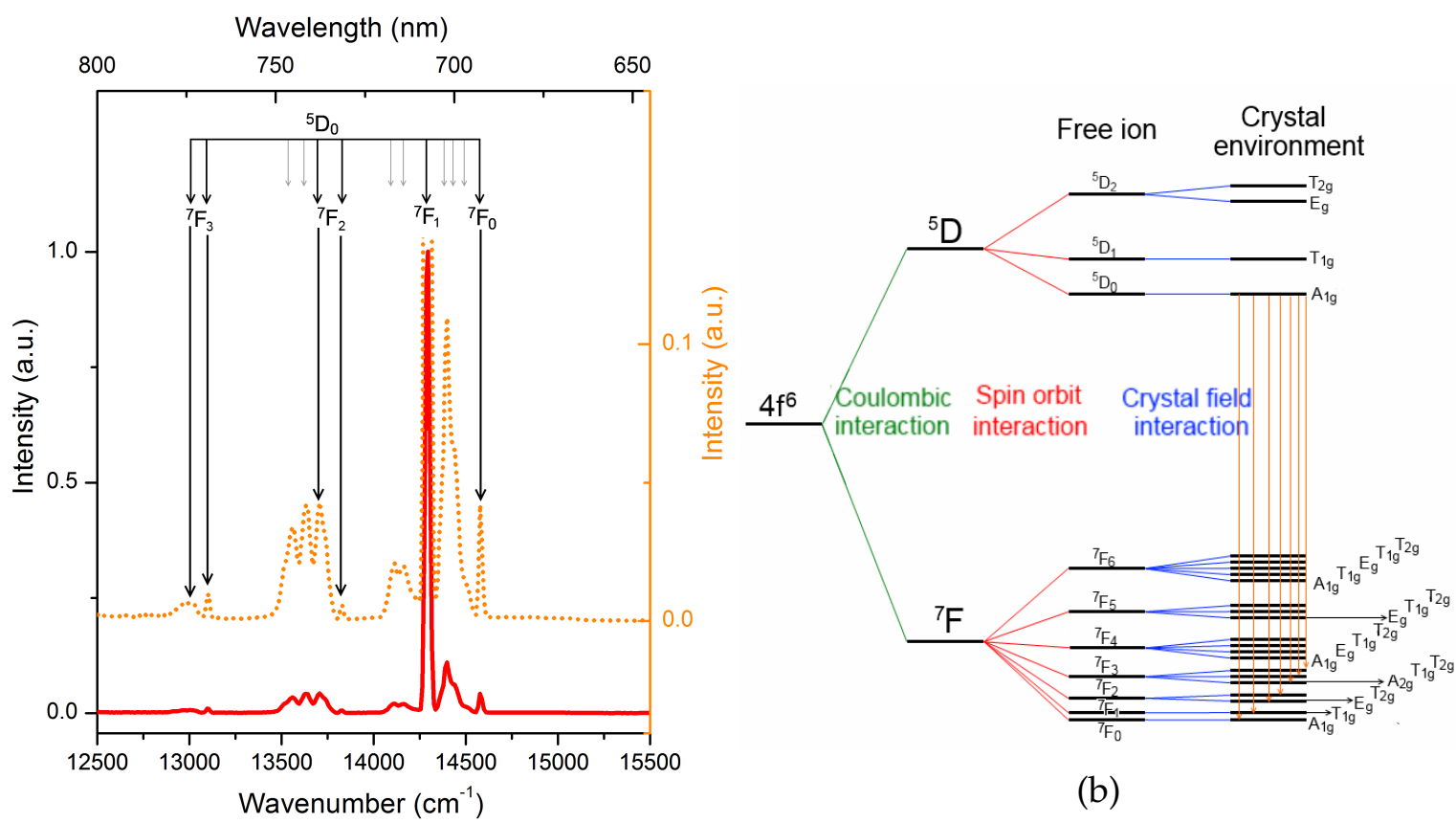

(b)

(a)

Figure 8.8: (a) The emission spectrum of $\mathrm{Ba}_{0.3} \mathrm{Sr}_{0.7} \mathrm{Cl}_{2}: \mathrm{Sm}$ at $12 \mathrm{~K}$ labelled above with the intra $4 \mathrm{f}^{6}$ configuration transitions occurring, the grey lines are phonon side bands of the labelled transitions. The spectrum is shown on a full scale (red) and an expanded scale (orange). (b) A diagram of the transitions observed from the emission spectrum at $12 \mathrm{~K}$.

figure 8.9 . The intensities were found by fitting the main emissions with multiple Gaussian functions and integrating those emissions from the $4 \mathrm{f}^{6}\left({ }^{5} \mathrm{D}_{0}\right)$ state and $4 \mathrm{f}^{5} 5 \mathrm{~d}^{1}$ configuration as a function of temperature, as was done for $\mathrm{LSCBC}: \mathrm{Sm}^{2+}$. At low temperatures the emission is entirely from the ${ }^{5} \mathrm{D}_{0}$ state, but as the temperature increases, and the non-radiative transitions

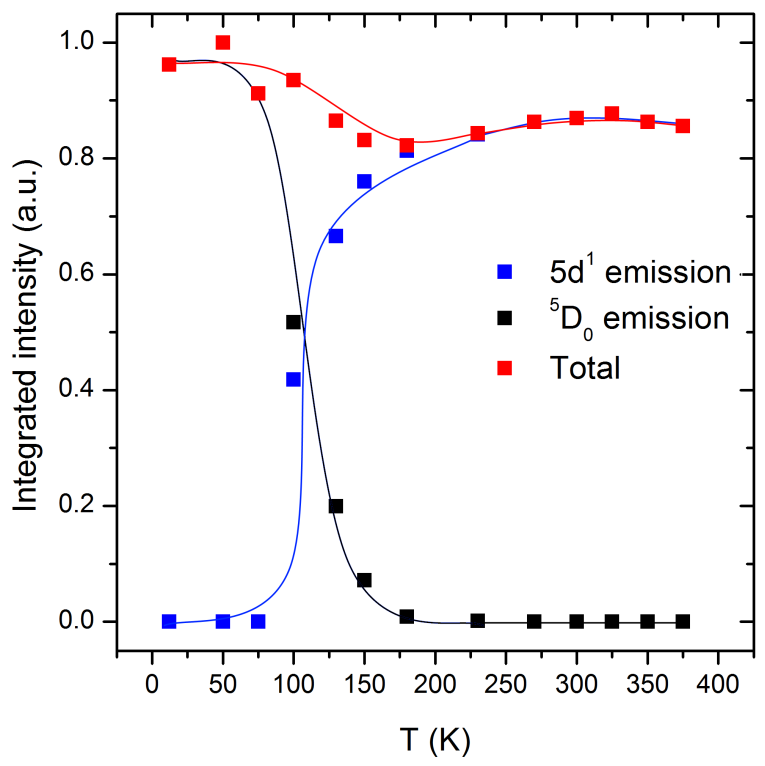

Figure 8.9: $\mathrm{Ba}_{0.3} \mathrm{Sr}_{0.7} \mathrm{Cl}_{2}: \mathrm{Sm}^{2+}$ integrated intensity of $4 \mathrm{f}^{5} 5 \mathrm{~d}^{1}, 4 \mathrm{f}^{6}\left({ }^{5} \mathrm{D}_{0}\right)$ and total photoluminescence emission as a function of temperature. The solid lines are provided as a guide to the eye. 

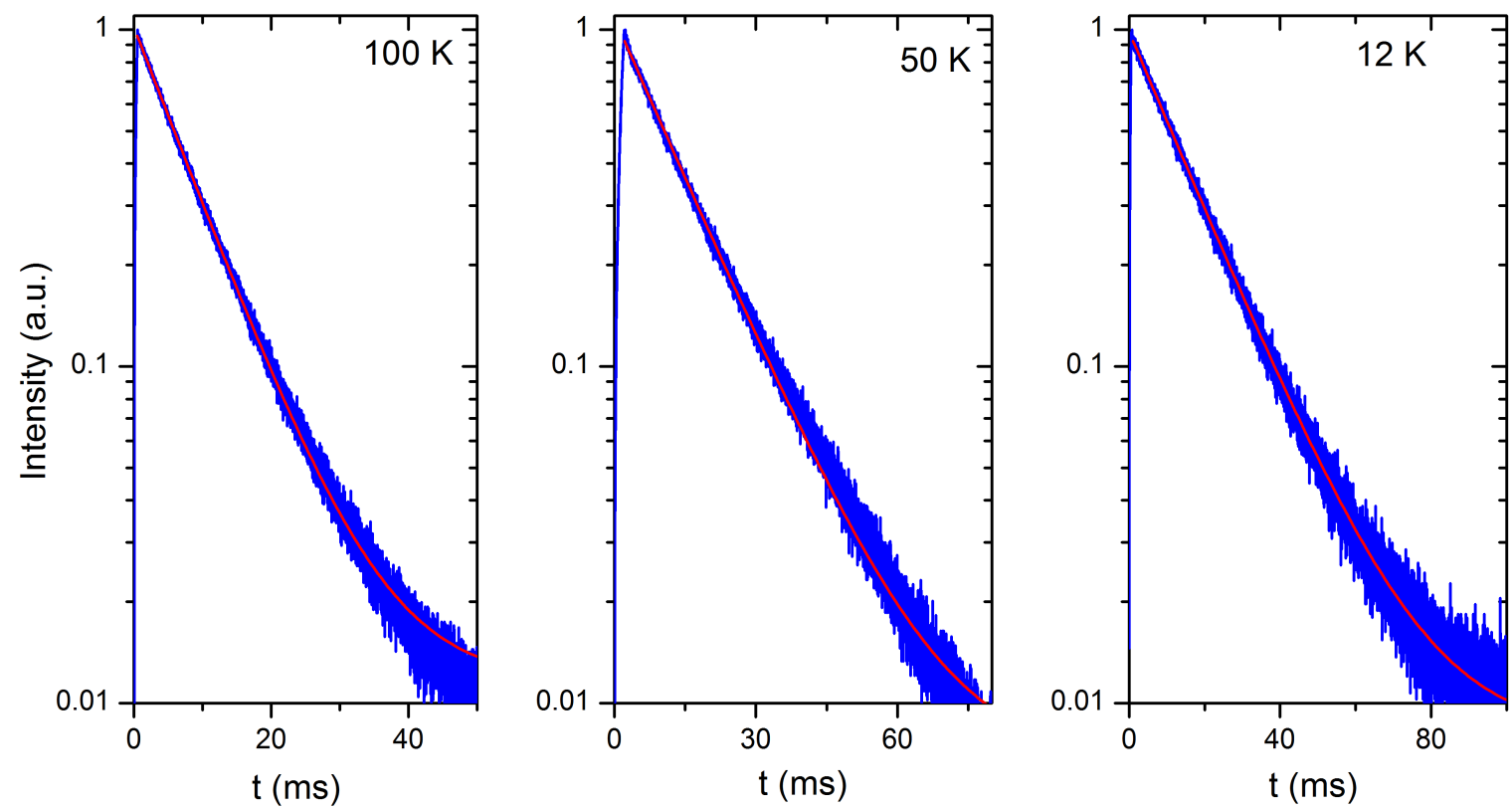

Figure 8.10: Decay profiles of the ${ }^{5} \mathrm{D}_{0}$ emission in $\mathrm{Ba}_{0.3} \mathrm{Sr}_{0.7} \mathrm{Cl}_{2}: \mathrm{Sm}(0.1 \%)$ at $12 \mathrm{~K}, 50 \mathrm{~K}$ and $100 \mathrm{~K}$ (left to right); all are well fitted by single exponentials (red lines). The lifetime was recorded at $685 \mathrm{~nm}$ with a $4 \mathrm{~nm}$ band pass and excited with a $375 \mathrm{~nm}$ LED.

$4 \mathrm{f}^{6}\left({ }^{5} \mathrm{D}_{0}\right) \rightarrow 4 \mathrm{f}^{5} 5 \mathrm{~d}^{1}$ transitions become more probable, the emission observed comes entirely from the $4 \mathrm{f}^{5} 5 \mathrm{~d}^{1}$ levels.
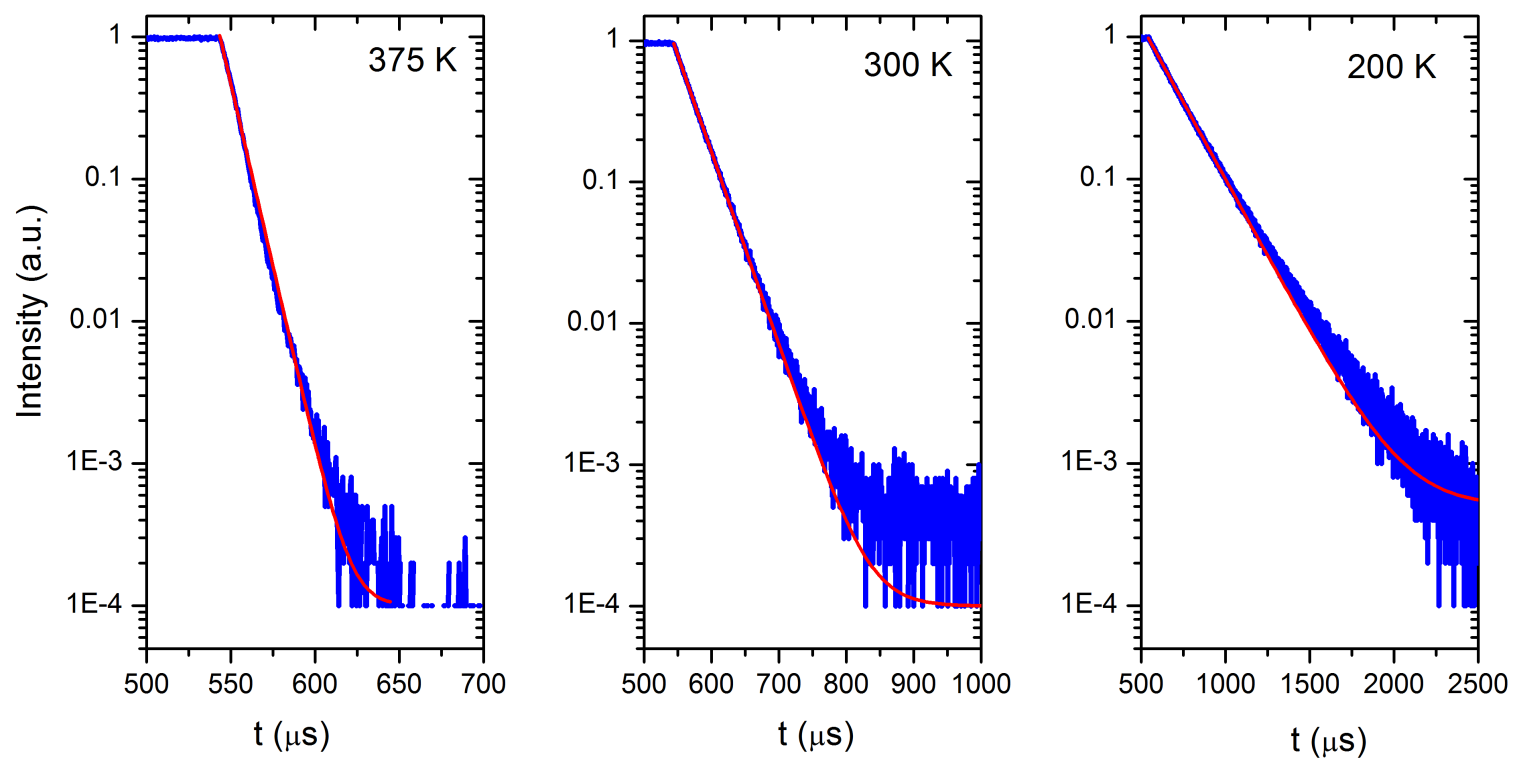

Figure 8.11: The decay profiles of the $4 \mathrm{f}^{5} 5 \mathrm{~d}^{1}$ emission in $\mathrm{Ba}_{0.3} \mathrm{Sr}_{0.7} \mathrm{Cl}_{2}: \mathrm{Sm}(0.1 \%)$ at $200 \mathrm{~K}, 300$ $\mathrm{K}$ and $375 \mathrm{~K}$ (left to right) all are well fitted by single exponentials (red lines). The lifetime was recorded at $700 \mathrm{~nm}$ with a $1 \mathrm{~nm}$ band pass and excited with a $375 \mathrm{~nm}$ LED. 


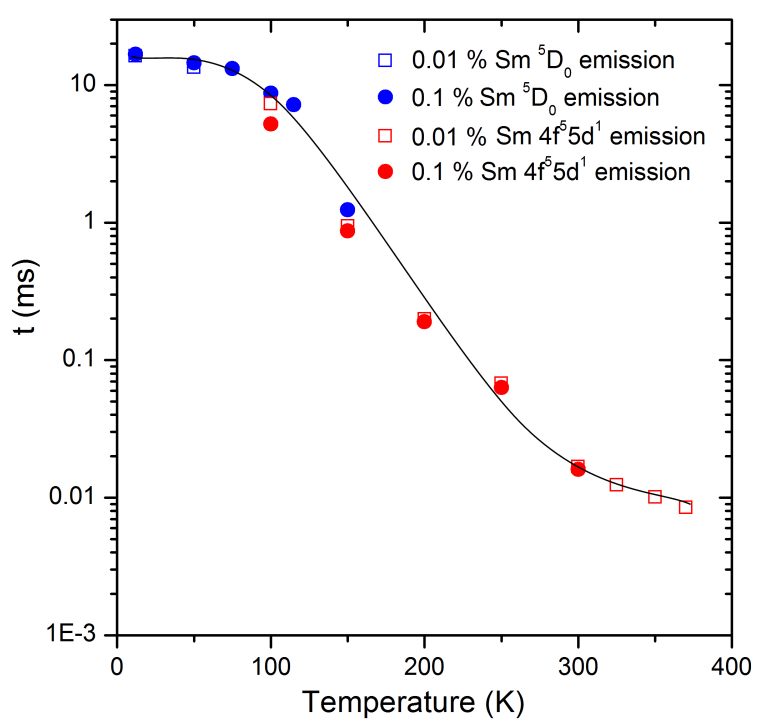

Figure 8.12: Summary of lifetime variation with temperature for both the $4 \mathrm{f}^{5} 5 \mathrm{~d}^{1}$ and ${ }^{5} \mathrm{D}_{0}$ emissions in samples with $0.1 \%$ and $0.01 \%$ samarium doping. The solid black line is provided as a guide to the eye.

\subsubsection{Lifetimes}

The time dependence of the UV excited broad band emission can be well fitted by a single exponential (with a baseline), and this is shown for various temperature between $150 \mathrm{~K}$ and $375 \mathrm{~K}$ in figure 8.11 . Figure 8.10 shows the lifetime of the ${ }^{5} \mathrm{D}_{0}$ emission fitted with a single exponential (with a baseline) for various temperatures from $12 \mathrm{~K}$ to $100 \mathrm{~K}$. At $\sim 100-125 \mathrm{~K}$ the intensity of the broad band $4 \mathrm{f}^{5} 5 \mathrm{~d}^{1}$ emission under the $4 \mathrm{f}^{6}\left({ }^{5} \mathrm{D}_{0}\right)$ emission line is negligible and both emissions can be recorded. A summary of the fitted lifetimes for both emissions is shown in figure 8.12 which shows the values for both $0.1 \%$ and $0.01 \%$ samarium samples; the overlap of data points indicates a lack of concentration dependence. As the temperature is lowered, the non radiative processes feeding the $4 \mathrm{f}^{5} 5 \mathrm{~d}^{1}$ level from the $4 \mathrm{f}^{6}\left({ }^{5} \mathrm{D}_{0}\right)$ state slow until the decay of the former simply mirrors that of the latter.

\subsubsection{Simulation}

We previously modelled the excited state populations and transition rates as a function of temperature for LSCBC:Sm ${ }^{2+}$ in chapter 7 , and we now do this for $\mathrm{Ba}_{0.3} \mathrm{Sr}_{0.7} \mathrm{Cl}_{2}: \mathrm{Sm}^{2+}$. Again we use a set of five levels, taking the both the ${ }^{5} \mathrm{D}_{0}$ and the lowest ${ }^{5} \mathrm{D}_{1}$ level and their radiative lifetimes from our experimental data. As we have not observed emission from the ${ }^{5} \mathrm{D}_{1}$ excited state we take the energy and lifetime we take to be $15920 \mathrm{~cm}^{-1}$ and $2.15 \mathrm{~ms}$ respectively as 


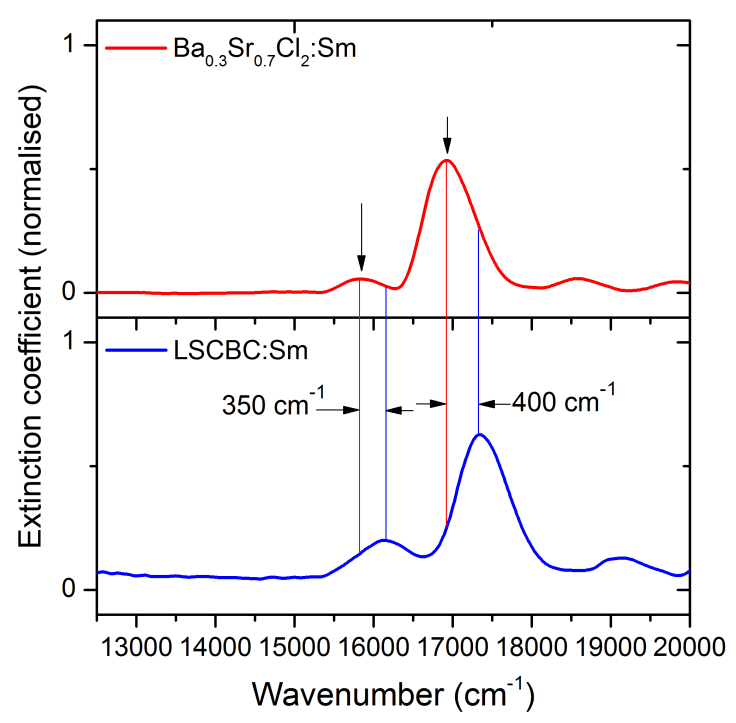

Figure 8.13: The shift in absorption bands of the lowest $4 \mathrm{f}^{5} 5 \mathrm{~d}^{1}$ levels between LSCBC:Sm ${ }^{2+}$ and $\mathrm{Ba}_{0.3} \mathrm{Sr}_{0.7} \mathrm{Cl}_{2}: \mathrm{Sm}^{2+}$.

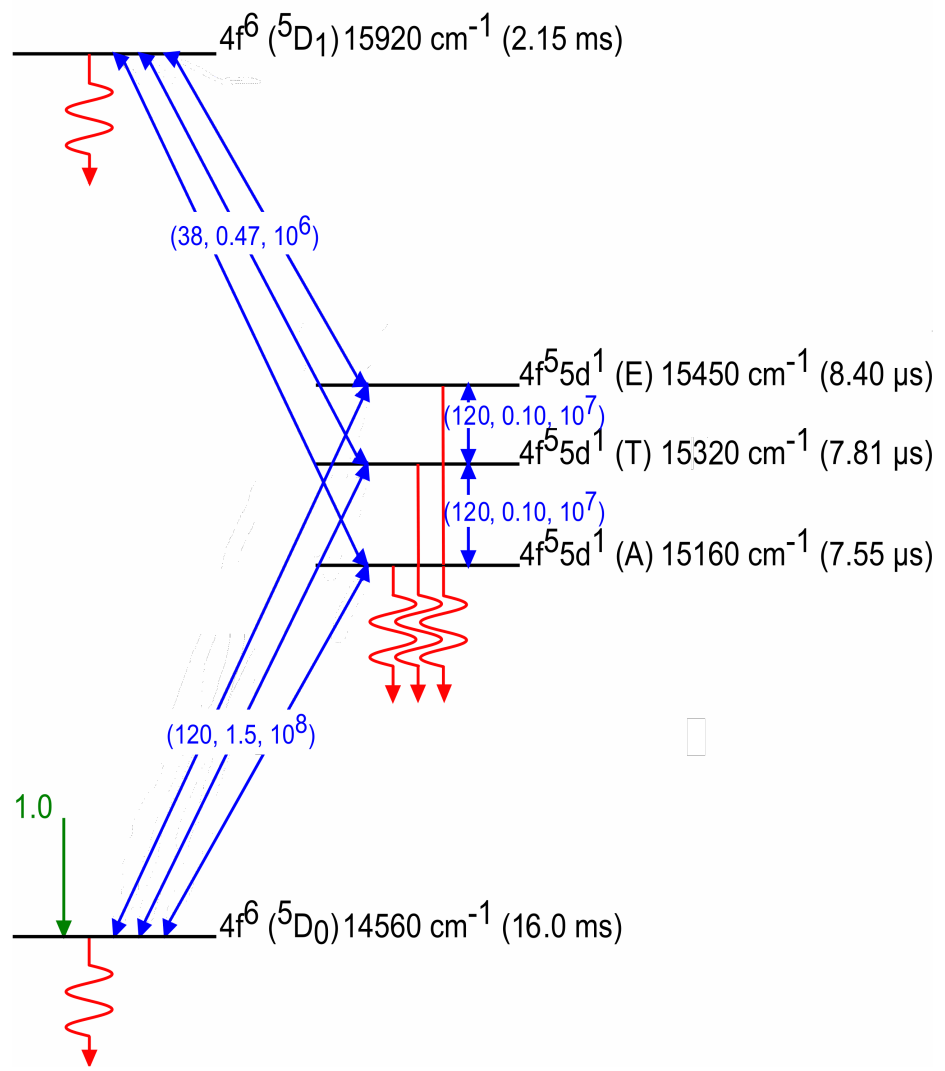

Figure 8.14: Summary of the energy levels and lifetimes used the photoluminescence simulation of $\mathrm{Ba}_{0.3} \mathrm{Sr}_{0.7} \mathrm{Cl}_{2}: \mathrm{Sm}^{2+}$. 
for LSCBC:Sm, and consistent with observations in similar materials [127, 128]. For the first, second and third lowest $4 \mathrm{f}^{5} 5 \mathrm{~d}^{1}$ levels we use the same energy gaps as for LSCBC:Sm ${ }^{2+}$ but apply an overall shift due to the change in the crystal field. We obtained a shift value of $-360 \mathrm{~cm}^{-1}$ by comparing the absorption bands for the lowest $4 \mathrm{f}^{5} 5 \mathrm{~d}^{1}$ levels in LSCBC:Sm${ }^{2+}$ and $\mathrm{Ba}_{0.3} \mathrm{Sr}_{0.7} \mathrm{Cl}_{2}: \mathrm{Sm}^{2+}$ as shown in figure 8.13 . The energy levels and lifetimes used are summarised in figure 8.14. The simulation process is as described in chapter 7. We assume the non-radiative decays from higher states solely populates the ${ }^{5} \mathrm{D}_{0}$ state as this is the only emission observed at $12 \mathrm{~K}$. The simulation provides an excellent match to data collected as seen in figure 8.15 which shows the simulated photoluminescence intensities and lifetimes alongside the experimental data.

\subsubsection{X-ray response}

\subsubsection{Radioluminescence}

The room temperature radioluminescence of the samarium doped materials is shown in figure 8.16. The emission replicates the photoluminescence seen at room temperature, consisting of a broad red emission at $14580 \mathrm{~cm}^{-1}(686 \mathrm{~nm})$. As well as the red emission band there is a very broad emission in the blue region $\left(\sim 25000 \mathrm{~cm}^{-1}\right)$ for the lightly doped samples. This is strongest for the lowest samarium concentrations then decreases in intensity as the samarium concentration increases due the overlap of the samarium absorption bands with this emission; the photons are absorbed by $\mathrm{Sm}^{2+}$ ions before they can escape the material. This effect is particularly clear for the $0.1 \%$ samarium sample, where the higher energy part of the blue emission band which overlaps with a strong samarium absorption band seen in figure 8.5 is preferentially absorbed. The $\mathrm{Sm}^{2+}$ ion luminescence shows a clear and strong concentration dependence. For samarium concentrations around $0.01 \%$ the emission is weak, it then becomes stronger to a maximum at about $0.1 \%$ before concentration quenching begins and the intensity decreases again for concentrations up to $1 \%$ and above.

The undoped material shows the same broad blue emission (see the black line in figure 8.16; this is a property of the host material independent of the samarium doping. The integrated intensity of the radioluminescence emission of the $0.1 \%$ samarium sample is $30 \%$ that of 


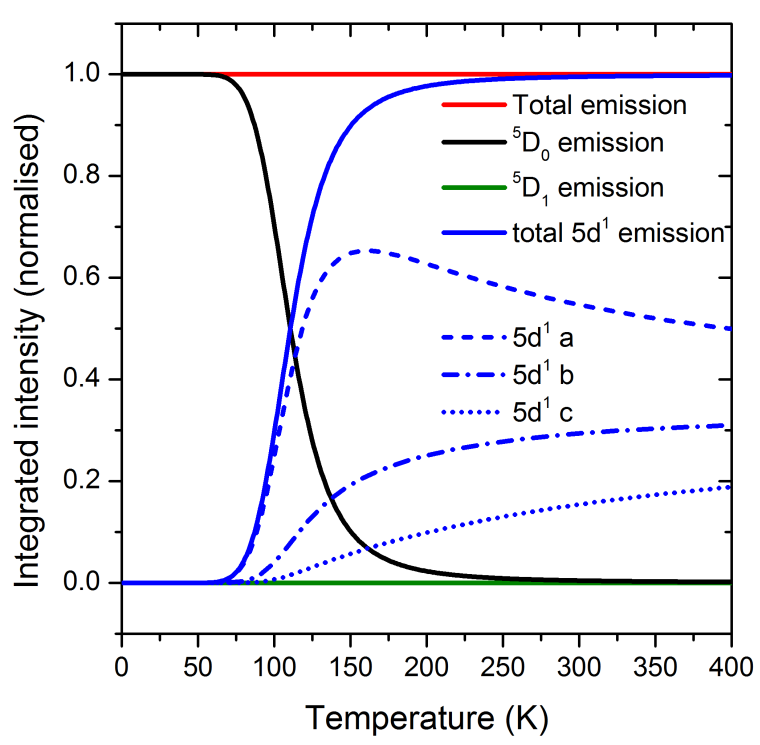

(a)

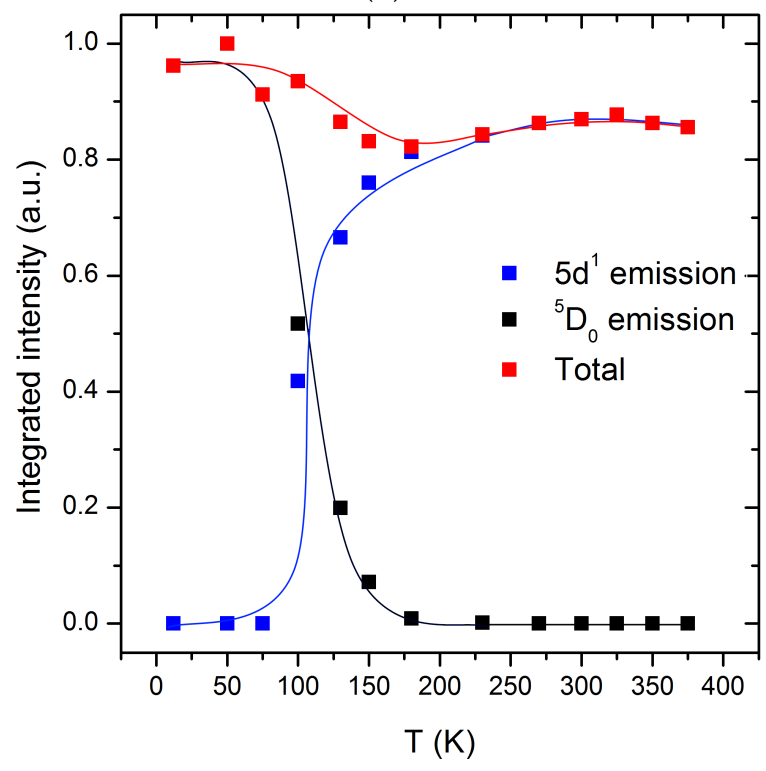

(c)

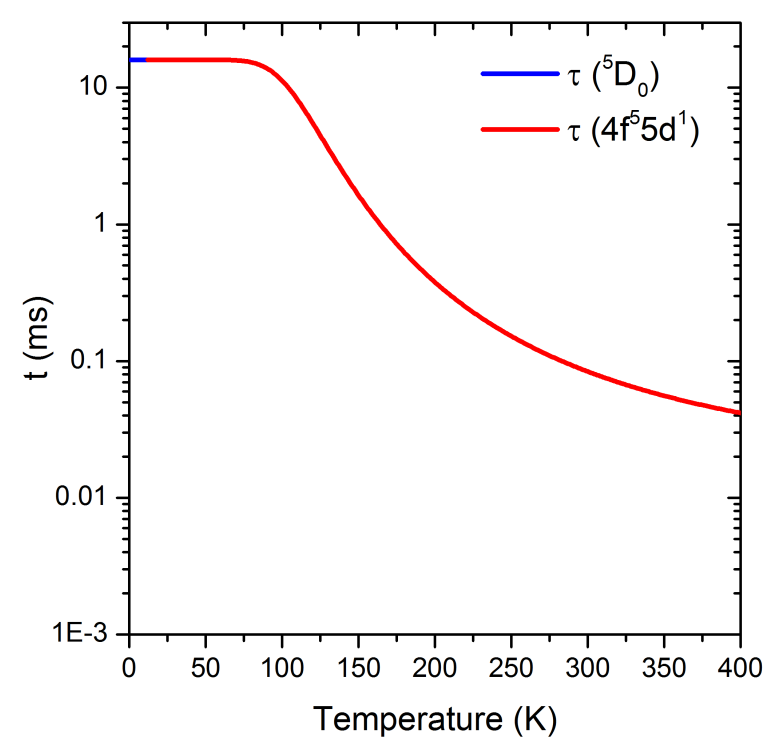

(b)

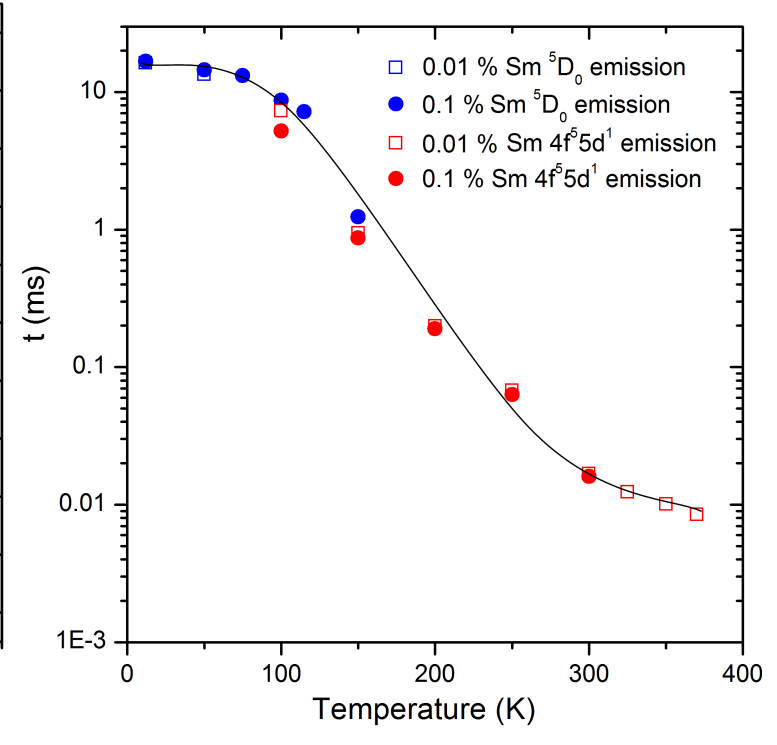

(d)

Figure 8.15: (a) Simulated integrated intensity of emission from the lowest five excited states in $\mathrm{Ba}_{0.3} \mathrm{Sr}_{0.7} \mathrm{Cl}_{2}: \mathrm{Sm}^{2+}$. (b) Simulated lifetimes of the ${ }^{5} \mathrm{D}_{0}$ and lowest $5 \mathrm{~d}^{1}$ excited states. (c) Experimental integrated intensity of emission from the $5 \mathrm{~d}^{1}$ and ${ }^{5} \mathrm{D}_{0}$ levels (repeated from figure 8.9). (d) Experimental lifetime variation with temperature for both the $4 f^{5} 5 d^{1}$ and ${ }^{5} D_{0}$ emission (repeated from figure 8.12). 


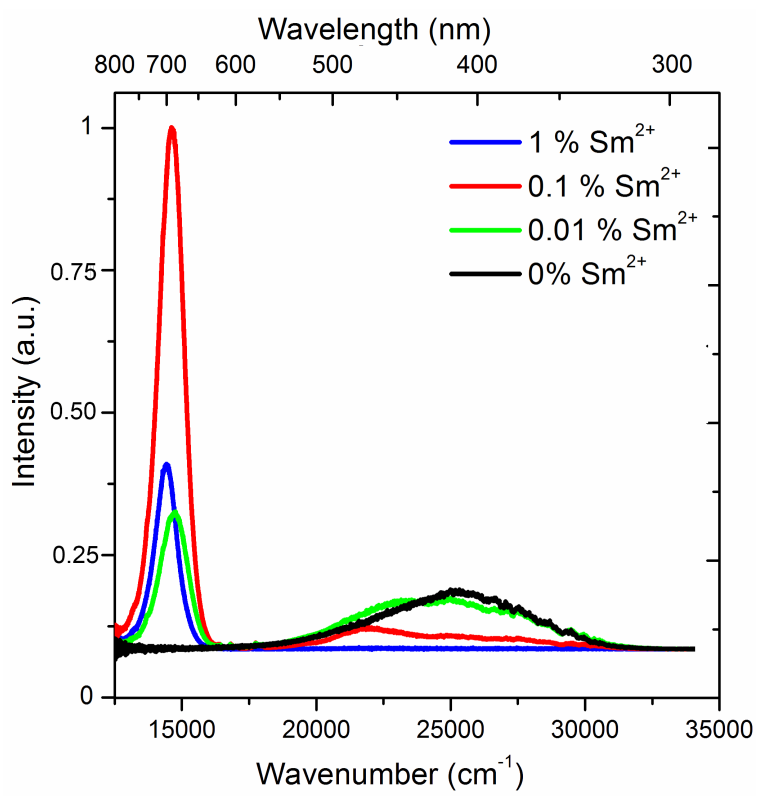

Figure 8.16: Radioluminescence spectra of $\mathrm{Ba}_{0.3} \mathrm{Sr}_{0.7} \mathrm{Cl}_{2}: \mathrm{Sm}^{2+}$. These spectra were recorded using a cobalt tube operated at $40 \mathrm{kV}$ and $30 \mathrm{~mA}$ and have been corrected for the spectral response of the detector and sample thickness.

the commercial material $\mathrm{Gd}_{2} \mathrm{O}_{2} \mathrm{~S}: \mathrm{Tb}^{3+}$ (DRX-STD by Kasei Optonix Ltd.) meaning these materials are very bright. As for LSCBC:Sm ${ }^{2+}$, we note that while the $\mathrm{Gd}_{2} \mathrm{O}_{2} \mathrm{~S}: \mathrm{Tb}^{3+}$ material is much thinner than our samples, both the samples of $\mathrm{Ba}_{0.3} \mathrm{Sr}_{0.7} \mathrm{Cl}_{2}$ and the $\mathrm{Gd}_{2} \mathrm{O}_{2} \mathrm{~S}$ : $\mathrm{Tb}^{3+}$ will be attenuating the $\mathrm{x}$-ray beam to $\sim 25 \%$ the initial intensity of the x-ray beam.

\subsubsection{Scintillation}

The blue emission from undoped $\mathrm{Ba}_{0.3} \mathrm{Sr}_{0.7} \mathrm{Cl}_{2}$ shows scintillation; the decay profile is shown in figure 8.17(a). This has been fitted with a triple exponential (with a baseline) with components of $0.11,0.70$ and $2.4 \mu$ s with relative integrated intensities of $0.1,1.0$ and 3.0. The samarium doped samples also show scintillation, an average of events from $\mathrm{Ba}_{0.3} \mathrm{Sr}_{0.7} \mathrm{Cl}_{2}: \mathrm{Sm}$ is shown in figure 8.17(b), these were recorded with a $550 \mathrm{~nm}$ longpass filter between the sample and photomultiplier so definitely arise from $\mathrm{Sm}^{2+}$ red emission. This averaged profile has been fitted with a single exponential (with a baseline) with a decay time of $30 \mu \mathrm{s}$. The spike at $t=0$ is a artefact which arises from the averaging of events.

Pulse height analysis (PHA) was recorded for the undoped sample and compared to the commercial material $\mathrm{NaI}(\mathrm{Tl})$. This was recorded with ${ }^{137} \mathrm{C}$ s as the source and an integration time of $4 \mu \mathrm{s}$. While there is no clear resolution of a strong photo peak the light output 
appears to be between 12,000 and 19,000 photons per MeV. Similarly PHA was recorded for a samarium doped sample $(0.1 \%)$, the integration time used was slightly longer at $7 \mu$ s to account for the longer scintillation time. There is evidence of a photo peak at 22,000 photons per $\mathrm{MeV}$. In obtaining this value we have included a correction for the spectral response of the photomultiplier tube as described in chapter 5. These are high efficiencies which are common among rare earth doped alkali halides, many scintillators in use have much lower efficiencies such as $\mathrm{Bi}_{4} \mathrm{Ge}_{3} \mathrm{O}_{12}$ which only has an efficiency of $\sim 8,000$ photons $/ \mathrm{MeV}$ [146]. The integrated intensity of the $\mathrm{NaI}(\mathrm{Tl})$ curves are much higher because this is a $25 \mathrm{~mm}$ diameter and length crystal compared to approximately $20 \mathrm{~mm}$ by $5 \mathrm{~mm}$ samples, hence the $\mathrm{NaI}(\mathrm{Tl})$ crystal interacts with a much higher fraction of the $\gamma$-rays.

\subsubsection{Thermally stimulated luminescence}

Figure 8.19 shows the glow curves of $\mathrm{Ba}_{0.3} \mathrm{Sr}_{0.7} \mathrm{Cl}_{2}$ and $\mathrm{Ba}_{0.3} \mathrm{Sr}_{0.7} \mathrm{Cl}_{2}: \mathrm{Sm}^{2+}$. The glow curves indicate up to three traps in the materials. These have been fitted with first order kinetic equations to extract the peak temperature and activation energy according to the method of Kitis [90], and the fitted parameters are shown in table 8.1. The thermoluminescence emissions match the emissions seen under $x$-irradiation for both samples at all temperatures. This

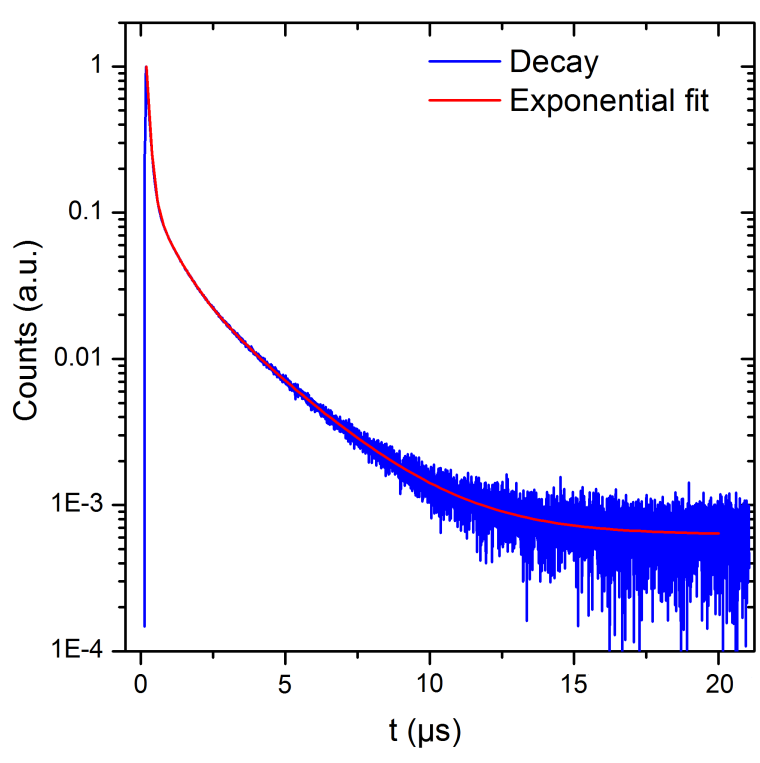

(a)

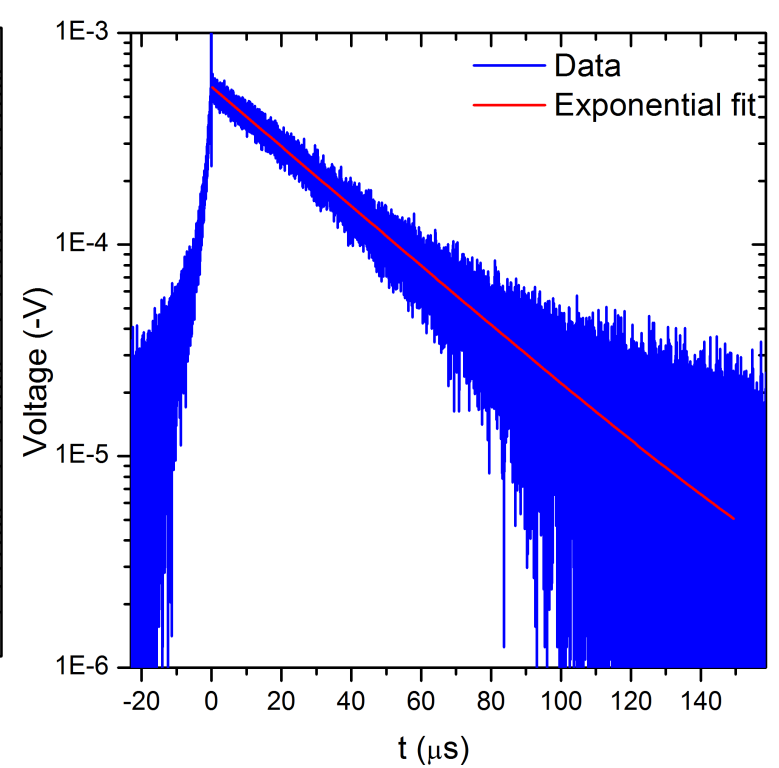

(b)

Figure 8.17: Scintillation profile of undoped $\mathrm{Ba}_{0.3} \mathrm{Sr}_{0.7} \mathrm{Cl}_{2}$ (a) recorded on the time correlated single photon counting system. Scintillation profile of $\mathrm{Ba}_{0.3} \mathrm{Sr}_{0.7} \mathrm{Cl}_{2}: \mathrm{Sm}^{2+}$ (b) recorded as an average of 2000 events on the fast oscilloscope. 


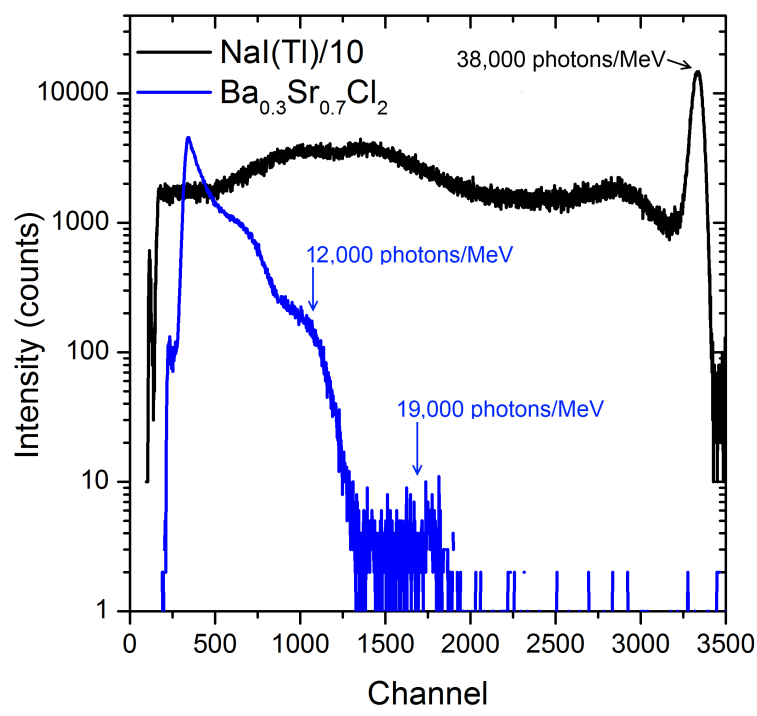

(a)

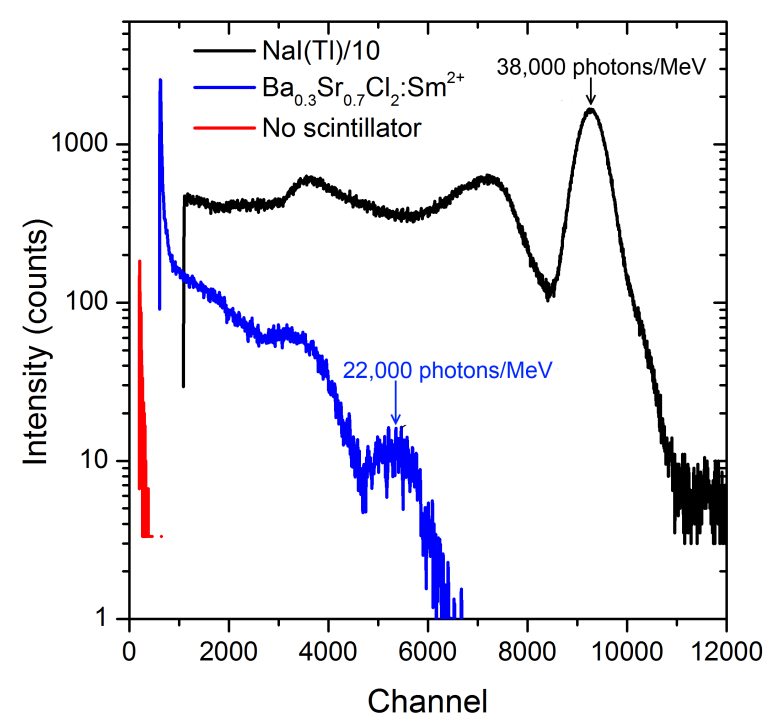

(b)

Figure 8.18: Pulse height analysis spectra for (a) $\mathrm{Ba}_{0.3} \mathrm{Sr}_{0.7} \mathrm{Cl}_{2}$ and (b) $\mathrm{Ba}_{0.3} \mathrm{Sr}_{0.7} \mathrm{Cl}_{2}: \mathrm{Sm}^{2+}$, both recorded with ${ }^{137} \mathrm{C}$ s sources and integration times of $4 \mu \mathrm{s}$ and $7 \mu$ s respectively.

Table 8.1: Fitted Kitis parameters to glow curves for $\mathrm{Ba}_{0.3} \mathrm{Sr}_{0.7} \mathrm{Cl}_{2}$ and $\mathrm{Ba}_{0.3} \mathrm{Sr}_{0.7} \mathrm{Cl}_{2}: \mathrm{Sm}^{2+}$. E refers to the trap depth and $\mathrm{T}$ the peak temperature of the glow curve corresponding to that trap.

\begin{tabular}{cccccc}
\hline Trap number & \multicolumn{2}{c}{ Undoped } & & \multicolumn{2}{c}{ Sm doped } \\
\cline { 2 - 3 } \cline { 6 - 6 } & $\mathrm{E}(\mathrm{eV})$ & $\mathrm{T}_{m}(\mathrm{~K})$ & & $\mathrm{E}(\mathrm{eV})$ & $\mathrm{T}_{m}(\mathrm{~K})$ \\
\hline 1 & $0.45 \pm 0.15$ & $370 \pm 15$ & & - & - \\
2 & $0.70 \pm 0.2$ & $440 \pm 20$ & & $0.80 \pm 0.1$ & $440 \pm 10$ \\
3 & - & - & $1.00 \pm 0.1$ & $485 \pm 20$ \\
\hline
\end{tabular}

was checked by running a second scan with a CCD detector instead of the PMT. Figure 8.20 shows snapshots of the emissions at the the glow curve peak temperatures for an undoped sample and a samarium doped sample. The undoped and samarium-doped samples have a common trap with a peak at around $440 \mathrm{~K}$ with a binding energy of $\sim 0.7 \mathrm{eV}$, however for the undoped material this is of much weaker intensity than the lower energy trap around 360 $\mathrm{K}$ with a depth of around $0.5 \mathrm{eV}$. This lower energy trap is absent for the samarium doped materials but there is a higher trap around $440 \mathrm{~K}$ of depth $\sim 0.7 \mathrm{eV}$ which is dominant for the samarium doped materials but not the undoped materials. These results are similar to those seen for LSCBC:Sm in chapter 7 . 


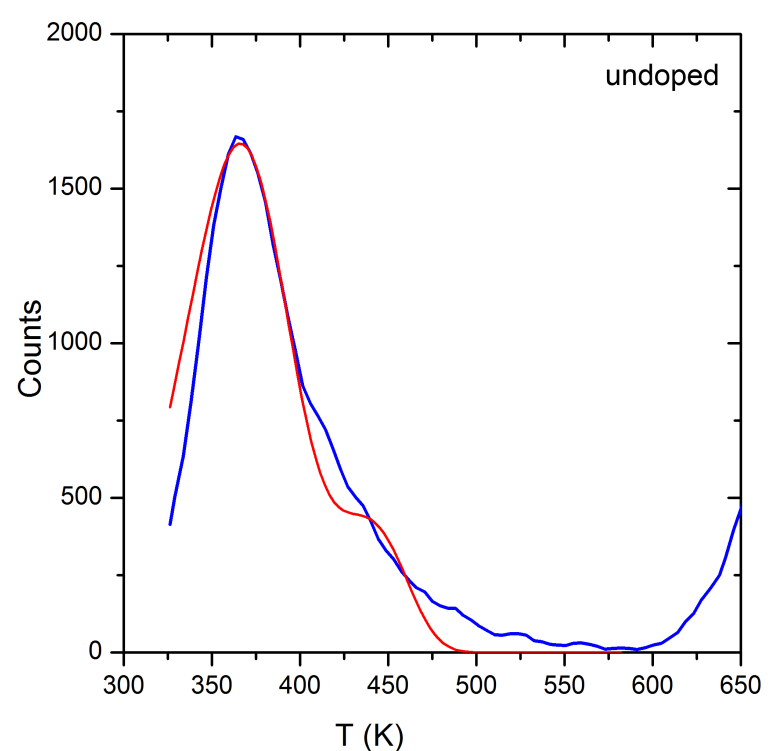

(a)

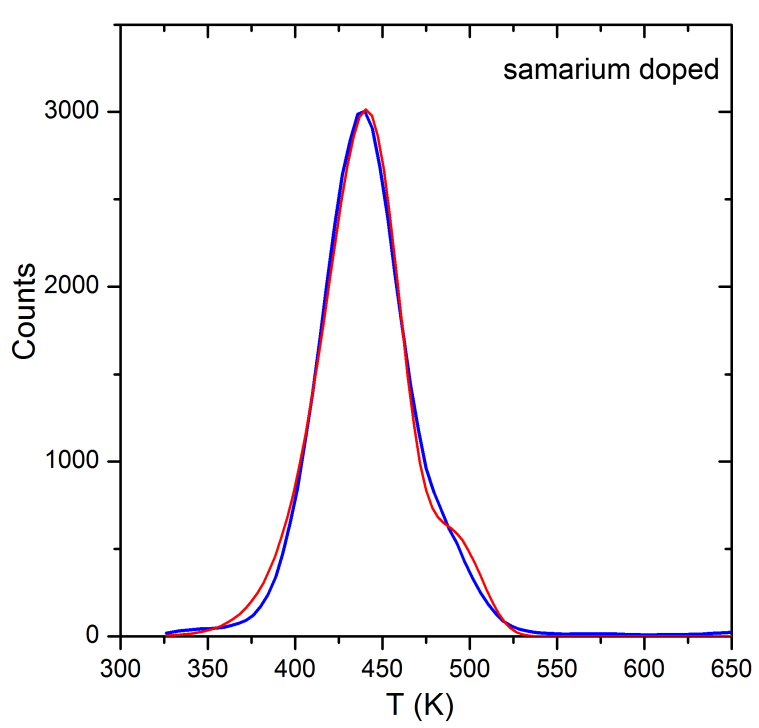

(b)

Figure 8.19: Thermally stimulated luminescence glow curves for $\mathrm{Ba}_{0.3} \mathrm{Sr}_{0.7} \mathrm{Cl}_{2}$ (a) and $\mathrm{Ba}_{0.3} \mathrm{Sr}_{0.7} \mathrm{Cl}_{2}: \mathrm{Sm}^{2+}(0.5 \%)$ (b). Both were irradiated for 15 minutes at $30 \mathrm{kV} 25 \mathrm{~mA}$. The red lines are the fitted curves, parameters of which are given in table 8.1. In (a) the high temperature signal is black body radiation.

\subsubsection{X-ray imaging}

The samarium doped materials can be used to form x-ray images. Figure 8.21(a) shows an image of an 8-pin integrated circuit (IC) chip in which the internal structure can be seen. Figure 8.21(b) shows an x-ray image of a standard lead grid (Nuclear Associates 07-553, 0.05 mm thick), taken using both $\mathrm{Gd}_{2} \mathrm{O}_{2} \mathrm{~S}: \mathrm{Tb}$ and $\mathrm{Ba}_{0.3} \mathrm{Sr}_{0.7} \mathrm{Cl}_{2}: \mathrm{Sm}^{2+}(0.1 \%)$, and recorded with the Canon 5D Mk 2 camera. Approximately 6 line pairs per millimetre (LP/mm) can be resolved by eye for $\mathrm{Ba}_{0.3} \mathrm{Sr}_{0.7} \mathrm{Cl}_{2}: \mathrm{Sm}^{2+}$, compared to approximately $4.2 \mathrm{LP} / \mathrm{mm}$ for the $\mathrm{Gd}_{2} \mathrm{O}_{2} \mathrm{~S}: \mathrm{Tb}$. Kasei quote a modulation transfer function (MTF) of 0.49 at $2 \mathrm{LP} / \mathrm{mm}$ for the $\mathrm{Gd}_{2} \mathrm{O}_{2} \mathrm{~S}: \mathrm{Tb}^{3+}$. The MTF is defined as the ratio of the Fourier transform of the output to the Fourier transform of the input. In practical terms the MTF is a measure of the spatial frequency response of the imaging system, in this case the phosphor plates.

\subsection{Europium doped $\mathrm{BaCl}_{2}-\mathrm{SrCl}_{2}$ solid solutions}

Because $\mathrm{Eu}^{2+}$ ions often shows very bright luminescence in alkali halide lattices, and given that we have found $\mathrm{Ba}_{0.3} \mathrm{Sr}_{0.7} \mathrm{Cl}_{2}: \mathrm{Sm}$ to be a bright $\mathrm{x}$-ray phosphor it would be reasonable 


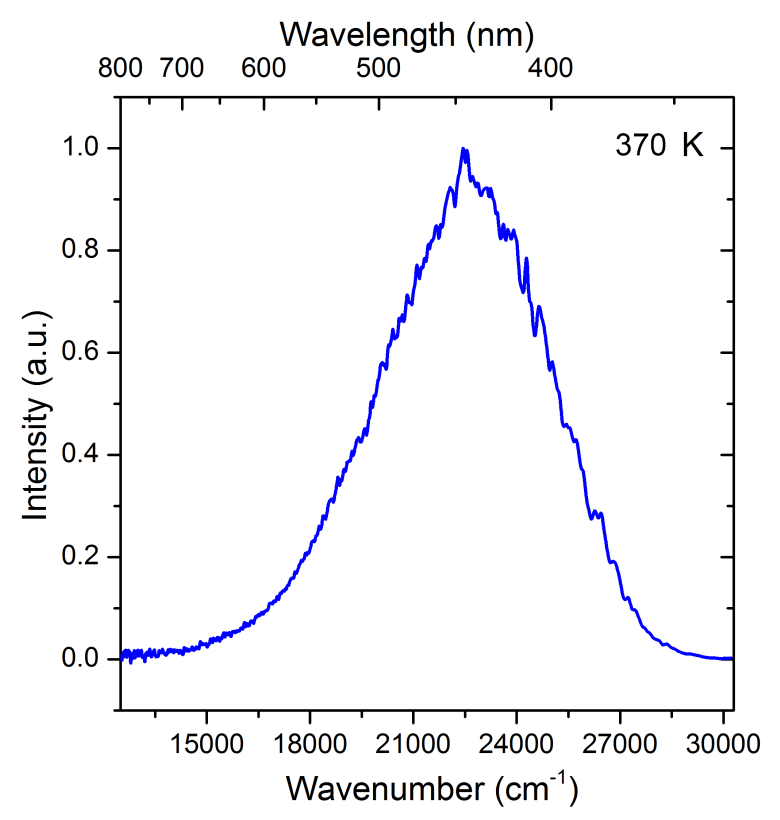

(a)

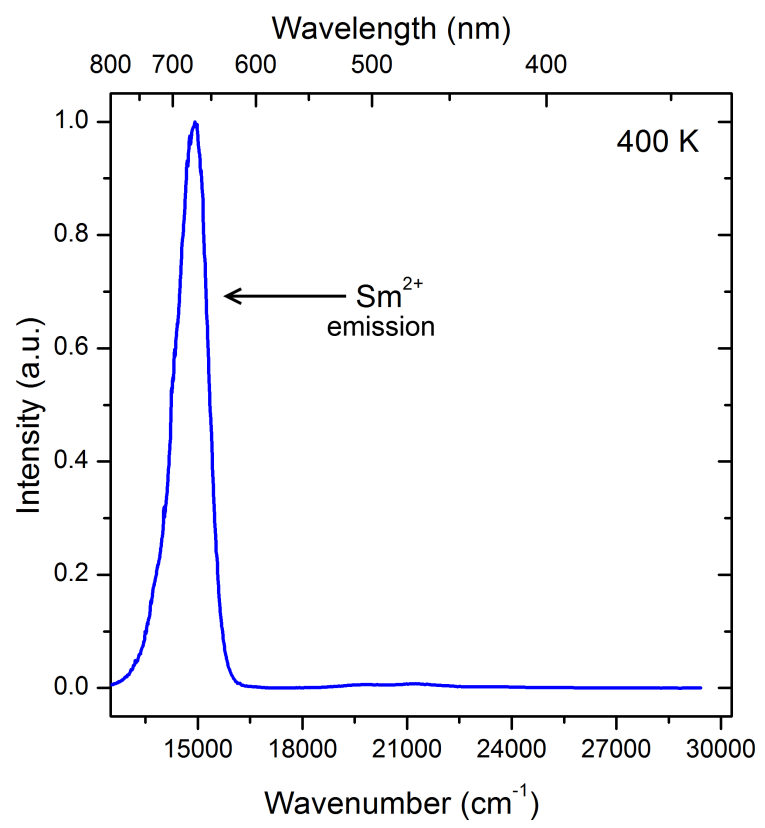

(b)

Figure 8.20: Emission spectra at the peak temperature of the glow curves for $\mathrm{Ba}_{0.3} \mathrm{Sr}_{0.7} \mathrm{Cl}_{2}$ (a) and $\mathrm{Ba}_{0.3} \mathrm{Sr}_{0.7} \mathrm{Cl}_{2}: \mathrm{Sm}^{2+}(0.5 \%)(\mathrm{b})$. The temperatures were $370 \mathrm{~K}$ and $400 \mathrm{~K}$ respectively. The emission in figure $8.20(\mathrm{a})$ is the intrinsic emission and in figure $8.20(\mathrm{~b})$ the $\mathrm{Sm}^{2+}$ ions.

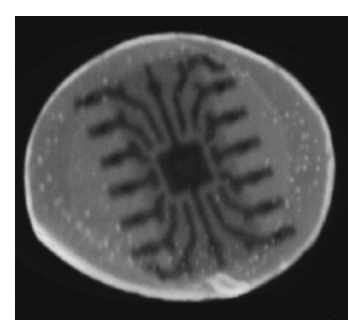

(a)

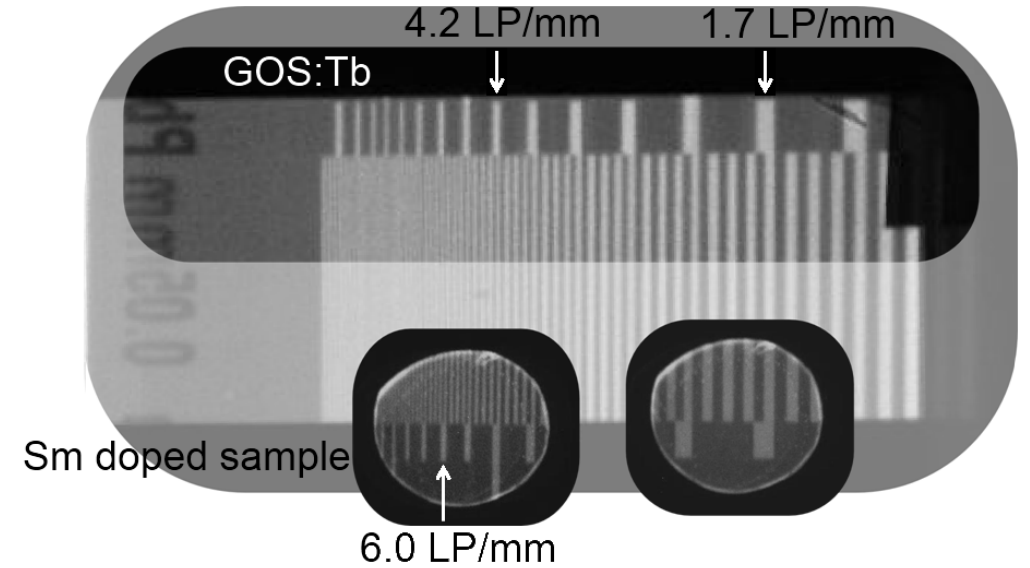

(b)

Figure 8.21: X-ray images of (a) a 8 pin IC chip taken with $\mathrm{Ba}_{0.3} \mathrm{Sr}_{0.7} \mathrm{Cl}_{2}: \mathrm{Sm}$ and (b) a lead grid showing the resolution compared to $\mathrm{Gd}_{2} \mathrm{O}_{2} \mathrm{~S}: \mathrm{Tb}$ 
to expect $\mathrm{Ba}_{0.3} \mathrm{Sr}_{0.7} \mathrm{Cl}_{2}: \mathrm{Eu}^{2+}$ to also be a bright $\mathrm{x}$-ray phosphor so we have also investigated $\mathrm{Ba}_{0.3} \mathrm{Sr}_{0.7} \mathrm{Cl}_{2}$ :Eu. The two distinct emission wavelengths avaliable with $\mathrm{Sm}^{2+}$ and $\mathrm{Eu}^{2+}$ doping would also offer a new type of dual layer detector with energy discrimination, and this will be discussed later in this chapter.

\subsubsection{Physical Properties}

$\mathrm{Eu}^{2+}$ doping was achieved by adding $\mathrm{EuCl}_{2}$ to the $\mathrm{BaCl}_{2}$ and $\mathrm{SrCl}_{2}$ mix prior to heating. This results in a cubic and transparent material, as shown earlier in figure 8.3 .

\subsubsection{Photoluminescence}

The room temperature photoluminescence emission and excitation spectra for $\mathrm{Ba}_{0.3} \mathrm{Sr}_{0.7} \mathrm{Cl}_{2}: \mathrm{Eu}$ are shown in figure $8.22(\mathrm{a})$. The europium emission corresponds to $4 \mathrm{f}^{6} 5 \mathrm{~d}^{1} \rightarrow 4 \mathrm{f}^{7}$ transitions and is peaked at $24630 \mathrm{~cm}^{-1}(406 \mathrm{~nm})$, the half width is $730 \mathrm{~cm}^{-1}$. The europium luminescence shows a decay which is well fitted by a single exponential (with a baseline) with a decay time of $1.0 \mu \mathrm{s}$, as shown in figure $8.22(\mathrm{~b})$. This value is similar to that for $\mathrm{SrCl}_{2}: \mathrm{Eu}^{2+}$ in which europium is reported to have a lifetime of $0.95 \mu \mathrm{s}$ [147] and LSCBC where the lifetime was reported as $0.358 \mu$ s for the band assigned to the site of cubic symmetry [118].

\subsubsection{X-ray response}

\subsubsection{Radioluminescence}

The radioluminescence follows the photoluminescence and is very bright; the room temperature spectrum is shown in figure 8.23 . For high europium concentrations the half width of the emission increases as can be seen in figure 8.23 . The integrated intensity of a $0.5 \%$ europium sample is around $40 \%$ that of $\mathrm{Gd}_{2} \mathrm{O}_{2} \mathrm{~S}: \mathrm{Tb}^{3+}$. 

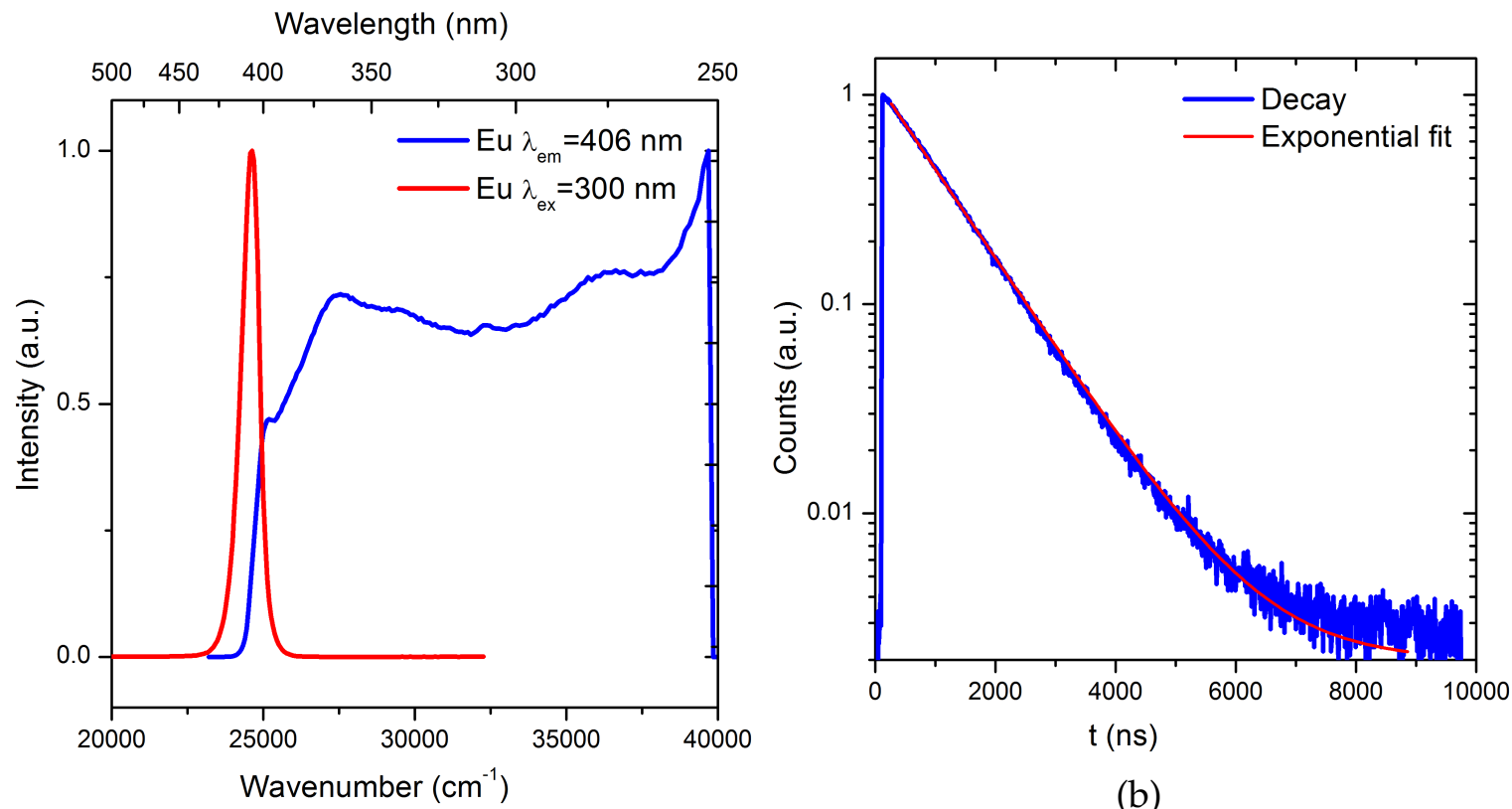

(a)

(b)

Figure 8.22: (a) Room temperature photoluminescence excitation and emission spectra of $\mathrm{Ba}_{0.3} \mathrm{Sr}_{0.7} \mathrm{Cl}_{2}: \mathrm{Eu}^{2+}$. (b) Room temperature photoluminescent lifetime of $\mathrm{Ba}_{0.3} \mathrm{Sr}_{0.7} \mathrm{Cl}_{2}: \mathrm{Eu}^{2+}$. In (a) both emission and excitation slits are $1 \mathrm{~nm}$. In (b) the photoluminescent lifetime was recorded at $406 \mathrm{~nm}$ with a $2 \mathrm{~nm}$ band pass and excited with a $295 \mathrm{~nm}$ LED.

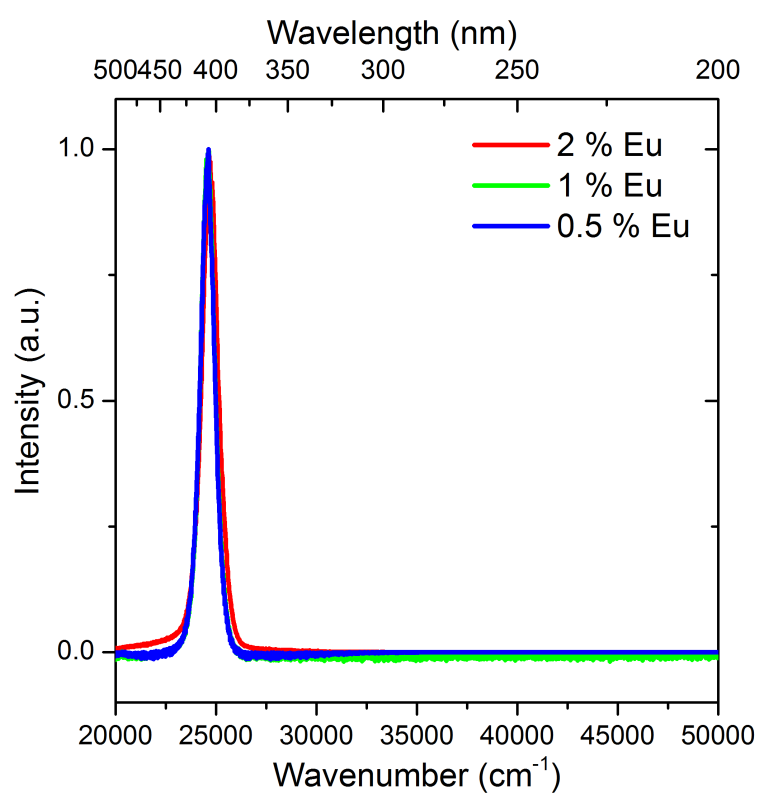

Figure 8.23: Normalised radioluminescence for $\mathrm{Ba}_{0.3} \mathrm{Sr}_{0.7} \mathrm{Cl}_{2}: \mathrm{Eu}^{2+}$ recorded using a cobalt tube operated at $40 \mathrm{kV}$ and $30 \mathrm{~mA}$ 


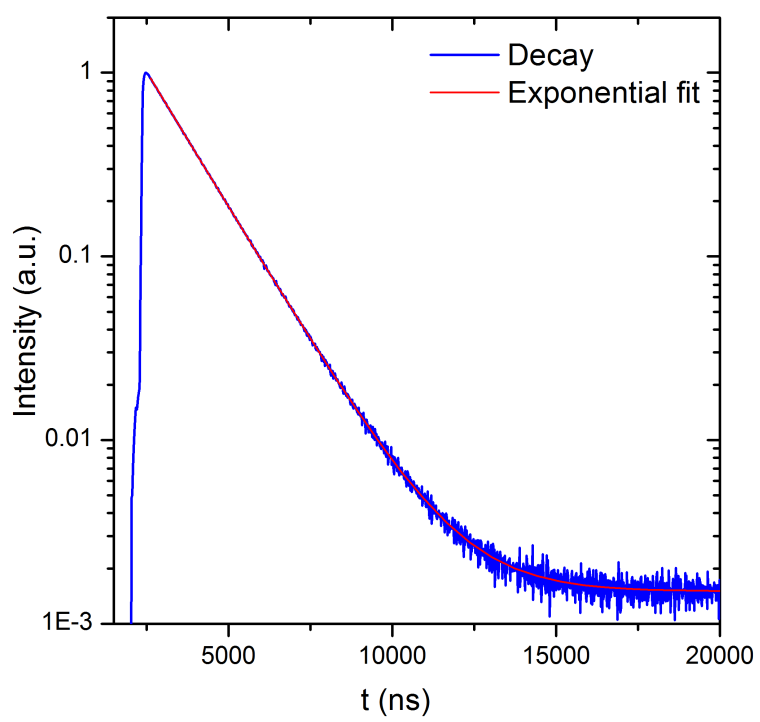

(a)

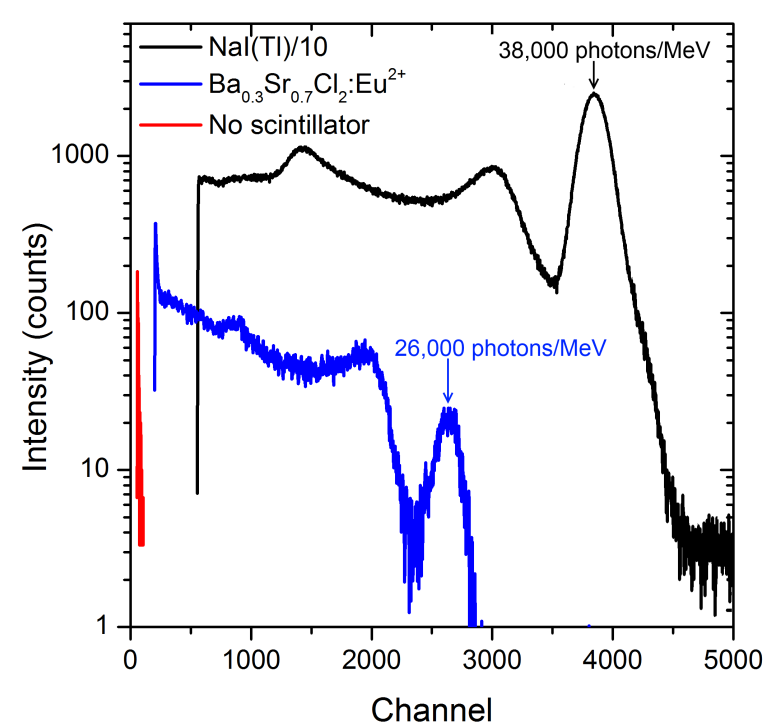

(b)

Figure 8.24: (a) The scintillation decay profile for $\mathrm{Ba}_{0.3} \mathrm{Sr}_{0.7} \mathrm{Cl}_{2}: \mathrm{Eu}^{2+}$ recorded using the TCSPC system. Also shown in red is the single exponential fit. (b) Pulse height analysis spectrum for $\mathrm{Ba}_{0.3} \mathrm{Sr}_{0.7} \mathrm{Cl}_{2}: \mathrm{Eu}^{2+}$ recorded using a ${ }^{137} \mathrm{Cs}$ gamma ray source, and shown compared to $\mathrm{NaI}(\mathrm{Tl})$ and the response from $\gamma$-rays with no scintillator present.

\subsubsection{Scintillation}

$\mathrm{Ba}_{0.3} \mathrm{Sr}_{0.7} \mathrm{Cl}_{2}$ :Eu shows reasonably bright scintillation, as previously mentioned in chapter 6 the decay time was recorded using the time correlated single photon counting system. Figure 8.24(a) shows the experimental decay which has been fitted with an exponential decay (with a baseline) of time constant of $1.6 \mu \mathrm{s}$. Pulse height analysis was recorded with an integration time of $4 \mu$ s and shown compared to the response of $\mathrm{NaI}(\mathrm{Tl})$ in figure $8.24(\mathrm{~b})$. The light output is around 26,000 photons per $\mathrm{MeV}$ by comparison with $\mathrm{NaI}(\mathrm{Tl})$. This compares to 52,000 and 70,000 photons per $\mathrm{MeV}$ reported for $\mathrm{BaCl}_{2}: \mathrm{Eu}^{2+}$ (orthorhombic) [148] and $\mathrm{SrCl}_{2}: \mathrm{Eu}^{2+}$ respectively [111].

\subsubsection{Thermally stimulated luminescence}

Shown in figure $8.25(\mathrm{a})$ is the glow curve recorded for $\mathrm{Ba}_{0.3} \mathrm{Sr}_{0.7} \mathrm{Cl}_{2}: \mathrm{Eu}^{2+}$. The glow curve has been fitted according to the Kitis technique and the fitted parameters are tabulated in table 8.2. Figure 8.25(b) shows the emission spectrum at $370 \mathrm{~K}$. The emission was the same over the whole glow curve changing only in intensity. A second order response for the emission can be seen around $800 \mathrm{~nm}$. In contrast to $\mathrm{Sm}^{2+}$ doping, $\mathrm{Ba}_{0.3} \mathrm{Sr}_{0.7} \mathrm{Cl}_{2}$ : $\mathrm{Eu}^{2+}$ shows a low energy 


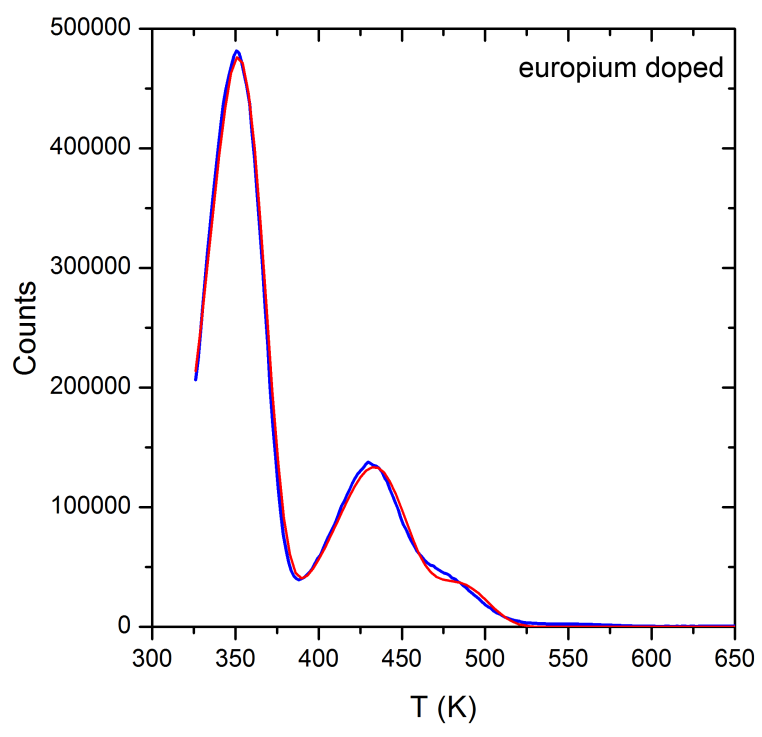

(a)

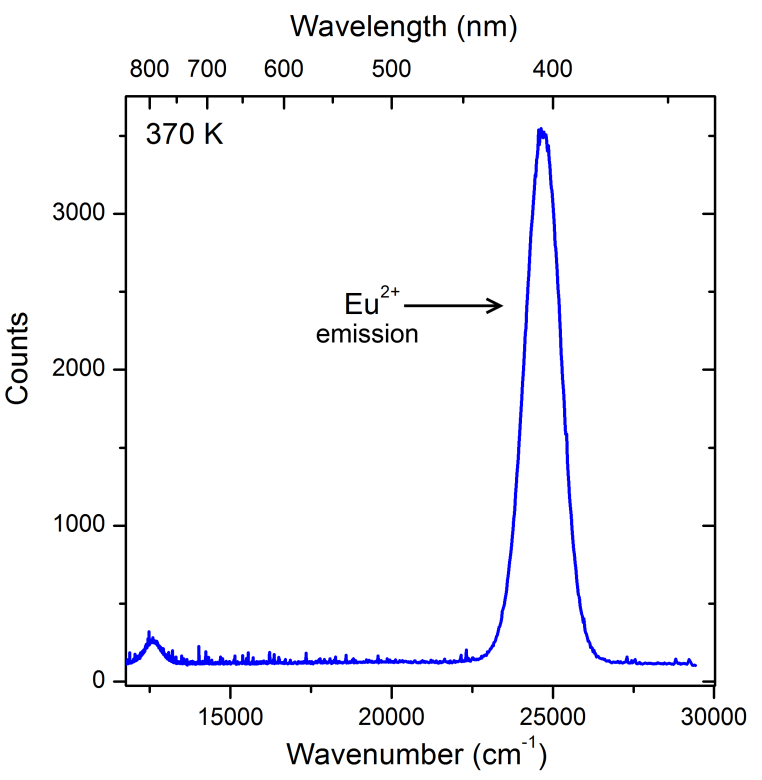

(b)

Figure 8.25: (a) Thermally stimulated luminescence glow curves for $\mathrm{Ba}_{0.3} \mathrm{Sr}_{0.7} \mathrm{Cl}_{2}$ : $\mathrm{Eu}^{2+}(0.5$ $\%)$, he red line is the fitted curves, parameters of which are given in table 8.2 (b) Emission spectra at the peak temperature $\left(370 \mathrm{~K}\right.$ ) of the glow curves for $\mathrm{Ba}_{0.3} \mathrm{Sr}_{0.7} \mathrm{Cl}_{2}: \mathrm{Eu}^{2+}$. Both (a) and (b) followed 15 minute irradiations at $30 \mathrm{kV}$ and $25 \mathrm{~mA}$.

Table 8.2: Kitis parameters fitted to glow curves for $\mathrm{Ba}_{0.3} \mathrm{Sr}_{0.7} \mathrm{Cl}_{2}: \mathrm{Eu}^{2+}$. The parameters for $\mathrm{Ba}_{0.3} \mathrm{Sr}_{0.7} \mathrm{Cl}_{2}$ and $\mathrm{Ba}_{0.3} \mathrm{Sr}_{0.7} \mathrm{Cl}_{2}: \mathrm{Sm}^{2+}$ are repeated from table 8.1 for comparison.

\begin{tabular}{|c|c|c|c|c|c|c|}
\hline \multirow[t]{2}{*}{ Trap number } & \multicolumn{2}{|c|}{ Undoped } & \multicolumn{2}{|c|}{ Sm doped } & \multicolumn{2}{|c|}{ Eu doped } \\
\hline & $\mathrm{E}(\mathrm{eV})$ & $\mathrm{T}(\mathrm{K})$ & $\mathrm{E}(\mathrm{eV})$ & $\mathrm{T}(\mathrm{K})$ & $\mathrm{E}(\mathrm{eV})$ & $\mathrm{T}(\mathrm{K})$ \\
\hline 1 & $0.45 \pm 0.15$ & $370 \pm$ & - & - & $0.60 \pm 0.1$ & $350 \pm 5$ \\
\hline 2 & $0.70 \pm 0.2$ & $440 \pm 20$ & $0.80 \pm 0.1$ & $440 \pm 10$ & $0.75 \pm 0.05$ & $430 \pm 5$ \\
\hline 3 & - & - & $1.00 \pm 0.1$ & $485 \pm 20$ & $1.00 \pm 0.1$ & $480 \pm 10$ \\
\hline
\end{tabular}

trap as for the undoped material.

\subsection{Discussion of $x$-ray response}

The undoped material shows a broad blue emission (see the black line in figure 8.16); this is a property of the host material independent of the samarium or europium doping. This host lattice emission is similar to that observed for the undoped LSCBC and reported for $\mathrm{SrCl}_{2}$ [76]. There is also a similar intrinsic emission observed in $\mathrm{BaBr}_{2}$ [149] and $\mathrm{BaCl}_{2}$ [109] which has been attributed to $\mathrm{F}$ centre and $\mathrm{V}_{k}$ centre radiative recombination. It was suggested this was also the cause of the similar emission in $\mathrm{SrCl}_{2}$ [76]. It would seem likely the emission observed 


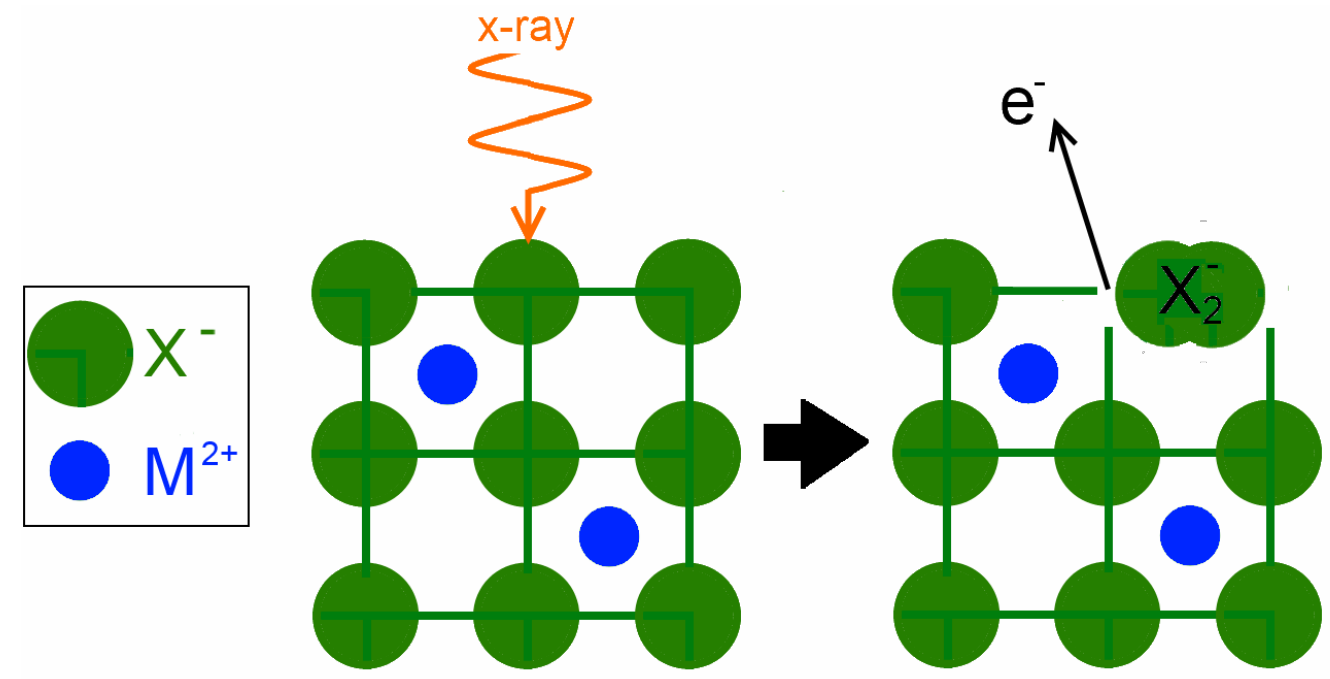

Figure 8.26: Depiction of possible trapping $\mathrm{H}$-F-centre recombination mechanism in $\mathrm{Ba}_{0.3} \mathrm{Sr}_{0.7} \mathrm{Cl}_{2}$.

here has a similar origin, but we propose that it involves $\mathrm{H}$ centres rather than $\mathrm{V}_{k}$ centres. The two both result in a $\mathrm{Cl}_{2}^{-}$molecular ion and have similar spectroscopic properties.

The suggested process is depicted in figure 8.26 , an incident $\gamma$-ray may simultaneously ionise and displace a $\mathrm{Cl}^{-}$ion from the lattice. The ionised electron may then be trapped at the anion vacancy left behind or at another distant anion vacancy. The displaced $\mathrm{Cl}^{-}$ion would likely move to a nearest neighbour interstitial site and associate with another lattice $\mathrm{Cl}^{-}$anion to form a $\mathrm{Cl}_{2}^{-}$molecular ion at an anion site, otherwise known as a $\mathrm{H}$ centre.

These traps would not be highly stable at room temperature and prompt recombination would occur as bound exciton emission (H centre + electron), this explains the broad blue emission in the undoped material.

This blue emission overlaps the $\mathrm{Sm}^{2+}$ excitation bands and to a lesser extent the $\mathrm{Eu}^{2+} \mathrm{ab}-$ sorption bands, and so in the samarium or europium doped material this blue emission may be absorbed by the $\mathrm{Sm}^{2+}$ or $\mathrm{Eu}^{2+}$ ions. In this case luminescence characteristic of $\mathrm{Sm}^{2+}$ or $\mathrm{Eu}^{2+}$ would be seen. The probability of seeing the host emission would decrease as the rare earth concentration increased as was observed. Direct excitation of $\mathrm{Sm}^{2+}$ or $\mathrm{Eu}^{2+}$ ions is also possible, an incident $\mathrm{x}$-ray is more likely to interact with these ions as their atomic numbers are larger than that of strontium, barium or chlorine. The $\mathrm{x}$-ray may ionise the $\mathrm{Sm}^{2+}$ or $\mathrm{Eu}^{2+}$ ion to give their trivalent forms $\left(\mathrm{Sm}^{3+}\right.$ or $\left.\mathrm{Eu}^{3+}\right)$, which could promptly recombine with the electron returning the ion to the divalent valence state in an excited state, resulting in $\mathrm{Sm}^{2+}$ 
or $\mathrm{Eu}^{2+}$ emission.

The typical concentrations used here for europium doping are much larger than those used for samarium doping because $\mathrm{Sm}^{2+}$ shows concentration quenching at relatively low concentrations. This matches with our observation of $\mathrm{Eu}^{2+}$ only luminescence for the europium doped samples.

The thermally stimulated luminescence (TSL) glow curves shows some interesting properties. The same low temperature trap $\left(\mathrm{T}_{\max } \sim 360 \mathrm{~K}\right)$ is observed for the undoped material and europium doped material. While the trap appears to be the same, the corresponding spectral emissions are different. In the europium doped material there must be some transfer of energy to the $\mathrm{Eu}^{2+}$ ions as it is the $\mathrm{Eu}^{2+}$ emission that is observed, whereas it is the blue emission observed from the same trap in the pure material. The samarium doped material does not have a glow curve peak corresponding to this low energy trap. The thermoluminescence signal intensities also vary between the materials. While we have no qualitative measure of this, the signal strength depends on many parameters such as the amount of material and the concentration, we can qualitatively say the signal from the europium doped material is very strong, that from the samarium doped material is moderate, and that from the undoped material is weak. Because the undoped material shows such a weak signal the emission due to black body radiation can be seen at high temperatures. For the samarium doping and europium doping this is dwarfed by the thermoluminescence signal.

Without extensive further measurements we cannot be unequivocal regarding the thermoluminescence mechanism. Here we consider just the dominant glow curve peaks and propose a mechanism which matches our low level experimental observations but would require further experimental verification.

It is widely recognised that alkaline earth halides commonly have impurities [150,151]. Eu ${ }^{3+}$ is a likely contaminant in our furnace/crucible infrastructure because it has been intensively studied at VUW. Assuming we have some unintentional $\mathrm{Eu}^{3+}$ impurities in our starting chemicals, this could provide an electron trap. Figure 8.27depicts our suggested trapping mechanism which is described in what follows. As a trivalent ion, this impurity will also have introduced extra anions. The extra anions may or may not be associated with the $\mathrm{Eu}^{3+}$ ion as 


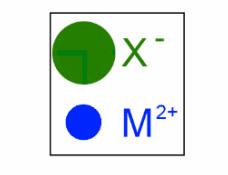

x-irradiation

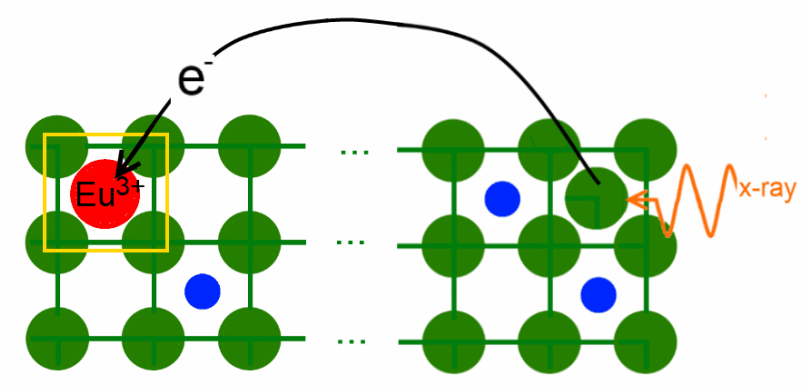

semi-stable

trap

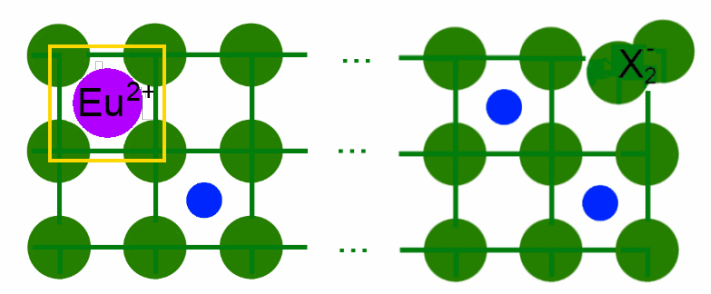

heating
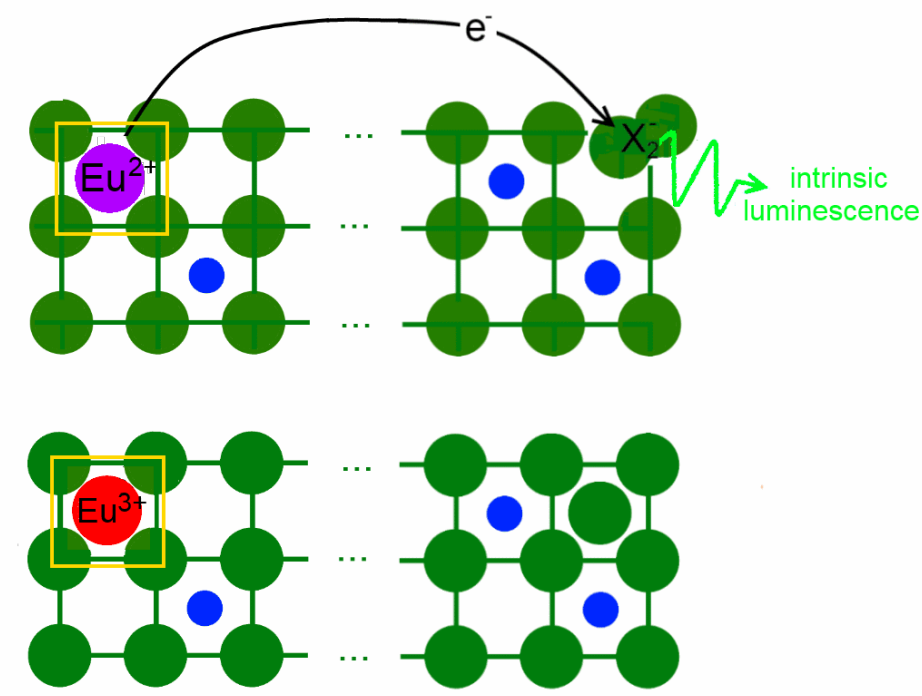

Figure 8.27: Depiction of possible TSL mechanism in nominally pure $\mathrm{Ba}_{0.3} \mathrm{Sr}_{0.7} \mathrm{Cl}_{2}$. The anions are shown in green, the cations in blue, $\mathrm{Eu}^{2+}$ ions in red and $\mathrm{Eu}^{3+}$ ions in purple. The anions surrounding the $\mathrm{Eu}^{3+}$ ions likely have some associated defect, this is indicated by the yellow square. 
it may or may not be charge compensated by a halide anion, or it may have some other charge compensation mechanism such as an $\mathrm{O}^{2-}$ ion; a mixture of compensated and uncompensated sites would likely occur [137]. For each $\mathrm{Eu}^{3+}$ not compensated by an interstitial anion there is an interstitial anion spatially distinct from the $\mathrm{Eu}^{3+}$ ion. On x-irradiation the interstitial anions may be ionised to form molecular ions, $\mathrm{H}$ centres. The ejected electron may then be attracted to the $\mathrm{Eu}^{3+}$ ion and become trapped. The trapped electron is bound to the $\mathrm{Eu}^{3+}$ ion giving a $\mathrm{Eu}^{2+}$ ion. This trap is stable at room temperature but on heating releases the electron, the electron may then find its way back to $\mathrm{H}$ centre where radiative recombination occurs and the intrinsic emission is seen.

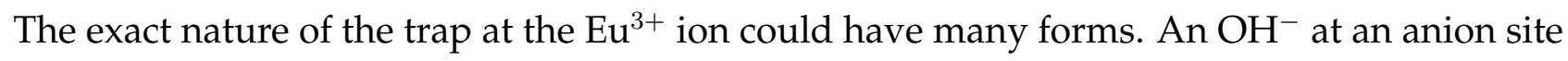
would seem likely; this allows for some level of charge compensation of the $\mathrm{Eu}^{3+}$ through the strong dipole of $\mathrm{OH}^{-}$molecular ion, but the electron released by the formation of an $\mathrm{H}$ centre would be strongly attracted to the trap as there is an overall positive charge. In contrast a $\mathrm{Eu}^{2+}-\mathrm{Cl}^{-}$pair is electrically neutral. $\mathrm{OH}^{-}$is a commonly observed impurity in alkaline earth halide lattices [152].

In the case of $\mathrm{Eu}^{2+}$ doping, we would expect many more traps. $\mathrm{EuCl}_{2}$ almost always has some fraction of $\mathrm{EuCl}_{3}$. Therefore the TSL signal would increase. The mechanism for $\mathrm{Eu}^{2+}$ doping is shown in figure 8.28 . The trap is the same, but the ionisation (and recombination) occur at a $\mathrm{Eu}^{2+}$ ion so the luminescence observed is that of $\mathrm{Eu}^{2+}$. The $\mathrm{Eu}^{2+}$ ion can be ionised by incoming $\mathrm{x}$-rays to leave an uncompensated $\mathrm{Eu}^{3+}$ ion. When the material is heated and the electron released from the trap it can return to the uncompensated $\mathrm{Eu}^{3+}$ ion and luminescence will be observed.

In the samarium doped material, a different trap depth is seen. In this case the mechanism would be the same as for the europium doped material but replacing all europium ions with samarium ions. There is some evidence of trap depths (above room temperature) changing with trivalent rare earth ion [137]. The TSL signal from $\mathrm{Ba}_{0.3} \mathrm{Sr}_{0.7} \mathrm{Cl}_{2}: \mathrm{Eu}^{2+}$ corresponding to this peak would correspond to samarium contamination in the preparation process.

While it is true we would expect to see the same $\mathrm{Eu}^{3+}$ traps in the samarium doped material as in the undoped material there are two reasons these might not be observed. Firstly they 


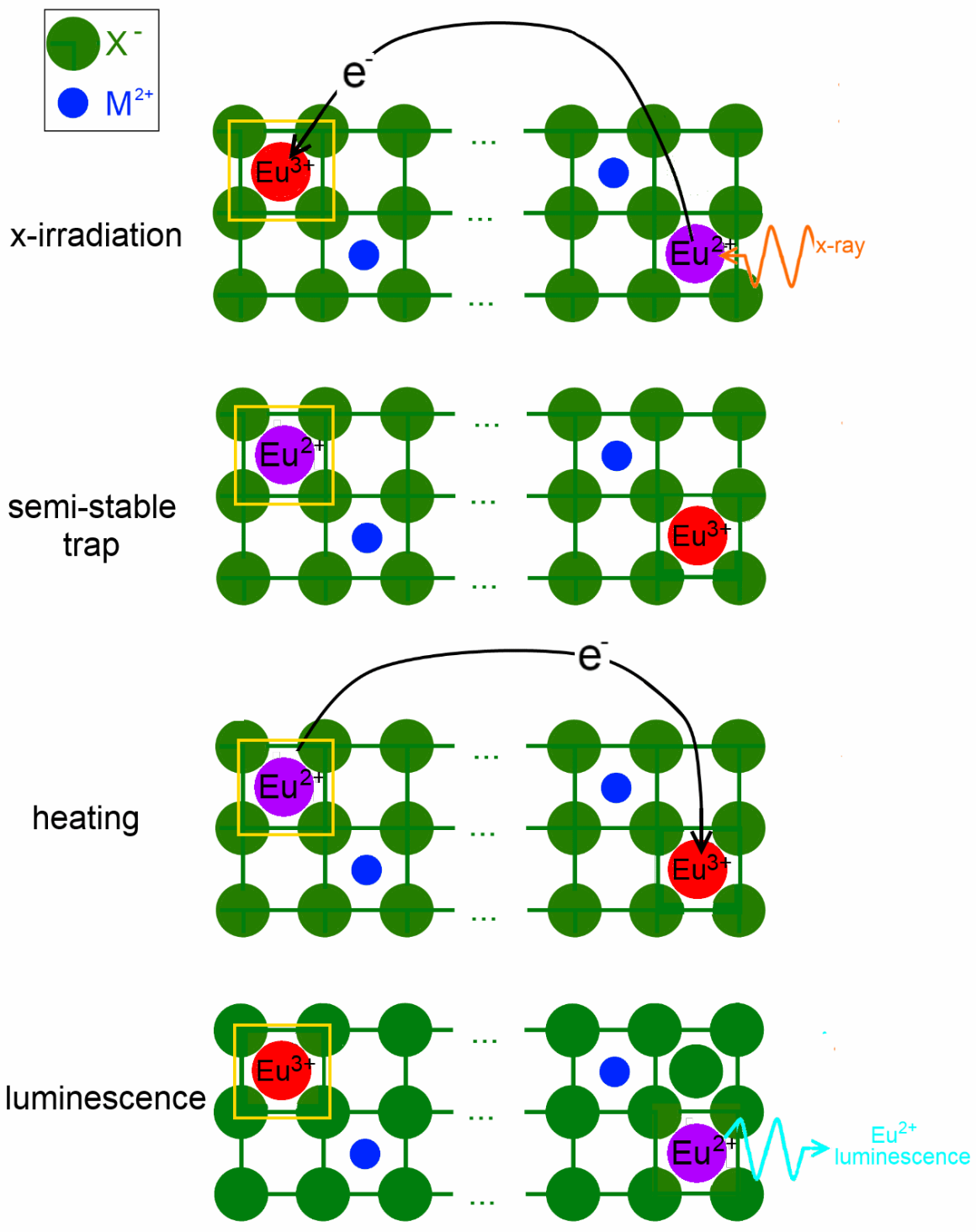

Figure 8.28: Depiction of possible TSL mechanism in europium doped $\mathrm{Ba}_{0.3} \mathrm{Sr}_{0.7} \mathrm{Cl}_{2}$. The red are cations, the green are fluorines, purple are blue are $\mathrm{Eu}^{3+}$ and $\mathrm{Eu}^{3+}$ respectively. The anions surrounding the $\mathrm{Eu}^{3+}$ ions likely have some associated defect, this is indicated by the yellow square.

could well be present but in a much lower concentration, as discussed above the signal from the undoped material is weak. Secondly the trapping probabilities would be expected to be different for $\mathrm{Eu}^{3+}$ and $\mathrm{Sm}^{3+}$.

This model allows the same trap depth for undoped and doped europium material but different emissions. It allows for a different trap depth for samarium doping. This model relies on the presence of $\mathrm{Eu}^{3+}$ impurities in undoped $\mathrm{Ba}_{0.3} \mathrm{Sr}_{0.7} \mathrm{Cl}_{2}$ and incomplete conversion of the trivalent dopant ions (samarium and europium). While we observed no evidence of trivalent samarium or europium in our materials, any spectroscopic absorption or emission 
from these ions would be very weak as the concentration would be low. Furthermore in the materials also containing divalent ion any trivalent ion emission or absorption would likely be dwarfed by the very strong allowed $5 \mathrm{~d} \rightarrow 4 \mathrm{f}$ transitions.

Sastry et al. [151] observed similar glow curves for $\mathrm{SrCl}_{2}$ and $\mathrm{SrCl}_{2}: \mathrm{Eu}^{2+}$ and proposed a similar trapping mechanism for the europium doped material. The difference between the models is the location of the hydroxyl group. Sastry et al. [151] associate this with the divalent europium ion as opposed to the trivalent ion which we suggest. We suggest it would be more likely to associate with the trivalent ion for both electrostatic and elastic reasons. We would expect the dipole of the hydroxyl group to mean it preferentially associates with the trivalent ion which has a charge mismatch to the lattice cations. As the trivalent ion is smaller than the divalent ion we would expect the hydroxyl group to 'fit' better at a site near the trivalent ion. Furthermore if the $\mathrm{Eu}^{2+}$ ion is associated with the hydroxyl group the emission observed should be of a perturbed site yet we see the same emission from photoluminescence and thermally stimulated luminescence.

\subsection{Summary}

Transparent polycrystalline $\mathrm{Sm}^{2+}$ and $\mathrm{Eu}^{2+}$ doped cubic $\mathrm{Ba}_{0.3} \mathrm{Sr}_{0.7} \mathrm{Cl}_{2}$ samples were easily produced without cracking. The spectroscopy of $\mathrm{Ba}_{0.3} \mathrm{Sr}_{0.7} \mathrm{Cl}_{2}: \mathrm{Sm}^{2+}$ is similar to that of LSCBC: $\mathrm{Sm}^{2+}$ but the crystal field appears stronger in $\mathrm{Ba}_{0.3} \mathrm{Sr}_{0.7} \mathrm{Cl}_{2}: \mathrm{Sm}^{2+}$. The effect of this is seen in a reduction of lifetime and red-shift of the emission band. The reduction of lifetime occurs as the $4 \mathrm{f}^{5} 5 \mathrm{~d}^{1}$ emission has a different, faster feeding rate. The materials are very bright phosphors with integrated intensities of $30 \%$ and $40 \%$ that of $\mathrm{Gd}_{2} \mathrm{O}_{2} \mathrm{~S}: \mathrm{Tb}^{3+}$ for $\mathrm{Sm}^{2+}$ and $\mathrm{Eu}^{2+}$ doping respectively. All the materials investigated show scintillation. The lifetime of the red scintillation from $\mathrm{Ba}_{0.3} \mathrm{Sr}_{0.7} \mathrm{Cl}_{2}: \mathrm{Sm}^{2+}$ is $\sim 30 \mu \mathrm{s}$, too long for most scintillation applications but satisfactory for a fast $\mathrm{x}$-ray phosphor. For $\mathrm{Ba}_{0.3} \mathrm{Sr}_{0.7} \mathrm{Cl}_{2}: \mathrm{Eu}^{2+}$ the scintillation lifetime was $1.6 \mu \mathrm{s}$. The imaging resolution is better than that for $\mathrm{Gd}_{2} \mathrm{O}_{2} \mathrm{~S}: \mathrm{Tb}^{3+}$. While this was measured for $\mathrm{Sm}^{2+}$ only, the resolution is a property of the polycrystalline character and the scattering, as opposed to the luminescent centre, so we expect the same for $\mathrm{Eu}^{2+}$ doping. There is an intrinsic host lattice emission of $\mathrm{Ba}_{0.3} \mathrm{Sr}_{0.7} \mathrm{Cl}_{2}$ which is likely due to $\mathrm{H}$ and 


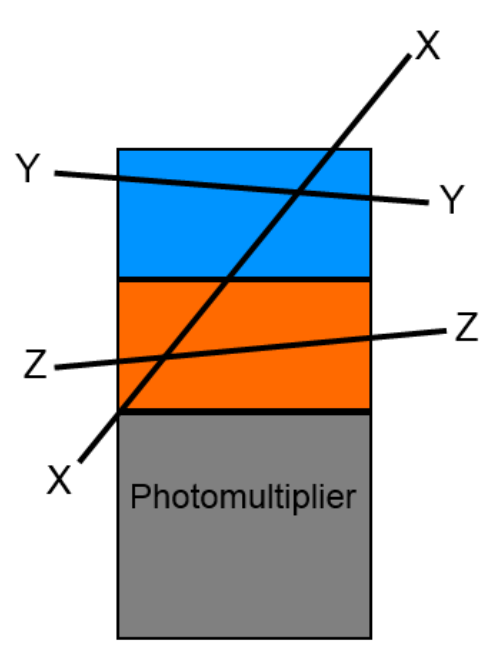

(a)

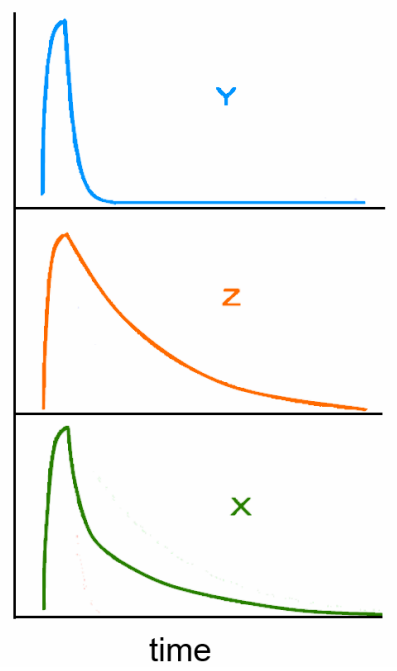

(b)

Figure 8.29: Phoswich design and expected pulse shapes, a $\gamma$-ray travelling on the path $Y-Y$ will give a fast pulse, $\gamma$-ray travelling on the path $Z-Z$ will give a long pulse and a $\gamma$-ray travelling on the path $X-X$ will give a composite pulse [153].

F centre recombination. There are significant traps, stable above room temperature, which are attributed to trivalent rare earth impurities and likely slow the scintillation process. The neutron sensitivity of these materials was also investigated, and this reported in appendix A.

\subsection{Phoswich application}

\subsubsection{Introduction}

The concept of a phoswich (phosphor sandwich) was originally introduced by D. H. Wilkinson in 1952 for directional sensing with one photodetector [153]. He proposed the basic phoswich system as shown in figure 8.29(a), where the top (blue) phosphor has a fast decay time and the lower (orange) phosphor has a much slower decay time, the pulse detected then depends on the path of the radiation. Figure $8.29(\mathrm{~b})$ shows the pulse variation depending on the path of the $\gamma$-ray. Wilkinson tested this set-up with $\mathrm{NaI}(\mathrm{Tl})$ and stillbene scintillators and was able to distinguish the three different shaped pulses depicted in figure 8.29 .

More recently phoswich detectors have been used for dual energy imaging [154]. This is a well known technique and is commonly used in commercial systems such as airport baggage 
scanners and bone density scans. The basic concept relies on the difference of the attenuation of x-rays through different materials. As discussed previously in chapter 3 , the attenuation depends on the chemical properties of the material, the energy of the x-ray and the thickness of the material. The number of photons of energy $E$ transmitted per second through a thickness $z$ of material with an attenuation coefficient of $\mu(E)$ is given by

$$
N=N_{0} \exp (-\mu(E) z)
$$

This means a standard x-ray image can be the same for a thick piece of material with a low attenuation coefficient or a thick piece with a high attenuation coefficient. One must know either the thickness or the material to extract the other. If one has two successive materials in the beam path the number of photons transmitted becomes

$$
N=N_{0}\left(\exp \left(-\mu_{1}(E) z_{1}\right) \times \exp \left(-\mu_{2}(E) z_{2}\right)\right.
$$

Knowledge of the materials, and so $\mu_{1}$ and $\mu_{2}$, is not sufficient to solve this equation. However, for a second x-ray beam at a different energy $\left(E^{\prime}\right)$ we have

$$
N=N_{0}\left(\exp \left(-\mu_{1}\left(E^{\prime}\right) z_{1}\right) \times \exp \left(-\mu_{2}\left(E^{\prime}\right) z_{2}\right)\right.
$$

With two equations and two unknowns (in this case the thicknesses $z_{1}$ and $z_{2}$ ) a unique solution can be found. This is important for diagnostics. For example the images can be subtracted to give separate tissue and bone maps, in medical radiography this can show if lesions have calcified. Figure 8.30 shows a commercial example of image subtraction.

In general dual energy imaging is done using either a one-shot or two shot technique. A two shot technique takes two distinct images with two different x-ray beams. This offers high signal to noise ratio but exposes the patient to two x-ray doses and allows for movement (for example respiration) between images which can blur features. The other main technique called 'one shot' uses just one x-ray beam with two detectors separated by a copper filter, this changes the beam by absorbing some of the lower energy component. This offers less contrast between the beams, but eliminates the effects of any patient movement between 

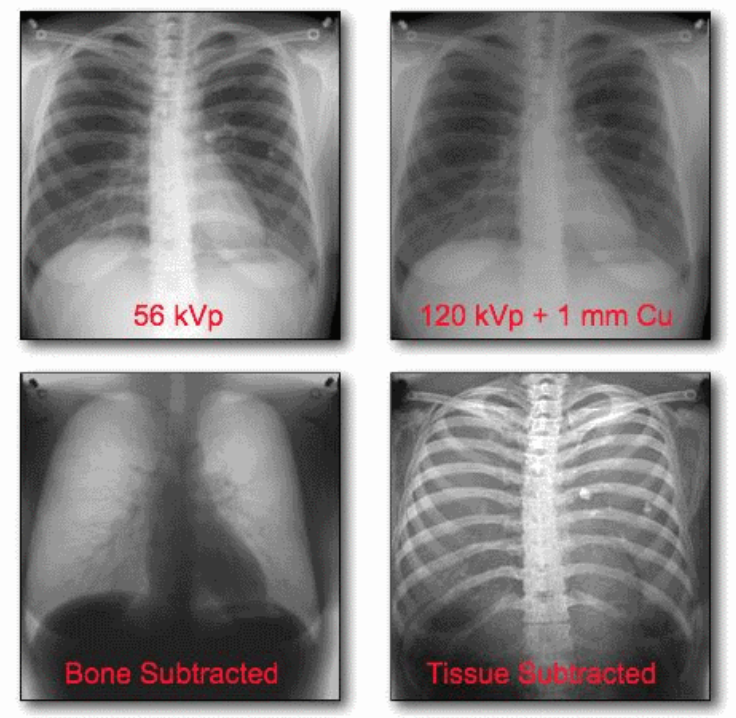

Figure 8.30: Commercial example of image subtraction providing separate bone and tissue maps of lungs and ribs [155]

images. Both these techniques require two read outs of the two images.

Using our new x-ray phosphor materials $\mathrm{Ba}_{0.3} \mathrm{Sr}_{0.7} \mathrm{Cl}_{2}:\left(\mathrm{Sm}^{2+}\right.$ or $\left.\mathrm{Eu}^{2+}\right)$ we propose a new phoswich system, in which the energy discrimination is based on the emission wavelengths as opposed to pulse shape analysis which is normally used [156, 157]. We can use the europium and samarium emissions in a two layer system. The attenuation of the x-ray beam in our top layer detector will be sufficient to change the beam to a different energy by the time it reaches the second layer. Using our set-up for imaging with a semi-professional grade CMOS digital camera we can utilise the three colour channels with responses peaked at 450 , 530 and $600 \mathrm{~nm}$ [96] to detect the different emissions in separate channels with a single read out.

\subsubsection{Energy discrimination with $\mathrm{Ba}_{0.3} \mathrm{Sr}_{0.7} \mathrm{Cl}_{2}: \mathrm{RE}\left(\mathrm{RE}=\mathrm{Sm}^{2+}\right.$ or $\left.\mathrm{Eu}^{2+}\right)$}

A 'phoswich' stratified detector was constructed from a thin disc of $\mathrm{Ba}_{0.3} \mathrm{Sr}_{0.7} \mathrm{Cl}_{2}$ : $\mathrm{Eu}^{2+}$ on top of a thicker $\mathrm{Ba}_{0.3} \mathrm{Sr}_{0.7} \mathrm{Cl}_{2}: \mathrm{Sm}^{2+}$ disc. As shown in figure $8.31(\mathrm{a})$ the relative intensities of the x-ray induced luminescence from these two layers of the phoswich depends on the energy of the incident beam. We find that the relative emission band intensities show a clear variation with mean x-ray energy, as shown in figure 8.31(b). This can be simply understood from the greater penetration of a higher energy x-ray beam so that the red emission from the lower 


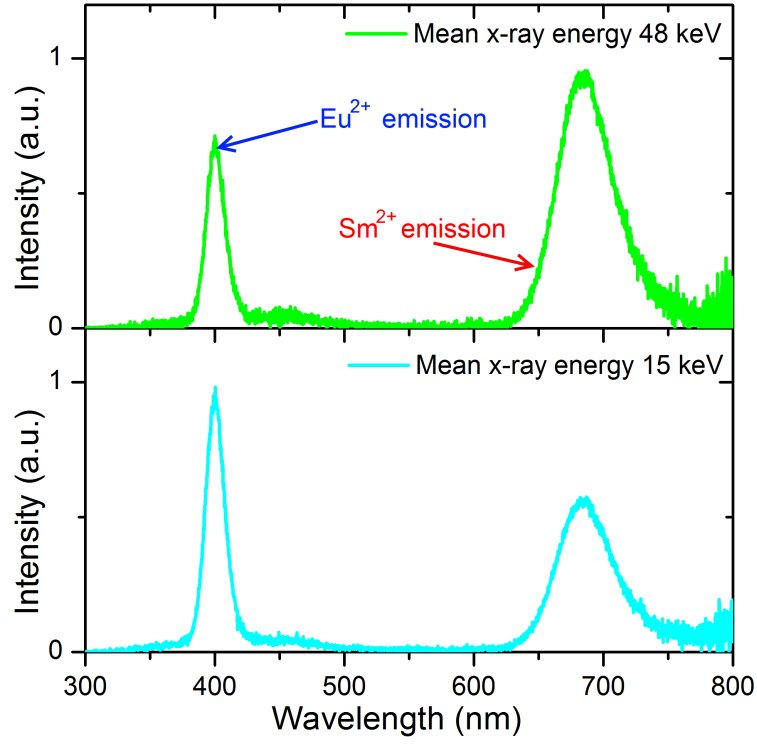

(a)

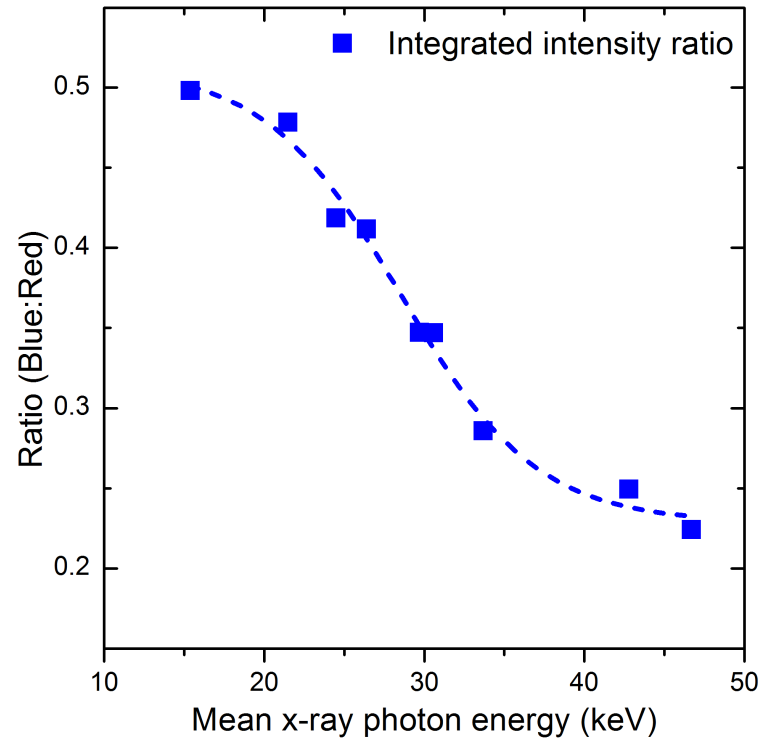

(b)

Figure 8.31: (a) $\mathrm{Ba}_{0.3} \mathrm{Sr}_{0.7} \mathrm{Cl}_{2}$ phoswich response to x-irradiation of different mean energies. (b) Plot of ratio of integrated intensities of $\mathrm{Eu}^{2+}$ emission to $\mathrm{Sm}^{2+}$ emission as a function of mean x-ray energy.

layer will increase relative to the blue from the top layer with x-ray energy. In this case the europium and samarium doped layers were 300 and $2000 \mu \mathrm{m}$ thick respectively.

Figure 8.32 shows an optical arrangement designed to make use of this energy discrimination in x-ray imaging, and which can potentially reveal composition information for a target object. Different elements in the target may be distinguished by the different dependencies of their x-ray absorption cross sections with energy. With a thin europium doped layer in front of a thicker samarium doped in the phoswich structure, two images are formed, one in the blue camera channel from the top disc, and one in the red channel from the bottom disc, and corresponding to different mean beam energies. This is because as the x-ray beam passes through the first layer the lower energy $x$-rays are preferentially absorbed leaving a hardened higher energy beam to be absorbed in the second thicker layer. Using mass energy attenuation coefficients to calculate the spectrum of x-rays absorbed in each layer and then integrating we have calculated the mean energy of the absorbed beam to be $30 \mathrm{keV}$ and 48 $\mathrm{keV}$ for a $500 \mu \mathrm{m}$ europium doped layer on a $1000 \mu \mathrm{m}$ samarium doped layer respectively. This calculation was based on the maximum energy of the polychromatic beam from the (tungsten anode) x-ray set being $70 \mathrm{keV}$. As our top europium doped layer is transparent to red light, the samarium emission can pass through it, and so we can record both the red and 

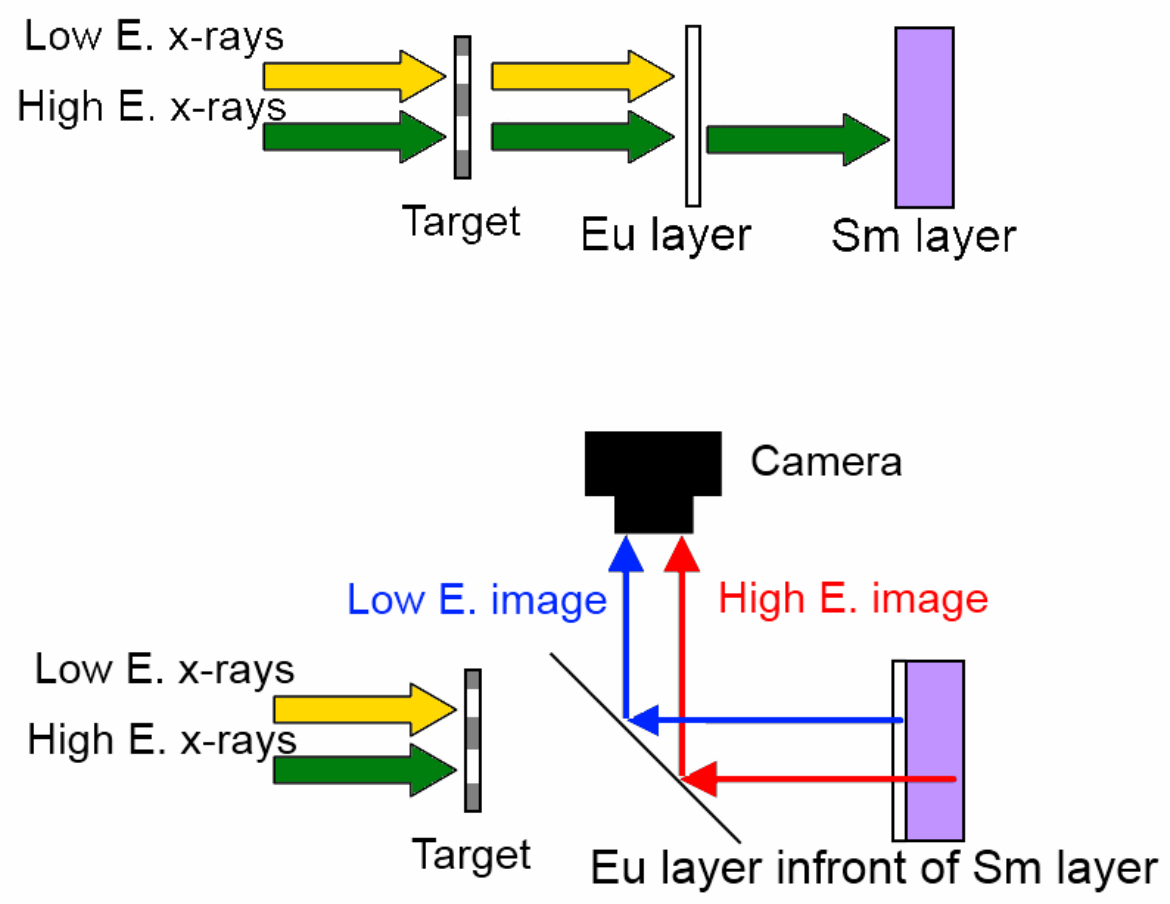

Figure 8.32: $\mathrm{Ba}_{0.3} \mathrm{Sr}_{0.7} \mathrm{Cl}_{2}$ phoswich set-up utilising camera detector.

blue images with the aid of a thin (x-ray transparent) plane mirror and a semi-professional grade digital SLR camera [96], as shown in figure 8.32 .

The different emissions are detected in different colour channels of the camera allowing for a single read out of the two images formed. The blue, green and red camera channels have peak sensitivity at 450, 530 and $600 \mathrm{~nm}$ [96]. While the phoswich emissions (samarium at $680 \mathrm{~nm}$ and europium at $406 \mathrm{~nm}$ ) are not perfectly matched to the peak channel wavelengths, there is minimal overlap of the bandpass and so it is possible to completely separate the images from the two layers of the phoswich. Figure 8.33 shows the low energy and high energy images of an IC chip, where the image is mainly generated by the metal tracks, legs and pads. The silicon chip at the centre of the IC is itself mounted on a metal pad. The low energy image shows little or no difference in brightness between the centre of the IC, where the silicon chip displaces most of the encapsulant, and the peripheral parts where the thickness comprises a conducting track and (mostly) the encapsulant. However the high energy image shows a clear contrast between those two zones.

We note the top europium doped layer image is much sharper than the bottom samarium doped layer image; this is due to both scattering and focusing effects. The camera is focused 

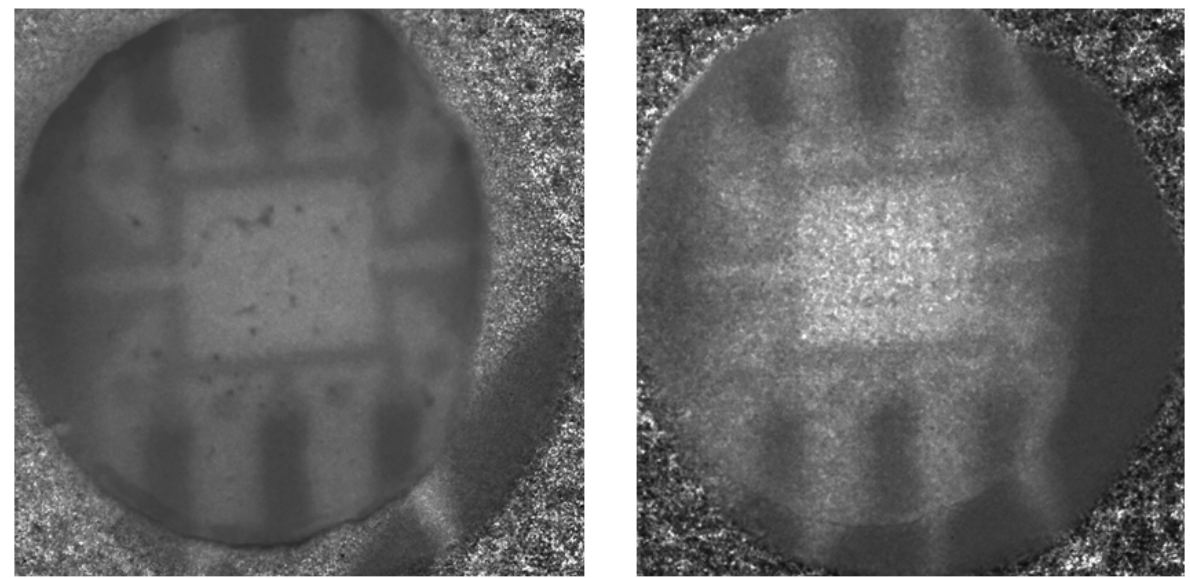

Figure 8.33: (a)Low and (b) high energy images of 8 pin integrated circuit chip.

on the top layer, so the finite depth of field of the camera means the bottom layer is not so well focused. Also as the lower layer is much thicker there is more scattering in that layer. Finally, the emission from the bottom layer must pass through the top layer which again increases the chance of scattering. Another potential cause of image distortion is the chance of the emission from the europium doped layer exciting photoluminescence in the samarium doped layer. To minimize this effect we interspersed a thin long-pass red cellophane filter between the two. This means the europium emission is blocked from reaching the red-emitting layer and exciting the $\mathrm{Sm}^{2+}$ ions, but the samarium emission is not prevented from reaching the detector. However this is a potential source of emission intensity loss due to internal reflections. 



\section{Chapter 9}

\section{Samarium doped calcium fluoride}

\subsection{Introduction}

In chapter 8 it was found that $\mathrm{Ba}_{0.3} \mathrm{Sr}_{0.7} \mathrm{Cl}_{2}: \mathrm{Sm}^{2+}$ showed red scintillation but the scintillation applications of this material are limited by the relatively long lifetime. For counting and coincidence applications, such as positron emission tomography (PET), a faster scintillation lifetime is required. While $\mathrm{Sm}^{2+}$ ions frequently show bright $4 \mathrm{f}^{5} 5 \mathrm{~d}^{1} \rightarrow 4 \mathrm{f}^{6}$ luminescence, the lifetime of this emission seems to be limited to the microsecond range. This was seen experimentally for $\mathrm{LSCBC}: \mathrm{Sm}^{2+}$ and $\mathrm{Ba}_{0.3} \mathrm{Sr}_{0.7} \mathrm{Cl}_{2}: \mathrm{Sm}^{2+}$ in the preceding chapters, and is generally consistent with the simulations (performed by Dr. Reid) described in chapter 7 . Further evidence of this is found when one looks at the $\mathrm{Sm}^{2+}$ ion emission in a range of alkali halide and alkaline earth halide lattices. Figure 9.1 shows the lifetime of the dominant $\mathrm{Sm}^{2+}$ emission in a range of alkaline earth halide host lattices as a function of temperature. These are recorded at the ${ }^{5} \mathrm{D}_{0} \rightarrow{ }^{7} \mathrm{~F}_{1}$ transition energy.

Two main trends can be observed from figure 9.1. Firstly, at the lowest temperatures the lifetime is generally $\sim 10 \mathrm{~ms}$. Secondly, as the temperature increases, the lifetime decreases. The strong temperature dependence of $\mathrm{Sm}^{2+}$ ion luminescence arises from the proximity of the $4 f^{5} 5 d^{1}$ and the $4 f^{6}\left({ }^{5} D_{0,1}\right)$ excited states as shown in chapter 7 and, in some lattices, the conduction band. Variations in crystal lattices change both the band gap and the crystal field influencing the $4 f^{5} 5 d^{1}$ excited state levels. While the exact level locations of the $4 f^{5} 5 d^{1}$ 


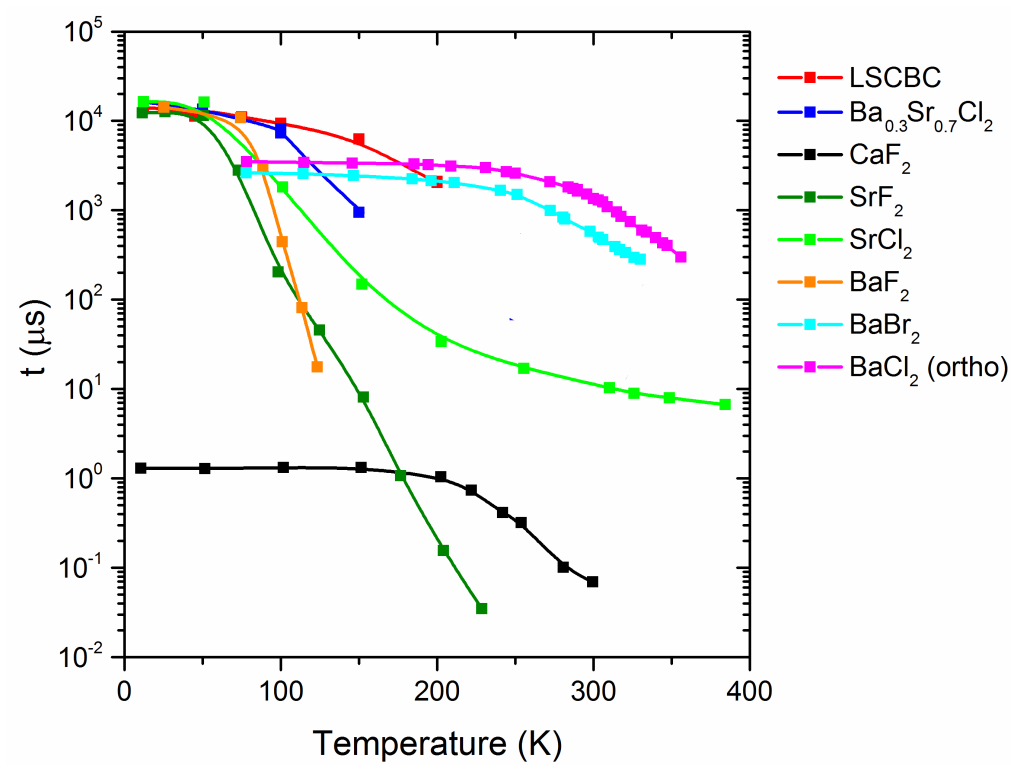

Figure 9.1: The lifetime of the dominant emission from $\mathrm{Sm}^{2+}$ ions in various alkaline earth halide lattices as a function of temperature. The lines are a guide to the eye. The data points for LSCBC and $\mathrm{Ba}_{0.3} \mathrm{Sr}_{0.7} \mathrm{Cl}_{2}$ are taken from chapters 7 and 8 of this work. The data points for $\mathrm{CaF}_{2}, \mathrm{SrF}_{2}, \mathrm{SrCl}_{2}$ and $\mathrm{BaF}_{2}$ are from reference [158] and for $\mathrm{BaBr}_{2}$ and $\mathrm{BaCl}_{2}$ from reference [128].

excited states vary, the lowest excited state is normally above the ${ }^{5} \mathrm{D}_{0}$ state. A generalised level structure is depicted in figure 9.2 .

At the lowest temperatures the main emission is from the lowest excited state, generally the ${ }^{5} \mathrm{D}_{0}$ state, and the $\sim 2$ - $20 \mathrm{~ms}$ lifetime recorded is the radiative lifetime of this state. As the temperature is increased the rate of competing non-radiative processes increases, and the overall lifetime decreases. In lattices where the $4 \mathrm{f}^{5} 5 \mathrm{~d}^{1}$ emission is seen the temperature dependence is very strong. This is the same behaviour seen in chapters 7 and 8 . While these trends hold for most alkaline earth halides, there is an anomaly: $\mathrm{CaF}_{2}: \mathrm{Sm}^{2+}$. At low temperatures the lifetime of $\mathrm{Sm}^{2+}$ ion luminescence in $\mathrm{CaF}_{2}$ is much faster than other $\mathrm{Sm}^{2+}$ doped alkali earth halides (1.3 $\mu$ s compared to $\left.\sim 10^{4} \mu \mathrm{s}\right)$. The crystal field is known to increase with cation size $[82-85]$ which makes the crystal field stronger in $\mathrm{CaF}_{2}$ than say $\mathrm{BaF}_{2}$, and so the crystal field in $\mathrm{CaF}_{2}$ is sufficiently large that the lowest $4 \mathrm{f}^{5} 5 \mathrm{~d}^{1}$ is actually below the $4 \mathrm{f}^{6}\left({ }^{5} \mathrm{D}_{0}\right)$ excited state [130, 159, 160]. This is more commonly seen in alkali halides and means the emission at the lowest temperatures is from the $4 \mathrm{f}^{5} 5 \mathrm{~d}^{1}$ configuration and is electric dipole allowed. As such, the lifetime of $\mathrm{Sm}^{2+}$ is much faster in $\mathrm{CaF}_{2}$ than in other alkaline earth halide lattices. 


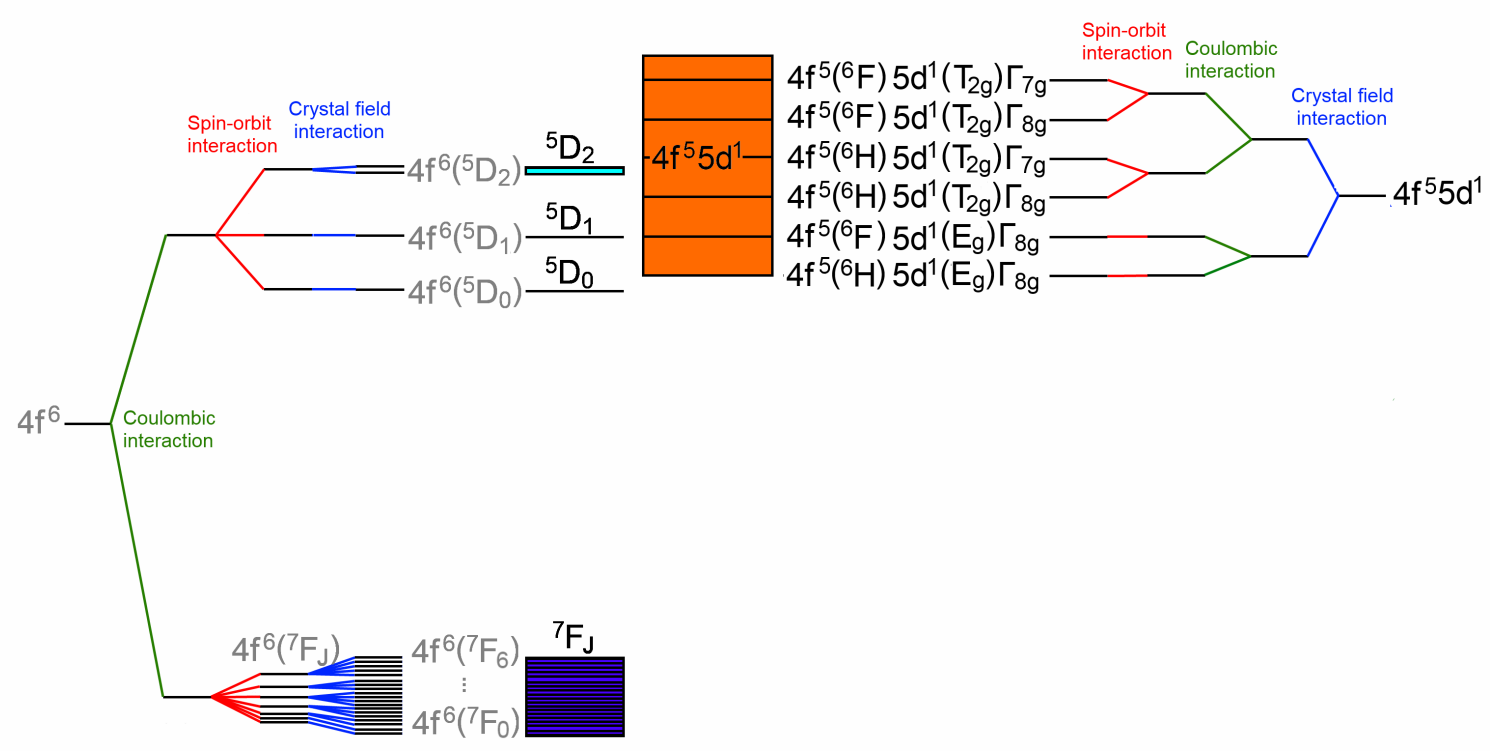

Figure 9.2: Depiction of the energy levels for $\mathrm{Sm}^{2+}$ in a cubic crystal site.

Above $200 \mathrm{~K}$ the lifetime of $\mathrm{Sm}^{2+}$ in $\mathrm{CaF}_{2}$ decreases as non-radiative transitions become significant. The rate of decrease in lifetime and the temperature at which the non-radiative processes become significant depends on the relative positions of the $4 \mathrm{f}^{5} 5 \mathrm{~d}^{1}$ emitting level, the $4 \mathrm{f}^{6}\left({ }^{5} \mathrm{D}_{0,1}\right)$ levels and the conduction band. In $\mathrm{CaF}_{2}: \mathrm{Sm}^{2+}$ luminescence is observed right up to $300 \mathrm{~K}$. In contrast the luminescence of $\mathrm{BaF}_{2}$ and $\mathrm{SrF}_{2}$ is completely quenched at room temperature as excitation to the conduction band becomes thermally activated below $300 \mathrm{~K}$. The band gap of $\mathrm{CaF}_{2}$ is approximately $12.1 \mathrm{eV}$ compared to $10.6 \mathrm{eV}$ for $\mathrm{BaF}_{2}$ [161], allowing for luminescence to be observed over a greater temperature range.

This decrease in luminescent lifetime of $\mathrm{CaF}_{2}: \mathrm{Sm}^{2+}$ comes at the cost of intensity. For a simple single level system the rate of change of population of the excited state $\left(N_{E}\right)$ is given by

$$
\frac{d N_{E}}{d t}=R_{\text {pump }}-N_{E}\left(R_{\text {rad }}+R_{\text {non-rad }}\right)
$$

Where $R_{\text {pump }}$ is the excitation rate into the excited state, and $R_{\text {rad }}$ and $R_{\text {non-rad }}$ the rates of de-excitation by radiative and non-radiative routes respectively. The light output $(I)$ observed will be proportional to the radiative de-excitation rate

$$
I \propto \int h v N_{E} R_{r a d} \mathrm{~d} v
$$

where $h v$ is the energy of the emitted photon. From setting equation 9.1 to zero for a steady 
state such as continuous wave excitation

$$
I \propto \int h v \mathrm{~d} v \frac{R_{\text {rad }} R_{\text {pump }}}{R_{\text {rad }}+R_{\text {non-rad }}}
$$

and as the observed lifetime $(\tau)$ is given by

$$
\tau=\frac{1}{R_{\text {rad }}+R_{\text {non-rad }}}
$$

the light output is proportional to

$$
\int h v \mathrm{~d} v R_{\text {rad }} R_{\text {pump }} \tau
$$

furthermore $R_{\text {rad }}$ is temperature independent so the change in light output as a function of temperature is directly proportional to the change in observed lifetime. As the lifetime of $\mathrm{CaF}_{2}$ decreases the light output must also decrease at the same rate. As discussed in chapter 3 . all scintillators have strengths and weaknesses, a high light output is required for computed tomography $(\mathrm{CT})$, but is desirable not critical for PET for example. Thus we investigate the possibility of using $\mathrm{CaF}_{2}: \mathrm{Sm}^{2+}$ as a fast red scintillator for coupling to semiconductor detectors.

$\mathrm{CaF}_{2}: \mathrm{Sm}^{2+}$ has been known to show luminescence for many years, and it was first investigated primarily due to the maser action of the material at low temperatures, discovered by Kaiser et al. in 1961 [162]. Both the spectroscopy of $\mathrm{CaF}_{2}: \mathrm{Sm}^{3+}[163,164]$ and $\mathrm{CaF}_{2}: \mathrm{Sm}^{2+}[130,162]$ have been reported previously. The low temperature thermally stimulated luminescence and effect of $\gamma$ irradiation have also been reported [165]. However, no account of the scintillation or x-ray phosphor characteristics of $\mathrm{CaF}_{2}: \mathrm{Sm}^{2+}$ could be found. $\mathrm{CaF}_{2}: \mathrm{Eu}^{2+}$ is a commercially available scintillator with a light output of 24,000 photons per MeV [166, 167]. Due to the low atomic numbers it offers low back-scattering, so is usually used for detecting $\beta$ particles and low-energy $\gamma$-rays.

In our investigation of this material we were first kindly lent some $\mathrm{CaF}_{2}: \mathrm{Sm}$ single crystals by Dr. John Campbell of Canterbury University to investigate before we took on the task of growing our own polycrystalline material. Preliminary investigations of these samples 


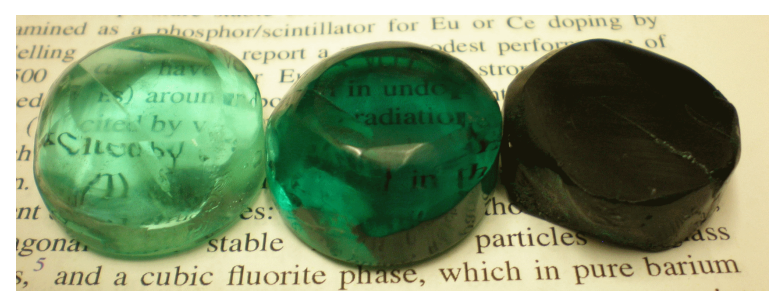

Figure 9.3: Photograph of $\mathrm{CaF}_{2}: \mathrm{Sm}$ samples for various samarium dopant concentrations. The concentrations are $0.01 \%, 0.1 \%$ and $1 \%$ from left to right.

showed we could indeed observe fast red scintillation from the materials, and so we undertook to make our own polycrystalline samples. All reported measurements are of samples produced at Victoria University.

\subsection{Physical properties}

The samples were made by melting anhydrous $\mathrm{CaF}_{2}$ single crystal chips, samarium metal powder and $\mathrm{PbF}_{2}$ powder. Samarium doping was also attempted with $\mathrm{SmF}_{3}$ but it was found the samarium metal was most effective at achieving $\mathrm{Sm}^{2+}$ doping. While the incorporation of $\mathrm{O}^{2-}$ ions in $\mathrm{CaF}_{2}$ does not change the luminescence of $\mathrm{CaF}_{2}: \mathrm{Sm}^{2+}$ [168], the lattice does incorporate oxygen easily and we wish to work with the pure material. Lead fluoride was therefore added to act as a oxygen scavenger via the reaction

$$
\mathrm{PbF}_{2}+\mathrm{O}^{2-} \rightarrow \mathrm{PbO}+2 \mathrm{~F}^{-}
$$

The constituent chemicals were heated up to $1465^{\circ} \mathrm{C}$ over a period of an hour in a glassy carbon crucible. The temperature was then held at $1465{ }^{\circ} \mathrm{C}$ for fifteen minutes. The melt was then slow cooled to $1210{ }^{\circ} \mathrm{C}$ over the period of eight hours before being cooled to room temperature over the period of ten hours. In this manner large (up to $15 \mathrm{~g}$ ) samples could be formed without cracking. Shorter cooling times resulted in cracking of the boules. As shown in figure 9.3 the colouration of samples ranged from light green for $0.01 \%$ samarium doping to almost black for $1 \%$ samarium doping.

Shown in figure 9.4 is the file x-ray diffraction pattern for $\mathrm{CaF}_{2}$ and a representative powder $\mathrm{x}$-ray diffraction pattern of our samples. The agreement between the two patterns is excellent 


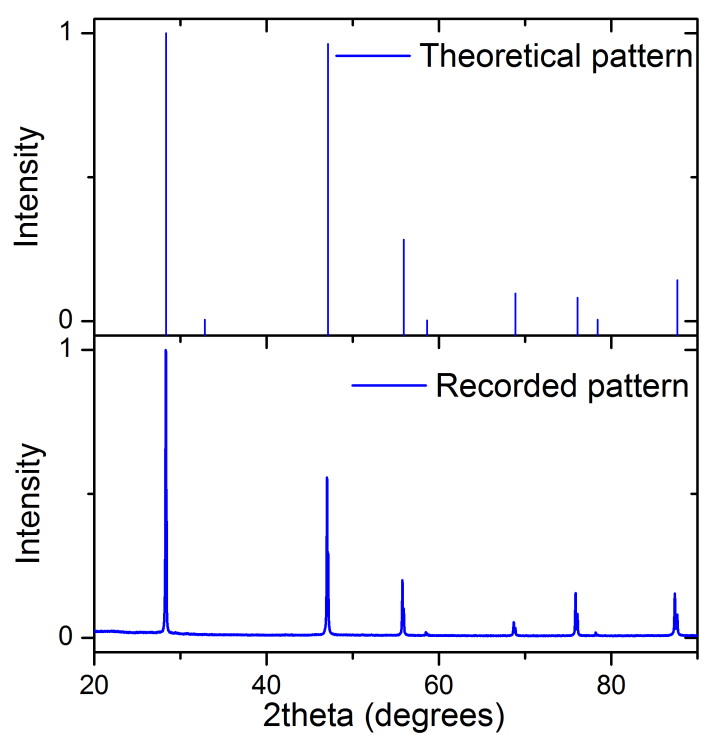

Figure 9.4: File x-ray diffraction pattern for $\mathrm{CaF}_{2}: \mathrm{Sm}(0.1 \%)$ (top) and experimental powder pattern.

confirming the samples are cubic $\mathrm{CaF}_{2}$. There is no evidence of any other phase or material.

Figure 9.5 shows the x-ray half thickness of $\mathrm{CaF}_{2}$ compared to that of the commercial scintillator $\mathrm{NaI}(\mathrm{Tl})$. While calcium and fluorine do not have particularly high atomic numbers, the x-ray half thickness is comparable to that of the $\mathrm{NaI}(\mathrm{Tl})$ in the range of 100 to $1000 \mathrm{MeV}$. Outside this energy range the half-thickness of $\mathrm{CaF}_{2}$ is an order of magnitude greater than that of $\mathrm{NaI}(\mathrm{Tl})$.

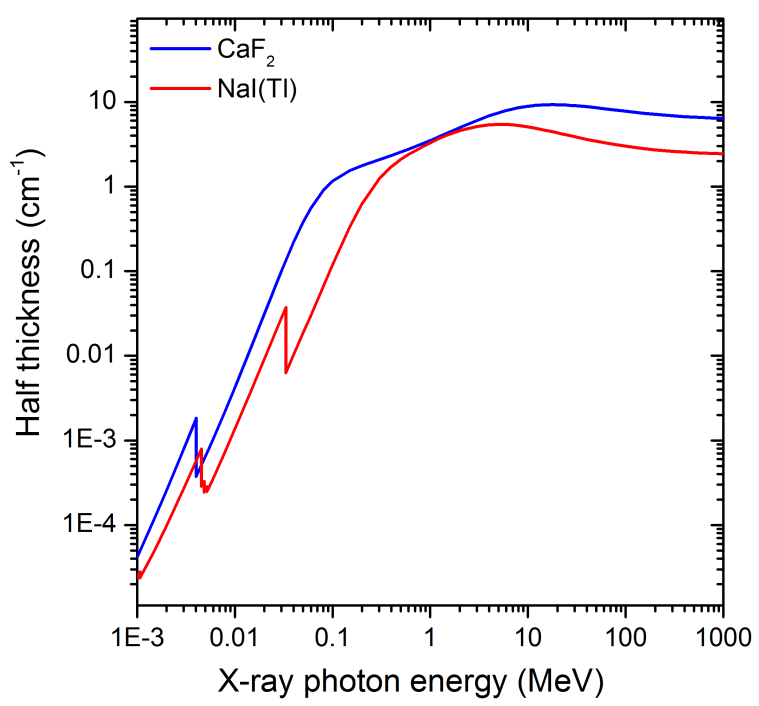

Figure 9.5: X-ray half thickness of $\mathrm{CaF}_{2}$ and $\mathrm{NaI}(\mathrm{Tl})$ as a function of x-ray energy. Data taken from the NIST database [145]. 


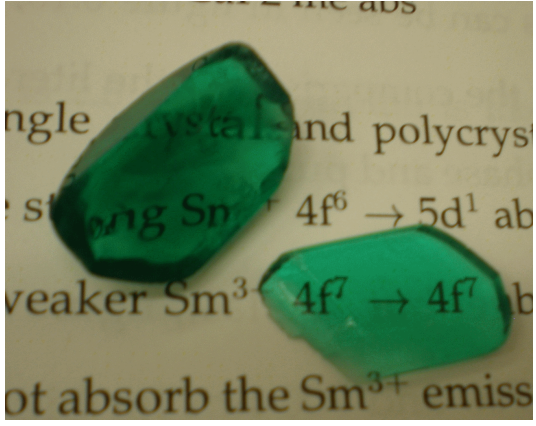

(a)

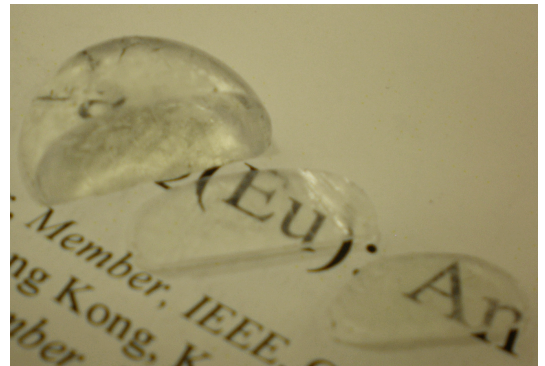

(b)

Figure 9.6: Photographs of $\mathrm{CaF}_{2}$ doped with $0.1 \%$ samarium (a) and $0.2 \%$ europium (b). Both samples show cleavage faces indicative of single crystals.

The samples produced where presumed to be polycrystalline, however, as shown in figure 9.6(a) they showed cleavage faces on fracturing. Cleavage faces are a feature of single crystals and not polycrystalline materials, and so it appeared that we had unintentionally produced large single crystals of $\mathrm{CaF}_{2}: \mathrm{Sm}$. Electron spin resonance (ESR) is one way of checking the crystallinity. The ESR spectrum of a material depends on the orientation of the magnetic field with respect to the crystal structure, thus the ESR spectrum of a rare earth doped single crystal is vastly different to the corresponding polycrystalline material and this technique can be used to clarify the crystallinity. Unfortunately $\mathrm{Sm}^{2+}$ does not show an ESR signal as there are an even number of electrons. Instead, as $\mathrm{Eu}^{2+}$ has on odd number of electrons $\left(4 \mathrm{f}^{7}\right.$ ground state configuration), europium doped $\mathrm{CaF}_{2}$ was prepared as for the samarium doped materials. As shown in figure 9.6(b) these were transparent and showed cleavage faces as for samarium doping.

Figure 9.7 shows the ESR spectrum recorded for $\mathrm{CaF}_{2}: \mathrm{Eu}^{2+}$ compared to the patterns simulated for a single crystal and for a polycrystalline material. The simulations were performed using Easyspin software [169] with parameters given in table 9.1. The recorded signal is an excellent match to that simulated for a single crystal. This is a very interesting result. We have produced large (15 g) single crystal samples in a RF furnace without any intention of doing so. This likely arises from a thermal gradient across the furnace due to a small gap in the insulation together with the slow cooling rate.

Given this surprising result, the materials in previous chapters were checked to ensure that they were indeed polycrystalline, and so ESR was also recorded for $\mathrm{Ba}_{0.3} \mathrm{Sr}_{0.7} \mathrm{Cl}_{2}$ : $\mathrm{Eu}^{2+}$. Figure 9.8 shows the ESR recorded for $\mathrm{Ba}_{0.3} \mathrm{Sr}_{0.7} \mathrm{Cl}_{2}: \mathrm{Eu}^{2+}$ compared to that simulated for $\mathrm{SrCl}_{2}: \mathrm{Eu}^{2+}$ 


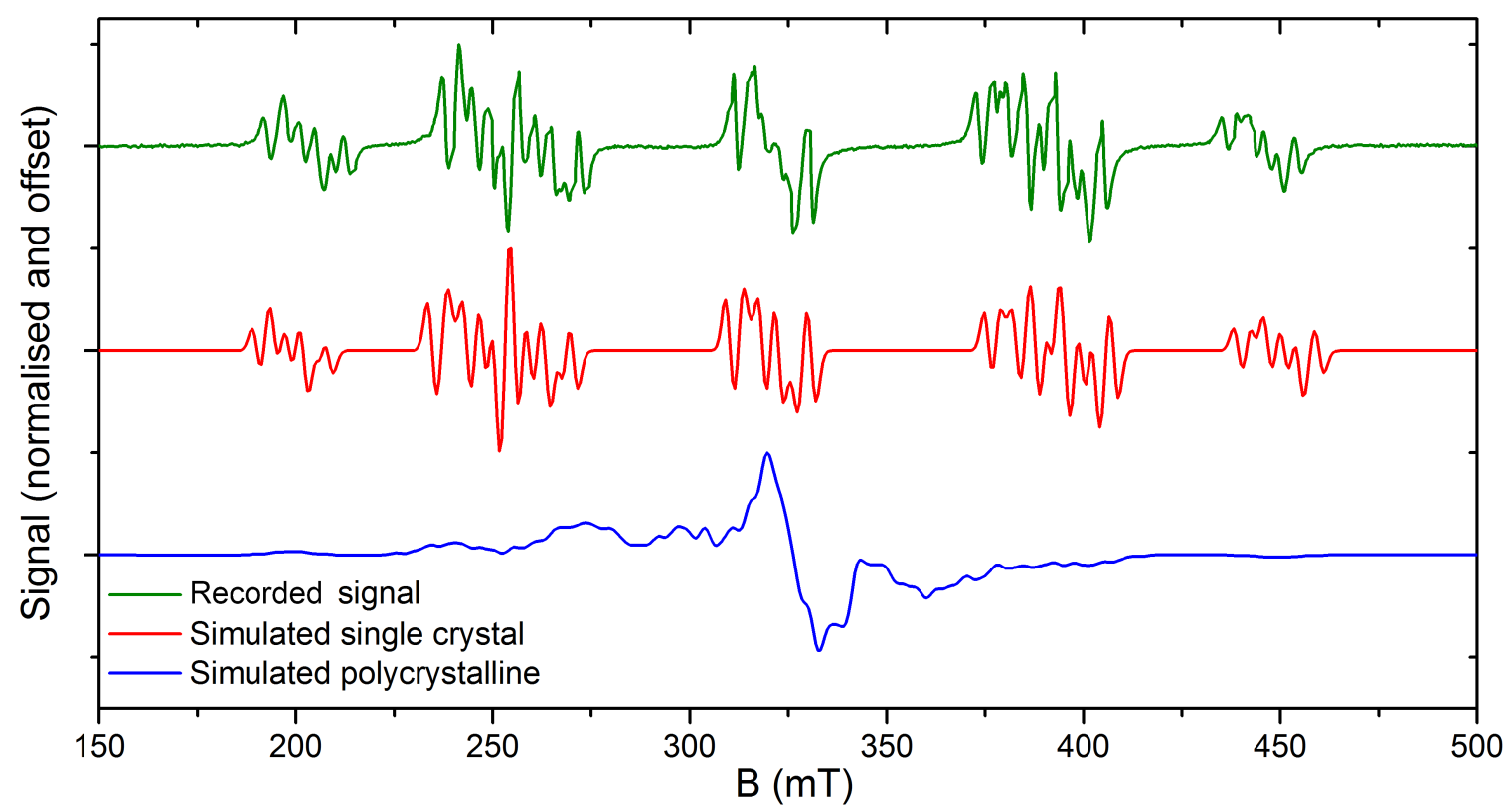

Figure 9.7: Electron spin resonance spectra of $\mathrm{CaF}_{2}: \mathrm{Eu}^{2+}$; the recorded spectrum (top), the simulated spectrum for a single crystal aligned with the magnetic field parallel to the $<100>$ axis (middle) and the simulated spectrum for a polycrystalline material (bottom).

Table 9.1: Electron spin resonance parameters used for Easyspin simulation.

\begin{tabular}{|c|c|c|c|c|c|c|c|}
\hline Lattice & $\mathrm{g}$ & $\begin{array}{c}\mathrm{A}_{151} \\
\left(10^{-4} \mathrm{~cm}^{-1}\right)\end{array}$ & $\begin{array}{c}\mathrm{A}_{153} \\
\left(10^{-4} \mathrm{~cm}^{-1}\right)\end{array}$ & $\begin{array}{c}\mathrm{b}_{4} \\
(\mathrm{MHz})\end{array}$ & $\begin{array}{c}\mathrm{b}_{6} \\
(\mathrm{MHz})\end{array}$ & $\begin{array}{c}\text { frequency } \\
(\mathrm{GHz})\end{array}$ & Reference \\
\hline $\mathrm{CaF}_{2}$ & 1.9926 & 36.9 & 16.4 & -176.12 & 0.78 & 9.004 & [170] \\
\hline $\mathrm{SrCl}_{2}$ & 1.995 & 32 & 14.2 & -178.10 & 0.12 & 9.014 & {$[171]$} \\
\hline
\end{tabular}

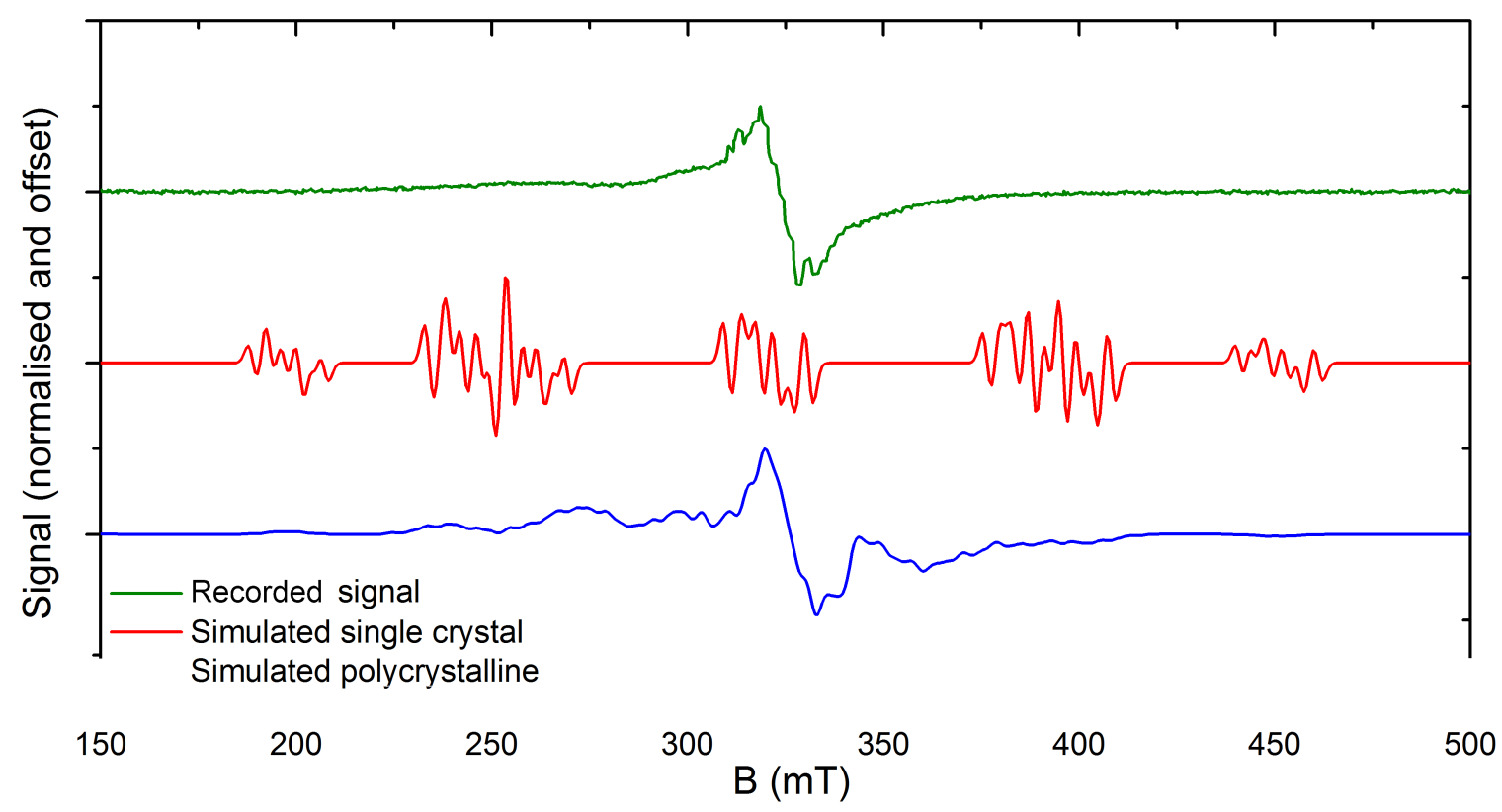

Figure 9.8: Electron spin resonance spectra of $\mathrm{Ba}_{0.7} \mathrm{Sr}_{0.3} \mathrm{Cl}_{2}: \mathrm{Eu}^{2+}$; the recorded spectrum (top), the simulated spectrum for a single crystal aligned with the magnetic field parallel to the $<100>$ axis (middle) and the simulated spectrum for a polycrystalline material (bottom). 
in single crystal and polycrystalline form. Again the simulations were performed using Easyspin software [169] and parameters used are given in table 9.1. Given the similarities between $\mathrm{Ba}_{0.3} \mathrm{Sr}_{0.7} \mathrm{Cl}_{2}: \mathrm{Eu}^{2+}$ and $\mathrm{SrCl}_{2}: \mathrm{Eu}^{2+}$ they should show similar ESR spectra. Figure 9.8 shows the $\mathrm{Ba}_{0.3} \mathrm{Sr}_{0.7} \mathrm{Cl}_{2}: \mathrm{Eu}^{2+}$ spectrum matches the polycrystalline spectrum of $\mathrm{SrCl}_{2}$ and not the single crystal.

We chose to work with polycrystalline materials because they are inexpensive and fast to produce. However, if we can produce large single crystals in the same manner there is no disadvantage. Single crystals have excellent optical properties, and should be potentially superior to a polycrystalline material.

\subsection{Optical spectroscopy}

\subsubsection{Absorption}

Figure 9.9 shows the absorption spectrum of $\mathrm{CaF}_{2}: \mathrm{Sm}$. This is dominated by the strong $\mathrm{Sm}^{2+}$ $4 \mathrm{f}^{6} \rightarrow 4 \mathrm{f}^{5} 5 \mathrm{~d}^{1}$ absorption bands which match those reported previously for $\mathrm{CaF}_{2}: \mathrm{Sm}^{2+}[130]$. There is no evidence of $\mathrm{Sm}^{3+}$ absorption, which is expected around $17800 \mathrm{~cm}^{-1}$ [132]. Any $\mathrm{Sm}^{3+} 4 \mathrm{f}^{5} \rightarrow 4 \mathrm{f}^{5}$ transitions would likely be much weaker than the $\mathrm{Sm}^{2+} 4 \mathrm{f}^{6} \rightarrow 5 \mathrm{~d}^{1}$ absorption bands.

\subsubsection{Photoluminescence excitation and emission spectra}

Shown in figure 9.10 is the photoluminescent excitation and emission spectra at room temperature and $12 \mathrm{~K}$. At room temperature the excitation spectrum follows the absorption spectrum and the emission spectrum shows two features; a broad band and some weaker sharp lines. The broad band emission is peaked at $13800 \mathrm{~cm}^{-1}(725 \mathrm{~nm})$. This is the $\mathrm{Sm}^{2+} 4 \mathrm{f}^{5} 5 \mathrm{~d}^{1} \rightarrow 4 \mathrm{f}^{6}$ transition. On cooling to $12 \mathrm{~K}$, the excitation spectrum shows some sharp lines superimposed on the broad bands, are these zero phonon absorption lines. In particular the lowest energy sharp line is at $14500 \mathrm{~cm}^{-1}(689 \mathrm{~nm})$ and is identified with the $4 \mathrm{f}^{6}\left({ }^{7} \mathrm{~F}_{0}\right) \rightarrow 4 \mathrm{f}^{5} 5 \mathrm{~d}^{1}\left(\Gamma_{8 g}\right)$ transition [130]. The emission spectrum at $12 \mathrm{~K}$ is dominated by one sharp line transition 


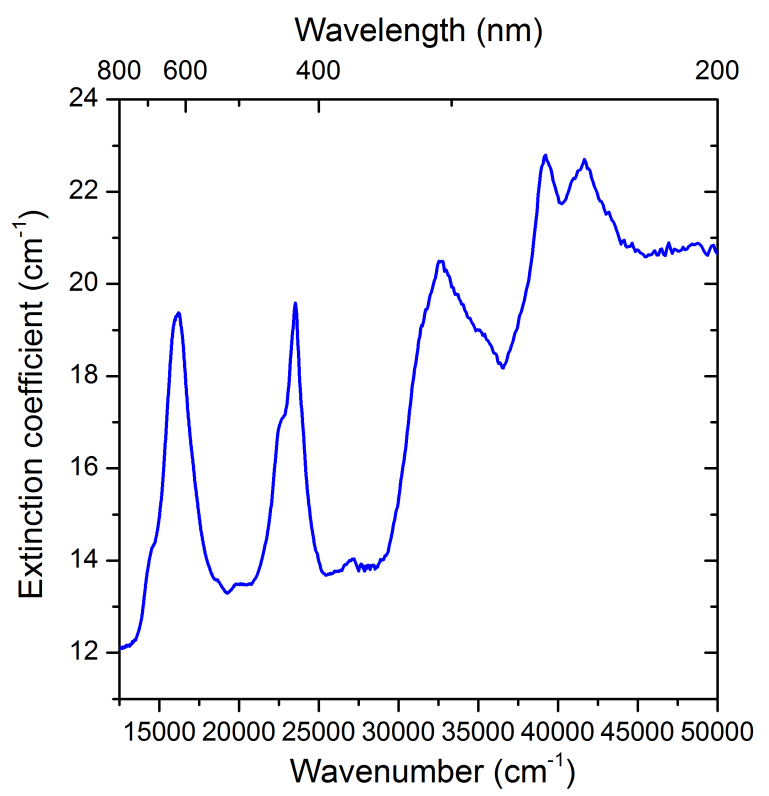

Figure 9.9: Absorption spectrum of $\mathrm{CaF}_{2}: \mathrm{Sm}(0.1 \%)$ at room temperature. The resolution is 1 nm.

at $14130 \mathrm{~cm}(708 \mathrm{~nm})$. Figure 9.11 shows the emission profile of $\mathrm{CaF}_{2}: \mathrm{Sm}^{2+}$ in more detail as the temperature is reduced from $300 \mathrm{~K}$ to $12 \mathrm{~K}$. The spectra are in good accordance with those previously reported [130]. The dominant sharp line is identified with the $4 \mathrm{f}^{5} 5 \mathrm{~d}^{1}\left(\Gamma_{8 g}\right) \rightarrow$ $4 \mathrm{f}^{6}\left({ }^{7} \mathrm{~F}_{1}\right)$ transition [130]. The continuum of lines of the low energy side of this transition are the transitions from the excited states of the $4 f^{5} 5 d^{1}$ configuration [130].

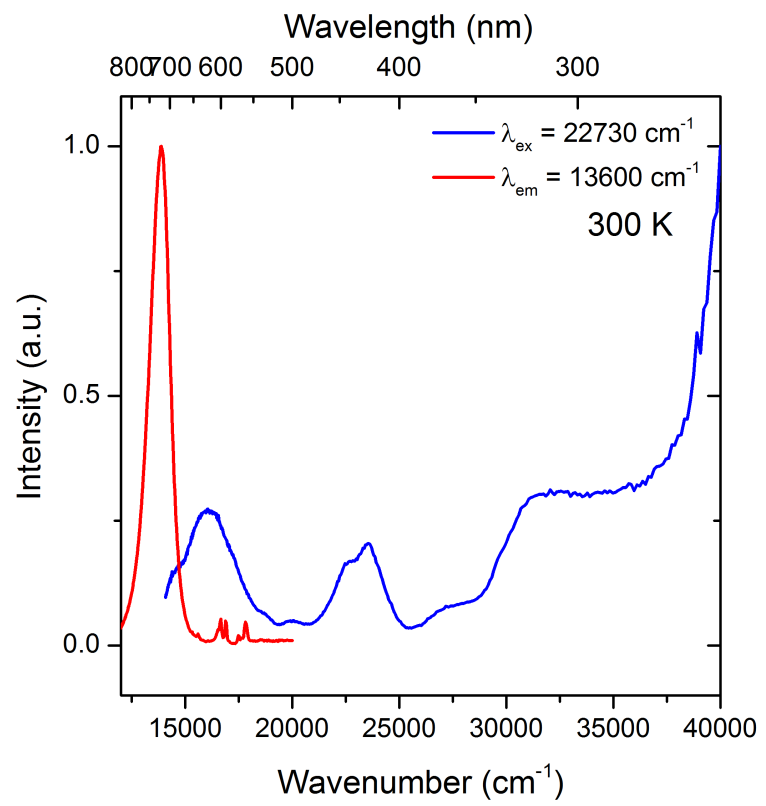

(a)

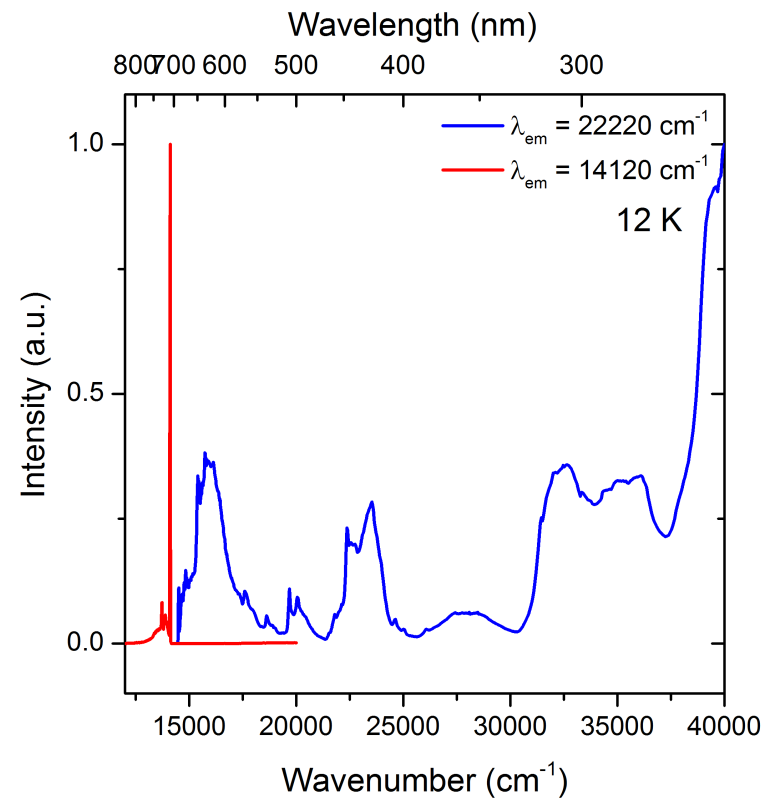

(b)

Figure 9.10: Photoluminescence excitation and emission spectra of $\mathrm{CaF}_{2}: \mathrm{Sm}(0.1 \%)$ at (a) 300 $\mathrm{K}$ and (b) $12 \mathrm{~K}$. The resolution is $1 \mathrm{~nm}$ for all spectra. 


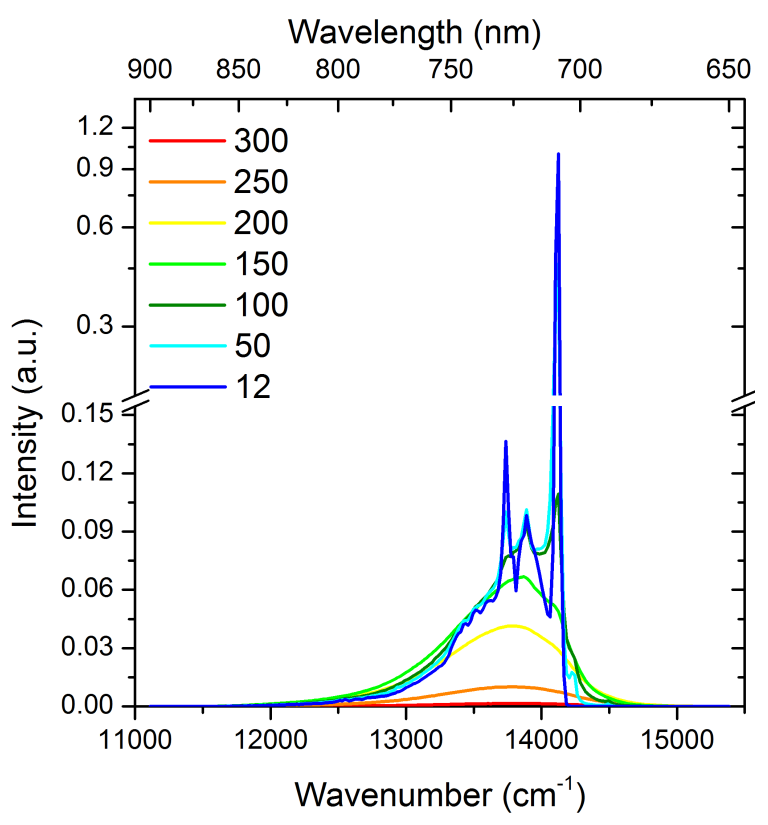

Figure 9.11: Photoluminescence emission spectra of $\mathrm{CaF}_{2}: \mathrm{Sm}(0.1 \%)$ between $300 \mathrm{~K}$ and $12 \mathrm{~K}$. The excitation energy was $22220 \mathrm{~cm}^{-1}$ and the resolution is $1 \mathrm{~nm}$ for all spectra.

The sharp lines visible in the room temperature emission spectrum (figure 9.10(a) between $16000 \mathrm{~cm}^{-1}(625 \mathrm{~nm})$ and $18000 \mathrm{~cm}^{-1}(556 \mathrm{~nm})$ match those reported for $\mathrm{CaF}_{2}: \mathrm{Sm}^{3+} 4 \mathrm{f}^{5} \rightarrow 4 \mathrm{f}^{5}$ transitions [132, 163]. At $12 \mathrm{~K}$ the $\mathrm{Sm}^{3+}$ luminescence is not visible.

The $\mathrm{Sm}^{3+}$ ions require charge compensation as they substitute for divalent cations. In $\mathrm{CaF}_{2}$ the dominant site for trivalent rare earth dopants at concentrations less than $0.1 \%$ is expected be a $\mathrm{C}_{4 v}$ site [132]. This site corresponds to charge compensation by a nearest neighbour interstitial fluorine along the $<100>$ direction [132]. At concentrations above $0.1 \%$ clusters preferentially form, these decay non-radiatively due to efficient cross-relaxation processes between the constituent $\mathrm{Sm}^{3+}$ ions [132]. A second site of $\mathrm{C}_{3 v}$ symmetry corresponding to charge compensation by an $\mathrm{O}^{2-}$ ion on an anion site is reported in the literature for $\mathrm{CaF}_{2}: \mathrm{Sm}^{3+}[132,163,172]$. Shown in figure 9.12 is the $\mathrm{Sm}^{3+}$ luminescence of samples of $\mathrm{CaF}_{2}: \mathrm{Sm}(0.1 \%)$ prepared with and without the addition of $\mathrm{PbF}_{2}$. The $\mathrm{Sm}^{3+}$ concentration is less than $0.1 \%$ as some conversion to $\mathrm{Sm}^{2+}$ has occurred and so, as the photoluminescence was weak only the dominant non-cluster related sites were observed. The emission lines which correspond to oxygen and fluorine charge compensation have been indicated above the spectra $[132,163,172]$. The lower spectrum, for a sample made without $\mathrm{PbF}_{2}$, shows emission lines corresponding to both sites. The upper spectrum, for a sample made with $\mathrm{PbF}_{2}$, shows predominantly the lines associated with an interstitial fluorine. This confirms 


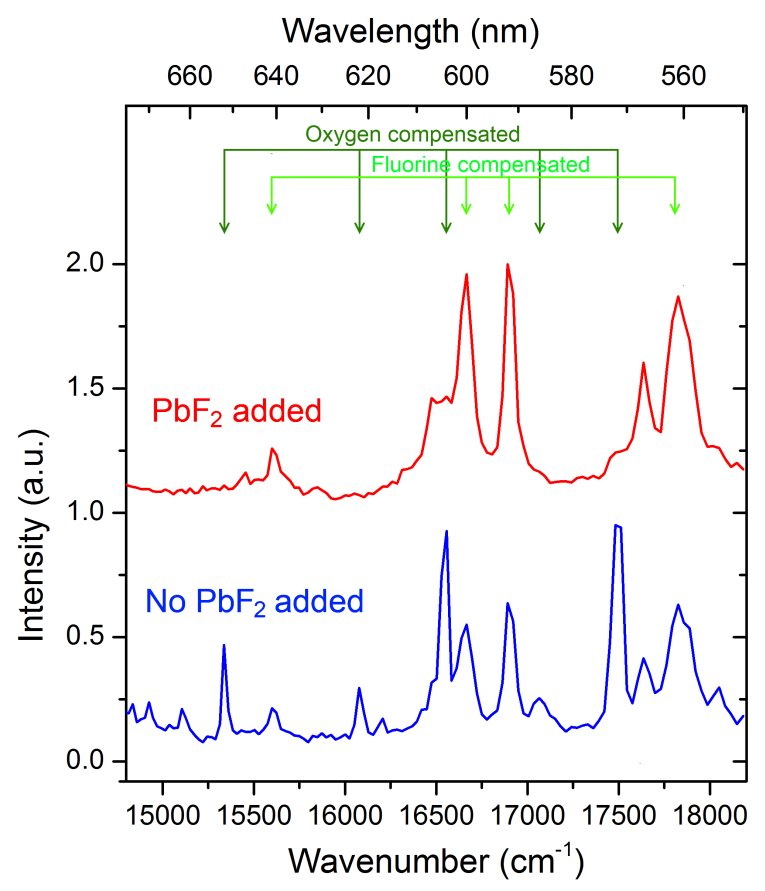

Figure 9.12: Photoluminescence emission of $\mathrm{CaF}_{2}: \mathrm{Sm}^{3+}$ prepared with and without $\mathrm{PbF}_{2}$ showing that different occur sites for the $\mathrm{Sm}^{3+}$ ion. Both spectra have been normalised. The bar diagrams above indicate the lines associated with the oxygen compensated site and fluorine interstitial compensated site [132, 163, 172]. These spectra are from samples doped with $\mathrm{SmF}_{3}$ and not samarium metal.

the addition of $\mathrm{PbF}_{2}$ is removing most of the $\mathrm{O}^{2-}$ ions present in the melt.

\subsubsection{Photoluminescent lifetimes}

Figure 9.13 shows the $\mathrm{CaF}_{2}: \mathrm{Sm}^{2+}$ photoluminescence decay profiles at $300 \mathrm{~K}$ and $12 \mathrm{~K}$. These have been fitted with single exponentials (with baselines) of $46 \mathrm{~ns}$ (300 K) and $1300 \mathrm{~ns}(12 \mathrm{~K}$ ) respectively. Figure 9.14 shows the room temperature decays of samples of concentrations $0.01 \%, 0.1 \%$ and $1 \%$ samarium doping. These have been fitted with exponential decays (with baselines) with time constants of 55 ns, 53 ns and 42 ns respectively. The lifetime decreases slightly with increasing concentration due to concentration quenching.

The decay was fitted with a single exponential decay (with a baseline) at various temperature between $12 \mathrm{~K}$ and $300 \mathrm{~K}$. A single exponential decay provides a good fit at all temperatures. Shown in figure $9.15(\mathrm{a})$ is the temperature variation of the lifetime recorded here as well as that reported in the literature [158]. In general the two data sets are similar, although the recorded lifetime at room temperature here is $46 \mathrm{~ns}$ compared to $\sim 90 \mathrm{~ns}$. The decay at 


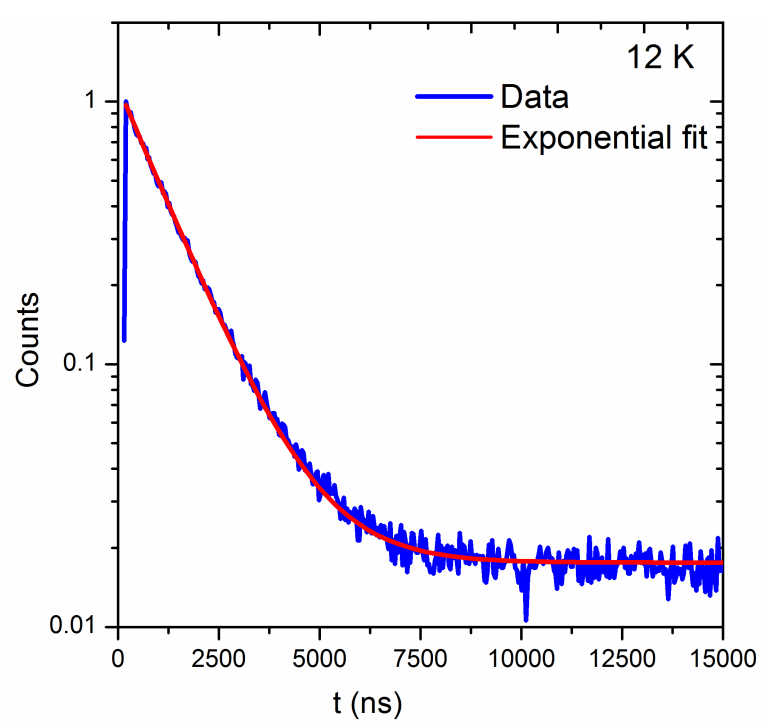

(a)

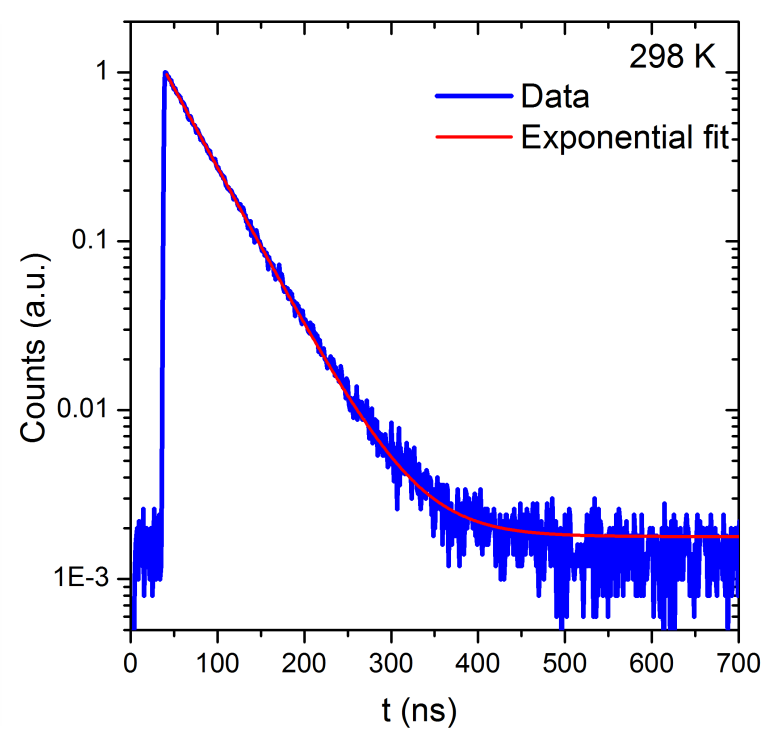

(b)

Figure 9.13: Photoluminescent lifetime of $\mathrm{CaF}_{2}: \mathrm{Sm}(0.5 \%)$ emission at (a) $12 \mathrm{~K}$ and (b) $300 \mathrm{~K}$. The excitation source was a $625 \mathrm{~nm}$ LED and the emission was recorded at $750 \mathrm{~nm}$ with a 10 $\mathrm{nm}$ bandpass. The decays have been fitted with exponential decays with lifetimes of $46 \mathrm{~ns}$ and $1.2 \mu$ s respectively.

room temperature was recorded with various excitation and emission wavelengths and no significant variation was found. The differences in lifetimes for measurements recorded here and in reference [158] are small, and likely arise from concentration differences.

Figure $9.15(\mathrm{~b})$ shows the normalised integrated intensity of the photoluminescence as a function of temperature, compared to the lifetime variation. As expected the temperature de-

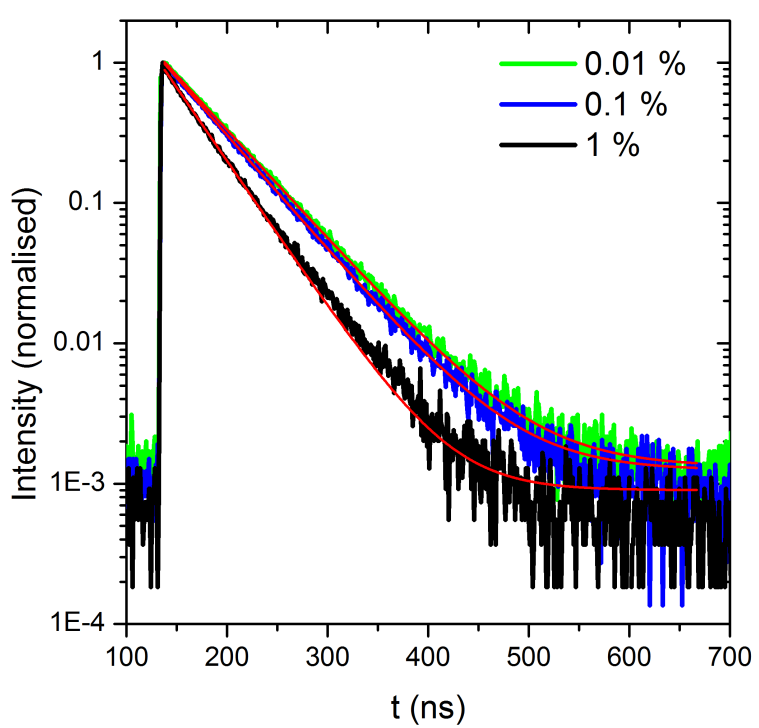

Figure 9.14: Photoluminescent lifetimes of $\mathrm{CaF}_{2}: \mathrm{Sm}$ for samarium concentrations of $0.01 \%$, $0.1 \%$ and $1 \%$ at $300 \mathrm{~K}$. These decays have been fitted with exponential decays with lifetimes of $55 \mathrm{~ns}, 53 \mathrm{~ns}$ and $42 \mathrm{~ns}$ respectively. 


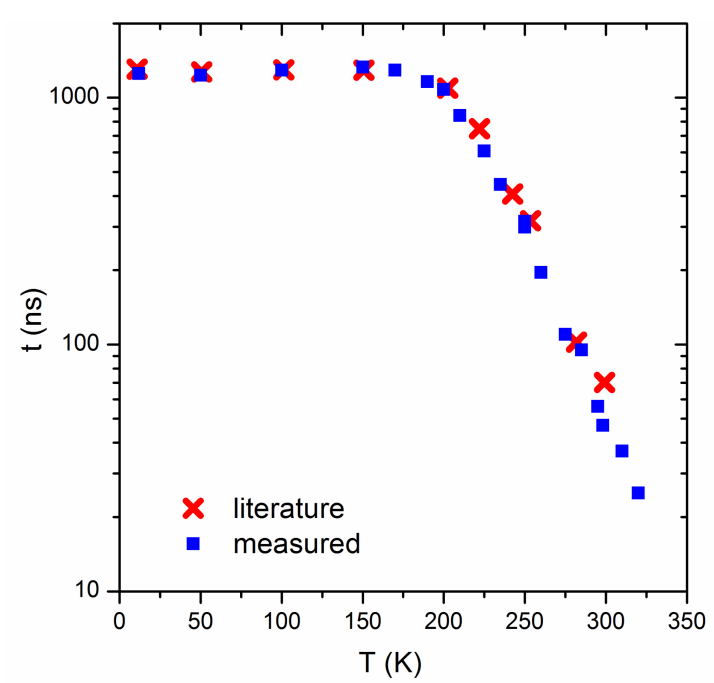

(a)

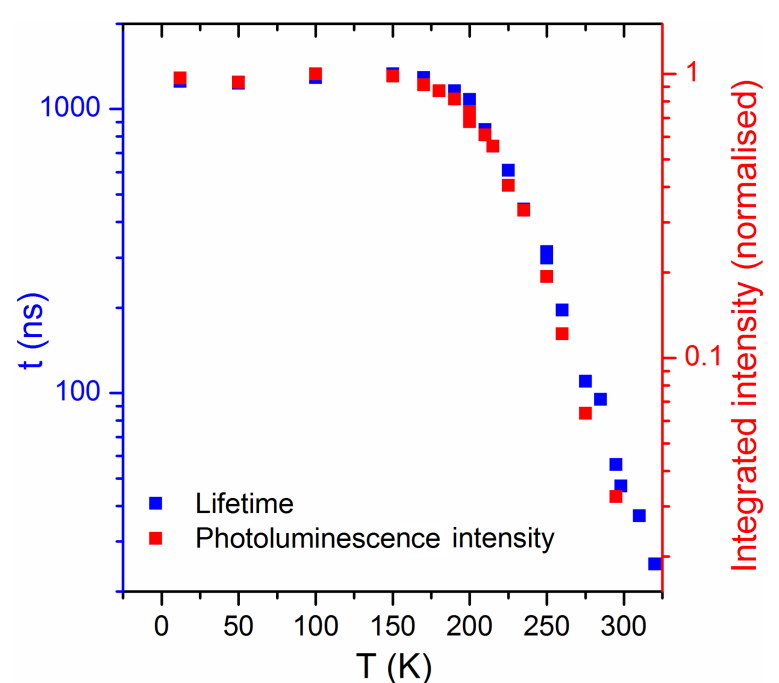

(b)

Figure 9.15: (a) $\mathrm{CaF}_{2}: \mathrm{Sm}$ photoluminescence lifetime as a function of temperature. (b) Normalised photoluminescence lifetime and integrated emission intensity as a function of temperature. The photoluminescence for lifetime measurements was excited at $15750 \mathrm{~cm}^{-1}$ and recorded at $136700 \mathrm{~cm}^{-1}$.

pendence of the photoluminescence intensity closely matches the photoluminescent lifetime. The decrease in both above $150 \mathrm{~K}$ indicates this is the point at which the thermal energy is sufficient to allow competing non-radiative processes. Specifically the quenching occurring above $150 \mathrm{~K}$ has been attributed to ionisation to the conduction band [158].

The large temperature variation in lifetime with temperature may be of application for fibre optic temperature sensing. Fibre optical temperature sensing is used when conditions, such as magnetic, electric and microwave fields mean measurement with electrical temperature sensors is not possible.

\subsubsection{Discussion of optical spectroscopy}

There is clear evidence of $\mathrm{Sm}^{3+}$ luminescence in the material from $16000-18000 \mathrm{~cm}^{-1}$ [132, 163, 164, 172]. Previous work on $\mathrm{CaF}_{2}: \mathrm{Sm}$ has noted difficulties in getting a single valence state of the samarium ion [130, 162, 165, 172], and in fact Royce et al. [165] estimate that at 200 ${ }^{\circ} \mathrm{C}$ the thermal energy is sufficient to allow $\mathrm{Sm}^{2+}$ conversion to $\mathrm{Sm}^{3+}$. In studying the valence state of samarium ions in oxide lattices, Mikhail et al. [173] noted one needs to make sure there are only sites of an appropriate size and charge for $\mathrm{Sm}^{2+}$ and not $\mathrm{Sm}^{3+}$. They found that while they could form $\mathrm{Sm}^{2+}$ in $\mathrm{SrB}_{4} \mathrm{O}_{7}: \mathrm{Sm}^{2+}$, substituting the $\mathrm{Sr}^{2+}$ cation for $\mathrm{Ba}^{2+}, \mathrm{Ca}^{2+}$, 
Table 9.2: The crystal radii of $\mathrm{Ba}^{2+}, \mathrm{Sr}^{2+}, \mathrm{Ca}^{2+}, \mathrm{Sm}^{2+}, \mathrm{Sm}^{3+}, \mathrm{Eu}^{2+}$ and $\mathrm{Eu}^{3+}$ ions. Data from references [120,121]. In the fluorite structures cations have a co-ordination number of 8.

\begin{tabular}{ccc}
\hline Ion & Co-ordination number & Crystal radius \\
\hline $\mathrm{Ba}^{2+}$ & 8 & 1.56 \\
$\mathrm{Sr}^{2+}$ & 8 & 1.4 \\
$\mathrm{Ca}^{2+}$ & 8 & 1.26 \\
$\mathrm{Sm}^{2+}$ & 8 & 1.41 \\
$\mathrm{Sm}^{3+}$ & 8 & 1.219 \\
$\mathrm{Eu}^{2+}$ & 8 & 1.39 \\
$\mathrm{Eu}^{3+}$ & 8 & 1.206 \\
\hline
\end{tabular}

$\mathrm{Pb}^{2+}$, or $\mathrm{Cd}^{2+}$ lead to only $\mathrm{Sm}^{3+}$ ions being present. They also found the preferred valence state to depend on the local geometry [173]. Various reduction techniques were used in an attempt to obtain only the divalent state of samarium, however, all techniques resulted in a mixture of valence states or only the trivalent state (if annealed in an inert or reducing atmosphere). This is in stark contrast to the similar materials LSCBC and $\mathrm{Ba}_{0.3} \mathrm{Sr}_{0.7} \mathrm{Cl}_{2}$ where the $\mathrm{Sm}^{3+}$ ions were reduced to $\mathrm{Sm}^{2+}$ without any attempt create a reducing atmosphere.The crystal radii of $\mathrm{Ba}^{2+}, \mathrm{Sr}^{2+}, \mathrm{Ca}^{2+}, \mathrm{Sm}^{3+}$ and $\mathrm{Sm}^{2+}$ ions are listed in table 9.2 .

From table 9.2 we can see that $\mathrm{Sm}^{3+}$ and $\mathrm{Ca}^{2+}$ have very similar radii and in fact are a much better match than $\mathrm{Sm}^{2+}$ and $\mathrm{Ca}^{2+}$. The opposite is true in LSCBC and $\mathrm{Ba}_{0.7} \mathrm{Sr}_{0.3} \mathrm{Cl}_{2}$. The main cations $\mathrm{Ba}^{2+}$ and $\mathrm{Sr}^{2+}$ are closer to the $\mathrm{Sm}^{2+}$ radius so this will preferentially form. In $\mathrm{CaF}_{2}$ the $\mathrm{Sm}^{3+}$ can be charge compensated by a $\mathrm{F}^{-}$interstitial; these $\mathrm{F}^{-}$interstitials are known to fit and occur in alkaline earth halide lattices [138]. That the trivalent ion preferentially forms because of lattice strain and constraints fits with the observation that any annealing, even in a reducing atmosphere, oxidises any $\mathrm{Sm}^{2+}$ ions present. The interstitial $\mathrm{F}^{-}$may be provided from the $\mathrm{PbF}_{2}$ oxygen scavenger added in the preparation process or from creating anion vacancies elsewhere in the material. Samarium metal was used as the samarium ion dopant as this gave a seemingly higher $\mathrm{Sm}^{2+}: \mathrm{Sm}^{3+}$ concentration ratio than techniques of a reducing atmosphere or pre reducing $\mathrm{SmF}_{3}$. Any $\mathrm{Sm}^{2+}$ will be readily detectable by the strong $4 \mathrm{f}^{6}$ $\leftrightarrow 4 \mathrm{f}^{5} 5 \mathrm{~d}^{1}$ transitions even for very low concentrations [160]. The $\mathrm{CaF}_{2}$ :Eu sample made in the same way showed only $\mathrm{Eu}^{2+}$ luminescence. The ionic radii of europium ions are very similar to samarium ions for both charge states, and so we would expect it form in a mixture of valence states too. However the stability of the $\mathrm{Eu}^{2+}$ ion is slightly higher than that of 


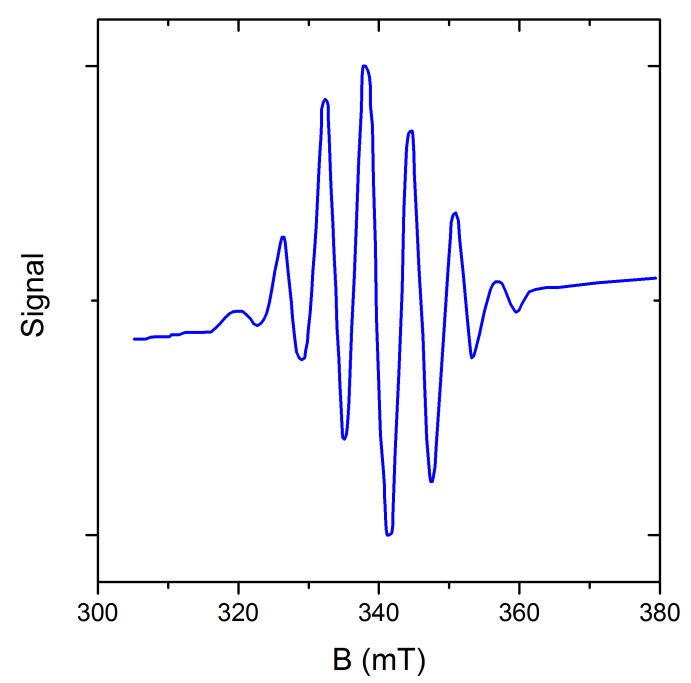

(a)

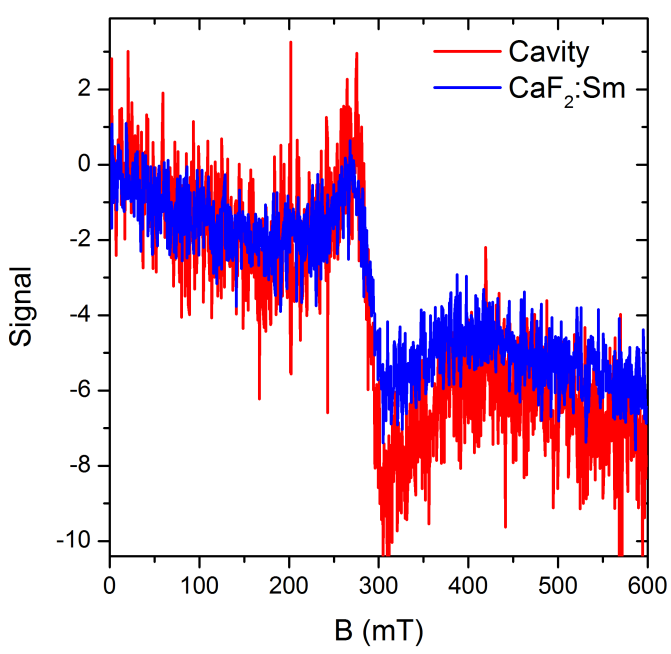

(b)

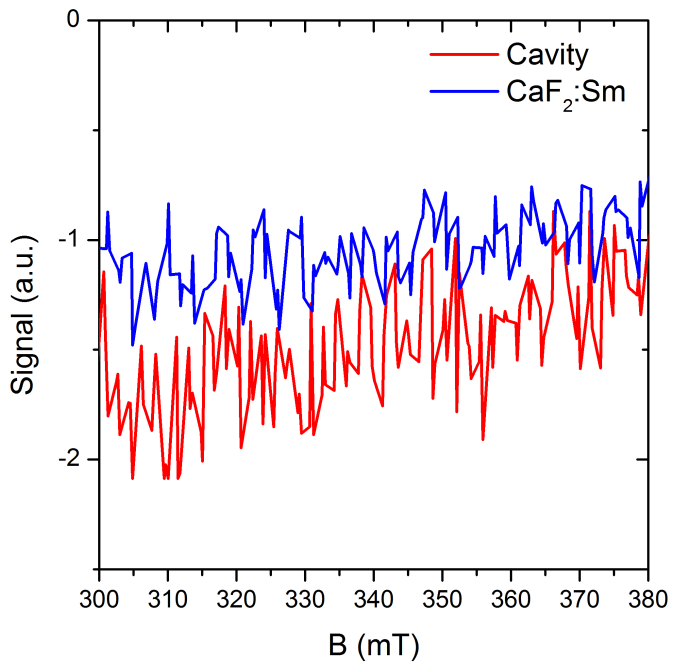

(d)

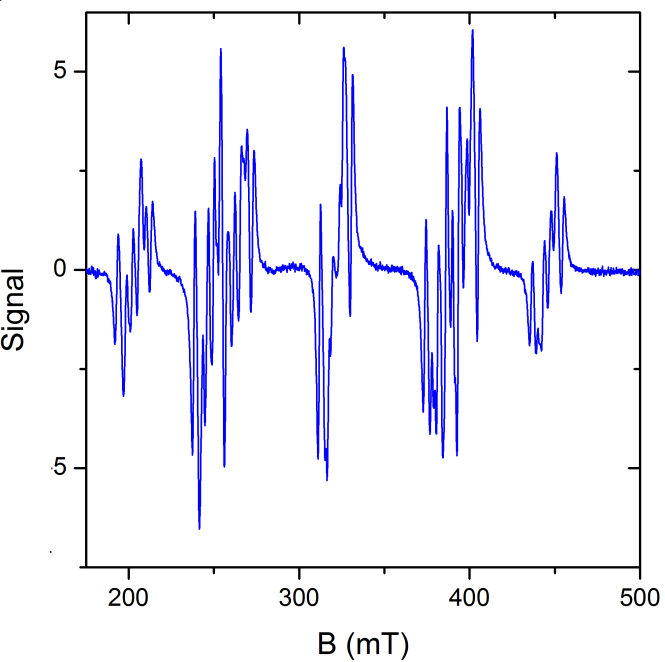

(c)

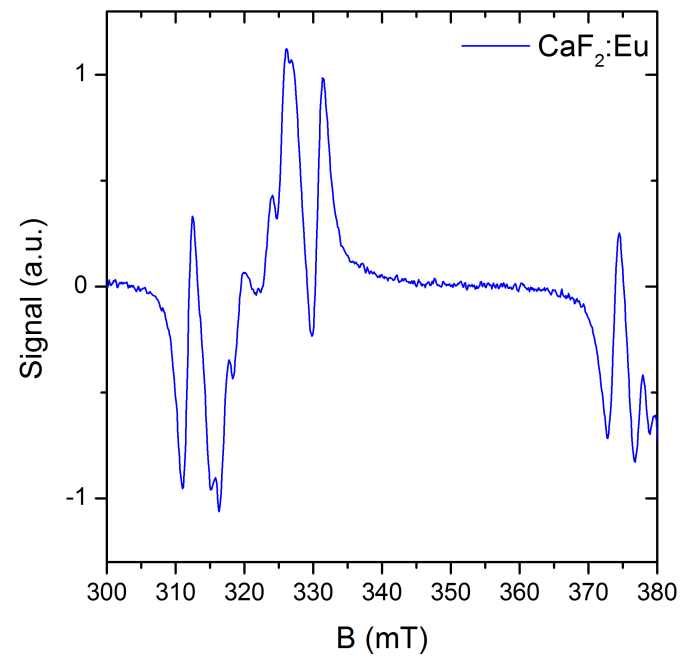

(e)

Figure 9.16: Electron spin resonance spectra; (a) of an $\mathrm{F}$ center in $\mathrm{CaF}_{2}$ [174] measured at $300 \mathrm{~K}$ and with the magnetic field parallel to the $<110>$ axis. The full recorded spectra for (b) $\mathrm{CaF}_{2}: \mathrm{Sm}$ and (c) $\mathrm{CaF}_{2}: \mathrm{Eu}$. Small section of the spectra for (d) $\mathrm{CaF}_{2}: \mathrm{Sm}$ and (e) $\mathrm{CaF}_{2}: \mathrm{Eu}$ showing just the region of interest for an $F$ centre. (b) - (e) were recorded with the magnetic field parallel to the $<100>$ axis. 
$\mathrm{Sm}^{2+}$ [125, 126]. Again the $\mathrm{Eu}^{2+}$ ion $4 \mathrm{f}^{7} \leftrightarrow 4 \mathrm{f}^{6} 5 \mathrm{~d}^{1}$ transitions will be much stronger than the $\mathrm{Eu}^{3+} 4 \mathrm{f}^{6} \leftrightarrow 4 \mathrm{f}^{6}$ transitions potentially masking small concentrations of $\mathrm{Eu}^{3+}$ ions.

As the dopant used was a metal, one would expect the material to have many fluorine vacancies. The metal is reduced via

$$
S m \rightarrow S m^{2+}+2 e^{-}
$$

or

$$
S m \rightarrow S m^{3+}+3 e^{-}
$$

thus adding cations to the melt but no anions are being added, so there must be anion vacancies. The electrons from the reduction would likely fill the anion vacancies to provide charge compensation and in doing so form F centres (electrons trapped at anion vacancies). To look for F centres we recorded ESR spectra of both europium and samarium doped materials. Figure 9.16(a) shows the ESR signal expected for a F centre in $\mathrm{CaF}_{2}$ as reported by Arends [174]. The recorded ESR signals of $\mathrm{CaF}_{2}: \mathrm{Eu}$ and $\mathrm{CaF}_{2}: \mathrm{Sm}$ are shown in figure 9.16. The samarium doped material shows no signal other than a weak resonance of the cavity. The europium doped material shows only transitions corresponding to $\mathrm{Eu}^{2+}$ and no $\mathrm{F}$ centres. Further, F centres would be expected to show optical absorption at $\sim 375 \mathrm{~nm}[174]$, but no such absorption is observed. Pairs of F centres, called M centres, would show no ESR signal due to exchange coupling leaving a singlet lowest state. But should still show optical absorption at $\sim 552 \mathrm{~nm}[138]$.

Thus the mechanism by which the reduction of samarium metal to $\mathrm{Sm}^{2+}$ and $\mathrm{Sm}^{3+}$ ions in $\mathrm{CaF}_{2}$ take place is as follows. As discussed in chapters 7 and 8 we suppose that the flow through gas system in the furnace is subject to small air leaks. As the temperature rises to over $150{ }^{\circ} \mathrm{C}$ the samarium metal is oxidised.

$$
4 \mathrm{Sm}+3 \mathrm{O}_{2} \rightarrow 2 \mathrm{Sm}_{2} \mathrm{O}_{3}
$$

We thus has a trivalent dopant. At higher temperatures the trivalent samarium is partially reduced by the $\mathrm{CO}$ atmosphere due to the reaction of the carbon and the leak induced 
oxygen.

\subsection{X-ray response}

\subsubsection{Radioluminescence}

Figure 9.17 shows the radioluminescence of $\mathrm{CaF}_{2}: \mathrm{Sm}$ for samarium concentration of 0.01 $\%, 0.1 \%$ and $1 \%$. The radioluminescence shows the $\mathrm{Sm}^{2+}$ and $\mathrm{Sm}^{3+}$ emissions seen in the photoluminescence. A new very broad blue emission is also seen. The blue emission is well documented as a self trapped exciton (STE) recombination luminescence [175, 176]. The intensity of this emission decreases with samarium concentration as the emission overlaps with the absorption of the samarium ions for both charge states. The $\mathrm{Sm}^{3+}$ emission is much stronger relative to the $\mathrm{Sm}^{2+}$ emission under x-ray excitation compared to photoexcitation. In studying the thermally stimulated luminescence of $\mathrm{CaF}_{2}: \mathrm{Sm}$, Royce et al. [165] noted it was feasible for a $\mathrm{Sm}^{2+}$ ion to capture a hole after $\mathrm{x}$-ray excitation, leading to a $\mathrm{Sm}^{3+}$ ion in an excited state. This $\mathrm{Sm}^{3+}$ could then return to the ground state by a radiative process. The intensity of the radioluminescence was constant over irradiations of up to fifteen minutes. If this process were occurring the $\mathrm{Sm}^{2+}$ emission should decrease in intensity, but this was not observed. The radioluminescence of $\mathrm{CaF}_{2}: \mathrm{Sm}(1 \%)$ shows no STE emission and no $\mathrm{Sm}^{3+}$ luminescence, most likely due to concentration quenching.

\subsubsection{Scintillation}

The material shows clear scintillation. Figure 9.18 shows an average of 5000 scintillation events for $\mathrm{CaF}_{2}: \mathrm{Sm}$ for both $0.1 \%$ and $0.01 \%$ samarium concentration. These were recorded at room temperature on the fast oscilloscope; a background spectrum with no scintillator is shown for comparison. The scintillation profiles clearly show two lifetimes and, as such, have been fitted with double exponential decays (with baselines). The parameters of the fitted profiles are given in table 9.3 .

The faster component ( $\sim 60 \mathrm{~ns})$, is likely from the $\mathrm{Sm}^{2+}$ ions and is similar to that observed 


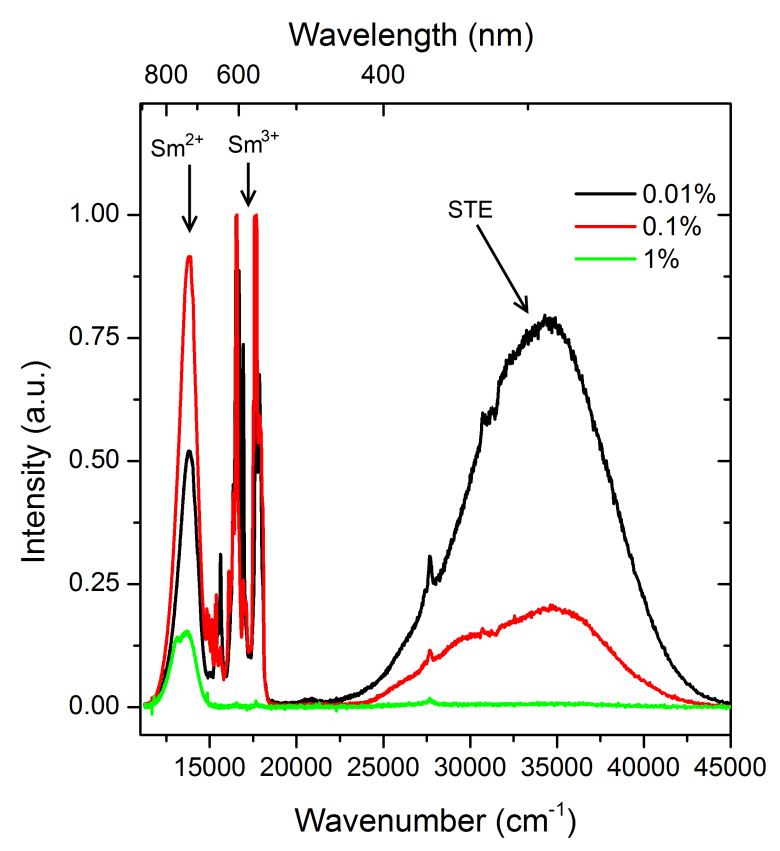

Figure 9.17: Radioluminescence of $\mathrm{CaF}_{2}: \mathrm{Sm}$ for various samarium concentrations, recorded with a tungsten tube operated at $30 \mathrm{kV}$ and $35 \mathrm{~mA}$ at room temperature.

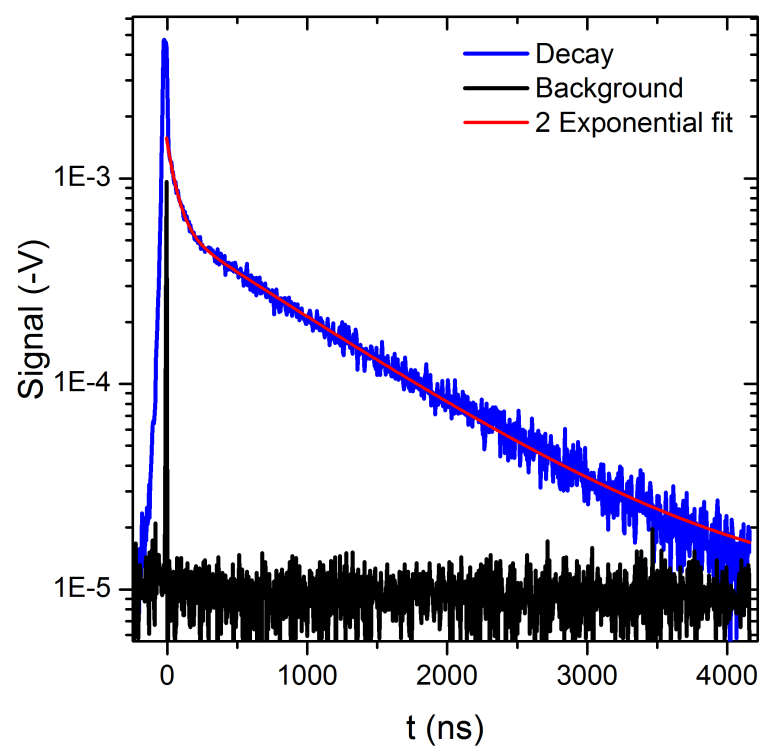

(a)

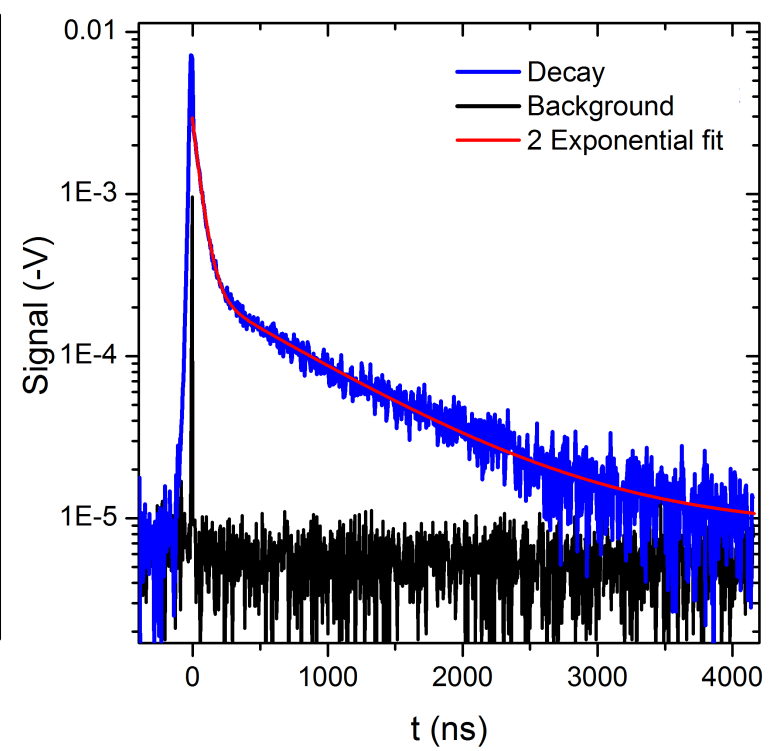

(b)

Figure 9.18: Scintillation decays for $\mathrm{CaF}_{2}: \mathrm{Sm}$ with (a) $0.01 \%$ samarium and (b) $0.1 \%$ samarium. The profiles are an average of 5000 events. The profiles have been fitted with double exponential decays, the parameters of which are presented in table 9.3 . The $\gamma$-ray source was ${ }^{137} \mathrm{Cs}$.

Table 9.3: Parameters of double exponential decays fitted to scintillation profiles.

\begin{tabular}{cccc}
\hline$\%$ samarium & $\tau_{1}(\mathrm{~ns})$ & $\tau_{2}(\mathrm{~ns})$ & integrated intensity ratio $\left(\frac{A_{1} \tau_{1}}{A_{2} \tau_{2}}\right)$ \\
\hline 0.1 & 58 & 870 & 0.72 \\
0.01 & 67 & 970 & 0.13 \\
\hline
\end{tabular}




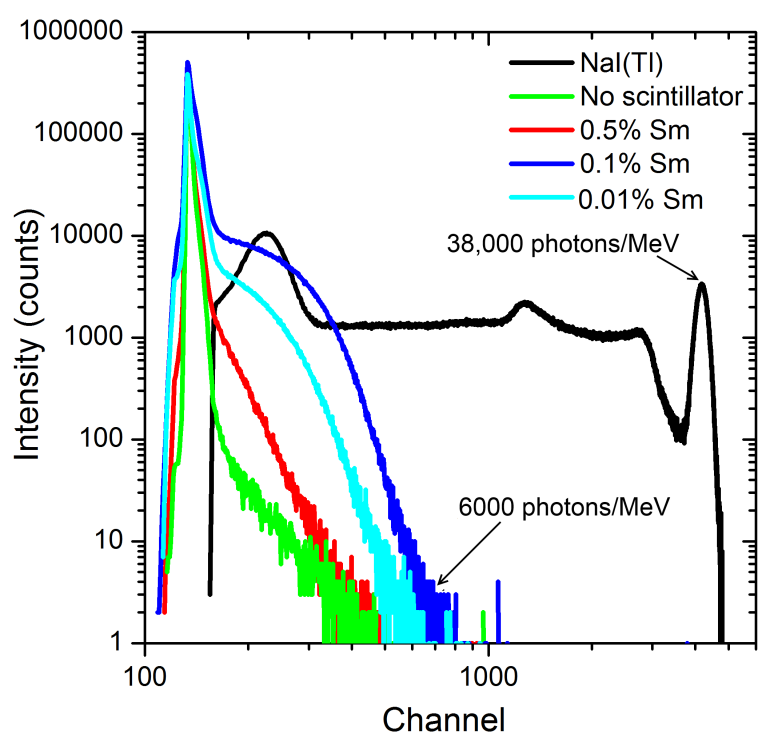

(a)

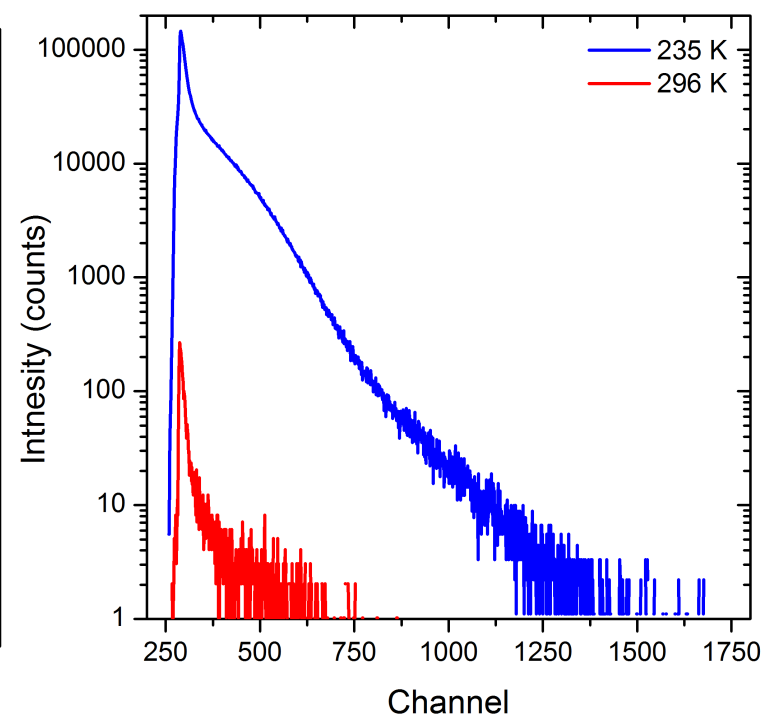

(b)

Figure 9.19: (a) Pulse height analysis spectra of $\mathrm{CaF}_{2}: \mathrm{Sm}$ of various concentrations compared to $\mathrm{NaI}(\mathrm{Tl})$. (b) Pulse height analysis spectra of $\mathrm{CaF}_{2}: \mathrm{Sm}(0.1 \%)$ at $296 \mathrm{~K}$ and $235 \mathrm{~K}$. For both spectra an integration time of $7 \mu$ s was used and ${ }^{137} \mathrm{Cs}$ was used as the $\gamma$-ray source $(0.66$ $\mathrm{MeV}$ ). In (a) a correction for the spectral response for the R5929 photomultiplier has been made and "No scintillator" refers to recording the effect of the $\gamma$-ray interaction with the photomultiplier tube.

under photoexcitation. The $\mathrm{Sm}^{3+}$ photoluminescence has a $\sim 10 \mathrm{~ms}$ decay time [132] so cannot be seen on scintillation time scale. Therefore the longer component is likely the blue STE emission contribution, as this contribution follows the relative intensities of the STE and $\mathrm{Sm}^{2+}$ radioluminescence in the two samples. The STE in $\mathrm{CaF}_{2}$ has a multicomponent long (> $50 \mu$ s) decay time [176], however the decay would likely decrease with rare earth doping as this provides different relaxation routes.

The pulse height spectrum recorded for $\mathrm{CaF}_{2}: \mathrm{Sm}$ is shown in figure 9.19(a), There is no clear photo peak for the $\mathrm{CaF}_{2}: \mathrm{Sm}$ samples. The relatively low atomic numbers of calcium and fluorine mean the Compton effect dominates the photoelectric effect and a photo peak is unlikely to be seen. This has been noted for $\mathrm{CaF}_{2}: \mathrm{Eu}^{2+}$ [166, 167].

The light output at room temperature is $\sim 6000$ photons / MeV. However, on cooling the light output increases substantially. Figure 9.19(b) shows the change in PHA spectrum for a 0.1 $\% \mathrm{Sm} \mathrm{CaF}_{2}$ sample on cooling from room temperature to $235 \mathrm{~K}$. The $\mathrm{x}$-intercept increases by a factor of 2.5 , this leads to an estimated light output of $\sim 15,000$ photons $/ \mathrm{MeV}$ at $235 \mathrm{~K}$. The area under the curve increases by an even larger factor, there is now $\sim 1000$ times more 


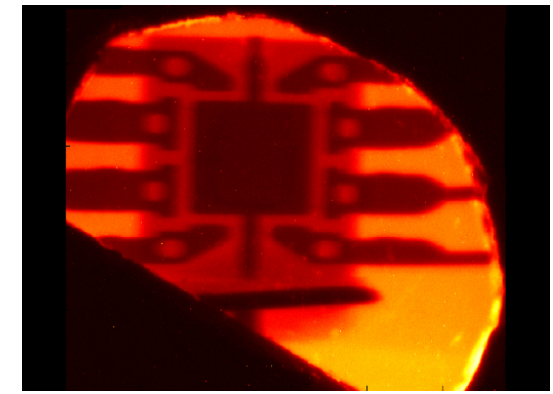

(a)

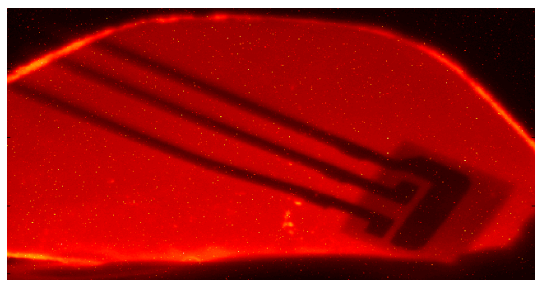

(c)

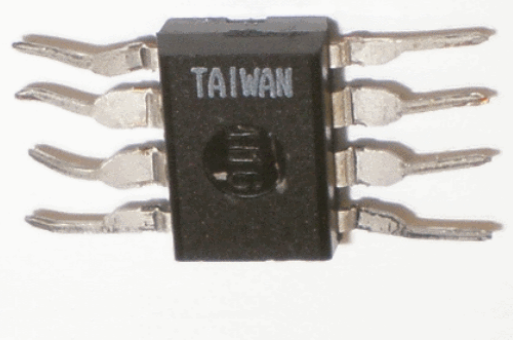

(b)

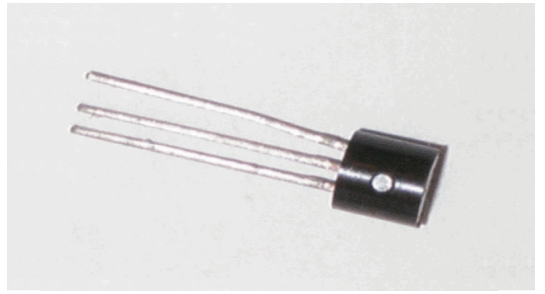

(d)

Figure 9.20: (a) X-ray image of 8 pin integrated circuit chip taken using $\mathrm{CaF}_{2}: \mathrm{Sm}(0.1 \%)$ and (b) optical image. (c) X-ray image of a transistor taken using $\mathrm{CaF}_{2}: \mathrm{Sm}(0.01 \%)$ and (d) and optical image (d). The $\mathrm{x}$-ray images were taken using a dental $\mathrm{x}$-ray set with a maximum x-ray energy of $70 \mathrm{keV}$. Both samples were $1 \mathrm{~mm}$ thick.

light being emitted. Cooling to $235 \mathrm{~K}$ was achieved using dry ice. Dry ice is inexpensive and readily available, and it would be feasible to operate the scintillator at $235 \mathrm{~K}$ in applications requiring a higher light output.

\subsection{X-ray imaging}

$\mathrm{CaF}_{2}: \mathrm{Sm}$ can be used as an x-ray phosphor in x-ray imaging. Figure 9.20 shows images of an 8-pin integrated circuit (IC) chip and a transistor. In both the internal structure can be seen. Figure 9.21 shows the x-ray image of a standard lead grid (Nuclear Associates 07-553, $0.05 \mathrm{~mm}$ thick), taken using $\mathrm{CaF}_{2}: \mathrm{Sm}(0.1 \%)$ and recorded with the CCD camera. The spatial resolution (line pairs per millimetre $(\mathrm{LP} / \mathrm{mm})$ ) of the grid are indicated in figure 9.21, and approximately $8.5 \mathrm{LP} / \mathrm{mm}$ can be resolved by eye. 


\subsection{Summary}

Large single crystals of $\mathrm{CaF}_{2}: \mathrm{Sm}$ and $\mathrm{CaF}_{2}: \mathrm{Eu}$ were produced. These were made by simply melting and cooling the constituent chemicals in a furnace. The $\mathrm{CaF}_{2}: \mathrm{Sm}$ materials showed evidence of both $\mathrm{Sm}^{2+}$ ions and $\mathrm{Sm}^{3+}$ ions. The $\mathrm{Sm}^{2+}$ ion luminescence has a fast $(\sim 50 \mathrm{~ns})$ lifetime under UV excitation. The radioluminescence spectra consist of samarium emissions as well as a self trapped exciton emission. The materials show scintillation, and there is a fast $(<100 \mathrm{~ns})$ component and a slower $(\sim 1 \mu \mathrm{s})$ component. The light output under $\gamma$-ray excitation is estimated to be 15,000 photons $/ \mathrm{MeV}$ when the sample is cooled to $235 \mathrm{~K}$. The materials can be used as x-ray phosphors to form x-ray images. The spatial resolution is high; up to $8.5 \mathrm{LP} / \mathrm{mm}$ could be resolved on an x-ray image of a standard lead grid. No charge conversion was observed under x-irradiation. In conclusion, $\mathrm{CaF}_{2}: \mathrm{Sm}^{2+}$ is an efficient red-emitting scintillator which has a decay time of $\leq 1 \mu$ s and high light output of 15,000 photons $/ \mathrm{MeV}$ when cooled by dry ice. Thus $\mathrm{CaF}_{2}: \mathrm{Sm}$ may find application either in $\gamma$-ray or $\beta$-particle detection systems.

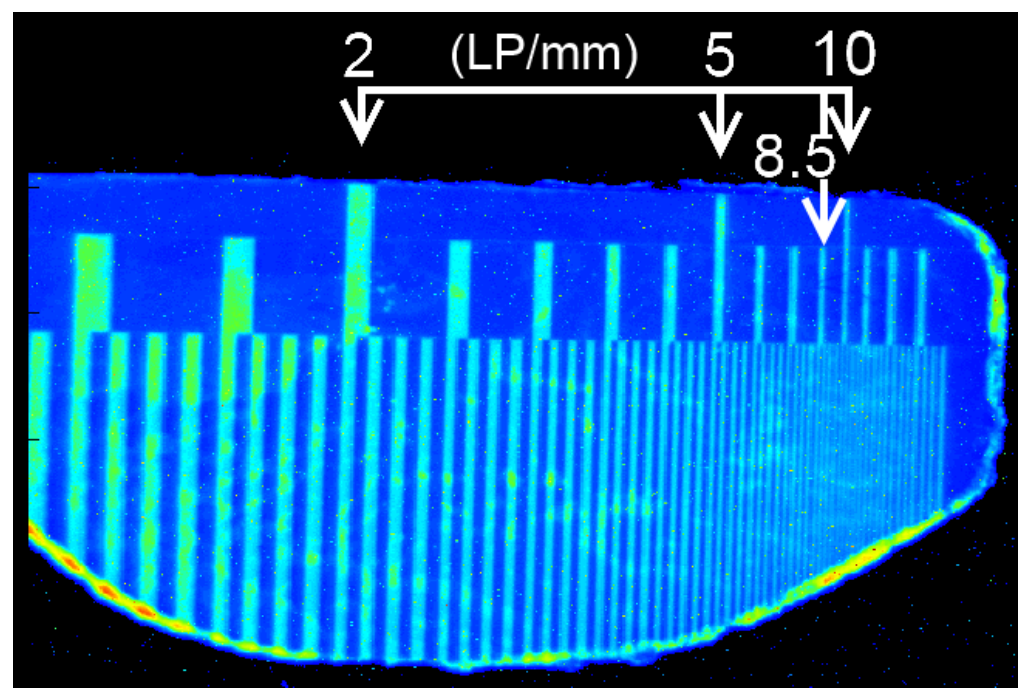

Figure 9.21: X-ray image of standard lead grid (Nuclear Associates 07-553, $0.05 \mathrm{~mm}$ thick). The x-ray image was taken with a $\mathrm{CaF}_{2}: \mathrm{Sm}(0.1 \%)$ sample which was $1 \mathrm{~mm}$ thick. Some $\mathrm{LP} / \mathrm{mm}$ markings are indicated in white. 


\section{Chapter 10}

\section{Conclusions and outlook}

\subsection{Conclusions}

A time correlated single photon counting system for recording scintillation decays has been built. The system was demonstrated to accurately record scintillation decays for a range of decay times ( $2.6 \mathrm{~ns}$ to $1600 \mathrm{~ns}$ ). In particular the scintillation decay of a commercial NE102 scintillator was recorded and a decay time of $2.5 \mathrm{~ns}$ obtained; this was in close agreement to the $2.4 \mathrm{~ns}$ specified by the manufacturer [51]. For $\mathrm{NaI}(\mathrm{Tl})$ the recorded decay time of $236 \mathrm{~ns}$ was also a close match to the manufacturers specification of $237 \mathrm{~ns}$ [105]. The decay recorded for a $\mathrm{Ba}_{0.3} \mathrm{Sr}_{0.7} \mathrm{Cl}_{2}: \mathrm{Eu}^{2+}$ developmental scintillator, which was not as bright as a commercial material, showed dramatically improved dynamic range and signal to noise ratio compared to measurements made using a fast oscilloscope.

The scintillator properties of three main materials were investigated, these were LSCBC:Sm${ }^{2+}$, $\mathrm{Ba}_{0.3} \mathrm{Sr}_{0.7} \mathrm{Cl}_{2}: \mathrm{Sm}^{2+}$ and $\mathrm{CaF}_{2}: \mathrm{Sm}^{2+}$. The emission of $\mathrm{Sm}^{2+}$ ions in each crystal is in the red spectral region and is well matched to the response of silicon photodiode photodetectors.

The emphasis in the thesis was on cubic structured materials since these are optically isotropic, and so, form the basis for low-cost polycrystalline host for rare earth doped scintillators. It was shown that the lanthanum stabilised cubic barium chloride (LSCBC) lattice could accommodate an additional $10 \%$ cation substitution for samarium ions while retaining the cubic phase. It was also found that, $12.5 \%$ doping with $\mathrm{PrCl}_{3}$ stabilised the cubic 
phase of $\mathrm{BaCl}_{2}$, in the same way as $\mathrm{La}^{3+}$. It was demonstrated that the $\mathrm{SrCl}_{2}$ lattice easily accommodates up to $30 \%$ cation substitution of $\mathrm{Sr}^{2+}$ for $\mathrm{Ba}^{2+}$ while retaining the cubic phase, and in addition rare earth ions can easily be incorporated into the lattice. Highly transparent samples of LSCBC, PSCBC and $\mathrm{Ba}_{0.3} \mathrm{Sr}_{0.7} \mathrm{Cl}_{2}$ were produced.

Large single crystals of $\mathrm{CaF}_{2}$ have been produced without the use of sophisticated equipment. Single crystals are normally only able to be grown by Bridgman-Stockbarger technique or Czochralski process.

$\mathrm{Sm}^{2+}$ was the primary rare earth dopant investigated. The temperature dependence of the photoluminescence intensity and lifetimes of $\mathrm{Sm}^{2+}$ ions in $\mathrm{LSCBC}$ and $\mathrm{Ba}_{0.3} \mathrm{Sr}_{0.7} \mathrm{Cl}_{2}$ was recorded. This was well described by a simple simulation which provided insight into the thermal processes which control the photoluminescence, and in particular the dramatic thermal crossover from broadband $4 \mathrm{f}^{5} 5 \mathrm{~d}^{1} \rightarrow 4 \mathrm{f}^{6}$ transitions to sharp line $4 \mathrm{f}^{6} \rightarrow 4 \mathrm{f}^{6}$ transitions. The LSCBC:Sm${ }^{2+}$ and $\mathrm{Ba}_{0.3} \mathrm{Sr}_{0.7} \mathrm{Cl}_{2}: \mathrm{Sm}^{2+}$ absorption and emission spectra could be fully described by a single site of $\mathrm{O}_{h}$ symmetry showing there is no close association between the $\mathrm{Sm}^{2+}$ ions and the extra $\mathrm{Cl}^{-}$ions implicit due to doping with $\mathrm{SmCl}_{3}$.

There was no evidence of $\mathrm{Sm}^{3+}$ in $\mathrm{LSCBC}$ or $\mathrm{Ba}_{0.3} \mathrm{Sr}_{0.7} \mathrm{Cl}_{2}$, showing these lattices accommodate (and favour) the divalent ion. It was argued that the production process used is reducing in character which also favours the divalent oxidation state. Contrastingly, the photoluminescence spectra of $\mathrm{CaF}_{2}: \mathrm{Sm}$ showed clear evidence of both $\mathrm{Sm}^{2+}$ and $\mathrm{Sm}^{3+}$. The difference was attributed to the differences in ionic radii; the crystal radius of $\mathrm{Sm}^{3+}$ is much closer to that of $\mathrm{Ca}^{2+}$ than the crystal radius of $\mathrm{Sm}^{2+}$ compared to $\mathrm{Ca}^{2+}$. In $\mathrm{LSCBC}$ and $\mathrm{Ba}_{0.3} \mathrm{Sr}_{0.7} \mathrm{Cl}_{2}$ the cation crystal radii are much closer to that of $\mathrm{Sm}^{2+}$ than $\mathrm{Sm}^{3+}$.

Across all three materials significant colour centre generation was observed under ionising radiation. This had the effect of slowing the scintillation lifetimes. In LSCBC:Sm ${ }^{2+}$ the scintillation luminescence occurred over $>100 \mu \mathrm{s}$; this was attributed to colour centre generation providing shallow traps and hence delayed luminescence. A trapping model was suggested to explain the results.

Despite colour centre generation limiting the scintillation of $\mathrm{Ba}_{0.3} \mathrm{Sr}_{0.7} \mathrm{Cl}_{2}: \mathrm{Sm}^{2+}$ the material was shown to be a bright and fast x-ray phosphor. The integrated intensity (per x-ray 
half thickness of material) of the radioluminescence is $\sim 30 \%$ that of $\mathrm{Gd}_{2} \mathrm{O}_{2} \mathrm{~S}: \mathrm{Tb}^{3+}$. The scintillation lifetime is $\sim 30 \mu$ s compared to milliseconds for $\mathrm{Gd}_{2} \mathrm{O}_{2} \mathrm{~S}: \mathrm{Tb}^{3+}$. Used in a imaging fashion the spatial resolution was significantly better than that of $\mathrm{Gd}_{2} \mathrm{O}_{2} \mathrm{~S}: \mathrm{Tb}^{3+}$.

Colour centre generation was observed for $\mathrm{CaF}_{2}: \mathrm{Sm}^{2+}$ also, however, it was still shown that $\mathrm{CaF}_{2}: \mathrm{Sm}^{2+}$ is a red-emitting scintillator with a decay time of $\leq 1 \mu \mathrm{s}$. The light output was shown to be $\sim 15,000$ photons $/ \mathrm{MeV}$ when cooled by dry ice. As the material was a single crystal and very transparent the spatial resolution for x-ray imaging was very high at 8.5 $\mathrm{LP} / \mathrm{mm}$.

The neutron sensitivities of LSCBC:Sm and $\mathrm{Ba}_{0.3} \mathrm{Sr}_{0.7} \mathrm{Cl}_{2}: \mathrm{RE}\left(\mathrm{RE}=\mathrm{Sm}^{2+}, \mathrm{Eu}^{2+}, \mathrm{Ce}^{3+}\right)$ were tested for performance as neutron detecting phosphors. The materials were additionally doped with neutron capture elements, such as gadolinium or lithium, to increase their sensitivity. The strongest emission observed was for $\mathrm{Ba}_{0.3} \mathrm{Sr}_{0.7} \mathrm{Cl}_{2}: \mathrm{Eu}^{2+}$ and was $6 \%$ that of the standard material ${ }^{6} \mathrm{LiI}\left(\mathrm{Eu}^{2+}\right)$. The strongest red-emitter was $\mathrm{Ba}_{0.3} \mathrm{Sr}_{0.7} \mathrm{Cl}_{2}: \mathrm{Sm}^{2+}$ sensitised with $5 \%$ lithium, however the lithium doping caused this material to have a mixture of cubic and orthorhombic phases.

\subsection{Outlook}

With regard to the materials investigated in this thesis, there are several possibilities for optimising the scintillator/phosphor properties. $\mathrm{CaF}_{2}: \mathrm{Sm}^{2+}$ showed good red scintillator properties but these can likely be improved. The samarium concentration needs optimisation for specific applications; increasing the samarium concentration may improve the scintillation decay time by quenching the slower host lattice luminescence at a cost of a slightly decreased light output. It would be interesting to try various dopants as a stabiliser of the divalent samarium ion to increase the $\mathrm{Sm}^{2+}: \mathrm{Sm}^{3+}$ ratio and also to further investigate the reduction process and effect of different samarium dopants. For example, it may be possible to quench the lattice emission with a co-dopant; in some halides it has been shown that $\mathrm{K}^{+}$doping can be used to reduce the intrinsic luminescence [177]. $\mathrm{CaF}_{2}: \mathrm{Sm}^{2+}$ may find application in $\gamma$-ray detection systems, as a $\beta$-particle detector, or as one element (the low $\mathrm{Z}$ component) of a 
phoswich. The strong temperature dependence of the photoluminescent lifetime may also be of interest for a fibre optic temperature sensor.

$\mathrm{Ba}_{0.3} \mathrm{Sr}_{0.7} \mathrm{Cl}_{2}: \mathrm{Sm}^{2+}$ was successfully produced in transparent polycrystalline form, this process was rapid and inexpensive. Work on forming a large transparent sheet of powdered $\mathrm{Ba}_{0.3} \mathrm{Sr}_{0.7} \mathrm{Cl}_{2}: \mathrm{Sm}^{2+}$ in a refractive-index matched resin would make the material highly attractive for imaging systems which require large detection areas. This material may find application in integrated imaging techniques which currently use $\mathrm{Gd}_{2} \mathrm{O}_{2} \mathrm{~S}: \mathrm{Tb}^{3+}$ such as computed tomography (CT) or security imaging. The high thermally stimulated luminescence signal from $\mathrm{Ba}_{0.3} \mathrm{Sr}_{0.7} \mathrm{Cl}_{2}: \mathrm{Eu}^{2+}$ suggests that it could be used as a sensitive $\mathrm{x}$-ray dosimeter, but this requires further testing.

While a bright red emitting x-ray phosphor $\left(\mathrm{Ba}_{0.3} \mathrm{Sr}_{0.7} \mathrm{Cl}_{2}: \mathrm{Sm}^{2+}\right)$ and a fast red emitting scintillator $\left(\mathrm{CaF}_{2}: \mathrm{Sm}^{2+}\right)$ have been found, a red emitting material in which the luminescence is both bright and fast remains elusive. The work in this thesis indicates this is unlikely to be achieved with $\mathrm{Sm}^{2+}$ as the luminescent centre; while a fast decay can be observed such as in $\mathrm{CaF}_{2}: \mathrm{Sm}^{2+}$ this is due to a large fraction of the light being lost to non-radiative processes, which decreases the efficiency of the scintillator. Of the rare earth ions that show allowed $5 \mathrm{~d}$ $\rightarrow 4 \mathrm{f}$ transitions in the visible range, $\mathrm{Ce}^{3+}$ shows the most promise of providing a fast and bright red-emitting luminescent centre. Dorenbos [81-85] has compiled extensive data on the emission wavelength of $\mathrm{Ce}^{3+}$ in various crystals, this indicates the most viable area for red emission is likely cerium doped oxides where the crystal field splitting is sufficient large to push the lowest $5 \mathrm{~d}^{1}$ levels into the red region. We carried out preliminary measurements on the cubic materials $\mathrm{CaO}: \mathrm{Ce}^{3+}, \mathrm{SrO}: \mathrm{Ce}^{3+}$ and $\mathrm{BaO}: \mathrm{Ce}^{3+}$; all showed fast red emission but this was very weak. There is some evidence that this could be sensitized with co-doping [178, 179]. Finding a cubic system with a strong crystal field in a narrow band gap material such a group II - group VI (VI = S, Se, Te) materials may also lead to a high efficiency material.

The neutron response of the materials investigated would likely increase with enriched neutron sensitive dopants. Optimisation of the neutron capture element doping to give materials with higher transparency would also increase the light output. 


\section{References}

[1] Su, M. Z. \& Zhao, W. Rare Earth Ions in Advanced X-ray Imaging Materials, vol. 83 of Springer Series in Materials Science, 500-529 (Springer Berlin Heidelberg, 2005).

[2] Ronda, C. Luminescence From theory to applications (Wiley-VCH, Germany, 2008).

[3] Duclos, C. G. \& S. Ceramic scintillators. Annual Review Materials Science 27, 69-88 (1997).

[4] Sells, R. T. W. \& L., R. Elementary Modern Physics (Allyn an Bacon, inc., Boston, 1960).

[5] Melcher, C. L. Perspectives on the future development of new scintillators. Nuclear Instruments and Methods in Physics Research Section A: Accelerators, Spectrometers, Detectors and Associated Equipment 537, 6-14 (2005).

[6] Yanagida, T. Study of rare-earth-doped scintillators. Optical Materials (2012). Doi=10.1016/j.optmat.2012.11.002.

[7] Eijk, C. W. E. v. Inorganic-scintillator development. Nuclear Instruments and Methods in Physics Research Section A: Accelerators, Spectrometers, Detectors and Associated Equipment 460, 1-14 (2001).

[8] Pichler, B. J., Wehrl, H. F. \& Judenhofer, M. S. Latest advances in molecular imaging instrumentation. Journal of Nuclear Medicine 49, 5S-23S (2008).

[9] XP2020Q photomultiplier tube data sheet (Accessed 13 June 2013). URL http: / / www. qsl.net/k0ff/1Manuals/PMT/Photonis/XP2020Q.pdf.

[10] Hamamatsu S2383 avalanchhe photodiode data sheet (Accessed 13 June 2013). 
URL http://www.hamamatsu.com/us/en/product/category/3100/4003/ 4110/S2383/index.html\#1328449178416.

[11] Moses, W. W. et al. Prospects for dense, infrared emitting scintillators. Nuclear Science, IEEE Transactions on 45, 462-466 (1998).

[12] Van t Spijker, J. Luminescence and Scintillation of $\mathrm{Ce}^{3+}$ Doped Inorganic Materials for Gamma-Ray Detection: In English and Dutch (Ios PressInc, 1999).

[13] Rodnyi, P. A. Physical Processes in Inorganic Scintillators (CRC Press, New York, 1997).

[14] Schotanus, P., Dorenbos, P. \& Ryzhikov, V. Detection of CdS(Te) and ZnSe(Te) scintillation light with silicon photodiodes. Nuclear Science, IEEE Transactions on 39, 546-550 (1992).

[15] Rodnyi, P. A., Gorohova, E. I., Mikhrin, S. B., Mishin, A. N. \& Potapov, A. S. Quest and investigation of long wavelength scintillators. Nuclear Instruments and Methods in Physics Research Section A: Accelerators, Spectrometers, Detectors and Associated Equipment 486, 244-249 (2002).

[16] Huber, G. et al. $\mathrm{Lu}_{2} \mathrm{~S}_{3}: \mathrm{Ce}^{3+}$, a new red luminescing scintillator. Nuclear Instruments and Methods in Physics Research Section B: Beam Interactions with Materials and Atoms 134, 304-309 (1998).

[17] Gamma-ray production (Accessed 13 June 2013). URL WwW . i onact ive.co . uk.

[18] Seibert, J. A. X-ray imaging physics for nuclear medicine technologists. Part 1: Basic principles of x-ray production. Journal of Nuclear Medicine Technology 32, 139-147 (2004).

[19] Hubbell, J. H. \& Seltzer, S. M. Tables of X-ray mass attenuation coefficients and mass energy-absorption coefficients $1 \mathrm{kev}$ to $20 \mathrm{Mev}$ for elements $Z=1$ to 92 and 48 additional substances of dosimetric interest. Tech. Rep., National Inst. of Standards and Technology-PL, Gaithersburg, MD (United States). Ionizing Radiation Div. (1995).

[20] Doug Reilly, H. S. J. S. K., Norbert Enslin. Passive Nondestructive Assay of Nuclear Materials (Office of Nuclear Regulartory Research, U.S. Nuclear Regulatory Commission, Washington, DC 20555, 1991). 
[21] Introduction to radiation monitors (Accessed 13 June 2013). URL http://www.equipcoservices.com/support/tutorials/ introduction-to-radiation-monitors/.

[22] Instruments, C. Gamma and X-ray detection (Accessed 13 June 2013). URL http://www.canberra.com/literature/fundamental-principles/ pdf/Gamma-Xray-Detection.pdf.

[23] Knoll, G. Radiation detection and measurement (Wiley, 2000).

[24] Eberth, J. \& Simpson, J. From Ge(Li) detectors to gamma-ray tracking arrays 50 years of gamma spectroscopy with germanium detectors. Progress in Particle and Nuclear Physics 60, 283-337 (2008).

[25] Dearnaley, G. \& Northrop, D. C. Semiconductor counters for nuclear radiations (Wiley, 1963).

[26] Kasap, S. O., Haugen, C., Nesdoly, M. \& Rowlands, J. A. Properties of $\alpha$-Se for use in flat panel x-ray image detectors. Journal of Non-Crystalline Solids 266269, Part 2, 1163-1167 (2000).

[27] Shah, K. S. et al. High energy resolution scintillation spectrometers. Nuclear Science, IEEE Transactions on $\mathbf{5 1 , 2 3 9 5 - 2 3 9 9 ~ ( 2 0 0 4 ) . ~}$

[28] Darbandi, A., Devoie, E., Matteo, O. D. \& Rubel, O. Modeling the radiation ionization energy and energy resolution of trigonal and amorphous selenium from first principles. Journal of Physics: Condensed Matter 24, 455502 (2012).

[29] Swiderski, L., Moszynski, M., Czarnacki, W., Syntfeld-Kazuch, A. \& Gierlik, M. Nonproportionality and energy resolution of $\mathrm{NaI}(\mathrm{Tl})$ at wide temperature range $\left(-40^{\circ} \mathrm{C}\right.$ to $\left.+23^{\circ} \mathrm{C}\right)$. In Nuclear Science Symposium Conference Record, 2006. IEEE, vol. 2, 1122-1128 (2006).

[30] Moszyski, M. et al. Temperature dependences of $\mathrm{LaBr}_{3}(\mathrm{Ce}), \mathrm{LaCl}_{3}(\mathrm{Ce})$ and $\mathrm{NaI}(\mathrm{Tl})$ scintillators. Nuclear Instruments and Methods in Physics Research Section A: Accelerators, Spectrometers, Detectors and Associated Equipment 568, 739-751 (2006). 
[31] Kerisit, S., Rosso, K. M., Cannon, B. D., Gao, F. \& Xie, Y. Computer simulation of the light yield nonlinearity of inorganic scintillators. Journal of Applied Physics 105, 114915-10 (2009).

[32] P. A. Russo, D. V. Gamma-ray detectors for nondestructive analysis (2007). URL http: //wWw.lanl.gov/orgs/n/n1/panda/,

[33] Ortec. Fast-timing discriminator introduction (Accessed 13 June 2013). URL www.ortec-online.com/download/ Fast-Timing-Discriminator-Introduction.pdf.

[34] RadEye PRD Personal Radiation Detector (Accessed 13 June 2013). URL http : / / www. thermoscientific.com/ecomm/

[35] Photomultiplier tube (Accessed 13 June 2013). URL http://elchem.kaist.ac. $\mathrm{kr} / \mathrm{vt} / \mathrm{chem}-\mathrm{ed} / \mathrm{optics} / \mathrm{detector/pmt} . \mathrm{htm}$

[36] J. Garcia Sole, L. B. \& Jaque, D. An Introduction to the Optical Spectroscopy of Inorganic Solids (John Wiley and Sons Ltd, England, 2005).

[37] Detectors (Accessed 13 June 2013). URL http://www . photonics . com/Article. aspx?AID $=25535$.

[38] Zavaleta, C. Photodiodes as substitutes for photomultiplier tubes (Accessed 13 June 2013). URL http: //ric.uthscsa.edu/personalpages/lancaster/ DI2_Projects_2003/Photodiode_vs_PMT.pdf

[39] Koren, B. Large-area avalanche photodiodes challenge PMTs. Laser focus world 34, 5 (1998).

[40] Moszyski, M., Szawlowski, M., Kapusta, M. \& Balcerzyk, M. Large area avalanche photodiodes in scintillation and x-rays detection. Nuclear Instruments and Methods in Physics Research Section A: Accelerators, Spectrometers, Detectors and Associated Equipment 485, 504-521 (2002).

[41] Lecoq, P., Annenkov, A., Gektin, A., Korzhik, M. \& Pedrini, C. Inorganic scintillators for detector systems: Physical principles and crystal engineering (2006). 
[42] (Accessed 13 June 2013). URL http: / / www.ndt-ed.org/EducationResources/ CommunityCollege/Radiography/Physics/attenuation.htm.

[43] Birowosuto, M. Novel Gamma-Ray and Thermal-Neutron Scintillators: Search for HighLight-Yield and Fast-Response Materials (Ios PressInc, 2008).

[44] Derenzo, S., Weber, M., Bourret-Courchesne, E. \& Klintenberg, M. The quest for the ideal inorganic scintillator. Nuclear Instruments and Methods in Physics Research Section A: Accelerators, Spectrometers, Detectors and Associated Equipment 505, 111-117 (2003).

[45] de Haas, J. T. M. \& Dorenbos, P. Advances in yield calibration of scintillators. Nuclear Science, IEEE Transactions on 55, 1086-1092 (2008).

[46] Kitai, A. Solid State Luminescence. Theory, materials and devices (Chapman and Hall, London, 1993).

[47] Dorenbos, P. Directions in scintillation materials research. In Radiation Detectors for Medical Applications, 191-207 (Springer, 2006).

[48] Dorenbos, P., de Haas, J. T. M. \& van Eijk, C. W. E. Non-proportionality in the scintillation response and the energy resolution obtainable with scintillation crystals. Nuclear Science, IEEE Transactions on 42, 2190-2202 (1995).

[49] Moses, W. W., Payne, S. A., Choong, W. S., Hull, G. \& Reutter, B. W. Scintillator nonproportionality: Present understanding and future challenges. Nuclear Science, IEEE Transactions on 55, 1049-1053 (2008).

[50] Duclos, S. J. et al. Development of the hilight ${ }^{T M}$ scintillator for computed tomography medical imaging. Nuclear Instruments and Methods in Physics Research Section A: Accelerators, Spectrometers, Detectors and Associated Equipment 505, 68-71 (2003).

[51] Gobain, S. Solid plastic scintillators (2012). Available:http://www.detectors.saintgobain.com/Plastic-Scintillator.aspx [ Accessed 13 June 2013] also bc430.

[52] University of Washington, I. R. L. Introduction to PET physics (2013). URL http: / / depts.washington.edu/nucmed/IRL/pet_intro/intro_src/ section5.html. 
[53] Grabmaier, B. C. Crystal scintillators. Nuclear Science, IEEE Transactions on 31, 372-376 (1984).

[54] Liu, Z., Liu, S., Wang, K. \& Luo, X. Measurement and numerical studies of optical properties of yag:ce phosphor for white light-emitting diode packaging. Appl. Opt. 49, 247-257 (2010).

[55] Ronda, C. Luminescece From theory to applications (Wiley-VCH, Germany, 2008).

[56] Mettler, F. A., Huda, W., Yoshizumi, T. T. \& Mahesh, M. Effective doses in radiology and diagnostic nuclear medicine: A catalog1. Radiology 248, 254-263 (2008).

[57] Klamra, W. et al. Properties of $\mathrm{CdWO}_{4}$ and $\mathrm{ZnWO}_{4}$ at liquid nitrogen temperature. IEEE Nuclear Science Symposium Conference Record (NSS/MIC) 1561 - 1565 (2009).

[58] Hook, J. \& Hall, H. Solid state physics (Wiley, 1991).

[59] Cho, G., Kang, D.-W. \& Kim, K. H. The performance of x-ray scanner using ceramic scintillator base detector module. Nuclear Science, IEEE Transactions on 55, 1321-1326 (2008).

[60] Kittel, C. Introduction to Solid State Physics (Wiley, 2004).

[61] Wyckoff, R. W. G. Crystal structures - Volume 1 (Interscience publishers, 1948).

[62] Vij, D. R. Handbook of Applied Solid State Spectrsocpy (Springer, 2006).

[63] Henderson, B. \& Imbusch, G. F. Optical Spectroscopy of Inorganic Solids (Oxford Science Publications, 1989).

[64] (Accessed 13 June 2013). URL rp-photonics. com.

[65] Chambers, M. \& Clarke, D. Doped oxides for high-temperature luminescence and lifetime thermometry. Annual Review of Materials Research 39, 325-359 (2009).

[66] Korthout, K., Parmentier, A. B., Smet, P. F. \& Dirk, P. A XAS study of the luminescent Eu centers in thiosilicate phosphors. Phys. Chem. Chem. Phys. (2013).

[67] Tsuboi, T. \& Silfsten, P. The lifetime of $\mathrm{Eu}^{2+}$ fluorescence in $\mathrm{CaF}_{2}: \mathrm{Eu}^{2+}$ crystals. Journal of Physics: Condensed Matter 3, 9163 (1991). 
[68] Grabmeier, G. B. \& C., B. Luminescent Materials (Springer-Verlag, Berlin, 1994).

[69] (2013). URL http://adg.llnl.gov/Research/qsg-090205/siliconDots_ stokes.html.

[70] Cho, D. H., Hirao, K. \& Soga, N. Persistent spectral hole burning of $\mathrm{Sm}^{2+}$ in borate glasses. Journal of Non-Crystalline Solids 189, 181-190 (1995).

[71] Riesen, H. \& Kaczmarek, W. A. Efficient x-ray generation of $\mathrm{Sm}^{2+}$ in nanocrystalline $\mathrm{BaFCl} / \mathrm{Sm}^{3+}$ : a photoluminescent $\mathrm{x}$-ray storage phosphor. Inorganic Chemistry 46, 7235-7237 (2007).

[72] Vahedi, S. et al. X-ray induced $\mathrm{Sm}^{3+}$ to $\mathrm{Sm}^{2+}$ conversion in fluorophosphate and fluoroaluminate glasses for the monitoring of high-doses in microbeam radiation therapy. Journal of Applied Physics 112, 073108-10 (2012).

[73] Axe, J. D. \& Sorokin, P. P. Divalent rare earth spectra selection rules and spectroscopy of $\mathrm{SrCl}_{2}: \mathrm{Sm}^{2+}$. Physical Review 130, 945 (1963).

[74] Kobayasi, T., Mroczkowski, S., Owen, J. F. \& Brixner, L. H. Fluorescence lifetime and quantum efficiency for $5 \mathrm{~d} \rightarrow 4 \mathrm{f}$ transitions in $\mathrm{Eu}^{2+}$ doped chloride and fluoride crystals. Journal of Luminescence 21, 247-257 (1980).

[75] van Loef, E. V. et al. Crystal growth and scintillation properties of strontium iodide scintillators. Nuclear Science, IEEE Transactions on 56, 869-872 (2009).

[76] Zych, A., Leferink op Reinink, A., van der Eerden, K., de Mello Doneg, C. \& Meijerink, A. Luminescence properties of lanthanide doped alkaline earth chlorides under (V)UV and x-ray excitation. Journal of Alloys and Compounds 509, 4445-4451 (2011).

[77] Oskam, K. D., Houtepen, A. J. \& Meijerink, A. Site selective 4f5d spectroscopy of $\mathrm{CaF}_{2}: \mathrm{Pr}^{3+}$. Journal of Luminescence 97, 107-114 (2002).

[78] Prez-Salas, R. et al. Cation size dependence of phosphorescence lifetime of $\mathrm{Eu}^{2+}$ doped alkali halide crystals. Optical Materials 35, 617-619 (2013).

[79] Rodnyi, P. A. et al. The observation of photon cascade emission in $\mathrm{Pr}^{3+}$-doped compounds under x-ray excitation. Optics Communications 204, 237-245 (2002). 
[80] Shao, L.-M. \& Jing, X.-P. Near-infrared luminescence of $\mathrm{Tb}^{3+}-\mathrm{Yb}^{3+}$ and $\mathrm{Ce}^{3+}-\mathrm{Yb}^{3+}$ co-doped $\mathrm{Y}_{3} \mathrm{Al}_{5} \mathrm{O}_{12}$. ECS Journal of Solid State Science and Technology 1, R22-R26 (2012).

[81] Dorenbos, P. The $5 \mathrm{~d}$ level positions of the trivalent lanthanides in inorganic compounds. Journal of Luminescence 91, 155-176 (2000).

[82] Dorenbos, P. 5d-level energies of $\mathrm{Ce}^{3+}$ and the crystalline environment. I. fluoride compounds. Physical Review B 62, 15640-15649 (2000).

[83] Dorenbos, P. 5d-level energies of $\mathrm{Ce}^{3+}$ and the crystalline environment. II. Chloride, bromide, and iodide compounds. Physical Review B 62, 15650-15659 (2000).

[84] Dorenbos, P. 5d-level energies of $\mathrm{Ce}^{3+}$ and the crystalline environment. III. Oxides containing ionic complexes. Physical Review B 64, 125117 (2001).

[85] Dorenbos, P. 5d-level energies of $\mathrm{Ce}^{3+}$ and the crystalline environment. IV. Aluminates and simple oxides. Journal of Luminescence 99, 283-299 (2002).

[86] McKeever, S. Thermoluminescence of Solids (Cambridge University Press, 1988).

[87] Bos, A. J. J. Theory of thermoluminescence. Radiation Measurements 41, Supplement 1, S45-S56 (2006).

[88] J.T. Randall, M. W. Phosphorescence and electron traps: I. The study of trap distributions. Proc. R. Soc. London A 184, 366389 (1945).

[89] J.T. Randall, M. W. Phosphorescence and electron traps: II. The interpretation of long-period phosphorescence. Proc. R. Soc. London A 184, 390407 (1945).

[90] Kitis, G., Gomez-Ros, J. M. \& Tuyn, J. W. N. Thermoluminescence glow-curve deconvolution functions for first, second and general orders of kinetics. Journal of Physics D: Applied Physics 31, 2636 (1998).

[91] ESR mage By "Astrochemist" at en.wikibooks, from Wikimedia Common (Accessed 13 June 2013). URL https://en.wikipedia.org/wiki/Electron_ paramagnetic_resonance.

[92] MacMillan, J. P. et al. Strontium and Strontium Compounds (Wiley-VCH Verlag GmbH \& Co. KGaA, 2000). 
[93] Fluorometer layout (Accessed 13 June 2013). URL http://photonicswiki.org/ index.php?title=Fluorometer

[94] Quilty, J. W., Robinson, J., Appleby, G. A. \& Edgar, A. Thermoluminescence apparatus using PT100 resistors as the heating and sensing elements. Review of Scientific Instruments 78, 083905-6 (2007).

[95] Instrumentation of ESR spectroscopy (Accessed 13 June 2013). URL http://www.pharmatutor.org/articles/ instrumentation-electron-spin-resonance-spectroscopy.

[96] Winch, N. M. \& Edgar, A. X-ray imaging using a consumer-grade digital camera. Nuclear Instruments and Methods in Physics Research Section A: Accelerators, Spectrometers, Detectors and Associated Equipment 654, 308-313 (2011).

[97] Hamamatsu R5929 photomultiplier data sheet (Accessed 13 June 2013). URL http://www.hamamatsu.com/jp/en/product/category/3100/3001/ R5929/index.html.

[98] Bartle, C. M. et al. Novel methods for measuring afterglow in developmental scintillators for x-ray and neutron detection. Nuclear Instruments and Methods in Physics Research, Section A: Accelerators, Spectrometers, Detectors and Associated Equipment 651, 105-109 (2011).

[99] Bollinger, L. M. \& Thomas, G. E. Measurement of the time dependence of scintillation intensity by a delayed coincidence method. Review of Scientific Instruments 32, 1044-1050 (1961).

[100] Becker, W. Advance Time-Correlated Single Photon Counting Techniques. Springer Series in Chemical Physics (Springer - Verlag Berline Heidelberg, Germany, 2005).

[101] Moses, W. W. A method to increase optical timing spectra measurement rates using a multi-hit TDC. Nuclear Instruments and Methods in Physics Research Section A: Accelerators, Spectrometers, Detectors and Associated Equipment 336, 253-261 (1993).

[102] Photonis. PHOTOMULTIPLIER TUBES principles and applications (Photonis, Brive, France, 2002). URL http: //www2.pv.infn.it//doc/Flyckt_Marmonier.pdf. 
[103] Hallam, A. Measurement of scintillation pulse shapes by the single-photon techniquean improved method. Journal of Physics E: Scientific Instruments 12, 298 (1979).

[104] Industries, Z. Oscilloscope measurement fundamentals (Accessed 13 June 2013). URL http://www.ztecinstruments.com/applications/ oscilloscope-measurement-fundamentals.php.

[105] Gobain, S. NaI(Tl) data sheet.

[106] Ghetti, R. et al. Calibration of a neutron time-of-flight multidetector system for an intensity interferometry experiment. Nuclear Instruments and Methods in Physics Research Section A: Accelerators, Spectrometers, Detectors and Associated Equipment 516, 492-501 (2004).

[107] Faham, C. H. Photomultiplier tube physics and operation (2013). URL http:// physics.bu.edu/NEPPSR/TALKS-2009/Faham_PMToperation.pdf.

[108] Technical rerint r/p0809 (Accessed 13 June 2013). URL http:// WwW.et-enterprises.com/files/file/technical-information/ rp089colour.pdf

[109] Selling, J., Birowosuto, M. D., Dorenbos, P. \& Schweizer, S. Europium-doped barium halide scintillators for x-ray and $\gamma$-ray detections. Journal of Applied Physics 101 (2007).

[110] Glodo, J. et al. Spectroscopy of selected alkaline earth halides. In Nuclear Science Symposium Conference Record (NSS/MIC), 2010 IEEE, 271-274.

[111] Bourret-Courchesne, E. D. et al. Crystal growth and characterization of alkali-earth halide scintillators. Journal of Crystal Growth 352, 78-83 (2012).

[112] Koshimizu, M., Onodera, K., Shibuya, K., Saito, H. \& Asai, K. Timing property of undoped $\mathrm{BaCl}_{2}$ single crystal scintillator. Journal of Applied Physics 105, 114912-6 (2009).

[113] Koshimizu, M. et al. X-ray detection capability of a $\mathrm{BaCl}_{2}$ single crystal scintillator. Journal of Applied Physics 111, 024906-5 (2012).

[114] Onodera, K., Koshimizu, M. \& Asai, K. Luminescent properties of $\mathrm{BaCl}_{2}$ under VUV excitation. Radiation Physics and Chemistry 78, 1031-1033 (2009). 
[115] Schweizer, S. et al. Photostimulated luminescence in Eu-doped fluorochlorozirconate glass ceramics. Applied Physics Letters 83, 449-451 (2003).

[116] Edgar, A., Bartle, M., Raymond, S. G., Williams, G. V. M. \& Varoy, C. Structural and scintillation properties of cerium-doped ceramics. Nuclear Science, IEEE Transactions on 55, 1160-1163 (2008).

[117] Edgar, A., Bartle, M., Varoy, C., Raymond, S. \& Williams, G. Structure and scintillation properties of cerium-doped barium chloride ceramics: Effects of cation and anion substitution. Nuclear Science, IEEE Transactions on 57, 1218-1222 (2010).

[118] Edgar, A., Zimmermann, J., von Seggern, H. \& Varoy, C. R. Lanthanum-stabilized europium-doped cubic barium chloride: An efficient x-ray phosphor. Journal of Applied Physics 107, 083516-7 (2010).

[119] Weast, R. C., Astle, M. J. \& Beyer, W. H. CRC Handbook of Chemistry and Physics (CRC Press, 1973).

[120] Shannon, R. Revised effective ionic radii and systematic studies of interatomic distances in halides and chalcogenides. Acta Crystallographica Section A 32, 751-767 (1976).

[121] Van Horn, D. J. Electronic Table of Shannon Ionic Radii (2001). [downloaded 31/07/2009].

[122] Karbowiak, M., Urbanowicz, A. \& Reid, M. F. $4 \mathrm{f}^{6} \rightarrow 4 \mathrm{f}^{5} 5 \mathrm{~d}^{1}$ absorption spectrum analysis of $\mathrm{Sm}^{2+}: \mathrm{SrCl}_{2}$. Physical Review B 76, 115125 (2007).

[123] Goldsmith, G. J. \& Pinch, H. L. Divalent rare-earth ions in optical maser materials. Tech. Rep., DTIC Document (1965).

[124] Carbon monoxide (Accessed 13 June 2013). URL https://en.wikipedia.org/ wiki/Carbon_monoxide.

[125] Dorenbos, P. \& Bos, A. Lanthanide level location and related thermoluminescence phenomena. Radiation Measurements 43, 139 - 145 (2008).

[126] Dorenbos, P. Locating lanthanide impurity levels in the forbidden band of host crystals. Journal of luminescence 108, 301-305 (2004). 
[127] He, Z.-y., Wang, Y.-s., Li, S. \& Xu, X.-r. Dynamic studies on the time-resolved fluorescence of $\mathrm{Sm}^{2+}$ in $\mathrm{BaCl}_{2}$. Journal of Luminescence 97, 102-106 (2002).

[128] Lauer, J. H. V. \& Fong, F. K. Role of the $4 \mathrm{f}^{5} 5 \mathrm{~d}$ band in the radiationless ${ }^{5} \mathrm{D}_{1} \rightarrow{ }^{5} \mathrm{D}_{0}$ coupling in $\mathrm{BaCl}_{2}: \mathrm{Sm}^{2+}$ and $\mathrm{BaBr}_{2}: \mathrm{Sm}^{2+}$. The Journal of Chemical Physics 65, 3108-3117 (1976).

[129] Alam, A. S. M. M. \& Bartolo, B. D. Thermal dependence of fluorescence and lifetimes of $\mathrm{Sm}^{2+}$ in several host lattices. The Journal of Chemical Physics 47, 3790-3801 (1967).

[130] Wood, D. L. \& Kaiser, W. Absorption and fluorescence of $\mathrm{Sm}^{2+}$ in $\mathrm{CaF}_{2}, \mathrm{SrF}_{2}$, and $\mathrm{BaF}_{2}$. Physical Review 126, 2079-2088 (1962).

[131] Wickleder, C. Excited states of $\mathrm{Sm}^{2+}$ in chloride host lattices. Journal of Luminescence 94-95, 127-132 (2001).

[132] Wells, J.-P. R. Laser Spectroscopy of Alkaline Earth Fluoride Crystals Doped With Trivalent Samarium and Europium Ions. Ph.D. thesis (1996).

[133] Reid, M. F. et al. Spectroscopy of high energy states of lanthanide ions. European Journal of Inorganic Chemistry 2010, 2649-2654 (2010).

[134] Duan, C., Meijerink, A., Reeves, R. \& Reid, M. The unusual temperature dependence of the $\mathrm{Eu}^{2+}$ fluorescence lifetime in $\mathrm{CaF}_{2}$ crystals. Journal of Alloys and Compounds 408, 784-787 (2006).

[135] Rebane, K. Impurity Spectra of Solids (Plenum Press New York, 1970).

[136] Struck, F. W., C.W. Understanding Luminescence Spectra and Efficiency Using Wp and Related Functions (Springer-Verlag Berlin, 1991).

[137] Maghrabi, M. \& Townsend, P. Thermoluminescence spectra of rare earth doped Ca, Sr and Ba fluorides. Journal of Physics: Condensed Matter 13, 5817 (2001).

[138] Hayes, W. Crystals with the Fluorite Structure: Electronic, Vibrational, and Defect Properties (Clarendon Press, 1974).

[139] Chrysochoos, J., Jacobs, P., Stillman, M. \& Chadwick, A. Laser induced emission spectra of $\mathrm{Pr}^{3+}$ in $\mathrm{CaF}_{2}$ at low temperatures. Journal of Luminescence 28, 177-190 (1983). 
[140] Tissue, B. M. \& Wright, J. C. Site-selective laser spectroscopy of $\mathrm{CaF}_{2}: \operatorname{Pr}^{3+}$ and $\mathrm{CaF}_{2}: \operatorname{Pr}^{3+}{ }_{,} R^{3+}\left(R^{3+}=\mathrm{Y}^{3+}, \mathrm{Gd}^{3+}, \mathrm{Nd}^{3+}\right)$. Phys. Rev. B 36, 9781-9789 (1987).

[141] Szymaski, L., Michalski, L., Krukowska-Fulde, B. \& Niemyski, T. Crystalline spectra of $\mathrm{Pr}^{3+}$ in $\mathrm{CdF}_{2}$. Materials Research Bulletin 8, 109-116 (1973).

[142] Köstler, W., Winnacker, A., Rossner, W. \& Grabmaier, B. C. Effect of Pr-codoping on the $\mathrm{x}$-ray induced afterglow of $(\mathrm{Y}, \mathrm{Gd})_{2} \mathrm{O}_{3}$ :Eu. Journal of Physics and Chemistry of Solids 56, 907-913 (1995).

[143] Schei, A. \& Flood, H. The phase diagram of the system $\mathrm{SrCl}_{2}-\mathrm{BaCl}_{2}$. Acta Chemica Scandinavica 14, 2067-2070 (1960).

[144] Gahane, D. H., Kokode, N. S., Muthal, P. L., Dhopte, S. M. \& Moharil, S. V. Effect of cation mixing on luminescence of $\mathrm{Eu}^{2+}$ in $\mathrm{SrCl}_{2}$. Journal of Luminescence 130, 254-257 (2010).

[145] Berger, M. et al. XCOM:photon cross sections database (version 1.5) (2010). URL http://physics.nist.gov/xcom.

[146] Mares, J. A. et al. Scintillation response of Ce-doped or intrinsic scintillating crystals in the range up to 1 mev. Radiation Measurements 38:353-357, 353-357 (2004).

[147] Kobayasi, T., Mroczkowski, S., Owen, J. F. \& Brixner, L. H. Fluorescence lifetime and quantum efficiency for $5 \mathrm{~d} \rightarrow 4 \mathrm{f}$ transitions in $\mathrm{Eu}^{2+}$ doped chloride and fluoride crystals. Journal of Luminescence 21, 247-257 (1980).

[148] Yan, Z., Bizarri, G. \& Bourret-Courchesne, E. Crystal Growth and Color Centers of Alkaline-Earth Halides. Nuclear Instruments and Methods in Physics Research Section A: Accelerators, Spectrometers, Detectors and Associated Equipment 698, 7-10 (2013).

[149] Secu, M., Schweizer, S., Rogulis, U. \& Spaeth, J.-M. Radiation-induced defects and their recombination processes in the x-ray storage phosphor $\mathrm{BaBr}_{2}: \mathrm{Eu}^{2+}$. Journal of Physics: Condensed Matter 15, 2061 (2003).

[150] Fong, F. K. \& P. Niel Yocom, J. Crystal Growth and Color Centers of Alkaline-Earth Halides. Journal of Chemical Physics 41, 805-815 (1983). 
[151] Sastry, M. D., Dalci, A. G. I., Page, A. G. \& Joshi, B. D. Thermoluminescence and EPR of rare-earth-doped $\mathrm{SrCl}_{2}$. The Journal of Chemical Physics 8, 3232-3240 (1975).

[152] Pastor, R. \& Arita, K. Crystal growth of alkaline earth fluorides in a reactive atmosphere. Materials Research Bulletin 10, 493 - 499 (1975).

[153] Wilkinson, D. H. The phoswich - a multiple phosphor. Review of Scientific Instruments 23, 414-417 (1952).

[154] Ziemons, K. et al. The clearPET ${ }^{T M}$ project: development of a 2nd generation highperformance small animal PET scanner. Nuclear Instruments and Methods in Physics Research Section A: Accelerators, Spectrometers, Detectors and Associated Equipment 537, 307-311 (2005).

[155] Image By Kieran Maher at en.wikibooks, from Wikimedia Common (Accessed 13 June 2013). URL https://en.wikibooks.org/wiki/Basic_Physics_of_ Digital_Radiography/The_Applications.

[156] Dahlbom, M. et al. Performance of a YSO/LSO phoswich detector for use in a PET/SPECT system. Nuclear Science, IEEE Transactions on 44, 1114-1119 (1997).

[157] Saoudi, A. et al. Investigation of depth-of-interaction by pulse shape discrimination in multicrystal detectors read out by avalanche photodiodes. Nuclear Science, IEEE Transactions on 46, 462-467 (1999).

[158] Chase, L., Payne, S. A. \& Wilke, G. D. Optical properties and nonradiative decay of $\mathrm{Sm}^{2+}$ in $\mathrm{CaF}_{2}-\mathrm{YF}_{3}$ and $\mathrm{CaF}_{2}-\mathrm{LaF}_{3}$ mixed crystals. Journal of Physics C: Solid State Physics 20, 953 (1987).

[159] Guzzi, M. \& Baldini, G. Luminescence and energy levels of $\mathrm{sm}^{2+}$ in alkali halides. Journal of Luminescence 6, 270-284 (1973).

[160] O., J. Doubly-valent rare-earth ions in halide crystals. Journal of Physics and Chemistry of Solids 52, 101-174 (1991).

[161] Zhukov, V. P. \& Zainullina, V. M. First principles calculations of the electronic structure 
of fluorite-type crystals $\left(\mathrm{CaF}_{2}, \mathrm{BaF}_{2}, \mathrm{SrF}_{2}\right.$, and $\left.\mathrm{PbF}_{2}\right)$ containing frenkel defects. analysis of optical and transport properties. Physics of the Solid State 40 (1998).

[162] Kaiser, W., Garrett, C. \& Wood, D. Fluorescence and optical maser effects in $\mathrm{CaF}_{2}$ : $\mathrm{Sm}^{++}$. Physical Review 123, 766-776 (1961).

[163] Rabbiner, N. Fluorescence of $\mathrm{Sm}^{3+}$ in $\mathrm{CaF}_{2}$. Physical Review 130, 502-506.

[164] Tsuboi, T. The Parity-Forbidden $4 \mathrm{f}^{5} \rightarrow 4 \mathrm{f}^{5}$ Transition of the $\mathrm{Sm}^{3+}$ Ion in $\mathrm{CaF}_{2}$. physica status solidi (b) 104, 697-701 (1981).

[165] Royce, G. et al. Radiation effects in samarium-doped calcium fluoride. Journal of Luminescence 29, 205 - 213 (1984).

[166] Holl, I., Lorenz, E. \& Mageras, G. A measurement of the light yield of common inorganic scintillators. Nuclear Science, IEEE Transactions on 35, 105-109 (1988).

[167] Plettner, C. et al. $\mathrm{CaF}_{2}(\mathrm{Eu})$ : An Old scintillator revisited. In Nuclear Science Symposium Conference Record (NSS/MIC), 2010 IEEE, 236-242 (IEEE, 2010).

[168] Forrester, P. A., Green, G. W. \& Sampson, D. F. The effects of oxygen on the properties of $\mathrm{CaF}_{2}$ as a laser host. British Journal of Applied Physics 16, 1209-1210 (1965).

[169] Easyspin (2013). URL http://www.easyspin.org/.

[170] Abragam, A. \& Bleaney, B. Electron paramagnetic resonance of transition ions (Clarendon P., 1970).

[171] Reynolds, R. W., Boatner, L. A. \& Abraham, M. M. EPR of ${ }^{153} \mathrm{Eu}^{2+}$ in Single Crystals of $\mathrm{SrCl}_{2}$. The Journal of Chemical Physics 52, 3851-3852 (1970).

[172] Ewanizky, T. F., Caplan, P. J. \& Pastore, J. R. Polarization of $\mathrm{CaF}_{2}: \mathrm{Sm}^{3+}$ Fluorescence. Journal of Chemical Physic 43, 4351-4355 (1965).

[173] Mikhail, P. \& Hulliger, J. Sm${ }^{2+}$ in oxide lattices: An unexplored chapter of crystal chemistry. Comments on Inorganic Chemisty: A Journal of Critical Discussion of the Current Literature 21:4-6, 263-283 (1999).

[174] Arends, J. Color centers in additively colored $\mathrm{CaF}_{2}$ and $\mathrm{BaF}_{2}$. physica status solidi (b) 7, 805-815 (1964). 
[175] Beaumont, J. H., Hayes, W., Kirk, D. L. \& Summers, G. P. An investigation of trapped holes and trapped excitons in alkaline earth fluorides. Proceedings of the Royal Society of London. A. Mathematical and Physical Sciences 315, 69-97 (1970).

[176] Williams, R. T., Kabler, M. N., Hayes, W. \& Stott, J. P. Time-resolved spectroscopy of self-trapped excitons in fluorite crystals. Phys. Rev. B 14, 725-740 (1976).

[177] Barland, M. \& Duval, E. Lattice relaxation, $V_{k}$ mobility and electron-hole recombination in $\mathrm{SrCl}_{2}$ doped with $\mathrm{K}^{+}$ions. Journal of Physics C: Solid State Physics 15, 6187 (1982).

[178] Brixner, L. H. New x-ray phosphors. Materials Chemistry and Physics 16, 253-281 (1987).

[179] Khare, R. \& Ranade, J. Sensitization of $\mathrm{Ce}^{3+}$ luminescence by $\mathrm{Gd}^{3+}$ in CaO. Journal of Materials Science 15, 1868-1869 (1980).

[180] Neutron capture cross sections (Accessed 13 June 2013). URL http://www. reconpetro.com/cmsAdmin/uploads/E_Appendix.pdf.

[181] Shen, Y. \& Bray, K. L. Effect of pressure and temperature on $4 \mathrm{f}-4 \mathrm{f}$ luminescence properties of $\mathrm{Sm}^{2+}$ ions in MFCL crystals (M=Ba, Sr and Ca). Physical Review B 58 (1998). 
Appendices 



\section{Appendix A}

\section{Neutron sensitivity}

\section{A.1 Neutron scintillators}

As with x-rays and $\gamma$-rays, neutrons are also useful in applications such as non destructive testing, e.g. for imaging organics and detection of radioactive elements. While the outcome is similar (a pulse of light out for incident radiation) the particle-solid interaction have differences. Neutrons are uncharged particles like photons, and so they do not interact with the electromagnetic field. In general free charge carriers can either be created by direct collisions with nuclei which sufficiently displace the nucleus or by nuclear reactions occurring within the material. This thesis solely focuses on thermal neutrons which have an energy of $0.025 \mathrm{eV}$; this is insufficient to displace the nucleus enough to create free charge carriers so the interaction must be by nuclear reactions. For neutron scintillators one wants to work with materials which have a high probability of reacting with the neutron, as characterised by the neutron capture cross section. A commonly used reaction is the radiative capture reaction $(\mathrm{n}, \gamma)$ such as that of gadolinium:

$$
\begin{aligned}
& { }^{155} G d+{ }_{0}^{1} n \rightarrow{ }^{156} G d+\gamma \\
& { }^{157} G d+{ }_{0}^{1} n \rightarrow{ }^{158} G d+\gamma
\end{aligned}
$$




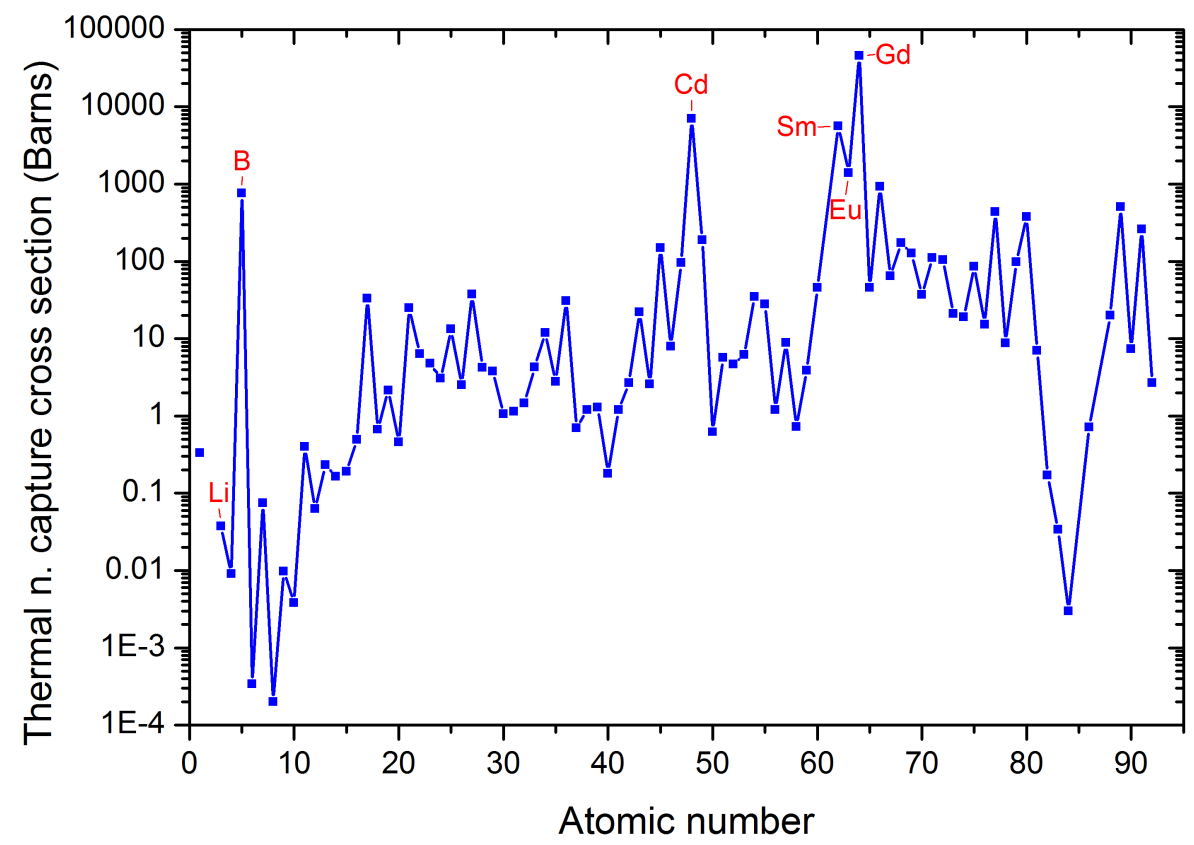

Figure A.1: Neutron capture cross sections for thermal neutrons plotted against atomic number [180].

These types of reactions generate $\gamma$-rays as the secondary product, which are often difficult to detect as they are of very high energy. For example in the above reactions they can have an energy of up to $6.5 \mathrm{MeV}$. Other options are neutron capture leading to proton or $\alpha$-particle emission $((n, p)$ and $(n, \alpha)$ reactions), and these are frequently used as they give charged particles which then interact strongly via the electromagnetic field in the material giving a large number of free charge carriers which can then excite the luminescent centre. ${ }^{6} \mathrm{Li}$ is a material which has such a reaction:

$$
{ }_{3}^{6} L i+{ }_{0}^{1} n \rightarrow{ }_{1}^{3} H+\alpha
$$

The resulting tritium and $\alpha$-particle have $4.8 \mathrm{MeV}$ of kinetic energy to create ionization tracks through the scintillator material. The cross sections for neutron absorption is very different to that for x-rays or $\gamma$ - rays. Neutrons are more likely to be absorbed or scattered by the lighter larger elements such as lithium and hydrogen; figure A.1. shows the capture cross section variation as the atomic number increases. This means neutron imaging can be used a complimentary technique to, or instead of, x-ray or $\gamma$-ray imaging, as it is sensitive to very different elements. The attenuation of an incident beam $I_{0}$ incident on a material of thickness $z$ is given by where $N$ is the concentration of target atoms and $\sigma$ the capture or scattering 
reaction cross section.

Much like $\gamma$-ray scintillation, neutron scintillators have key performance indicators such as a high neutron sensitivity, a low $\gamma$-ray sensitivity, a high light yield, a fast lifetime, transparency and ease of production. Often neutron-scintillators can be created from $\gamma$-ray scintillators with added elements of high neutron capture cross sections; these are called neutron sensitizers (commonly lithium, boron or gadolinium is used). Here we investigate the neutron responses of LSCBC:Sm and $\mathrm{Ba}_{0.3} \mathrm{Sr}_{0.7} \mathrm{Cl}_{2}: \mathrm{RE}$ ( $\mathrm{RE}=\mathrm{Sm}$, Eu or Ce), both with and without additional neutron sensitiser dopants. Samarium and europium both have high neutron capture cross sections as seen in figure A.1. so we expect the samarium and europium doped materials to be neutron sensitive without additional co-doping. For the samarium doped materials we expect broad red luminescence as for the photoluminescence and radioluminescence. As for $\gamma$-ray detection, a red emission is desirable to match the spectral response of silicon photodiodes.

\section{A.2 Neutron sensitised lanthanum stabilised cubic barium chloride}

\section{A.2.1 Structure}

Samples of LSCBC:Sm were prepared, and some were additionally doped with $\mathrm{LiCl}$ or $\mathrm{GdCl}_{3}$ in order to increase neutron sensitivity by adding anhydrous $\mathrm{LiCl}$ or $\mathrm{GdCl}_{3}$ to the starting materials. Figure A.2 shows the XRD patterns for various samples attempted. The patterns are indicative of the cubic fluorite structure for LSCBC:Sm and for LSCBC:Sm co-doped with $3 \% \mathrm{Gd}^{3+}$. The higher concentration $(5 \%)$ of $\mathrm{Gd}^{3+}$ ions and both concentrations of $\mathrm{Li}^{+}$ions (3 $\%$ and $5 \%$ ) resulted in a mixed phase materials as indicated by by the multitude of lines in the XRD patterns. There are large differences between the $\mathrm{Gd}^{3+}$ ion and and $\mathrm{Li}^{+}$ion; first the charge, $\mathrm{Gd}^{3+}$ is trivalent and $\mathrm{Li}^{+}$is monovalent. Secondly the $\mathrm{Gd}^{3+}$ ion has a crystal ionic radius of $1.078 \AA$ compared to $0.900 \AA$ of the $\mathrm{Li}^{+}$ion [120, 121]. This is a better match to the $\mathrm{Ba}^{2+}$ and $\mathrm{La}^{3+}$ ions with radii of $1.032 \AA$ and $1.35 \AA$ respectively [120,121]. So it is not 


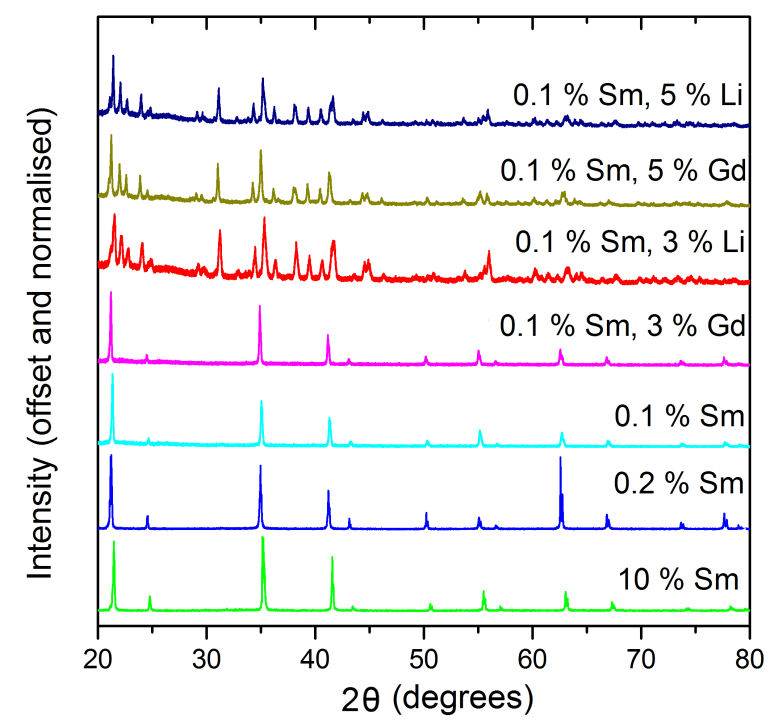

Figure A.2: Powder XRD patterns of LSCBC neutron sensitized samples. The lower four patterns are cubic while the upper three are orthorhombic.

surprising to find small amounts of $\mathrm{Gd}^{3+}$ ions can be accommodated in the LSCBC lattice while the same fraction of $\mathrm{Li}^{+}$ions causes a phase separation.

\section{A.2.2 X-ray response}

Figure A.3 shows the radioluminescence of the samples prepared for neutron sensitivity tests. The emissions from the cubic materials match those seen earlier in this chapter. For the non-cubic materials, the radioluminescence spectra are all similar and are dominated by sharp line emissions. This is the emission seen for $\mathrm{Sm}^{2+}$ ion in orthorhombic $\mathrm{BaCl}_{2}$ [127] and is consistent with the XRD patterns in figure A.2. There is a sharp line emission observable in the gadolinium doped samples at $311 \mathrm{~nm}$. This is a characteristic emission of $\mathrm{Gd}^{3+}\left({ }^{6} \mathrm{P}_{7 / 2} \rightarrow\right.$ ${ }^{8} S_{7 / 2}$ ) [68]; it appears weaker in the sample of higher gadolinium concentration only because the spectra have each been normalised and the sharp line orthorhombic $\mathrm{BaCl}_{2}: \mathrm{Sm}^{2+}$ emission has a much higher peak intensity than the broad band emission in the cubic material.

\section{A.2.3 Neutron response}

The light output under neutron irradiation was measured as described in chapter 5 wherever the intensity was sufficient to make a meaningful measurement. The relative sensitivities compared to ${ }^{6} \mathrm{LiI}\left(\mathrm{Eu}^{2+}\right)$ (a commercial material) are tabulated in table A.1. The ${ }^{6} \mathrm{LiI}\left(\mathrm{Eu}^{2+}\right)$ 


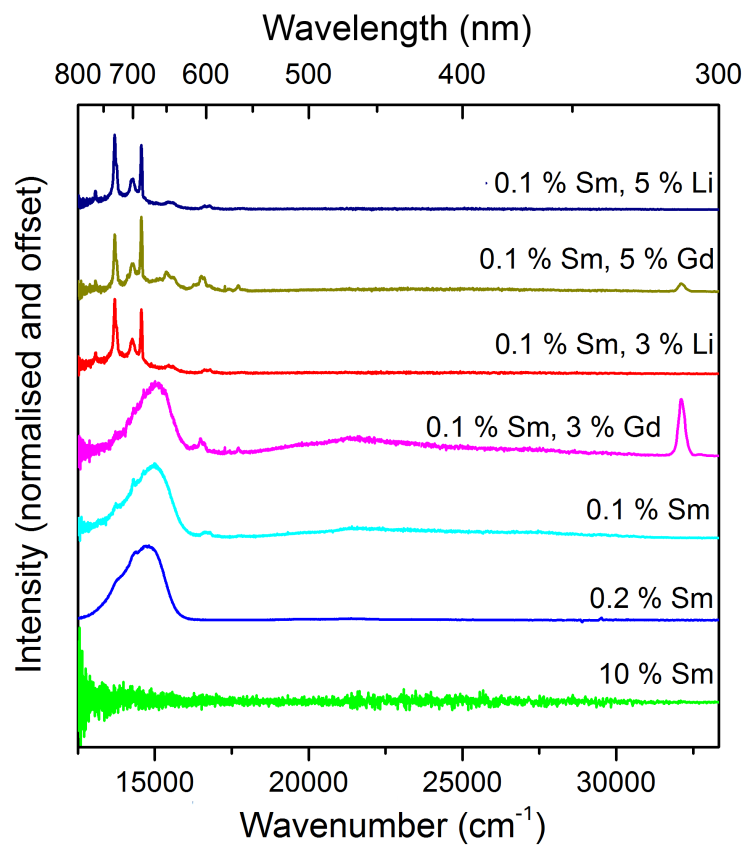

Figure A.3: Normalised radioluminescence of neutron sensitized samples, offset for clarity. The spectra were recorded under $\mathrm{x}$-irradiation by a copper tube operated at $35 \mathrm{kV}$, and with a $2 \mathrm{~mm}$ aluminium filter.

Table A.1: Summary of luminescent ion, additional neutron sensitiser, crystal structure, neutron response relative to that of ${ }^{6} \mathrm{LiI}\left(\mathrm{Eu}^{2+}\right)$ and fitted afterglow exponential decay time constant. The afterglow time is based on the fitted exponential decay to the light output after the shutter was closed. The neutron sensitiser percentages correspond to natural abundances not enriched materials.

\begin{tabular}{cccccc}
\hline Sm \% & n sensitiser & $\%$ & Structure & n sensitivity & Afterglow \\
\hline 10 & \multicolumn{5}{c}{ cubic } \\
0.2 & & & cubic & $0.6 \%$ & $2.5 \mathrm{~ms}$ \\
0.1 & & & cubic & $1.5 \%$ & $6.5 \mathrm{~ms}$ \\
0.1 & $\mathrm{Gd}$ & $3 \%$ & cubic & $2.1 \%$ & $6.5 \mathrm{~ms}$ \\
0.1 & $\mathrm{Li}$ & $3 \%$ & mixed & $0.3 \%$ & \\
0.1 & $\mathrm{Gd}$ & $5 \%$ & mixed & $0.6 \%$ & \\
0.1 & $\mathrm{Li}$ & $5 \%$ & mixed & $0.3 \%$ & \\
\hline
\end{tabular}

scintillator has been doped with enriched lithium (with a higher fraction of ${ }^{6} \mathrm{Li}$ ) which makes it highly sensitive, our materials have only the natural abundances of the neutron sensitive isotopes. The afterglow was recorded and fitted as described by Bartle et al. [98]. Table A.1. also notes the structure and the afterglow.

Doping with gadolinium increased the sensitivity slightly, however not as much as one would expect for the $3 \%$ gadolinium added. This is likely due to the $\gamma$-rays emitted in the nuclear capture reaction being of too high an energy and passing right through the material without being absorbed. For samarium doping, as with radioluminescence, the $0.1 \%$ sample 
showed a higher light output than the $0.2 \%$ sample with outputs of $1.5 \%$ and $0.6 \%$ that of commercial material ${ }^{6} \mathrm{LiI}\left(\mathrm{Eu}^{2+}\right)$. The increase in samarium concentration would increase the neutron capture rate substantially, but this must be offset by a significant self absorption as the light output decreases. This is in line with the concentration quenching observed for the $\mathrm{x}$-ray luminescence. Lithium co-doping resulted in a non-transparent material with different emission so the results are not comparable. Figure A.4 shows an example of the change in the output of the PMT coupled to a $\mathrm{Sm}^{2+}$ and $\mathrm{Gd}^{3+}$ co-doped sample with the shutter open and shut corresponding to neutron irradiation and the background signal. One can see that there is a significant change in signal despite the light output being only $2.1 \%$ that of ${ }^{6} \mathrm{LiI}\left(\mathrm{Eu}^{2+}\right)$, this may show improvement with the use of enriched gadolinium.

\section{A.3 Neutron sensitised $\mathrm{Ba}_{0.3} \mathrm{Sr}_{0.7} \mathrm{Cl}_{2}: \mathrm{RE}(\mathrm{RE}=\mathrm{Sm}, \mathrm{Eu}$ or $\mathrm{Ce})$}

\section{A.3.1 Structure}

Samples of $\mathrm{Ba}_{0.3} \mathrm{Sr}_{0.7} \mathrm{Cl}_{2}$ doped with samarium, europium or cerium as a luminescent ion and some additionally doped with lithium or gadolinium in order to increase neutron sensitivity were produced. Lithium or gadolinium doping was achieved by adding $3-5$ mole \% of $\mathrm{LiCl}$ or $\mathrm{GdCl}_{3}$ to the materials before melting. Figure A.5 shows the XRD patterns for

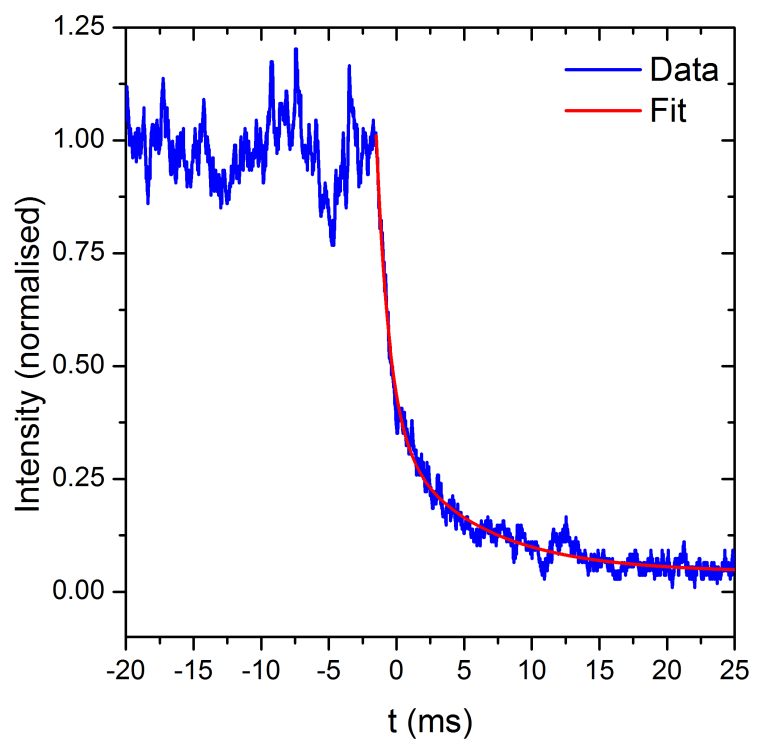

Figure A.4: Example of the response to the neutron beam and the fitting of an afterglow profile. This is for a $\mathrm{Sm}^{2+}(0.1 \%)$ and $\mathrm{Gd}^{3+}(3 \%)$ co-doped LSCBC sample. 


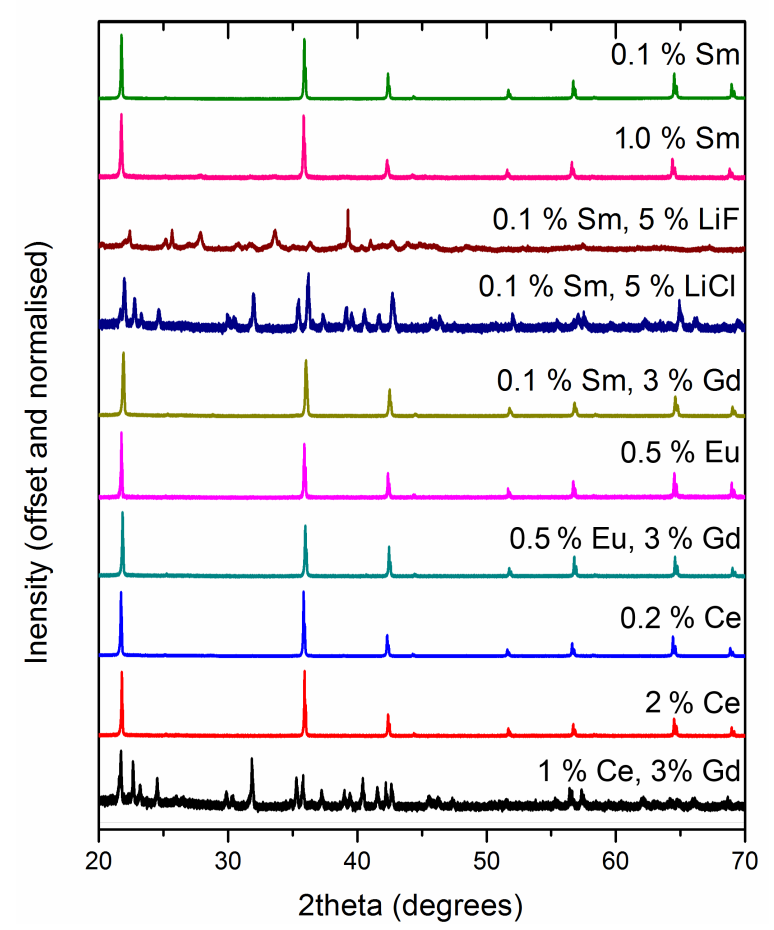

Figure A.5: Powder x-ray diffraction patterns of various neutron sensitised $\mathrm{Ba}_{0.3} \mathrm{Sr}_{0.7} \mathrm{Cl}_{2}: \mathrm{RE}$ $(\mathrm{RE}=\mathrm{Sm}, \mathrm{Eu}$ or $\mathrm{Ce})$ samples.

various samples produced. As for LSCBC:Sm it can be seen that additional doping with lithium results in a non-cubic material. Samples doped with europium or samarium and $3 \%$ gadolinium formed in the cubic phase but the cerium doped sample with an additional $3 \%$ gadolinium shows evidence of the orthorhombic phase. As cerium is trivalent compared to divalent europium and samarium ions it may be that the lattice can accommodate up to $3 \%$ trivalent ions but not $4 \%$.

\section{A.3.2 X-ray sensitivity}

Figure A.6 shows the radioluminescence of the samples prepared for neutron sensitivity tests. The emissions match those seen earlier in this chapter except for the lithium doped materials in which we see sharp line emission at room temperature. This was also seen in LSCBC:Sm and by comparison with reference [181] it corresponds to the emission of $\mathrm{Sm}^{2+}$ ions in the orthorhombic phase of $\mathrm{BaCl}_{2}$. As in the case for LSCBC:Sm a sharp line emission is present in the gadolinium doped samples at $311 \mathrm{~nm}$, this a characteristic ${ }^{6} \mathrm{P}_{7 / 2} \rightarrow{ }^{8} \mathrm{~S}_{7 / 2}$ emission of the $\mathrm{Gd}^{3+}$ ion [68]. It appears much stronger in the samarium sample than europium as it is dwarfed by the strong europium emission in the normalised spectra. 


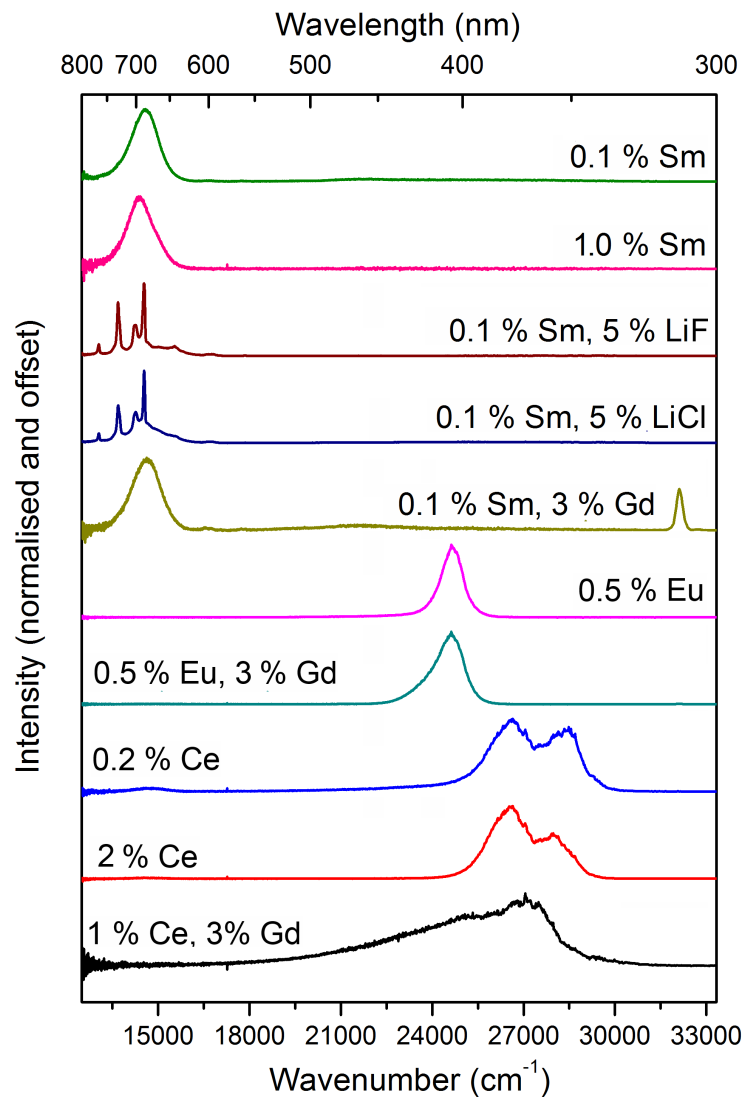

Figure A.6: Normalised radioluminescence of various neutron sensitised samples, offset for clarity.

\section{A.3.3 Neutron sensitivity}

The light output under neutron irradiation was measured as described in chapter 5. The relative sensitivities compared to ${ }^{6} \mathrm{LiI}\left(\mathrm{Eu}^{2+}\right)$ are tabulated in table A.2. This table also notes the structure and afterglow.

For samarium doping, as with radioluminescence the $0.1 \%$ sample had higher light output than the $1.0 \%$ samarium (without additional neutron activator, note samarium is neutron sensitive itself) with outputs of $1.2 \%$ and $0.1 \%$ that of commercial material ${ }^{6} \mathrm{LiI}\left(\mathrm{Eu}^{2+}\right)$. Lithium co-doping resulting in a non-transparent material with different emission so the results are not comparable. Gadolinium co-doping (3\%) did not increase the light output. Either the $\gamma$ rays were too higher energy and passed right through or it was insignificant increase on samarium sensitivity. Europium doped $\mathrm{Ba}_{0.3} \mathrm{Sr}_{0.7} \mathrm{Cl}_{2}$ gave the best result overall, with just $0.5 \%$ europium this gave $6 \%$ the light output of ${ }^{6} \mathrm{LiI}\left(\mathrm{Eu}^{2+}\right)$. The addition of $3 \%$ $\mathrm{Gd}^{3+}$ resulted in slightly lower light output $\left(4.9 \%{ }^{6} \mathrm{LiI}\left(\mathrm{Eu}^{2+}\right)\right)$ but this is most likely due to a slightly lowered optical quality. 
Table A.2: Summary of luminescent ion, additional neutron sensitiser, crystal structure, neutron response relative to that of ${ }^{6} \mathrm{LiI}\left(\mathrm{Eu}^{2+}\right)$ and fitted afterglow exponential decay time constant. The neutron sensitiser percentages correspond to natural abundances not enriched materials.

\begin{tabular}{|c|c|c|c|c|c|}
\hline Lum. ion (\%) & $\mathrm{n}$ sensitiser & $\%$ & Structure & n sensitivity & Afterglow \\
\hline $\operatorname{Sm}(0.1 \%)$ & & & cubic & $3.6 \%$ & $2 \mathrm{~ms}$ \\
\hline Sm (1.0 \%) & & & cubic & $0.3 \%$ & \\
\hline $\operatorname{Sm}(0.1 \%)$ & $\mathrm{LiF}$ & $6 \%$ & other & $3 \%$ & $3 \mathrm{~ms}$ \\
\hline $\operatorname{Sm}(0.1 \%)$ & $\mathrm{LiCl}$ & $5 \%$ & cubic and ortho. & $5.4 \%$ & $4 \mathrm{~ms}$ \\
\hline $\operatorname{Sm}(0.1 \%)$ & $\mathrm{GdCl}_{3}$ & $3 \%$ & cubic & $3.3 \%$ & $1.5 \mathrm{~ms}$ \\
\hline $\mathrm{Eu}(0.5 \%)$ & & & cubic & $6 \%$ & $<1 \mathrm{~ms}$ \\
\hline $\mathrm{Eu}(0.5 \%)$ & $\mathrm{GdCl}_{3}$ & $3 \%$ & cubic & $4.9 \%$ & 1.7 \\
\hline $\mathrm{Ce}(0.2 \%)$ & & & cubic & $0.1 \%$ & \\
\hline $\mathrm{Ce}(2.0 \%)$ & & & cubic & $0.6 \%$ & \\
\hline Ce $(1.0 \%)$ & $\mathrm{GdCl}_{3}$ & $3 \%$ & cubic and ortho. & $1.3 \%$ & \\
\hline
\end{tabular}

Figure A.7 shows an example of the change in the output of the PMT coupled to a samarium and gadolinium co-doped sample with the shutter open and shut corresponding to neutron irradiation and background. It also shows the afterglow profile after the shutter is closed. One can see that while the light output was orders of magnitude less than that of $\mathrm{LiI}(\mathrm{Eu})$ there is a significant change in signal. The afterglow is relatively low, being well fitted by a decay constant of $1.5 \mathrm{~ms}$. One can see that there is a significant change in signal despite the light output being only $2.1 \%$ that of ${ }^{6} \mathrm{LiI}\left(\mathrm{Eu}^{2+}\right)$, this may show improvement with the use of enriched gadolinium.

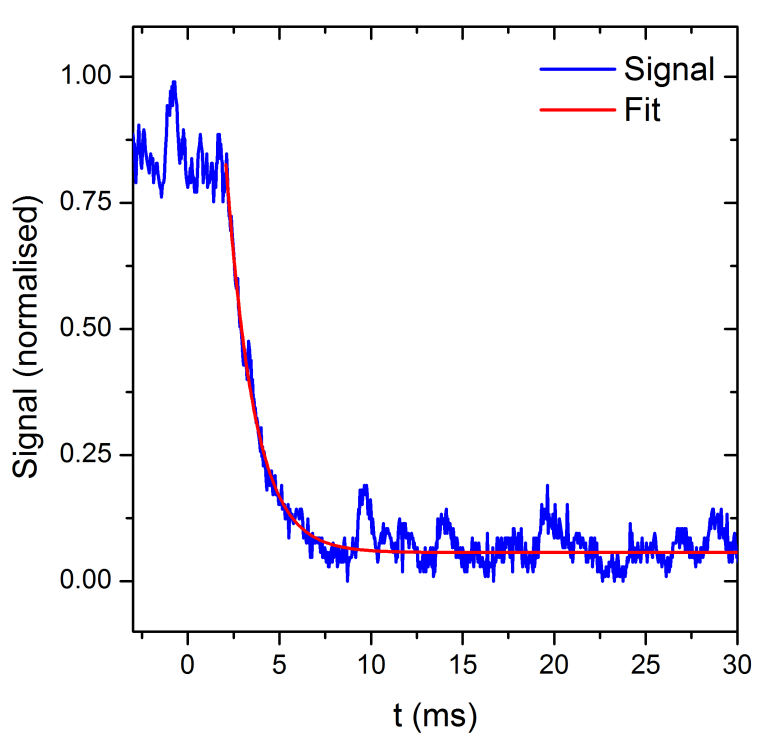

Figure A.7: Example of response to neutron beam of a $\mathrm{Sm}^{2+}$ and $\mathrm{Gd}^{3+}$ co-doped sample of $\mathrm{Ba}_{0.3} \mathrm{Sr}_{0.7} \mathrm{Cl}_{2}$. The afterglow profile after the shutter has closed has been fitted with an exponential decay with a decay constant of $1.5 \mathrm{~ms}$. 



\section{Appendix B}

\section{List of publications}

1. L. C. Dixie, A. Edgar, M. Bartle, Energy resolved imaging with a stratified phosphor detector, Radiation Measurements 56 (2013)

2. L. C. Dixie, A. Edgar, M.F. Reid, $\mathrm{Sm}^{2+}$ fluorescence and absorption in cubic $\mathrm{BaCl}_{2}$ : strong thermal crossover of fluorescence between $4 f^{6}$ and $4 f^{5} 5 d^{1}$ configurations, Journal of Luminescence 132 (2012)

3. C. M. Bartle, A. Edgar, L. Dixie, C. Varoy, R. Piltz, S. Buchanan, K. Rutherford, Novel methods for measuring afterglow in developmental scintillators for $x$-ray and neutron detection, Nuclear Instruments and Methods in Physics Research (2011)

4. L. Dixie, A. Edgar, and M. Bartle, Luminescence and X-ray phosphor properties of samarium and lanthanum-doped cubic barium chloride, Physica Status Solidi (c) C8 (2011) 